DEPARTMENT OF THE INTERIOR

UNITED STATES GEOLOGICAL SURVEY

GEORGE OTIS SMITH, DIRECTOR

Buthetin 507

\title{
THE MINING DISTRICTS OF THE WESTERN UNITED STATES \\ BY
}

JAMES M. HILL

WITH A

\section{GEOLOGIC INTRODUCTION}

BY

\author{
WALDEMAR LINDGREN
}

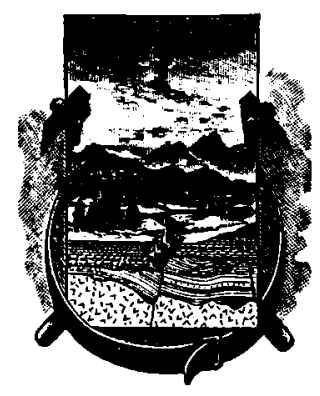

WASHINGTON

GOVERNMENT PRINTING OFFIOE

1912 
68743 


\section{CONTENTS.}

PART I. Geologic introduction, by Waldemar Lindgren...............

Origin and scope of this work..................................

Distribution of mining districts................................ 6

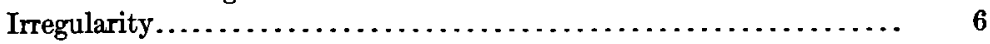

Distribution in relation to geologic structure................ $\quad 7$

Form and content.......................................... 9

Classification of deposits..................................... 9

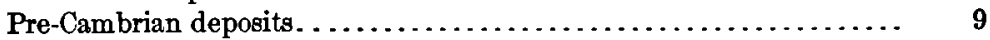

Post-Cambrian deposits................................... 10

Deposits in sedimentary rocks. . . . . . . . . . . 10

Deposits connected with igneous rocks................... 11

Local geology of western ore deposits........................ 14

Arizona. . . . . . . . . . . . .

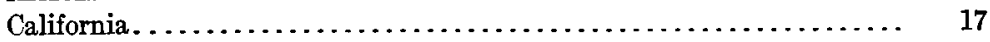

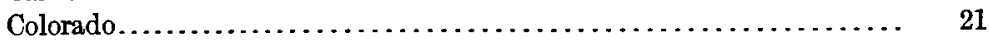

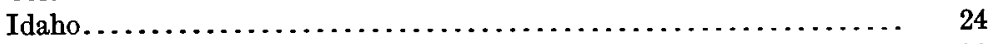

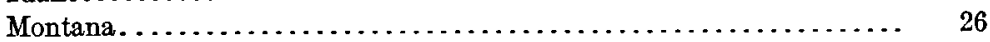

Nevada............................................. 29

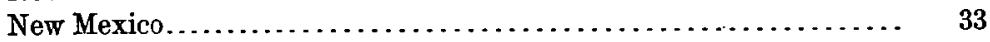

Oregon................................................. 36

South Dakota......................................... 37

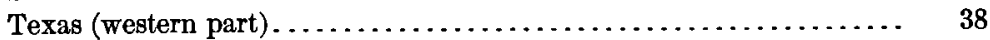

Utah................................................. 39

Washington............................................. 41

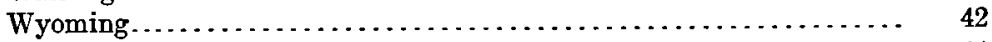

Part II. Catalogue of mining districts, by J. M. Hill................. 44

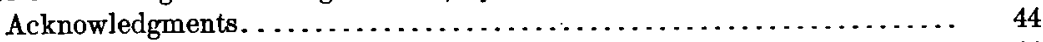

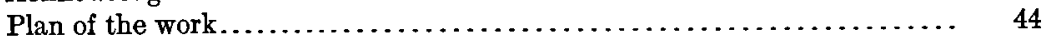

Classification of mining districts.............................. 52

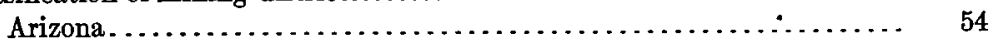

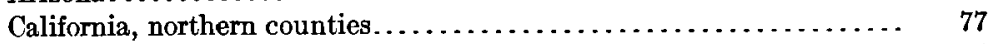

California, southern counties............................... 114

Colorado............................................ 134

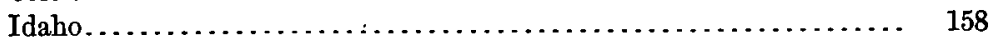

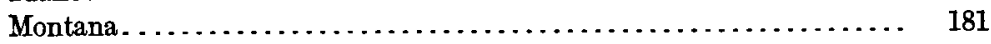

Nevada ............................................... 199

New Mexico.......................................... 229

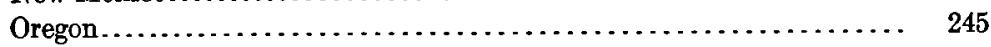

South Dakota....................................... 257

Texas............................................... 262

Utah............................................... 265

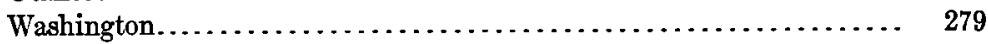

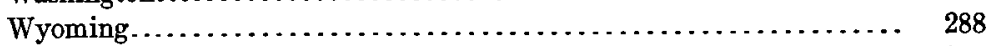

Alphabetic index of mining districts. . . . . . . . . . 


\section{ILLUSTRATIONS.}

PLATE I Map of Western States showing areas covered by topographic surveys. Page.

II. Map of Western States showing areas covered by geologic surveys... 50

III. Map of Arizona, showing location of mining districts........... 54

IV. Map of northern counties of California, showing location of mining

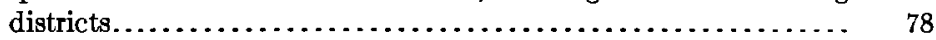

V. Map of southern counties of California, showing location of mining districts........................................ 114

VI. Map of Colorado, showing location of mining districts.......... 134

VII. Map of Idaho, showing location of mining districts............ 158

VIII. Map of Montana, showing location of mining districts........... 182

IX. Map of Nevada, showing location of mining districts. ......... 200

X. Map of New Mexico, showing location of mining districts......... 230

XI. Map of Oregon, showing location of mining districts ............. 246

XII. Map of South Dakota, showing location of mining districts........ 258

XIII. Map of Texas, showing location of mining districts............. 262

XIV. Map of Utah, showing location of mining districts.............. 266

XV. Map of Washington, showing location of mining districts......... 280

XVI. Map of Wyoming, showing location of mining districts........... 288

Frgure 1. Map of the Western States showing the general distribution of the mining districts.................................. 7 


\title{
THE MINING DISTRICTS OF THE WESTERN UNITED STATES.
}

\section{PART I. GEOLOGIC INTRODUCTION.}

\author{
By Waldemar Lindgren.
}

\section{ORIGIN AND SCOPE OF THIS WORK.}

The mining districts of the Western or Cordilleran States are numerous and scattered over wide areas. The first attempt to locate all of them on a single map was carried out by the writer in 1907, with the aid of Mr. J. M. Hill and the statisticians in charge of the offices of the Survey at Denver, Salt Lake City, and San Francisco (Messrs. Chester Naramore, V. C. Heikes, and C. G. Yale). This map of the Western States was included in the annual volume of Mineral Resources of the United States. The large area covered and the necessity of adding explanatory tables made this map cumbersome to handle. When a revision was decided upon in 1910 and assigned to Mr. J. M. Hill, it was found advisable to map the several States separately and publish the maps, with more extensive explanatory text, in the convenient form of a bulletin.

At the outset not a little difficulty was experienced in the definition of the mining districts and in deciding their proper names and extent. Mineral deposits rarely appear singly; on the contrary, a number of them usually cluster in certain localities. In general in the Western States, when such a mineralized area is discovered and locations have been made, a miners' meeting is called, regulations are adopted beyond the simple statutory laws of the Federal and State governments, the limits of the district are defined, and a name is given to it. For many districts these limits are uncertain and vague and can not always be ascertained, though the data are supposed to be on file with the county authorities. The name does not always correspond with that of the locality; for instance, the mining district 
surrounding Butte, Mont., is called Summit Valley. At many places little attention has been given to these legal districts and the original area has by common consent been subdivided into several more or less definite subdistricts. The real reason for the adoption of mining districts is to have an easily accessible record of claims, for, as is well known, the Federal authorities keep no list of claims located, and this duty is relegated to the recorder of each district.

In California, except in the desert region, few well-defined mining districts exist, and each center of mining operations has been given a geographic name appropriate to the locality.

It is impossible, therefore, to apply the term "mining district" consistently, but in the present work wherever practicable the local designations and rules have been followed. A large mineralized area is designated as a mining region; for instance, the "San Juan region," embracing the mountainous area of southwestern Colorado. The mining region consists of a number of mining districts. These vary greatly in size, but such a representative unit would perhaps include from 12 to 36 square miles. Many districts are informally subdivided into smaller units, referred to as "camps," but as a rule the camps can not be shown owing to the small scale of the map.

\section{DISTRIBUTION OF MINING DISTRICTS.}

IRREGULARITY.

In order to show comprehensively the distribution of the districts they have been represented on a smaller-scale map of the Western States (fig. 1). The first impression is that of irregular distribution, a clustering at some places, a scattering at others, with certain areas almost devoid of mineral deposits. Except along the Pacific coast in California there is little evidence of any arrangement in regular zones or "belts."

The most easterly indications of the metallic wealth of the Cordillera are found in western Texas and Oklahoma, where copper ores are thinly scattered through certain "Permo-Carboniferous" sandstones. Slightly farther west rise small groups of mountains, sentinels in front of the Rocky Mountains, such as the Black Hills of South Dakota, the Little Rockies of Montana, or some of the short ranges of western Texas; these contain some ore deposits.

The most westerly districts lie in the Coast Range of California, which contains quicksilver ores, and along the beaches of Oregon and Washington, where the sands yield gold and platinum.

There are several notable "blanks" on the map where mining districts are almost absent. One of these occupies areas in Oregon and Washington. Another barren area embraces parts of Utah, Arizona, New Mexico, and Colorado. 


\section{DISTRIBUTION IN RELATION TO GEOLOGIC STRUCTURE.}

As the deposits of metallic ores are the results of geologic processes, there is necessarily a close connection between the geologic structure and the mineralization of an area. On a map of the scale of figure 1

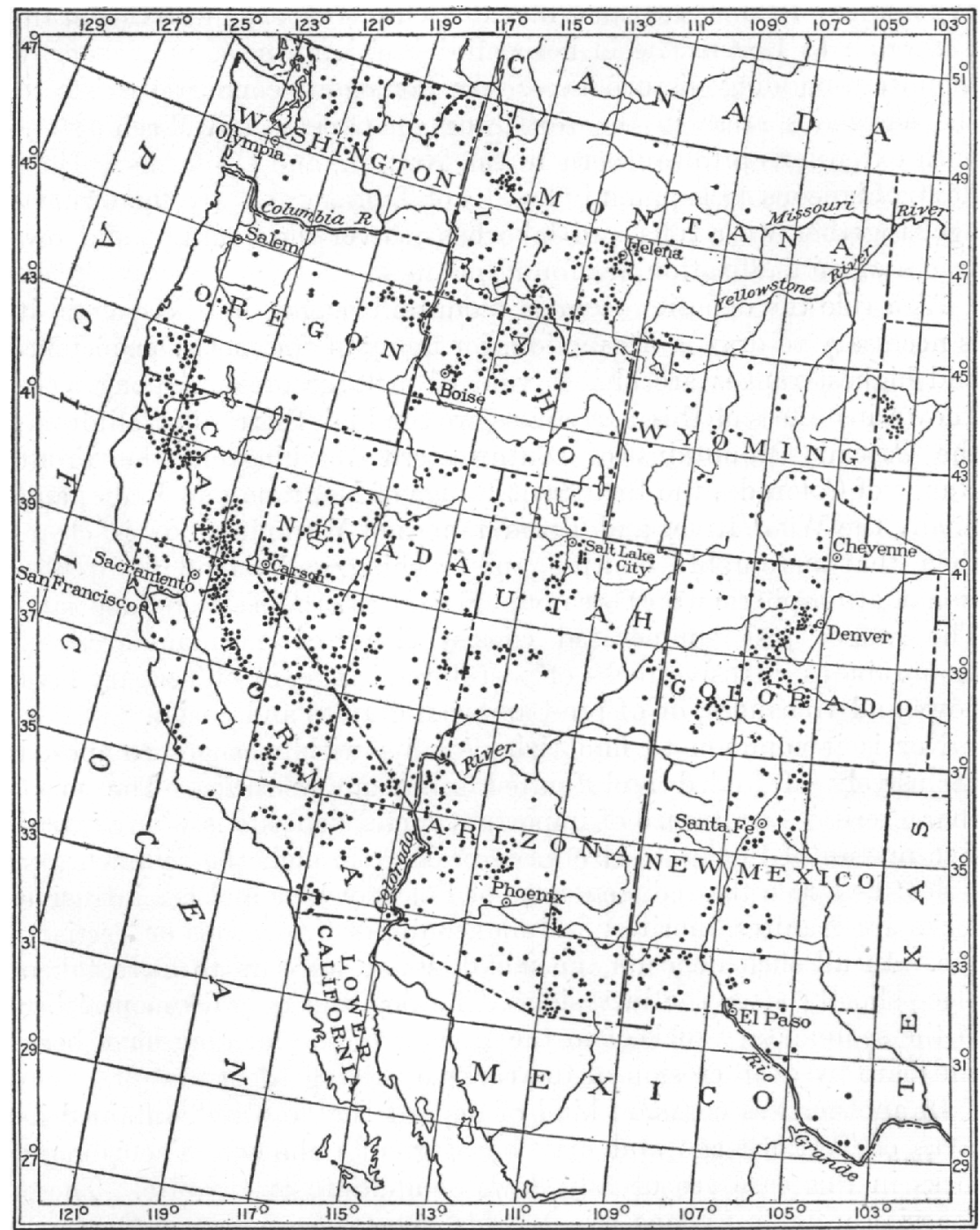

$\begin{array}{lllll}100 & 0 & 100 & 200 & 300 \text { Miles }\end{array}$

Figure 1.-Map of the Western States, showing the general distribution of the mining districts.

it is scarcely possible to bring out these relations clearly, especially where the structure is complicated. The first broad principle which holds for the Cordilleran region is that where the rocks lie horizontal and no disturbance has taken place ore deposits are rare, poor, or 
absent. The last is of course true for alluvial areas like that of the Sacramento Valley in California. Few mining districts are found in the vast plateau province of southern and eastern Utah, western Colorado, northern Arizona, and western New Mexico, already referred to; this barren area also extends into western and central Wyoming. In this great province the sediments, ranging in age from Cambrian to Eocene, lie in horizontal position almost unbroken by mountain-building disturbances. An analogous condition exists in the enormous areas in the Northwest, in Oregon and Washington, with extensions into southern Idaho, Nevada, and California. Here flows of igneous rocks, mainly basalts of Tertiary to early Quaternary age, together with tuffs and lake beds, cover the country, and few traces of mineralization are found in them.

As a rule the deposits occur in mountain ranges, but here again it is necessary to draw a distinction, for many of the most conspicuous and highest ranges are almost void of metallic ores. Among wellknown instances of this barrenness are the high Sierras of California, the Cascade Mountains of southwestern Washington, the Front Range of Colorado, the Bitterroot Range of Montana and Idaho, and finally the Wind River and Teton ranges of Wyoming. It is clear, then, that mere uplift, faulting, and crushing, and attendant circulation of atmospheric water are not sufficient to produce metal deposits. The ranges just enumerated consist either of enormous areas of mountainous intrusive rocks of post-Jurassic age, or of Tertiary lava flows and volcanoes, or of pre-Cambrian granite and gneiss.

Nor is it common to find rich deposits within ranges composed exclusively of folded and faulted sedimentary rocks. The most characteristic occurrence of important metal deposits is where intrusive masses of moderate size have broken through the sedimentary series; as a rule the sediments are of Paleozoic age and the intrusive rocks are granites, diorites, and monzonite of Cretaceous or Tertiary age. In all such deposits the metallization appears to have taken place shortly after the intrusion. The deposits may develop either in the sedimentary rock or in the igneous rock, and they have been laid bare by deep erosion of the rocks overlying them.

In another less common kind of deposit the ores are contained in lavas of Tertiary age and may be of great richness. The igneous rocks in this case are usually flows of andesite or rhyolite. These deposits occur in regions like Nevada, where there have been eruptions from many local vents and where the rocks have been extensively decomposed by solution and gases of volcanic origin. Many of these deposits have suffered little erosion since their formation, which followed closely the end of the epoch of eruptions.

There are many other kinds of deposits, but those mentioned are the sources of the largest part of the metallic production of the West. 


\section{FORM AND CONTENT.}

The form of the deposits is manifold and depends on the spaces provided for their reception or on the manner in which they made place for themselves. Practically all of them were formed by deposition from water solutions, usually ascending hot water, and as the path of such waters commonly follows fissures the resulting deposits are in large part fissure veins. The walls of the fissures were soaked by the solutions and ores were deposited in them. Some rocks like limestone are peculiarly susceptible to chemical alteration, and where traversing such rocks the mineralizing solutions spread far and wide, causing the deposition of metallic ores. Such occurrences are called replacement deposits.

The ore minerals were in the first place sulphides of the baser metals, such as galena, pyrite, or zinc blende, or more rarely oxides such as magnetite. Gold alone has originally been deposited as native metal; more rarely it is combined with tellurium. Through the oxygen of surface waters the sulphides have usually been oxidized to various other compounds such as cerusite, hematite, and calamine; this so-called oxidized zone ordinarily reaches down to the permanent water level and in some places considerably below it. In regions of heavy precipitation the oxidized zone is thin. Where there is little rainfall the water level lies far below the surface. Within the Cordilleran region the water level is rarely more than a few hundred feet below the surface; in exceptional places such as Tintic, Utah, it may exceed 2,200 feet in depth.

The ores of the oxidized zone are, as a rule, richer than the underlying sulphides. Just below the oxidized belt secondary sulphides may be formed through concentration by descending surface waters and this part of the deposit is likely to be exceptionally rich. Such secondary sulphides are chalcocite or copper glance and varjous rich silver minerals. In many copper deposits, such as those at Ely, Ner., and Miami and Ray, Ariz., the only workable part of the deposits consists of a layer of this secondary copper glance. In silver veins no such sharp division between the two zones is found. Much of the richest ore mined near the surface in the Cordilleran region is the result of secondary enrichment by descending waters.

\section{CLASSIFICATION OF DEPOSITS.}

The first broad division that can be established among the mineral deposits of the West is between those of pre-Cambrian and postCambrian age. As a matter of fact the latter are almost wholly postJurassic and a large majority are post-Cretaceous.

\section{PRE-CAMBRIAN DEPOSITS.}

The ores of pre-Cambrian age were formed before the earliest Cambrian sediments were laid down in the Paleozoic seas which 
covered a large part of the Cordilleran region. They had then been subjected to considerable erosion and therefore only the deep-seated deposits are now exposed. They are of many different kinds, but it is believed that they were mainly the result of after action of preCambrian intrusions or were leached from old rocks by hot waters at considerable depth. The rocks in which they are contained are mainly granites, gneisses, and schists of various kinds; most commonly the ores are inclosed in amphibolite, diorite, or gabbro. The mere fact that ores occur in pre-Cambrian rocks is not enough to establish their age, for deposition could have been effected in these rocks during subsequent periods. They are in places directly overlain by Paleozoic sediments, and this of course constitutes a good proof of the age of the ore deposits. The pre-Cambrian rocks have usually participated in and been pressed by dynamometamorphic processes and are now schistose. This is another criterion, for except along the Pacific coast in California, Oregon, Washington, and western Nevada, there has been no regional metamorphism in the Cordilleran region since pre-Cambrian time. The association of the ore minerals with heavy silicates such as garnet and tourmaline is common in preCambrian deposits, though such an association is also found in deposits formed during later epochs of metallization.

The pre-Cambrian deposits include segregations in igneous magmas, such as those of Iron Mountain, in Wyoming, but this type is rare. More common are irregular fissure veins and disseminations in schist. The metal contained is mainly copper in the form of chalcopyrite, with small amounts of gold and silver; lead deposits are of rare occurrence,

As the pre-Cambrian rocks are largely covered by younger sediments and igneous rocks, these ancient deposits are very irregularly distributed and do not form distinct belts or zones. They occur in South Dakota, Wyoming, Colorado, New Mexico, and Arizona; probably also in southwestern California. Compared to the later deposits they are on the whole poor.

\section{POST-CAMBRIAN DEPOSITS.}

The deposits formed later than the earliest Paleozoic sediments may be divided into two groups-(1) those contained in sedimentary rocks with no traceable connection with igneous rocks; (2) those occurring in igneous rocks, or in the sedimentary rocks surrounding them.

\section{DEPOSITS IN SEDIMENTARY ROCKS.}

The first group is much the smaller one. Aside from some poor and irregular deposits of copper and lead in limestone-for instance, in northeastern Utah and adjacent parts of Idaho-the origin of which is in doubt, this group consists of the widespread copper ores 
in sandstone found in Oklahoma, Texas, New Mexico, Colorado, Arizona, Utah, and Idaho. The ores are disseminated in Jurassic, Triassic, or Carboniferous sandstones, and the most prominent ore mineral below the zone of oxidation consists of chalcocite. In western Colorado and eastern Utah these sandstones also contain vanadium and uranium minerals. Successful exploitation has been carried on at some places, but on the whole the deposits are poor. The metals sparsely disseminated as salts in the sedimentary rocks are believed to have been leached by surface waters. The concentration has probably proceeded since an active water circulation was established in these strata-that is, since the beginning of the Tertiary.

DEPOSITS CONNECTED WITH IGNEOUS ROCKS.

The large group of deposits connected with igneous rocks yields almost the whole of the Cordilleran production. Practically all the deposits are post-Jurassic, for in the Rocky Mountain region the Paleozoic and the larger part of the Mesozoic were eras of quiet sedimentation. Only along the Pacific coast, in the Sierra Nevada, do we find evidence of Paleozoic eruptions, which were mostly surface flows, and it is not impossible that a few ore deposits in that region may be found to be of Paleozoic age. During Triassic and Jurassic time eruptions of basic lava took place in California, Oregon, and western Nevada, and it is possible that they were closely followed by some metallization. The only definite evidence of this kind is from southeastern Oregon, where the Triassic or Jurassic basic flows east of Baker City, on Powder River, contain chalcocite and native copper in connection with zeolites, calcite, and epidote.

At the end of the Jurassic period or in the earliest part of the Cretaceous an era of igneous activity began in the Cordilleran region, which culminated in the middle of the Tertiary and continued in sporadic outbursts up to a comparatively recent time. Small eruptions are believed to have taken place near Lassen Peak, in northern California, within the last 200 years. Most of the ore deposits now worked in the Western States were formed within this era of volcanism.

Geologic evidence has shown that each epoch of. eruptions was followed by a more or less intense mineralization, consisting in the development of ore minerals by the filling of fissures or by the replacement of the rocks themselves in or about the igneous rocks.

Volcanism manifests itself by the eruption of lavas at the surface and by the intrusion of magmas into deeper portions of the crust. The lavas, cooling quickly, usually consolidate as fine-grained porphyritic rocks, commonly containing more or less of a glassy base; the intrusive magmas, cooling slowly and under pressure, result in coarser-grained granular or porphyritic rocks without a glassy base. As the intrusions take place at a considerable depth, it is clear that 
such masses and the ore deposits which may accompany them will not be exposed at the surface unless it happens that the region has been subjected to deep erosion. The eruption of lava flows at the surface is no doubt usually accompanied by intrusions underneath them, and there is likewise little doubt that the great masses of postJurassic intrusions were accompanied by now eroded lava flows. In the San Juan region of southwestern Colorado, at Tintic, Utah, and at some other places erosion has exposed the intrusive bodies below the earlier Tertiary lava flows.

The mineral deposits formed at considerable depth in and around intrusive masses differ both structurally and mineralogically from those formed near the surface, though it is possible, and in fact likely, that the latter may be the upper parts of a deeper body of mineralized rock and that they would be found to change their character if they could be followed to great depths. Therefore, though a distinction must be made between the two classes, there are intermediate forms and probably no radical genetic difference.

The oldest intrusions are the great early Cretaceous granitic batholiths of the Sierra Nevada, extending northward into British Columbia and Alaska. Less extensive intrusive masses of the same age are found in Oregon, Nevada, and Arizona, but on the whole toward the east such masses are less conspicuous and smaller and date from a later time. Most of the relatively small granitic and monzonitic stocks of Colorado and New Mexico are probably of latest Cretaceous or earliest Tertiary age. After they had been exposed by erosion, the middle Tertiary lavas in many places covered their outcrops.

The volcanic flows which cover large areas in the Western States are generally of Tertiary age, ranging from Eocene to latest Pliocene, though the latter are mainly nonproductive basalts. The productive areas are mostly in Eocene or Miocene flows of andesite or rhyolite. Late Cretaceous lavas occur in Montana and Colorado, but so far as known they contain few mineral deposits. Although mineralization, more or less intense, is the rule in the vicinity of intrusive bodies, especially those of moderate or small size, it is rather exceptional in the lava flows and enormous areas of them show no trace of metallic ores.

From the relations explained above it follows that the deposits in or near intrusive bodies are apt to be of greater age than those in the more recent lava flows; on the whole their age ranges from the earliest Cretaceous to the earliest Tertiary. Among the occurrences are the gold-quartz veins of the Sierra Nevada and Oregon and many similar deposits in Nevada; the gold, silver, and base-metal deposits extending from Leadville to Boulder, in Colorado, and the similar deposits in the great batholiths of central Idaho and western Montana and at 
many places in Arizona, for instance near Kingman and Prescott; and the copper deposits of Clifton, Globe, and Bisbee, in Arizona; of Ely, in Nevada; and of Bingham, in Utah. The deposits may take the form of contact-metamorphic bodies, which usually carry copper and zinc, more rarely lead; or replacement deposits of lead ores some distance away from the intrusive contacts; or normal veins and shear zones with simple sulphides of the base metals, carrying more or less gold and silver; or of native gold or irregular disseminations of sulphides in altered igneous rocks. Very commonly all these modifications occur in the same district and form what has been termed a metallic aureole around the intrusive rock. Structurally the veins are likely to be clear cut and well defined, with regular strike and dip. The filling is massive and drusy structure is less common than in the veins formed near the surface, the principal gangue mineral being a milky quartz.

The deposits formed near the surface and usually appearing in volcanic flow rocks are almost exclusively veins or disseminations; most of the productive occurrences are typical veins. As the rocks are more extensively shattered near the surface than at greater depths, many wide areas of volcanic rock are deeply altered by ore-bearing solutions and transformed into quartzose, sericitic, or kaolinitic masses or less completely altered to greenish "propylite" by the development of chlorite and pyrite. The gangue of many deposits contains calcite, barite, and fluorite in addition to the common usually finegrained and locally chalcedonic quartz. The structure of the veins is likely to be irregular, brecciated, and drusy. These veins, many of which are exceedingly rich, carry mainly native gold in fine distribution, rarely coarse, of ten alloyed with much silver; also argentite and various silver antimonites and arsenites.

Base metals are ordinarily not abundant in the deposits formed near the surface. These deposits occur in Nevada, Washington, Idaho, Colorado, New Mexico, Utah, and Arizona, but Nevada contains more of them than any other State. As examples may be cited, in Nevada the Comstock, Tonopah, Bullfrog, Tuscarora, Rawhide, Fairview, and National veins, as well as the more irregular deposits of Goldfield; in Washington, the Monte Cristo and Republic veins; in Idaho, the Custer and Silver City veins; in Colorado, the Cripple Creek and Silver Cliff veins; in New Mexico, the Mogollon veins; in Arizona, the veins of the Black Range, near Colorado River; and in Utah, the veins of the Mount Baldy district, near Richfield and Marysvale. The veins of the San Juan region in Colorado are found in a deeply dissected volcanic dome or plateau and some of the types here occurring are unusually rich in lead, copper, and zinc. 


\section{LOCAL GEOLOGY OF WESTERN ORE DEPOSITS.}

ARIZONA.

The valuable ore deposits of Arizona are confined mainly to the southwestern part of the State. The portion which lies northeast of a line drawn from a point a little north of Clifton to Peach Springs and thence in the same direction to the Nevada line is covered by the horizontal beds of the plateau province or by lavas and contains only a few and unimportant copper deposits in sedimentary rocks. Wherever, as in parts of the canyon of Colorado River, the preCambrian crystalline rocks are exposed, mineral deposits may be found, though the prospects thus far uncovered do not indicate strong metallization.

The southwestern and larger part of the State is characterized by ore deposits of great variety and abundance, scattered irregularly through detached ranges rising above desert plains. No mineral belts of great persistence and length can be traced.

The ranges of Arizona are of manifold structure and composition. In the eastern half of the mineral-bearing area there is as a rule a succession of several thousand feet of Paleozoic rocks ranging from Cambrian to Carboniferous, which rest on a pre-Cambrian basement that is in many places exposed. In the extreme southeast Cretaceous rocks are also present, though rarely mineralized. Toward the west the exposures of pre-Cambrian schist increase in area and a large number of ranges west of the one hundred and twelfth meridian are wholly built up of such rocks, locally intruded by monzonites or covered by Tertiary lavas.

The geologic section of the Paleozoic and Mesozoic rocks varies considerably in different parts of the State. At Bisbee, in the southeast corner, the Cambrian consists of 430 feet of quartzite overlain by 770 feet of limestone. The Carboniferous includes both Mississippian limestone 700 feet thick and heavy beds of Pennsylvanian limestone, of which 3,000 feet are exposed. The Cretaceous (Comanche) formations aggregate 4,500 feet in thickness. Farther north, near Clifton, the total thickness of the Paleozoic and later rocks is very much less.

Pre-Cambrian deposits of several types containing copper, gold, and silver are found in the districts adjoining Colorado River, near Parker, which is south of Williams Fork, and probably at other places farther south, in the little-explored country in Yuma County. They also appear in the crystalline schists of Yavapai County, and it has been suggested ${ }^{1}$ that the great copper deposit of the United Verde mine at Jerome, which is contained in amphibolitic schists, also belongs to this class. Deposits connected with granular intru-

I Graton, L. C., Mineral Resourees U. S. for 1907, pt. 1, U. S. Geol. Survey, 1908, p. 597. 
sive rocks of Mesozoic or early Tertiary age are abundant in all parts of the region and consist of veins, contact-metamorphic deposits, and replacement deposits in limestone away from the immediate vicinity of igneous rocks.

Contact-metamorphic deposits in zones of garnet and other silicates adjoining intrusive rocks are particularly abundant, and as excellent examples may be mentioned those of the Clifton, Bisbee, Saddle Mountain, Twin Buttes, Silver Bell, and Washington districts. The gold-bearing veins of Yavapai County are probably of Mesozoic age and appear around smaller monzonite intrusions in pre-Cambrian schists. The veins near Kingman are likewise associated with intrusions of porphyry in pre-Cambrian schist. Copper, gold, silver, lead, and zinc are the principal metals found in the veins of these classes, but tungsten and molybdenum also occur. Chalcocite blanket deposits, due to concentration of lean primary disseminations by descending surface waters, connected with the same epochs of mineralization are mined at Clifton, Ray, and Globe, and prospected at a number of other places.

The deposits of the late Tertiary epoch are generally veins and are contained in rhyolitic and andesitic lavas; they are widely scattered, the most prominent examples being those of the Black Range, along Colorado River north of Needles, where the noted Gold Road mine is situated. Similar veins occur in the Kofa district, Yuma County; in the Mammoth district, Pinal County; and at some places in the Santa Rita Range, Santa Cruz County. The well-known Commonwealth mine, in the Pearce district, Cochise County, also belongs to the same class. These veins generally carry gold and silver, but in places also copper, lead, and molybdenum. Placers are known principally in regions of metallic aureoles around intrusions of granular rocks, but they can rarely be worked, on account of the scarcity of water; dry washing is often resorted to on a small scale. Among the best-known placer regions are those of Yuma and La Paz, along Colorado River; Red Hill, in Yavapai County; Quijotoa and Greaterville, in Pima County; and Dos Cabezas, in Cochise County.

After this general review the individual ranges and their mineralization may be briefly mentioned.

The southeastern mountains, including the Peloncillo, Chiricahua, and Pinaleno ranges, are not rich in valuable deposits, though mineralized at several points, notably at Dos Cabezas and Paradise. The well-known copper deposits of the Clifton-Morenci district are situated near the New Mexico line, near the southern boundary of the plateau province, but in no distinct structural range.

Next to the west, in Cochise and Graham counties, follow from south to north the Mule Pass and Dragoon ranges, both strongly mineralized and containing many types of deposits, most prominent 
among which are the contact-metamorphic copper deposits of Bisbee (Warren district) and the gold-silver-lead replacement deposits of Tombstone. The Dragoon Range and its foothills have gold-silver deposits in lavas at Pearce and contact-metamorphic and replacement deposits at Dragoon, Turquoise, and Courtland. To the northnorthwest these ranges are continued by the Galiuro Mountains, which contain copper deposits at Copper Creek and undeveloped gold deposits in rhyolite. Still farther to the north-northwest, in Gila County and adjoining parts of Pinal County, are the veins and blanket deposits of copper ores at Globe, in a region of general uplift in which the range structure is less well defined. At short distances west and south of Globe are a number of contact-metamorphic copper deposits, among which the Saddle Mountain is the most prominent; also the chalcocite blankets of Ray and the now worked-out silver mine of Silver King.

The next tier of mountains begins at the Mexican line with the Huachuca and Canelo ranges, neither of which contains important mines. Just west of the Canelo lie the Patagonia and Santa Rita ranges, both of which are strongly mineralized and show deposits of many kinds. Contact-metamorphic copper deposits are found at Washington and Helvetia, here and there accompanied by chalcocite deposits. Silver deposits in andesite occur in the Harshaw district and mixed ores in rhyolite in the same vicinity; lead ores occur as replacements of limestone at the Mowry mines.

North of the Santa Rita extends the rugged Santa Catalina Range, with few valuable deposits; at its northern foot, in the San Pedro Valley, lies the Mammoth district, with gold-molybdenum ores in rhyolite.

West of Tucson and Santa Cruz River, in Pima, Pinal, Maricopa, and Yuma counties, the desert plains become more prominent. Numerous short ranges rise above the plains and are flanked by sloping alluvial fans. The region is extremely arid. Some of these ranges, especially those toward the west, contain only pre-Cambrian rocks. Others are built up of Paleozoic folded and faulted sediments with intrusive Mesozoic granitic rocks. Still others are composed mainly of Tertiary flow rocks. Among the more prominent deposits in Pima County may be mentioned the contact-metamorphic copper deposits of Twin Buttes and Silver Bell and the gold-bearing veins of Quijotoa, said to occur in Tertiary lavas. In the extreme western part of the county are the little-known districts of Ajo, Growler, and Gunsight, in which copper ores are said to occur in contact-metamorphic deposits.

In Yuma County there are also a considerable number of low desert ranges containing some mining districts. The gold-bearing vein of the Fortuna district, said to be in gneissoid rock, is southeast of 
Yuma; the lead ores of Castle Dome are in a detached range northeast of Yuma. Farther away in the same direction are the gold-silver mines of Kofa, which are worked on vein deposits in Tertiary lava flows.

In the northern part of Yuma County the ranges are mainly of preCambrian crystalline rocks. In the Harcuvar and Harquahala ranges intrusions of probably Mesozoic granite have caused considerable mineralization and the development of gold-bearing quartz veins, the best known district being that of Harquahala. A little farther east, in Maricopa County, also in a small desert range, lies the well-known gold deposit of the Vulture mine.

In the central portion of the State, in Yavapai County, rise a group of mountains, the most prominent of which are known as the Bradshaw Range. In this region cluster a great number of deposits of gold, silver, and copper. Among the gold mines the Congress is the best known, and the copper deposit of the United Verde is situated in the same region.

The mining districts in Mohave County comprise those of the Cerbat, Black, and Hualpai ranges, all of which trend north and south. The Black Range is continued north of Colorado River for some distance into Nevada. The Cerbat ores are mainly base metals associated with lead, zinc, and copper and differ greatly from the more recent gold veins of the Black Range, which outcrop in Tertiary lavas.

\section{CALIFORNIA.}

The dependence of mineral belts on geologic structure is particularly well marked in California.

Along the Pacific coast, from the vicinity of Santa Barbara to Humboldt County, rise the Coast Ranges, most recent of the great structural features of the State. They are largely built up of folded and crushed Cretaceous, Jurassic, and Tertiary sediments, in places broken through by andesite and basalt, and an older series of diabasic and serpentinoid rocks. The Coast Ranges are singularly poor in deposits of the ordinary metals, but a persistent belt of quicksilver ores follows them throughout and reaches its greatest development just north and south of San Francisco Bay. The quicksilver deposits are of late Tertiary or Quaternary age and seem to have been formed shortly after the eruption of basalt and andesite. The ores were formed near the surface and in all probability by hot ascending springs, of which the Coast Range contains a great number. The gangue usually contains much opal. Among the best-kpown districts are those of the Clear Lake region and those of New Almaden and New Idria, in Santa Clara and San Benito counties.

To the east of the central valleys and separating them from the Great Basin lies the long and imposing mountain block of the Sierra

21528 - Bull. 507-12-2 
Nevada. Geologically it consists of clayey and sandy sediments, ranging from earlier Paleozoic to later Jurassic in age, closely folded and forming a belt along the western part that greatly increases in width toward the north. In early Cretaceous time these sediments were intruded by enormous masses of granitic rocks, mainly granodiorite, which since the late Cretaceous and Tertiary movements along the eastern border of the range form a zone that is of moderate extent in the northern part, but widens toward the south until in Tulare and Kern counties the intrusive rocks form nearly the whole range.

The gold belt of California, a succession of deposits of unusual persistence, begins as a narrow fringe along the foot of the Sierra Nevada in Tulare and Kern counties, but widens greatly toward the north, and in Butte and Plumas counties is over 60 miles broad. Along this belt are located a great number of well-known mining districts. In the southern counties from Eldorado to Mariposa it contains the numerous and strong veins of the Mother Lode region, on which a number of well-known mines are being worked. The Kennedy, with a depth of 3,600 feet, is the deepest mine in California. Farther north, in Nevada County, the celebrated Nevada City and Grass Valley districts are situated within this belt. Placer deposits abound throughout the gold belt, having accumulated along the present rivers and in the far older Tertiary gravels now exposed as erosion remnants high up above the canyons; in part the Tertiary gravels are covered by andesite and rhyolite, likewise of Tertiary age but without evidence of mineralization. The Tertiary gravels are still mined at many places by hydraulic or drifting methods. The Quaternary gravels at the foot of the range are now mined extensively by dredging at Oroville, Marysville, Folsom, and several points in Calaveras County.

The primary gold deposits, as distinguished from the secondary placers derived from their destruction by erosion, are for the most part quartz veins containing free gold. They were formed shortly after the intrusion of smaller masses of granite, granodiorite, gabbro, and peridotite into the sedimentary series, probably in early Cretaceous time.

The parts of the veins now exposed give evidence of having been deposited at considerable depth. Many thousand feet of overlying rock masses, together with the former outcrops of the veins, have been removed by erosion. Toward the north, in Tehama, northern Plumas, and Lassen counties, the gold belt is covered by a broad tongue of Tertiary volcanic rocks. Equally abruptly it emerges from this mantle above Redding and continues northward to the Oregon line, its width being from 50 to 60 miles. In this area, comprising parts of Shasta, Trinity, and Siskiyou counties and generally 
known as the Klamath Mountains, gold-bearing veins and pıacers are widely distributed. The placers have been the more productive. The richest veins occur in the Coffee Creek region. Hydraulic mining is still actively pursued along Klamath River and in the older Tertiary gravels. The deposits and their geologic conditions are similar to those of the Sierra Nevada.

Toward the south end of the State gold-quartz veins, probably of about the same age as those of central California, appear in San Diego, Riverside, and Los Angeles counties, in the mountains which form the continuation of the Sierra of Lower California. Except in San Diego, where the formerly productive Banner and Julian districts are located, the output from these deposits has been small. The veins occur in or near masses of metamorphic schist, which lie embedded in granitic rock.

A less well-defined belt of copper-bearing deposits follows the foothills of the Sierra Nevada and continues toward the north-northwest into the Klamath Mountains. Deposits of economic value have been found in Calaveras County at Copperopolis and Campo Seco; in Placer County at Dairy Farm; in Nevada County at Spenceville; and at several other places. At present the most important copper mines are those at Keswick and Bully Hill, in Shasta County. Copper deposits are also found in the northern part of Siskjyou County, near the Oregon line. The copper deposits as a rule occur in connection with igneous rocks, including both basic rocks like the diabase porphyries and Mesozoic andesites of the Sierra Nevada and acidic rocks like the alaskite porphyry of Shasta County. In places they also occur in genetic connection with peridotite and gabbro. They appear to have been formed a little earlier than the gold-bearing quartz veins in late Jurassic or earliest Cretaceous time, at considerable depths below the surface. Some of the smaller copper deposits, however, probably resulted from concentration by deep water from the surrounding rocks. All these deposits carry chalcopyrite with pyrite; only exceptionally is chalcocite present.

That part of California which lies to the east of the Sierra Nevada and the Peninsular Range is very different from the rest of the State. It is an arid region with detached ranges, generally trending northward; geologically it belongs to the Great Basin region of Nevada and the desert province of Arizona. The map shows that the irregular and scattered distribution of the mining districts in this area is similar to that of the region mentioned. In many localities the geologic structure is as yet unknown. In general, the deposits may be grouped into those of pre-Cambrian age, those connected with granitic intrusions of late Mesozoic age, and lastly, those of late Tertiary age, which usually occur in surface lava flows. The few pre, Cambrian deposits are probably limited to the vicinity of the Arizona 
boundary line and contain mainly copper ores and gold ores. The gold mines at Hedges, near Yuma, Ariz., probably belong in this class, as do those of Whipple Mountain, in San Bernardino County. The Mesozoic deposits are widely distributed from Mono County southward and appear as aureoles around smaller intrusions of granodiorite. They are in large part gold-bearing quartz veins, exemplified by the deposits at Randsburg, in Kern County. A number of small districts in the desert range of San Bernardino and Riverside counties contain gold and quartz veins in granitic country rock.

A smaller number of deposits are replacements of limestone by galena, as at Tecopa, Cerro Gordo, and Panamint, in Inyo County. Still fewer are the contact-metamorphic deposits, of which examples may be found in the copper-bearing garnet zones of Ubehebe, in Inyo County, and the Standard mine at Cima, in San Bernardino County. In the western part of California contact-metamorphic deposits are very rare, though a few unimportant examples are found in the Sierra Nevada and the Peninsular Range. In the northern part of the State, in Shasta County, important contactmetamorphic deposits carrying magnetite occur between limestone and granitic rocks north of Pit River, and similar deposits carrying copper have been discovered on the same contact south of the river.

More characteristic of the eastern region are the deposits-generally quartz veins-in Tertiary rhyolite and andesite. Isolated examples are found along the eastern boundary of the State, as at Hayden Hill in Lassen County, in Alpine County, at Bodie and several other places in Mono County, in Inyo County, and in San Bernardino County. They usually carry both gold and silver, but in places (as at Calico) also silver alone. Several deposits of this kind are found near Mohave, in San Bernardino County.

The iron deposits of California are scattered from the southern to the northern part of the State. Few of them are of present economic importance. Most of them carry magnetite and were formed at the contacts or elsewhere shortly after the intrusion of deep-seated igneous rocks. The contact-metamorphic deposit of Herault, in Shasta County, has already been mentioned. Other magnetite deposits in granite occur at Dale, San Bernardino County, and in the Eagle Mountains, Riverside County, both connected with granitic intrusions. Another iron deposit is found at the Minarets, in the western part of Madera County.

Manganese deposits of minor importance as well as prospects of chromite are found at several places in the Coast Range. The chromite is contained in serpentine country rock.

Deposits of rare metals are not abundant in California. Platinum occurs in the gold-bearing gravels of northern California and in the Sierra Nevada, and a small quantity is saved each year. Deposits of scheelite are worked near Randsburg, Kern County. 
COLORADO.

The ore deposits of Colorado are confined to the western or mountainous half of the State, but within this area they are not by any means uniformly distributed. Parts of the area near the western and northwestern borders belong to the plateau province of little-disturbed rocks. These contain only a special class of copper and raremetal deposits. The southeastern part of the mountainous area is also poorly mineralized. The important deposits are confined largely to a diagonal belt trending northeast and southwest, beginning in Boulder County and ending in the San Juan Mountains.

The mines of Colorado yield principally gold, silver, lead, and zinc. There are few distinctly copper districts and none of great importance. With some exceptions, like those of Cripple Creek, which are almost exclusively gold bearing, the ores of Colorado are of complex nature and in many places they contain all five of the metals mentioned.

The rugged Front Range, composed mainly of pre-Cambrian rocks, extends from the Wyoming line to the Sangre de Cristo Range, at the New Mexico boundary. On the whole, it is very poor in ore deposits.

West of the Front Range lies a wide belt of uplifts of complicated structure, trending mainly northeastward, in which the basal preCambrian is exposed at many places but elsewhere covered by a folded and faulted sedimentary series consisting of Paleozoic and Mesozoic beds of great thickness. As exemplified at Aspen, the sedimentary rocks consist of relatively thin formations of Cambrian, Ordovician, and Devonian age and several thousand feet of Carboniferous beds, in part sandy. The upper and thicker part of the Carboniferous is of dark-red color and may be considered as part of the "Red Beds." Above this lie 2,000 feet of Triassic "Red Beds" and the section is capped by Cretaceous sandstones and shales, which are said to reach a thickness of 3,500 feet at Breckenridge. At Leadville the basal formation is the Cambrian quartzite, 200 feet thick. This is overlain by the Silurian white limestone, 100 feet thick, covered by 50 feet of the "Parting" quartzite of Devonian age. Above this comes about 4,000 feet of Carboniferous rocks, divided into the Leadville or "Blue" limestone, 400 feet thick, the "Weber shales and grit," 2,500 feet thick, and 1,000 to 1,500 feet of the limestone and red beds of the "Upper Coal Measures."

With these sediments are interbedded sheets and dikes of monzonite porphyries of many kinds, and these intrusive rocks as a rule indicate the presence of mineral deposits.

The southwestern part of the State, known as the San Juan region, is marked by a large area of Tertiary (in part early Tertiary) volcanic flows of rhyolite and andesite. Intruded in this series are smaller masses of monzonite porphyries, and surrounding it in slightly upturned position is a thick mass of sediments, including a thin body of 
Devonian rocks, about 4,000 feet of sandstone and limestone of Carboniferous age, including the lower part of the "Red Beds," about 1,000 feet of Jurassic and Triassic "Red Beds," and 1,500 feet or more of Cretaceous formations.

The northeast corner of the State is covered by flat-lying Eocene beds except where the east end of the Uinta uplift has brought older rocks to view.

As in other States, the deposits may be conveniently divided into those of pre-Cambrian and those of post-Cambrian age. The latter are by far the more important.

The pre-Cambrian ores occur, of course, only in the rocks of the same age, but such occurrence alone is not a sufficient criterion for determining the age of the deposits. Near the northern boundary line and extending into Wyoming are a number of low-grade copper deposits-for instance, those of Pearl, in Larimer County-which, on good grounds, are held to be of this early age. Little production has thus far been obtained from these deposits. They are in part veins, in part irregular masses of pyrite and chalcopyrite, usually connected with intrusions of diorite or gabbro. Considerably further south, in Jefferson, Fremont, and Chaffee counties, are several copper deposits, also in close connection with basic igneous rocks to which a similar age has been attributed. The most prominent among these deposits is that of the Sedalia mine, which also yields zinc ore. A considerable number of small deposits of copper ore in the Front Range and the Sangre de Cristo Range probably belong in the same class.

As elsewhere in the Western States, there was a long interval between the pre-Cambrian and the later epochs of metallization, which may be assumed to begin with the close of the Cretaceous or the beginning of the Tertiary. The division between the earlier Tertiary mineralization which followed the intrusion of monzonite porphyries and the later development of deposits in surface lava is less strongly marked in Colorado than elsewhere.

The principal mineral belt, extending from Boulder to Leadville and Gunnison, appears to be of earliest Tertiary age and its ores occur in a great variety of rocks from pre-Cambrian to Cretaceous, constantly associated with intrusive dikes, sheets, and masses. Most of the deposits bear clearly the stamp of having been formed at considerable depths below the surface. None of the deposits occur in surface flows.

Beginning from the northeast, we have, first, the gold and silver bearing quartz veins in the pre-Cambrian rocks of Boulder, Gilpin, and Clear Creek counties. Associated with them and of about the same age are the tungsten veins of Boulder County, which yield the larger part of the tungsten ores produced in this country. There is little free gold, the precious metals occurring mainly in pyritic ores, 
galena, or zinc blende. The veins are accompanied by a system of porphyry dikes. Beyond the Divide are the veins of Breckenridge, in Cretaceous rocks, accompanied by sheets of monzonite porphyry. A number of smaller districts connect with the great deposits of Leadville, in which complex pyritic ores replace limestone and carry silver, gold, lead, zinc, and copper. The intrusives have here taken the form of thick sheets forced into the Paleozoic sediments. The Red Cliff and Tenmile districts are also characterized by replacement ores, as is Aspen, west of Leadville. At Aspen the silver-lead-zinc ores follow a network of fault fissures in limestone and are not so intimately connected with intrusive rocks; it is probable that the mineralization here is of more recent date than elsewhere.

To the southwest of Leadville, in Gunnison and Chaffee counties, lie a considerable number of less important districts exhibiting considerable variety in structure and mineralogy. A short barren stretch separates the Gunnison region from the San Juan region and is occupied mainly by surface lavas and tuffs originating in the volcanic center of the San Juan Mountains.

The San Juan region is rich in ore deposiis, which carry mainly gold and silver, but also to some extent lead, copper, and zinc. The ore deposits are typically fissure veins which have a northeast or northwest trend. At Telluride and Ouray these quartz veins carry gold and some silver. At Silverton and in the Rico Mountains base metals are also present in abundance. The veins cut most of the volcanic flows of the region as well as the still later intrusive monzonites and in places the sedimentary and pre-Cambrian rocks. Toward the eastern border of the region is the Lake City district and, still farther east, the Creede district, the quartz veins of which cut rhyolite and carry gold, silver, lead, and zinc.

The veins of San Juan County bear the marks of having been formed relatively near to the surface, but, as is natural from the great depth to which erosion has exposed the lower rocks, some of them present features characteristic of deposition under conditions of higher temperature and pressure.

Practically no contact-metamorphic deposits of economic importance exist in Colorado. The ores of Leadville recall this type in some ways, but lack the characteristic contact minerals.

Outside of the main mineral belt are the districts of Cripple Creek and Silver Cliff, both situated in the Front Range south of Denver. The ores of these districts were deposited relatively near the surface of old volcanic centers. At Cripple Creek, well known as one of the principal gold-producing districts of the United States, gold tellurides occur in veins in a dissected volcano, which, in late Tertiary times, emitted phonolitic lavas. At Silver Cliff the silver ores occur in andesite and rhyolite, in part cementing breccia-filled pipes in the 
volcanic rock. The age of this mineralization is thought to be Eocene.

In many of the gold districts mentioned detrital or placer deposits are found in the valleys below the outcrops of the ore bodies. The richest parts of these placers were washed out many years ago, but they still yield some gold by modern processes of dredging and hydraulic work.

There are few placer deposits in the San Juan region and at Cripple Creek. Most of them are in the main northeast-southwest belt from Boulder to Leadville and the most important now worked are situated at Breckenridge. Some gold is also mined from the Recent and Tertiary gravels of Routt County.

Colorado is poor in iron deposits. A few small magnetite deposits occur in Gunnison County, generally at the contact of sedimentary rocks and granite. The oxidized ores of Leadville in part are rich in iron and manganese and are utilized as flux and in the manufacture of spiegeleisen, an alloy of iron and manganese.

It remains to mention an entirely different class of deposits, carrying copper, vanadium, and uranium. The ores occur disseminated in sedimentary beds, mainly in the "Red Beds" of southwestern and central Colorado, and were probably formed by concentration by means of meteoric waters from small quantities of the metals disseminated through the beds. Vanadium and uranium bearing sandstones of Jurassic age are mined in San Miguel and Montrose counties and also occur in Routt County. In places copper occurs together with the metals mentioned, and chalcocite ores have been found disseminated in the conglomerates, sandstones, and carbonaceous shales of Red Gulch, in Fremont County. They are also reported from Huerfano County, in the south-central part of the State. The tungsten-bearing veins of Nederland, in Boulder County, have already been mentioned

\section{IDAHO.}

Ore deposits are irregularly distributed in Idaho. The extreme southern and eastern parts of the State are almost barren. The mining districts are most abundantly clustered in a region extending from the vicinity of Boise in a northeasterly direction to Lemhi County and the Salmon River valley. The most important output is that of lead and silver from the Cour d'Alene district, in the northern part of the State. Gold and silver are derived from the belt mentioned and from veins in Owyhee County, in the southwest corner of the State.

The most prominent geologic feature is the great central mountain mass bordering Montana and continued on the north by the Clearwater and Cour d'Alene Mountains. The eastern part of this 
mountain mass consists of pre-Cambrian and Paleozoic strata, the extreme western part of Triassic sediments. Between these two areas lies a great intrusive mass of granitic rocks, principally quartz monzonite, continued on the north and east by smaller intrusive areas. The northern or panhandle part of the State is mainly built up of the steeply inclined sedimentary strata of a thick pre-Cambrian complex known as the Belt series. The southern and southwestern parts of the State are covered by thick volcanic flows of Tertiary age and this area connects on the east with the lavas of the Yellowstone Park region and Utah. Toward the Nevada line rise a number of short ranges of the type of the Basin Ranges of that State, and the extreme southeastern part of Idaho is occupied by north-south ranges built up of folded and faulted Paleozoic and Mesozoic rocks which may be considered as a northward extension of the Wasatch Mountain system.

Pre-Cambrian deposits are not present in Idaho, except possibly in some of the less important districts in the northern part of the State, where mineralization seems to have accompanied the intrusion of diabase sills in the Belt series. Here as elsewhere two important kinds of deposits may be recognized-those which were formed shortly after the intrusion of the great central batholith of Jurassic rocks, probably in late Cretaceous time, and those of late Tertiary age which developed after the outburst of the Tertiary lavas in the southern and central parts of the State. The latter are confined to the gold and silver veins of Owyhee County and to a belt of lavas in the central part of the State, including such deposits as the Custer, in Custer County; the Singiser veins, in Lemhi County; and the Thunder Mountain deposits, in Idaho County.

A few unimportant deposits of copper and lead ores of uncertain age occur in the Paleozoic limestones of the ranges in the extreme southeast corner of the State.

The upper parts of the late Cretaceous ore deposits have to a considerable extent been removed by erosion and the parts now exposed contain ores formed at considerable depths. They are mainly fissure veins, but in places, as in southern Lemhi County and at the Wood River (Hailey) district, these merge into replacement deposits of galena in limestone. Contact-metamorphic deposits containing - copper ores are developed on a rather extensive scale at Seven Devils, near the Oregon boundary line, and at White Knob, in the southeastern part of Custer County, but neither place has proved a large or steady producer.

The veins are contained in a great number of districts situated in and around the borders of the central granitic mass. Most of them carry gold and silver in a quartz gangue with abundant sulphides. Among the best-known districts are those of the Idaho Basin and 
the vicinity of Boise, adjoined on the east by the Sawtooth, Atlanta, and Rocky Bar districts. To the northeast are a number of minor districts in Lemhi County. To the north are those of Warren, Florence, Buffalo Hump, Dixie, Elk City, and Pierce. Still farther north, surrounding a smaller granitic intrusive mass, cluster the lead-silver veins of the Cour d'Alene district. The late Cretaceous gold-bearing veins have usually yielded placer deposits of importance, many of which are still producing. The placer districts of greatest present importance are in the Idaho Basin, along the eastern boundary of Lemhi County, and at Pierce. The now largely exhausted placers of Warren, Florence, Elk City, and the Cour d'Alene region should also be mentioned. The fine gold occurring in the sands of Snake River has been described frequently, but the actual production from these deposits is very small. Placers of diminishing importance are situated along Salmon and Boise rivers.

Among the Tertiary veins those near Silver City and De Lamar, in Owyhee County, have been and are still important producers. These veins cut through early Tertiary rhyolites and basalt and bear clear evidence of having been formed within short distances of the present surface.

Less well known are the veins at Custer and also those of a few districts like Singiser, in Lemhi County, which occur under similar conditions. Low-grade gold ores have been mined at Thunder Mountain, in Idaho County, and are said to form disseminations in rhyolitic tuffs. The Tertiary veins carry gold and silver with a very small amount of sulphides and no important amounts of the base metals.

Idaho contains few deposits of iron ore and none of them are mined at present. Iron Mountain, in Washington County, near Snake River, is the principal locality. No rare-metal deposits of importance occur in Idaho.

\section{MONTANA.}

The ore deposits of Montana occur in the western or mountainous half of the State. Though a few are contained in the detached front ranges, the major part are situated in a cluster in the southwestern part of the State, practically within the area included by Helena, Dillon, Missoula, and Virginia City. A few are situated north of the Yellowstone Park, and some others near the north end of the boundary line between Idaho and Montana, but the northern part of the Rocky Mountain area, though traversed by several high ranges, such as the Main Range and the Mission Range, is singularly barren of mineral deposits.

The great copper production of the Butte mines (Summit Valley district) overshadows all the rest of Montana's metallic output, but 
the State also produces annually several million dollars in gold and much silver and lead.

The geologic structure is complicated. The eastern part of the Rocky Mountain region shows a succession of dome-shaped uplifts in which the Mesozoic and Paleozoic rocks are successively exposed; the central core consists of igneous rocks or the pre-Cambrian complex. Of such character are the Bighorn, Little and Big Belt, Moccasin, Judith, Little Rocky, and Bearpaw mountains. The main mass of mountains west of Helena and Livingston consists of similar systems of faulted and interlocking uplifts which expose a great thickness of sedimentary rocks. As measured near Philipsburg, the sedimentary rocks consist of a great thickness of the Algonkian (preCambrian) Belt series, amounting to about 13,000 feet of slates, limestones, and quartzite, then 1,200 feet of Cambrian, 300 feet of Silurian, 1,000 feet of Devonian, 1,200 feet of Mississippian, 700 feet of Pennsylvanian, 400 feet of Jurassic, and about 2,000 feet of Cretaceous rocks. In this western province, which seems to have suffered more erosion than the area farther east, a number of batholiths, or great masses of granite rocks, have been intruded. They are mainly quartz monzonites of late Cretaceous or earliest Tertiary age, and the largest of them extends from Helena for about 70 miles in a southsouthwest direction to Butte and Dillon. Several smaller intrusive masses surround this large body. Extensive eruptions of andesite lavas preceded the main epoch of intrusion.

The thick Belt series, which begins in the latitude of Butte, occupies a large part of the northern mountains of Montana, but it is as a rule poor in mineral deposits and contains few intrusive bodies.

The ore deposits of Montana mainly cluster in and around the great intrusive bodies in the southwestern part of the State. They were evidently formed shortly after the intrusive activity, at considerable depths below the surface, and their upper parts have been removed by erosion.

Few pre-Cambrian deposits have been observed in Montana, and the probable occurrences seem to be limited to copper ores associated with intrusive sills in the Belt series on the east side of Missouri River.

Important deposits of late Tertiary age in flows of lavas are also scarce, though some veins occur in rhyolite between Helena and Butte. Some veins in the early Tertiary andesites southcast of Helena are probably genetically connected with the monzonite intrusions mentioned above as occurring in this region. Near Helena, Boulder, and Radersburg there is some indication that hotspring activity and ore deposition have continued until a late geologic period, but definite information on the subject has not yet been published. 
The deposits connected with intrusive bodies assume a great variety of form and content. Contact-metamorphic deposits are rare, but are known in the Elkhorn, Bannack, Argenta, Bear Gulch, Highland, and Philipsburg districts; the Cable gold mine, which has been a large producer, is located in the district last named. Contact deposits are also reported to exist near Garnet, in Granite County.

A number of deposits of the same general class are quartz veins, carrying gold, silver, or both. Of this kind are the formerly highly productive veins of Gloster and Marysville, near Helena; the GraniteBimetallic mine, near Philipsburg; and many others. More commonly the quartz veins are rich in sulphides, which carry silver and gold in intimate combination with pyrite, galena, chalcopyrite, and zinc blende. Examples of this class are plentiful in almost all the mining counties, especially between Helena and Boulder, where the old camps of Alta, Comet, and Lump Gulch are located, and near Dillon, in Beaverhead County.

The vein systems of the Summit Valley district at Butte carry mainly copper, with small amounts of gold and silver, but there are also veins rich in silver and zinc.

Replacement deposits of silver-bearing galena occur in the Elkhorn district, southeast of Helena; at Barker, in the Little Belt Mountains; at Castle, in Meagher County, and at some other places, but the production obtained from these sources is not large at the present time.

The outlying uplifts in front of the main range contain some goldbearing deposits of importance associated with intrusions of granitic or monzonitic porphyries. Most prominent are those of the Moccasin and Warm Springs districts in Fergus County, which are replacement deposits of gold-bearing quartz and fluorite in limestone, and in the Little Rocky Mountains, where the altered porphyry itself contains disseminated free gold in irregular bodies.

In Park County, north of the Yellowstone Park, are a number of little-known mining districts that contain gold-bearing quartz veins in places associated with sulphides and at one locality (in the Sheepeater district) with tungsten ores.

Near the north end of the Idaho boundary line, in the slates of the Belt series, are a number of vein deposits which show a close relationship to the Cœur d'Alene types, and contain galena and in places chalcopyrite. Genetically they are probably connected with intrusions of quartz monzonites. Among these districts the Libby, Iron Mountain, and St. Regis are the best known.

Wherever the deposits carry visible gold, placers have been formed in the gravels at lower elevations. Though the placer production is not large now and is derived mainly from dredging operations in the Alder Gulch (Virginia City) district, Montana was formerly noted for its rich gold-bearing gravels, which were worked not only at the place 
mentioned but also near Helena, Diamond City, Bannock, and at many other places.

No important deposits of iron are known in Montana. Manganese has been mined at one or two places in Jefferson County. There are few notable deposits of rare metals, though tungsten ores are found at Butte and in the Sheepeater district.

\section{NEVADA.}

Nevada is one of the most important metal-mining States of the West and has produced large quantities of gold, silver, and lead. At present her production of precious metals is again rising rapidly, and although the output of lead is much smaller than formerly the State is now an important producer of copper. The mining districts number about 200 , and a glance at the map (PI. IX, p. 200) will show that they are irregularly scattered over the State. The Great Basin, of which Nevada forms the larger part, is characterized by a great number of north-south ranges of complicated structure, separated by gravel-filled valleys. There is scarcely one of these ranges that is not mineralized to some extent. The great variety and the scattered distribution of the districts renders it difficult to give an adequate description of the metallic resources of the State within the space here allotted.

The northern boundary line of Nevada lies within the province of the Columbia River lava, and these flows of basalt and rhyolite extend for some distance into the State. At the bend of the California State line the Sierra Nevada forms the boundary of Nevada and a small part of that great range is included within its limits. A small area at the extreme south end of the State is occupied by the horizontal strata of the plateau province. Elsewhere the Basin Ranges are the dominating features, except where they are partly obliterated by Tertiary lava flows.

The ranges are built up of a great variety of rocks. The pre-Cambrian basement is visible at few places except in the extreme southern part of the State, close to Colorado River, where granitic rocks of that age appear. East of a line drawn a little east of Winnemucca through Austin to a point somewhat west of Tonopah, Paleozoic strata are the predominating rocks and there is little or no Mesozoic material present. The typical section at Eureka shows 7,600 feet of Cambrian shale, quartzite, and limestone, 5,000 feet of Ordovician limestone with some quartzite, 6,000 feet of Devonian limestone, and 11,300 feet of Carboniferous shale, quartzite, limestone, and conglomerate. This enormous thickness indicates the ricinity of a shore line, and we find, in fact, that west of the line mentioned above the Paleozoic disappears and is supplanted by a thick series of Triassic and Jurassic sediments. During the Paleozoic era western Nevada was a land area discharging sediments to the east. The Mesozoic 
sediments that accumulated in western Nevada were derived from a land area of uplifted Paleozoic strata in the eastern part of the State. The Triassic is typically exposed in the Humboldt Range and its limestone, slate, sandstones, and associated flow rocks attain a thickness of 10,000 feet. The Jurassic is present in the range about Winnemucca, and also in the Humboldt Range. Its total thickness is considerable but not exactly known on account of extensive folding and metamorphism; it consists mainly of slates with some limestones.

Intrusive igneous rocks of the type of granodiorite, quartz monzonite, or corresponding porphyries are abundant but form smaller areas. These irregular masses or batholiths are most common in the western part of the State adjacent to California, but they also occur in the Paleozoic ranges of eastern Nevada. Their age appears to range from latest Jurassic to earliest Tertiary; those near the Sierra Nevada are probably the older. Tertiary flow rocks - andesite, rhyolite, and basalt - are widely distributed and cover large areas, some ranges being entirely built up of them. The lavas near the northern boundary have already been referred to. Another large field lies adjacent to the southwest corner of Utah, and from that vicinity toward the California boundary the lava flows are especially prominent. Some of the western flows are probably of Eocene age, but it is clear that the eruptions continued throughout the Tertiary period. The old lavas contain in some places small intrusions of monzonite.

The sedimentary rocks of the ranges have been folded and faulted. At some places the faulting is comparatively old, but at other places it is later than some of the lavas and is still visible in the topographic forms; in this case the ranges are apt to be monoclinal in structure.

The ore deposits fall into three classes according to their age and occurrence-pre-Cambrian, Cretaceous or early Tertiary, and middle or late Tertiary.

The pre-Cambrian deposits are very poorly represented. There is, in fact, only one occurrence which may with some confidence be placed in this class. It is in the Copper King district, near Bunkerville, Clark County, where basic dikes carry copper, nickel, and platinum.

The Cretaceous or early Tertiary deposits are invariably connected with intrusions of granodiorite or quartz monzonite, and as they have suffered much erosion since their formation the now accessible parts were formerly deeply buried. Gold, silver, copper, lead, and zinc are the principal metals.

There are several types in this class. Contact-metamorphic deposits between or near the boundary of limestone and intrusive rocks are not abundant nor have they yielded a great production; copper is the most prominent metal. They have been noted at Yerington (Mason district), in Lyon County; at Coppereid, in Churchill County; at Adelaide and the Jackson Mountains, in Humboldt County; at 
Cortez, in Lander County; at Bullion, Lone Mountain, Lime Mountain, and Contact, in Elko County; and at Ely and Ward, in White Pine County.

Replacement deposits of argentiferous galena in limestone or quartzite, usually situated at some distance from the contacts but apparently connected genetically with intrusive masses, have yielded a large but now diminishing output. At many places transitions into normal fissure veins are observed. To this class belong the celcbrated deposits at or near Pioche, in southern Nevada and those at Eureka, in Eureka County, although in the latter place there is some doubt about the genetic connection, for both rhyolite and acidic intrusive rocks occur in the district. Other districts are the Lida, Montezuma, and Southern Klondike districts, in Esmeralda County; the Rye Patch mine in the Humboldt Range, Humboldt County; Cortez and Mill Canyon in Lander County; Mineral Hill, in Eureka County; and many others.

There are all transitions between these replacement deposits and silver and lead bearing veins in quartz gangue, often accompanied by rich silver minerals; these may cut through the sedimentary or the intrusive rocks and they occur in so many places that it would be difficult to enumerate all. At Austin, in the Reese River district, silver-bearing veins cut granite; at Belmont, Nye County, they are contained in Paleozoic sediments in which granite is intruded; at the Horn Silver district, 26 miles south-southwest of Goldfield, the veins are contained in early Paleozoic beds. In the oxidized parts of these veins horn silver and rich sulphantimonites and arsenites usually occur in abundance, changing to poorer sulphides below water level.

In still another group of veins gold predominates, locally as native gold accompanjed by quartz gangue but more commonly in association with more or less sulphides and some silver. These veins are usually found in or near intrusive bodies. Examples of their occurrence are in the Silver Peak district, Esmeralda County; at Edgemont, in Elko County; at Osceola, in White Pine County; and in the Humboldt and Santa Rosa ranges, in Humboldt County. Many of the gold-bearing quartz veins occur in quartzite. At De Lamar, Lincoln County, for instance, a bed of quartzite penetrated by basic dikes has been impregnated with gold-bearing solutions and during its oxidation the gold was concentrated into a valuable mass of ore which for many years yielded a large production.

Pyritic disseminations, usually in porphyry, accompany this type of mineralization in many places, and where, as at Ely, White Pine County, these disseminations have been worked over by descending waters, important low-grade blanket deposits of chalcocite may be developed.

The various types mentioned may all occur in the same district, together forming a metallic aureole in and around intrusive masses. 
The Tertiary mineralization of lavas in Nevada occurred on a scale and with a diversity not equaled elsewhere in the Western States. It took place at several epochs, and in places each eruption was followed by the ascension of metal-bearing solutions, which penetrated and altered great areas of the porous volcanic rocks, causing what is termed propylitization. The main epoch of mineralization appears to have been in the Miocene. The deposits are in veins or shear zones; some of them in extensive and irregular masses of silicified lavas. They occur in andesite, latite, dacite, or rhyolite, but few of them in basalt. The metals are gold and silver, which in many places occur together, in a quartz and adularia gangue, more rarely, as at Bullfrog, accompanied by calcite. Native gold is not common below the water level, though it is present, for instance, in the Round Mountain and National districts; above the water level the oxidized material usually carries gold. Copper, lead, and zinc are subordinate.

Districts of this type are abundant in western Nevada but are in fact scattered all over the State. Veins in andesite constitute the great Comstock deposits, in Washoe County; those of Tonopah, Kawich, and Gold Crater, in Nye County; Tuscarora, Cornucopia, and Stafford, in Elko County; and Seven Troughs, in Humboldt County. Veins in rhyolite are abundant and usually carry gold and silver. As examples of such districts may be mentioned Fairview and Wonder, in Churchill County; Bullfrog, Silverbow, Eden, Cactus Springs, Wilson's Camp, Stonewall Mountain, and Wellington, in Nye County; Rawhide, Esmeralda County; Gold Circle, Elko County; and National, Humboldt County. The Goldfield mines, which in late years have yielded a large production of gold, are working irregular bodies of altered and silicified rocks in latite.

By far the greater part of the gold and silver production of Nevada is derived from these Tertiary veins.

Placers have yielded large amounts of gold in Nevada, but in the absence of an abundant supply of water they are difficult to work. Both Cretaceous and Tertiary deposits have contributed to their formation, though they are practically absent from many of the richest Tertiary deposits, such as the Comstock lode and the deposits of Tonopah and Goldfield.

The gold-bearing gravels have been worked at Tuscarora, in the Humboldt Range, at Round Mountain, at Battle Mountain, and at numerous other places.

Iron ores are not of frequent occurrence. Brown iron ores are reported from Montello, Cobre, and Moor, Elko County, and from Winnemucca, Humboldt County. Magnetite deposits are said to occur some distance east of Lovelocks, Humboldt County; in the Cortez Mountains, Eureka County; and near Bullion and Dolly Varden, Elko County. Little is known about their geologic relations. 
Nickel and cobalt ores occur in diorite and andesite of probably Mesozoic age, in the Cottonwood Canyon district, in the Humboldt Range.

Tungsten ores as wolframite or hübnerite are worked near Osceola, White Pine County, and near Browns station, in southwestern Humboldt County. In minor quantities the ore occurs in many Tertiary veins, for instance, at Tonopah and Round Mountain.

Quicksilver ore (cinnabar) is of widespread occurrence in western Nevada, in Humboldt, Nye, and Esmeralda counties, but its exploitation has been attempted only at Ione, in Nye County. The ore usually occurs in Tertiary deposits in rhyolite or andesite associated with stibnite, locally also with gold and silver. At Steamboat Springs, in Washoe County, the decomposed granite contains some cinnabar evidently deposited by hot springs. The deposits in the Humboldt Range are of uncertain age and occur in Triassic or Jurassic sedimentary rocks.

\section{NEW MEXICO.}

The ore deposits of New Mexico form a gradually widening belt extending through the central portion of the State from Colfax County, at the north, to the southwest corner; in this southern part the districts are scattered over a wide area between El Paso and Silver City. In spite of the fact that there are numerous districts, the production of New Mexico is not large. Gold, silver, copper, lead, and zinc are the principal metals of the output, and Grant and Socorro are the principal productive counties.

New Mexico contains several well-defined geologic provinces. Over the smaller eastern portion extend high plains or plateaus of horizontal or gently dipping Tertiary, Cretaceous, and Carboniferous sediments. About the middle of the State these almost connect with the similar strata of the plateau province which cover the northwestern part, their monotony being broken only by several minor uplifts like the Zuni Mountains.

Through the center of the State and trending north and south are a succession of ranges. Toward the Colorado boundary they are high and assume the type of the Rocky Mountain uplifts, with a central core of pre-Cambrian rocks surrounded by the upturned edges of Paleozoic and Mesozoic strata. Toward the south they are lower and are of the monoclinal type of the Basin Ranges, being limited on the east or west side by prominent faults. The preCambrian is less prominently exposed and the sediments comprise a thick series. In the central part of the State a thin bed of Cambrian quartzite is covered by a few hundred feet of Ordovician, 200 feet of Devonian shale, 100 feet of Mississippian, and 3,000 feet of Pennsylvanian limestone and sandstone, the upper part including some of the "Red Beds." Above this lies a small thickness of prob- 
ably Jurassic strata, and these are in turn covered by at least 3,000 feet of Cretaceous coal-bearing sandstones and shales. The southwest corner of New Mexico is occupied by a number of low ranges of the Arizona type, trending north-northwest, separated by wide desert plains and built up of folded and faulted Paleozoic and Mesozoic sediments; these ranges rarely have pronounced monoclinal structure. Lavas of late Tertiary age, chiefly andesitic and rhyolitic, cover a large space in the southwest, separating the province of the desert ranges from the plateau province. These lavas also extend toward the northeast and occupy large areas north of Santa Fe, but are here mainly basalts of latest Tertiary or even Quaternary age. Minor intrusions of quartz monzonites of late Cretaceous or early Tertiary age are known in nearly all the ranges and follow closely in their distribution the trend of the main mineral-bearing districts.

Deposits of pre-Cambrian age are known to occur in the northern ranges in Colfax, Taos, and Rio Arriba counties. Copper and gold are the principal metals. The deposits form either lenticular quartz veins or disseminations of sulphides in basic igneous rocks. At the Hamilton mine, in the Pecos district, Carboniferous strata cover the decomposed outcrops of a copper deposit of the latter kind. Many of the deposits contain minerals like garnet, tourmaline, and amphibole. The principal locality is that of the Hopewell and Bromide districts, in Rio Arriba County, where veins of this kind and placers derived from them have been mined.

In New Mexico, as elsewhere in the Western States, the most abundant deposits are those which are formed at the end of the Cretaceous or in the earliest Tertiary and which stand in close connection with granite or monzonite intrusions, surrounding them like metallic aureoles. There are several subdivisions of this genetic class which yield ores of gold, silver, copper, lead, and zinc.

Contact-metamorphic deposits in the silicate zone between limestone and intrusive rocks are more abundant than in any of the other States except possibly Arizona. They carry copper ores in the Elizabethtown, San Pedro, Organ, Jarilla, Hanover, and Hachita districts; lead and zinc ores are developed in the Magdalena and Tres Hermanas districts; iron ores at Hanover, Jones Camp, and other places. In all, 13 districts with such ores are known. Magdalena, San Pedro, and Hanover have yielded a considerable production of base metals.

Quartz veins almost invariably accompany the intrusions and are chiefly of the gold-bearing pyritic type, though in places they also carry silver, lead, and copper. The principal producing districts of this kind are Elizabethtown, Ortiz, White Oaks, and Pinos Altos. The silver veins form a smaller group, most of them occurring in Grant County Replacement deposits of argentiferous galena or sil- 
ver ores are found at some little distance from the intrusive contact and have been mined in the Organ, Hermosa, Kingston, Hillsboro, Lake Valley, Georgetown, Victorio, Chloride Flat, and Granite Gap districts.

In connection with this general type of mineralization near intrusive bodies, there are at a few places, such as the Burro Mountains and Santa Rita, in Grant County, disseminations of cupriferous pyrite which are too poor to mine in themselves, but which have been enriched by a Recent or late Tertiary deposition of chalcocite formed by descending waters. These deposits now form large bodies of low-grade copper ore which are the main source of the copper production of the State.

Placer deposits which formerly were highly productive are worked at Elizabethtown, in Colfax County, in the Ortiz and San Pedro Mountains, at Hillsboro, and at Pinos Altos. Most of them are derived from the veins of early Tertiary age.

A distinctly later mineralization than that described above developed in some places in the lavas of middle or late Tertiary age, shortly after their eruption. - It produced quartz veins carrying chiefly gold and silver and with certain characteristics indicating deposits near the surface. The principal districts are at Cochiti, in Sandoval County; at Hillsboro and in the Black Range, in Sierra County; and in the Mogollon district, in Socorro County. At present the principal production of gold and silver of the State is derived from the Mogollon district.

Concentrations of chalcocite in the sandstones of the "Red Beds" of Triassic or late Carboniferous age are found at Tecolote and other places in San Miguel County; in the Nacimiento and Zuni mountains, in the northwestern part of the State; at Estey, in Lincoln County; at Tularosa, in Otero County; and at several other places. The origin of these copper ores is still under discussion, but they have probably been concentrated by meteoric waters from small amounts of metal contained in the sandstones and this concentration has most likely been in progress ever since a water circulation was established in the strata involved. The deposits are in general independent of igneous rocks.

The iron ores consist chiefly of magnetite, occurring in contactmetamorphic deposits. They have been mined at Fierro, in Grant County. A bed of limonite in Cretaceous strata has also been mined near Glorieta, in Santa Fe County.

Bismuth ores occur in the San Andreas Range. Vanadium ores occur near Hillsboro and in the Caballo Range, in both places in connection with galena deposits in limestone. Some wolframite has been recovered from a quartz vein in limestone in the Victorio district, Luna County. 


\section{OREGON.}

The map (Pl. XI, p. 246) shows plainly that the metal deposits of Oregon are confined to two small and widely separated regions. The northeastern part of the State, including Baker and Grant counties, contains a number of districts yielding mainly gold and silver but also some copper. The southwest corner, including parts of Douglas, Josephine, Jackson, and Coos counties, holds the extreme north end of the California gold belt, and the mines here yield gold, though there are also a few copper-bearing districts.

With the exception of these relatively small areas, the whole State is covered or underlain by flows of late Tertiary lavas, mainly andesites and basalts, or, as along the western coast, by Tertiary or Cretaceous sediments. Here and there the older rocks are exposed under the lavas, as in the Puebla Mountains, near the Nevada boundary line, and here some older mineralization may appear. In a few places, as in the Bohemia district, in the southern Cascade Mountains, and at a few points to the north in the same range, mineralization appears in the andesite rocks, resulting in deposits of gold and silver or quicksilver, but this, too, is unusual.

In geologic structure both regions belong to the Pacific type in which considerable regional metamorphism has been superimposed upon Paleozoic and Mesozoic rocks.

In southwestern Oregon the rocks are essentially the same as in the Sierra Nevada and in northern California. The structural trend here swings around from a northwesterly to a northeasterly direction. The sedimentary rocks comprise Devonian, Carboniferous, and Jurassic strata, the thickness of which is difficult to measure on account of the intense compression to which they have been subjected. These beds are intruded by granodiorite and its porphyries, as well as by serpentine and greenstone; the intrusions are earlier than the Cretaceous rocks, which also have a considerable development in the region. The gold-bearing quartz veins and the placers derived from their eroded outcrops stand in close connection with the intrusive porphyries and greenstones. Most of them are situated near Ashland and Jacksonville and especially north of Grants Pass. There are a great number of loosely defined districts, among which may be mentioned the Greenback, Gold Hill, Applegate, Jalico, Grave Creek, and Waldo. In all these districts placers accompany the veins. A few scattered gold deposits are situated in Coos and Curry counties. Gold-bearing black sands are mined at Gold Beach, Port Orford, and other places, the fine gold having probably been transported to the sea by the river which drains the auriferous region.

Of the rarer metallic products, nickel ores have been mined at Riddles, in Douglas County. Platinum occurs rather commonly but 
only in small quantities in the gold-bearing gravels and beach sands and is probably derived from the serpentine rocks. Small quantities of this metal are recovered. Quicksilver occurs as low-grade cinnabar ore at Black Butte, in Douglas County.

In the Blue Mountains of northeastern Oregon, which stand out above the surrounding lava flows, there is a highly compressed and not easily measured section of Paleozoic rocks and an overlying less highly altered series of Triassic sediments, the latter extending northeastward across Snake River and into Idaho, by the Seven Devils copper district. These sedimentary rocks are intruded by a number of smaller masses of granodiorite, in and around which the gold-bearing quartz veins cluster. The veins as a rule carry more or less sulphides and contain more silver than the gold veins of the southwestern part of the State. The richest veins are those of the Cracker Creek district, near Sumpter; others in the same vicinity in Baker County are the Bonanza, Cable Cove, Elkhorn, Connor Creek, Virtue, and Cornucopia. The adjoining Grant County contains similar veins at Susanville, Quartzburg, Granite, and Canyon City. Placers accompany the veins, especially at Sumpter, Baker City, and Canyon City. The last-named place marks the west end of this goldbearing region, whose southeastern limit is at Humboldt Basin, in Malheur County.

\section{SOUTH DAKOTA.}

Cretaceous and Tertiary horizontal sediments cover almost the whole of the State of South Dakota, but in the southwest corner rises a prominent outlier of the Rocky Mountains, known as the Black Hills, which contains gold deposits of great economic importance. This group is similar to the uplifts of Wyoming and Montana, its center being an elliptical mass of pre-Cambrian schists and granites surrounded by outward-dipping Cambrian, Carboniferous, Jurassic, and Cretaceous beds. In the northern part of this uplift are masses, sheets, laccoliths, and dikes of porphyries of phonolitic, rhyolitic, and andesitic types, the age of which is considered to be Eocene.

Both pre-Cambrian and Tertiary (probably Eocene) ore deposits are found in the Black Hills. The pre-Cambrian deposits include lenticular veins in the schists, with quartz, native gold, and sulphides. Of these the most prominent example is the great Homestake mine at Lead, which is a low-grade but very large deposit of lenses in mica schist. These lenses of altered rock contain quartz stringers and much amphibole and carry native gold and auriferous sulphides. Somewhat similar but as yet not productive deposits occur in other parts of the pre-Cambrian area, in Lawrence, Pennington, and Custer counties. There are also low-grade copper depos- 
its consisting of pyritic disseminations in schists, but at present none of these are productive.

The placer deposits, which now yield but little, were derived from veins in the schists. The oldest placers were accumulated in the conglomerate at the base of the Cambrian, when the Paleozoic sea first transgressed over the disintegrated pre-Cambrian rocks. Quaternary gold-bearing gravels lie in the present stream beds.

In the southern part of the Black Hills the granitic pre-Cambrian rocks hold pegmatite dikes which contain cassiterite, wolframite, and various tantalum-columbium minerals, also amblygonite and other lithia-bearing minerals.

A type of gold ore entirely different from that of the pre-Cambrian is connected with the intrusive porphyries, and the mineralization which produced it is probably of early Tertiary age. These gold ores, which do not carry visible metal, are siliceous replacements of Cambrian dolomite limestone, locally also of quartzite and shale; they occur in flat shoots following the stratification and are connected with usually barren vertical fissures that are believed to have formed the ducts for the passage of the solutions which caused the deposits. Cyanidation is the usual process of treatment and the ores, though now of medium to low tenor in gold, contribute largely to the production of the region. The ores are confined mainly to the northern part of the Black Hills, in Lawrence County. Wolframite occurs in one or two places in connection with these refractory siliceous ores.

\section{TEXAS (WESTERN PART).}

The western or trans-Pecos part of Texas contains a number of small mining districts in El Paso, Presidio, and Brewster counties. The principal metal production is limited to silver and quicksilver.

From eastern New Mexico a chain of short ranges indicated by the White Mountains, the Sacramento Range, and the Guadalupe Mountains extends southward into western Mexico; they are built up of Carboniferous and Cretaceous strata with small bodies of igneous rocks. A little farther south, in Presidio and Brewster counties, is a large area of volcanic flows which extends southward into Mexico.

The scattered mining districts in El Paso County have not yet proved of importance. In Presidio County is the Shafter district, which for several years has produced a notable amount of silver from an irregular oxidized deposit in Carboniferous limestone. East of the volcanic area mentioned the Cretaceous strata contain many small areas of volcanic flow rocks, in or near which, at Mariscal and Terlingua, important quicksilver deposits are located. Tin ore is mined near El Paso in the Franklin Mountains, where it occurs in a granitic rock. 
The ore deposits of Utah are in the main confined to the western part of the State. They begin near the southwest corner of the State; thence the line between the productive and the barren region trends north-northwest, and in the latitude of Utah Lake and Salt Lake follows the crest of the Wasatch Range. The extreme northern part of the State, adjoining Idaho, contains few deposits. In the eastern region lie a few deposits of copper and rare metals in sandstone, on Grand River, a few iron deposits, and the gold-bearing district of the La Sal Mountains, near the Colorado boundary line.

Utah produces gold, silver, copper, and lead in large quantities and in the last few years the output of zinc ore has also been increasing.

The distribution of the districts corresponds to the two geologic provinces into which the State is divided. The unproductive area lies in the plateau province, in which Paleozoic, Mesozoic, and Tertiary strata are almost horizontal and igneous intrusions are generally absent. The ore-bearing area corresponds to the eastern part of the Great Basin, which is characterized by northward-trending ranges separated by desert plains. These ranges are built up of a thick series of Paleozoic sediments similar to that of eastern Nevada. Along the Wasatch Range Triassic, Jurassic, and Cretaceous rocks are also present. A typical section of the sedimentary strata comprises 10,000 or 12,000 feet of Cambrian quartzite, 1,500 feet of Silurian limestone, 1,250 feet of Devonian quartzite, 10,000 to 15,000 feet of Carboniferous limestone and quartzite, and finally about 5,000 feet of uppermost Carboniferous (Permian) or Triassic shales, sandstone, and limestone, the upper portion of which grades into the Mesozoic and consists in part of "Red Beds." In these folded and faulted sediments are numerous intrusions of granodiorite and quartz monzonite, and in places they are covered by Tertiary flow rocks like andesite, latite, and rhyolite. The largest area of intrusive rock is in Cottonwood Canyon, in the Wasatch Range, but even this is of comparatively small dimensions. The largest area of lavas is that of Beaver and adjacent counties in the southern part of the State, and this covers several thousand square miles. Toward the west it is continued across the Nevada line in the direction of Pioche.

The intrusive rocks are of post-Jurassic age; probably most of them date back to the late Cretaceous, but there is also reason to believe that granular rocks were intruded during early Tertiary time into some of the lofty volcanoes which at that time were situated in front of the Wasatch Range. . The effusive rocks-the lavas-began to pour out early in the Tertiary period, and the maximum stage of their eruption falls in the later part of that period, though the basaltic eruptions which followed the andesite and rhyolite continued well into Quaternary time. 
No pre-Cambrian ore deposits are known in Utah. As elsewhere the ores are, in a broad way, divided into those of Cretaceous age connected with intrusive rocks and those contained in lavas of late Tertiary age. Owing to the presence at some places of early Tertiary intrusions into volcanic cones, the line between these two classes is here and there difficult to define strictly.

Contact-metamorphic deposits carrying copper and some gold have been noted in the Frisco district, Beaver County; at Clifton, Tooele County; and in Cottonwood Canyon, Salt Lake County. Copper deposits allied to them are also found at Bingham. Replacement deposits of silver-bearing galena occur in many districts and are as a rule intimately connected with fissure veins. Examples are furnished by the districts of Park City, Tintic, West Tintic, Cottonwood, American Fork, Frisco, Star, Fish Springs, and Willow Springs. Replacement deposits of copper ores in limestone are worked at Bingham and Tintic. Copper ores disseminated in monzonite and accompanied by tourmaline are mined at the Cactus mine, in the Frisco district. Gold-bearing replacement deposits of limestone are mined on an extensive scale at Mercur, in the Oquirrh Range, and appear to be genetically connected with intrusive sheets of porphyry.

Gold and silver bearing fissure veins in or near intrusive rocks occur at many places, among which may be mentioned Bingham and Tintic, but they do not contribute a large share to the gold production of the State. At Bingham there are widespread impregnations of cupriferous pyrite, and its enrichment by chalcocite deposited by descending solutions has given rise to the great low-grade copper deposits now worked by open-cut methods.

Placers derived from veins and other deposits connected with intrusive rocks have yielded a small production in Utah. Such gravels have been worked at Bingham, in the La Sal Mountains, and along San Juan River.

Veins formed during the volcanic activity in middle Tertiary time occur mainly in andesites and rhyolites and carry chiefly gold and silver; the principal localities are in the Kimberly, Mount Baldy, and Ohio districts, in the volcanic center situated in Piute County; similar veins are found in the Stateline district, bordering on Nevada, in Iron County.

Differing from all the preceding types are the metal-bearing sandstone ("Red Beds") of the plateau province. They show no evidence of genetic connection with igneous rocks. Silver and copper ores of this kind have been mined at Harrisburg (Silver Reef), in Washington County, and similar copper ores in sandstone are reported from Uinta County, in northeastern Utah. Vanadium and uranium ores occur on Grand River near the Colorado line.

Some quicksilver is found in the gold ores of the Mercur or Camp Floyd district, from which some production of this metal has been 
reported, and a selenide and a sulphoselenide of mercury occur in veins of the Ohio district, near Marysvale.

Iron ores are found at several points. In the Iron Springs district, Iron County, and at Bull Valley, Washington County, are considerable bodies of magnetite in deposits of contact-metamorphic origin, between porphyry and limestone. A hematite deposit in Carboniferous limestone occurs at Rhodes Plateau, in Summit County, in the Uinta Mountains. Brown iron ores in small masses are not uncommon. One of these deposits, contained in probably Mesozoic sandstone, occurs in the northeast corner of the State near Vernal

\section{WASHINGTON.}

The metal production of the State of Washington includes gold, silver, copper, and lead, but the output of each metal is comparatively small. The southern half of the State and the Olympic Peninsula are almost wholly covered by Tertiary basaltic flows or by Cretaceous and Tertiary sediments barren of metal deposits. In contrast to this, the northern part of the Cascade Range, as well as the mountainous region extending eastward to Idaho along the international boundary, is built up of metamorphosed Paleozoic sediments, mainly slates with smaller masses of limestone, into which a number of granitic masses were intruded during late Cretaceous and early Tertiary time. Lavas of Tertiary age cover these rocks in a number of places.

Ore deposits of pre-Cambrian age have not been reported from Washington but may possibly occur in the Kettle River region, near the Canadian boundary. In the late Tertiary lavas, in spite of the large space they occupy, mineralization is of rare occurrence. There are, however, three prominent districts in the deposits of this kind, all in andesitic rocks. The first is at Monte Cristo, in Snohomish County, in the Cascade Range, where gold-bearing veins occur with abundant sulphides and arsenopyrite. The second is the Republic district, in Ferry County, in the northeastern part of the State, where gold-bearing veins with chalcedonic quartz still yield a notable production. The third is the Pierre Lake district, at Orient, in Stevens County, the output of which has been rather large for several years.

Practically all the other metal deposits of Washington are of greater geologic age and were probably formed shortly after the intrusion of granodiorite and similar rocks in the Paleozoic sediments at the close of the Cretaceous period. Extensive erosion of the upper parts of the veins has left exposed the deeper-seated portions. There are scattered districts of this kind in the Cascade Range, but none of them have as yet an important production. The Blewett or Peshastin district, on the eastern slope of the Cascades in Chelan County, is the best known. Rich gold ores have also been found in 
the Mount Baker district, in the northern part of the Cascade Mountains, where gold-bearing quartz veins associated with dikes of granodiorite have cut through peridotite and Paleozoic sediments.

Most of the producing districts are situated in Okanogan, Ferry, and Stevens counties, and many of them lie close to the Canadian boundary. Contact-metamorphic deposits carrying copper are represented by those at Buckhorn Mountain, in Okanogan County, and at the Belcher Camp, in Ferry County, near Republic. Silver-lead veins occur at Conconully (Ruby Hill), in Okanogan County; auriferous quartz veins on the Methow, in the same county, and in many other smaller districts. Lead ores, without much silver, occurring as replacements in limestone, are present near Bossburg and Colville and in the Metaline district, all in Stevens County, in the northeast corner of the State. There exists here between the town of Chewelah and Clark Fork of the Columbia a large body of granite intrusive in Paleozoic slates and limestones, and it is held that the numerous deposits in this part of the State were formed shortly after this intrusion.

The State contains few placer-mining districts of importance. Among those which yielded much gold in the early days may be mentioned the Swauk and Peshastin districts.

Iron ores are present at a number of places, but are not as yet utilized. Magnetite is found at Snoqualmie Pass, in King County, in connection with metamorphosed limestone, and on Skagit River, in the northern Cascades, as lenses in slate. Chromiferous magnetite appears at Clealum, in Kittitas County, on the contact between sandstone and serpentine. Brown iron ore and bog iron is found at several places in Stevens County-for instance, near Colville and Chewelah.

Of the rarer metals, nickel occurs in quartzose ores near Keller, Ferry County. Cassiterite or oxide of tin has been found in granite near Spokane. Wolframite, an important tungsten ore, occurs at the same place, and also in larger quantities, together with some bismuth minerals, in the quartz veins of the Deer Park and Spring Dale (Cedar Canyon) districts, in Stevens County. Arsenic has been recovered from the pyritic veins of the Monte Cristo district. A quartz vein carrying molybdenite has been discovered in the granite near Lake Chelan.

\section{WYOMING.}

The State of Wyoming contains few and scattered metal-bearing districts, and its production is limited to a little gold and copper. Wide stretches of high plains or high plateaus underlain by horizontal Tertiary and Cretaceous sediments extend over the eastern and southwestern parts. The several uplifts of the Rocky Mountains traverse the State in a northwesterly direction and comprise a number of high ranges, usually exposing a large core of pre-Cambrian 
rocks surrounded by upturned Paleozoic and Mesozoic sediments. There is little or no evidence of the late Mesozoic intrusions which in other parts of the West were so effective in promoting mineralization.

In the southern part of the State are the Laramie Hills, the Medicine Bow Range, and the Sierra Madre. In the northwestern part rise the Wind River, Teton, and Bighorn ranges, as well as the high plateaus of the Yellowstone Park region, the latter covered by a large area of effusive Tertiary lavas. If uplift and active water circulation produce mineral deposits, they surely ought to be abundant in this State, and if the metals could be concentrated by such circulation the pre-Cambrian igneous rocks, which undoubtedly contain traces of metals, would offer a source for such concentration. The general absence of ores of date later than the uplifts goes far to prove that metallization is dependent on other conditions.

Near the eastern boundary of the Yellowstone Park are some districts which have produced little and whose geologic features are not well known. Of the districts investigated practically all contain deposits of pre-Cambrian age, inclosed in the highly altered pre-Cambrian sediments or in the large masses of granitic and basic rocks intruded with them before the beginning of the Paleozoic era. The Laramie Hills contain many low-grade copper deposits in lenticular veins and disseminations in schists and granite. In the Medicine Bow Range are several districts yielding gold and copper; in the adjacent Sierra Madre, near the Colorado line, is the well-known Encampment district, which has yielded much copper and gold from veins and irregular deposits of pyritic ores enriched by chalcocitization in or near pre-Cambrian gabbros. Copper deposits are also found in similar rocks south of Casper. At several places the pre-Cambrian contains lenticular quartz veins with native gold; the best known deposits of this kind are at Atlantic and South Pass, in Fremont County. Placers below rich veins have been worked at the places mentioned and in several districts in the Medicine Bow Range. The basal conglomerate of the Cambrian in the region adjacent to the Black Hills is said to contain some placer gold.

Iron ores are found in the pre-Cambrian rocks. Iron Mountain, north of Cheyenne, in Laramie County, is a huge dike of ilmenite, in anorthositic rock, as yet not utilized. At Hartville, in the same county, lenses of hematite are worked, lying between the pre-Cambrian and the overlying Carboniferous. They are considered to be of preCambrian age. Similar deposits are said to occur near Rawlins and in the Seminoe Mountains, in Carbon County.

There are few deposits of rare metals in Wyoming. Platinum, palladium, and other platinum metals are found associated with copper minerals in the Rambler mine, near Holmes, in Albany County. They are reported to be present in the concentrates in paying quantities. 


\title{
PART II. CATALOGUE OF MINING DISTRICTS.
}

\author{
By James M. Hit.L.
}

\section{ACKNOWLEDGMENTS.}

The writer is indebted to members of the United States Geological Survey who have supplied much first-hand information on many mining districts about which little has been known or published. The maps have been inspected by the men most familiar with the regions covered by them and have thus gained much in accuracy of location. To Mr. H. D. McCaskey the writer is indebted for advice as to method and arrangement and for constant cooperation throughout the work of compilation. Mr. Waldemar Lindgren has been a never-failing source of information. His careful serutiny of the maps, lists, and text has added greatly to their value. Sincere thanks are due to him for his help and for the geologic introduction to this bulletin.

\section{PLAN OF THE WORK.}

In 1907 the United States Geological Survey issued, as a part of its publication "Mineral resources of the United States," a map showing by means of dots the relative location of the gold, silver, copper, lead, zinc, and quicksilver mining districts of the Western States. This map was published in one large sheet covering all the States west of the one hundred and third meridian, and the symbols used on it made no distinction for the predominant metal produced in each camp, with the exception of quicksilver.

Since the publication of that map many new districts have been added to the lists of producers. The Survey has also had the opportunity to study many regions about which there was little authentic information. The present bulletin is a revision and correction of the original map, based on the latest available information, with some added features which it is hoped will be useful to engineers, geologists, and others interested in the metal-mining industry of the Western States.

It is the hope of the writer that those interested will cooperate in the correction of mistakes and omissions in both maps and lists by notifying the Geological Survey of any errors they may discover. 
The map of each State is published separately in order that it may be more easily handled. The scale of each map is about 40 miles to the inch. The State of California, on account of its large size, has been mapped in two parts, the dividing line being an entirely arbitrary one along the county lines south of the Mother Lode region. In California there are no mining districts as the term is used in other States-that is. there are no recorded boundaries to a particular district. The Survey has arbitrarily made the subdivision into districts for convenience of reference, the mines in a certain locality or near a particular town or camp being included in one district.

The location of the mining district is indicated on the maps by a symbol and a number. The different symbols indicate as far as possible the most important metal produced in each camp. This classification, however, was not always possible, as in some districts several metals are of equal importance, and other districts are in the prospect stage and their predominant metals are not certain or are unknown.

The number with each symbol refers to the list of the districts printed on the margin of each map and to the bibliography of that State. These lists are arranged alphabetically by counties and each map has one consecutive series of numbers.

The map of each State is accompanied by a few pages in which more information is given than can be shown on the map. This consists of the following items:

1. A number, which corresponds to the number given on the map.

2. The name and subnames of the district.

3. A list of the abbreviations for the metals produced in the camp, in the order of their importance. In this list the following abbreviations are used for the different metals:

\begin{tabular}{|c|c|c|}
\hline $\begin{array}{l}\text { Au. . . . . Gold. } \\
\text { Ag. . . . . . Silver. } \\
\text { Cu. . . . . Copper. } \\
\text { Pb.... . . Lead. } \\
\text { Zn . . . . . Zinc. } \\
\text { Hg. . . . . Quicksilver. } \\
\text { Fe . . . . . Iron. } \\
\text { Cr. . . . . Chromium. }\end{array}$ & $\begin{array}{l}\text { Ti..... . Titanium. } \\
\text { Mn .... . Manganese. } \\
\text { Ni...... Nickel. } \\
\text { Co...... Cobalt. } \\
\text { W..... Tungsten. } \\
\text { Sb..... Antimony. } \\
\text { As.......Arsenic. }\end{array}$ & $\begin{array}{l}\text { Bi. . . . . Bismuth. } \\
\text { U... . . . Uranium. } \\
\text { V...... . Vanadium. } \\
\text { Sn ... . . . Tin. } \\
\text { Mo . . . . Molybdenum. } \\
\text { Pt.... . . Platinum. } \\
\text { Pd. . . . . Palladium. }\end{array}$ \\
\hline
\end{tabular}

The last 12 metals are classed as rare, and where they are the most important metals the district is shown on the map as a rare-metal district. Metals found in the district but of relatively slight importance are indicated by the appropriate abbreviations in parentheses.

For some districts the list of metals produced is followed by "Pl," signifying that the production is largely from placer mines, or by "Pl and D" or " $\mathrm{D}$ and $\mathrm{Pl}$," signifying that the production is from both deep and placer mines, the more important source being placed first. In 
all districts not marked in this way the production is derived from deep mines.

4. The shipping point of the camp, with the distance and direction of the camp from it and the railroad on which it is located-for example, " 14 miles NNE. Winnemucca, S. P. R. R." The distance is estimated from a town in or from the center of the mining district, and where possible is the number of miles along the route followed by the United States mail. It is entirely possible and in many cases probable that there may be some shorter means of approach to the camp than that given, but some uniform method had to be adopted for the measurement of distances. Many of the distances given do not represent the distances as measured on the map, because roads are winding and a mail route may touch several other towns in its course to a given locality.

The following abbreviations are used for the railroads of the Western States:

A. \& M. R. R. R. . . . . . . . . . . Arcata \& Mad River Railroad.

A. \& N. M. R. R................. Arizona \& New Mexico Railway.

A. \& S. R. R..................... Arizona \& Swansea Railroad.

A. E. R. R....................... Arizona Eastern Railroad.

Am. C. R. R................... Amador Central Railroad.

Ar. C. R. R.................. Argentine Central Railway.

A. S. R. R.....................Arizona Southern Railroad.

A. T. \& S. F. R. R.................Atchison, Topeka \& Santa Fe Railway.

B. \& L. R. R.................... . Boca \& Loyalton Railroad.

B. \& S. R. R . . . . . . . . . . . . . . Barnwell \& Searchlight Railway.

B. A. \& P. R. R.................. . Butte, Anaconda \& Pacific Railway.

B. B. \& B. C. R. R................ Bellingham Bay \& British Columbia Railroad.

B. C. R. R.................... Butte County Railroad.

B. G. R. R.................. Bullfrog Goldfield Railroad.

B. H. \& F. P. R. R............. Black Hills \& Fort Pierre Railway (Chicago, Burlington \& Quincy Railroad).

C. \& N. W. R. R................ Chicago \& North Western Railway.

C. \& P. S. R. R.................. Columbia \& Puget Sound Railroad.

C. \& S. R. R.................. Colorado \& Southern Railway.

C. \& W. R. R.................... Colorado \& Wyoming Railway.

C. B. \& Q. R. R.................. Chicago, Burlington \& Quincy Railroad.

G. G. W. R. R................... Chicago Great Western Railroad.

C. M. \& P. S. R. R............... . . Chicago, Milwaukee \& Puget Sound Railway.

C. M. R. R................... Colorado Midland Railway.

C. R. \& S. J. R. R... . . . . . . . . . . Crystal River \& San Juan Railway.

C. R. I. \& P. R. R................ Chicago, Rock Island \& Pacific Railway.

C. R. R. of O ................ Central Railroad of Oregon.

C. S. \& C. C. R. R............. . . Colorado Springs \& Cripple Creek District Railway.

D. \& R. G. R. R................. Denver \& Rio Grande Railroad.

D. B. \& W. R. R................. Denver, Boulder \& Western Railroad.

D. N. W. \& P. R. R................ Denver, Northwestern \& Pacific Railway.

E. \& P. R. R................... Eureka \& Palisade Railway.

E. P. \& S. W. R. R.............. El Paso \& Southwestern Railroad.

E. R. R. of N. M................ Eastern Railway of New Mexico. 
G. \& P. R. R

Gilmore \& Pittsburgh Railroad.

G. C. R. R

Grand Canyon Railway.

G. N. R. R

Great Northern Railway.

G. V. G. \& N. R. R.............. Gila Valley, Globe \& Northern Railway.

I. \& W. N. R. R................. Idaho \& Washington Northern Railroad.

I. N. R. R..................... Idaho Northern Railway.

L. H. P. \& P. R. R............... Laramie, Hahns Peak \& Pacific Railway.

I. V. \& T. R. R.................. Las Vegas \& Tonopah Railroad.

N. C. B. R. R .................. Nevada Copper Belt Railroad.

N. C. N. G. R. R................. Nevada County Narrow Gage Railroad.

N. C. O. R. R . . . . . . . . . . . . . Nevada-California-Oregon Railway.

N. C. R. R

Nevada Central Railroad.

N. N. R. R

Nevada Northern Railway.

N.P.R. R

Northern Pacific Railway.

N. W. P. R. R

Northwestern Pacific Railroad.

O. \& E. R. R

Oregon \& Eureka Railroad.

O. \& S. E. R. R

Oregon \& Southeastern Railroarl.

O. S. L. R. R

Oregon Short Line Railroad.

O. W. R. R. \& N. Co

Oregon-Washington Railroad \& Navigation Co.

P. \& I. N. R. R

. Pacific \& Idaho Northern Railway.

P. C. R. R

Pacific Coast Railway.

P. R. R. I. \& P. Co

Portland Railway, Light \& Power Co.

P. T. S. R. R

Port Townsend Southern Railroad.

R. C. B. H. \& W. R. R........... . Rapid City, Black Hills \& Western Railroad.

R. G. S. R. R.................. Rio Grande Southern Railroad.

R. G. W. R. R ................. Rio Grande Western Railway.

R. R. V. R. R.................. Rogue River Valley Railway.

St. L. R. M. \& P. R. R............ . St. Louis, Rocky Mountain \& Pacific Railway.

S. \& E. R. R................. Saratoga \& Encampment Railway.

S. \& I. E. R. R................ Spokane \& Inland Empire Railroad.

S. D. \& C. R. R................. San Diego \& Cuyamaca Railway.

S. F. C. R. R................... Santa Fe Central Railway (New Mexico Central Railroad).

S. F. P. \& P. R. R................ . Santa Fe, Prescott \& Phoenix Railway.

S. I. \& M. R. R................ . . . Salt I Lake \& Mercur Railroad

S. N. R. R

Silverton Northern Railroad.

Sil. P. R. R

Silver Peak Railroad.

S. P. \& S. R. R.................. Spokane, Portland \& Seattle Railway.

S. P. L. A. \& S. L. R. R.......... San Pedro, Los Angeles \& Salt Irake Railroad.

S. P. R. R...................... Southern Pacific Railroad.

S. R. R......................... Silverton Railway.

S. R. R. of Cal .................. Sierra Railway of California.

Sier. V. R. R.................. Sierra Valley Railroad (Sierra \& Mohawk Railway).

Sump. V. R. R................. Sumpter Valley Railway (Oregon).

S. V. \& E. R. R................. Sacramento Valley \& Eastern Railroad.

T. \& G. R. R..................... Tonopah \& Goldfield Railroad.

T. \& P. R. R................... Texas \& Pacific Railway.

T. \& T. R. R.................. Tonopah \& Tidewater Railroad.

T. B. R. R.................... Twin Buttes Railroad.

T. E. R. R.................... Tacoma Eastern Railroad.

U. P. R. R.................... Union Pacific Railroad.

U. R. R....................... Uintah Railway.

U. V. \& P. R. R.................. United Verde \& Pacific Railway.

V. \& T. R. R..................... Virginia \& Truckee Railway. 
W. \& M. R. R. R................ Wyoming \& Missouri River Railroad.

W. \& N. W. R. R . . . . . . . . . . . . . Wyoming \& Northwestern Railway.

W. I. \& M. R. R................. Washington, Idaho \& Montana Railway.

W. P. R. R.................. Western Pacific Railway.

Y. R. R.................... Yreka Railroad.

Y. V. R. R.................... Yosemite Valley Railroad.

5. A statement of the geologic formation of the region, intended to give simply a general idea of the rocks in which the deposits occur. The attempt has been made to name the oldest rocks first, using the terms pre-Cambrian schists, granite, or complex. The term complex is used in districts where schist, gneiss, and granite are found in intimate association. The Paleozoic or Mesozoic limestones, slates, quartzites, etc., are all combined under the terms Paleozoic or Mesozoic sediments. Intrusive rocks are said to "cut" the older formations, and volcanic flows "cap" the other formations. Where the relation is not known only the names of the rocks are given, as Paleozoic sediments, granite, andesite. In placer districts the gravels have been classed as follows:

Stream gravels, those of the present stream channel.

Terrace gravels, those of the present stream but found above the present level of the stream.

Beach gravels, confined to the shores of the Pacific Ocean.

Glacial gravels, moraines and outwash from the glaciers.

Tertiary river gravels, like the buried channels of the Sierra Nevada in California.

6. The kind of deposits found in the region. No attempt has been made to discuss the deposits; simply their general character is mentioned in the belief that it may be of use. The following terms have been used with the meanings indicated, which are sanctioned by the United States Geological Survey:

Vein.-A single body of minerals occupying or following a fissure, both walls of which generally, though not invariably, are well defined. Where several veins are so closely spaced that the ground between them becomes in places ore bearing and in its whole width constitutes an ore body the assemblage is called a lode. In this bulletin the term vein is used for both veins and lodes.

Contact-metamorphic deposits.-Ore deposits which occur at or near the contacts of intrusive rocks with sedimentary beds and which carry minerals characteristic of contact metamorphism, such as garnet, pyroxene, and epidote.

Replacement deposits.-Masses of ore and gangue formed by the alteration of limestone, dolomite, and other rocks. Usually irregular in form and in many places grading into country rock.

Disseminated deposits.-Deposits containing ore minerals scattered throughout the rock, such as chalcocite occurring in grains through granite porphyry. The term "impregnations" is sometimes applied to deposits of this type.

Stockwork.-A deposit consisting of a complex system of small fissure veins.

Lenses.--Roughly tabular bodies which diminish in size vertically and horizontally.

7. A bibliography of Survey publications relative to the district. It is fully realized that many articles bearing on the metal industry which appear in mining, engineering, and scientific journals are of great 


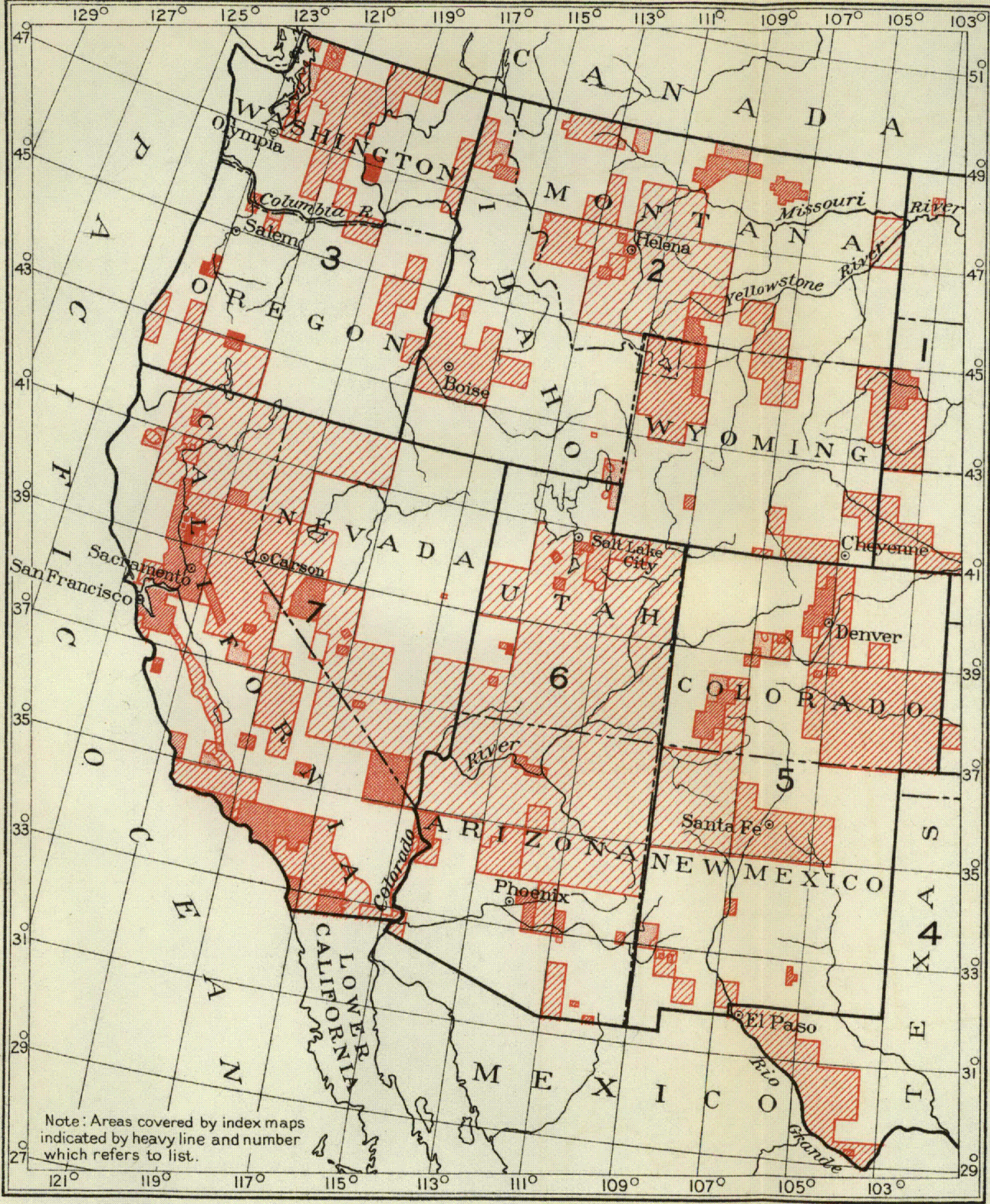

MAP OF THE WESTERN STATES, SHOWING AREAS COVERED BY

TOPOGRAPHIC SURVEYS AND THE SCALE EMPLOYED FOR EACH AREA

$100 \quad 0 \quad 100 \quad 200 \quad 300$ Miles




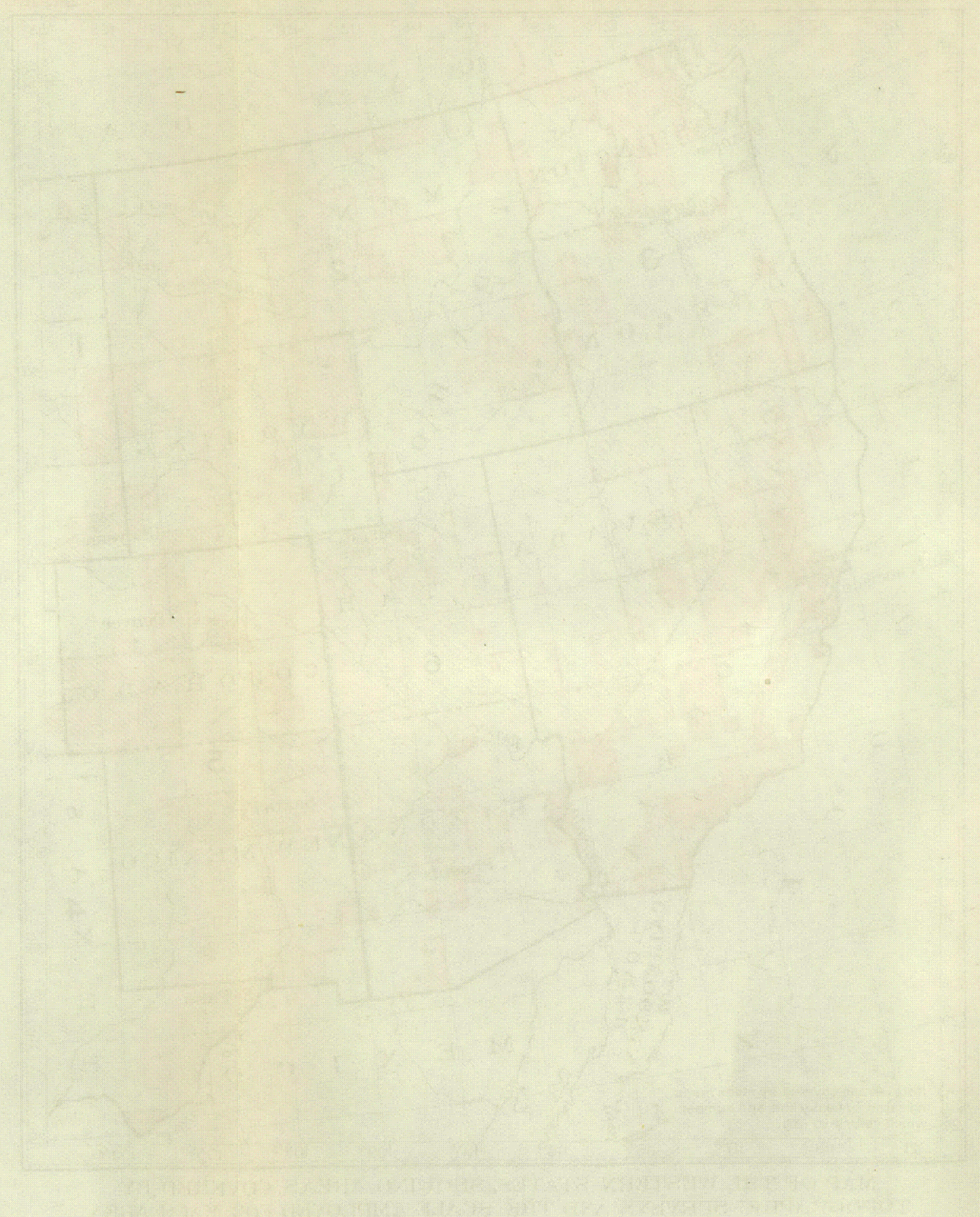


value and should be included in any complete bibliography of the mining industry of the United States. The great mass of literature bearing on the subject of mining and the relatively short time available for this revision and compilation made it necessary to limit the publications listed, except as stated below, to those issued by the United States Geological Survey. These consist of several series, which are indicated by the following abbreviations:

M......... . Monograph.
P. P....... Professional Paper.
Bull...... Bulletin.'
W. S. P.... . Water-Supply Paper.

Min. Res.....Mineral Resources. ${ }^{1}$ Folio........Folio of Geologic Atlas.

Top. sheet.. Sheet of Topographic Atlas.

Colorado, South Dakota, Washington, and Wyoming are the only States of the Cordilleran region which maintain geological surveys. Their publications are referred to in the list.

The following example will show the method of presenting the information indicated in the preceding paragraphs. The district is in New Mexico.

9. Organ. $\mathrm{Pb}, \mathrm{Ag}, \mathrm{Cu}$.

15 miles NE. Las Cruces, A. T. \& S. F. R. R., stage line.

Paleozoic sediments cut by quartz monzonite.

Veins, contact metamorphic, replacements.

Lindgren, W., and Graton, L. C., A reconnaissance of the mineral deposits in

New Mexico: Bull. 285, 1906, pp. 74-86.

This interpreted means: The deep mines of the Organ district, which is numbered 9 on the map of New Mexico, produce lead, silver, and copper, lead predominating. The central part of the district is 15 miles northeast of the shipping point, Las Cruces, on the Atchison, Topeka \& Santa Fe Railway, with which it is connected by stage. The region is underlain by Paleozoic sediments intruded by quartz monzonite. The ores occur in veins, in contact-metamorphic deposits at the contacts of the sediments and intrusive rock, and as replacement bodies in the sediments. Notes on the district are given on pages 74 to 86 of Bulletin 285 of the Geological Survey, published in 1906. (Other Survey publications on this district are cited in the list on p. 230.)

The Thirty-second Annual Report of the Director of the Survey eontains two maps showing the areas covered by geologic and topographic surveys. Plates I and II are adapted from these maps and show what has been accomplished by the Survey in the mapping and study of the geology of the Western States. Plate I shows the areas covered by topographic surveys. In this work the unit of survey is a quadrangle 15 minutes, 30 minutes, or 1 degree in extent each way, covering an area of one-sixteenth, one-fourth, or one

\footnotetext{
'In the series of bulletins entitled "Contributions to economic geology" and in the volumes of Mineral Resources are given bibliographies on the several metals.
} 


\section{Explanation of Plate II.}

The numbers indicate Survey publications, as shown in the following list:

\section{ARIZONA.}

1. Bisbee, P. P. 21, Folio 112.

2. Bradshaw Mountains, Folio 126.

3. Clifton, P. P. 43, Folio 129.

4. Globe, P. P. 12, Folio 111. CALIFORNIA.

1. Bidwell Bar, Folio 43 .

2. Big Trees, Folio 51.

3. Colfax, Folio 66 .

4. Downieville, Folio 37.

5. Jackson, Folio 11.

Mother Lode district, Folio 63.

6. Lassen Peak, Folio 15.

7. Marysville, Folio 17.

8. Placerville, Folio 3.

9. Pyramid Peak, Folio 31.

10. Redding, Folio 138.

11. Sacramento, Folio 5.

12. San Iuis, Folio 101.

13. Santa Cruz, Folio 163.

14. Smartsville, Folio 18.

Nevada City, Folio 28.

15. Sonora, Folio 41.

16. Truckee, Folio 39.

\section{COLORADO.}

1. Anthracite, Folio 9.

2. Apishapa, will be folio.

3. Aspen, M. XXXI, $\$ 3.60$.

4. Crested Butte, Folio 9.

5. Durango, will be folio.

6. Elmoro, Folio 58.

7. Engineer Mountain, Folio 171.

8. Georgetown, P. P. 63.

9. La Plata, Folio 60.

10. Leadville, M. XII, $\$ 8.40$.

11. Needle Mountains, Folio 131.

12. Nepesta, Folio 135.

13. Ouray, Folio 153.

14. Pikes Peak, Folio 7. Cripple Creek, P. P. 54.

15. Pueblo, Folio 36 .

16. Rico, Folio 130.

17. Silverton, Folio 120.

18. Spanish Peaks, Folio 71.

19. Telluride, Folio 57.

20. Tenmile, Folio 48.

21. Walsenburg, Folio 68.

22. Breckenridge, P. P. 75. IDAFO.

1. Bitterroot Range and Clearwater Mountains, P. P. 27

2. Boise, Folio 45 .

3. Cour d'Alene, P. P. 62.

4. Nampa, Folio 103 .

5. Silver City, Folio 104.

\section{MONTANA.}

1. Butte, Folio 38, P. P. 74.

2. Fort Benton, Folio 55.

4. Little Belt Mountains, Folio 56.

5. Livingston, Folio 1.

6. Marysville, P. P. 57.

7. Philipsburg, will be folio and P. P. 78.

8. Three Forks, Folio 24.

\section{NEVADA.}

1. Bullfrog, Bull. 407.

2. Comstock lode, M. III, $\$ 11 ;$ IV, $\$ 1.50$.

3. Ely.

4. Eureka, M. VII, $\$ 1.20$; VIII, $\$ 1.10$; $\mathrm{XX}, \$ 5.25$.

5. Goldfield, P. P. 66 .

6. Silver Peak, P. P. 55.

7. Tonopah, P. P. 42.

8. Southwestern Nevada and eastern California, Bull. 308.

\section{NEW MEXICO.}

Geology and ore deposits, P. P. 68.

1. Silver City, will be folio.

\section{OREGON}

1. Coos Bay, Folio 73.

2. Crater Iake, P. P. 3.

3. Port Orford, Folio 89.

4. Riddles, will be folio.

5. Roseburg, Folio 49.

\section{SOUTH DAKOTA.}

1. Belle Fourche, Folio 164, P. P. 65

2. Deadwood, P. P. 26, will be folio.

3. Edgemont, Folio 108.

4. Oelrichs, Folio 85.

5. Rapid, P. P. 26, will be folio.

TEXAS.

1. El Paso, Folio 166.

UTAF.

1. Bingham, P. P. 38.

3. Tintic, Folio 65 .

WASHINGTON.

1. Ellensburg, Folio 86, P. P. 19.

2. Mount Stuart, Folio 106.

3. Snoqualmie, Folio 139, P. P. 19.

4. Tacoma, Folio 54.

WYOMING.

1. Aladdin, Folio 128, P. P. 65.

2. Bald Mountain, Folio 141.

3. Canyon, M. XXXII, $\$ 2.45$.

4. Cloud Peak, Folio 142.

5. Crandall, M. XXXII, $\$ 2.45$.

6. Dayton, Folio 141.

7. Devils Tower, Folio 150.

8. Encampment, P. P. 25.

9. Fort McKinney, Folio 142.

10. Gallatin, M. XXXII, $\$ 2.45$.

11. Hartville, Folio 91.

12. Ishawooa, M. XXXII, $\$ 2.45$.

13. Lake, M. XXXII, $\$ 2.45$.

14. Laramie, Folio 173.

15. Newcastle, Folio 107.

16. Sherman, Folio 173.

17. Shoshone, M. XXXII, $\$ 2.45$.

18. Sundance, Folio 127.

19. Bighorn Basin, P. P. 53.

20. Bighorn Mountains, P. P. 51. 

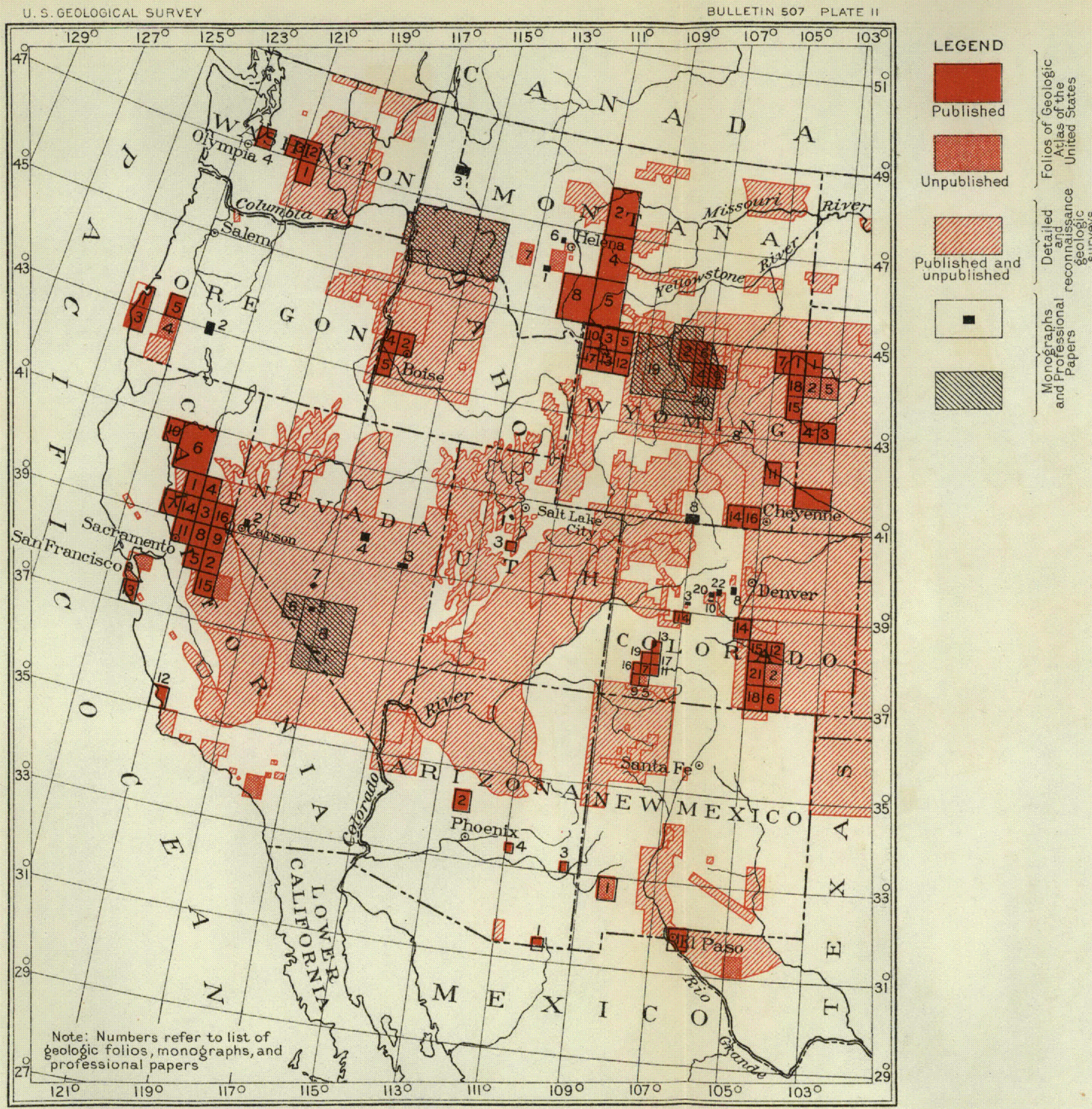

MAP OF THE WESTERN STATES, SHOWING AREAS COVERED BY GEOLOGIC SURVEYS AND KIND OF WORK DONE

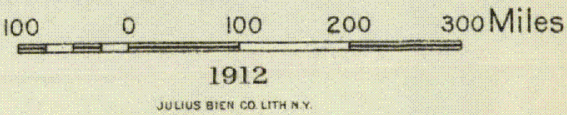



square degree. A quadrangle is designated by the name of some well-known place or feature in it. The unit of publication is an atias sheet $16 \frac{1}{2}$ by 20 inches, and each sheet is a topographic map of one quadrangle. As the atlas sheets are uniform in size, the greater the area covered the smaller the scale of the map. The scale of the 1degree sheet is $1: 250,000$, that of the 30-minute sheet is $1: 125,000$, and that of the 15-minute sheet is $1: 62,500$. The names of adjoining quadrangles for which sheets have been published are printed on the margins. The cultural features, such as roads, railroads, cities, and towns, as well as all lettering, are shown in black; the water features in blue; the features of relief-hills, mountains, etc.-by brown contour lines. The contour interval varies with the scale of the map and the relief of the country. Some maps of areas that are economically important are not in conformity with the general scheme outlined above.

"Index maps" on a scale of about 40 miles to the inch, showing the location and names of quadrangles represented by published topographic sheets, also names and numbers of geologic folios, have been prepared and may be obtained free on application to the Director of the Survey at Washington. As it is impossible to show distinctly the names of the quadrangles on Plate $I$, the areas covered by index maps are indicated by heavy lines and by numbers which correspond to those in the subjoined list:

Form No.

1. North and South Dakota..................... 9-323 q

2. Montana, Idaho, and Wyoming.................... 9-323 k

3. Oregon and Washington......................... 9-323o

4. Texas and Oklahoma........................... 9-323 j

5. Colorado and New Mexico.................... 9-323 m

6. Utah and Arizona............................... 9-323 1

7. California and Nevada....................... 9-323 n

The topographic maps are sold at 5 cents a sheet of standard size, or $\$ 3$ a hundred if at least 100 are ordered at one time. Prepayment is required, and may be made by money order payable to the order of the Director of the United States Geological Survey, or in stamps or cash-the exact amount.

Plate II shows the areas covered by geologic surveys. The numbers refer to the list of folios, professional papers, and monographs on the page facing the plate.

Geologic maps of the quadrangles covered by the topographic maps are generally published in the form of folios. On these maps the areal geology, underground structure, and mineral deposits are represented by colors and patterns. Each folio contains other maps, descriptive text, etc. The price of folios is 25 cents each, as a rule; a few that are unusually large are sold for 50 cents. A general circular on geologic folios may be had on application. 
Professional papers are distributed free of charge on application to the Director of the Geological Survey, It sometimes happens, however, that they are out of stock; in that case they can usually be obtained from the Superintendent of Documents, Government Printing Office, Washington, D. C., at a nominal cost. Monographs are sold at the prices indicated in the list.

It is impossible to indicate the areas described in the other publications of the Survey. Those which deal particularly with mining are "Mineral Resources" and some of the bulletins. A complete list of Survey publications, revised quarterly, may be had on application to the Director.

\section{CLASSIFICATION OF MINING DISTRICTS.}

Fourteen States west of the one hundred and third meridian are represented in this report as producers of gold, silver, copper, lead, zinc, quicksilver, iron, chromium, manganese, and the rare metals.

In these States there are more than 1,479 mining districts recognized by the United States Geological Survey, that number being shown on the maps. Some districts are not shown owing to lack of knowledge or lack of space. The scale of the map limits the number of mining districts which can be shown as distinct units. In Gilpin County, Colo., there are about 12 districts, which have been grouped into three on the map. The Mother Lode region of California is another example of the necessity for combining districts on account of lack of space.

The classification of the districts by predominant metals was made on the basis of the production of the districts for the period from 1902 to 1908, as reported to the United States Geological Survey. The following table shows the number of districts in each State which produce the several metals, as thus classified:

Distribution of the mining districts in the Western States according to the predominant metal.

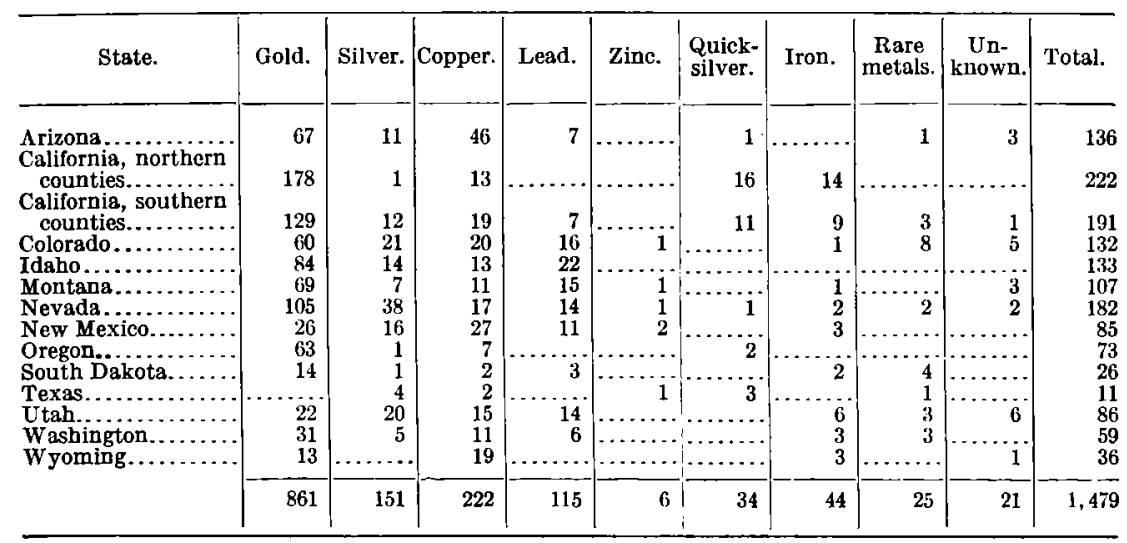


This table takes no account of the other metals found in a camp. In most regions several metals are produced, and in many districts it is difficult to say which is the most important product. Where there are few mines the production of one of them may greatly influence the classification. For instance, in a copper camp one producer may obtain a large amount of gold from his ore. In case this gold value exceeds the value of all the copper produced in the camp it would be classed as a gold district, even though it is generally spoken of as belonging to the copper class. The silver camps are particularly open to question, for there are few mines producing principally silver, yet in 151 districts in the Western States silver has a greater value than the other metals produced. The table is inserted for the reason that it gives the number of districts in each State in which one metal has a greater value than the others produced.

The above statement is applicable to the tabulated statements by counties for each State.

For a truer record of the relative importance of the metals produced by the mining districts of the Western States the reader is referred to the volumes of "Mineral Resources" published by the United States Geological Survey. 


\section{ARIZONA.}

In 12 counties of Arizona there are 136 mining districts. Of these the predominant metal is unknown in 3, and in 1 tungsten minerals are.produced exclusively; 67 districts produce more gold than any other metal, 46 more copper, 11 more silver, and 7 more lead. Quicksilver is recovered in 1 district.

Distribution of the predominant metals produced in the mining districts of Arizona.

\begin{tabular}{|c|c|c|c|c|c|c|c|c|}
\hline County. & Gold. & Silver. & Copper. & Lead. & $\begin{array}{l}\text { Quick- } \\
\text { silver. }\end{array}$ & $\begin{array}{l}\text { Rare } \\
\text { metals. }\end{array}$ & $\begin{array}{c}\text { Un- } \\
\text { known. }\end{array}$ & Total. \\
\hline \multirow{9}{*}{ 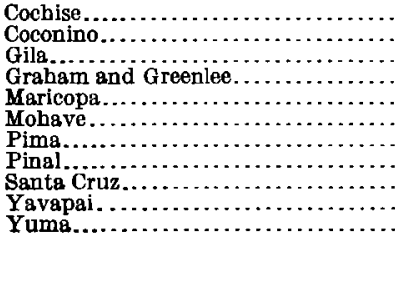 } & 5 & & 5 & 1 & & 1 & 1 & 13 \\
\hline & 4 & & $\begin{array}{l}3 \\
6\end{array}$ & & & & & 10 \\
\hline & $\mathbf{1}$ & 1 & 5 & 1 & & & & 8 \\
\hline & $\begin{array}{r}4 \\
19\end{array}$ & 2 & $\begin{array}{l}1 \\
2\end{array}$ & 2 & & & $\cdots$ & $\begin{array}{r}5 \\
25\end{array}$ \\
\hline & 5 & 1 & 8 & 1 & & & $\ddot{2}$ & 17 \\
\hline & 4 & 1 & 5 & & & & & 10 \\
\hline & 3 & & 2 & & & & & 11 \\
\hline & $\begin{array}{r}16 \\
6\end{array}$ & & $\begin{array}{l}4 \\
5\end{array}$ & $\dddot{2}$ & $\dddot{i}$ & & & $\begin{array}{l}20 \\
14\end{array}$ \\
\hline & 67 & 11 & 46 & 7 & 1 & 1 & 3 & 136 \\
\hline
\end{tabular}

\section{MINING DISTRICTS IN ARIZONA.}

[See Pl. III. Additional references may be found in Mineral Resources for 1910 and 1911.]

\section{COCHISE COUNTY.}

1. California (Paradise). $\mathrm{Pb}, \mathrm{Ag}, \mathrm{Cu}, \mathrm{Au}$.

15 miles NW. Rodeo, N. Mex., E. P. \& S. W. R. R.

Paleozoic (?) limestones cut by granite porphyry.

Contact metamorphic, veins.

Min. Res. 1905, pp. 144-145.

1906, p. 154.

1907, pt. 1, p. 160.

1908, pt. 1, p. 297.

1909, pt. 1, p. 242.

2. Courtland (Turquoise). $\mathrm{Cu}, \mathrm{Au}, \mathrm{Ag}$.

Station E. P. \& S. W. R. R.

Paleozoic sediments cut by granite porphyry.

Replacements, veins in porphyry.

Ransome, F. L., The Turquoise copper-mining district, Arizona: Bull. 530, 1912.

Min. Res. 1905, p. 146.

1906, p. 156.

1907, pt. 1, p. 162.

1908 , pt. 1, pp. 205, 298.

1909 , pt. 1, pp. 167, 243.

3. Dos Cabezas. Au, Ag, Pb, Fe, Cu. (D, P1.)

15 miles E. Willcox, S. P. R. R.

Min. Res. 1906, p. 155.

1907, pt. 1, p. 161.

1908, pt. 1, p. 297.

1909 , pt. 1, p. 242. 
UST OF MINING DISTRICTS

COCHISE COUNTY

California (Paradise)

Dos Cabezas

Huachuca (Hartford)

Johnson
Pearce
Teviston

Tombstone
Warren (Bisbe)

Whetstone (Benson)

Winchester

COCONINO COUNTY

Francis

5. Grand Canyon
6. Jacobs Lake (Kaibab

GILA COUNTY

Banner (Troy)
Black Warrior

Christmas (Saddle

Mountain, San Carlos)
Dripping Springs

1.. Ellison

23. Green Valley (Payson

25. Miami
26. Pinto Creek

GRAHAM AND GREENLEE

27. Aravaipa

Claek)

Copper Mountain

Greenlee (Metcalf)

Lone St
Stanley

MARICOPA COUNTY

Agua Fria
Cave Creek

San Domingo (location

Vulture

MOHAVE COUNTY

(Omitted)

Bontley

Cedar Valley 1Owens
Cerbat (Hualpai)

Chemehuevis

Eldorado Pas

48. Gold Basin

50. Gold Road (Sar

51. Hackberry

52. Lual pai

Mineral Park (Hualpai) Music Mountains

Signal (Greenwood)

Union Pass (Sanalpa

Francisco)

64. Vivian (San Francisco)

PIMA COUNTY

66. Ajo

9aboquivari
Cababi (Comobabi)

1. Empire

3. Gunsight

15. Montezuma (Sonoita

Mountains

78. Pima(Olive T

9. Rincon

8.. Silver Bell

PINAL COUNTY

3. Bunker Hill (Copper Creek)
Gold Mountains (Galiuro)

5. Mammoth

Mineral Hill (location

8. Old Hat (Oracle)

S. Silver King
2. Vekol (Casa Grande)

SANTA CRUZ COUNTY

93. Harshaw

94. Mowry
95. Nogales
96. Oro Blanco

97. Palmetto

San Cayetano

Washington (Duquesne)
Whatec)

Wrightson

Agua Fria

105. Big Bug
106. Black Canyon

107. Black Hills

8. Black Rock
B. Cherry Creek

Congress

2. Del Rio

13. Eureka

15. Peck

8. Tiger (Hatrington)

. Tip Top

Weaver (Rich Hill)

White-Picacho

YUMA COUNTY

4. Castle Dom
5. Cienega

Harcuvar

27. Harquahala
128. Kofa (Humbug, Polaris)

129. La Fortuna
130. Laguna

Planet

32. Plomosa

Swansea

6. Vicksburg
Weaver (La Paz)

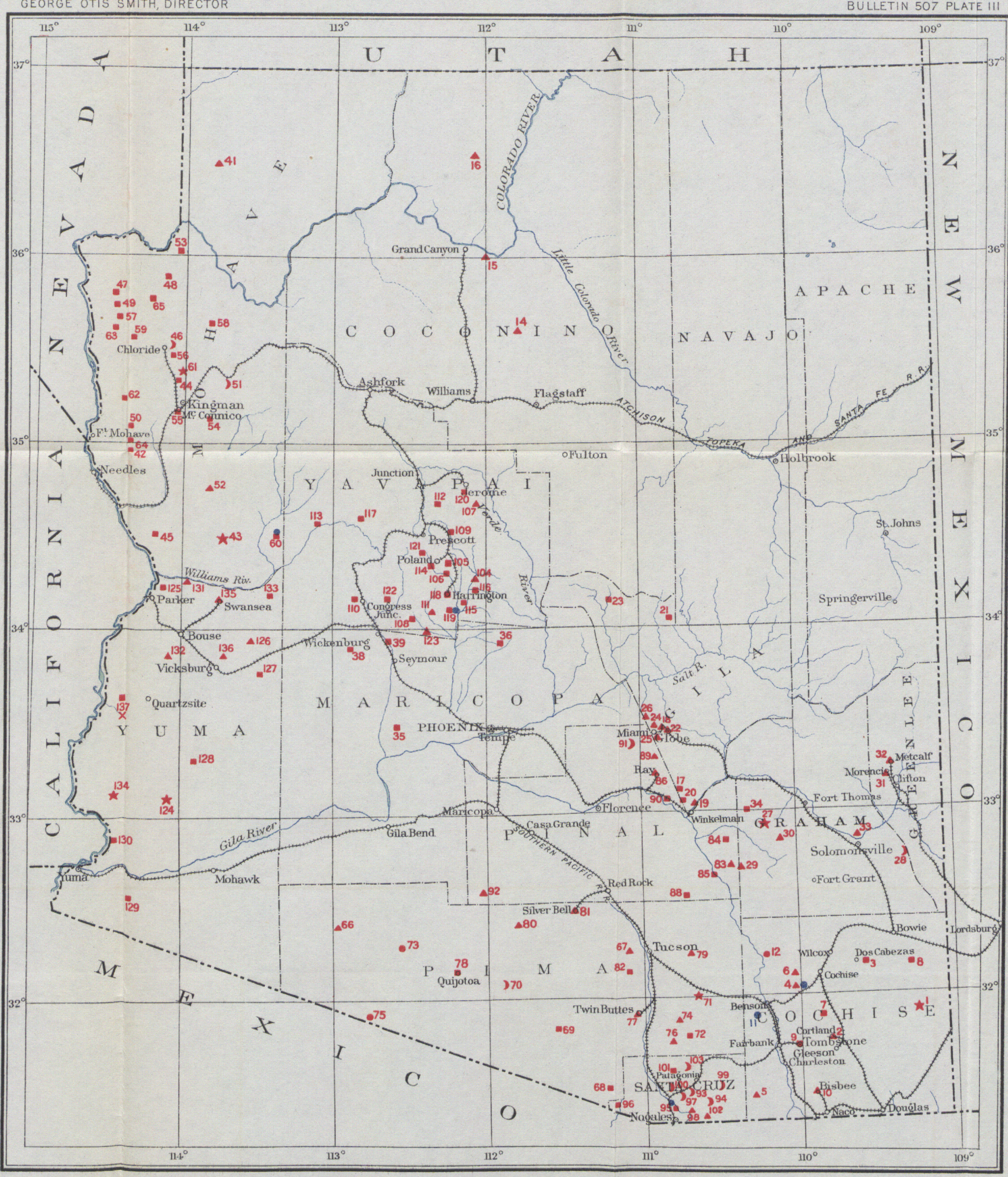

MAP OF ARIZONA, SHOWING LOCATION OF MINING DISTRICTS

Scale $\frac{1,500,000}{2,500}$

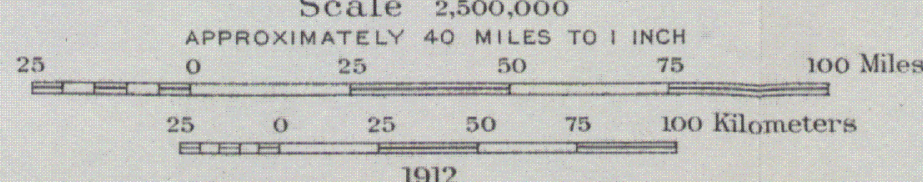

LEGEND

old predominant

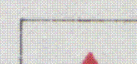

Copper predominant

>

$+$

Lead predominant

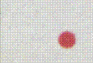

Gold, silver, copper, lead

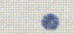

Quicksilve 

COCHISE COUNTY-Continued.

4. Dragoon. $\mathrm{Cu}, \mathrm{Au}, \mathrm{Ag}, \mathrm{W}$.

Station S. P. R. R.

Paleozoic limestone cut by granite.

Contact metamorphic, veins.

Min. Res. 1899, p. 300.

1900 , p. 257.

1904, pp. 329-330.

1905, p.p. 145, 412 .

1906, p. 525.

1908, pt. 1, p. 725 .

1909 , pt. 1, p. 577.

5. Huachuca (Hartford). $\mathrm{Cu}, \mathrm{Ag}, \mathrm{Au}$.

12 miles W. Hereford, E. P. \& S. W. R. R.

Min. Res. 1907, pt. 1, p. 161.

1908 , pt. 1, p. 297.

1909, pt. 1, p. 242.

6. Johngon (Cochise). $\mathrm{Cu}, \mathrm{Au}, \mathrm{Ag}, \mathrm{Pb}$.

9 miles W. Cochise, S. P. R. R., stage.

Paleozoic sediments cut by granite porphyry.

Replacements, contact metamorphic.

Min. Res. 1905, p. 145.

1906, pp. 154-155.

1907 , pt. 1, p. 160.

1908 , pt. 1, p. 297.

1909, pt. 1, p. 242.

7. Pearce. Au, Ag.

Station A. E. R. R.

Rhyolite.

Veins.

8. Teviston (?). All, Ag, $\mathrm{Pb}$.

16 miles SE. Bowie, 10 miles WSW. San Simon, S. P. R. R.

Min. Res. 1905, p. 145.

1908 , pt. 1, p. 298.

1909 , pt. 1, p. 242.

9. Tombstone. $\mathrm{Au}, \mathrm{Ag}, \mathrm{Pb}, \mathrm{Zn}, \mathrm{Cu}$.

Station E. P. \& S. W. R. R.

Paleozoic and Mesozoic sediments cut by porphyry.

Veins, replacements.

Harder, E. C., Manganese deposits of the United States: Bull. 380, 1908, p. 274; Bull. 427, 1910, pp. 151-152.

Min. Res. $1883-84$, p. 425.

1885 , p. 258.

1905, pp. 145-146.

1906, pp. 155-156.

1907, pt. 1, p. 161.

1908, pt. 1, p. 298.

1909 , pt. 1, pp. $242-243$.

Top. sheet Tombstone special.

0. Warren (Bisbee). $\mathrm{Cu}, \mathrm{Au}, \mathrm{Ag}$.

Station E. P. \& S. W. R. R.

Paleozoic limestone cut by granite porphyry.

Replacements, probably of contact-metamorphic origin. 
COCHISE COUNTY-Continued.

10. Warren (Bisbee)-Continued.

Douglas, J., Cupola smelting of copper in Arizona: Min. Res. 1883-84, pp. 397-410.

Ranzome, F. L., Copper deposits of Bisbee, Ariz.: Bull. 213, 1903, pp. 149157.

The geology and ore deposits of the Bisbee quadrangle, Arizona: P. P. 21, 1904

Weed, W. H., The copper mines of the United States in 1905: Bull. 285, 1906, pp. 99-100.

Min. Res. 1882, pp. 221-224.

1883-84, pp. 334-336.

1885 , p. 215.

1886 , p. 116.

1887 , p. 74 .

1888 , p. 58.

1904, pp. 234-235, 385-387.

1905 , pp. 143-144, 352.

1906, pp. 156-158.

1907 , pt. 1, pp. 162-164, 593-594.

1908, pt. 1, pp. 201-202, 298-299.

Folio 112, 1904.

1909 , pt. 1, pp. 166, 243-244.

11. Whetstone (Benson). W.

12 miles SSW. Benson, S. P. R. R.

Granite.

Veins.

Hess, F. L., Wolframite in the Whetstone Mountains, Arizona: Bull. 380, 1909, pp. 164-165.

Min. Res. 1906, p. 525.

1908, pt. 1, p. 725.

12. Winchester.

20 miles N. Benson, S. P. R. R.

Min. Res. 1909, pt. 1, p. 245.

13. Yellowstone (?). $\mathrm{Au}, \mathrm{Ag}, \mathrm{Cu}, \mathrm{Pb}$.

Location not known.

Min. Res. 1907, pt. 1, p. 164.

\section{COCONIRO COUNTY.}

14. Francis. $\mathrm{Cu}, \mathrm{Ag}, \mathrm{Au}$.

Williams, A. T. \& S. F. R. R.

Min. Res. 1906, p. 158.

1907, pt. 1, p. 164 .

1909 , pt. 1, p. 245.

Top. sheet San Francisco Mountains.

15. Grand Canyon. $\mathrm{Cu}, \mathrm{Au}$.

12 miles E. Grand Canyon, A. T. \& S. F. R. R.

Carboniferous sediments.

Replacement shear zones.

Emmons, S. F., The copper in the red beds of the Colorado Plateau region:

Bull. 260, 1905, pp. 221-232.

Min. Res. 1906, p. 158.

1907 , pt. 1, p. 164

1908, pt. 1, p. 299.

Top. sheet San Francisco Mountains. 


\section{cocorno codNTY-Continued.}

16. Jacobs Lake (Kaibab Plateau). Cu.

180 miles S. Marysville, Utah, R. G. W. R. R.

Paleozoic sediments.

Veins, disseminations.

Emmons, S. F., The copper in the red beds of the Colorado Plateau region Bull. 260, 1905, p. 229.

Weed, W. H., The copper deposits of the United States in 1905: Bull. 285, 1906, p. 103.

Top. sheet Kaibab.

GIIA COUNTY.

17. Banner (Troy). $\mathrm{Au}, \mathrm{Ag}, \mathrm{Cu}$.

8 miles NE. Kelvin, A. E. R. R.

Paleozoic sediments cut by diabase, quartz monzonite, and porphyry.

Replacements.

Top. sheet Ray.

18. Black Warrior. $\mathrm{Cu}, \mathrm{Ag}$.

Station A. E. R. R.

Pre-Cambrian schist, dacite, and dacite tuff.

Disseminated.

Min. Res. 1905, p. 147.

Top. sheet Globe.

Folio 111.

19. Christmas (Saddle Mountain, San Carlos). $\mathrm{Cu}, \mathrm{Au}, \mathrm{Ag}$.

9 miles N. Winkelman, A. E. R. R.

Altered Paleozoic sediments cut by porphyry.

Contact metamorphic.

Min. Res. 1905, pp. 154-155.

1906, pp. 160, 169-170.

1907, pt. 1, pp. 167, 177, 598.

1908 , pt. 1, p. 300 .

20. Dripping Springs. Au. 1909 , pt. 1, p. 246.

15 miles E. Kelvin, A. E. R. R.

Paleozoic sediments cut by diabase and monzonite porphyry.

Veins.

Top. sheet Ray.

21. Ellison. Au, Ag.

112 miles SE. Jerome, U. V. \& P. R. R.

Top. sheet Holbrook.

22. Globe. $\mathrm{Cu},(\mathrm{Au}, \mathrm{Ag})$.

Station A. E. R. R.

Pre-Cambrian schist; Paleozoic sediments cut by granite, quartz monzonite, and diabase.

Replacements, veins, and disseminations.

Douglas, J., Cupola smelting of copper in Arizona: Min. Res. 1883-84, pp. $397-410$.

Ransome, F. L., The Globe copper district, Arizona: P. P. 12, 1904.

Weed, W. H., The copper mines of the United States in 1905: Bull. 285, 1906, pp. 100-101.

Min. Res. 1882, pp. 221-224.

1883-84, pp. 334-338.

1885 , p. 215. 
GILA CoUTTY-Continued.

22. Globe--Continued.

Min. Res. 1887, p. 74.

1888 , p. 58.

1904, pp. 236-237.

1905, pp. 146-147, 353 .

1906, pp. 159-160, 390-391.

1907 , pt. 1, pp. 165-167, 595-597.

1908 , pt. 1, pp. 204, 300.

1909, pt. 1, pp. 166, 245-246.

Top. sheet Globe.

Folio 111, 1905.

23. Green Valley (Payson). All, Ag.

104 miles SE. Jerome, U. V. \& P. R. R.

Min. Res. 1909, pt. 1, p. 246.

Top. sheet Verde.

24. Lost Gulch. $\mathrm{Cu}, \mathrm{Pb}, \mathrm{Au}, \mathrm{Ag}$.

8 miles NW. Black Warrior, A. E. R. R.

Paleozoic limestone cut by quartz monzonite and diabase.

Replacements.

Min. Res. 1907, pt. 1, p. 167.

1908, pt. 1, p. 300 .

1909, pt. 1, p. 246.

25. Miami. $\mathrm{Cu}, \mathrm{Ag}$.

Station A. E. R. R.

Pre-Cambrian schist; Paleozoic sediments cut by granite and granite porphyry.

Disseminated.

Douglas, J., Cupola smelting of copper in Arizona: Min. Res. 1883-84, pp. $397-410$.

Ransome, F. I., The Globe copper district, Arizona: P. P. 12, I904.

Weed, W. H., The copper mines of the United States in 1905: Bull. 285, 1906, pp. 100-101.

Min. Res. 1882, pp. 221-224.

1883-84, pp. 334-338.

1885, p. 215.

1887, p. 74.

1888 , p. 58.

1904, pp. 236-237.

1905, pp. 146-147, 353.

1906, pp. 159-160, 370-371.

1907, pt. 1, pp. 165-167, 595-597.

1908 , pt. 1, pp. 204, 300.

Top. sheet Globe.

Folio 111, 1904.

26. Pinto Creek. $\mathrm{Cu}, \mathrm{Ag}, \mathrm{Au}$.

10 miles NW. Black Warrior, A. E. R. R.

Pre-Cambrian schists cut by granite and granite porphyry.

Disseminations.

Min. Res. 1908, pt. 1, p. 300. 
GRAFAM AND GREENLEE COUNTEES.

27. Aravaipa. $\mathrm{Pb}, \mathrm{Cu}, \mathrm{Ag}, \mathrm{Au}$.

75 miles NW. Willcox, S. P. R. R.; 30 miles SSE. San Carlos, A. E. R. R. Min. Res. 1909, pt. 1, p. 247.

28. Ash Peak. Ag, Au.

12 miles W. Seldon, A. \& N. M. R. R.

Min. Res. 1906, p. 161.

1907 , pt. 1, p. 168.

1908, pt. 1, p. 301.

1909 , pt. 1, p. 247.

29. Bunker Hill (Copper Creek). $\mathrm{Cu}, \mathrm{Au}, \mathrm{Ag}, \mathrm{Pb}, \mathrm{Fe}$.

36 miles SE. Winkelman, A. E. R. R.

Limestone, diorite, rhyolite.

Disseminated, contact metamorphic.

Min. Res. 1905, p. 148.

1907 , pt. 1, p. 168

30. Clark. $\mathrm{Cu}, \mathrm{Al}, \mathrm{Ag}, \mathrm{Pb}$.

35 miles W. Safford, A. E. R. R.

Min. Res. 1906, p. 161.

1909 , pt. 1, p. 247.

31. Copper Mountain (Morenci). Cu, Au, Ag.

Station A. \& N. M. R. R.

Paleozoic sediments cut by acidic porphyry.

Contact metamorphic, veins, disseminated.

Douglas, J., Cupola smelting of copper in Arizona: Min. Res. 1883-84, pp. 397-410.

Lindgren, W., Copper deposits at Clifton, Ariz.: Bull. 213, 1903, pp. 133-140. 1905 .

Lindgren, W., and Hillebrand, W. F., Minerals from (lifton-Morenci, Ariz.: Bull. 262, 1905, pp. 42-54.

Weed, W. H., The copper mines of the United States in 1905: Bull. 285, 1906, pp. 101-102.

Min. Res. 1882, pp. 221-224.

1885 , p. 215.

1886 , p. 116.

1887, p. 75.

1888 , p. 58.

1904, pp. 235-236.

1905, pp. 148-149, 353.

1906, pp. 161-162, 387-389.

1907 , pt. 1, pp. 168-170, 594-595.

1908 , pt. 1, pp. 203-204, 301.

Top. sheet Clifton.

1909 , pt. 1, pp. 166, 247.

Folio 129, 1905.

32. Greenlee (Metcalf). $\mathrm{Cu}, \mathrm{Au}, \mathrm{Ag}$.

Station A. \& N. M. R. R.

Paleozoic sediments cut by acidic porphyries.

Contact metamorphic, veins, and disseminated.

Douglas, J., Cupola smelting of copper in Arizona: Min. Res. 1883-84, pp. 397-410.

Lindgren, W., Copper depoeits at Clifton, Ariz.: Bull. 213, 1903, pp. 133-140. 
GRAHAM AND GREENLEE COUNTIES-Continued.

32. Greenlee (Metcalf)-Continued.

Lindgren, W., The copper deposits of the Clifton-Morenci district, Arizona: P. P. 43, 1905.

Lindgren, W., and Hillebrand, W. F., Minerals from the Clifton-Morenci district, Arizona: Bull. 262, 1905, pp. 42-54.

Weed, W. H., The copper mines of the United States in 1905: Bull. 285, 1906, pp. 101-102.

Min. Res. 1882, pp. 221-410.

1885 , p. 215.

1886, p. 116.

1887 , p. 75 .

1888 , p. 58.

1904, pp. 235-236.

1905, pp. 148-149, 353.

1906, pp. 161-162, 387-389.

1907, pt. 1, pp. 168-170, 594-595.

1908, pt. 1, pp. 203-204, 301.

Top. sheet Clifton.

1909, pt. 1, pp. 166, 247.

Folio 129, 1905.

33. Lone Star. $\mathrm{Cu}, \mathrm{Au}, \mathrm{Ag}$.

7 miles N. Solomonsville, A. E. R. R.

Min. Res. 1906, p. 162.

1907, pt. 1, p. 170

1908 , pt. 1, p. 301

1909 , pt. 1, p. 248

34. Stanley. Au, Ag, Pb.

18 miles SSE. San Carlos, A. E. R. R.

Min. Res. 1905, p. 149.

1906, p. 162.

MARICOPA COUNTY.

35. Agua Fria. Au.

25 miles W. Phoenix, S. F. P. \& P. R. R., A. E. R. R.

Veins (?).

Min. Res. 1905, p. 149.

1906, p. 162.

1907, pt. 1, p. 171.

1908, pt. 1, p. 302.

36. Cave Creek. $\mathrm{Au}, \mathrm{Cu}, \mathrm{Ag}$.

30 miles NNE. Phoenix, S. F. P. \& P. R. R., A. E. R. R.

Pre-Cambrian granite and schists cut by basic dikes.

Veins.

Min. Res. 1904, p. 149.

1908, pt. 1, p. 302.

$\cdot 1909$, pt. 1, p. 248.

37. San Domingo. $\mathrm{Cu}, \mathrm{Au}, \mathrm{Ag}$.

Hot Springs Junction, S. F. P. \& P. R. R.

Min. Res. 1908, pt. 1, p. 302.

1909, pt. 1, p. 248. 
MARICOPA COUNTY-Continued.

38. Vulture. $\mathrm{Au}(\mathrm{Cu}, \mathrm{Ag})$.

20 miles SW. Wickenburg, S. F. P. \& P. R. R.

Pre-Cambrian schist cut by diabase.

Veins.

Min. Res. 1905, p. 150.

1908 , pt. 1, p. 302.

1909 , pt. 1, p. 248.

39. Wickenburg. Au, Ag.

Station S. F. P.\& P. R. R.

40. Omitted.

MOHAVE COUNTY.

41. Bentley. $\mathrm{Cu}, \Lambda \mathrm{g}(\Lambda \mathrm{u})$.

65 miles ESE. Moapa, Nev., S. P. L. A. \& S. L. R. R.

Sediments.

Replacements.

Min. Res. 1905, p. 150.

1906, p. 163.

1907, pt. 1, p. 172.

1908 , pt. 1 , p. 303 .

1909 , pt. 1, p. 249.

Top. sheet Mount Trumbull.

42. Boundary Cone. Au, Ag.

12 miles NE. Needles, Cal., A. T. \& S. F. R. R.

Tertiary volcanics.

Veins.

Schrader, F. C., Mineral deposits of the Cerbat Range, Black Mountains, and Grand Wash Cliffs, Mohave County, Ariz.: Bull. 340, 1908, p. 82; Bull. 397, 1909, pp. 198-203.

Min. Res. 1905, pp. 151-152.

Top. sheet Needles special.

43. Cedar Valley (McCracken). $\mathrm{Pb}, \Lambda \mathrm{g}, \mathrm{Cu}, \mathrm{Au}, \mathrm{W}$.

35 miles SE. Yucca, A. T. \& S. F. R. R.

Pre-Cambrian gneiss and schist.

Veins.

Bancroft, H., Reconnaissance of the ore deposits in northern Yuma County, Ariz.: Bull. 451, 1911, pp. 12, 44, 123-126.

Schrader, F. C., Mineral deposits of the Cerbat Range, Black Mountains, and Grand Wash Cliffe, Mohave County. Ariz.: Bull. 397, 1909, p. 141.

Min. Res. 1905, pp. 150, 412.

1906 , p. 163.

1907, pt. 1, p. 172.

1908, pt. 1, p. 725 .

44. Cerbat (Hualpai). $\mathrm{Au}, \mathrm{Ag}, \mathrm{Pb}$.

14 miles NW. Kingman, A. T. \& S. F. R. R.

Pre-Cambrian gneiss and schist cut by granite porphyry.

Veins.

Schrader, F. C., Mineral deposits of the Cerbat Range, Black Mountains, and Grand Wash Cliffs, Mohave County, Ariz.: Bull. 340, 1908, pp. 67-69; Bull. 397, 1909, pp. 91-107.

Min. Res. 1905, p. 152.

1906, pp. 164-165.

1907, pt. 1, p. 173.

1908, pt. 1, pp. 303-304. 


\section{MOHAVE COUNTY-Continued.}

45. Chemehuevis. $\mathrm{Au}, \mathrm{Ag}, \mathrm{Pb}$. (D, Pl.) 40 miles SSW. Franconia, A. T. \& S. F. R. R.

Top. sheet Parker.

46. Chloride (Hualpai). $\mathrm{Ag}, \mathrm{Pb}, \mathrm{Au}, \mathrm{Cu}$.

Station A. T. \& S. F. R. R.

Pre-Cambrian granite, gneiss, and schist cut by granite porphyry.

Veins.

Schrader, F. C., Mineral deposits of the Cerbat Range, Black Mountains, and

Grand Wash Cliffs, Mohave County, Ariz.: Bull. 340, 1908, pp. 62-65;

Bull. 397, 1909, pp. 51-80.

Min. Res. 1905, p. 752.

1906, pp. 164-165.

1907, pt. 1, p. 173.

1908 , pt. 1, pp. 303-304.

Top. sheet Camp Mohave.

47. Eldorado Pass. $\mathrm{Au}, \mathrm{Ag}, \mathrm{Cu}$.

50 miles NE. Searchlight, B. \& S. R. R.

Pre-Cambrian granite, gneiss, and schist cut by Tertiary volcanics.

Veins.

Schrader, F. C., Mineral deposits of the Cerbat Range, Black Mountains, and Grand Wash Cliffs, Mohave County, Ariz.: Bull. 340, 1908, p. 72; Bull. 397, 1909, p. 218.

Top. sheet Camp Mohave.

48. Gold Basin. $\mathrm{Au}, \mathrm{Pb}, \mathrm{Cu}, \mathrm{Mo}, \mathrm{W}$.

40 miles N. Hackberry, A. T. \& S. F. R. R.

Pre-Cambrian gneiss, schist, and granite.

Veins.

Schrader, F. C., Mineral deposits of the Cerbat Range, Black Mountains, and Grand Wash Cliffs, Mohave County, Ariz.: Bull. 340, 1908, pp. 69-70; Bull. 397, 1909, pp. 118-127.

Min. Res. 1905, pp. 150-160.

1906, p. 163.

1908 , pt. 1 , p. 303.

1909, pt. 1, p. 249.

Top. sheet Camp Mohave.

49. Gold Bug. Au, Ag.

40 miles NW. Chloride, A. T. \& S. F. R. R.

Tertiary volcanics.

Veins.

Schrader, F. C., Mineral deposits of the Cerbat Range, Black Mountains, and Grand Wash Cliffs, Mohave County, Ariz.: Bull. 340, 1908, pp. 72-73; Bull. 397,1909 , p. 217.

Top. sheet Camp Mohave.

50. Gold Road (San Francisco). Au.

16 miles NE. Needles, Cal., 24 miles W. Kingman, A. 'T. \& S. F. R. R.

Tertiary Volcanics.

Veins.

Schrader, F. C., Mineral deposits of the Cerbat Range, Black Mountains, and Grand Wash Cliffs, Mohave County, Ariz.: Bull. 340, 1908, pp. 75-78; Bull. 397, 1909, pp. 152-180.

Min. Res. 1905, pp. 151-152.

1906, pp. 163-164.

1907 , pt. 1 , pp. $172-173$.

1908, pt. 1, p. 303.

Top. sheet Camp Mohave. 
MOHAVE COUNTY-Continued.

51. Hackberry. Ag.

Station A. T. \& S. F. R. R.

Schrader, F. C., Mineral deposits of the Cerbat Range, Black Mountains, and Grand Wash Cliffs, Mohave County, Ariz.: Bull. 397, 1909, pp. 47, 141.

Min. Res. 1907, pt. 1, p. 172.

Top. sheet Diamond Creek.

52. Hualpai. $\mathrm{Cu}$.

20 miles E. Yucca, A. T. \& S. F. R. R.

Pre-Cambrian granite.

Veins.

Min. Res. 1909, pt. 1, p. 249.

53. Lost Basin. $\mathrm{Au}, \mathrm{Ag}, \mathrm{Cu}$.

60 miles N. Chloride, A. T. \& S. F. R. R.

Pre-Cambrian granite.

Veins.

Schrader, F. C., Mineral deposits of the Cerbat Range, Black Mountains, and Grand Wash Cliffs, Mohave County, Ariz.: Bull. 340, 1908, p. 72; Bull. 397, 1909, pp. 150-151.

Top. sheet St. Thomas.

54. Maynard. $\mathrm{Au}, \mathrm{Cu}(\mathrm{Mo}, \mathrm{Hg}, \mathrm{W})$.

15 miles SE. Kingman, A. T. \& S. F. R. R.

Pre-Cambrian granite cut by porphyry.

Veins.

Sckrader, F. C., Mineral deposits of the Cerbat Range, Black Mountains, and Grand Wash Cliffs, Mohave County, Ariz.: Bull. 340, 1908, p. 70; Bull. 397, 1909, pp. 139-142.

Min. Res. 1908, pt. 1, pp. 303, 725.

Top. sheet Diamond Creek.

55. McConnico. Au.

Station A. T. \& S. F. R. R.

Pre-Cambrian granite and pegmatite.

Shear zones.

Schrader, F. C., Mineral deposits of the Cerbat Range, Black Mountains, and Grand Wash Cliffs, Mohave County, Ariz.: Bull. 340, 1908, pp. 70-71.

Top. sheet Camp Mohave.

56. Mineral Park (Hualpai). $\mathrm{Au}, \mathrm{Ag}, \mathrm{Pb}, \mathrm{Cu}$.

5 miles SSE. Chloride, A. T. \& S. F. R. R.

Pre-Cambrian complex cut by granite porphyry.

Veins.

Schrader, F. C., Mineral deposits of the Cerbat Range, Black Mountains, and Grand Wash Cliffs, Mohave County, Ariz.: Bull. 340, 1908, pp. 65-66; Bull. 397,1909 , pp. 80-91.

Min. Res. 1905, p. 152.

1906, pp. 164-165.

1907 , pt. 1, p. 173 .

1908 , pt. 1, pp. 303-304.

57. Mocking Bird (Weaver). Au.

24 miles NW. Chloride, A. T. \& S. F. R. R.

Pre-Cambrian granite, gneiss, schist, cut by Tertiary volcanics.

Veins.

Schrader, F. C., Mineral deposits of the Cerbat Range, Black Mountains, and Grand Wash Cliffs, Mohave County, Ariz.: Bull. 340, 1908, pp. 73-74. Bull. 397, 1909, pp. 215-217.

Min. Res. 1907, pt. 1, pp. 173-174.

1908 , pt. 1, p. 304.

Top. sheet Camp Mohave. 


\section{MOHAVE COUNTY-Continued.}

58. Music Mountains. $\mathrm{Au}, \mathrm{Cu}$.

25 miles N. Hackberry, A. T. \& S. F. R. R.

Pre-Cambrian complex cut by acidic and basic dikes.

Veins.

Schrader, F. C., Mineral deposits of the Cerbat Range, Black Mountains, and Grand Wash Cliffs, Mohave County, Ariz.: Bull. 340, 1908, p. 71; Bull. 397, 1909, pp. 142-151.

Min. Res. 1905, p. 151.

1908 , pt. 1, p. 303

1909, pt. 1, p. 249.

Top. sheet Diamond Creek.

59. Pilgrim. Au.

12 miles W. Chloride, A. T. \& S. F. R. R.

Rhyolite and granite porphyry.

Veins.

Schrader, F. C., Mineral deposits of the Cerbat Range, Black Mountains, and Grand Wash Cliffs, Mohave County, Ariz.: Bull. 340, 1908, p. 74; Bull. 397, 1909, p. 214.

Top. sheet Camp Mohave.

60. Signal (Greenwood). Au, W.

107 miles SSE. Kingman, A. T. \& S. F. R. R.

Schrader, F. C., Mineral deposita of the Cerbat Range, Black Mountains, and Grand Wash Cliffs, Mohave County, Ariz.: Bull. 397, 1909, p. 47.

61. Stockton Hill (Hualpai). $\mathrm{Pb}, \mathrm{Ag}, \mathrm{Au}, \mathrm{Cu}$.

10 miles NNW. Kingman, A. T. \& S. F. R. R.

Pre-Cambrian complex cut by acidic and basic dikes.

Veins.

Schrader, F. C., Mineral deposits of the Cerbat Range, Black Mountains, and Grand Wash Cliffs, Mohave County, Ariz.: Bull. 397, 1909, pp. 107-118.

Min. Res. 1905, p. 152.

1906, pp. 164-165.

1907, pt. 1, p. 173.

1908 , pt. 1, pp. 303-304.

Top. sheet Camp Mohave.

62. Union Pass (San Francisco). Au.

30 miles WNW. Kingman, A. T. \& S. F. R. R.

Pre-Cambrian complex cut by Tertiary volcanics.

Veins.

Schrader, F. C., Mineral deposits of the Cerbat Range, Black Mountains, and Grand Wash Cliffs, Mohave County, Ariz.: Bull. 340, 1908, pp. 74-75; Bull. 397, 1909, pp. 203-214.

Min. Res. 1905, p. 151.

1906, pp. 163-164.

1907, pt. 1, pp. 173-174

1908 , pt. 1, p. 303 .

1909, pt. 1, p. 249.

Top. sheet Camp Mohave.

63. Virginia (Weaver). Au.

25 miles $\mathrm{NW}$. Chloride, A. T. \& S. F. R. R.

Tertiary volcanics.

Veins.

Schrader, F. C., Mineral deposits of the Cerbat Range, Black Mountains, and Grand Wash Cliffs, Mohave County, Ariz.: Bull. 340, 1908, p. 74; Bull. 397, 1909, p. 214.

Min. Res. 1907, pt. 1, pp.173-174. 
MOHAVE COUNTY-Continued.

63. Virginia (Weaver)-Continued.

Min. Reg. 1908, pt. 1, p. 304.

1909, pt. 1, p. 250.

Top. sheet Camp Mohave.

64. Vivian (San Francisco). Au.

14 miles NE. Needles, Cal., A. T. \& S. F. R. R.

Tertiary volcanics.

Veins.

Schrader, F. C., Mineral deposits of the Cerbat Range, Black Mountains, and Grand Wash Cliffs, Mohave County, Ariz.: Bull. 340, 1908, pp. 78-81; Bull. 397, 1909, pp. 180-198.

Min. Res. 1905, p. 151.

1906, pp. $163-164$.

1907, pt. 1, pp. 172-173.

1908 , pt. 1, p. 303.

1909 , pt. 1, p. 249.

65. White Hills (Indian Secret). Au, Ag.

30 miles NNW. Chloride, A. T. \& S. F. R. R.

Pre-Cambrian complex.

Veins.

Schrader, F. C., Mineral deposits of the Cerbat Range, Black Mountains, and Grand Wash Cliffs, Mohave County, Ariz.: Bull. 397, 1909, pp. 127-139.

Min. Res. 1906, p. 163.

Top. sheet Camp Mohave.

66. Ajo. $\mathrm{Cu}, \mathrm{Au}, \mathrm{Ag}(\mathrm{W})$.

PRAA COUNTY.

45 miles SSW. Gila Bend, S. P. R. R.

Eruptives (?).

Replacements (?)

Min. Res. 1905, p. 153.

1906, p. 166.

1907, pt. 1, p. 174.

1908, pt. 1, p. 304.

1909, pt. 1, p. 250.

67. Amole. $\mathrm{Cu}, \mathrm{Au}, \mathrm{Ag}$.

10 miles WNW. Tucson, S. P. R. R.

Tertiary volcanics.

Veins.

Min. Res. 1905, pp. 152-153.

1906, p. 166.

1907, pt. 1, p. 174.

1908, pt. 1, p. 304.

1909 , pt. 1, p. 250.

68. Arivaca. $\mathrm{Au}, \mathrm{Cu}, \mathrm{Ag}, \mathrm{Pb}, \mathrm{W}$.

25 miles SW. Twin Buttes, 70 miles SSW. Tucson, S. P. R. R.

Granite.

Veins.

Min. Res. 1899 (Twenty-first Ann. Rept., pt. 6), p. 300.

1905, p. 153.

1906 , pp. 166, 525 .

1907, pt. 1, p. 174.

1908, pt. 1, pp. 305, 725.

1909 , pt. 1, p. 251.

$21528^{\circ}-$ Bull. $507-12-5$ 
PIMA coUNTY-Continued.

69. Baboquivari. $\mathrm{Au}, \mathrm{Ag}$.

60 miles SW. Tucson, S. P. R. R.

Min. Res. 1906, p. 166.

1908, pt. 1, p. 305.

70. Cababi (Comobabi). $\mathrm{Ag}, \mathrm{Cu}$.

75 miles WSW. Tucson, S. P. R. R.

Min. Res. 1905, p. 166.

71. Empire. $\mathrm{Pb}, \mathrm{Ag}, \mathrm{Cu}$.

1907, pt. 1, p. 174.

9 miles S. Pantano, S. P. R. R.

Paleozoic sediments cut by acidic intrusives.

Replacements.

Min. Res. 1906, p. 166.

1907, pt. 1, p. 175.

1908 , pt. 1, p. 305 .

1909 , pt. 1, p. 251.

Top. sheet Patagonia.

72. Greaterville. Au, Ag. (D, Pl.)

15 miles NW. Sonoita, S. P. R. R.

Granite (pre-Cambrian?), Paleozoic sediments cut by acidic intrusives.

Veins and replacements.

Hill, J. M., Placer deposits of Greaterville, Ariz.: Bull. 430, 1910, pp. 11-22.

Min. Res. 1905, p. 153.

1906, pp. 166-167.

1908 , pt. 1, p. 305.

1909 , pt. 1, p. 251.

Top. sheet Patagonia.

73. Gunsight.

60 miles S. Gila Bend, S. P. R. R.

Contact metamorphic (?).

74. Helvetia. $\mathrm{Cu}, \mathrm{Au}, \mathrm{Ag}, \mathrm{Pb}, \mathrm{Mo}$.

17 miles S. Vail, S. P. R. R.

Granite (pre-Cambrian?), Paleozoic sediments cut by acidic intrusives.

Veins, replacements, contact metamorphic.

Schrader, F. C., and Hill, J. M., Some occurrences of molybdenite in the

Santa Rita and Patagonia mountains, Ariz.: Bull, 430, 1910, pp. 154-158.

Min. Res. 1905, p. 153.

1906, pp. 167-168, 392.

1907 , pt. 1, p. 175 .

1908 , pt. 1, p. 305.

1909 , pt. 1, p. 251.

Top. sheet Patagonia.

75. Montezuma (Sonoita Mountains).

100 miles S. Gila Bend, S. P. R. R.

76. Old Baldy. $\mathrm{Cu}, \mathrm{Au}, \mathrm{Ag}$, (Fe, Mo).

45 miles SSE. Tucson, S. P. R. R.

Pre-Cambrian granite and schist cut by acidic volcanics, capped by Tertiary volcanics.

Veins.

Schrader, F. C., and Hill, J. M., Some occurrences of molybdenite in the

Santa Rita and Patagonia mountains, Ariz.: Bull. 430, 1910, pp. 158-159.

Top. sheet Patagonia. 
PIMA COUNTY-Continued.

77. Pima (Olive, San Xavier, Twin Buttes). $\mathrm{Cu}, \mathrm{Ag}, \mathrm{Pb}, \mathrm{Au}$.

Station Twin Buttes, S. P. R. R.

Paleozoic sediments cut by granite.

Cortact metamorphic.

Min. Res. 1905, p. 153.

1906, pp. 168, 391 .

1907, pt. 1, p. p, 175.

1908 , pt. 1 , p. 305 .

1909 , pt. 1, p. 251.

78. Quijotoa. $\mathrm{Au}, \mathrm{Cu}, \mathrm{Ag}, \mathrm{Pb} . \quad(\mathrm{D}, \mathrm{Pl}$.)

70 miles SSW. Casa Grande, S. P. R. R.

Min. Res. 1905, p. 153.

1906, p. 167.

1907, pt. 1, 175.

1908 , pt. 1, p. 305 .

79. Rincon. Cu.

1909 , pt. 1, p. 251.

18 miles E. Tucson, S. P. R. R.

Min. Res. 1907, pt. 1, p. 175.

Top. sheet Tucson.

80. Santa Rosa. Cu, Au, Ag.

15 miles WSW. Silver Bell, A. S. R. R.

81. Silver Bell. $\mathrm{Cu}, \mathrm{Pb}, \mathrm{Ag}, \mathrm{Au}$.

Station A. S. R. R.

Paleozoic sediments cut by granite porphyry.

Contact metamorphic, disseminated, veins.

Weed, W. H., The copper mines of the United States in 1905: Bull. 285, 1906, pp. 102-103.

Min. Res. 1905, p. 153.

1906, pp. 167, 391.

1907 , pt. 1, pp. $175-176,598$.

1908 , pt. 1, pp. 204, 305.

82. Silver Hill. $\mathrm{Au}, \mathrm{Ag}, \mathrm{Cu}, \mathrm{Pb}$.

1909 , pt. 1, pp. 167, 251.

18 miles SW. Tucson, S. P. R. R.

\section{PINAL COUNTY.}

83. Bunker Hill (Copper Creek). $\mathrm{Cu}, \mathrm{Au}, \mathrm{Ag}, \mathrm{Pb}, \mathrm{Fe}$.

33 miles SSE. Winkelman, A. E. R. R.

Limestone, diorite, rhyolite.

Disseminated, contact metamorphic.

Min. Res. 1908, pt. 1, p. 306.

1909, pt. 1, p. 252.

84. Gold Mountains (Galiuro). Au.

10 miles SE. Winkelman, A. E. R. R.

Tertiary volcanics.

Veins (?).

85. Mammoth. Au, Mo.

21 miles SSE Winkelman, A. E. R. R.

Tertiary volcanics.

Veins.

Min. Res. 1904, p. 341. 
PINAL COUNTY-Continued.

86. Mineral Creek (Ray, Kelvin). Cu.

7 miles N. Kelvin, A. E. R. R.

Pre-Cambrian schist, Paleozoic sediments.

Disseminated.

Min. Res. 1904, p. 342.

1905 , p. 154.

1906 , p. 169.

1907, pt. 1, pp. 176-177, 599.

1908 , pt. 1, pp. 205, 306.

1909 , pt. 1, pp. 167, 252.

Top. sheet Ray.

87. Mineral Hill. $\mathrm{Cu}, \mathrm{Ag}, \mathrm{Au}, \mathrm{Pb}$.

Location uncertain.

Min. Res. 1908, pt. 1, p. 306.

88. Old Hat (Oracle). Au, Ag, Pb, W. (D, P1.)

38 miles N. Tucson, S. P. R. R.

Pre-Cambrian granite.

Veins.

Min. Res. 1905, p. 154

1906, p. 169.

1907 , pt. 1, p. 177.

1908 , pt. 1, pp. 306, 725-726.

1909 , pt. 1, pp. 253, 577.

89. Pioneer. $\mathrm{Cu}, \mathrm{Pb}, \mathrm{Ag}, \mathrm{Au}, \mathrm{Zn}$.

10 miles NE. Kelvin, A. E. R. R.

Paleozoic sediments cut by diabase.

Veins.

Min. Res. 1905, p. 154.

1906, p. 369.

1907, pt. 1, p. 177.

1908 , pt. 1, p. 306.

1909, pt. 1, p. 253.

Top. sheet Ray.

90. Riverside. $\mathrm{Au}, \mathrm{Ag}, \mathrm{Cu}, \mathrm{Pb}$.

5 miles E. Kelvin, A. E. R. R.

Paleozoic sediments cut by diabase and monzonite porphyry.

Veins (?).

Min. Res. 1905, p. 154.

1906 , p. 169.

1907, pt. 1, p. 177.

1908 , pt. 1, p. 307.

Top. sheet Ray.

91. Silver King. $\mathrm{Ag}, \mathrm{Cu}$.

39 miles NE. Florence, A. E. R. R.

Pre-Cambrian schist and Paleozoic sediments cut by syenite.

Veins.

Min. Res. 1909, pt. 1, p. 253.

Top. sheet Florence.

92. Vekol (Casa Grande). $\mathrm{Cu}, \mathrm{Au}, \mathrm{Ag}, \mathrm{Pb}$.

28 miles S. Casa Grande, S. P. R. R.

Contact metamorphic (?).

Min. Res. 1905, p. 154. 
PINAI COUNTY-Continued.

92. Vekol (Casa Grande)-Continued.

Min. Res. 1907, pt. 1, p. 176.

1908 , pt. 1, p. 306 .

1909 , pt. 1, p. 252.

SANTA CRUZ COUNTY.

93. Harshaw. $\mathrm{Ag}, \mathrm{Au}, \mathrm{Cu}, \mathrm{Pb}$.

12 miles S. Patagonia, S. P. R. R.

Paleozoic sediments and Tertiary volcanics.

Veins, replacements.

Min. Res. 1905, p. 155.

1906, pp. 170-171.

1908, pt. 1, p. 307.

1909 , pt. 1, p. 253.

Top. sheet Nogales.

94. Mowry. $\mathrm{Ag}, \mathrm{Pb}, \mathrm{Au}, \mathrm{Cu}$.

15 miles S. Patagonia, S. P. R. R.

Granite (pre-Cambrian?), Paleozoic sediments.

Veins.

Min. Res. 1905, pp. 155, 156.

1906, p. 170.

1907, pt. 1, p. 178.

1908, pt. 1, p. 307.

Top. sheet Nogales.

95. Nogales. $\mathrm{Au}, \mathrm{Cu}, \mathrm{W}$.

8 miles N. Nogales, S. P. R. R.

Granite (pre-Cambrian?), cut by acidic and basic dikes.

Veins.

Hill, J. M., Notes on the occurrence. of tungsten minerals near Calabasas, Ariz.: Bull. 430, 1910, pp. 164-166.

Min. Res. 1908, pt. 1, p. 725.

Top. sheet Nogales.

96. Oro Blanco. $\mathrm{Au}, \mathrm{Ag}, \mathrm{Cu}$.

30 miles W. Nogales, 77 miles SSW. Tucson, S. P. R. R.

Min. Res. 1905, pp. 155-156.

1907 , pt. 1, p. 178

1908 , pt. 1, p. 307

1909 , pt. I, p. 253.

97. Palmetto. $\mathrm{Ag}, \mathrm{Pb}, \mathrm{Cu}$.

15 miles SW. Patagonia, S. P. R. R.

Granite (pre-Cambrian?), cut by acidic and basic dikes.

Veins and disseminations.

Min. Res. 1907, pt. 1, p. 178.

1908 , pt. 1, p. 307.

Top. sheet Nogales.

98. Patagonia. $\mathrm{Cu}, \mathrm{Au}, \mathrm{Ag}, \mathrm{Pb}, \mathrm{Mo}$.

15 miles E. Nogales, S. P. R. R.

Granite (pre-Cambrian?), cut by acidic and basic dikes.

Veins.

Schrader, F. C., and Hill, J. M., Some occurrences of molybdenite in the Santa Rita and Patagonia mountains, Arizona: Bull. 430, 1910, pp. 159-161. 
SANTA CRUZ COUNTY-Continued.

98. Patagonia-Continued.

Min. Res. 1906, p. 171.

1908 , pt. 1, p. 307.

1909 , pt. 1, p. 253.

Top. sheet Nogales.

99. Red Rock. $\mathrm{Ag}, \mathrm{Pb}, \mathrm{Cu}, \mathrm{Au}, \mathrm{Fe}$.

18 miles E. Patagonia, S. P. R. R.

Tertiary volcanics.

Veins.

Top. sheets Patagonia, Nogales.

100. San Cayetano. $\mathrm{Ag}, \mathrm{Pb}, \mathrm{Cu}$.

8 miles N. Calabasas, S. P. R. R.

Granite (pre-Cambrian?), Tertiary volcanics.

Veins.

Top. sheet Patagonia.

101. Tyndall (Aztec). $\mathrm{Au}, \mathrm{Ag}, \mathrm{Cu}, \mathrm{Pb}$.

20 miles NNW. Patagonia, S. P. R. R.

Granite (pre-Cambrian?) cut by diorite, capped by Tertiary volcanics.

Veins.

Min. Res. 1906, p. 170.

1907 , pt. 1 , p. 178.

1908, pt. 1, p. 307.

1909, pt. 1, p. 254.

Top. sheet Patagonia.

102. Washington (Duquesne). $\mathrm{Cu}, \mathrm{Au}, \mathrm{Ag}, \mathrm{Pb}, \mathrm{Zn}$.

20 miles E. Nogales, 21 miles S. Patagonia, S. P. R. R.

Granite (pre-Cambrian?) Paleozoic sediments cut by acidic dikes.

Veine, contact metamorphic.

Schrader, F. G., and Hill, J. M., Some occurrences of molybdenite in the

Santa Rita and Patagonia mountains, Arizona: Bull. 430, 1910, pp. 161-162.

Min. Res. 1903, p. 330.

1905 , p. 156.

1907, pt. 1, p. 178.

1908, pt. 1, p. 307.

Top. sheet Nogales.

103. Wrightson. $\mathrm{Ag}, \mathrm{Au}, \mathrm{Pb}, \mathrm{Cu}$.

14 miles N. Patagonia, S. P. R. R

Tertiary volcanics.

Veins.

Min. Res. 1906, p. 171.

1907 , pt. 1, p. 178.

1908 , pt. 1, pp. 307-308.

1909, pt. 1, p. 254.

Top. sheet Patagonia.

YAVAPAI COUNTY.

104. Agua Fria. Cu.

3 miles E. Mayer, S. F. P. \& P. R. R.

Schists.

Impregnations. 
Tavarar countr-Continued:

to4. Água Fria-Continued.

Min. Res. 1905, p. 158.

1906, p. 172 .

1907, pt. 1, p. 180 .

1908, pt. 1, p. 309 .

1909 , pt. 1, p. 255 .

Top. sheet Bradshaw Mountains.

Folio 126, 1905.

105. Big Bug. Au, Ag, Cu, $\mathrm{Pb}, \mathrm{Zn}$. (D, Pl.)

4 miles SE. Mayer, S. F. P. \& P. R. R.

Schists.

Veins, disseminations.

Weed, W. H., The copper mines of the United States in 1905: Bull. 285, 1906, p. 103.

Min. Res. 1905, pp. 158-159.

1906, pp. 172-174.

1907, pt. 1, pp. 180-181.

1908, pt. 1, p. 309.

1909 , pt. 1, p. 255.

Top. sheet Bradshaw Mountains.

Folio 126, 1905.

106. Black Canyon. Au, Ag.

10 miles SE. Turkey, S. F. P. \& P. R. R.

Schists cut by granite.

Veins.

Min. Res. 1905, p. 159.

1906, p. 174.

1907, pt. 1, p. 181.

1908 , pt. 1, p. 310.

1909 , pt. 1, p. 255.

Top. sheet Bradshaw Mountains.

Folio 126, 1905.

107. Black Hills. $\mathrm{Cu}, \mathrm{Au}, \mathrm{Ag}$.

15 miles S. Jerome, U. V. \& P. R. R.

Min. Res. 1905, p. 159.

1906, p. 174.

1907, pt. 1, p. 181.

1908, pt. 1, p. 310.

1909, pt. 1, pp. 255-256.

Top. sheet Jerome.

108. Black Rock. Au, Ag, Cu.

14 miles NE. Wickenburg, S. F. P. \& P. R. R.

Schists.

Veins.

Min. Res. 1905, p. 159.

1906, p. 174.

1907, pt. 1, pp. 181-182.

1908 , pt. 1, p. 310 .

1909 , pt. 1, p. 256.

109. Cherry Creek. Au, Ag.

18 miles E. Dewey, S. F. P. \& P. R. R.

Schist and granite, pre-Cambrian.

Veins. 
YAVAPAI COUNTY-Continued.

109. Cherry Creek-Continued.

Min. Res. 1907, pt. 1, p. 182. 1908 , pt. 1, p. 310. 1909, pt. 1, p. 256.

110. Congress. Au, Ag.

Station S. F. P. \& P. R. R.

Granite.

Veins.

Min. Res. 1905, p. 159.

1906, p. 175.

1907, pt. 1, pp. 183-184.

1908, pt. 1, p. 311.

1909 , pt. 1, p. 256.

Top. sheet Congress.

111. Copper Basin. $\mathrm{Cu}, \mathrm{Au}, \mathrm{Ag}$.

8 miles SW. Harrington, S. F. P. \& P. R. R.

Schist cut by granite and quartz diorite.

Veins.

Min. Res. 1888, p. 59 .

1906, p. 174 .

1907 , pt. 1, p. 183.

1908 , pt. 1, p. 310 .

1909 , pt. 1, p. 256.

112. Del Rio. Au. (Pl.)

10 miles SSE. Junction, S. F. P. \& P. R. R.

Wash gravels.

Top. sheet Jerome.

113. Eureka. $\mathrm{Au}, \mathrm{Ag}, \mathrm{Cu}$.

28 miles NW. Hillside, S. F. P. \& P. R. R.

Min. Res. 1906, pp. 174-175.

1907, pt. 1, p. 183.

1908 , pt. 1, p. 310.

1909 , pt. 1, p. 256.

114. Hassayampa. $\mathrm{Au}, \mathrm{Ag}, \mathrm{Cu}, \mathrm{Pb}$.

Poland station, S. F. P. \& P. R. R.

Schist and amphibolite cut by quartz diorite.

Veins.

Min. Res. 1905, p. 159.

1906, p. 175.

1907, pt. 1, p. 183.

1908, pt. 1, pp. 310-311.

1909 , pt. 1, p. 256.

Top. sheet Bradshaw Mountains.

Folio 126, 1905.

115. Peck. $\mathrm{Au}, \mathrm{Ag}, \mathrm{Cu}, \mathrm{Pb}$.

8 miles SE. Turkey, S. F. P. \& P. R. R.

Slate and quartzite.

Vein.

Min. Res. 1905, pp. 159-160.

1906, p. 175.

1907, pt. 1, pp. 184, 185.

1908 , pt. 1, p. 311.

1909 , pt. 1, p. 256. 
IAVAPAI COUNTY-Continued.

115. Peck-Continued.

Top. sheet Bradshaw Mountains.

Folio 126, 1905.

116. Squaw Creek. Au, Ag.

15 miles E. Turkey, S. F. P. \& P. R. R.

Schist and granite.

Veins.

Min. Res. 1906, p. 175.

117. Thumb Butte. Au, Ag.

18 miles NNW. Hillside, S. F. P. \& P. R. R.

Min. Res. 1905, p. 160.

1907, pt. 1, p. 184

Top. sheet Prescott.

118. Tiger (Crown King, Harrington). Au, $\mathrm{Ag}, \mathrm{Pb}, \mathrm{Zn}, \mathrm{Cu}$.

Station S. F. P. \& P. R. R.

Schist cut by granite and quartz diorite.

Veins.

Min. Res. 1905, p. 160.

1906, p. 176.

1907 , pt. 1, pp. 184-185.

1908 , pt. 1, p. 311

1909 , pt. 1, p. 256

Top. sheet Bradshaw Mountains.

Folio 126, 1905.

119. Tip Top (Bradshaw Mountains). Au (W).

8 miles S. Crown King, S. F. P. \& P. R. R.

Granite.

Veins.

Weed, W. H., The copper mines of the United States in 1905: Bull. 285, 1906, p. 103.

Top. sheet Bradshaw Mountains.

Folio 126, 1905.

120. Verde. $\mathrm{Au}, \mathrm{Cu}, \mathrm{Ag}$.

Jerome station, U. V. \& P. R. R.

Amphibolite schist.

Replacements.

Weed, W. H., The copper mines of the United States in 1905: Bull. 285, 1906, p. 103.

Min. Res. 1887, p. 75.

1888, pp. 58-59.

1904, p. 237.

1905 , pp. $160,353-354$.

1906, pp. 176-177, 389-390.

1907 , pt. 1, pp. $185,597-598$.

1908, pt. 1, pp. 202-203, 311-312.

1909, pt. 1, pp. 166, 257.

Top. sheet Jerome.

121. Walker. $\mathrm{Au}, \mathrm{Ag}, \mathrm{Pb}, \mathrm{Cu}$. (D, Pl.)

8 miles NW. Poland, S. F. P. \& P. R. R.

Schist and granite cut by quartz diorite.

Veins.

Min. Res. 1905, p. 160.

1906, p. 176. 
YAVAPAI COUNTY-Continued.

121. Walker-Continued.

Min. Res. 1907, pt. 1, p. 185.

1908 , pt. 1, p. 312.

Top. sheet Bradshaw Mountains.

Folio 126, 1905.

122. Weaver (Rich Hill). $\mathrm{Au}, \mathbf{A g}, \mathrm{Pb} . \quad(\mathrm{D}, \mathrm{Pl}$.)

10 miles E. Congress, S. F. P. \& P. R. R.

Min. Res. 1905, pp. 160-161.

1906 , p. 176.

1907, pt. 1, p. 185.

1908 , pt. 1, p. 312

1909, pt. 1, p. 257.

Top. sheet Congress.

123. White Picacho. $\mathrm{Cu}, \mathrm{Au}, \mathrm{Ag}$.

24 miles NE. Morristown, S. F. P. \& P. R. R.

Min. Res. 1907, pt. 1, pp. 185-186.

YUMA COUNTY.

124. Castle Dome. $\mathrm{Pb}, \mathrm{Ag}(\mathrm{Au}, \mathrm{Cu})$.

25 miles NNE. Yuma, S. P. R. R.

Pre-Cambrian granite and schist cut by granite and monzonite porphyry,

Tertiary volcanics.

Veins (?).

Bancroft, II., Ore deposits in northern Yuma County, Ariz.: Bull. 451, 1911, pp. 32,45 .

Min. Res. 1905, p. 161.

1906, p. 177.

1907, pt. 1, p. 186.

1908, pt. 1, p. 313.

1909 , pt. 1, p. 258.

125. Cienega. $\mathrm{Au}, \mathrm{Cu}, \mathrm{Ag}(\mathrm{Pb})$.

15 miles NE. Parker, A. T. \& S. F. R. R.

Pre-Cambrian schist capped by Tertiary volcanics.

Veins.

Bancroft, H., Ore deposits in northern Yuma County, Ariz.: Bull, 451, 1911, pp. $68-73$.

Min. Res. 1905, p. 162.

1908 , pt. 1, p. 313

1909, pt. 1, p. 258.

Top. sheet Parker.

126. Harcuvar. $\mathrm{Cu}, \mathrm{Au}$.

12 miles N. Wenden, A. T. \& S. F. R. R.

Pre-Cambrian schist cut by granite.

Veins, contact metamorphic.

Bancroft, H., Ore deposits in northern Yuma County, Ariz.: Bull. 451, 1911, pp. 95-103.

Min. Res. 1888 , p. 59.

1909 , pt. I, p. 258.

127. Harquahala. Au.

9 miles S. Salome, A. T. \& S. F. R. R.

Pre-Cambrian complex cut by basic dikes. 
TUMA COUNTY-Continued.

127. Harquahala - Continued.

Veins.

Bancroft, H., Ore deposits in northern Yuma County, Ariz.: Bull. 451, 1911, pp. 104-105.

Min. Res. 1905, p. 161 .

1906, p. 177.

1907, pt. 1, pp. 186-187.

1908 , pt. 1, p. 313.

128. Kola (Humbug, Polaris). Au, Ag.

45 miles NNW. Mohawk, S. P. R. R.

Pre-Cambrian complex and Tertiary volcanics.

Veins.

Bancroft, H., Ore deposits in northern Yuma County, Ariz.: Bull. 451, 1911, p. 45.

Min. Res. 1905, pp. 161-162.

1906, p. 177.

1907, pt. 1, p. 187.

1908 , pt. 1, p. 313.

1909 , pt. 1, p. 256.

129. La Fortuna. Au.

36 miles SE. Yuma, S. P. R. R.

Pre-Cambrian schists.

Veins.

130. Laguna. Au. (Pl.)

14 miles NE. Yuma, S. P. R. R.

Wash gravels.

131. Planet. Cu, Fe.

28 miles NNW. Bouse, A.\& S. R. R.

Pre-Cambrian schists and sediments.

Replacements.

Douglas, J., Cupola smelting of copper in Arizona: Min. Res. 1883-84, pp. $397-410$.

Bancroft, H., Ore depositg in northem Yuma County, Ariz.: Bull. 451, 1911, pp. $47-55$.

Min. Res. 1909, pt. 1, pp. 167, 258.

132. Plomosa. $\mathrm{Cu}, \mathrm{Ag}, \mathrm{Au}$. (D, Pl.)

18 miles WSW. Vicksburg, A. T. \& S. F. R. R.

Pre-Cambrian schists capped by Tertiary volcanics.

Veins.

Bancroft, H., Ore deposits in northern Yuma County, Ariz.: Bull. 451, 1911, pp. 21-22, 33, 87-95.

Min. Res. 1905, pp. 162, 398.

1906, pp. 177, 495.

1907, pt. 1, p. 187.

1908, pt. 1, pp. 313,692 .

1909 , pt. 1, p. 258.

133. Santa Maria (Bighorn). $\mathrm{Au}, \mathrm{Ag}, \mathrm{Cu}$.

30 miles NNE. Wenden, A. T. \& S. F. R. R.

Bancroft, H., Ore deposits in northern Yuma County, Ariz.: Bull. 451, 1911, pp. 122-123.

134. Silver. $\mathrm{Pb}, \mathrm{Ag}$.

30 miles NNE. Yuma, S. P. R. R. 
YUMA COUNTY-Continued.

135. Swansea. $\mathrm{Cu}, \mathrm{Au}, \mathrm{Ag}$.

Station, A. \& S. R. R.

Pre-Cambrian schists and sediments.

Replacements.

Bancroft, H., Ore deposits in northern Yuma County, Ariz.: Bull. 451, 1911, p. 59.

136. Vicksburg. $\mathrm{Cu}, \mathrm{Au}$.

Station, A. T. \& S. F. R. R.

Pre-Cambrian schists cut by granite.

Contact metamorphic, veins.

Bancroft, H., Ore deposits in northern Yuma County, Ariz.: Bull. 451, 1911, pp. 95-104.

137. Weaver (La Paz). $\mathrm{Hg}, \mathrm{Cu}, \mathrm{Au}$.

55 miles WSW. Vicksburg, A. T. \& S. F. R. R.

Pre-Cambrian schists capped by Tertiary volcanics.

Veins.

Bancroft, H., Ore deposits in northern Yuma County, Ariz.: Bull. 451, 1911. - Notes on the occurrence of cinnabar in west-central Arizona: Bull. 430, 1910, pp. 151-153.

Min. Res. 1908, pt. 1, p. 692. 


\section{CALIFORNIA, NORTHERN COUNTIES.}

The State of California maintains a Bureau of Mines, from which a large number of reports dealing with the mining industry of the State have come. These reports contain much information relative to the mining districts which has not been listed in this bibliography.

In the 31 northern counties of California there are 222 areas called mining districts by the United States Geological Survey. In 178 of these districts gold is the principal metal produced; in 16 districts quicksilver, in 14 iron or chromium, in 13 copper, and in 1 silver.

Distribution of the predominant metals produced in the mining districts of the northern counties of California.

\begin{tabular}{|c|c|c|c|c|c|c|}
\hline County. & Gold. & Silver. & $\begin{array}{l}\text { Cop- } \\
\text { per. }\end{array}$ & $\begin{array}{l}\text { Quick- } \\
\text { silver. }\end{array}$ & Iron. & Total. \\
\hline 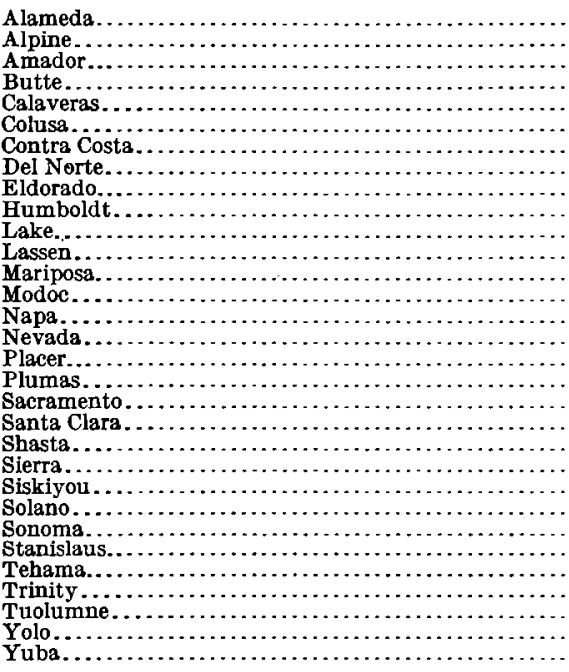 & 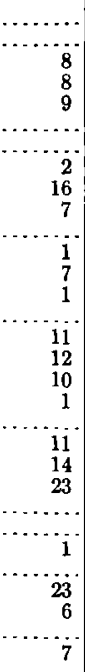 & 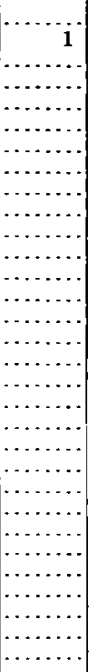 & 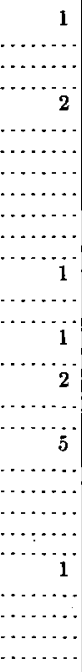 & 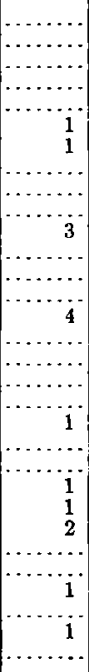 & 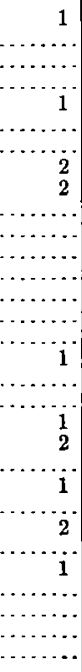 & $\begin{array}{r}1 \\
8 \\
1 \\
4 \\
12 \\
13 \\
12 \\
1 \\
2 \\
2 \\
18 \\
14 \\
25 \\
1 \\
4 \\
1 \\
2 \\
24 \\
6\end{array}$ \\
\hline & 178 & 1 & 13 & 16 & 14 & 22 \\
\hline
\end{tabular}

MINING DISTRICTS IN NORTHERN COUNTIES OF CALIFORNIA.

[See Pl. IV. Additional references may be found in Mineral Resources for 1910 and 1911.]

1. Alma. $\mathrm{Cu}$.

ALAMEDA COURTY.

10 miles W. Oakland, S. P. R. R., A. T. \& S. F. R. R.

Serpentine.

Lenses.

Top. sheet Concord.

2. Livermore (Tesla). $\mathrm{Mn}, \mathrm{Cr}$

15 miles SE. Livermore, S. P. R. R.

Serpentine and chert.

Lenses.

Harder, E. C., Manganese deposits of the United States: Bull. 380, 1909, p. 271; Bull. 427, 1910, pp. 159-163. 
ALAMEDA COUNTY-Continued.

2. Livermore (Tesla)-Continued.

Harder, E. C., Chromite deposits in California: Bull. 430, 1910, pp. 173-174.

Min. Res. 1885, pp. 349-350, 357.

1907, pt. 1, p. 101.

1908, pt. 1, p. 767.

Top. sheet Tesla.

\section{AIPINE COUNTY.}

3. Silver Mountain (Leviathan). Ag, Au.

25 miles SSW. Minden, Nev., V. \& T. R. R.

Tertiary volcanics.

Veins.

Lindgren, W., Tertiary gravels of the Sierra Nevada: P. P. 73, 1911, p. 191.

Min. Res. 1907, pt. 1, p. 233.

Top. sheet Markleeville.

\section{AMADOR COUNTY.}

4. Jackson. Au.

Station Am. C. R. R.

Slates and greenstones.

Veins.

Min. Res. 1905, p. 174.

1906, p. 186.

1907, pt. 1, p. 201.

1908 , pt. 1, p. 327.

1909, pt. 1, pp. 270-271.

Top. sheet Jackson.

Folio 11, 1894.

5. Iancha Plana. Au. (Pl.)

8 miles NE. Wallace, S. P. R. R.

Bench gravels.

Min. Res. 1905, p. 174.

1906, p. 186.

1907, pt. 1, p. 201.

1908 , pt. 1 , p. 328 .

Top. sheet Jackson.

Folio 11, 1894.

6. Middle Bar. Au. (Pl.)

10 miles SW. Jackson, Am. C. R. R.

Terrace gravels.

Top. sheet Jackson.

Folio 11, 1894.

7. Oleta. Au. (Pl.)

21 miles NE. May, S. P. R. R.

Quaternary and Tertiary gravels.

Lindgren, W., Tertiary gravels of the Sierra Nevada: P. P. 73, 1911, p. 199.

Min. Res. 1905, p. 174.

1906, p. 186.

1907, pt. 1, p. 201.

1908 , pt. 1, p. 328 .

1909, pt. 1, pp. $270-271$.

Top. sheet Placerville.

Folio 3, 1894. 
BULLETIN 507 PLATE IV

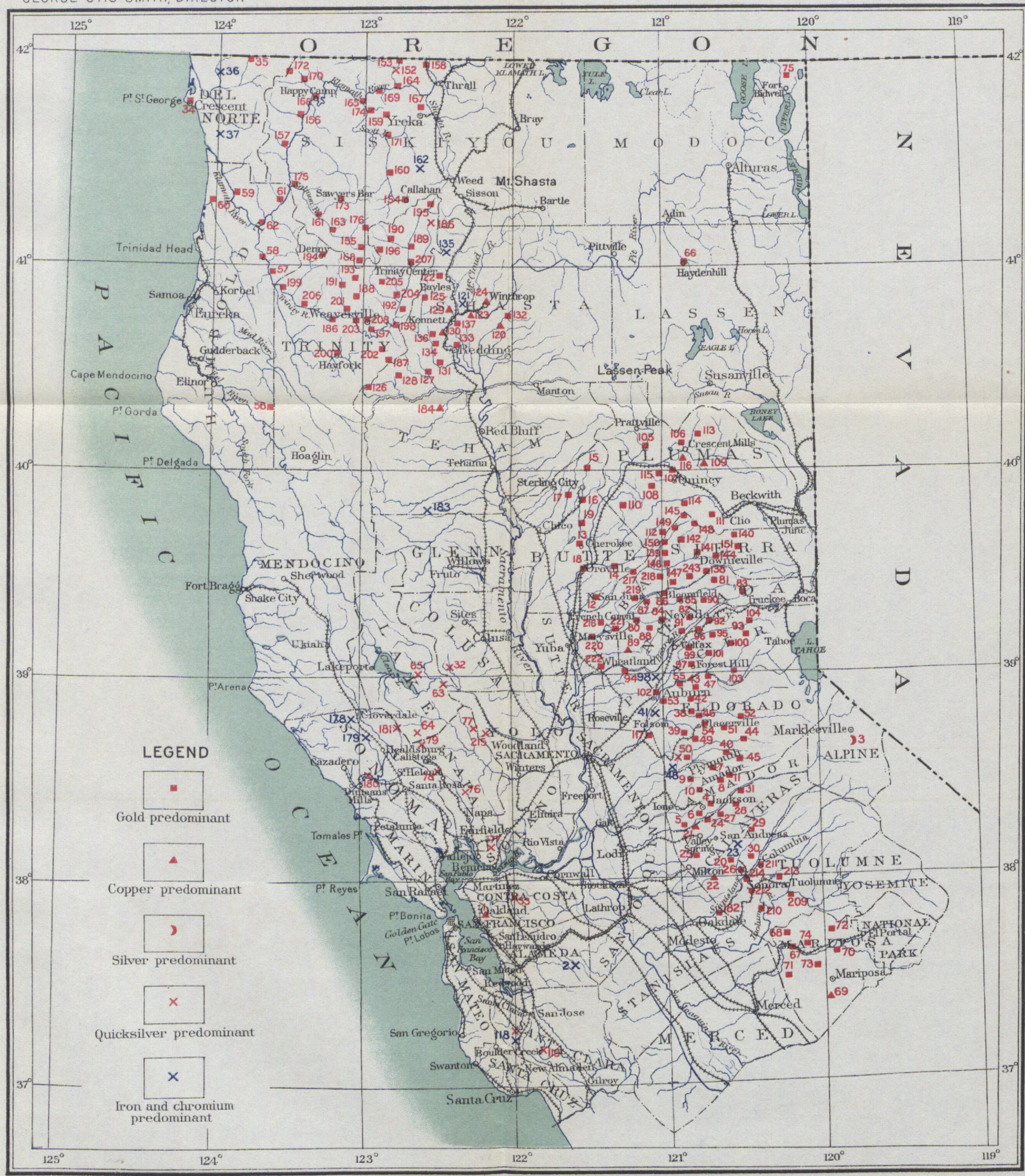

MAP OF NORTHERN COUNTIES OF CALIFORNIA,SHOWING LOCATION OF MINING DISTRICTS Scale $2,500,000$
ALAMEDA COUNTY

Livermore (Tesla)

ALPINE COUNTY

Silver Mountain
(Leviathan)

AMADOR COUNTY

4. Jackson

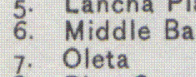

7. Oleta

9. Plymouth (Drytown) Soltano

BUTTE COUNTY

Bangor
3. Cherokee
Forbestown

15. Inskip
16. Magalia

17. Nimshew
18. Oroville

9. Yankee Hill

CALAVERAS COUNTY

20. Angels
21. Campo Seco (Valley

22. Copperopolis

. Esmeralda (Murphy

24. Gwin
25. Jenny Lind
26. Melones (Carson Hill)
27. Mokelumne Hill

27. Mokelumne Hill

29. Sheep Ranch (Eldorado)

Vallecito (Douglas Flat)
West Point

COLUSA COUN

32. Sulphur Creek

CONTRA COSTA COUNTY
Mount Diablo (Ryne)

DEL NORTE COUNTY

Crescent City
Diamond Creek

. Rattlesnake Divide

ELDORADO COUNTY

3. Eldorado (Shingle

Springs)

41. Flagstaff Hill

Georgetown

44. Grizzly Flat
45. Indian Digging

46. Kelsey

48. Latrobe

No. Nashville
1. Newtown (South Fork

Webber Cocic

52. Pacific

54. Placerville
55. Spanish Digging

HUMBOLDT COUNTY

6. Blocksburg
57. China Flat

9. Koopa

6o. Orick

61. Orleans
62. Weitchpec

LAKE COUNTY

63. Baker (Clear Lake)

64. Mayacmas (Great
65. Sulphur Bank

LASSEN COUNTY

Hayden Hill

MARIPOSA COUNTY

67. Bagby

69. Green Mountain (Ben Hur)

70. Hite Cove

71. Hornitos
72. Kinsley
73. Mt. Bullion (Whitlock

44. Quartz Mountain

MODOC COUNTY

Hoag

NAPA COUNTY

6. Bella Union

78. La Joya (Oat Hill)

9. Mayacmas (Napa

NEVADA COUNTY

80. French Corral

82. Lowell Hill

83. Meadow Lake
84. Nevada City (Grass

85. North Bloomfield (Relief)
86. North Columbia (Grizzly

87. North'San Juan (Badger

88. Rough and Ready

9.. Washington (Omega)
9. You Bet (Little York)

PLACER COUNTY

92. Blue Canyon
93. Canada Hill

95. Damascus (Trent)

Dutch Flat (Gold Run,

97. Forest Hill

98. Hotaling

99. Last Chance
o. Michigan Bluff

. Ophir (Aubur

103. Ralston D
104. Westrille

PLUMAS COUNTY

5. Butte Valley
o6. Crescent Mills

(Greenville)
107. Elizabethtown
O8. Edmanton (Meadow

Valley)
Genesee Valley

110. Granite Basin

11. Johnsville (Janis
i2. La Porte

13. Lights Canyon
14. Sawpit Flat

15. Spanish Ranch

SACRAMENTO COUNTY

Folsom (Blue Ravine

18. Los Gatos

SHASTA COUNTY

20. Afterthought (Ingot)

22. Bayles (Lamoine)

23. Black Diamond
23. Bully Hill (Winthrop)

25. French Gulch

127. Igo (South Clear Creek)

129. Kennett (Squaw Creek

. Keswick (Iron Mount

13. Larkin
132. Omitted

133. Redding

13. Shotgun Creek

. Shita Sitehouse (Buckey

American Hill

139. Brandy City

14'. Downieville (Goodyea

Eureka

Flats, Minnesota) Gibsonville (Whis
Diggings)
Indian Hill

Poker Flat (Table Rock)

St. Louis) (Scales,
Povery Hill (Scals)

Mount Pleasant)

SISKIYOU COUNTY

52. Beaver Creek
53. Blue Ledge

154. Callahans
Cecilville

Clear Creek

58. Cottonwood Creek

Forks of Salmon

Gazita

Hamburg

Happy Camp (Nolton)

Knownothing
Oak Bar

O'Mears (Walker)

. Preston Peak

75. Somes Bar

SOLANO COUNTY

St. Johns

SONOMA COUNTY

Cloverdale

Goyserville

Eastern)
Mayacmas (Pine Flat)

STANISLAUS COUNTY

Knights ferry

83. Newville

TRINITY COUNTY

85. Altoona (Allens Camp)

187. Bully Choop
88. Canyon City

188. Canyon City
189. Carrville

91. Colridge
192. Deadwood

93. Dedrick

95. Dodge

97. Douglas City

kins Gulch(Lewiston)

0. Hay Fork

200. Hay Fork

Indian Creek
Junction City

Slate Creek

Swedes Point
Trinity Center
Weaverville

TUOLUMNE COUNTY

209. Big Oak Flat

211. Columbsonvilie)

12. Jamestown (Rawhide,

Soulsbyville (Carters)

YOLO COUNTY

15. Knoxville

COUNTY

216. Browns Valley

218. Camptonville

220. Marysville (Yuba Basin

221. Smartsville
222. Wheatland 
AMADOR COUNTY-Continued.

8. Pine Grove. Au. (Pl.)

10 miles NE. Jackson, Am. C. R. R.

Terrace gravels.

Min. Res. 1905, p. 174.

1906 , p. 186.

1907, pt. 1, p. 201.

Top. sheet Jackson.

Folio 11, 1894.

9. Plymouth (Drytown). Au. (D, Pl.)

12 miles ENE. of May, S. P. R. R.

Slates and greenstone.

Veins.

Min. Res. 1905, p. 174.

1906, p. 186.

1907, pt. 1, p. 201.

1908 , pt. 1, pp. $327,328$.

1909 , pt. 1, pp. 270-271.

Top. sheet Jackson.

Folios 11, 1894; 63, 1900.

10. Sutter Creek (Amador). Au.

5 miles N. Martell, Am. C. R. R.

Slates and greenstone.

Veins.

Min. Res. 1905, p. 174.

1906 , p. 186.

1907, pt. 1, p. 201.

1908, pt. 1, pp. 327-328.

1909, pt. 1, pp. 270-271.

Top. sheet Jackson.

Foliog 11, 1894; 63, 1900.

11. Volcano. Au. (Pl.)

13 miles NE. Jackson, Am. C, R, R.

Quaternary and Tertiary gravels.

Lindgren, W., Tertiary gravels of the Sierra Nevada: P. P. 73, 1911, p. 199.

Min. Res. 1905, p. 174.

1906, p. 186.

1907, pt. 1, p. 201.

1908, pt. 1, p. 328 .

1909 , pt. 1, p. 271.

Top. sheet Jackson.

Folio 11, 1894.

12. Bangor. Au. (Pl.)

BUTTE COUNTY.

15 miles SE. Oroville, S. P. R. R.

Tertiary river gravels.

Min. Res. 1906, p. 187.

Top. sheet Smartsville.

Folio 18, 1895.

13. Cherokee. Au, Pt. (Pl.)

12 miles N. Oroville, S. P. R. R.

Tertiary river gravels.

Lindgren, W., Tertiary gravels of the Sierra Nevada: P. P. 73, 1911, pp. 86-88.

Min. Res. 1883-84, p. 576.

1906 , p. 187. 


\section{BUTTE COUNTY-Continued}

13. Cherokee-Continued.

Min. Res. 1908, pt. 1, p. 329.

Top. sheet Chico.

14. Forbestown. $\mathrm{Au}, \mathrm{Cr}$.

22 miles E. Oroville, S. P. R. R.

Diorite cut by granite.

Veins.

Turner, H. W., Further contributions to the geology of the Sierra Nevada:

Seventeenth Ann. Rept., pt. 1, 1896, p. 587.

Min. Res. 1905, p. 176.

1906, p. 187.

1908 , pt. 1, pp. 328,769 .

1909 , pt. 1, pp. 271-272.

Top. sheet Bidwell Bar.

Folio 43, 1898.

15. Inskip. $\mathrm{Au}$.

7 miles N. Sterling City, B. C. R. R.

Diorite cut by granite.

Veins.

Min. Res. 1906, p. 187.

Top. sheet Chico.

1908 , pt. 1, p. 329 .

16. Magalia. Au. (Pl.)

Station B. C. R. R.

Tertiary river gravels.

Turner, H. W., Further contributions to the geology of the Sierra Nevada: Seventeenth Ann. Rept., pt. 1, 1896, p. 544.

Lindgren, W., Tertiary gravels of the Sierra Nevada: P. P. 73, 1911, pp. 90-93.

Min. Res. 1906, p. 187.

1907, pt. 1, p. 202.

1908 , pt. 1, p. 329.

Top. sheet Chico.

17. Nimshew. Au. (Pl.)

21 miles NE. Chico, S. P. R. R.

Tertiary river gravels.

Lindgren, W., Tertiary gravels of the Sierra Nevada: P. P. 73, 1911, pp. 95-98.

Min. Ree. 1906, p. 187.

Top. sheet Chico.

1907, pt. 1, p. 203.

18. Oroville. Au, Pt. (Pl.)

Station S. P. R. R.

Quaternary gravels.

Lindgren, W., Tertiary gravels of the Sierra Nevada: P. P. 73, 1911, pp. 86-90.

Min. Res. 1883-84, p. 576.

1905, p. 176.

1906, p. 187.

1907, pt. 1, p. 203.

1908 , pt. 1, p. 329.

1909, pt. 1, pp. 271-272.

Top. sheet Chico.

19. Yankee Hill. Au. (D, P1.)

21 miles N. Oroville, S. P. R. R.

Schists and greenstones.

Veins and Tertiary river gravels. 
19. Yankee Hill-Continued.

Turner, H. W., Further contributions to the geology of the Sierra Nevada: Seventeenth Ann. Rept., pt. 1, 1896, p. 601.

Lindgren, W., Tertiary gravels of the Sierra Nevada: P. P. 73, 1911, pp. 150-154.

Min. Res. 1906, p. 187.

1909, pt. 1, pp. 271-272.

Top. sheets Chico, Bidwell Bar.

Folio 43, 1898.

20. Angels. $A u$.

CALAVERAS COUNTY.

Station S. R. R. of Cal.

Slates and greenstones.

Veins.

Turner, H. W., Further contributions to the geology of the Sierra Nevada:

Seventeenth Ann. Rept., pt. 1, 1896, p. 675.

Min. Res. 1.905, pp. 174-175.

1906, p. 188.

1907, pt. 1, pp. 204-205.

1908, pt. 1, pp. 330-331.

1909 , pt. 1, p. 273.

Top. sheet Jackson.

Folios 11, 1894; 63, 1900.

21. Campo Seco (Valley Springs). Cu, Au, Ag, Fe, Cr.

4 miles N. Valley Springs, S. P. R. R.

Schists and greenstones.

Veins and lenses.

Weed, W. H., The copper mines of the United States in 1905: Bull. 285, 1906, p. 106.

Harder, E. C., Chromite deposits of California: Bull. 430, 1910, pp. 174-176.

- Iron ores of western and central California: Bull. 430, 1910, pp. 223-225.

Min. Res. 1882, pp. 226-227.

1883-84, pp. $341,570-571$.

1905, pp. 174-175.

1906, pp. 187-188, 394.

1907, pt. I, pp. 205, 601 .

1908, pt. 1, pp. 331, 768.

Top. sheet Jackson. 1909, pt. 1, p. 273.

Folios 11, 1894; 63, 1900.

22. Copperopolis. $\mathrm{Cu}, \mathrm{Au}, \mathrm{Ag}$.

18 miles ESE. Milton, S. P. R. R.

Schists cut by greenstones and granite.

Veins and lenses.

Turner, H. W., Further contributions to the geology of the Sierra Nevada: Seventeenth Ann. Rept., pt. 1, 1896, p. 680.

Weed, W. H., The copper mines of the United States in 1905: Bull. 285, 1906, p. 106.

Min. Res. 1887, p. 76.

1888 , p. 59.

1906, pp. 187-188, 394.

1907, pt. 1, pp. 204, 601.

21528 - Bull. $507-12-6$ 
CALAVERAS COURTY-Continued.

22. Copperopolis-Continued.

Min. Res. 1908, pt. 1, p. 331 .

1909 , pt. 1, p. 273.

23. Esmeralda (Murphy, Sperry). Fe, Au.

20 miles ESE. Valley Springs, S. P. R. R.

Slates and greenstones, Tertiary gravels.

Lenses, Tertiary river gravels.

Harder, E. C., Iron ores of western and central California: Bull. 430, 1910, pp. 223-224.

Lindgren, W., Tertiary gravels of the Sierra Nevada: P. P. 73, 1911, p. 201.

Top. sheets Jackson and Big Trees.

Folios 11, 1894; 51, 1898.

24. Gwin. $\Lambda u$.

8 miles NE. Valley Springs, S. P. R. R.

Slates and greenstones.

Veins.

Min. Res. 1905, p. 175.

1906, p. 188.

1907, pt. 1, p. 205.

1908, pt. 1, p. 331.

Top. sheet Jackson.

Folios 11, 1894; 63, 1900.

25. Jenny Lind. Au. (Pl.)

6 miles N. Milton, S. P. R. R.

Tertiary gravels.

Min. Res. 1905, pp. 174-175.

1906 , p. 188.

1907, pt. 1, p. 205.

1908 , pt. 1, p. 768.

1909, pt. 1, p. 273.

Top. sheet Jackson.

Folios 11, 1894; 63, 1900.

26. Melones (Carson Hill). Au.

Station S. R. R. of Cal.

Slates and greenstones.

Veins.

Min. Res. 1905, pp. 174-175.

1907, pt. 1, p. 205.

1908, pt. 1, p. 331.

1909, pt. 1, p. 273.

Top. sheet Big Trees.

Folios 51, 1898; 63, 1900.

27. Mokelumne Hill. Au. (Pl.)

16 miles NE. Valley Springs, S. P. R. R.

Tertiary river gravels.

Lindgren, W., Tertiary gravels of the Sierra Nevada: P. P. 73, 1911, pp. 205-209.

Min. Res. 1905, p. 174.

1907, pt. 1, p. 205.

1908 , pt. 1, pp. 331-332.

1909, pt. 1, p. 273.

Top. sheet Jackson.

Folios 11, 1894; 63, 1900. 
CaIaveras courTy-Continued.

28. Railroad Flat. Au. (Pl.)

24 miles NE. Valley Springe, S. P. R. R.

Tertiary river gravels.

Lindgren, W., Tertiary gravels of the Sierra Nevada: P. P. 73, 1911, pp. 210-212.

Min. Res. 1907, pt. 1, p. 205.

Top. sheet Jackson.

1908 , pt. 1, p. 332.

Folio 11, 1894.

29. Sheep Ranch (Eldorado). Au. (D, Pl.)

27 miles E. Valley Springs, S. P. R. R.

Slates and greenstone.

Veins and Tertiary river gravels.

Lindgren, W., Tertiary gravels of the Sierra Nevada: P. P. 73, 1911, p. 211.

Turner, H. W., Further contributions to the geology of the Sierra Nevada:

Seventeenth Ann. Rept., pt. 1, 1896, p. 707.

Min. Res. 1905, pp. 174-175.

1906 , p. 188.

1907, pt. 1, pp. 205-206.

1909 , pt. 1, p. 273.

Top. sheet Big Trees.

Folio 51, 1898.

30. Vallecito (Douglas Flat). Au. (PI.)

7 miles NE. Angles Camp, S. R. R. of Cal.

Tertiary river gravels.

Lindgren, W., Tertiary gravels of the Sierra Nevada: P. P. 73, 1911, pp. 199-201, 202.

Min. Res. 1907, pt. 1, p. 206.

1909, pt. 1, p. 273.

Top. sheet Big Trees.

Folios 51, 1898; 63, 1900.

31. West Point. Aur. (D, Pl.)

30 miles NE. Valley Springs, S. P. R. R.

Granite.

Veins and Tertiary river gravels.

Lindgren, W., Tertiary gravels of the Sierra Nevada: P. P. 73, 1911, pp. 210-211.

Min. Res. 1907, pt. 1, p. 206.

1908 , pt. 1, p. 332.

1909 , pt. 1, p. 273.

Top. sheet Jackson.

Folio 11, 1894.

32. Sulphur Creek. Hg.

COLUSA COUNTY.

25 miles SW. Williams, S. P. R. R.

Schists and slates.

Stockworks.

Becker, G. F., Geology of the quicksilver deposits of the Pacific slope: M. XIII, 1888, p. 367.

Min. Res. 1908, pt. 1, p. 685.

1909, pt. 1, pp. 289, 552. 
CONTRA COSTA COUNTY.

33. Mount Diablo (Ryne). Hg (Ag, Au).

12 miles SE. Martinez, S. P. R. R.

Schists and slates.

Stockworks.

Becker, G. F., Geology of the quicksilver deposits of the Pacific slope:

M. XIII, 1888, pp. 378-379.

Top. sheet Mount Diablo.

\section{DEL NORTE COUNTY.}

34. Crescent City. Au, Ag, Pt. (Pl.)

97 miles SW. Grants Pass, Oreg., S. P. R. R.

Stream gravels.

Min. Res. 1897 (Nineteenth Ann. Rept., pt. 6), p. 266.

1906, p. 188.

1907, pt. 1, p. 233.

1909, pt. 1, p. 274.

35. Diamond Creek. Au, $\mathrm{Ag}, \mathrm{Cu}, \mathrm{Hg}$. (D, Pl.)

65 miles SW. Grants Pass, Oreg., S. P. R. R.

Serpentine.

Disseminated.

36. Low Divide. Cr.

117 miles SW. Grants Pass, Oreg., S. P. R. R.

Serpentine.

Lenses.

Min. Res. 1908, pt. 1, p. 768.

37. Rattlesnake Divide. Cr.

120 miles SW. Grants Pass, Oreg., S. P. R. R.

Serpentine.

Lenses.

Min. Res. 1908, pt. 1, p. 768.

38. Coloma. Au. (D, Pl.)

ELDORADO COUNTY.

7 miles NNW. Placerville, S. P. R. R.

Schists cut by granodiorite.

Veins, stream gravels.

Min. Res. 1907, pt. 1, p. 206.

1909 , pt. 1, pp. 273-274.

Top. sheet Placerville.

Folio 3, 1894.

39. Eldorado (Shingle Springs). Au. (D, Pl.)

Station S. P. R. R.

Schists cut by diorite.

Veins, stream gravels.

Min. Res. 1906, p. 188.

1908, pt. 1, p. 333.

1909 , pt. 1 , pp. $273-274$.

Top. sheet Placerville.

Folio 3, 1894.

40. Fairplay. Au. (D, Pl.)

20 miles SE. Placerville, S. P. R. R.

Granodiorite and andesite.

Veins and Tertiary river gravels.

Lindgren, W., Tertiary gravels of the Sierra Nevada: P. P. 73, 1911, pp. 180-181. 
ELDORADO COUNTY-Continued.

40. Fairplay-Continued.

Min. Res. 1905, p. 175.

Top. sheet Placerville.

Folio 3, 1894.

41. Flagstaff Hill. Cr.

9 miles S. Auburn, S. P. R. R.

Amphibolite and serpentine.

Lenses.

Min. Res. 1908, pt. 1, p. 768.

Top. sheet Sacramento.

Folio 5, 1894.

42. Garden Valley (Greenwood). Au. (D, PI.)

12 miles N. Placerville, S. P. R. R.

Slates and greenstone.

Veins and stream gravels.

Min. Res. 1908, pt. 1, pp. 332, 333.

1909, pt. 1, pp. 273-274.

Top. sheet Placerville.

Folio 3, 1894.

43. Georgetown. Au. (D, Pl.)

16 miles N. Placerville, S. P. R. R.; 19 miles E. Auburn, S. P. R. R.

Slates and greenstone.

Veins and stream and Tertiary river gravele.

Lindgren, W., Tertiary gravels of the Sierra Nevada: P. P. 73, 1911, pp. 168-169.

Min. Res. 1906, p. 188.

1908, pt. 1, pp. 332, 333.

1909 , pt. 1, pp. 273-274.

Top. sheet Placerville.

Folio 3, 1894.

44. Grizzly Flat. Au. (D, Pl.)

21 miles ESE. Placerville, S. P. R. R.

Schists cut by granodiorite.

Veins, Tertiary river gravels.

Lindgren, W., Tertiary gravels of the Sierra Nevada: P. P. 73, 1911, pp. 180-181.

Min. Res. 1907, pt. 1, p. 206.

1908 , pt. 1, pp. $332,333$.

1909 , pt. 1, pp. 273-274.

Top. sheet Placerville.

Falio 3, 1894.

45. Indian Diggings (Brownsville). Au. (P1.)

33 miles SE. Placerville, S. P. R. R.

Tertiary river gravels.

Lindgren, W., Tertiary gravels of the Sierra Nevada: P. P. 73, 1911, pp. 180-181.

Min. Res. 1908, pt. 1, p. 333.

1909, pt. 1, pp. 273-274.

Top. sheet Placerville.

Folio 3, 1894.

46. Kelsey. Au.

5 miles N. Placerville, S. P. R. R.

Slates and greenstone.

Veins. 
46. Kelsey-Continued.

Top. sheet Placerville.

Folio 3, 1894.

47. Kentucky Flat (Volcanoville). Au. (D, Pl.)

31 miles E. Auburn, S. P. R. R.

Slates and andesite.

Veins and Tertiary river gravels.

Min. Res. 1909, pt. 1, pp. 273-274.

Top. sheet Placerville.

Folio 3, 1894.

48. Latrobe. Cr.

Station S. P. R. R.

Serpentine.

Lenses.

Min. Res. 1908, pt. 1, p. 768.

Top. sheet Placerville.

Folio 3, 1894.

49. Logtown. Au. (D, Pl.)

5 miles S. El Dorado, S. P. R. R.

Slates cut by diabase and quartz porphyry.

Veins and stream gravels.

Top. sheet Placerville.

Folio 3, 1894.

50. Nashville. $\mathrm{Au}, \mathrm{Hg}$.

12 miles S. El Dorado, S. P. R. R.

Slates and diabase.

Veins.

Min. Res. 1905, p. 175.

1907 , pt. 1, p. 206.

1908 , pt. 1 , p. 332 .

Top. sheet Placerville.

Folio 3, 1894.

51. Newtown (South Fork Webber Creek). Au. (PI.)

8 miles E. Placerville, S. P. R. R.

Tertiary river gravels.

Lindgren, W., Tertiary gravels of the Sierra Nevada: P. P. 73, 1911, p. 178.

Top. sheet Placerville.

Folio 3, 1894.

52. Pacific. Au. (Pl.)

19 miles E. Placerville, S. P. R. R.

Tertiary river gravels.

Lindgren, W., Tertiary gravels of the Sierra Nevada: P. P. 73, 1911, pp. $169-171$.

Top. sheet Placerville.

Folio 3, 1894.

53. Pilot Hill. Au, Fe. (D, Pl.)

10 miles SE. Auburn, S. P. R. R.

Schists and slates.

Tertiary river gravels.

Lenses.

Harder, E. C., Chromite deposits in California: Bull. 430, 1910, p. 176.

Lindgren, W., Tertiary gravels of the Sierra Nevada: P. P. 73, 1911, p. 164.

Min. Res. 1883-84, p. 286.

1907, pt. 1, p. 206. 
ELDORADO COUNTY-Continued.

53. Pilot Hill-Continued.

Top. sheet Sacramento.

Folio 5, 1894.

54. Placerville. Au. (D, P1.)

Station, S. P. R. R.

Slates and greenstone; andesite.

Veins and Tertiary river gravels.

Lindgren, W., Tertiary gravels of the Sierra Nevada: P. P. 73, 1911, pp. 171-180.

Min. Res. 1905, p. 175.

1906, p. 188.

1907, pt. 1, p. 206.

1908, pt. 1, pp. 332-333.

1909, pt. 1, pp. 273-274.

Top. sheet Placerville.

Folio 3, 1894.

55. Spanish Diggings. Au.

14 miles E. Auburn, S. P. R. R.

Schists and slates.

Veins.

Min. Res. 1909, pt. 1, pp. 273-274.

Top. sheet Placerville.

Folio 3, 1894.

\section{HOMBOLDT COUNTY.}

56. Blocksburg. Au, Ag. (Pl.)

76 miles N. Sherwood, 42 miles SSE. Carlotta, N. W. P. R. R.

Stream gravels.

Min. Res. 1906, p. 188.

57. China Flat. Au, Ag, Pt. (Pl.)

45 miles ENE. Korbel, A. \& M. R. R. R.

Stream gravels.

Min. Res. 1906, p. 188.

1907, pt. 1, p. 207.

1908, pt. ], p. 333.

58. Hoopa. $\mathrm{Au}, \mathrm{Ag}$. (PI.)

33 miles NE. Korbel, A. \& M. R. R. R.

Stream gravels.

Min. Res. 1906, p. 188.

59. Klamath. Au, Ag. (Pl.)

51 miles NNE. Trinidad, O. \& E. R. R.

Stream gravels.

Min. Res. 1906, p. 188.

60. Orick. Au, Ag. (Pl.)

25 miles N. Trinidad, O. \& E. R. R.

Beach gravels.

Min. Res. 1906, p. 188.

61. Orleans. $\mathrm{Au}, \mathrm{Ag}, \mathrm{Pt}$. (Pl.)

62 miles NE. Korbel, A. \& M. R. R. R.

Stream gravels.

Min. Res. 1906, p. 188.

1907, pt. 1, p. 207.

1909, pt. 1, p. 247. 
HOMBOLDT COUNTY-Continued.

62. Weitchpec. Au, Ag, Pt. (Pl.)

45 miles NE. Korbel, A: \& M. R. R. R.

Stream gravels.

Min. Res. 1906, p. 188.

1907, pt. 1, p. 207.

63. Baker (Clear Lake). Hg.

\section{LAKE COUNTY.}

44 miles NNE. Calistoga, S. P. R. R.

Metamorphosed sediments cut by andesite.

Stockworks.

Becker, G. F., Geology of the quicksilver deposits of the Pacific slope:

M. XIII, 1888, pp. 233-250; Fighth Ann. Rept., pt. 2, 1889, pp. 974-979.

Min. Res. 1909, pt. 1, p. 553.

64. Mayacmas (Great Western). Hg.

16 miles NNW. Calistoga, S. P. R. R.

Mesozoic sediments, greenstone and serpentine, cut by basalt.

Veins, stockworks, and disseminations.

Becker, G. F., Geology of the quicksilver deposits of the Pacific slope:

M. XIII, 1888, pp. 368-377; Eighth Ann. Rept., pt. 2, 1889, p. 980.

Min. Res. 1882, pp. 394-398.

$$
\begin{aligned}
& 1883-84 \text {, pp. } 492-493 . \\
& 1887 \text {, pp. } 119-121 . \\
& 1908 \text {, pt. } 1 \text {, p. } 686 . \\
& 1909 \text {, pt. } 1 \text {, p. } 552 .
\end{aligned}
$$

65. Sulphur Bank. Hg.

33 miles N. Calistoga, S. P. R. R.

Metamorphosed sediments cut by andesite and basalt.

Disseminations and stockworks.

Becker, G. F., Geology of the quicksilver deposits of the Pacific slope:

M. XIII, 1888, pp. 251-270; Eighth Ann. Rept., pt. 2, 1889, p. 975.

Min. Res. 1882, pp. 394-398. 1883-84, pp. 492-493.

1887, pp. 119-121.

66. Hayden Hill. $\mathrm{Au}, \mathrm{Ag}$.

\section{LASSEN COUNTY.}

40 miles W. Madeline, N. C. \& O. R. R.

Tertiary volcanics (?).

Veins (?).

Min. Res. 1908, pt. 1, p. 358.

1909, pt. 1, p. 289.

Top. sheet Alturas.

67. Bagby. Au.

\section{MARIPOSA COUNTY.}

Station, Y. V. R. R.

Schists and slates.

Veins.

Min. Res. 1906, p. 190.

1907, pt. 1, p. 211.

1909 , pt. 1, p. 277.

Top. sheet Sonora.

Folio 41, 1897.

68. Coulterville. Au.

12 miles NE. Varain, Y. V. R. R.

Schists, slates, and serpentines. 
MARIPOSA COUNTY-Continued.

68. Coulterville-Continued.

Veins.

Turner, H. W., Further contributions to the geology of the Sierra Nevada: Seventeenth Ann. Rept., pt. 1, 1896, p. 675.

Min. Res. 1905, p. 175 .

1906 , p. 190.

1907 , pt. 1, p. 211.

1908 , pt. 1 , p. 337.

1909 , pt. 1, p. 277.

Top. sheet Sonora.

Folio 41, 1897.

69. Green Mountain (Ben Hur). Cu.

35 miles SSW. Bagby, Y. V. R. R.

Diorite, greenstones.

Veins.

Min. Res. 1907, pt. 1, pp. 210-211.

1908 , pt. 1, p. 337.

Top. sheet Mariposa (to be issued).

70. Hite Cove. Au.

15 miles E. Briceburg, Y. V. R. R.

Slates.

Veins.

Min. Res. 1905, p. 175.

1907, pt. 1, p. 211.

1909 , pt. 1, p. 277.

Top. sheet Yosemite.

71. Hornitos (Indian Gulch). $\mathrm{Au}, \mathrm{Cu}$.

8 miles E. Merced Falls, Y. V. R. R.

Slates and greenstones.

Veins.

Turner, H. W., Further contributions to the geology of the Sierra Nevada:

Seventeenth Ann. Rept., pt. 1, 1896, p. 661.

Min. Res. 1905, p. 175.

1906, p. 190.

1907, pt. 1, p. 211.

1908 , pt. 1, p. 337.

1909, pt. 1, p. 277 .

Top. sheet Sonora.

Folio 41, 1897.

72. Kinsley. Au.

12 miles NNE. Mountain King, Y. V. R. R.

Slates (?).

Veins (?).

Min. Res. 1905, p. 175.

1906, p. 190.

1907, pt. 1, p. 211.

1908 , pt. 1, p. 337.

Top. sheet Yocemite.

73. Mount Bullion (Whitlock, Mariposa). Au. (D, Pl.)

25 miles SE. Bagby, Y. V. R. R.

Slates and augite porphyry.

Veins and stream gravels.

Turner, H. W., Further contributions to the geology of the Sierra Nevada:

Seventeenth Ann. Rept., pt. 1, 1896, p. 695. 
maRIPOSA COUNTY-Continued.

73. Mount Bullion (Whitlock, Mariposa)-Continued.

Min. Res. 1905, p. 175.

1906, p. 190.

1907, pt. 1, p. 211.

1908 , pt. 1, p. 337.

1909, pt. 1, p. 277.

Top. sheet Sonora.

Folio 41, 1897.

74. Quartz Mountain. Au.

Mountain King station, Y. V. R. R.

Slates cut by augite porphyry.

Veins.

Top. sheet Sonora.

Folio 41, 1897.

\section{MODOC COUNTY.}

75. Hoag. $\mathrm{Au}, \mathrm{Ag}$.

57 miles NNE. Alturas, N. C. \& O. R. R.

Tertiary volcanics

Veins.

Min. Res. 1907, pt. 1, p. 234.

Top. sheet Alturas.

76. Bella Union. Hg.

\section{NAPA COUNTY.}

12 miles N. Napa, S. P. R. R.

Serpentine, greenstone, and Mesozoic sediments cut by andesite and basalt. Disseminations and stockworks.

Becker, G. F., Geology of the quicksilver deposits of the Pacific slope:

M. XIII, 1888 , p. 377 .

Min. Res. 1909 , pt. 1, p. 553

Top. sheet Napa.

77. Knoxville. Hg.

15 miles NW. Knoxville, S. P. R. R.

Serpentine, greenstone, and Mesozoic sediments cut by basalt.

Impregnations and stockworks.

Becker, G. F., Geology of the quicksilver deposits of the Pacific slope:

M. XIII, 1888, pp. 271-290; Eighth Ann. Rept., pt. 2, 1889, p. 977.

Melville, W. H., and Lindgren, W., Contributions to the mineralogy of the

Pacific coast: Bull. 61, 1890, pp. 22, 23-25.

Min. Res. 1887, pp. 119-121.

1908 , pt. 1, p. 686 .

1909 , pt. 1, p. 553 .

78. La Joya (Oat Hill). Hg.

St. Helena station, S. P. R. R.

Serpentine, greenstone, and Mesozoic sediments cut by andesite and basalt.

Stockworks and impregnations.

Becker, G. F., Geology of the quicksilver deposits of the Pacific slope: M. XIII, 1888, pp. 354-358; Eighth Ann. Rept., pt. 2, 1889, p. 980.

79. Mayacmas (Napa Consolidated). Hg.

17 miles N. St. Helena, S. P. R. R.

Serpentine, greenstone, and Mesozoic sediments cut by andesite and basalt.

Stockworks and impregnations.

Becker, G. F., Geology of the quicksilver deposits of the Pacific slope: M. XIII, 1888, pp. 368-377. 
NAPA COUNTY-Continued.

79. Mayacmas (Napa Consolidated)-Continued.

Min. Res. 1882, pp. 394-398.

1887, pp. 119-121.

1888, pp. 97-99.

1908, pt. 1, p. 686.

1909, pt. 1, p. 553.

NEVADA COUNTY.

80. French Corral. Au. (D, PI.)

10 miles NW. Nevada City, N. C. N. G. R. R.

Granodiorite and diabase.

Veins and Tertiary river gravels.

Lindgren, W., Tertiary gravels of the Sierra Nevada: P. P. 73, 1911, pp. 123-125.

Min. Res. 1905, p. 179.

1906, p. 191.

1907 , pt. 1, p. 215.

1908, pt. 1, p. 341 .

Top. sheet Smartsville.

Folio 18, 1895.

81. Graniteville. Au.

31 miles ENE. Nevada City, N. C. N. G. R. R.

Slates cut by granite, andesite.

Veins and Tertiary river gravels.

Lindgren, W., Tertiary gravels of the Sierra Nevada: P. P. 73, 1911, pp. 99-101.

Min. Res. 1905, p. 179.

1906, p. 191.

1907, pt. 1, p. 215.

1908 , pt. 1, p. 341 .

1909 , pt. 1, p. 280.

Top. sheet Colfax.

Folio 66, 1900.

82. Lowell Hill. Au. (Pl.)

6 miles N. Dutch Flat, S. P. R. R.

Tertiary river gravels.

Lindgren, W., Tertiary gravels of the Sierra Nevada: P. P. 73, 1911, pp. 146-147.

Min. Res. 1905, p. 179.

1906, p. 191.

1907, pt. 1, p. 215.

1908 , pt. 1, p. 341.

Top. sheet Colfax.

Folio 66, 1900.

83. Meadow Lake. Au.

30 miles NE. Emigrant Gap, S. P. R. R.

Schist cut by granodiorite and capped by andesite.

Veins.

Lindgren W., Tertiary gravels of the Sierra Nevada: P. P. 73, 1911, pp. 98-99.

Min. Res. 1907, pt. 1, p. 215.

Top. sheet Truckee.

Folio 39, 1897. 
NEVADA COUNTY-Continued.

84. Nevada City (Grass Valley). Au. (D, Pl.)

Station, N. C. N. G. R. R.

Slates, schists, and granodiorite capped by andesite.

Veins and Tertiary river gravels.

Lindgren, W., Gold-quartz veins of Nevada City and Grass Valley districts, California: Seventeenth Ann. Rept., pt. 2, 1896, pp. 1-262.

- Tertiary gravels of the Sierra Nevada: P. P. 73, 1911, pp. 125-132.

Min. Res. 1905, p. 178.

1906, p. 191.

1907, pt. 1, pp. 213-215.

1908 , pt. 1, pp. $339,340-341$.

1909 , pt. 1, pp. 278-279.

Top. sheets Smartsville, Nevada City special.

Folios 18, 1895; 29, 1896.

85. North Bloomfield (Relief). Au. (Pl.)

14 miles NE. Nevada City, N. C. N. G. R. R.

Tertiary river gravels.

Lindgren, W., Tertiary gravels of the Sierra Nevada: P. P. 73, 1911, pp. 139-141.

Min. Res. 1905, p. 179.

1906, p. 191.

Top. sheet Colfax.

1907 , pt. 1, p. 215.

Folio 66, 1900.

86. North Columbia (Grizzly Hill). Au. (D, Pl.)

21 miles N. Nevada City, N. C. N. G. R. R.

Slates, schists, and diorite.

Veins and Tertiary river gravels.

Lindgren, W., Tertiary gravels of the Sierra Nevada: P. P. 73, 1911, p. 139.

Min. Res. 1908, pt. 1, p. 341.

Top. sheet Colfax.

Folio 66, 1900.

87. North San Juan (Badger Hill). Au. (D, Pl.)

13 miles N. Nevada City, N. C. N. G. R. R.

Slate, granodiorite, and diabase.

Veins and Tertiary river gravels.

Lindgren, W., Tertiary gravels of the Sierra Nevada: P. P. 73, 1911, pp. 121-125.

Min. Res. 1905, p. 179.

1906, p. 192.

1907, pt. 1, p. 215.

Top. sheet Smartsville.

Folio $18,1895$.

88. Rough and Ready. Au. (D, Pl.)

4 miles SW. Grass Valley, N. C. N. G. R. R.

Granodiorite and amphibolite.

Veins and Tertiary river gravels.

Lindgren, W., Tertiary gravels of the Sierra Nevada: P. P. 73, 1911, pp. 127-132.

Min. Res. 1907, pt. 1, p. 215.

Top. sheet Smartsville.

Folio 18, 1895.

89. Spenceville. $\mathrm{Cu}, \mathrm{Ag}, \mathrm{Au}$.

17 miles NE. Wheatland, S. P. R. R.

Diabase and granodiorite. 
NEVADA COUNTY-Continued.

89. Spenceville-Continued.

Veins.

Min. Res. 1882, pp. 226-227.

1883-84, pp. 340-341.

1887 , p. 76.

1907, pt. 1, p. 215.

Top. sheet Smartsville.

Folio 18, 1895.

90. Washington (Omega). Au. (D, Pl.)

19 miles ENE. Nevada City, N. C. N. G. R. R.

Slates and serpentine.

Veins and stream gravels.

Lindgren, W., Tertiary gravels of the Sierra Nevada: P. P. 73, 1911, pp. 139-141.

Min. Res. 1905, p. 179.

1906 , p. 215.

1908 , pt. 1, p. 341.

Top. sheet Colfax.

Folio 66, 1900.

91. You Bet (Little York). Au. (PI.)

4 miles NW. Gold Run, S. P. R. R.

Tertiary river gravels.

Lindgren, W., Tertiary gravels of the Sierra Nevada: P. P. T, 1911, p. 144.

Min. Res. 1907, pt. 1, p. 215.

1909 , pt. 1, p. 278.

Top. sheet Collax.

Folio 66, 1900.

PLACER COUNTY.

92. Blue Canyon. Au. (D, Pl.)

Station, S. P. R. R.

Slates and andesite.

Veins and Tertiary gravels.

Lindgren, W., Tertiary gravels of the Sierra Nevada: P. P. 73, 1911, p. 146.

Min. Res. 1905, p. 179.

1906, p. 192.

1907, pt. 1, p. 218.

1908, pt. 1, pp. 342-343.

Top. sheet Colfax.

Folio 66, 1900.

93. Canada Hill. Au. (D, P1.)

40 miles E. Colfax, S. P. R. R.

Slates and andesite.

Veins and Tertiary river gravels.

Lindgren, W., Tertiary gravels of the Sierra Nevada: P. P. 73, 1911, pp. 157-158.

Top. sheets Colfax, Truckee.

Folios 66, 1900; 39, 1897.

94. Dairy Farm (Trent). Au, Cu.

10 miles NE. Sheridan, S. P. R. R.

Greenstone.

Veins.

Min. Res. 1905, p. 179.

1907, pt. 1., p. 216.

Top. sheet Smartsville.

Folio 18, 1895 . 
PLACER CoUNTY-Continued.

95. Damascus. Au. (Pl.)

12 miles E. Colfax, S. P. R. R.

Tertiary river gravels.

Lindgren, W., Tertiary gravels of the Sierra Nevada: P. P. 73, 1911, pp. 156-157.

Min. Res. 1905, p. 179.

1906, p. 192.

1907 , pt. 1, p. 217

Top. sheet Colfax.

Folio 66, 1900.

96. Dutch Flat (Gold Run, Towle). Au, Cr. (D, Pl.)

Station, S. P. R. R.

Schists and gabbro capped by andesite.

Veins and Tertiary river gravels.

Lindgren, W., Tertiary gravels of the Sierra Nevada: P. P. 73, 1911, pp. 144-145.

Min. Res. 1905, p. 179.

1906, p. 192.

1907 , pt. 1, pp. 216-218.

1908 , pt. 1, pp. 342-343, 768.

1909, pt. 1, p. 280.

Top. sheet Colfax.

Folio 66, 1900.

97. Forest Hill. Au. (D, Pl.)

15 miles SE. Colfax, S. P. R. R.

Slates, schists, and serpentine capped by andesite.

Veins and Tertiary river gravels.

Lindgren, W., Tertiary gravele of the Sierra Nevada: P. P. 73, 1911, pp. 149-151, 155-156, 164.

Min. Res. 1905, p. 179.

1906, p. 192.

1907, pt. 1, pp. 216-217.

1908, pt. 1, pp. 343, 768 .

1909, pt. 1, p. 280.

Top. sheet Colfax.

Folio 66, 1900 .

98. Hotaling. Fe.

$3 \frac{1}{2}$ miles NW. Clipper Gap, S. P. R. R.

Slates, granodiorite, and diabase.

Contact metamorphic.

Harder, E. C., Iron ores of western and central California: Bull. 430, 1910, pp. 225-227.

Min. Res. 1882, pp. 148, 428.

1883-84, pp. 286, 570-571.

1885, pp. 197-199.

Top. sheet Sacramento.

Folio 5, 1894.

99. Iowa Hill. Au. (D, Pl.)

9 miles E. Colfax, S. P. R. R.

Slates and amphibolite capped by andesite.

Veins and Tertiary river gravels.

Lindgren, W., Tertiary gravels of the Sierra Nevada: P. P. 73, 1911, pp. 148-140.

Min. Res. 1905, p. 179. 
PLACER COUNTT-Continued.

99. Iowa Hill-Continued.

Min. Res. 1906, p. 192.

1907 , pt. 1, p. 218

1908 , pt. 1, p. 343.

1909 , pt. 1, p. 280

Top. sheet Colfax.

Folio 66, 1900.

100. Last Chance. Au. (D, Pl.)

42 miles ENE. Auburn, S. P. R. R.

Slates capped by andesites.

Veins and Tertiary river gravels.

Lindgren, W., Tertiary gravels of the Sierra Nevada: P. P. 73, 1911, p. 158.

Min. Res. 1909, pt. 1, p. 280.

Top. sheet Colfax.

Folio 66, 1900.

101. Michigan Bluff. Au, Cr. (D, Pl.)

22 miles SE. Colfax, S. P. R. R.

Slates and serpentine capped by andesite.

Veins and Tertiary river gravels.

Lindgren, W., Tertiary gravels of the Sierra Nevada: P. P. 73, 1911, pp. $152-154$.

Min. Res. 1905, p. 179.

1906, p. 192.

1907, pt. 1, pp. 216, 218.

1908 , pt. 1, pp. 343,768 .

1909 , pt. 1, p. 280.

Top. sheet Colfax.

Folio 66, 1900.

102. Ophir (Auburn). Au, Ag. (D, PI.)

Station, S. P. R. R.

Amphibolite and granodiorite capped by andesite.

Veins, Tertiary and stream gravels.

Lindgren, W., The gold-silver mines at Ophir, Cal.: Fourteenth Ann. Rept., pt. 2,1894 , pp. $243-284$. Tertiary gravels of the Sierra Nevada: P. P. 73, 1911, p. 164.

Min. Res. 1905, p. 179.

1906, p. 192.

1907, pt. 1, p. 216.

1908 , pt. 1, p. 342 .

Top. sheet Sacramento.

Folio 5, 1894.

103. Ralston Divide. Au. (Pl.)

40 miles E. Auburn, S. P. R. R.

Tertiary river gravels.

Top. sheets Placerville and Colfax.

Folios 5 and 66, 1894.

104. Westville. Au. (D, Pl.)

40 miles NE. Auburn, S. P. R. R.

Slates capped by andesite.

Veins and Tertiary river gravels.

Min. Res. 1907, pt. 1, p. 216.

Top. sheet Colfax.

1908 , pt. 1, p. 342.

Folio 66, 1894. 
PLUMAS COUNTY.

105. Butte Valley (Sunnyside). Au. (D, Pl.)

49 miles NE. Sterling City, B. C. R. R.; 71 miles NW. Beckwith, S. V. R. R. and B. \& L. R. R.

Slates and greenstone capped by basalt.

Veins and stream gravels.

Top. sheet Lassen Peak.

Folio 15, 1895.

106. Crescent Mills (Greenville). $\mathrm{Au}, \mathrm{Ag}, \mathrm{Cu} . \quad(\mathrm{D}, \mathrm{P1}$ )

47 miles NW. Beckwith, S. V. R. R.

Paleozoic sediments cut by granodiorite.

Veins.

Diller, J. S., Geology of the Taylorsville region, California: Bull. 353, 1908, pp. 114-115.

Lindgren, W., Tertiary gravels of the Sierra Nevada: P. P. 73, 1911, pp. 114-116.

Min. Res. 1905, p, 180.

1907, pt. 1, p. 219.

1908 , pt. 1, p. 344 .

1909 , pt. 1, p. 281.

Top. sheets Indian Valley, Taylorsville, Honey Lake.

107. Elizabethtown. Au. (Pl.)

39 miles NW. Clio, S. V. R. R.

Stream gravels.

Diller, J. S., Geology of the Taylorsville region, California: Bull. 353, 1908.

Top. sheets Taylorsville, Indian Valley, Honey Lake.

108. Edmanton (Meadow Valley). Au, Mn. (D, Pl.)

38 miles NW. Clio, S. V. R. R.

Slates, amphibolites, granite.

Veins and Tertiary river gravels.

Harder, E. C., Manganese deposits of the United States: Bull. 380, 1909, pp. 270-271.

Lindgren, W., Tertiary gravels of the Sierra Nevada: P. P. 73, 1911, pp. 98-99.

Min. Res. 1907, pt. 1, p. 101.

1909 , pt. 1, p. 281.

Top. sheet Bidwell Bar.

Folio 43, 1898.

109. Genesee Valley. $\mathrm{Cu}, \mathrm{Au}$.

34 miles NW. Beckwith, S. V. R. R., B. \& L. R. R.

Paleozoic and Mesozoic sediments cut by granodiorite and capped by andesite.

Veins

Diller, J. S., Mineral resources of the Indian Valley region: Bull. 260, 1905, pp. 45-49; Geology of the Taylorsville region: Bull. 353, 1908, pp. 111-121.

Min. Res. 1905, p. 180.

1907, pt. 1, p. 219.

Top. sheets Indian Valley, Genesee, Honey Lake.

110. Granite Basin. Au. (D, Pl.)

57 miles NE. Oroville, S. P. R. R.

Amphibolite and granite.

Veins and Tertiary river gravels.

Top. sheet Bidwell Bar.

Folio 43, 1898 . 
PLUMAS COUNT - Continued.

111. Johnsville (Jamison). Au.

9 miles W. Clio, S. V. R. R., W. P. R. R.

Slates, gabbro, augite porphyry.

Veins and Quaternary gravels.

Lindgren, W., Tertiary gravels of the Sierra Nevada: P. P. 73, 1911, p. 111.

Min. Res. 1905, p. 180.

1906, p. 192.

1907, pt. 1, p. 218.

1908 , pt. 1, p. 344.

1909 , pt. 1, p. 281.

Top. sheet Downieville.

Folio 37, 1897.

112. La Porte. Au. (Pl.)

47 miles NE. Oroville, S. P. R. R.

Tertiary river gravels.

Lindgren, W., Tertiary gravels of the Sierra Nevada: P. P. 73, 1911, p. 105.

Min. Res. 1907, pt. 1, p. 218.

1908 , pt. 1, p. 344

1909 , pt. 1, p. 281 .

Top. sheet Downieville.

Folio 37, 1897.

113. Lights Canyon. Au. (Pl.)

50 miles NE. Beckwith, S. V. R. R., W. P. R. R.

Stream gravels.

Diller, J. S., Geology of the Taylorsville region: Bull. 353, 1908.

Lindgren, W., Tertiary gravels of the Sierra Nevada: P. P. 73, 1911, pp. 114-116.

Top. sheets Indian Valley, Genesee, Honey Lake.

114. Sawpit Flat. Au. (PI.)

60 miles NE. Oroville, S. P. R. R.; 49 miles W. Clio, S. V. R. R.

Tertiary river gravels.

Top. sheet Downieville.

Folio 37, 1897.

115. Spanish Ranch. Au, Cr. (Pl.)

36 miles NW. Clio, S. V. R. R.

Seipentine.

Lenses, Tertiary river gravels.

Lindgren, W., Tertiary gravels of the Sierra Nevada: P. P. 73, 1911, pp. 98-99.

Turner, H. W., Further contributions to the geology of the Sierra Nevada:

Seventeenth Ann. Rept., pt. 1, 1896, p. 557.

Min. Res. 1907, pt. 1, p. 218.

1908 , pt. 1, p. 768 .

Top. sheet Bidwell Bar.

Folio 43, 1898.

116. Taylorsville. Cu, Au. (D, Pl.)

40 miles NW. Beckwith, S. V. R. R., B. \& L. R. R.

Slates, andesite, granodiorite.

Veins, stream gravels.

Min. Res. 1905, p. 180.

1907, pt. 1, p. 219.

Top. sheets Taylorsville, Indian Valley, Honey Lake.

$21528^{\circ}-$ Bull. $507-12-7$ 
SACRAMENTO COUNTY.

117. Folsom (Blue Ravine, Michigan Bar). Au. (Pl.)

Stations S. P. R. R.

Quaternary gravels.

Lindgren, W., Tertiary gravels of the Sierra Nevada: P. P. 73, 1911, pp. 164$165,222$.

Min. Res. 1905, p. 181.

1906, p. 193.

1907, pt. 1, pp. 219-220.

1908 , pt. 1, p. 345.

1909, pt. 1, p. 282.

Top. sheet Sacramento.

Folio 5, 1894.

SANTA CLARA COUNTY.

118. Los Gatos. Cr, Hg.

Station S. P. R. R.

Serpentine.

Lenses.

Becker, G. F., Geology of the quicksilver deposits of the Pacific slope: M. XIII, 1888, p. 379.

Min. Res. 1908, pt. 1, p. 768.

119. New Almaden. Hg.

13 miles S. San Jose, S. P. R. R.

Mesozoic sediments.

Stockworks.

Becker, G. F., Geology of the quicksilver deposits of the Pacific slope: M. XIII, 1888, pp. 310-330; Eighth Ann. Rept., 1889, p. 978.

Christy, S. B., Quicksilver reduction at New Almaden, Cal.: Min. Res. 1883-84, pp. 503-536.

Min. Res. 1882, pp. 394-396.

$1883-84$, p. 492 .

1885 , p. 284.

1886, pp. $160-165$.

1887, pp. 119-121.

1888, pp. $97-99$.

1908, pt. 1, p. 690 .

1909, pt. 1, p. 553.

SHASTA COUNTY.

120. Afterthought (Little Cow Creek, Ingot). $\mathrm{Cu}, \mathrm{Zn}(\mathrm{Pb})$.

26 miles NE. Redding, S. P. R. R.

Paleozoic sediments cut by alaskite porphyry.

Replacements.

Diller, J. S., Copper deposits of the Redding district, California: Bull. 213, 1903, p. 126.

Mining and mineral resources in the Redding quadrangle, California, in 1903: Bull. 225, 1904, pp. 169-179.

Graton, L. C., Occurrence of copper in Shasta County, Cal.: Bull. 430, 1910, pp. 71-111.

Min. Res. 1906, pp. 195, 394.

1907 , pt. I, pp. 224, 599-560.

1908, pt. 1, pp. 205-206, 349.

Top. sheet Redding.

Folio 138, 1906. 
SHASTA COUNTY-Continued.

121. Baird (Heroult). Fe.

4 miles N. Heroult, S. V. \& E. R. R.

Paleozoic limestone cut by augite diorite.

Contact metamorphic.

Diller, J. S., Iron ores of the Redding quadrangle, California: Bull. 213, 1903, pp. 219-220.

Top. sheet Redding.

Folio 138, 1906.

122. Bayles (Lamoine). Au. (Pl.)

Station, S. P. R. R.

Stream gravels.

Min. Res. 1907, pt. 1, p. 225.

1908 , pt. 1, p. 349 .

Top. sheets Redding, Red Bluff.

Folio 138, 1906.

123. Black Diamond. $\mathrm{Cu}, \mathrm{Au}, \mathrm{Ag}$.

14 miles NE. Redding, S. P. R. R.

Paleozoic sediments cut by augite diorite.

Contact metamorphic.

Diller, J. S., Copper deposits of the Redding district, California: Bull. 213, 1903, p. 130.

Top. sheet Redding.

Folio 138, 1906.

124. Bully Hill (Winthrop). $\mathrm{Cu}, \mathrm{Au}, \mathrm{Ag}$.

Station, S. V. \& E. R. R.

Paleozoic slates, alaskite porphyry.

Replacements.

Diller, J. S., Copper deposits of the Redding district, California: Bull. 213, 1903, pp. 126-130.

Mining and mineral resources in the Redding quadrangle, California, in 1903: Bull. 225, 1904, pp. 169-179.

Graton, L. C., The occurrence of copper in Shasta County, Cal.: Bull. 430, 1910, pp. 71-111.

Weed, W. H., The copper mines of the United States in 1905: Bull. 285, 1906, p. 104.

Min. Res. 1904, p. 239.

1905 , p. 182.

1906, pp. 195, 392-394.

1907, pt. 1, pp. 223-224, 599-600.

1908 , pt. 1, pp. 205-206, 349.

1909, pt. 1, pp. 167, 284.

Top. sheets Redding, Red Bluff.

Folio 138, 1906.

125. French Gulch. $\mathrm{Au}, \mathrm{Cu}$.

21 miles NW. Redding, S. P. R. R.

Alaskite porphyry.

Veins.

Min. Res. 1906, p. 195.

1907, pt. 1, p. 224.

1908, pt. 1, p. 348.

1909 , pt. 1, p. 284.

Top. sheet Red Bluff. 
SHASTA CoUITYY-Continued.

126. Harrison Gulch (Knob). Au, Ag. 53 miles SW. Redding, S. P. R. R.

Min. Res. 1907, pt. 1, pp. 224-225. 1908 , pt. 1, p. 348. 1909 , pt. 1, p. 284.

Top. sheet Red Bluff.

127. Igo (South Clear Creek). Au. (D, Pl.)

13 miles SW. Redding, S. P. R. R.

Greenstones and slates cut by granodiorite.

Veins, stream gravels.

Min. Res. 1907, pt. 1, p. 225.

1908 , pt. 1, p. 348

Top. sheet Red Bluff.

128. Jerusalem Creek (Ono). Au. (Pl.)

21 miles SW. Redding, S. P. R. R.

Stream gravels.

Top. sheet Red Bluff.

129. Kennett (Squaw Creek, Little Backbone). Cu, Au, Ag.

Station S. P. R. R.

Paleozoic sediments and alaskite porphyry.

Veins and replacements.

Diller, J. S., Mining and mineral resources in the Redding quadrangle, California, in 1903: Bull. 225, 1904, pp. 169-179.

Graton, L. C., The occurrence of copper in Shasta County, Cal.: Bull. 430, 1910 , pp. 71-111.

Min. Res. 1904, p. 239.

1905 , pp. 182, 355 .

1906, pp. 195, 392-394.

1907 , pt. 1 , pp. $223,599-600$.

1908, pt. 1, pp. 205-206, 348, 349.

1909, pt. 1, p. 284.

Top. sheets Redding, Red Bluff.

Folio 138, 1906.

130. Keswick (Iron Mountain). $\mathrm{Cu}, \mathrm{Au}, \mathrm{Ag}$.

Station S. P. R. R.

Paleozoic sediments and alaskite porphyry.

Veins and replacements.

Diller, J. S., Copper deposits of the Redding district, California: Bull. 213, 1903, pp. 131-132.

Mining and mineral resources in the Redding quadrangle, California, in 1903: Bull. 225, 1904, pp. 169-179.

Graton, L. C., The occurrence of copper in Shasta County, Cal.: Bull. 430, 1910, pp. 71-111.

Weed, W. H., The copper mines of the United States in 1905: Bull. 285, 1906, pp. 103-105.

Min. Res. 1895 (Seventeenth Ann. Rept., pt. 3), p. 103.

1905 , pp. 181-182, 355 .

1906, pp. 195, 392-394.

1907 , pt. 1, pp. 222-223, 599-600.

1908 , pt. 1 , pp. 205-206, 348, 349.

1909, pt. 1, p. 284.

Top. sheet Redding.

Folio 138, 1906. 
SHASTA COUNTY-Continued.

131. Larkin. $\mathrm{Au}, \mathrm{Ag}, \mathrm{Cu} . \quad(\mathrm{D}, \mathrm{Pl}$.

8 miles SW. Redding, S. P. R. R.

Paleozoic sediments, meta-andesite, and diorite.

Veins and stream gravels.

Min. Res. 1908, pt. 1, p. 348.

Top. sheet Redding.

Folio 138, 1906.

132. Omitted.

133. Redding. Au. (Pl.)

Station S. P. R. R.

Quaternary gravels.

Diller, J. S., Copper deposits of the Redding district, California: Bull. 213, 1903, pp. 123-132.

- Iron ores of the Redding quadrangle, California: Bull. 213, 1903, pp. 219, 220.

Mining and mineral resources in the Redding quadrangle, California, in 1903: Bull. 225, 1904, pp. 169-179.

Min. Res. 1904, p. 239.

Top. sheets Redding, Red Bluff.

Folio 138, 1906.

134. Shasta. Au. (D, Pl.)

6 miles W. Redding, S. P. R. R.

Quartz diorite.

Veins and replacements.

Min. Res. 1907, pt. 1, p. 225.

Top. sheet Redding.

Folio 138, 1906.

135. Shotgun Creek. Cr.

15 miles NNW. Lamoine, S. P. R. R.

Serpentine.

Lenses.

Min. Res. 1908, pt. 1, pp. 766-767.

Top. sheet Shasta.

136. Stella. $\mathrm{Au}, \mathrm{Cu}$.

20 miles NW. Redding, S. P. R. R.

Alaskite porphyry.

Veins.

Min. Res. 1907, pt. 1, p. 225.

1908, pt. 1, p. 349.

Top. sheet Red Bluff.

137. Whitehouse (Buckeye). Au. (D, Pl.)

5 miles $N$. Keswick, S. P. R. R.

Meta-andesite.

Veins.

Min. Res. 1908, pt. 1, p. 349.

1909, pt. 1, p. 284.

Top. sheet Redding.

Folio 138, 1906.

SIERRA COUNTY.

138. American Hill. Au. (Pl.)

31 miles NE. Nevada City, N. C. N. G. R. R.

Tertiary river gravels. 
SIERRA COUNTY-Continued.

138. American Hill-Continued.

Lindgren, W., Tertiary gravels of the Sierra Nevada: P. P. 73, 1911, pp. 142-143.

Top, sheet Colfax.

Folio 66, 1900.

139. Brandy City. Au. (P1.)

31 miles N. Nevada City, N. C. N. G. R. R.

Tertiary river gravels.

Lindgren, W., Tertiary gravels of the Sierra Nevada: P. P. 73, 1911, p. 101. Top. sheet Bidwell Bar.

Folio 43, 1898.

140. Church Meadows. Au. (P1.)

20 miles W. Loyalton, B. \& L. R. R.

Glacial gravels.

Top. sheet Downieville.

Folio 37, 1897.

141. Downieville (Goodyears Bar). Au. (D, PI.)

63 miles NW. Marysville, S. P. R. R.; 40 miles N. Nevada City, N. C. N. G. R. R.

Slates and serpentine.

Veins, stream gravels.

Min. Res. 1905, p. 182.

1906, p. 196.

1907, pt. 1, pp. 225-226.

1908 , pt. 1 , pp. 350,768 .

1909 , pt. I, pp. 284-285.

Top. sheet Downieville.

Folio 37, 1897.

142. Eureka. Au. (Pl.)

70 miles NW. Marysville, S. P. R. R.; 50 miles N. Nevada City, N. C. N. G. R. R.

Tertiary river gravels.

Lindgren, W., Tertiary gravels of the Sierra Nevada: P. P. 73, 1911, p. 110.

Top. sheet Downieville.

Folio 37, 1897.

143. Forest (Alleghany, Chips Flats, Minnesota). Au. (D, Pl.)

65 miles ENE. Marysville, S. P. R. R.

Amphibolite and serpentine capped by andesite.

Veins, Tertiary river gravels.

Lindgren, W., Tertiary gravels of the Sierra Nevada: P. P. 73, 1911, pp. 112, $113,142$.

Min. Res. 1905, p. 182.

1906, pp. 195-196.

1907 , pt. 1, pp. 225-226.

1908 , pt. 1, p. 350.

1909, pt. 1, p. 285.

Top. sheet Colfax.

Folio 66, 1900.

144. Furnier. Au.

69 miles NW. Marysville, S. P. R. R.

Slates capped by andesite and basalt.

Veins.

Top. sheet Downieville.

Folio 37, 1897. 
SIERRA COUNTY-Continued.

145. Gibsonville (Whisky Diggings). Au. (D, Pl.)

68 miles NNE. Marysville, S. P. R. R.

Tertiary river gravels.

Lindgren, W., Tertiary gravels of the Sierra Nevada: P. P. 73, 1911, pp. 106-107.

Min. Res. 1907, pt. 1, p. 226.

1908 , pt. 1, p. 351.

1909 , pt. 1, pp. 284-285.

Top. sheet Downieville.

Folio 37, 1897.

146. Indian Hill. Au. (Pl.)

50 miles NE. Marysville, S. P. R. R.

Tertiary river gravels.

Lindgren, W., Tertiary gravels of the Sierra Nevada: P. P. 73, 1911, p. 111.

Top. sheet Downieville.

Folio 37, 1897.

147. Pike. Au, Cr. (D, P1.)

52 miles ENE. Marysville, S. P. R. R.

Slates and diabase capped by andesite.

Veins, Tertiary river gravels.

Min. Res. 1907, pt. 1, pp. 225-226. 1908, pt. 1, p. 769.

Top. sheet Colfax.

Folio 66, 1900.

148. Poker Flat (Table Rock). Au. (Pl.)

75 miles NE. Marysville, S. P. R. R.

$\mathrm{T} \epsilon$ :tiary river gravels.

Lindgren, W., Tertiary gravels of the Sierra Nevada: P. P. 73, 1911, p. 105.

Min. Res. 1909, pt. 1, pp. 284-285.

Top. sheet Downieville.

Folio 37, 1897.

149. Port Wine (Queen City, St. Louis). Au. (D, P1.)

69 miles NE. Marysville, S. P. R. R.

Slates, andesite, and amphibolite.

Veins, Tertiary river gravels.

Lindgren, W., Tertiary gravels of the Sierra Nevada: P. P. 73, 1911, pp. 108-110.

Turner, H. W., Further contributions to the geology of the Sierra Nevada: Seventeenth Ann. Rept., pt. 1, 1896, p. 602.

Min. Res. 1908, pt. 1, p. 351.

Top. sheet Downieville.

Folio 37, 1897.

150. Poverty Hill (Scales, Mount Pleasant). Au. (Pl.)

59 miles NE. Marysville, S. P. R. R.

Tertiary river gravels.

Lindgren, W., Tertiary gravels of the Sierra Nevada: P. P. 73, 1911, pp. 105-108.

Turner, H. W., Further contributions to the geology of the Sierra Nevada:

Seventeenth Ann. Rept., pt. 1, 1896, p. 600.

Min. Res. 1908, pt. 1, p. 351.

1909 , pt. 1, pp. 284-285.

Top. sheet Downieville.

Folio 37, 1897. 
STERRA COUNTY-Continued.

151. Sierrá City (Sierra Butte). Au. (D, P1.)

46 miles NW. Truckee, 80 miles ENE. Marysville, S. P. R. R.

Slates, serpentine, quartz porphyry.

Veins, terrace gravels.

Lindgren, W., Tertiary gravels of the Sierra Nevada: P. P. 73, 1911, pp. 117118.

Turner, H. W., Further contributions to the geology of the Sierra Nevada:

Seventeenth Ann. Rept., pt. 1, 1896, p. 653.

Min. Res. 1905, p. 182.

1907, pt. 1, pp. 225-226.

1908, pt. 1, p. 350.

1909 , pt. 1, pp. 284-285.

Top. sheet Sierraville.

\section{SISKIYOU COUNTY.}

152. Beaver Creek. Hg.

28 miles W. Hornbrook, S. P. R. R.

Granodiorite (?).

Impregnations (?).

Min. Res. 1908, pt. 1, p. 690. 1909 , pt. 1, p. 554 .

Top. sheet Shasta.

153. Blue Ledge. $\mathrm{Au}, \mathrm{Cu}$.

40 miles NW. Hornbrook, S. P. R. R.

Granodiorite (?).

Veins (?).

Top. sheet Shasta.

154. Callahans. Au. (D, Pl.)

27 miles SW. Gazelle, S. P. R. R.

Paleozoic sediments cut by acidic and basic intrusives.

Stream gravels and veins.

Min. Res. 1907, pt. 1, p. 227.

1908 , pt. 1, p. 351.

1909 , pt. 1, pp. 285-286.

Top. sheet Shasta.

155. Cecilville. Au. (D, P1.)

57 miles SW. Gazelle, S. P. R. R.

Greenstones.

Veins and stream gravels.

Min. Res. 1905, p. 183.

1907, pt. 1, p. 227.

1908, pt. 1, p. 352 .

1909 , pt. 1, p. 286.

156. Clear Creek. Au, Cu. (D, P1.)

82 miles W. Yreka, Y. R. R.

Paleozoic sediments cut by acidic and basic intrusives.

Veins and stream gravels.

157. Cottage Grove. Au. (Pl.)

100 miles W. Yreka, Y. R. R.

Stream and terrace gravels.

158. Cottonwood Creek (Henley, Hornbrook). Au, Cu. (D, P1.)

Station S. P. R. R.

Metamorphosed Paleozoic sediments cut by granite and diorite.

Veins, stream and terrace gravels. 
SISKIYOU COUITY-Continued.

158. Cottonwood Creek (Henley, Hornbrook)-Continued. Min. Res. 1908, pt. 1, p. 352.

Top. sheet Shasta.

159. Deadwood. Au. (Pl.)

20 miles SW. Yreka, Y. R. R.

Stream gravels.

Top. sheet Shasta.

160. Etna. Au. (Pl.)

31 miles SW. Yreka, Y. R. R.

Stream gravels.

Min. Res. 1909, pt. 1, p. 286.

Top. sheet Shasta.

161. Forks of Salmon. Au. (PI.)

74 miles SW. Yreka, Y. R. R.

Stream and terrace gravels.

Min. Res. 1905, p. 183.

1907 , pt. 1, p. 227.

1909, pt. 1, p. 286.

162. Gazelle. Cr.

10 miles SW. Gazelle, S. P. R. R.

Serpentine.

Lenses.

Min. Res. 1908, pt. 1, p. 769.

Top. sheet Shasta.

163. Gilta. Au. (Pl.)

83 miles SW. Yreka, Y. R. R.

Stream gravels.

Min. Res. 1907, pt. 1, p. 227.

1908 , pt. 1, p. 352.

164. Gottville. Au. (Pl.)

18 miles WSW. Hornbrook, S. P. R. R.

Terrace gravels.

Min. Res. 1908, pt. 1, p. 352.

1909, pt. 1, p. 286.

Top. sheet Shasta.

165. Hamburg. Au. (Pl.)

50 miles W. Hornbrook, S. P. R. R.

Stream and terrace gravels.

Min. Res. 1905, p. 183. 1907, pt. 1, p. 227.

166. Happy Camp (Nolton). Au. (Pl.)

80 miles W. Hornbrook, S. P. R. R.

Stream and terrace gravels.

Min. Res. 1907, pt. 1, p. 227.

167. Hawkinsville (Yreka). Au. (D, Pl.)

Station Y. R. R.

Paleozoic sediments cut by acidic and basic intrusives.

Veins and stream gravels.

Min. Res. 1905, p. 183.

1907 , pt. 1, p. 227.

1908 , pt. 1, p. 352 .

1909 , pt. 1, p. 286.

Top. sheet Shasta. 
168. Knownothing. Au. (Pl.)

60 miles WNW. Bayles, S. P. R. R.

Stream gravels.

169. Oak Bar. Au, Ag. (D, Pl.)

33 miles W. Hornbrook, S. P. R. R.

Schists, Paleozoic sediments cut by acidic and basic intrusives.

Stream and terrace gravels, veins.

Min. Res. 1907, pt. 1, p. 228.

1908 , pt. 1, p. 690 .

Top. sheet Shasta.

170. O'Mears. Au. (Pl.)

72 miles W. Hornbrook, S. P. R. R.

Stream and terrace gravels.

171. Oro Fino (Walker). Au, Cu. (D, PI.)

23 miles SW. Yreka, Y. R. R.

Schists, Paleozoic sediments cut by acidic and basic intrusives.

Stream and terrace gravels, veins.

Top. sheet Shasta.

172. Preston Peak. Au, Cu. (D, Pl.)

80 miles W. Hornbrook, S. P. R. R.

Paleozoic sediments cut by acidic and basic intrusives.

Veins, stream and terrace gravels.

173. Sawyers Bar (Rollins, Black Bear). Au. (Pl.)

57 miles SW. Yreka, Y. R. R.

Stream and terrace gravels.

Min. Res. 1907, pt. 1, p. 227.

1908, pt. 1, p. 352 .

1909 , pt. 1, p. 286.

174. Scotts Bar. Au. (Pl, D.)

45 miles SW. Hornbrook, S. P. R. R.

Paleozoic sediments cut by acidic and basic intrusives.

Stream and terrace gravels, veins.

Min. Res. 1907, pt. 1, pp. 227, 228.

175. Somes Bar. Au. (Pl.)

93 miles $\mathrm{SW}$. Yreka, Y. R. R.

Stream and terrace gravels.

176. Summerville. Au. (Pl.)

50 miles WNW. Bayles, S. P. R. R.

Stream gravels.

\section{SOLANO COUNTY.}

177. St. Johns. Hg.

4 miles SE. Vallejo, S. P. R. R.

Metamorphosed sediments.

Stockworks.

Becker, G. F., Geology of the quicksilver deposits of the Pacific slope:

M. XIII, 1888, p. 378.

Min. Res. 1908, pt. 1, p. 690. 1909, pt. 1, p. 554 .

Top. sheet Carquinez.

SONOMA COUNTY.

178. Cloverdale. $\mathrm{Cr}$.

Station N. W. P. R. R.

Serpentine. 
SONOMA COUNTY-Continued.

178. Cloverdale-Continued.

Lenses.

Min. Res. 1908, pt. 1, p. 769.

179. Geyserville. Cr.

Station N. W. P. R. R.

Serpentine.

Lenses.

Min. Res. 1908, pt. 1, p. 769.

180. Guerneville (Great Eastern). Hg.

Station N. W. P. R. R.

Mesozoic sediments and serpentine cut by andesite and basalt.

Stockworks, veins, and disseminations.

Becker, G. F., Geology of the quicksilver deposits of the Pacific slope:

M. XIII, 1888, pp. 362-364; Eighth Ann. Rept., pt. 2, 1889, p. 980.

Min. Res. 1908, pt. 1, pp. 690-691.

181. Mayacmas (Pine Flat). $\mathrm{Hg}$.

20 miles E. Geyserville, N. W. P. R. R.

Mesozoic sediments cut by andesite and basalt.

Stockworks, veins, and disseminations.

Becker, G. F., Geology of the quicksilver deposits of the Pacific slope:

M. XIII, 1888, pp. 368-377.

Min. Res. 1908, pt. 1, p. 691.

1909, pt. 1, p. 554 .

STANISLAUS COUNTY.

182. Knights Ferry. Au. (Pl.)

12 miles NE. Oakdale, A. T. \& S. F. R. R., S. P. R. R.

Quaternary gravels.

Lindgren, W., Tertiary gravels of the Sierra Nevada: P. P. 73, 1911, p. 201.

TEHAMA COUNTY.

183. Newville. Cr.

70 miles SW. Redding, S. P. R. R.

Serpentine.

Lenses.

Min. Res. 1908, pt. 1, p. 769.

184. Tom Head. $\mathrm{Cu}$.

25 miles NW. Red Bluff, S. P. R. R.

Greenstone.

Veins.

Top. sheet Red Bluff.

\section{TRINITY COUNTY.}

185. Altoona (Allens Camp). $\mathrm{Hg}, \mathrm{Au}$. (D, PI.)

50 miles NW. Bayles, S. P. R. R.

Greenstone, slate, serpentine, and sandstone.

Impregnations and veins.

Becker, G. F., Geology of the quicksilver deposits of the Pacific slope:

M. XIII, 1888, p. 366.

Min. Res. 1908, pt. 1, p. 691.

Top. sheet Shasta.

186. Big Bar. Au. (Pl.)

78 miles WNW. Redding, S. P. R. R.

Stream gravels. 


\section{TRINITY COUNTY-Continued.}

186. Big Bar-Continued.

Diller, J. S., The auriferous gravels of the Trinity River basin, California: Bull. 470, 1911, p. 22.

Min. Res. 1907, pt. 1, p. 229.

1909 , pt. 1, p. 287.

187. Bully Choop. Au.

70 miles W. Redding, S. P. R. R.

Basic intrusives.

Veins.

Min. Res. 1909, pt. 1, p. 287.

Top. sheet Red Bluff.

188. Canyon City. Au. (Pl, D.)

56 miles NW. Redding, S. P. R. R.

Greenstone.

Stream gravels, veins.

189. Carrville. $\mathrm{Au}, \mathrm{Ag}, \mathrm{Cu}$. (D, Pl.)

33 miles NW. Bayles, S. P. R. R.

Greenstones and slates cut by acidic and basic intrusives.

Stream and terrace gravels, veins, and replacements.

MacDonald, D. F., Notes on the gold lodes of the Carrville district, Trinity County, Cal.: Bull. 530, 1912.

Min. Res. 1907, pt. 1, pp. 228-229.

1908, pt. 1, p. 353.

1909 , pt. 1, p. 287.

Top. sheet Shasta.

190. Coffee. Au. (Pl, D.)

40 miles NW. Bayles, S. P. R. R.

Serpentine and granodiorite cut by baeic dikes.

Veins and stream gravels.

MacDonald, D. F., The Weaverville-Trinity Center gold gravele, Trinity County, Cal.: Bull. 430, 1910, pp. 48-58.

Min. Res. 1905, p. 184.

1907, pt. 1, p. 229.

1909, pt. 1, p. 287.

Top. sheet Shasta.

191. Colridge. Au, Hg. (D, PI.)

77 miles NW. Redding, S. P. R. R.

Paleozoic sediments cut by acidic and basic intrusives.

Stream gravels, veins.

192. Deadwood. Au.

31 miless NW. Redding, S. P. R. R.

Paleozoic sediments cut by acidic and basic intrusives.

Veins (?).

Min. Res. 1907, pt. 1, p. 229.

1908, pt. 1, p. 353.

1909 , pt. 1, p. 287.

Top. sheet Red Bluff.

193. Dedrick. Au. (D, Pl.)

48 miles WNW. Bayles, S. P. R. R.

Hornblende schist.

Veins, terrace gravels.

Min. Res. 1907, pt. 1, p. 229.

1908, pt. 1, p. 353.

1909, pt. 1, p. 287. 
TRINITY COUNTY-Continued.

194. Denny. Au, Ag, Pt, Cu. (D, Pl.)

150 miles NW. Redding, S. P. R. R.

Paleozoic sediments cut by basic intrusives.

Veins, stream gravels.

Min. Res. 1907, pt. 1, p. 229.

1908, pt. 1, p. 353.

1909 , pt. 1, p. 287.

195. Dodge. Au. (PI.)

47 miles SSW. Gazelle, S. P. R. R.

Terrace and stream gravels.

Top. sheet Shasta.

196. Dorleska. Au. (D, Pl.)

55 miles WNW. Bayles, S. P. R. R.

Serpentine, limestone, and shale cut by basic intrusives.

Veins.

MacDonald, D. F., Notes on the gold lodes of the Carrville district, Trinity

County, Cal.: Bull. 530, 1912.

Min. Res. 1905, p. 184.

1907 , pt. 1, p. 229.

1908 , pt. 1, p. 353 .

1909 , pt. 1, p. 287.

Top. sheet Shasta.

197. Douglas City. Au. (D, P1.)

57 miles W. Redding, S. P. R. R.

Mica and hormblende shist.

Stream and terrace gravels, veins.

Diller, J. S., The auriferous gravels of the Trinity River basin, California:

Bull. 470, 1911, pp. 18-20.

Min. Res. 1907, pt. 1, p. 229.

1908 , pt. 1 , pp. $353-354$.

1909 , pt. 1 , p. 287.

Top. sheet Red Bluff.

198. Eastman Gulch (Lewiston). Au. (D, Pl.)

36 miles NW. Redding, S. P. R. R.

Paleozoic sediments cut by greenstone.

Stream gravels, veins.

Min. Res. 1909, pt. 1, p. 287.

Top. sheet Red Bluff.

199. Hawkins Creek (Burnt Ranch). Au. (Pl.)

105 miles WNW. Redding, S. P. R. R.

Terrace and stream gravels.

Min. Res. 1907, pt. 1, pp. 228, 229.

1908, pt. 1, p. 353.

200. Hay Fork. Au, Pt. (Pl.)

78 miles W. Redding, S. P. R. R.

Stream and terrace gravels.

Diller, J. S., The auriferous gravels of the Trinity River basin, California:

Bull. 470, 1911, p. 21.

Min. Res. 1897-98, p. 267.

1907 , pt. 1, p. 229.

1909 , pt. 1, p. 287.

201. Helena. Au. (Pl.)

67 miles NW. Redding, S. P. R. R. 
TRINITY COUNTY-Continued.

201. Helena-Continued.

Stream and terrace gravels.

Min. Res. 1907, pt. 1, p. 229.

1909 , pt. 1, p. 287.

202. Indian Creek. Au. (P1.)

85 miles W. Redding, S. P. R. R.

Stream and terrace gavels.

Min. Res. 1907, pt. 1, p. 229.

Top. sheet Red Bluff.

203. Junction City. Au, Pt. (Pl.)

59 miles WNW. Redding, S. P. R. R.

Stream and terrace gravels.

Kemp, J. F., The geological relations and distribution of platinum and associated metals: Bull. 193, 1902, pp. 51-54.

MacDonald, D. F., The Weaverville-Trinity Center gold gravels, Trinity County, Cal.: Bull. 430, 1910, pp. 48-58.

Min. Res. 1905, p. 184.

1909 , pt. 1, p. 287.

204. Minersville. Au. (Pl.)

44 miles NW. Redding, S. P. R. R.

Stream and terrace gravels.

MacDonald, D. F., The Weaverville-Trinity Center gold gravels, Trinity County, Cal.: Bull. 430, 1910, pp. 48-58.

Min. Res. 1905, p. 184.

1907 , pt. 1, p. 229.

1909, pt. 1, p. 287.

Top. sheet Red Bluff.

205. Slate Creek. Au. (Pl.)

54 miles NW. Redding, S. P. R. R.

Stream gravels.

Top. sheet Red Bluff.

206. Swedes Point. Au. (Pl.)

88 miles WNW. Redding, S. P. R. R.

Terrace gravels.

207. Trinity Center. Au. (Pl.)

29 miles W. Bayles, S. P. R. R.

Stream and terrace gravels.

MacDonald, D. F., The Weaverville-Trinity Center gold gravels, Trinity County, Cal.: Bull. 430, 1910, pp. 48-58.

Notes on the gold lodes of the Carrville district, Trinity County, Cal.: Bull. 530, 1912.

Min. Res. 1905, p. 184.

1907 , pt. 1, pp. 228, 229.

1908 , pt. 1, p. 354 .

1909 , pt. 1, p. 287.

Top. sheet Shasta.

208. Weaverville. Au. (Pl.)

50 miles NW. Redding, S. P. R. R.

Terrace and stream gravels.

Diller, J. S., The auriferous gravels of the Trinity River basin, California:

Bull. 470, 1911, pp. 15-18, 25.

MacDonald, D. F., The Weaverville-Trinity Center gold gravels, Trinity County, Cal.: Bull. 430, 1910, pp. 48-58, 1909. 
TRINTTY COUNTY-Continued.

208. Weaverville-Continued.

Min. Res. 1905, pp. 183, 184.

1907, pt. 1, pp. 228, 229.

1909 , pt. 1, p. 287.

Top. sheet Red Bluff.

\section{TUOLUMNE COUNTY.}

209. Big Oak Flat. Au. (D, Pl.)

16 miles E. Chinese Camp, S. R. R. of Cal.

Slates cut by granodiorite.

Veins.

Lindgren, W., Tertiary gravels of the Sierra Nevada: P. P. 73, 1911, p. 217.

Min. Res. 1905, p. 175.

1907 , pt. 1, p. 230.

1908 , pt. 1, p. 355.

1909 , pt. 1, p. 288.

Top. sheet Sonora.

Folio 41, 1897.

210. Chinese Camp (Jacksonville). Au, Cr. (Pl, D.)

Station S. R. R. of Cal.

Slates and serpentine.

Tertiary river gravels, lenses.

Lindgren, W., Tertiary gravels of the Sierra Nevada: P. P. 73, 1911, p. 217.

Min. Res. 1905, p. 175.

1907, pt. 1, p. 232.

1908 , pt. 1, p. 769 .

1909 , pt. 1, p. 288.

Top. sheet Sonora.

Folios 41, 1897; 63, 1900.

211. Columbia. Au. (Pl.)

4 miles N. Sonora, S. R. R. of Cal.

Tertiary river gravels.

Lindgren, W., Tertiary gravels of the Sierra Nevada: P. P. 73, 1911, pp. 212-213.

Min. Res. 1905, p. 175.

1906 , p. 355.

1909 , pt. 1, p. 288

Top. sheet Big Trees.

Folios 51, 1898; 63, 1900.

212. Jamestown (Rawhide, Stent). Au. (Pl, D.)

Station S. R. R. of Cal.

Slates, serpentine, and amphibolites.

Veins and Tertiary river gravels.

Lindgren, W., Tertiary gravels of the Sierra Nevada: P. P. 73, 1911, pp. 217-218.

Min. Res. 1905, pp. 175-176.

1907 , pt. 1, p. 231.

1908, pt. 1, pp. 355-356. 769.

1909 , pt. 1, p. 288.

Top. sheet Sonora.

Folios 41, 1897; 63, 1900.

213. Soulsbyville (Carters). Au

Station S. R. R. of Cal.

Granodiorite. 
TUOLUMNE coUNTr-Continued.

213. Soulsbyville (Carters)-Continued

Veins.

Lindgren, W., Tertiary gravels of the Sierra Nevada: P. P. 73, 1911, p. 217.

Turner, H. W., Further contributions to the geology of the Sierra Nevada:

Seventeenth Ann. Rept., pt. 1, 1896, pp. 676-677.

Min. Res. 1905, p. 175.

1907, pt. 1, pp. 230-231, 232.

1908, pt. 1, p. 356 .

1909 , pt. 1, p. 288.

Top. sheet Sonora.

Folio 41, 1897.

214. Tuttletown. Au.

Station S. R. R. of Cal.

Slates and amphibolites.

Veins.

Lindgren, W., Tertiary gravels of the Sierra Nevada: P. P. 73, 1911, p. 217. Turner, H. W., Further contributions to the geology of the Sierra Nevada:

Seventeenth Ann. Rept., pt. 1, 1896, p. 675.

Min. Res. 1908, pt. 1, p. 356.

1909 , pt. 1, p. 288.

Top. sheet Sonora.

Folios 41, 1897; 63, 1900.

YOLO COUNTY.

215. Knoxville. Hg.

5 miles W. Tancred, S. P. R. R.

Mesozoic sediments and serpentine cut by andesite and basalt.

Stockworks and disseminations.

Becker, G. F., Geology of the quicksilver deposits of the Pacific slope:

M. XIII, 1888, pp. 271-290; Eighth Ann. Rept., pt. 2, 1889, p. 976.

YUBA COUNTY.

216. Browns Valley. Au.

13 miles NW. Marysville, S. P. R. R.

Diabase.

Veins.

Min. Res. 1907, pt. 1, p. 233.

1909, pt. 1, p. 289.

Top. sheet Smartsville.

Folio 18, 1895.

217. Brownsville. Au. (Pl, D.)

34 miles NE. Marysville, 36 miles E. Oroville, S. P. R. R.

Greenstone and diorite.

Tertiary river gravels and veins.

Min. Res. 1907, pt. 1, p. 233.

1908, pt. 1, p. 357.

1909 , pt. 1, p. 289.

Top. sheet Smartsville.

Folio 18, 1895.

218. Camptonville. Au. (P1.)

47 miles NE. Marysville, S. P. R. R.; 23 miles N. Nevada City, N. C. N. G. R. R.

Tertiary river gravels. 
YUBA COUNTY-Continued.

218. Camptonville-Continued.

Lindgren, W., Tertiary gravels of the Sierra Nevada: P. P. 73, 1911, pp. 105-108, 123.

Min. Res. 1909, pt. 1, p. 289.

Top. sheet Smartsville.

Folio 18, 1895.

219. Dobbins. Au.

30 miles NE. Marysville, S. P. R. R.

Granodiorite.

Veins.

Min. Res. 1907, pt. 1, p. 233.

1909, pt. 1, p. 289.

Top. sheet Smarteville.

Folio 18, 1895.

220. Marysville (Yuba Basin). Au. (Pl.)

Station S. P. R. R.

Quaternary river gravels.

Lindgren, W., Tertiary gravels of the Sierra Nevada: P. P. 73, 1911, pp. 120, 221-222.

Min. Res. 1907, pt. 1, p. 232.

1909 , pt. 1, p. 289.

Top. sheet Smartsville.

Folio 18, 1895.

221. Smartsville. Au. (Pl.)

27 miles E. Marysville, S. P. R. R.; 14 miles W. Grass Valley, N. C. N. G. R. R.

Tertiary river gravels.

Lindgren, W., Tertiary gravels of the Sierra Nevada: P. P. 73, 1911, pp. 123-125.

Min. Res. 1907, pt. 1, p. 232.

1908 , pt. 1, p. 357.

Top. sheet Smartsville.

Folio 18, 1895.

222. Wheatland. Au. (Pl.)

Station S. P. R. R.

Quaternary gravels.

Min. Res. 1907, pt. 1, pp. 232, 233.

Top. sheets Smartsville, Wheatland.

Folio 18, 1895.

$21528^{\circ}-$ Bull. $507-12-8$ 


\section{CAIIFORNIA, SOUTHERN COUNTIES.}

In the 17 southern counties of California there are 191 regions which the Survey has called mining districts for convenience of reference. Of this number 9 produce mainly iron or chromium, 11 quicksilver, 3 rare metals, 129 gold, 19 copper, 12 silver, and 7 lead. In one district the principal metal is unknown.

Distribution of the predominant metals produced in the mining districts of the southern counties of California.

\begin{tabular}{|c|c|c|c|c|c|c|c|c|c|}
\hline County. & Gold. & Silver. & Copper. & Lead. & Iron. & $\begin{array}{l}\text { Quick- } \\
\text { silver. }\end{array}$ & $\begin{array}{c}\text { Rare } \\
\text { metals. }\end{array}$ & $\begin{array}{c}\text { Un- } \\
\text { known. }\end{array}$ & Total. \\
\hline 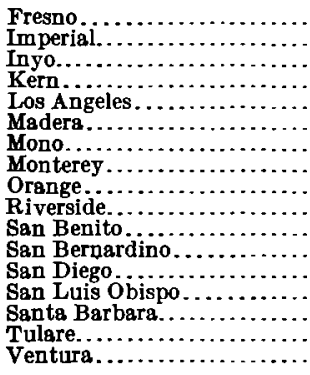 & $\begin{array}{r}3 \\
4 \\
19 \\
22 \\
7 \\
6 \\
13 \\
2 \\
9 \\
2 \\
2 \\
9 \\
1 \\
5 \\
2\end{array}$ & 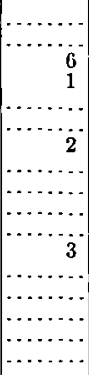 & $\begin{array}{r} \\
\ldots \\
\ldots \ldots \\
4 \\
2 \\
\ldots \ldots \\
1 \\
\cdots \ldots \ldots \\
\cdots \ldots \ldots\end{array}$ & $\left.\begin{array}{l}\cdots \\
\cdots\end{array}\right]$ & 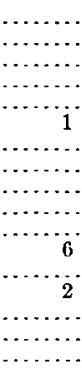 & 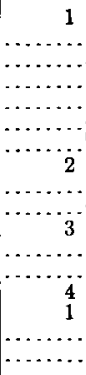 & 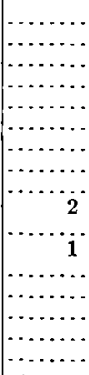 & 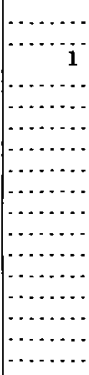 & $\begin{array}{r}4 \\
36 \\
25 \\
7 \\
8 \\
15 \\
4 \\
1 \\
11 \\
3 \\
45 \\
10 \\
7 \\
1 \\
6 \\
2\end{array}$ \\
\hline & 129 & 12 & 19 & 7 & 9 & 11 & 3 & . 1 & $\begin{array}{ll}-191 \\
\end{array}$ \\
\hline
\end{tabular}

MINING DISTRICTS IN SOUTHERN COUNTIES OF CALIFORNIA.

[See Pl. V. Additional references may be found in Mineral Resources for 1910 and 1911.]

FRESNO COUNTY.

1. Big Dry Creek. Cu, Au.

11 miles ENE. Clovis, S. P. R. R.

Mica schists and granite cut by basic dikes.

Lenses.

2. Mexican. Hg.

25 miles W. Mendota, S. P. R. R.

Upper Cretaceous (Chico) sandstone.

- Dissemination.

Min. Res. 1908, pt. 1, p. 685.

3. Mill Creek. Au, Ag. (D, Pl.)

22 miles W. Sanger, S. P. R. R.

Schists and granite.

Veins.

4. Pollasky. Cu.

Friant station, S. P. R. R.

Schists and granite.

Veins.

Min. Res. 1909, pt. 1, pp. 274-275.

5. Sycamore. Au.

30 miles NE. Clovis, S. P. R. R.

Granite, slate, and schist.

Veins.

Top. sheet Kaiser. 
FRESNO COUNTY-Continued.

6. Temperance Flat. Au.

12 miles E. Friant, S. P. R. R.

IMPERLAL COUNTY.

7. Cargo Muchacho (Hedges, Ogilby). Au.

5 miles NE. Ogilby, S. P. R. R.

Schists cut by pegmatite.

Veins.

Min. Res. 1905, p. 181.

1907, pt. 1, p. 234.

1909, pt. 1, p. 289.

Top. sheet Yuma.

8. Picacho. Au. (D, Pl.)

25 miles NNW. Yuma, S. P. R. R.

Schist and granite capped by Tertiary volcanics.

Impregnations, and wash gravels.

Min. Res. 1909, pt. 1, p. 289.

9. White Gold Basin. Au, Ag. (D, Pl.)

22 miles NNW. Yuma, S. P. R. R.

Min. Res. 1907, pt. 1, p. 234.

Top. sheet Yuma.

10. Potholes. Au. (Pl, D.)

12 miles NNE. Yuma, S. P. R. R.

Gneiss.

Veins.

Top. sheet Yuma.

11. Beveridge. Au.

INYO COUNTY.

10 miles NE. Whitney, S. P. R. R.

Paleozoic sediments cut by granite.

Veins.

Spurr, J. E., Geology of Nevada south of the fortieth parallel: Bull. 208, 1903 , pp. 206-212.

Top. sheet Mount Whitney.

12. Big Pine. $\mathrm{Au}, \mathrm{Ag}, \mathrm{Pb}$.

Alvord station, S. P. R. R.

Paleozoic sediments cut by granite.

Veins.

Spurr, J. E., Geology of Nevada south of the fortieth parallel: Bull. 208, 1903, pp. 206-212.

Min. Res. 1907, pt. 1, p. 207.

1908, pt. 1, p. 334

1909 , pt. 1, p. 275.

13. Bishop Creek. $\mathrm{Au}, \mathrm{Ag}, \mathrm{Cu}$.

5 miles SW. Laws, S. P. R. R.

Paleozoic sediments cut by granite.

Veins.

Spurr, J. E., Geology of Nevada south of the fortieth parallel: Bull. 208, 1903, pp. 206-212.

Min. Res. 1907, pt. 1, p. 207.

1908, pt. 1, p. 335.

14. Cerro Gordo. $\mathrm{Ag}, \mathrm{Pb}, \mathrm{Au}, \mathrm{Cu}, \mathrm{Fe}$.

28 miles NE. Keeler, S. P. R. R.

Paleozoic sediments cut by granite.

Contact metamorphic, replacements. 
INYO COUNTY-Continued.

14. Cerro Gordo-Continued.

Spurr, J. E., Geology of Nevada south of the fortieth parallel: Bull. 208, 1903, pp. 206-212.

Min. Res. 1907, pt. 1, p. 208.

1908 , pt. 1, p. 334 .

15. Chloride Cliff. $\mathrm{Au}, \mathrm{Pb}, \mathrm{Cu}$.

15 miles S. Bullfrog, Nev., B. G. R. R.

Paleozoic sediments.

Replacements and veins.

Ball, S. H., Notes on ore deposits of southwestern Nevada and eastern California: Bull. 285, 1906, pp. 72-73.

- A geologic reconnaissance in southwestern Nevada and eastern California: Bull. 308, 1907, pp. 161, 162, 173-175.

Spurr, J. E., Geology of Nevada south of the fortieth parallel: Bull. 208, 1903, pp. 187-195.

Top. sheet Furnace Creek.

16. Darwin. $\mathrm{Au}, \mathrm{Ag}, \mathrm{Cu}, \mathrm{Pb}$.

24 miles SE. Keeler, S. P. R. R.

Paleozoic sediments cut by monzonite.

Veins.

Spurr, J. E., Geology of Nevada south of the fortieth parallel: Bull. 208, 1903, pp. 212-213.

Min. Res. 1907, pt. 1, pp. 207-208.

1908, pt. 1, p. 334 .

1909 , pt. 1, p. 275.

17. Daylight. $\mathrm{Ag}, \mathrm{Pb}(\mathrm{Au}, \mathrm{Cu})$.

15 miles SW. Bullfrog, Nev., B. G. R. R.

Tertiary volcanics.

Veins.

Ball, S. H., A geologic reconnaissance in southwestern Nevada and eastern California: Bull. 308, 1907, p. 176.

Spurr, J. E., Geology of Nevada south of the fortieth parallel: Bull. 208, 1903, pp. 187-195.

Top. sheet Furnace Creek.

18. Deep Springs (Pine Mountain). $\mathrm{Ag}, \mathrm{Pb}(\mathrm{Au}, \mathrm{Cu})$.

15 miles E. Laws, S. P. R. R.

Paleozoic sediments cut by granite.

Veins.

Spurr, J. E., Geology of Nevada south of the fortieth parallel: Bull. 208, 1903, pp. 206-212.

19. Echo Canyon (Schwaub). Au.

12 miles NW. Death Valley, T. \& T. R. R.

Paleozoic sediments.

Veins.

Ball, S. H., A geologic reconnaissance in southwestern Nevada and eastern California: Bull. 308, 1907, p. 175.

Spurr, J. E., Geology of Nevada south of the fortieth parallel: Bull. 208, 1903, pp. 187-195.

Top. sheet Furnace Creek.

20. Emigrant. Au.

75 miles NE. Brown, S. P. R. R.

Paleozoic sediments and Tertiary volcanics.

Veins. 
INYO COUNTY-Continued.

20. Emigrant-Continued.

Spurr, J. E., Geology of Nevada south of the fortieth parallel: Bull. 208, 1903, pp. 200-205.

Top. sheet Ballarat.

21. Furnace Creek (Greenwater). $\mathrm{Cu}, \mathrm{Au}, \mathrm{Ag}$.

20 miles S. Death Valley, T. \& T. R. R.

Paleozoic sediments cut by granite.

Veins.

Ball, S. H., A geologic reconnaissance in southwestern Nevada and eastern California: Bull. 308, 1907, p. 201.

Spurr, J. E., Geology of Nevada south of the fortieth parallel: Bull. 208, 1903, pp. 187-195.

Min. Res. 1907, pt. 1, p. 208.

Top. sheet Greenwater.

22. Goldbelt. $\mathrm{Cu}, \mathrm{Au}$.

30 miles E. Keeler, S. P. R. R.

Paleozoic sediments cut by monzonite.

Contact metamorphic, veins.

Ball, S. H., Notes on ore deposits of southwestern Nevada and eastern California: Bull. 285, 1906, p. 73.

- A geologic reconnaissance of southwestern Nevada and eastern California: Bull. 308, 1907, p. 211.

Spurr, J. E., Geology of Nevada south of the fortieth parallel: Bull. 208, 1903, pp. 200-205.

Top. sheet Ballarat.

23. Grapevine Canyon. $\mathrm{Au}, \mathrm{Ag}$.

20 miles NW. Bullfrog, Nev., B. G. R. R.

Paleozoic sediments and Tertiary volcanice.

Veins.

Ball, S. H., A geologic reconnaissance in southwestern Nevada and eastern California: Bull. 308, 1907, p. 176.

Spurr, J. E., Geology of Nevada south of the fortieth parallel: Bull. 208, 1903, pp. 187-195.

24. Harrisburg. $\mathrm{Cu}, \mathrm{Au}, \mathrm{Ag}, \mathrm{Pb}$.

70 miles NE. Brown, S. P. R. R.

Paleozoic sediments cut by quartz monzonite.

Veins.

Ball, S. H., A geologic reconnaissance in southwestern Nevada and eastern California: Bull. 308, 1907, p. 211.

Spurr, J. E., Geology of Nevada south of the fortieth parallel: Bull. 208, 1903, pp. 200-205.

Top. sheet Harrisburg.

25. Independence (Paiute, Waucoba). $\mathrm{Ag}, \mathrm{Pb}, \mathrm{Au}$.

10 miles NE. Citrus, S. P. R. R.

Paleozoic sediments cut by granite.

Veins.

Spurr, J. E., Geology of Nevada south of the fortieth parallel: Bull. 208, 1903, pp. 206-212.

Min. Res. 1907, pt. 1, p. 208.

Top. sheet Mount Whitney.

26. Kearsarge. Au, Ag.

10 miles W. Citrus, S. P. R. R.

Paleozoic sediments cut by granite. 
ITYO COUNTY-Continued.

26. Kearsarge-Continued.

Veins.

Top. sheet Mount Whitney.

27. Kelley. Au, Ag.

20 miles NE. Brown, S. P. R. R.

28. Lee. $\mathrm{Au}, \mathrm{Ag}$.

30 miles SSE. Keeler, S. P. R. R.

Paleozoic sediments cut by granite.

Veins and replacements.

Spurr, J. E., Geology of Nevada south of the fortieth parallel: Bull. 208, 1903, pp. 212-213.

Top. sheet Ballarat.

29. Lees Camp. Au, Ag.

6 miles W. Leeland, T. \& T. R. R.

Paleozoic sedimente.

Veins.

Ball, S. H., A geologic reconnaissance in southwestern Nevada and eastern California: Bull. 308, 1907, p. 175.

Spurr, J. E., Geology of Nevada south of the fortieth parallel: Bull. 208, 1903, pp. 187-195.

Min. Res. 1907, pt. 1, p. 208.

30. Lone Pine.

Top. sheet Lees Camp.

4 miles W. Whitney, S. P. R. R.

Top. sheet Mount Whitney.

31. Lookout (Modoc). $\mathrm{Pb}, \mathrm{Ag}$.

45 miles NE. Brown, S. P. R. R.

Paleozoic sediments, Tertiary volcanics.

Replacements.

Spurr, J. E., Geology of Nevada south of the fortieth parallel: Bull. 208, 1903, pp. 212-213.

Top. sheet Ballarat.

32. New Coso. $\mathrm{Pb}, \mathrm{Ag}(\mathrm{Cu}, \mathrm{Au})$.

30 miles NE. Brown, S. P. R. R.

Paleozoic sediments, Tertiary volcanics.

Replacements.

Spurr, J. E., Geology of Nevada south of the fortieth parallel: Bull. 208, 1903, pp. 212-213.

33. Panamint (Ballarat). $\mathrm{Ag}, \mathrm{Pb}(\mathrm{Cu}, \mathrm{Sb})$.

50 miles NE. Brown, S. P. R. R.

Paleozoic sediments.

Veins, replacements.

Spurr, J. E., Geology of Nevada south of the fortieth parallel: Bull. 208, 1903, pp. 200-205.

Min. Res. $1883-84$, p. 642.

1907, pt. 1, p. 207.

1908 , pt. 1, p. 334.

1909 , pt. 1, p. 275.

Top. sheet Ballarat.

34. Poison Spring. Au.

16 miles W. Leeland, T. \& T. R. R.

Paleozoic sediments.

Veins.

Ball, S. H., A geologic reconnaissance in southwestern Nevada and eastern California: Bull. 308, 1907, p. 175.

Top. sheet Furnace Creek. 
35. Russ. $\mathrm{Pb}, \mathrm{Ag}$.

INYO COUNTY-Continued.

15 miles NE. Whitney, S. P. R. R.

Paleozoic sediments cut by granite.

Replacements and veins.

Spurr, J. E., Geology of Nevada south of the fortieth parallel: Bull. 208, 1903, pp. 206-212.

36. Saratoga (Tecopa Mountain). $\mathrm{Pb}, \mathrm{Cu}, \mathrm{Ag}, \mathrm{Au}, \mathrm{Zn}$.

12 miles NE. Tecopa, T. \& T. R. R.

Paleozoic sediments, granite and Tertiary volcanics.

Veins.

Spurr, J. E., Geology of Nevada south of the fortieth parallel: Bull. 208, 1903, pp. 195-200.

Min. Res. 1907, pt. 1, pp. 207, 208.

37. Sherwin. Au.

20 miles W. Bishop, S. P. R. R.

Granite.

Veins.

38. Skidoo. $\mathrm{Au}, \mathrm{Pb}, \mathrm{Ag}$.

75 miles NE. Brown, S. P. R. R.; 45 miles SW. Rhyolite, Nev., B. G. R. R.

Granite cut by syenite, diorite, and monzonite.

Veins.

Spurr, J. E., Geology of Nevada south of the fortieth parallel: Bull. 208, 1903, pp. 200-205.

Min. Res. 1907, pt. 1, p. 208.

1908 , pt. 1, p. 334.

1909 , pt. 1, p. 275.

Top. sheet Ballarat.

39. Swansea (Keeler, Kruger). $\mathrm{Pb}, \mathrm{Ag}$.

Keeler station S. P. R. R.

Paleozoic sediments cut by granite.

Veins and replacements.

Spurr, J. E., Geology of Nevada south of the fortieth parallel: Bull. 208, 1903, pp. 206-212.

Min. Res. 1909, pt. 1, p. 275.

40. Tibbets. $\mathrm{Au}, \mathrm{Ag}$.

16 miles N. Citrus, S. P. R. R.

Paleozoic sediments cut by granite.

Veins.

Spurr, J. E., Geology of Nevada south of the fortieth parallel: Bull. 208, 1903, pp. 206-212.

Top. sheet Mount Whitney.

41. Tinemaha (Fish Springa). Au.

10 miles S. Alvord, S. P. R. R.

Paleozoic sediments cut by granite.

Veins.

Min. Res. 1907, pt. 1, p. 208.

42. Tucki Mountain. Au.

80 miles NE. Brown, S. P. R. R.; 50 miles SW. Death Valley, T. \& T. R. R.

Paleozoic sediments cut by granite.

Veins.

Ball, S. H., A geologic reconnaissance in southwestern Nevada and eastern California: Bull. 308, 1907, p. 211.

Spurr, J. E., Geology of Nevada south of the fortieth parallel: Bull. 208, 1903, pp. 200-205.

Top. sheet Ballarat. 
INYO COUNTY-Continued.

43. Ubehebe. $\mathrm{Cu}(\mathrm{Au}, \mathrm{Ag})$.

60 miles E. Laws, S. P. R. R.

Paleozoic limestone cut by acidic intrusives.

Contact metamorphic.

44. Union. $\mathrm{Pb}, \mathrm{Ag}$.

12 miles SSE. Laws, S. P. R. R.

Paleozoic sediments cut by granite.

Replacements and veins.

Spurr, J. E., Geology of Nevada south of the fortieth parallel: Bull. 208, 1903, pp. 206-212.

45. Wildrose. $\mathrm{Ag}, \mathrm{Pb}$

70 miles NE. Brown, S. P. R. R.

Paleozoic sediments cut by granite.

Veins and replacements.

Spurr, J. E., Geology of Nevada south of the fortieth parallel: Bull. 208, 1903, pp. 200-205.

Top. sheet Ballarat.

46. Willow. $\mathrm{Au}, \mathrm{Ag}$.

15 miles NW. Zabriskie, T. \& T. R. R.

Paleozoic sedimente, Tertiary volcanics.

Veins.

Spurr, J. E., Geology of Nevada south of the fortieth parallel: Bull. 208, 1903, pp. 187-195.

\section{EERA COUNTY.}

47. Agua Caliente. Au.

20 miles E. Caliente, A. T. \& S. F. R. R.

Veins.

48. Amalie (Paris). $\mathrm{Ag}, \mathrm{Au}, \mathrm{W}, \mathrm{Pb}$.

15 miles E. Caliente, A. T. \& S. F. R. R.

Limestone and schist.

Veins.

Min. Res. 1905, p. 177.

1906, pp. 188,525 .

49. Big Dixie. Au.

1908, pt. 1, pp. 335, 336, 724 .

15 miles NE. Cantil, S. P. R. R.

50. Black Mountain. Au, Mo.

15 miles NW. Johannesburg, A. T. \& S. F. R. R.

51. Garlock. Au.

11 miles SW. Johannesburg, A. T. \& S. F. R. R.

Granite, schist.

Veins.

Hess, F. L., Gold mining in the Randsburg quadrangle, California: Bull. 430,1910 , pp. 23-47.

52. Goler. Au. (Pl.)

6 miles W. Searles, S. P. R. R.

Wash gravels.

Hess, F. L., Gold mining in the Randsburg quadrangle, California: Bull. 430,1910 , pp. $23-47$.

53. Greenhorn Mountains. Au.

40 miles N. Caliente, A. T. \& S. F. R. R. 


\section{KERN COUNTY-Continued.}

54. Green Mountain. Au, Ag.

48 miles NNE. Caliente, A. T. \& S. F. R. R.

Top. sheet Kernville.

55. Havilah. Au.

25 miles N. Caliente, A. T. \& S. F. R. R.

Metamorphosed slates and limestones cut by granite.

Vèins.

Min. Ree. 1908, pt. 1, p. 336 .

56. Isabella. $\mathrm{Au}, \mathrm{Ag}$.

37 miles N. Caliente, A. T. \& S. F. R. R.

Min. Res. 1909, pt. 1, p. 276.

Top. sheet Kernville.

57. Kernville. $\mathrm{Au}, \mathrm{Ag}, \mathrm{Cu}$.

42 miles N. Caliente, A. T. \& S. F. R. R.

Top. sheet Kernville.

58. Keysville. $\mathrm{Au}, \mathrm{Ag}$.

31 miles N. Caliente, A. T. \& S. F. R. R.

59. Long Tom. $\mathrm{Au}(\mathrm{Pb}, \mathrm{Cu})$.

15 miles ENE. Bakersfield, A. T. \& S. F. R. R., S. P. R. R.

60. Mohave. Au, Ag.

Mojave station, A. T. \& S. F. R. R., S. P. R. R.

Tertiary volcanics.

Veins.

Min. Res. 1905, p. 177.

1906, p. 188.

1907, pt. 1, p. 209.

1908 , pt. 1, p. 336.

$\cdot 1909$, pt. 1, p. 276.

61. Piute Mountain. Au.

35 miles N.NE. Caliente, A. T. \& S. F. R. R.

Min. Res. 1908, pt. 1, p. 336.

1909 , pt. 1, p. 276.

Top. sheet Kernville.

62. Rademacher. $\mathrm{Cu}, \mathrm{Ag}, \mathrm{Au}$.

5 miles N. Searles, S. P. R. R.

Granite and schist.

Veins.

Hess, F. L., Gold mining in the Randsburg quadrangle, California: Bull. 430,1910 , pp. $23-47$.

63. Randsburg. Au, Ag, W.

$1 \frac{1}{4}$ miles W. Johannesburg, A. T. \& S. F. R. R.

Schist and granite.

Veins.

Hess, F. L., Gold mining in the Randsburg quadrangle, California: Bull. 430, 1910, pp. 23-47.

Spurr, J. E., Geology of Nevada south of the fortieth parallel: Bull. 208, 1903, pp. 216-218.

Min. Res. 1905, pp. 177, 412.

1906, pp. 189,525 .

1907 , pt. 1, pp. $209-210$.

1908, pt. 1, pp. 336, 723.

1909, pt. 1, p. 276.

Top. sheet Randsburg. 
KERI COUNTY-Continued.

64. Red Rock. Au. (Pl.)

12 miles NNW. Cantil, S. P. R. R.

Wash gravels.

65. Rosamond. Au.

9 miles W. Rosamond, S. P. R. R.

Min. Res. 1905, p. 177.

66. San Emigdio. Au, Sb.

30 miles NE. Caliente, A. T. \& S. F. R. R.

Min. Res. 1882, pp. 438-439.

67. Searles. $\mathrm{Au}, \mathrm{Cu}$.

$1883-84$, p. 641 .

1885 , p. 387 .

Station S. P. R. R.

68. Slate Range. Au.

28 miles SSE. Portersville, S. P. R. R.

Veins.

69. Summit. Au. (Pl.)

6 miles N. Johannesburg, A. T. \& S. F. R. R.

Wash gravels.

Hess, F. L., Gold mining in the Randsburg quadrangle, California: Bull.

430, 1910, pp. 23-47.

Top. sheet Randsburg.

70. Vaughn. Au.

33 miles N. Caliente, A. T. \& S. F. R. R.

71. Woody. $\mathrm{Cu}, \mathrm{Au}$.

34 miles NE. Martensdale, S. P. R. R.

Granodiorite, aplite.

Vein.

LOS ANGELES COUNTY.

72. Arrastre Canjon (Newhall). Au. (Pl.)

Station S. P. R. R.

Wash gravels.

Top. sheet Santa Susana.

73. Azusa (Kern). Au, Ag (Ni, Co).

10 miles N. San Dimas, $\Lambda$. T. \& S. F. R. R., S. P. R. R.

Schist and greenstones.

Veins.

Top. sheet Pomona.

74. Baldy Mountain. Au.

22 miles SE. Palmdale, S. P. R. R.

Top. sheet Rock Creek.

75. Cedar. Au, Ag.

8 miles N. Acton, S. P. R. R.

Schist and greenstones.

Veins.

76. Gleason Mountain. $\mathrm{Au}, \mathrm{Ag}(\mathrm{Cu})$.

15 miles S. Acton, S. P. R. R.

Granite and schist.

Veins.

Top. sheet Tujunga.

77. Soledad (Acton). Au. (D, Pl.)

Station S. P. R. R.

Granite and schist. 
LOS ANGELES COUNTY-Continued.

77. Soledad (Acton)-Continued.

Veins, stream gravels.

Min. Res. 1907, pt. 1, p. 233.

Top. sheet Tujunga.

78. Tujunga. Au, Ag.

15 miles SSE. Acton, S. P. R. R.

Granite and schist.

Veins.

Top. sheet Tujunga.

MADERA COUNTY.

79. Coarse Gold. Au. (D, Pl.)

44 miles NE. Madera, S. P. R. R.

Turner, H. W., Further contributions to the geology of the Sierra Nevada:

Seventeenth Ann. Rept., pt. 1, 1896, pp. 684, 695.

Min. Res. 1906, p. 190.

1907, pt. 1, p. 234.

1908, pt. 1, p. 359.

80. Daulton. Cu.

Station S. P. R. R.

Granite and schist.

Veins.

Min. Res. 1907, pt. 1, p. 234.

81. Fine Gold. Au. (D, P1.)

42 miles E. Madera, S. P. R. R.

Veins and stream gravels.

Turner, H. W., Further contributions to the geology of the Sierra Nevada:

Seventeenth Ann. Rept., pt. 1, 1896, pp. 684, 695.

82. Grub Gulch. Au, W. (D, Pl.)

14 miles NE. Raymond, S. P. R. R.

Schist.

Veins.

Hess, F. L., Note on a tungsten-bearing vein near Raymond, Cal.: Bull. 340, 1908, p. 271.

Turner, H. W., Further contributions to the geology of the Sierra Nevada: Seventeenth Ann. Rept., pt. 1, 1896, p. 695.

83. Hildreth. Au, Ag.

35 miles E. Madera, S. P. R. R.

Granite and slate.

Veins.

84. Minarets (Mount Raymond). Fe, Ag.

90 miles NE. Friant, S. P. R. R.

Turner, H. W., Further contributions to the geology of the Sierra Nevada:

Seventeenth Ann. Rept., pt. 1, 1896, p. 711.

Top. sheet Mount Lyell.

85. North Fork. Au.

45 miles ENE. Madera, S. P. R. R.

Top. sheet Mount Lyell.

86. Potter Ridge. Au.

10 miles NE. Madera, S. P. R. R.

Granite and slate.

Veins. 


\section{MONO COUNTY.}

87. Blind Spring. $\mathrm{Au}, \mathrm{Ag}, \mathrm{Cu}, \mathrm{Pb}$.

Benton station, S. P. R. R.

Granite, limestone, and Tertiary volcanice.

Veins.

88. Bodie. Au, Ag.

46 miles WSW. Thorn, Nev., S. P. R. R.

Tertiary volcanics.

Veins.

Min. Res. 1905, p. 178.

1906, p. 190.

1907, pt. 1, pp. 211-212.

1908 , pt. 1, p. 338.

89. Castle Peak. Ag. 1909 , pt. 1, p. 278.

90 miles S. Minden, Nev., V. \& T. R. R.

90. Cloverpatch. Ag, Au.

20 miles $S W$. Benton, S. P. R. R.

91. Dogtown Diggings. Au. (P1.)

85 miles $\mathrm{S}$. Minden, Nev., V. \& T. R. R.

92. Homer (Lundy). Au, Ag.

63 miles SW. Thorn, Nev., S. P. R. R.

Granite.

Veins.

Min. Res. 1906, p. 190.

1907, pt. 1, p. 212.

1908 , pt. 1, p. 338.

93. Indian. $\mathrm{Au}, \mathrm{Ag}$. 1909, pt. 1, p. 278.

20 miles W. Benton, S. P. R. R.

94. Jordan. $\mathrm{Au}, \mathrm{Cu}$.

50 miles SW. Thorn, Nev., S. P. R. R.

95. Omitted.

96. Keith. Au.

70 miles S. Minden, Nev., V. \& T. R. R.; 66 miles S. Thorn, Nev., S. P. R. R.

97. Lakes (Mammoth). $\mathrm{Au}, \mathrm{Ag}$.

50 miles SW. Benton, S. P. R. R.

Granite and schist.

Veins.

Top. sheet Mount Lyell.

98. Masonic. $\mathrm{Au}, \mathrm{Ag}$.

65 miles S. Minden, Nev., V. \& T. R. R.

Min. Res. 1906, p. 190.

1907, pt. 1, p. 212.

1908 , pt. 1, p. 338 .

1909 , pt. 1, p. 278.

99. Patterson. $\mathrm{Au}, \mathrm{Ag}$.

65 miles S. Minden, Nev., V. \& T. R. R.

100. Tioga. Au, Ag.

75 miles SW. Thorn, Nev., S. P. R. R.

Granite.

Veins.

Turner, H. W., Further contributions to the geology of the Sierra Nevada: Seventeenth Ann. Rept., pt. 1, 1896, p. 713. 
Moro coUNTY-Continued.

101. West Walker. $\mathrm{Au}(\mathrm{Ag})$.

36 miles S. Minden, Nev., V. \& T. R. R.

102. White Peak. Au, Cu.

Benton station, S. P. R. R.

MONTEREY COUNTY.

103. Dutro. Hg. 40 miles WSW. King City, S. P. R. R.

104. Jolon. Au. (Pl, D.) 21 miles S. King City, S. P. R. R. Min. Res. 1909, pt. 1, p. 290.

105. Los Burros. Au. (Pl, D.) 43 miles W. King City, S. P. R. R. Slates, serpentine, and limestone. Veins.

106. Table Mountain. Hg. 30 miles ENE. San Miguel, S. P. R. R.

ORATGE COUNTY.

107. Trabuco Canyon. $\mathrm{Pb}, \mathrm{Zn}, \mathrm{Au}, \mathrm{Ag}$. 25 miles N. San Juan Capistrano, A. T. \& S. F. R. R. Clay, slate, and porphyry.

Veins.

Top. sheets Corona, Elsinore.

108. Chuckwalla. Au.

\section{RIVERSIDE COUNTY.}

50 miles ENE. Mecca, S. P. R. R.

109. Corona. Mo.

4 miles N. Corona, A. T. \& S. F. R. R.

Granite.

Veine.

Hess, F. L., Some molybdenum deposits of Maine, Utah, and California: Bull. 340, 1908, pp. 238-239.

110. Dos Palmas. Au.

20 miles E. Mecca, S. P. R. R.

111. Ironwood. $\mathrm{Au}, \mathrm{Ag}, \mathrm{Cu}, \mathrm{Pb}$. 40 miles S. Randolph, A. T. \& S. F. R. R.

Porphyry.

Veins.

112. Iost Horse. Au. 30 miles $N$. Indio, S. P. R. R.

113. Menifee (Auld). $\mathrm{Au}, \mathrm{Ag}$. 10 miles S. Winchester, A. T. \& S. F. R. R. Top. sheet Elsinore.

114. Monte Negro (Eagle Mountain). $\mathrm{Au}, \mathrm{Ag}, \mathrm{Cu}, \mathrm{Fe}$. 50 miles S. Amboy, A. T. \& S. F. R. R.; 40 miles NE. Mecca, S. P. R. R. Granite and metamorphosed sediments cut by granite porphyry. Veins.

Harder, E. C., The Iron Age iron-ore deposit near Dale, San Bernardino County, Cal.: Bull. 430, 1910, p. 229.

115. Onward. Au.

55 miles NE. Mecca, S. P. R. R.

Granite.

Veins. 


\section{RIVERSIDE COUNTY-Continued}

116. Perris. Au.

Station A. T. \& S. F. R. R.

Slate and granite.

Veins.

Top. sheet Elsinore.

117. Pinyon Mountain. Au.

15 miles N. Indio, S. P. R. R.

117a. Temescal Mountains (San Jacinto). Sn.

10 miles SE. Corona, A. T. \& S. F. R. R.

Metamorphosed sediments cut by granite.

Veins.

Hess, F. L., and Graton, L. C., The occurrence and distribution of tin: Bull. 260, 1905, pp. 165-166.

Min. Res. 1882, pp. 434-435.

1883-84, pp. 614-615.

1891, p. 161.

1894 (Sixteenth Ann. Rept., pt. 3), pp. 536-538.

118. Central Benito. Hg.

\section{SAN BENITO COUNTY.}

26 miles SSE. Tres Pinos, S. P. R. R.

Mesozoic sediments.

Stockworks.

Becker, G. F., Geology of the quicksilver deposits of the Pacific slope: M. XIII, 1888, p. 380.

Min. Res. 1909, pt. 1, p. 553.

119. New Idria. $\mathrm{Hg}$.

64 miles SE. Tres Pinos, S. P. R. R.

Cretaceous shales and sandstones.

Stockworks, veins, and disseminations.

Becker, G. F., Geology of the quicksilver deposits of the Pacific slope:

M. XIII, 1888, pp. 291-309; Eighth Ann. Rept., 1889, p. 977.

Melville, W. H., and Lindgren, W., Contributions to the mineralogy of the

Pacific coast: Bull. 61, 1890, p. 21.

Min. Res. 1882, pp. 394-398.

1883-84, p. 492.

1887, pp. 119-120.

1908 , pt. 1, pp. $686-689$.

1909 , pt. 1, p. 553.

120. Stayton (McLeod, Panoche). $\mathrm{Hg}, \mathrm{Sb}$.

15 miles NE. Hollister, S. P. R. R.

Metamorphosed slates.

Stockworks.

Becker, G. F., Geology of the quicksilver deposits of the Pacific slope:

M. XIII, 1888, pp. 379-381.

Min. Res. 1883-84, p. 642 .

1885, p. 387.

1909 , pt. 1, p. 553.

\section{SAN BERNARDINO COUNTY.}

121. Alvord. Au.

35 miles NE. Daggett, A. T. \& S. F. R. R., S. P. L. A. \& S. L. R. R.

Limestone cut by porphyry.

Veins. 
SAN BERTARDINO COUNTY-Continued.

122. Arondo (Slate Range). Au, Ag.

50 miles NE. Johannesburg, A. T. \& S. F. R. R.

Tertiary volcanics.

Veins.

Spurr, J. E., Geology of Nevada south of the fortieth parallel: Bull. 208, 1903, p. 213.

123. Arrowhead. Au.

20 miles SE. Kelso, S. P. L. A. \& S. L. R. R.; 20 miles NNW. Dabney, A. T. \& S. F. R. R.

Granite.

Veins.

124. Atolia. W, Au.

Station A. T. \& S. F. R. R.

Granite and schist.

Veins.

Hess, F. L., Gold mining in the Randsburg quadrangle, California: Bull. 430,1910, pp. $23-47$.

Min. Res. 1906, p. 525.

1907 , pt. 1, p. 712 .

1908 , pt. 1, p. 723.

1909 , pt. 1, pp. 577-578.

Top. sheet Randsburg.

125. Blackhawk (Silver Reef). Au, Ag.

40 miles ESE. Victorville, S. P. L. A. \& S. I. R. R.

Sediments and granodiorite.

Veins and replacements.

Top. sheet San Gorgonio.

126. Brightwood (Providence Mountains). $\mathrm{Ag}, \mathrm{Pb}, \mathrm{Cu}$.

15 miles NE. Kelso, S. P. L. A. \& S. L. R. R.

Granite and schist cut by diorite and porphyry.

Veins.

127. Bullion (Standard). $\mathrm{Cu}, \mathrm{Ag}, \mathrm{Pb}, \mathrm{Au}$.

14 miles N. Cima, S. P. L. A. \& S. L. R. R.

Paleozoic sediments cut by granite.

Contact metamorphic.

Min. Res. 1908, pt. 1, p. 724.

1909, pt. 1, p. 282.

128. Calico (Daggett). Ag.

10 miles N. Daggett, A. T. \& S. F. R. R., S. P. L. A. \& S. L. R. R.

Rhyolite.

Replacements and veins.

Min. Res. 1907, pt. 1, p. 221.

1908 , pt. 1, p. 346 .

129. Cave Canyon. Fe.

1 mile N. Scott, S. P. L. A. \& S. L. R. R.

Paleozoic sediments cut by granite.

Contact metamorphic.

Harder, E. C., and Rich, J. L., The Iron Age iron-ore deposit near Dale, San Bernardino County, Cal.: Bull. 430, 1910, p. 229.

Leith, C. K., Iron ores of western United States and British Columbia: Bull. 285, 1905, p. 198. 
130. Cima. Ag, Au.

Cima station, S. P. L. A. \& S. L. R. R.

Granite.

Veins.

Min. Res. 1906, p. 194.

1907, pt. 1, p. 221.

1908 , pt. 1, p. 346 .

131. Cottonwood. Au, Ag.

5 miles N. Judson, S. P. L. A. \& S. L. R. R.

132. Crackerjack. Au.

10 miles W. Silver Lake, T. \& T. R. R.

133. Dry Lake. Cu.

18 miles NE. Crucero, T. \& T. R. R., S. P. L. A. \& S. L. R. R.

134. Garlic Spring. Fe.

25 miles N. Barstow, A. T. \& S. F. R. R., S. P. L. A. \& S. L. R. R.

Harder, E. C., and Rich, J. L., The Iron Age iron-ore deposit near Dale, San Bernardino County, Cal.: Bull. 430, 1910, p. 229.

135. Grapevine. $\mathrm{Au}, \mathrm{Ag}, \mathrm{Pb}$.

10 miles N. Barstow, A. T. \& S. F. R. R., S. P. L. A. \& S. L. R. R.

Veins.

136. Hart. Au.

3 miles S. Hitt, B. \& S. R. R.

Rhyolite.

Veins.

Min. Res. 1907, pt. 1, p. 221.

137. Helen. $\mathrm{Au}, \mathrm{Ag}$.

Judson station, S. P. L. A. \& S. L. R. R.

138. Holcomb. $\mathrm{Au}, \mathrm{Ag}$.

20 miles NE. Patton, A. T. \& S. F. R. R.

Top. sheet San Gorgonio.

139. Ibex. Au.

15 miles NW. Needles, A. T. \& S. F. R. R.

140. Iron Mountain. Fe.

20 miles N. Crucero, S. P. L. A. \& S. L. R. R., T. \& T. R. R.

Harder, E. C., and Rich, J. L., The Iron Age iron-ore deposit near Dale,

San Bernardino County, Cal.: Bull. 430, 1910, p. 229, map.

141. Ivanpah (Copper World). $\mathrm{Cu}, \mathrm{Au}, \mathrm{Ag}, \mathrm{Pb}$.

Station A. T. \& S. F. R. R.

Paleozoic sediments cut by granite.

Veins and contact metamorphic.

Min. Res. 1898 (Twentieth Ann. Rept., pt. 6), p. 185.

1907, pt. 1, pp. 221, 712.

142. Kane Springs. $\mathrm{Cu}, \mathrm{Au}, \mathrm{Ag}$.

20 miles SE. Daggett, A. T. \& S. F. R. R., S. P. L. A. \& S. L. R. R.

Granite.

Veins.

143. Kelso. Fe.

10 miles S. Kelso, S. P. L. A. \& S. L. R. R.

144. Kewanee. Au.

6 miles W. Leastalk, S. P. L. A. \& S. L. R. R.

Veins.

Min. Res. 1907, pt. 1, p. 221. 
SAN BERTARDITO COUNTY-Continued.

145. Kingston Range. Fe.

25 miles SE. Tecopa, T. \& T. R. R.

Harder, E. C., and Rich, J. L., The Iron Age iron-ore deposit near Dale, San

Bernardino County, Cal.: Bull. 430, 1910, p. 229. (Shows the location.)

146. Knob Peak. Au, Cu.

35 miles SE. Tecopa, T. \& T. R. R.

147. Lava Beds (Newberry). $\mathrm{Au}, \mathrm{Ag}, \mathrm{Cu}$.

12 miles W. Ludlow, A. T. \& S. F. R. R., T. \& T. R. R.

Granodiorite.

Veins.

Harder, E. C., and Rich, J. L., The Iron Age iron-ore deposit near Dale, San Bernardino County, Cal.: Bull. 430, 1910, p. 229.

148. Monument Peak (Whipple Mountain). Cu, Au.

14 miles N. Vidal, A. T. \& S. F. R. R.

Pre-Cambrian gneiss, schist; diorite capped by Tertiary volcanics.

Veins.

Bancroft, H., Reconnaissance of the ore deposits in northern Yuma County, Ariz.: Bull. 451, 1911, pp. 68-73.

Top. sheet Parker.

149. Morongo (Lone Valley?). Au, Ag.

I5 miles N. Palm Springs, S. P. R. R.

Limestone and slate.

Vein.

Top. sheet San Gorgonia.

150. Needles. $\mathrm{Au}, \mathrm{Cu}$.

15 miles W. Mellen, A. T. \& S. F. R. R.

Veins.

151. New York (Manvel, Barnwell). Au, Ag, $\mathrm{Cu}(\mathrm{Pb}, \mathrm{Zn}, \mathrm{W})$.

Barnwell station, A. T. \& S. F. R. R.

Granite and schist.

Veins.

Min. Res. 1906, p. 525.

1907, pt. 1, p. 221.

1908 , pt. 1, pp. 346, 724 .

152. Ord. $\mathrm{Au}, \mathrm{Ag}, \mathrm{Cu}$.

30 miles S. Daggett, A. T. \& S. F. R. R., S. P. L. A. \& S. L. R. R.

Granite.

Veins.

153. Oro Grande. $\mathrm{Au}, \mathrm{Ag}, \mathrm{Pb}$.

10 miles W. Hesperia, S. P. L. A. \& S. L. R. R.

Paleozoic sediments cut by diorite and quartz porphyry.

Replacements and veins.

Top. sheet Hesperia.

154. Ow1 Holes. Fe.

70 miles NW. Silver Lake, T. \& T. R. R.

Harder, E. C., and Rich, J. L., The Iron Age iron-ore deposit near Dale, San

Bernardino County, Cal.: Bull. 430,1910, p. 229. (Shows the location.)

155. San Antonio. Au.

15 milès W. Cajon, A. T. \& S. F. R. R.

Top. sheet San Antonio.

156. Shadow Mountains. $\mathrm{Cu}, \mathrm{Au}, \mathrm{Ag}, \mathrm{Pb}, \mathrm{W}$.

20 miles NW. Cima, S. P. L. A. \& S. L. R. R.

Min. Res. 1908, pt. 1, p. 724.

21528 - Bull. 507-12-9 
157. Signal (Vontrigger). $\mathrm{Cu}, \mathrm{Au}, \mathrm{W}$.

8 miles E. Vontrigger, A. T. \& S. F. R. R.

Min. Res. 1908, pt. 1, p. 724.

158. Silver Mountain. $\mathrm{Au}, \mathrm{Ag}, \mathrm{Cu}$.

20 miles E. Victorville, S. P. L. A. \& S. L. R. R.

Granite, limestone.

Veins.

159. Spangler. $\mathrm{Au}, \mathrm{Ag}, \mathrm{Cu}$.

24 miles NE. Johannesburg, A. T. \& S. F. R. R.

160. Stedman. $\mathrm{Cu}, \mathrm{Au}, \mathrm{Ag}$.

15 miles S. Ludlow, A. T. \& S. F. R. R., T. \& T. R. R.

Min. Res. 1905, p. 180.

1906, p. 194.

1907, pt. 1, p. 220.

161. Stringer. $\mathrm{Au}, \mathrm{W}(\mathrm{Pb}, \mathrm{Zn}, \mathrm{Cu})$.

Atolia station, A. T. \& S. F. R. R.

Schist and granite.

Veins.

Hess, F. L., Gold mining in the Randsburg quadrangle, California: Bull. 430,1910 , pp. 23-47.

Min. Res. 1906, p. 525.

1907, pt. 1, p. 712 .

Top. sheet Stringer.

162. Omitted.

163. Trojan (Providence). Au, Cu.

20 miles W. Blake, A. T. \& S. F. R. R.

Veins.

164. Twentynine Palms. Au, Ag. 40 miles SSW. Amboy, A. T. \& S. F. R. R. Veins.

165. Vanderbilt. $\mathrm{Au}, \mathrm{Cu}$. Barnwell station, A. T. \& S. F. R. R.

Granite and limestone.

Veins.

166. Omitted.

167. Virginia Dale (Monte Negro). $\mathrm{Au}, \mathrm{Ag}, \mathrm{Cu}, \mathrm{Fe}$.

45 miles S. Amboy, A. T. \& S. F. R. R.; 40 miles NE. Mecca, S. P. R. R.

Sediments cut by granite, diorite, and syenite.

Veins.

Harder, E. C., and Rich, J. L., The Iron Age iron-ore deposit near Dale,

San Bernardino County, Cal.: Bull. 430, 1910, pp. 228-239.

Min. Res. 1907, pt. 1, p. 221.

1908 , pt. 1 , p. 346.

1909 , pt. 1, p. 282.

168. Omitted.

\section{SAT DIEGO COUNTY.}

169. Banner. Au.

30 miles NE. Lakeside, S. D. \& C. R. R.

Schist and granite.

Veins.

Min. Res. 1909, pt. 1, p. 290.

Top. sheet Ramona. 
SAN DIEGO COUNTY-Continued.

170. Cuyamaca. Au.

25 miles ENE. Lakeside, S. D. \& C. R. R.

Schist and granite (?).

Veins.

Min. Res. 1907, pt. 1, p. 234.

Top. sheet Cuyamaca.

171. Dulzura. Au.

24 miles SE. Spring Valley, S. D. \& C. R. R.

Metamorphosed sediments cut by acidic and basic dikes.

Veins.

Min. Res. 1907, pt. 1, p. 234.

Top. sheet Cuyamaca.

172. Escondido. $\mathrm{Au}, \mathrm{Ag}$.

Station A. T. \& S. F. R. R.

Granite.

Veins.

Min. Res. 1907, pt. 1, p. 234.

1909, pt. 1, p. 290.

Top. sheet Escondido.

173. Foster (San Vicente). Au.

Foster station, S. D. \& C. R. R.

Top. sheets Ramona, Cuyamaca.

174. Julian. Au.

25 miles NE. Foster, S. D. \& C. R. R.

Slates, schist, and granite.

Veins.

Min. Res. 1905, p. 181.

1907, pt. 1, p. 234.

1909, pt. 1, p. 290.

Top. sheet Ramona.

175. Mesa Grande. Au.

25 miles NNE. Foster, S. D. \& C. R. R.

Top. sheet Ramona.

176. Pine Valley. Au, Ag.

30 miles E. Lakeside, S. D. \& C. R. R.

Granite and schist.

Veins.

177. Rice. Au.

40 miles ENE. Foster, S. D. \& C. R. R.

178. San Mateo (Pinacate). Cu.

25 miles E. San Juan Capistrano, A. T. \& S. F. R. R.

Schists, gneiss, granite.

Veins.

Top. sheets San Luis Rey, Capistrano.

SAN IUIS OBISPO COUNTY.

179. Adelaida (Klau). Hg.

16 miles W. Paso Robles, S. P. R. R.

Mesozoic slates and sandstones.

Stockworks.

Becker, G. F., Geology of the quicksilver deposits of the Pacific slope:

M. XIII, 1888, pp. 381-382.

Min. Res. 1908, pt. 1, pp. 689-690.

1909 , pt. 1, p. 553. 


\section{SAN IUIS OBISPO COUNTY-Continued.}

180. La Panza. Au. (Pl.)

50 miles SE. Santa Margarita, S. P. R. R.

Min. Res. 1907, pt. 1, p. 234.

181. Oceanic (Santa Rosa). Hg.

25 miles NW. San Luis Obispo, S. P. R. R.

Mesozoic sandstone.

Impregnations.

Becker, G. F., Geology of the quicksilver deposits of the Pacific slope:

M. XIII, 1888, pp. 381-382.

Min. Res. 1887, pp. 119-121.

1908 , pt. 1, p. 689.

1909 , pt. 1, p. 553.

182. Ocean View (Pine Peak). Hg.

44 miles NW. San Luis Obispo, S. P. R. R.

Metamorphosed slates.

Stockworks.

Becker, G. F., Geology of the quicksilver deposita of the Pacific slope: M. XIII, 1888, pp. 381-382.

183. Perfumo Canyon. Fe, Cr.

5 miles WSW. San Luis Obispo, S. P. R. R.

Jurassic sediments.

Lenses.

Harder, E. C., Iron ores of western and central California: Bull. 430, 1910, pp. 220-223.

Min. Res. 1882, p. 428.

1883-84, pp. 570-571.

1885, pp. 357-358.

1886, p. 176.

184. Rinconada (Santa Margarita). Hg.

Station S. P. R. R.

Metamorphosed slates and serpentine.

Disseminations.

Becker, G. F., Geology of the quicksilver deposits of the Pacific slope: M. XIII, 1888, pp. 381-382.

Top. sheet San Luis Obispo.

185. San Luis Obispo (Santa Lucia). Fe, Cr.

20 miles NW. San Luis Obispo, S. P. R. R.

Metamorphosed slates and serpentine.

Lenses.

Harder, E. C., Some chromite deposits in western and central California:

Bull. 430, 1910, pp. 168-173.

Min. Res. $1883-84$, pp. $570-571$

1908 , pt. 1, p. 767 .

Top. sheet San Luis Obispo.

Folio 101, 1904

SANTA BARBARA COUNTY.

186. Los Prietos. Hg.

5 miles N. Santa Barbara, S. P. R. R.

Serpentine.

Stockworks.

Becker, G. F., Geology of the quicksilver deposits of the Pacific slope:

M. XIII, 1888, p. 382.

Top. shêet Santa Barbara special. 
TULARE COUNTY.

187. Bald Mountain. $\mathrm{Au}, \mathrm{Cu}$.

37 miles SE. Plano, S. P. R. R.

Serpentine and limestone.

Vein (?).

188. Finger Rock. Au.

30 miles NE. Exeter, S. P. R. R.

Top. sheet Tehipite.

189. Globe. Au.

14 miles E. Portersville, S. P. R. R.

Top. sheet Kaweah.

190. Mineral King. $\mathrm{Au}, \mathrm{Cu}, \mathrm{Sb}$.

48 miles ENE. Exeter, S. P. R. R.

Metamorphosed sediments and granite.

Contact metamorphic.

Min. Res. 1883-84, p. 642.

Top. sheet Kaweah.

191. Portersville. Cu.

Station S. P. R. R.

192. White River. $\mathrm{Au}, \mathrm{Cu}$.

23 miles SE. Plano, S. P. R. R.

VENTURA COUNTY.

193. Frazier Mountain. Au.

46 miles SSE. Maricopa, S. P. R. R.

Gneiss, slate, and granite.

Vein.

Top. sheet Tejon.

194. Snowy Creek (Piru Creek). Au.

35 miles N. Piru, S. P. R. R.

Granite.

Vein.

Top. sheet Tejon. 


\section{COLORADO.}

In 35 counties of Colorado there are 132 mining districts, as shown by Plate VI. This is not accurate in that in Gilpin and Clear Creek counties and some other localities the mining districts are small and several have been combined under one symbol and number. In 5 of the 132 districts the predominant metal is unknown, in 1 zinc is the most important product, in 1 iron, in 8 rare metals, in 60 gold, in 21 silver, in 20 copper, and in 16 lead.

Distribution of the predominant metals produced in the mining districts of Colorado.

\begin{tabular}{|c|c|c|c|c|c|c|c|c|c|}
\hline County. & Gold. & Silver. & Copper. & Lead. & Zinc. & Iron. & $\begin{array}{c}\text { Rare } \\
\text { metals. }\end{array}$ & $\begin{array}{c}\text { Un- } \\
\text { known. }\end{array}$ & Total. \\
\hline 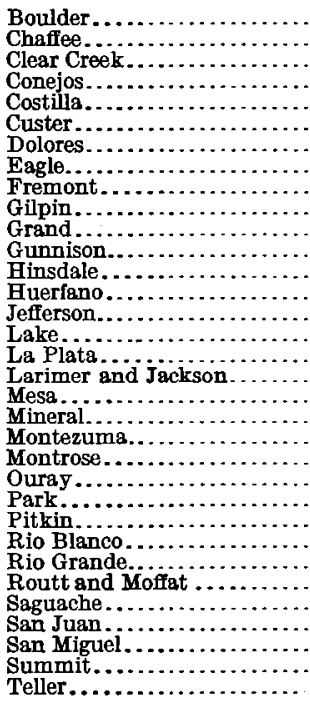 & 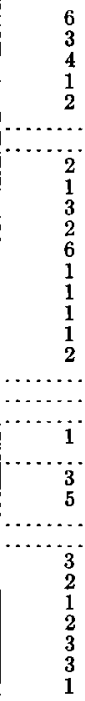 & 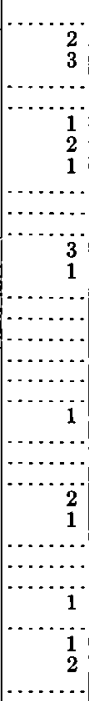 & 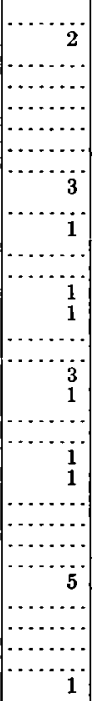 & 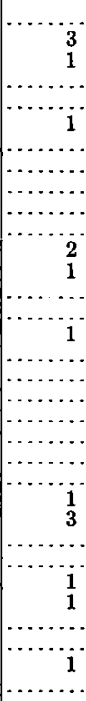 & 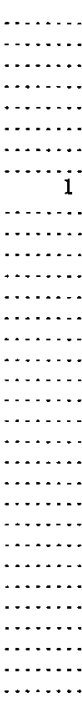 & 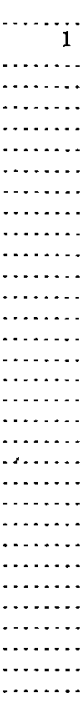 & 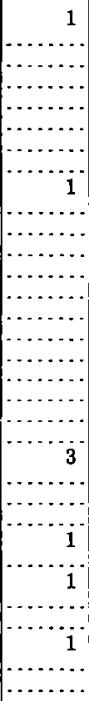 & 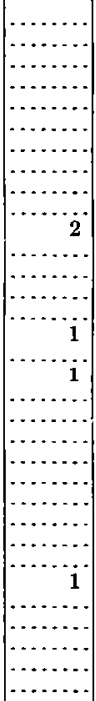 & $\begin{array}{r}7 \\
11 \\
8 \\
1 \\
2 \\
2 \\
2 \\
3 \\
6 \\
3 \\
5 \\
11 \\
3 \\
2 \\
2 \\
3 \\
2 \\
4 \\
1 \\
1 \\
1 \\
4 \\
4 \\
8 \\
4 \\
1 \\
3 \\
10 \\
3 \\
2 \\
5 \\
6 \\
2\end{array}$ \\
\hline & 60 & 21 & 20 & 16 & 1 & 1 & 8 & 5 & 132 \\
\hline
\end{tabular}

\section{MINING DISTRIC'TS IN COLORADO.}

[See Pl. VI. Additional references may be found in Mineral Resources for 1910 and 1911.]

1. Central. Au, Ag.

\section{BOULDER COUNTT.}

7 miles NW. Boulder, C. \& S. R. R., U. P. R. R.

Pre-Cambrian complex and basic dikes.

Veins.

Min. Res. 1905, p. 194.

1906, p. 208.

1907, pt. 1, p. 245.

1908, pt. 1, p. 371.

1909 , pt. 1, p. 303. 
LIST OF MINING DISTRICTS Gold Hill

4. Magnolia
5. Nederland
5. Sugarloaf

Sugarloaf
Ward

8. Alpine

11. Cottonwood

La Plata (Winfield)
Monarch-Garfield

5iverside

South Arkansas

CLAR CREEK COUNTY

Argentine
Griffith (Goorgetown, silve
Plume, QQuens, etcc)

- Idaho Springs

2. Jackson (Corral, Cascado, Dom
3. Lincoln (Alice, Yannkee)

5. Trail Union (Empire)
6pper Unios COUNYY

to costituro countr

Ba. Prayback (Russell)

Haruscrabble (Silver Cliff)

Spaulding CounTY
DOLRES Count

Pioner (Rico)

Battle Mountain (Red Clifí,
Fuliman)

Holy Cross (Eagle River)
FREMONT COUTY

Canon City

Cotopaxi
Greent Creek (Micanite)
Greenhorn (Grape Creek)

Whitehorn (Manoas Calumet)

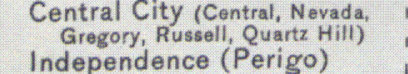

43. Pine (Kingston, Apex)
44. GRAND COUNT

Blue Ridge
5. Corral Creek

Grand Lake (Wolverine)

. La Plata

Box Canyon COUNTY

Cochetopa a an, Doming

Elk Mountain (Ruby)
Gold Brick

5. Quartr Creek

7. Ruby reek

Tincup (Pieplant)

HINSDALE COUNTY

G. Galena
2. Lake (San Cristobal)
Park (Sherman)

3. Park
HUER ERA ANO COUNTY
4uerfano (Malachite)

jacKSOON COUNTY (Soe Lar

66. Evergreen (Malachite)
67. Golden

14. Manhattan

8o. Hydraulic
8i. La Sal

82. Naturita (Vixen)
83. Roc Creek

5. Red Mountain
6. Sneffels
9. Uncompahgre

88. Buckskin
89. Consolidated Motro

Hartsel

Horseshoe
Mosquito
Sacramento

Fryingpa

Decatur
Summitville

17. Animas

1. Mount Wilson

Peru Rive
Swan Riv

3o. Cripple Creek
31. East Beaver

LARE COUNTY
68. California (Leadville)
69. Harrington (Tennesseg

9. Harrington (Tonneos
7win Lakes
LA PLATA COUNTY

1. California (Oro Fino)
12. Needle Mountains (Florid

73. Empire (Howes Gulch)

76. Steamboat Rock (Gray Rock)

77. Unaweep
MiNERLA counTY
7. Sunnyside (Creede)

MOFFAT COUNTY ISOe Routt)
MONTEUUMA COUNTY

79. East Mandos
MONTROSE COUNTY

OURAY COUNTY
4. Imogene Basin (Camp Bir

Montgomery

Tarryall PITKIN countr

Columbia (Ashcroff)
Con

Roaring Fork (Aspen)
RIO BANCO CAUNTY

Roal Creek COUNTY

ROUTT AND MOFFAT COUNTIES

Hahns Peak (Columbine)

Oak Creek (Red Gorge)
Rock Creek (Gore Ridge)

Rock Creek (Gore Ridge)
Skull Creek (Blue Mountain)

Slavonia
Spring Creek (Steam (Sporti
S

Baca Grant (Crestone)
Blake (Kerber Creek)

Crystal Hill G Creek
SAN JUAN CONTY

Ig. Iron Spring (Ophir)

. Lower San Miguel

Snyderville (Cedar)
Upper San Miguel To

Breckenridge (Bevan Uni

Montezuma (Snake Rive)

Tenmile (Kokomo)

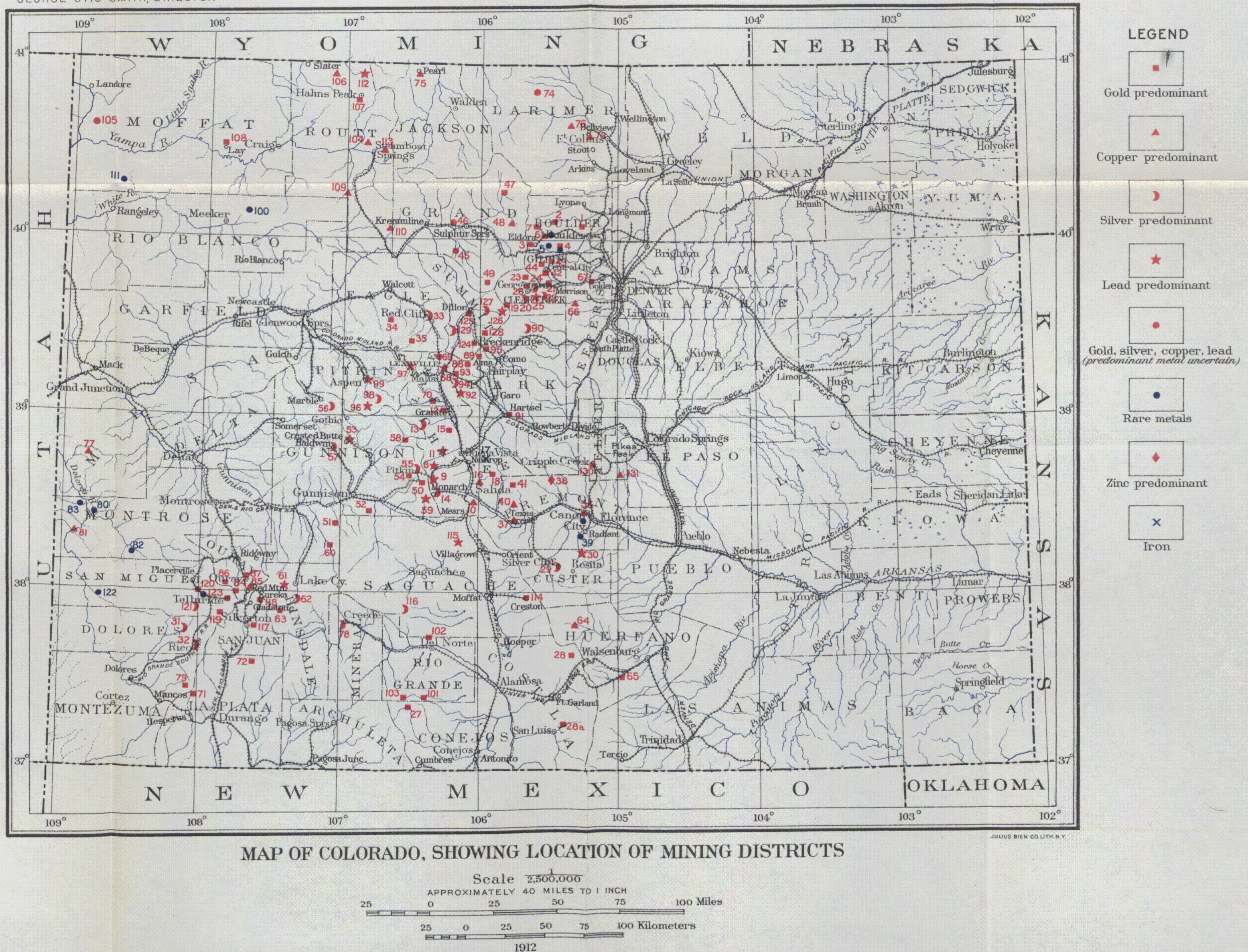


BOULDER COUNTY-Continued.

2. Gold Hill. $\mathrm{Au}, \mathrm{Ag}, \mathrm{Pb}, \mathrm{Cu}$.

6 miles NNW. Crisman, D. B. \& W. R. R.

Pre-Cambrian complex cut by acidic and basic dikes.

Veins.

Min. Res. 1905, p. 194.

1906, p. 208.

1907 , pt. 1, p. 245.

1908 , pt. 1, p. 371.

1909 , pt. 1, p. 303.

3. Grand Island (Caribou). $\mathrm{Au}, \mathrm{Ag}, \mathrm{Pb}(\mathrm{Mn}, \mathrm{Cu})$.

2 miles W. Cardinal, D. B. \& W. R. R:

Pre-Cambrian complex cut by acidic and basic dikes.

Veins.

Min. Res. 1905, p. 195.

1906, p. 208.

1907, pt. 1, p. 245.

1908 , pt. 1, p. 371.

1909 , pt. 1, p. 304.

Top. sheet Central City.

4. Magnolia. $\mathrm{Au}, \mathrm{Ag}, \mathrm{W}$.

18 miles SW. Boulder, C. \& S. R. R., U. P. R. R.

Pre-Cambrian complex cut by acidic and basic dikes.

Veins.

George, R. D., and Crawford, R. D., The main tungsten area of Boulder

County, Colo.: First Ann. Rept. Colorado Geol. Survey, 1909, pp. 7-105.

Min. Res. 1906, p. 208.

1907, pt. 1, pp. 245, 712.

1908, pt. 1, pp. 371, 722 .

1909, pt. 1, p. 304.

Top. sheet Central City.

5. Nederland. W.

$2 \frac{1}{2}$ miles E. Cardinal, D. B. \& W. R. R.

Pre-Cambrian complex cut by acidic and basic dikes.

Veins.

George, R. D., and Crawford, R. D., The main tungsten area of Boulder

County, Colo.: First Ann. Rept. Colorado Geol. Survey, 1909, pp. 7-105.

Min. Res. 1904, pp. 330-331.

1905 , p. 411.

1907, pt. 1, p. 712 .

1908 , pt. 1 , p. 722 .

1909, pt. 1, pp. 578-579.

Top. sheet Central City.

6. Sugarloaf. $\mathrm{Au}, \mathrm{Ag}, \mathrm{W}, \mathrm{Pb}, \mathrm{Cu}$.

Station D. B. \& W. R. R.

Pre-Cambrian complex cut by acidic and basic dikes

Veins.

Min. Res. 1904, p. 330.

1905 , pp. 195, 411.

1906, p. 208.

1907, pt. 1, pp. 245, 246, 712.

1908 , pt. 1, p. 372 .

1909, pt. 1, pp. 304, 579. 
7. Ward. $\mathrm{Au}, \mathrm{Ag}, \mathrm{Cu}, \mathrm{Pb}$.

Station D. B. \& W. R. R.

Pre-Cambrian complex cut by acidic and basic dikes.

Veins.

Min. Res. 1905, p. 195.

1906, p. 209.

1907, pt. 1, p. 246.

1908, pt. 1, p. 372 .

1909 , pt. 1, p. 304 .

\section{CHAFFEE COUNTY.}

8. Alpine. $\mathrm{Pb}, \mathrm{Ag}, \mathrm{Au}, \mathrm{Cu}$.

Station C. \& S. R. R.

Pre-Cambrian complex.

Veins.

Spurr, J. E., and Garrey, G. H., Economic geology of the Georgetown quadrangle, Colorado: P. P. 63, 1908, p. 106.

9. Chalk Creek. $\mathrm{Pb}, \mathrm{Ag}, \mathrm{Au}, \mathrm{Zn}$.

St. Elmo station, C. \& S. R. R.

Min. Res. 1906, p. 209.

1907 , pt. 1, p. 247.

1908 , pt. 1, p. 373.

1909 , pt. 1, p. 305.

10. Cleora. $\mathrm{Cu}, \mathrm{Au}, \mathrm{Ag}, \mathrm{Zn}, \mathrm{Pb}$.

4 miles S. Salida, D. \& R. G. R. R.

Pre-Cambrian complex.

Veins.

Lindgren, W., Notes on copper deposits in Chaffee, Fremont, and Jefferson counties, Colo.: Bull. 340, 1908, pp. 157-174.

Min. Res. 1906, p. 209.

1908, pt. 1, p. 373.

1909 , pt. 1, p. 305.

11. Cottonwood. $\mathrm{Pb}, \mathrm{Ag}$.

10 miles W. Buena Vista, D. \& R. G. R. R., C. M. R. R.

Pre-Cambrian complex (?).

Veins (?).

Min. Res. 1905, p. 195.

12. Granite. $\mathrm{Au}, \mathrm{Ag}, \mathrm{Pb}$. (D, Pl.)

Station D. \& R. G. R. R., C. M. R. R.

Pre-Cambrian complex cut by dikes.

Veins.

Min. Res. 1908, pt. 1, p. 373.

1909 , pt. 1, p. 305.

Top. sheet Leadville.

13. La Plata (Winfield). $\mathrm{Ag}, \mathrm{Au}, \mathrm{Cu}, \mathrm{Pb}$.

15 miles WSW. Granite, D. \& R. G. R. R., C. M. R. R.

Min. Res. 1905, p. 195.

1906, p. 209.

1907, pt. 1, p. 247.

1908 , pt. 1, p. 373.

1909, pt. 1, p. 305. 
CHATFEe COUNTY-Continued.

14. Monarch-Garfeld. $\mathrm{Ag}, \mathrm{Au}, \mathrm{Cu}, \mathrm{Pb}, \mathrm{Zn}$.

19 miles W. Salida, D. \& R. G. R. R.

Pre-Cambrian granite and Paleozoic sediments cut by quartz monzonite.

Veins and replacemente.

Crawford, R. D., A preliminary report on the geology of the Monarch mining

district, Chaffee County, Colo.: Bull. Colorado Geol. Survey No. 1, 1910.

Min. Res. 1885, p. 256.

1887 , p. 105.

1888, p. 87.

1905 , p. 195.

1906, p. 210.

1907 , pt. 1, p. 247.

1908, pt. 1, p. 373 .

1909, pt. 1, p. 305.

15. Riverside. $\mathrm{Au}, \mathrm{Ag}, \mathrm{Pb}, \mathrm{Cu}$.

15 miles NNW. Buena Vista, D. \& R. G. R. R., C. M. R. R.

Min. Res. 1906, p. 210.

1907, pt. 1, p. 247.

1908 , pt. 1, p. 373.

16. Sedalia. $\mathrm{Cu}, \mathrm{Zn}$. 1909 , pt. 1, p. 305.

6 miles ENE. Salida, D. \& R. G. R. R.

Pre-Cambrian schists and gneiss.

Lenses.

Lindgren, W., Notes on copper deposits in Chaffee, Fremont, and Jefferson counties, Colo.: Bull. 340, 1908, pp. 157-174.

Min. Res. 1883-84, p. 341.

1907, pt. 1, pp. 247-248.

1908 , pt. 1, p. 373.

17. South Arkansas. Fe.

19 miles NNE. Salida, D. \& R. G. R. R.

Min. Res. 1883-84, p. 282. 1887 , p. 53.

18. Turret. $\mathrm{Au}, \mathrm{Ag}, \mathrm{Cu}$.

14 miles NNE. Salida, D. \& R. G. R. R.

Pre-Cambrian complex.

Veins and lenses.

Lindgren, W., Notes on copper deposits in Chaffee, Fremont, and Jefferson counties, Colo.: Bull. 340, 1908, pp. 157-174.

Min. Res. 1905, p. 196.

1906, p. 210.

1907, pt. 1, pp. $247,248,602$.

1908 , pt. 1, p. 373.

1909, pt. 1, p. 305 .

CLEAR CREEK COUNTY.

19. Argentine. $\mathrm{Au}, \mathrm{Pb}, \mathrm{Ag}, \mathrm{Cu}, \mathrm{Zn}$.

Waldorf station, Ar. C. R. R.

Pre-Cambrian complex cut by acidic dikes.

Veins.

Patton, H. B., The Montezuma mining district of Summit County, Colo.: First Ann. Rept. Colorado Geol. Survey, 1909, pp. 105-145.

Spurr, J. E., and Garrey, G. H., Economic geology of the Georgetown quadrangle, Colorado: P. P. 63, 1908, pp. 69, 104, 136, 246, 267, 270, 271. 
CLEAR CREEK COUNTT-Continued.

19. Argentine-Continued.

Min. Res. 1885, p. 256.

1905, pp. 197-198.

1906, p. 212.

1907, pt. 1, p. 249.

1908, pt. 1, p. 375.

1909, pt. 1, p. 307.

Top. sheet Georgetown.

20. Georgetown (Griffith, Silver Plume, Queens). $\mathrm{Ag}, \mathrm{Zn}, \mathrm{Pb}, \mathrm{Au}, \mathrm{Cu}$.

Silver Plume station, C. \& S. R. R.

Pre-Cambrian complex cut by acidic dikes.

Veins.

Spurr, J. E., and Garrey, G. H., Preliminary report on the ore deposits of the Georgetown mining district, Colorado: Bull. 260, 1905, pp. 99-120.

- Economic geology of the Georgetown quadrangle, Colorado: P. P. 63, 1908, pp. 278-290.

Min. Res. 1882, pp. 310-311.

1885 , p. 256.

1905, p. 197.

1906, pp. 211-212.

1907, pt. 1, p. 249.

1908 , pt. 1 , pp. $375-376$.

1909 , pt. 1, pp. 307-308.

21. Idaho Springs (Virginia). $\mathrm{Au}, \mathrm{Ag}, \mathrm{Pb}, \mathrm{Cu}, \mathrm{Zn}$.

Idaho Springs station, C. \& S. R. R.

Pre-Cambrian complex cut by acidic dikes.

Veins.

Garrey, G. H., The Idaho Springs mining district, Colorado: Bull. 285, 1906, pp. 35-40.

Spurr, J. E., and Garrey, G. H., Preliminary report on the ore deposits of the Georgetown mining district, Colorado: Bull. 260, 1905, pp. 99-120.

_ Economic geology of the Georgetown quadrangle, Colorado: P. P. 63, 1908, pp. 311-382.

Min. Res. $1883-84$, p. 341.

1885 , p. 256.

1905, pp. 196-197.

1906, p. 211.

1907, pt. 1, p. 250.

1908 , pt. 1, pp. $276-377$.

1909, pt. 1, pp. 308-309.

Top. sheets Georgetown, Idaho Springs special.

22. Jackson (Corral, Cascade, Democrat). $\mathrm{Ag}, \mathrm{Au}, \mathrm{Pb}, \mathrm{Cu}$.

Idaho Springs station, C. \& S. R. R.

Pre-Cambrian complex cut by acidic dikes.

Veins.

Spurr, J. E., and Garrey, G. H., Preliminary report on the ore deposits of the Georgetown mining district, Colorado: Bull. 260, 1905, pp. 99-120.

Economic geology of the Georgetown quadrangle, Colorado: P. P. 63, 1908, pp. 311-382.

Min. Res. 1885, p. 256.

1906, p. 212.

1907, pt. 1, p. 250

1908, pt. 1, pp. 376-377.

1909, pt. 1, p. 309.

Top. sheet Georgetown. 


\section{CLEAR CREEK COURTY-Continued.}

23. Iincoln (Alice, Yankee). Au, Ag, Cu, Pb. (D, Pl.)

12 miles NW. Idaho Springs, C. \& S. R. R.

Pre-Cambrian complex cut by acidic and basic dikes.

Veins, stream and terrace gravels.

Min. Res. 1905, p. 197.

1908, pt. 1, p. 377.

1909 , pt. 1, p. 307.

Top. sheet Central City.

24. Montana (Lawson, Dumont). $\mathrm{Ag}, \mathrm{Au}, \mathrm{Cu}, \mathrm{Pb}, \mathrm{Zn}$.

Lawson and Dumont stations, C. \& S. R. R.

Pre-Cambrian complex cut by acidic dikes.

Veins.

Spurr, J. E., and Garrey, G. H., Preliminary report on the ore deposits of the Georgetown mining district, Colorado: Bull. 260, 1905, pp. 99-120. 1908.

Min. Res. 1885, p. 256.

1905 , p. 197.

1906, p. 213.

1907, pt. 1, pp. 250-251.

1908 , pt. 1, p. 377.

1909, pt. 1, p. 309.

Top. sheet Central City.

25. Trail. $\mathrm{Pb}, \mathrm{Au}, \mathrm{Ag}, \mathrm{Cu}, \mathrm{Zn}$.

6 miles WSW. Idaho Springs, C. \& S. R. R.

Pre-Cambrian complex cut by acidic dikes.

Veins.

Spurr, J. E., and Garrey, G. H., Preliminary report on the ore deposits of the Georgetown mining district, Colorado: Bull. 260, 1905, pp. 99-120.

1908, pp. 311-340.

Min. Res. 1883 , p. 341 .

1885 , p. 256.

1905, p. 196.

1906, p. 212.

1907, pt. 1, p. 251.

1908 , pt. 1, p. 377.

1909, pt. 1, p. 309.

Top. sheet Georgetown.

26. Upper Union (Empire). $\mathrm{Au}, \mathrm{Ag}, \mathrm{Cu}, \mathrm{Pb}$.

3 miles NW. Empire, C. \& S. R. R.

Pre-Cambrian complex cut by acidic dikes.

Veins.

Spurr, J. E., and Garrey, G. H., Economic geology of the Georgetown quadrangle, Colorado: P. P. 63, 1908, pp. 383-410.

Min. Res. 1906, pp. 212-213.

1907 , pt. 1, p. 251.

1908 , pt. 1, p. 377 .

1909, pt. 1, p. 307.

Top. sheet Central City.

CONEJOS COURTY.

27. Ute (Platoro). Au, Ag.

45 miles SW. Monte Vista, D. \& R. G. R. R.

Tertiary volcanics.

Veins. 
CONEJOS OOUHTY-Continued.

27. Ute (Platoro)-Continued.

Min. Res. 1905, p. 198.

1906, p. 213.

1907, pt. 1, p. 251.

costilla coJatr.

28. Grayback (Russell). Au. (D, Pl.)

3 miles N. Russell station, D. \& R. G. R. R.

Pre-Cambrian complex and Paleozoic sediments cut by monzonite.

Veins and replacements, stream gravels.

Patton, H. B., and others, Geology of the Grayback mining district; Costilla County, Colo.: Bull. Colorado Geol. Survey No. 2, 1910.

Min. Res. 1906, p. 213.

1907 , pt. 1, p. 251.

Top. sheet Huerfano Park.

28a. Plomo. Au, (Pl.)

17 miles S. Fort Garland, D. \& R. G. R. R.

Pre-Cambrian gneiss cut by quartz porphyry.

Replacements.

CUSTER COUNTY.

29. Hardscrabble (Rosita, Silver Clifi). $\mathrm{Ag}, \mathrm{Au}, \mathrm{Pb}, \mathrm{Cu}, \mathrm{Zn}$.

Westcliff station, D. \& R. G. R. R.

Pre-Cambrian complex, Tertiary volcanics.

Veins and pipes.

Cross, W., Geology of Silver Cliff and the Rosita Hills, Colorado: Seventeenth Ann. Rept., pt. 2, 1896, pp. 263-403.

Emmons, S. F., The mines of Custer County, Colo.: Seventeenth Ann. Rept., pt. 2, 1896, pp. 405-472.

Min. Res. 1883-84, p. 549.

1885 , p. 256.

1905, p. 198.

1906, p. 213.

1907, pt. 1, pp. 251-252.

1908, pt. 1, p. 378.

1909, pt. 1, p. 309.

Top. sheet Canon City.

30. Spaulding. $\mathrm{Pb}, \mathrm{Au}, \mathrm{Ag}$.

14 miles SW. Florence, D. \& R. G. R. R.

Min. Res. 1905, p. 198.

1906, p. 213.

1907, pt. 1, pp. 251-252.

1908, pt. 1, p. 378.

Top. sheet Canon City.

DOLORES COUNTY.

31. Lone Cone (Dunton). $\mathrm{Ag}, \mathrm{Au}, \mathrm{Pb}, \mathrm{Zn}, \mathrm{Cu}$.

$16 \frac{1}{2}$ miles NW. Rice, R. G. S. R. R.

Min. Res. 1905, p. 199.

1906, p. 214.

1907 , pt. 1, p. 252.

1908 , pt. 1, p. 378.

1909, pt. 1, p. 310.

32. Pioneer (Rico). Ag, $\mathrm{Cu}, \mathrm{Pb}, \mathrm{Zn}, \mathrm{Au}$.

Station R. G. S. R. R.

Pre-Cambrian and Paleozoic sediments domed by monzonite porphyry. 


\section{DOLORES COUNTY-Continued.}

32. Pioneer (Rico)-Continued.

Veins, replacements, and stockworks.

Cross, W., and Spencer, A. C., Geology of the Rico Mountains, Colorado:

Twenty-first Ann. Rept., pt. 2, 1900, pp. 7-165.

Ransome, F. L., The ore deposits of the Rico Mountains, Colorado: Twentysecond Ann. Rept., pt. 2, 1901, pp. 229-297.

Min. Res. 1886, p. 145.

1905, p. 199.

1906, pp. 214, 476.

1907, pt. 1, p. 252.

1908 , pt. 1, pp. $378,379$.

1909 , pt. 1, p. 310.

Top. sheets Rico, Rico special.

Folio 130, 1905.

\section{EAGLE COUNTY.}

33. Battle Mountain (Red Cliff, Gilman). $\mathrm{Ag}, \mathrm{Zn}, \mathrm{Au}, \mathrm{Cu}, \mathrm{Pb}$.

Red Cliff station, D. \& R. G. R. R.

Pre-Cambrian granite, Paleozoic sediments cut by acidic porphyry.

Veins and replacements.

Min. Res. 1882, pp. 310-311.

1883-84, p. 422 .

1885, p. 255.

1886, p. 144.

1905 , p. 199.

1906, p. 215.

1907, pt. 1, p. 253.

1908, pt. 1, p. 378 .

1909, pt. 1, p. 311.

34. Fulford. $\mathrm{Au}, \mathrm{Cu}$.

18 miles SE. Eagle, D. \& R. G. R. R.

Min. Res. 1905, p. 199.

1906, p. 215.

1907, pt. 1, p. 253.

1908, pt. 1, p. 379.

35. Holy Cross (Eagle River). Au, Ag. (D, PI.)

18 miles SW. Red Cliff, D. \& R. G. R. R.

Min. Res. 1906, p. 215.

1907, pt. 1, p. 253.

1908 , pt. 1, p. 379 .

1909 , pt. 1, p. 311.

Top. sheet Leadville.

FREMONT COUNTY.

36. Canon City. $\mathrm{Cu}, \mathrm{W}$.

Station D. \& R. G. R. R.

Pre-Cambrian complex.

Veins.

Min. Res. 1906, p. 216.

Top. sheet Canon City.

37. Cotopaxi. $\mathrm{Cu}, \mathrm{Zn}, \mathrm{Pb}$.

Station D. \& R. G. R. R.

Pre-Gambrian complex. 
37. Cotopaxi-Continued.

Lenses.

Lindgren, W., Notes on copper in Chaffee, Fremont, and Jefferson counties, Colo.: Bull. 340, 1908, pp. 166-167.

38. Currant Creek (Micanite). Zn (Au, Ag).

26 miles $\mathrm{S}$. Howbert, C. M. R. R.

Min. Res. 1906, p. 215.

39. Greenhorn (Grape Creek). Ni, Co, Fe.

15 miles SW. Canon City, 3 miles S. Radiant, D. \& R. G. R. R.

Min. Res. 1882, p. 404.

1883-84, pp. 281-282.

1887, p. 53.

1906, p. 216.

Top. sheet Canon City.

40. Red Gulch. $\mathrm{Cu}, \mathrm{Ag}$.

9 miles N. Cotopaxi, D. \& R. G. R. R.

Paleozoic sediments, "red beds."

Disseminated.

Lindgren, W., Notes on copper in Chaffee, Fremont, and Jefferson counties, Colo.: Bull. 340, 1908, pp. 170-174.

Min. Res. 1907, pt. 1, pp. 253-254.

1908 , pt. 1, p. 380

41. Whitehorn (Manoa, Calumet). $\mathrm{Au}, \mathrm{Ag}(\mathrm{Cu})$.

26 miles NE. Salida, D. \& R. G. R. R.

\section{GILPIN COUNTY.}

42. Central City (Central, Nevada, Gregory, Russell, Quartz Mountain). $\mathrm{Au}, \mathrm{Ag}, \mathrm{Cu}, \mathrm{Pb}$.

Station C. \& S. R. R.

Pre-Cambrian complex cut by acidic dikes.

Vëins.

Min. Res. 1882, pp. 227-228.

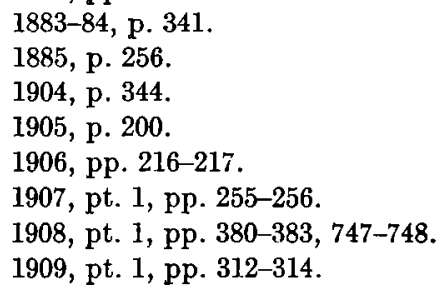

Top. sheet Central City.

43. Independence (Perigo). $\mathrm{Au}, \mathrm{Ag}, \mathrm{Cu}, \mathrm{Pb}$.

6 miles N. Central City, C. \& S. R. R.; 5 miles SSW. Rollinsville, D. N. W. \& P. R. R.

Pre-Cambrian complex cut by acidic dikes.

Veins.

Min. Res. 1883-84, p. 341.

1905, p. 200.

1906, p. 217.

1907, pt. 1, p. 255.

1908 , pt. 1, pp. $383,389$.

1909 , pt. 1, p. 314 .

Top. sheet Central City. 
GILPIN COUNTY-Continned.

44. Pine (Kingaton, Apex). $\mathrm{Au}, \mathrm{Pb}, \mathrm{Cu}, \mathrm{Ag}$.

7 miles NW. Central City, C.\& S. R. R.; 5 miles S. Tolland, D. N. W.\& P. R.R.

Pre-Cambrian complex cut by acidic dikes.

Veins.

Min. Res. 1883-84, p. 341 .

1905 , p. 200.

1906, p. 217.

1907, pt. 1, p. 256.

1908, pt. 1, pp. 383-384.

1909 , pt. 1, p. 314 .

Top. sheet Central City.

45. Blue Ridge.

\section{GRATD COUHTY.}

11 miles S. Parshall, D. N. W. \& P. R. R.

46. Corral Creek.

Parshall station, D. N. W. \& P. R. R.

47. Grand Lake (Wolverine). $\mathrm{Au}, \mathrm{Cu}, \mathrm{Ag}, \mathrm{Pb}$.

16 miles NNE. Granby, D. N. W. \& P. R. R.

Pre-Cambrian complex.

Veins.

48. Harmon. Cu.

Min. Res. 1905, p. 201.

12 miles E. Granby, D. N. W. \& P. R. R.

Pre-Cambrian.

Veins.

Min. Res. 1905, p. 201. 1906, p. 218.

49. La Plata. $\mathrm{Au}, \mathrm{Ag}, \mathrm{Cu}$.

24 miles SSE. Granby, D.N. W. \& P. R. R.

Pre-Cambrian complex.

Veins.

Min. Res. 1906, p. 218.

\section{GUNIISON COUNTY.}

50. Box Canyon. $\mathrm{Au}, \mathrm{Ag}(\mathrm{Cu})$.

7 miles S. Pitkin, C. \& S. R. R.

Pre-Cambrian schists cut by diorite.

Lenses.

Hill, J. M., Notes on the economic geology of southeastern Gunnison County, Colo.: Bull. 380, 1909, pp. 21-40.

Min. Res. 1907, pt. 1, p. 257.

1908 , pt. 1, p. 384 .

1909 , pt. 1, p. 315 .

51. Cebolla (Vulcan, Domingo). $\mathrm{Au}, \mathrm{Ag}, \mathrm{Fe}$.

18 miles S. Iola, D. \& R. G. R. R.

Pre-Cambrian schists, Cretaceous sediments.

Veins.

Harder, E. C., Manganese deposits of the United States: Bull. 380, 1909, p. 273; Bull. 427, 1910, p. 150.

Leith, C. K., Iron ores of the western United States and British Columbia: Bull. 285, 1906, p. 197.

Min. Res. 1882, pp. 144-147.

1883-84, p. 283.

1907 , pt. 1, pp. 105, 257.

1908, pt. 1, p. 111. 
52 Cochetopa. Au, Ag.

5 miles S. Parlin, D. \& R. G. R. R.

Pre-Cambrian granite cut by acidic and basic dikes.

Veins.

Hill, J. M., Notes on the economic geology of southeastern Gunnison County, Colo.: Bull. 380, 1909, pp. 21-40.

Min. Res. 1906, p. 218.

1907, pt. 1, p. 257.

1908 , pt. 1, p. 384 .

53. Elk Mountain (Ruby). $\mathrm{Pb}, \mathrm{Au}, \mathrm{Ag}$.

Crested Butte station, D. \& R. G. R. R.

Cretaceous sediments cut by acidic and basic dikes.

Veins.

Harder, E. C., The Taylor Peak and White Pine iron-ore deposits, Colorado: Bull. 380, 1909, pp. 188-198.

Min. Res. 1906, p. 218.

1907, pt. 1, p. 257.

1908, pt. 1, p. 385 .

1909 , pt. 1, p. 315.

Top. sheet Crested Butte. Folio 9, 1894.

54. Gold Brick. $\mathrm{Au}, \mathrm{Ag}, \mathrm{Pb}$.

3 miles N. Ohio, C. \& S. R. R.

Pre-Cambrian granite schiste.

Veins.

Hill, J. M., Notes on the economic geology of southeastern Gunnison County,

Colo.: Bull. 380, 1909, pp. 21-40.

Min. Res. 1905, p. 202.

1906, p. 219.

1907 , pt. 1, p. 257

1908 , pt. 1, p. 385

1909 , pt. 1, p. 315 .

55. Quartz Creek. $\mathrm{Ag}, \mathrm{Au}, \mathrm{Pb}$.

3 miles N. Pitkin, C. \& S. R. R.

Pre-Cambrian complex, Paleozoic sediments cut by acidic dikes.

Veins and replacements.

Hill, J. M., Notes on the economic geology of southeastern Gunnison County,

Colo.: Bull. 380, 1909, pp. 21-40.

Min. Res. 1905, p. 202.

1906, p. 219.

1907, pt. 1, p. 257

1908 , pt. 1, p. 385 .

1909 , pt. 1, p. 315.

56. Rock Creek. $\mathrm{Ag}, \mathrm{Pb}, \mathrm{Cu}, \mathrm{Zn}, \mathrm{Au}$.

Marble station, C. R. \& S. J. R. R.

Min. Res. 1905, p. 201.

1906, pp. 218-219.

1907 , pt. 1, p. 257.

1908 , pt. 1, p. 385 .

Top, sheet Anthracite.

Folio 9, 1894.

57. Ruby. Ag.

Floresta station, D. \& R. G. R. R.

Cretaceous sediments eut by aeidic and basic dikes.

Veins. 
57. Ruby-Continued.

Min. Res. 1905, p. 201.

1908, pt. 1, p. 385 .

Top. sheet Anthracite.

Folio 9, 1894.

58. Tincup (Pieplant). $\mathrm{Au}, \mathrm{Ag}, \mathrm{Pb}(\mathrm{Cu})$.

37 miles SSW. Buena Vista, C. \& S. R. R., C. M. R. R., D. \& R. G. R. R.

Pre-Cambrian complex, Paleozoic sediments cut by acidic porphyry.

Veins and replacements.

Hill, J. M., Notes on the economic geology of southeastern Gunnison County,

Colo.: Bull. 380, 1909, pp. 21-40.

Min. Res. 1905, pp. 201-202.

1906, p. 219.

1907, pt. 1, pp. 257, 258.

1908, pt. 1, p. 385 .

1909, pt. 1, p. 315.

59. Tomichi (Whitepine). $\mathrm{Pb}, \mathrm{Ag}, \mathrm{Zn}, \mathrm{Cu}, \mathrm{Au}, \mathrm{Fe}$.

12 miles NNE. Sargents, D. \& R. G. R. R.

Pre-Cambrian granite, Paleozoic sediments cut by acidic dikes.

Veins and replacements.

Harder, E. C., The Taylor Peak and Whitepine iron-ore deposits, Colorado:

Bull. 380, 1909, pp. 188-198.

Hill, J. M., Notes on the economic geology of southeastern Gunnison County, Colo.: Bull. 380, 1909, pp. 21-40.

Leith, C. K., Iron ores of the western United States and British Columbia:

Bull. 285, 1905, pp. 196-198.

Min. Rea. 1885, p. 256.

1887, p. 28.

1905, p. 202.

1906, p. 219.

1907, pt. 1, p. 258.

1908, pt. 1, p. 385 .

60. White Earth. Au.

1909 , pt. 1, p. 315 .

18 miles S. Iola, D. \& R. G. R. R.

HITSDALE COUNTY.

61. Galena. $\mathrm{Pb}, \mathrm{Ag}, \mathrm{Cu}, \mathrm{Au}$.

5 miles W. Lake City, D. \& R. G. R. R.

Tertiary volcanics.

Veins.

Irving, J. D., Ore deposits in the vicinity of Lake City, Colo.: Bull. 260, 1905, pp. 78-84.

Irving, J. D., and Bancroft, H., Geology and ore deposits near Lake City, Colo.: Bull. 478, 1911, pp. 81-99.

Min. Res. 1905, p. 202.

1906, p. 220.

1907, pt. 1, pp. 258-259.

1908, pt. 1, p. 386.

1909 , pt. 1, p. 316.

Top. sheet Lake.

62. Lake (San Cristobal). $\mathrm{Ag}, \mathrm{Au}, \mathrm{Pb}, \mathrm{Cu}, \mathrm{Zn}$.

6 miles S. Lake City, D. \& R. G. R. R.

Tertiary volcanics.

$21528^{\circ}-$ Bull. $507-12-10$ 
HINSDALE COUNTY-Continued.

62. Lake (San Cristobal)-Continued.

Veins.

Irving, J. D., Ore deposits in the vicinity of Lake City, Colo.: Bull. 260, 1905, pp. 78-84.

Irving, J. D., and Bancroft, H., Geology and ore deposits near Lake City, Colo.: Bull. 478, 1911, pp. 99-116

Min. Res. 1905, p. 202.

1906, p. 220.

1907, pt. 1, p. 259.

1908, pt. 1, p. 386.

1909, pt. 1, p. 316.

Top. sheet Lake.

63. Park (Sherman). Au, Ag.

22 miles SW. Lake City, D. \& R. G. R. R.

Tertiary volcanics.

Veins.

Irving, J. D., Ore deposits in the vicinity of Lake City, Colo.: Bull. 260, 1905, pp. 78-84.

Larsen, E. S., The economic geology of Carson Camp, Hinsdale County, Colo.: Bull. 470, 1911, pp. 30-38.

Woolsey, L. H., Lake Fork extension of the Silverton mining area, Colorado: Bull. 315, 1907, pp. 26-30.

Min. Res. 1905, p. 202.

Top. sheet San Cristobal.

HUERFAKO COUNTY.

64. Huerfano (Malachite). Cu, Ag.

32 miles WNW. Walsenburg, D. \& R. G. R. R., C. \& S. R. R.

Paleozoic sediments, "Red Beds."

Disseminated.

Min. Res. 1909, pt. 1, p. 316.

Top. sheet Huerfano Park.

65. La Veta. Au, Ag.

Station D. \& R. G. R. R.

Tertiary volcanics, acidic and basic dikes.

Veins.

Top. sheets Walsenburg, Spanish Peaks.

Folios 68, 1900; 71, 1901.

JACKSON COUNTY. (See Larimer.)

JEFFERSON COUNTY.

66. Evergreen (Malachite). $\mathrm{Cu}, \mathrm{Ag}, \mathrm{Au}, \mathrm{Ni}$.

11 miles W. Mount Morrison, C. \& S. R. R.

Pre-Cambrian complex cut by basic dikes.

Veins.

Lindgren, W., Notes on copper in Chaffee, Fremont, and Jefferson counties, Colo.: Bull. 340, 1908, pp. 167-179.

Min. Res. 1905, pp. 202-203.

1906, p. 220.

1907, pt. 1, p. 259 .

1909 , pt. 1, p. 316 .

Top. sheet Denver.

67. Golden. Au. (Pl.)

Station C. \& S. R. R.

Stream gravels. 
JEFFERSON COUNTY-Continued.

67. Golden-Continued.

Min. Res. 1905, p. 203.

Top. sheet Denver.

\section{LAKE COUNTY.}

68. California (Leadville). $\mathrm{Pb}, \mathrm{Ag}, \mathrm{Au}, \mathrm{Zn}, \mathrm{Cu}, \mathrm{Fe}, \mathrm{Mn}$.

Leadville station, D. \& R. G. R. R.

Pre-Cambrian complex, Paleozoic sediments cut by acidic intrusives.

Replacements and veins.

Emmons, S. F., Geology and mining industry of Leadville, Colo.: M. XII, 1895.

Emmons, S. F., and Irving, J. D., The Downtown district of Leadville, Colo.: Bull. 320, 1907.

Harder, E. C., Manganese deposits of the United States: Bull. 380, 1909, p. 273; Bull. 427, 1910, pp. 137-143.

Min. Res. 1882, pp. 310-311.

1883-84, pp. $419-422$.

1885 , pp. $250-255,348$.

1886, p. 144.

1887 , p. 105.

1888 , pp. $53,87$.

1894 (Sixteenth Ann. Rept., pt. 3), pp. 407-410.

1904, p. 276.

1905, pp. 203-204.

1906, pp. 221-223, 475, 517.

1907, pt. 1, pp. 105, 259-261, 601.

1908, pt. 1, pp. 152, 386-389.

1909 , pt. 1, pp. 317-319.

Top. sheets Leadville, Leadville special.

69. Harrington (Tennessee Pass).

Tennessee Pass station, D. \& R. G. R. R.

Top. sheet Leadville.

70. Twin Lakes. Au, Ag, Pb. (D, Pl.)

9 miles NW. Granite, D. \& R. G. R. R., C. M. R. R.

Pre-Cambrian complex.

Veins, glacial gravels.

Top. sheet Leadville.

IA PLATA COUNTY.

71. California (Oro Fino). $\mathrm{Au}, \mathrm{Ag}, \mathrm{Pb}, \mathrm{Cu}$.

14 miles NW. Durango, D. \& R. G. R. R.

"Red Beds" cut by diorite or monzonite porphyry.

Veins.

Emmons, W. H., The Neglected mine and near-by properties, Durango quadrangle, Colorado: Bull. 260, 1905, pp. 121-127.

Min. Res. 1905, p. 205.

1906 , p. 224.

1907, pt. 1., p. 262.

1908 , pt. 1 , p. 389 .

Top. sheets Durango and La Plata.

Folio 60, 1899.

72. Needle Mountains (Florida). $\mathrm{Au}, \mathrm{Ag}, \mathrm{Cu}(\mathrm{Pb})$.

25 miles ENE. Tacoma, D. \& R. G. R. R.

Pre-Cambrian complex cut by granite.

Veins. 
LA PLATA COUNTY-Continued.

72. Needle Mountains (Florida)-Continued

Min. Res. 1905, p. 205.

1906, p. 224.

1908 , pt. 1, p. 389.

Top. sheet Needle Mountains.

Folio 131, 1905.

LARIMER AND JACKSON COUNTIES.

73. Empire (Howes Gulch). $\mathrm{Cu}, \mathrm{Au}$.

8 miles W. Fort Collins, C. \& S. R. R.

Pre-Cambrian complex.

Veins.

74. Manhattan.

45 miles NW. Fort Collins, C. \& S. R. R.

75. Pearl. $\mathrm{Cu}(\mathrm{Au}, \mathrm{Ag})$.

33 miles SE. Riverside, Wyo., S. \& E. R. R.

Pre-Cambrian complex.

Veins.

Spencer, A. C., Reconnaissance examination of the copper deposits at Pearl, Colo.: Bull. 213, 1903, pp. 163-169.

Min. Res. 1906, p. 224.

76. Steamboat Rock (Gray Rock). Cu, Au.

NW. Fort Collins, C. \& S. R. R.

Pre-Cambrian complex.

Veins.

\section{MESA COUNTY.}

77. Unaweep. $\mathrm{Cu}, \mathrm{Au}, \mathrm{Ag}$.

25 miles SSW. Grand Junction, D. \& R. G. R. R., C. M. R. R.

Pre-Cambrian schists and Paleozoic and Cretaceous sediments.

Disseminated.

Emmons, S. F., The copper in the red beds of the Colorado Plateau region: Bull. 260, 1905, p. 227.

MINERAL COUNTY.

78. Sunnyside (Creede). $\mathrm{Ag}, \mathrm{Pb}, \mathrm{Au}, \mathrm{Zn}$.

Station D. \& R. G. R. R.

Tertiary volcanics.

Veins.

Emmons, W. H., and Larsen, E. S., Preliminary report on the geology and ore deposits of Creede, Colo.: Bull. 530, 1912.

Min. Res. 1905, p. 205.

1906, pp. 224-225.

1907, pt. 1, pp. 262-263.

1908, pt. 1, p. 390.

1909, pt. 1, p. 320 .

\section{MOFFAT COUNTY. (Seo Rontt.)}

MONTEZUYA COUNTY.

79. East Mancos. Au, Ag.

12 miles NE. Mancos, R. G. S. R. R.

"Red Beds" cut by diorite and monzonite.

Veins.

Min. Res. 1907, pt. 1, p. 263.

Top. sheet La Plata.

Folio 60, 1899. 
80. Hydraulic. V, U.

MONTROSE COUNTY.

74 miles WNW. Placerville, R. G. S. R. R.

Jurassic sediments."

Disseminated.

Boutwell, J. M., Vanadium and uranium in Utah: Bull. 260, 1905, pp. 202-203.

Min. Res. 1908, pt. 1, p. 748.

81. La Sal. $\mathrm{Cu}, \mathrm{Ag}, \mathrm{V}, \mathrm{U}$.

76 miles W. Placerville, D. \& R. G. R. R.

Jurassic sediments.

Veins and disseminations.

Boutwell, J. M., Vanadium and uranium in Utah: Bull. 260, 1905, pp. 202-203.

Emmons, W. H., The Cashin mine, Montrose County, Colo.: Bull. 285, 1906, pp. $125-128$.

Hillebrand, W. F., and Ransome, F. L., On carnotite and associated vanadiferous minerals in western Colorado: Bull. 262, 1905, pp. 14-16.

Min. Res. 1900, p. 261.

1907, pt. 1, p. 264.

1908, pt. 1, pp. $741,748$.

82. Naturita (Vixen). V, U.

43 miles WNW. Placerville, R. G. S. R. R.

Jurassic sediments.

Diseeminations.

Boutwell, J. M., Vanadium and uranium in Utah: Bull. 260, 1905, p. 203.

Min. Res. 1908 , pt. 1, p. 748.

83. Roc Creek. V, U.

80 miles WNW. Placerville, R. G. S. R. R.

Jurassic sediments.

Disseminations.

Boutwell, J. M., Vanadium and uranium in Utah: Bull. 260, 1905, p. 203.

Hillebrand, W. F., and Ransome, F. L., On carnotite and associated vanadiferous minerals in western Colorado: Bull. 262, 1905, p. 16.

Min. Res. 1900, p. 261.

1908, pt. 1, pp. $741,748$.

OURAY COUNTY.

84. Imogene Basin (Camp Bird). Au, Ag.

8 miles SW. Ouray, D. \& R. G. R. R.

Tertiary volcanics.

Veins.

Ransome, F. L., A report on the economic geology of the Silverton quadrangle, Colorado: Bull. 182, 1901, pp. 1-265.

Min. Res. 1905, p. 206.

1906 , p. 226.

1907 , pt. 1, p. 264.

1908, pt. 1, p. 391.

1909 , pt. 1, p. 322.

Top. sheet Silverton.

Folio 120, 1905.

85. Red Mountain. $\mathrm{Cu}, \mathrm{Ag}, \mathrm{Au}, \mathrm{Pb}$.

Station S. R. R.

Tertiary volcanics.

Veins. 
OURAT COUNTY-Continued.

85. Red Mountain-Continued.

Ransome, F. L., A report on the economic geology of the Silverton quadrangle, Colorado: Bull. 182, 1901, pp. 1-265.

Min. Res. 1905, pp. 206-207.

1906 , p. 227.

1907, pt. 1, p. 265.

1908, pt. 1, pp. 391-392.

1909 , pt. 1, p. 322 .

Top. sheet Silverton.

Folio 120, 1905.

86. Sneffels. $\mathrm{Au}, \mathrm{Ag}, \mathrm{Pb}, \mathrm{Cu}$.

8 miles W. Ouray, D. \& R. G. R. R

Tertiary volcanics.

Veins.

Irving, J. D., Ore deposits of the Ouray district, Colorado: Bull. 260, 1905, pp. 50-77.

Min. Res. 1905, p. 206.

1906 , p. 226.

1907 , pt. 1, p. 265.

1908, pt. 1, p. 391.

1909 , pt. 1, p. 322 .

Top. sheet Ouray.

Folio 153, 1907.

87. Uncompahgre. $\mathrm{Au}, \mathrm{Ag}, \mathrm{Pb}, \mathrm{Cu}, \mathrm{Zn}$.

Ouray station, D. \& R. G. R. R.

Paleozoic sediments and Tertiary volcanics.

Veins and replacements.

Irving, J. D., Ore deposits of the Ouray district, Colorado: Bull. 260, 1905, pp. 50-77.

Min. Res. 1905, p. 206.

1906, p. 227.

1907 , pt. 1, pp. 265-266.

1908 , pt. 1, p. 392.

1909, pt. 1, p. 322.

Top. sheet Ouray.

Folio 153, 1907.

PARK COUNTY.

88. Buckskin. $\mathrm{Au}, \mathrm{Ag}, \mathrm{Cu}, \mathrm{Pb}, \mathrm{Zn}$.

6 miles NW. Alma, C. \& S. R. R.

Pre-Cambrian complex and Paleozoic sediments.

Veins.

Min. Res. 1905, p. 207.

1906, p. 228.

1907 , pt. 1, p. 266.

1908 , pt. 1, pp. $392-393$

1909 , pt. 1, p. 323 .

Top. sheet Leadville.

89. Consolidated-Montgomery. $\mathrm{Au}, \mathrm{Ag}, \mathrm{Pb}, \mathrm{Cu}$.

6 miles N. Alma, C. \& S. R. R.

Pre-Cambrian complex.

Veins.

Min. Res. 1906, p. 228.

1907, pt. 1, pp. 266-267.

1908, pt. 1, p. 393. 


\section{PARK COUNTY-Continued.}

89. Consolidated-Montgomery-Continued.

Min. Res. 1909, pt. 1, p. 323.

Top. sheet Leadville.

90. Halls Gulch. $\mathrm{Ag}(\mathrm{Au})$.

5 miles N. Grant, C. \& S. R. R.

Pre-Cambrian complex cut by acidic dikes.

Veins.

Min. Res. 1900, p. 228.

1907, pt. 1, p. 267.

1908, pt. 1, p. 393.

91. Hartsel. Au.

Station C. M. R. R.

92. Horseshoe. $\mathrm{Pb}, \mathrm{Ag}, \mathrm{Au}, \mathrm{Cu}, \mathrm{Zn}$.

7 miles W. Garo, C. \& S. R. R.

Pre-Cambrian complex, Paleozoic sediments.

Veins and replacements.

Min. Res. 1906, p. 228.

1907, pt. 1, p. 267.

1908, pt. 1, p. 393.

Top. sheet Leadville.

93. Mosquito. $\mathrm{Au}, \mathrm{Ag}, \mathrm{Pb}$. (D, Pl.)

6 miles W. Alma, C. \& S. R. R.

Paleozoic sediments.

Veins and glacial gravels.

Min. Res. 1905, p. 207.

1906, p. 228.

1907, pt. 1, p. 267.

1908, pt. 1, p. 393.

1909 , pt. 1, p. 323.

Top. sheet Leadville.

94. Sacramento. $\mathrm{Ag}, \mathrm{Au}, \mathrm{Pb}(\mathrm{Cu})$.

8 miles NW. Garo, C. \& S. R. R.

Pre-Cambrian complex.

Veins.

Min. Res. 1909, pt. 1, p. 323

Top. sheet Leadville.

95. Tarryall. Au, Ag. (D, Pl.)

Como station, C. \& S. R. R.

Paleozoic and Cretaceous sediments cut by monzonite.

Veins and glacial gravels.

Min. Res. 1906, p. 228.

1907, pt. 1, p. 267.

1908, pt. 1, p. 393 .

1909 , pt. 1, p. 323 .

\section{PITEIN COUNTY.}

96. Columbia (Asheroft). $\mathrm{Pb}, \mathrm{Ag}, \mathrm{Cu}, \mathrm{Fe}$.

12 miles S. Aspen, D. \& R. G. R. R., C. M. R. R.

Cretaceous sediments cut by acidic and basic dikes.

Veins.

Leith, C. K., Iron ores in western United States and British Columbia: Bull. 285, 1906, pp. 196-198.

Min. Res. 1887, p. 28.

1888 , p. 33.

1906, p. 230. 
PITEW CoUNTY-Continued.

96. Columbia (Ashcroft)-Continued.

Min. Res. 1907, pt. 1, p. 269.

1908 , pt. 1, p. 394.

1909 , pt. 1, p. 324.

Top. sheet Aspen.

97. Frying Pan. $\mathrm{Pb}, \mathrm{Au}, \mathrm{Ag}, \mathrm{Cu}$.

Nast station, C. M. R. R.

Pre-Cambrian granite; Paleozoic sediments cut by acidic and basic dikes.

Veins and replacements.

98. Lincoln. Ag, $\mathrm{Pb}, \mathrm{Au}$.

15 miles SSE. Aspen, C. M. R. R., D. \& R. G. R. R.

Min. Res. 1905, p. 208.

1906, p. 230.

1907 , pt. 1, pp. 268-269.

1908 , pt. 1, p. 294.

1909 , pt. 1, p. 324 .

99. Roaring Fork (Aspen). $\mathrm{Pb}, \mathrm{Ag}, \mathrm{Zn}, \mathrm{Cu}$.

Aspen station, D. \& R. G. R. R., C. M. R. R.

Paleozoic sediments cut by dikes.

Veins and replacements.

Spurr, J. E., Geology of the Aspen mining district, Colorado: M. XXXI, 1898.

Min. Res. $1883-84$, p. 422.

$$
\begin{aligned}
& 1885, \text { p. } 256 . \\
& 1886, \text { p. } 145 . \\
& 1887, \text { pp. } 105-107 . \\
& 1888, \text { pp. } 87-88 . \\
& 1905, \text { p. } 208 . \\
& 1906 \text {, pp. } 229-230 . \\
& 1907, \text { pt. } 1, \text { p. } 268 . \\
& 1908 \text {, pt. } 1 \text {, p. } 294 . \\
& 1909, \text { pt. } 1 \text {, p. } 324 .
\end{aligned}
$$

Top. sheets Aspen, Aspen special.

100. Coal Creek. V, U.

RIO BLANCO COUNTY.

50 miles N. Rifle, D. \& R. G. R. R.

Jurassic sediments.

Disseminated.

Gale, H. S., Carnotite in Rio Blanco County, Colo.: Bull. 315, 1907, pp. , 110-117.

Min. Res. 1908, pt. 1, pp. 741, 748.

101. Decatur. $\mathrm{Au}, \mathrm{Ag}$.

\section{RIO GRANDE COUNTY.}

33 miles SW. Monte Vista, D. \& R. G. R. R.

102. Embargo. $\mathrm{Au}, \mathrm{Ag}, \mathrm{Pb}, \mathrm{Cu}$.

Del Norte station, D. \& R. G. R. R.

Min. Res. 1908, pt. 1, p. 395.

103. Summitville. $\mathrm{Au}, \mathrm{Ag}$.

28 miles SW. Del Norte, D. \& R. G. R. R.

Tertiary volcanics.

Veins.

Min. Res. 1905, p. 198.

1906, p. 213. 


\section{ROUTT AND MOFFAT COUNTIES.}

104. Copper Ridge. $\mathrm{Cu}, \mathrm{Au}$.

9 miles NW. Steamboat Springs, D. N. W. \& P. R. R.

105. Douglas Mountain.

40 miles NE. Dragoon, Utah, U. R. R.

106. Elkhorn (Three Forks, Slater). $\mathrm{Cu}, \mathrm{Pb}, \mathrm{Ag}, \mathrm{Au}$. 70 miles SE. Wamsutter, Wyo., U. P. R. R.

Pre-Cambrian complex.

Veins.

107. Hahns Peak (Columbine). Au, Ag, Pb. (D, PI.)

26 miles N. Steamboat Springs, D. N. W. \& P. R. R.

Pre-Cambrian complex and Cretaceous sediments cut by rhyolite porphyry.

Veins.

Gale, H. S., The Hahns Peak gold field, Colorado: Bull. 285, 1906, pp. 28-34.

George, R. D., and Crawford, R. D., The Hahns Peak region, Routt County,

Colo.: First Ann. Rept. Colorado Geol. Survey, 1909, pp. 221-226.

Min. Res. 1905, p. 208.

1906 , p. 230.

1907 , pt. 1, p. 269.

1908 , pt. 1, p. 395.

1909 , pt. 1, p. 324 .

108. Lay (Jackrabbit). Au. (P1.)

8 miles W. Steamboat Springs, D. N. W. \& P. R. R.

River gravels.

Gale, H. G., Gold placers near Lay, Routt County, Colo.: Bull. 340, 1908, pp. 84-85.

White, C. A., On the geology and physiography of a portion of northwestern Colorado and adjacent parts of Utah and Wyoming: Ninth Ann. Rept., 1889, p. $69 \mathrm{I}$.

Min. Res. 1906, p. 230.

1907, pt. 1, p. 269.

1908 , pt. 1 , p. 395 .

1909 , pt. 1, p. 324.

109. Oak Creek (Red Gorge). Cu.

Station D. N. W. \& P. R. R.

110. Rock Creek (Gore Range). Cu.

16 miles E. Yampa, D. N. W. \& P. R. R.

111. Skull Creek (Blue Mountain). V, U, Cu.

80 miles NE. Dragoon, Utah, U. R. R.

Jurassic sediments.

Disseminations along shear zones.

Gale, H. S., Carnotite and associated minerals in western Routt County, Colo.:

Bull. 340, 1908, pp. 257-262.

Min. Res. 1908, pt. 1, pp. 741, 748.

112. Slavonia. $\mathrm{Pb}(\mathrm{Ag}, \mathrm{Cu}, \mathrm{Au})$.

40 miles N. Steamboat Springs, D. N. W. \& P. R. R.

Granite, gneiss, and schist cut by granite porphyry and diorite.

Veins.

George, R. D., and Crawford, R. D., The Hahns Peak region, Routt County,

Colo.: First Ann. Rept. Colorado Geol. Survey, 1909, pp. 221-226.

Min. Res. 1906, p. 230.

113. Spring Creek (Steamboat Springs). Cu, Au.

Steamboat Springs station, D. N. W. \& P. R. R. 


\section{SAGUACHE COUNTY.}

114. Baca Grant (Crestone). Au, Ag.

13 miles E. Moffat, D. \& R. G. R. R.

Min. Res. 1905, p. 209.

1906, p. 231.

1907, pt. 1, p. 270.

1908 , pt. 1, p. 394.

1909 , pt. 1, p. 324 .

115. Blake (Kerber Creek). $\mathrm{Pb}, \mathrm{Ag}, \mathrm{Au}(\mathrm{Cu})$.

16 miles W. Villagrove, D. \& R. G. R. R.

Min. Res. 1905, p. 209.

1906, p. 231.

1907, pt. 1, p. 270.

1909 , pt. 1, p. 324 .

116. Crystal Hill. Ag, Au.

25 miles NW. Del Norte, D. \& R. G. R. R.

Min. Res. 1907, pt. 1, p. 270.

SAT JUAY COUTTY.

117. Animas. $\mathrm{Au}, \mathrm{Ag}, \mathrm{Cu}, \mathrm{Pb}, \mathrm{Zn}$.

Silverton station, D. \& R. G. R. R.

Tertiary volcanics.

Veins stockworks.

Emmons, W. H., Ore deposits of Bear Creek, near Silverton, Colo.: Bull. 285,1906 , pp. 25-27.

Ransome, F. L., A report on the economic geology of the Silverton quadrangle, Colorado: Bull. 182, 1901, pp. 1-265.

Min. Res. 1905, pp. 209-210.

1906, p. 232.

1907, pt. 1, pp. 271-272.

1908, pt. 1, pp. 396-397.

1909 , pt. 1, p. 326.

Top. sheet Silverton.

Folio 120, 1905.

118. Eureka. $\mathrm{Au}, \mathrm{Ag}, \mathrm{Pb}, \mathrm{Cu}, \mathrm{Zn}$.

Station S. N. R. R.

Tertiary volcanics.

Veins, stockworks.

Ransome, F. L., A report on the economic geology of the Silverton quadrangle, Colorado: Bull. 182, 1901, pp. 1-265.

Min. Res. 1905, pp. 209-210.

1906 , pp. 232-233.

1907, pt. 1, p. 272 .

1908 , pt. 1, p. 297.

1909 , pt. 1, p. 326.

Top. sheet Silverton.

Folio 120, 1905.

SAN MIGUEL COUNTT.

119. Iron Spring (Ophir). $\mathrm{Au}, \mathrm{Ag}, \mathrm{Pb}, \mathrm{Cu}$.

Ophir station, R. G. S. R. R.

Tertiary volcanics.

Veins, stockworks.

- Purington, C. W., Preliminary report on the mining industries of the Telluride quadrangle, Colorado: Eighteenth Ann. Rept., pt. 3, 1898, pp. 745-850. 
SAN MIGUEL COUNTT-Continued.

119. Iron Spring (Ophir)-Continued.

Min. Res. 1886, p. 145.

1905, pp. 210-211.

1906, p. 234.

1907, pt. 1, pp. 273-274.

1908 , pt. 1, p. 399.

1909 , pt. 1, p. 328 .

Top. sheet Telluride.

Folio 57, 1899.

120. Lower San Miguel (Placerville, Sawpit, Newmire). $\mathrm{Au}, \mathrm{Ag}, \mathrm{Pb}, \mathrm{Cu}, \mathrm{V}$. $(\mathrm{D}, \mathrm{Pl}$.

Sawpit and Newmire stations, R. G. S. R. R.

Cretaceous sediments cut by dikes.

Veins and replacements.

Hess, F. L., Notes on the vanadium deposits near Placerville, Colo.: Bull. $530,1912$.

Hillebrand, W. F., and Ransome, F. L., On carnotite and associated minerals in western Colorado: Bull. 262, 1905, pp. 9-14.

Purington, C. W., Preliminary report on the mining industries of the Telluride quadrangle, Colorado: Eighteenth Ann. Rept., pt. 3, 1898, p. 827.

Min. Res. 1900, pp. 262-265.

1905 , p. 211.

1906, p. 234.

1907, pt. 1, p. 274

1908 , pt. 1, p. 299.

1909 , pt. 1, p. 328.

Top. sheet Telluride.

Folio 57, 1899.

121. Mount Wilson. $\mathrm{Ag}, \mathrm{Au}, \mathrm{Pb}, \mathrm{Cu}$.

12 miles S. Newmire, R. G. S. R. R.

Cretaceous sediments and Tertiary volcanics.

Veins.

Purington, C. W., Preliminary report on the mining industries of the Telluride quadrangle, Colorado: Eighteenth Ann. Rept., pt. 3, 1898, pp. 745-850.

Min. Res. 1905, p. 211.

1907, pt. 1, p. 274.

1908, pt. 1, p. 399.

1909 , pt. 1, p. 328.

Top. sheet Telluride.

Folio 57, 1899.

122. Snyderville (Cedar). V, U.

35 miles WSW. Placerville, R. G. S. R. R.

Jurassic sediments.

Disseminations.

Min. Res. 1906, pp. 531-532.

1908, pt. 1, pp. 741, 748 .

123. Upper San Miguel (Telluride). $\mathrm{Au}, \mathrm{Ag}, \mathrm{Pb}, \mathrm{Cu}, \mathrm{Zn}$.

Telluride station, R. G. S. R. R.

Tertiary volcanics.

Veins, stockworks.

Purington, C. W., Preliminary report on the mining industries of the Telluride quadrangle, Colorado: Eighteenth Ann. Rept., pt. 3, 1898, pp. $745-850$. 
SAN MIGUEL COUNTY-Continued.

123. Upper San Miguel (Telluride)-Continued.

Min. Res. 1905, p. 210.

1906, pp. 233-234.

1907, pt. 1, p. 273.

1908, pt. 1, pp. 398-399.

1909 , pt. 1, pp. 327-328.

Top. sheet Telluride.

Folio 57, 1899.

\section{SUMMIT COUNTY.}

124. Breckenridge (Bevan, Union, Minnesota). Au, $\mathrm{Zn}, \mathrm{Pb}, \mathrm{Ag} . \quad$ (D, Pl.)

Station C. \& S. R. R.

Paleozoic and Cretaceous sediments cut by porphyry dikes.

Veins, replacements, and disseminations.

Ransome, F. L., Geology and ore deposits of the Breckenridge district, Colorado: P. P. 75, 1911.

Min. Res. 1905, p. 211.

1906, pp. 235-236, 475.

1907, pt. 1, p. 274.

1908, pt. 1, pp. 400-401.

1909 , pt. 1, p. 329 .

Top. sheets Leadville, Breckenridge special.

125. Frisco. $\mathrm{Au}, \mathrm{Ag}(\mathrm{Pb}, \mathrm{Zn})$.

Station C. \& S. R. R.

Min. Res. 1905, p. 211.

1908 , pt. 1, p. 401.

126. Montezuma (Snake River). $\mathrm{Pb}, \mathrm{Ag}, \mathrm{Cu}, \mathrm{Zn}, \mathrm{Au}$.

12 miles E. Dillon, C. \& S. R. R.

Pre-Cambrian complex cut by acidic dikes.

Veins.

Patton, H. B., The Montezuma mining district of Summit County, Colo.:

First Ann. Rept. Colorado Geol. Survey, 1909, pp. 112-444.

Min. Res. 1905, p. 211.

1906, pp. 236, 475.

1907, pt. 1, p. 275.

1908 , pt. I, p. 401.

1909, pt. 1, p. 329.

127. Peru. $\mathrm{Ag}, \mathrm{Pb}, \mathrm{Au}$.

8 miles E. Dillon, C. \& S. R. R.

Min. Res. 1905, p. 211.

1909 , pt. 1, p. 329.

128. Swan River. Au. (D, Pl.)

12 miles NE. Breckenridge, C. \& S. R. R.

Pre-Cambrian complex cut by acidic porphyries.

Veins.

Top. sheet Breckenridge special.

129. Tenmile (Kokomo). $\mathrm{Ag}, \mathrm{Au}, \mathrm{Pb}, \mathrm{Zn}, \mathrm{Cu}$.

Kokomo station, C. \& S. R. R.

Paleozoic sediments cut by acidic dikes.

Veins and replacements.

Min. Res. 1905, pp. 211-212.

1906, p. 236.

1907 , pt. 1, p. 275 .

1908, pt. 1, p. 401.

1909, pt. 1, p. 329 . 
SUMmIT COUNTY-Continued.

129. Tenmile (Kokomo)-Continued.

Top. sheet Tenmile.

Folio 48, 1898.

TELLER COUNTY.

130. Cripple Creek. Au, Ag.

Station C. S. \& C. C. R. R.

Tertiary volcanics, mainly phonolite, in pre-Cambrian granite.

Veins.

Cross, W., and Penrose, R. A. F., jr., Geology and mining industries of the Cripple Creek district, Colorado: Sixteenth Ann. Rept., pt. 2, 1895, pp. 13-209.

Hillebrand, W. F., Two tellurium minerals from Colorado: Bull. 262, 1905, pp. 55-57.

Lindgren, W., The geological resurvey of the Cripple Creek district, Colorado: Bull. 260 , 1905, pp. 85-98.

Lindgren, W., and Ransome, F. L., Report of progress in the geological resurvey of the Cripple Creek district, Colorado: Bull. 254, 1904.

Geology and gold deposits of the Cripple Creek district, Colorado: P. P. 54, 1906.

Min. Res. 1905, pp. 212-214.

1906, pp. 236-240.

1907, pt. 1, pp. 275-278.

1908 , pt. 1, pp. $401-405$.

1909 , pt. 1, pp. $330-333$.

Top. sheets Pikes Peak, Cripple Creek special.

Folio 7, 1894.

131. East Beaver. $\mathrm{Cu}, \mathrm{Au}, \mathrm{Ag}$.

8 miles S. Rosemont, C. S. \& C. C. R. R.

Pre-Cambrian complex cut by basic dikes.

.Veins. 


\section{IDAHO.}

In the State of Idaho there are 133 mining districts in 20 counties. Gold is the predominant metal produced in 84 districts, silver in 14, lead in 22, and copper in 13.

Distribution of the predominant metals produced in the mining districts of Idaho.

\begin{tabular}{|c|c|c|c|c|c|}
\hline County. & Gold. & Silver. & Copper. & Lead. & Total. \\
\hline 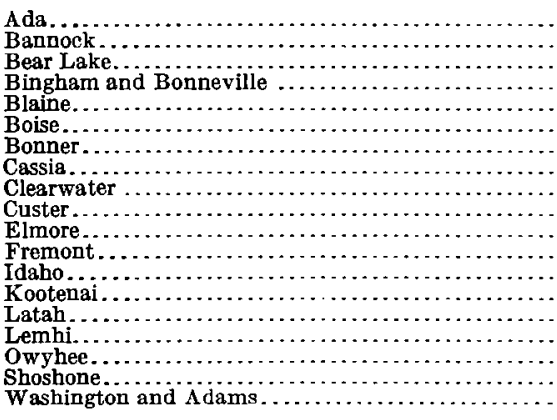 & $\begin{array}{r}3 \\
1 \\
5 \\
11 \\
3 \\
2 \\
4 \\
5 \\
6 \\
10 . \\
10.3 \\
3 \\
13 \\
3 \\
4 \\
4\end{array}$ & 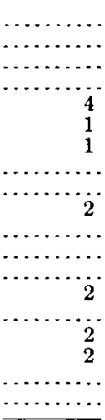 & $\begin{array}{c}1 \\
1 \\
1 \\
1 \\
1 \\
1 \\
\cdots \\
1 \\
1 \\
1 \\
1\end{array}$ & 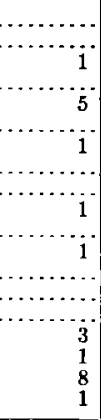 & $\begin{array}{r}3 \\
1 \\
2 \\
2 \\
15 \\
12 \\
6 \\
2 \\
4 \\
9 \\
6 \\
2 \\
17 \\
3 \\
3 \\
19 \\
6 \\
14 \\
7\end{array}$ \\
\hline & 84 & 14 & 13 & 22 & 133 \\
\hline
\end{tabular}

MINING DISTRICTS IN IDAHO.

[See Pl. VII. Additional references may be found in Mineral Resources for 1910 and 1911.]

ADA COUNTY.

1. Black Hornet (Highland Valley, Shaw Mountain). Au, Ag. (D, Pl.)

8 miles E. Boise, O. S. L. R. R.

Granite cut by granite porphyry.

Veins.

Lindgren, W., The mining districts of Idaho Basin and Boise Ridge, Idaho:

Eighteenth Ann. Rept., pt. 3, 1898, pp. 703, 705, 707.

Min. Res. 1905, pp. 224-225.

1906, pp. 249-250.

1907, pt. 1, pp. 288-289.

1908, pt. 1, p. 414

1909 , pt. 1, p. 341 .

Top. sheet Boise.

Folio 45, 1898.

2. Boise (McIntyre). Au, Ag.

3 to 5 miles E. Boise, O. S. L. R. R.

Granite cut by granite porphyry and lamprophyre dikes.

Veins.

Lindgren, $W$., The mining districts of Idaho Basin and Boise Ridge, Idaho:

Eighteenth Ann. Rept., pt. 3, 1898, p. 705. 158 


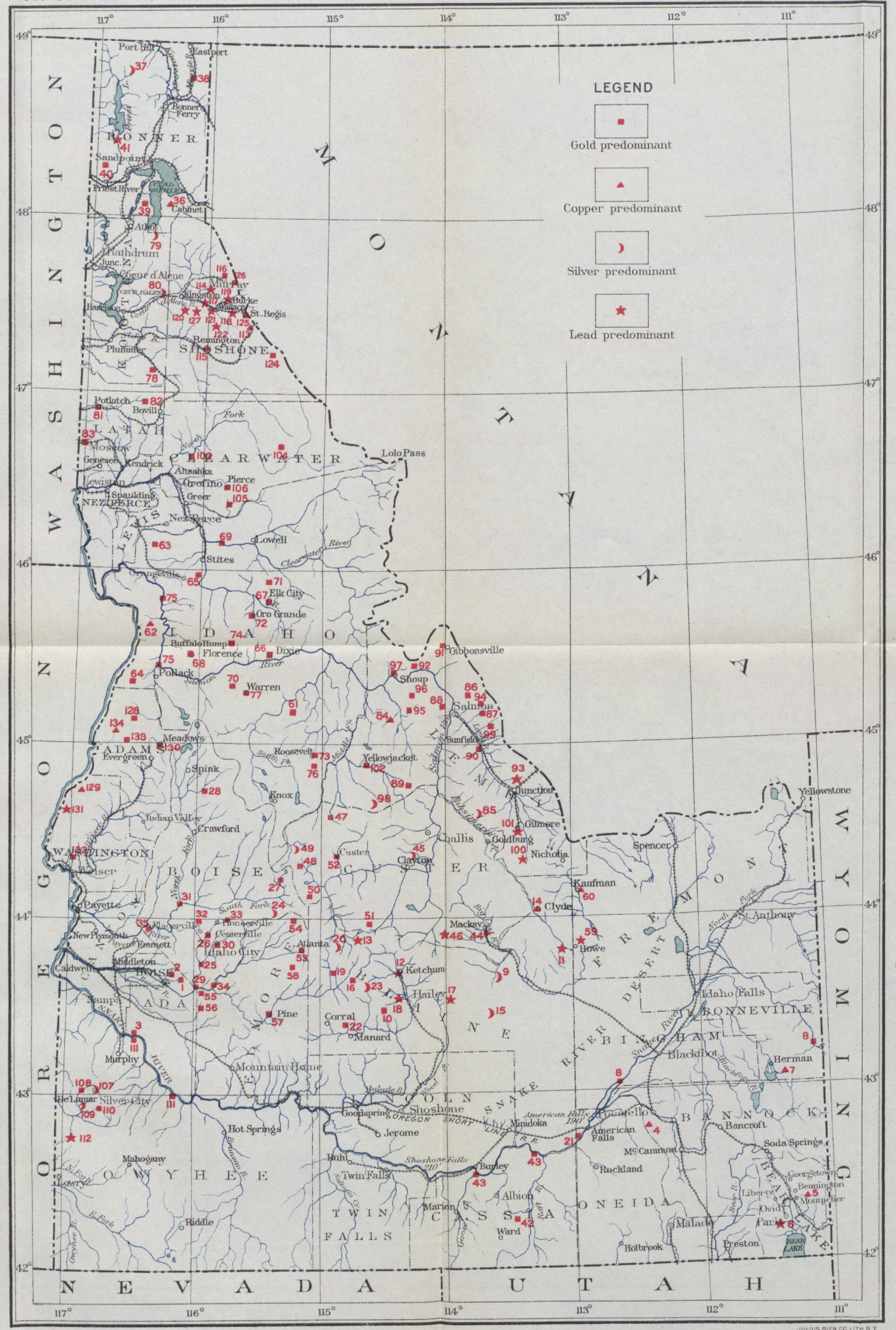

MAP OF IDAHO, SHOWING LOCATION OF MINING DISTRICTS
LIST OF MINING DISTRICTS

ADA COUNTY

FREMONT COUNTY

Black Hornet (Highland 59. Little Lost River (Howe)

Boise (Mclntyre)

Snake River placers

ADAMS COUNTY

(See Washington)

BANNOCK COUNTY

Fort Hall

BEAR LAKE COUNTY

Bear Lake

NGHAM AND COUNTIES

Mt. Pisgah (

Snake River placers

BLAINE COUNTY

Antelope

Dome

Elkhorn

Hamilton (Clyde)

. Lava Creek (Martin)

Little Smoky

Little Wood Rive

Mineral Hill (

9. Rosetta

Sawtooth

Snake River Placer (Neeley)

. Warm Springs BOISE COUNT

4. Banner

26. Centerville (1dat

27. Deadwood

8. Gold Fork (Roseberry)

Highland Valley Payette River placers (Jacobs Gulch)

SummitFlat(Pioneerville)

Twin Springs

Westview (Willow Creek
5. Win Springs Rock Creek, Pearl) BONNER COUNTY

East Shore (Clark Fork)

Kootenai

Pend Oreil

. Pine Creek

. Priest Lake (Coolin) BONNEVILLE COUN (See Bingham) CASSIA COUNTY

Connor Creek (Cumora, Stokes) CLEARWATER COUNTY formerly in Nez Perce County, recently subdivided)

3. Burnt Creek

04. Moose City

5. Musselshell Creek (Weippe)

Pierce

CUSTER COUNTY

45. Bay Horse

6. East Fork

47. Loon Creek (Casto)

8. Seafoam

49. Sheep Mount

50. Stanley Basin

1. Washington Basin

Yankee Fork (Custer) ELMORE COUNTY

3. Atlanta

54. Biack Warrior

5. Highland Valle

Grove

8. Rocky Bar (Bear Creek)
. Skull Canyon (Kaufman)

DAHO COUNTY

61. Big Creek

62. Camp Howard (White

Bird)

. Cottonwood Buttes

64. Crooks Corral

5. Dewey (Harpster)

列

68. Florence

9. Maggie

1. Newsome

2. Orogrande

74. Robbins (Buffalo Hump)

75. Salmon River placers

(Simpson)

6. Thunder Mountain

KOOTENAI COUNTY

7. Camas Cove (Tyson)

79. Lakeview

LATAH COUNTY

Gold Creek (Potlatch)

82. Hoodoo (Blackbird)

83. Moscow

LEMHI COUNTY

4. Blackbird

5. Bluewing (Patterson Creek)

86. Carmen Creek

87. Eldorado (Geerston)

88. Eureka

89. Gravel Range (Forney)

9o. McDevitt

1. Gibbonsville

92. Indian Creek (Ulysses)

93. Junction

94. Kirtley Creek

95. Leesburg (Arnett Creek)

6. Mackinaw

Mineral Hill (Shoup)

Parker Mountain

9. Pratt Creek

. Spring Mountain

1. Texas Creek (Gilmore)

Yellowjacket

OWYHEE COUNTY

07. Carson (War Eagle, Silver

City, Florida Mtn.)

108. De Lamar

og. Flint

i. Snake River placers

12. South Mountain

SHOSHONE COUNTY

13. Bald Mountain (Monitor)

4. Beaver (Cœur d'Alene)

5. Black Prince

i16. Eagle (Murray, Cosur d'Alene)

17. Evolution (Cœeur d'Alene)

8. Hunter (Mullan, Cœur d'Alene)

9. Leland (Burke, Cœur d'Alene)

20. Pine Creek
21. Placer Center (Wallace Cour d'Alene)

2late Creek

23. Omitted

124. St. Joe

25. St. Regis (Coour d'Alene)

(Cour d'Alene)

127. Yroka (Wardner, Coeur d'Alene)

WASHINGTON AND ADAMS COUNTIES

128. Black Lake

129. Heath

130. Meadow

32. Monroe Creek (Weiser)

133. Placer Basin

34. Seven Devils
Marshall (Resort) 

ADA COUNTY-Continued.

2. Boise (McIntyre)-Continued.

Min. Res. 1907, pt. 1, p. 288.

1908 , pt. 1, p. 414

1909, pt. 1, p. 341.

3. Snake River placers. Au. (Pl.)

Stream gravels.

Min. Res. 1905, p. 224.

1907, pt. 1, p. 289.

Top. shee+ Bisuka.

\section{ADAMS COUNTY. (See Washington.)}

BANTOCK COUNTY.

4. Fort Hall. $\mathrm{Cu}, \mathrm{Ag}, \mathrm{Au}, \mathrm{Pb}$.

9 miles E. Pocatello, $1 \frac{1}{2}$ miles W. Portneuf, O. S. I. R. R.

Paleozoic sediments and diabase.

Veins.

Weed, W. H., Copper mines in the United States in 1905: Bull. 285, 1906, pp. 107-108.

Weeks, F. B., and Heikes, V. C., Notes on the Fort Hall mining district, Idaho: Bull. 340, 1908, pp. 175-183.

Min. Res. 1905, p. 225.

1906, p. 250.

1907, pt. 1, p. 289.

1908 , pt. 1, p. 414

1909 , pi. 1, p. 341 .

BEAR LAKE COUNTY.

5. Bear Lake. Cu.

4 miles E. Montpelier, O. S. L. R. R.

Triassic "Red Beds."

Disseminations.

Gale, II. S., Geology of the copper deposits near Montpelier, Idaho: Bull. 430,1910 , pp. 112-121.

Weeks, F. B., and Ferrier, W. F., Phosphate deposits in the western United States: Bull. 315, 1907, pp. 460-461.

Weeks, F. B., Phosphate deposits in the western United States: Bull. 340, 1908, pp. 441-444.

Min. Res. 1905, p. 225.

1906, p. 250.

1907, pt. 1, pp. 289-290.

6. St. Charles. $\mathrm{Pb}, \mathrm{Ag}, \mathrm{Cu}$.

5 miles S. Paris, O. S. L. R. R.

Paleozoic sediments.

Veins and replacements.

Richards, R. W., Notes on lead and copper deposits in the Bear River Range, Idaho and Utah: Bull. 470, 1911, pp. 177-187.

BINGHAM AND BONNEVILLE COUNTIES.

7. Mount Pisgah (Caribou). $\mathrm{Cu}, \mathrm{Au}, \mathrm{Ag}$. (Pl, D.)

42 miles NNE. Soda Springs, O. S. L. R. R.

Min. Res. 1905, p. 225.

1906, p. 250.

1907, pt. 1, p. 290.

1908, pt. 1, p. 415 .

1909 , pt. 1, p. 342 . 
8. Snake River placers. Au. (Pl.)

Stream gravels.

Min. Res. 1905, p. 225.

1906, p. 250.

1907 , pt. 1, p. 290

1908 , pt. 1, p. 415

1909, pt. 1, p. 342 .

\section{BLAINE COUNTY.}

9. Antelope. $\mathrm{Ag}, \mathrm{Pb}$.

17 miles SW. Darlington, O. S. L. R. R.

Min. Res. 1908, pt. 1, p. 416.

10. Camas. Au.

20 miles SW. Hailey, O. S. L. R. R.

Granite and diorite.

Veins.

Lindgren, W., The gold and silver veins of Silver City, De Lamar, and other mining districts in Idaho: Twentieth Ann. Rept., pt. 3, 1900, p. 208.

11. Dome. $\mathrm{Pb}, \mathrm{Ag}, \mathrm{Cu}, \mathrm{Au}$.

28 miles NE. Arco, O. S. L. R. R.

Paleozoic sediments and granite.

Veins and replacements.

Eldridge, G. H., A geological reconnaissance across Idaho: Sixteenth Ann. Rept., pt. 2, 1895, pp. 264, 271.

Min. Res. 1906, p. 251.

1907 , pt. 1, p. 291.

1908 , pt. 1, p. 416.

1909 , pt. 1, pp. 342-343.

12. Elkhorn (Ketchum). $\mathrm{Pb}, \mathrm{Ag}$.

Station O. S. I. R. R.

Paleozoic sediments.

Veins.

Lindgren, W., The gold and silver veins of Silver City, De Lamar, and other mining districts in Idaho: Twentieth Ann. Rept., pt. 3, 1900, pp. 209-211.

Min. Res. 1882, pp. 311-312.

1883-84, pp. 424-425.

1885 , p. 258.

Top. sheet Hailey.

13. Galena. $\mathrm{Pb}, \mathrm{Ag}$.

26 miles NW. Ketchum, O. S. L. R. R.

Min. Res. 1882, pp. 311-312.

1883-84, pp. 424-425.

1885, p. 258.

1886, p. 146.

Top. sheet Sawtooth.

14. Hamilton (Clyde). $\mathrm{Cu}, \mathrm{Pb}, \mathrm{Ag}, \mathrm{Au}$.

28 miles NE. Mackay, O. S. L. R. R.

Paleozoic sediments.

Veins.

Min. Res. 1907, pt. 1, p. 291.

1908 , pt. 1, p. 416.

15. Lava Creek (Martin). $\mathrm{Ag}, \mathrm{Cu}, \mathrm{Pb}$.

24 miles SW. Arco, O. S. L. R. R.

Paleozoic sediments, granite. 
BLANKE COONTY-Continued.

15. Lava Creek (Martin)-Continued.

Veins and replacements.

Eldridge, G. H., A geological reconnaissance across Idaho: Sixteenth Ann.

Rept., pt. 2, 1895, pp. 264-271.

Min. Res. 1906, p. 251.

1907, pt. 1, p. 291.

1908 , pt. 1, p. 416.

16. Little Smoky. $\mathrm{Au}, \mathrm{Pb}, \mathrm{Ag}$.

28 miles W. Hailey, O. S. L. R. R.

Paleozoic sediments cut by granite.

Veins and replacements.

Lindgren W., The gold and silver veins of Silver City, De Lamar, and other mining districts in Idaho: Twentieth Ann. Rept., pt. 3, 1900, p. 209.

Min. Res. 1908, pt. 1, p. 416.

1909 , pt. 1, p. 343.

Top. sheet Sawtooth.

17. Little Wood River (Muldoon). Pb, Ag.

20 miles E. Bellevue, O. S. L. R. R.

Paleozoic sediments.

Veins and replacements.

Min. Res. 1882, pp. 311-312.

1883-84, pp. 424-425.

1885 , p. 258.

1908, pt. 1, p. 416

1909 , pt. 1, p. 343

18. Mineral Hill (Hailey). $\mathrm{Pb}, \mathrm{Ag}, \mathrm{Zn}, \mathrm{Cu}, \mathrm{Au}$.

Station O. S. L. R. R.

Paleozoic sediments cut by granitic rocks.

Veins.

Lindgren W., The gold and silver veins of Silver City, De Lamar, and other mining districts in Idaho: Twentieth Ann. Rept., pt. 3, 1900, pp. 190-209.

Min. Res. 1882, pp. 311-312.

1883-84, pp. 424,425 .

1887 , p. 107.

1905 , pp. 226-227.

1906, pp. 251-252.

1907, pt. 1, pp. 291-292.

1908 , pt. 1, pp. $416-417$.

1909 , pt. 1, p. 343.

Top. sheet Hailey.

19. Rosetta. $\mathrm{Au}, \mathrm{Ag}, \mathrm{Pb}, \mathrm{Zn}$.

30 miles W. Ketchum, O. S. L. R. R.

Paleozoic sediments cut by granitic rocks.

Veins.

Min. Reg. 1905, p. 227.

1906, p. 252.

1907, pt. 1, p. 292

1909, pt. 1, p. 334

Top. sheet Sawtooth.

20. Sawtooth. Ag.

40 miles NW. Ketchum, O. S. L. R. R.

Granite.

$21528^{\circ}-$ Bull. $507-12-11$ 
BLAINE COUNTY-Continued.

20. Sawtooth-Continued.

Veins.

Min. Res. 1885, p. 258.

Top. sheet Sawtooth.

21. Snake River placer (Neeley). Au. (Pl.)

Neeley station, O. S. L. R. R.

Stream gravels.

Min. Res. 1905, p. 227.

1906, p. 252.

22. Soldier. Au. (Pl.)

33 miles N. Gooding, O. S. L. R. R.

Stream gravels.

Min. Res. 1908, pt. 1, p. 417.

1909 , pt. 1, p. 343.

23. Warm springs. $\mathrm{Ag}, \mathrm{Pb}, \mathrm{Zn}, \mathrm{Au}$.

12 miles W. Ketchum, O. S. I. R. R.

Min. Res. 1882, pp. 311-312.

1883-84, pp. 424-425.

1905 , p. 227.

1906, p. 253.

1907, pt. 1, p. 292.

1908 , pt. 1, p. 417

1909 , pt. 1, p. 343.

Top. sheet Sawtooth.

BOISE COUNTY.

24. Banner. Ag.

76 miles NE. Boise, O. S. L. R. R.

Granite.

Veins.

Top. sheet Bear Valley.

25. Moore Creek. Au, monazite. (Pl.)

Stream gravels.

Top. sheet Idaho Basin.

26. Centerville (Idaho Basin). Au. (D, Pl.)

44 miles NE. Boise, O. S. L. R. R.

Granite cut by aplite and lamprophyres.

Veins, stream gravels.

Lindgren, W., The mining districts of Idaho Basin and Boise Ridge, Idaho:

Eighteenth Ann. Rept., pt. 3, 1898, pp. 617-744.

Min. Res. 1905, pp. 228-229.

1906, pp. 253-254.

1907 , pt. 1, p. 293.

1908, pt. 1, pp. 418-419.

1909 , pt. 1, p. 344 .

Top. sheet Idaho Basin.

27. Deadwood. Au. (P1.)

80 miles NE. Boise, O. S. L. R. R.

Stream gravels.

Min. Res. 1907, pt. 1, p. 293.

1908, pt. 1, p. 419.

1909, pt. 1, p. 345 .

Top. sheet Bear Valley. 
BOISE COUNTY-Continued

28. Gold Fork (Roseberry). Au. (PI.)

41 miles SE. Evergreen, P. \& I. N. R. R.

Tertiary gravels.

Lindgren, W., The gold and silver veins of Silver City, De Iamar, and other mining districts in Idaho: Twentieth Ann. Rept., pt. 3, 1900, p. 242.

29. Highland Valley. Au. (D, PI.)

18 miles ESE. Boise, O. S. L. R. R.

Granite cut by granite porphyry.

Veins.

Min. Res. 1908, pt. 1, p. 414.

1909 , pt. 1, p. 345 .

Top. sheet Boise.

Folio 45, 1898.

30. Idaho City (Idaho Basin). Au, monazite. (D, Pl.)

36 miles NE. Boise, O. S. L. R. R.

Granite cut by aplite and lamprophyres.

Veins, stream gravels.

Lindgren, W., The mining districts of Idaho Basin and Boise Ridge, Idaho:

Eighteenth Ann. Rept., pt. 3, 1898, pp. 617-744.

Min. Res. 1905, pp. 228-229.

1906, pp. 253-254.

1907, pt. 1, p. 293.

1909 , pt. 1, p. 344 .

Top. sheet Idaho Basin.

Moore Creek. See No. 25.

31. Payette River placers (Jacobs Gulch). Au. (PI.)

Stream gravels.

Min. Res. 1908, pt. 1, p. 419.

1909, pt. 1, p. 345.

Top. sheets Garden Valley, Squaw Creek.

32. Quartzburg (Idaho Basin). Au, monazite. (D, PI.)

51 miles NNE. Boise, O. S. L. R. R.

Granite cut by diorite porphyry.

Veins, stream gravels.

Lindgren, W., The mining districts of Idaho Basin and Boise Ridge, Idaho:

Eighteenth Ann. Rept., pt. 3, 1898, pp. 617-744.

Min. Res. 1905 , pp. $228-229$.

1906, pp. 253-254.

1907 , pt. 1, p. 293.

1908 , pt. 1 , pp. $418-419$.

1909 , pt. 1, p. 344 .

Top. sheet Idaho Basin.

33. Summit Flat (Pioneerville). Au, Ag.

50 miles NNE. Boise, O. S. L. R. R.

Granite cut by aplite and lamprophyre.

Veins.

Lindgren, W., The mining districts of Idaho Basin and Boise Ridge, Idaho:

Eighteenth Ann. Rept., pt. 3, 1898, pp. 617-744.

Min. Res. 1907, pt. 1, p. 294.

1908 , pt. 1, p. 420.

1909 , pt. 1, p. 345.

Top. sheets Idaho Basin, Garden Valley. 
BOISE COUNTY-Continued.

34. Twin Springs. Au. (P1.)

41 miles E. Boise, O. S. L. R. R.

Stream gravels.

Top. sheet Twin Springs.

35. Westview (Willow Creek, Pearl, Rock Creek). $\mathrm{Au}, \mathrm{Ag}, \mathrm{Pb}$.

8 miles NE. Emmett, P. \& I. N. R. R.; 15 miles N. Eagle, O. S. L. R. R.

Granite cut by granite and diorite porphyry dikes.

Veins.

Lindgren, W., The mining districts of Idaho Basin and Boise Ridge, Idaho:

Eighteenth Ann. Rept., pt. 3, 1898, p. 707.

Min. Res. 1905, p. 229.

1906, p. 255.

1907, pt. 1, p. 294.

1908 , pt. 1, p. 420.

Top. sheet Boise.

1909, pt. 1, p. 345 .

Folio 45, 1898.

BONHER COUNTY.

36. East Shore (Clark Fork), $\mathrm{Cu}, \mathrm{Au}, \mathrm{Ag}, \mathrm{Pb}$.

Clark Fork and Hope stations, N. P. R. R.

Paleozoic sediments.

Veins.

Calkins, F. C., and MacDonald, D. F., A geological reconnaissance in northern

Idaho and northwestern Montana: Bull. 384, 1909, pp. 95-97.

MacDonald, D. F., Economic features of northern Idaho and northwestern Montana: Bull. 285, 1906, pp. 47-48.

Min. Res. 1907, pt. 1, p. 294.

1908, pt. 1, p. 420.

37. Kootenai. $\mathrm{Ag}, \mathrm{Pb}$. 1909 , pt. 1, p. 345 .

40 miles NW. Iola, G. N. R. R.

38. Mooyie Yaak. $\mathrm{Au}, \mathrm{Pb}, \mathrm{Ag}$.

19 miles NE. Bonners Ferry, G. N. R. R.

Calkins, F. C., and MacDonald, D. F., A geological reconnaissance in northern

Idaho and northwestern Montana: Bull. 384, 1909, pp. 107-108.

39. Pend Oreille (Blacktail). $\mathrm{Au}, \mathrm{Ag}, \mathrm{Cu}, \mathrm{Pb}$.

5 miles $\mathrm{E}$. Westmond, N. P. R. R.

Pre-Cambrian sediments cut by monzonites.

Veins.

Calkins, F. C., and MacDonald, D. F., A geological reconnaissance in northern

Idaho and northwestern Montana: Bull. 384, 1909, pp. 97-99.

MacDonald, D. F., Economic features of western Idaho and northwestern Montana: Bull. 285, 1906, pp. 46-47.

Min. Res. 1907, pt. 1, p. 294.

1908, pt. 1, pp. $420,421$.

1909 , pt. 1, p. 345 .

Top. sheet Sandpoint.

40. Pine Creek. $\mathrm{Au}, \mathrm{Ag}, \mathrm{Pb}, \mathrm{Zn}$.

12 miles NNW. Priest River, G. N. R. R.

Pre-Cambrian sediments. 
BONMER COUNTY-Continued.

40. Pine Creek-Continued.

Veins.

MacDonald, D. F., Economic features of northern Idaho and northwestern Montana: Bull. 285, 1906, pp. 45-46.

Top. sheet Sandpoint.

41. Priest Lake (Coolin). $\mathrm{Pb}, \mathrm{Ag}, \mathrm{Cu}, \mathrm{Au}$.

25 miles N. Priest River, G. N. R. R.

Pre-Cambrian sediments, granite.

Veins.

Min. Res. 1908, pt. 1, p. 420.

1909 , pt. 1, p. 345 .

Top. sheet Sandpoint.

\section{BONNEVILLE COUNTY. (See Bingham.)}

Cassia codrty.

42. Connor Creek (Cumora, Stokes). $\mathrm{Au}, \mathrm{Ag}, \mathrm{Pb}, \mathrm{Cu}$.

38 miles SSE. Burley, O. S. I. R. R.

Veins.

Min. Res. 1905, pp. 229-230.

1906 , p. 255.

1907, pt. 1, p. 295.

1908, pt. 1, p. 421.

43. Snake River placers. Au. (Pl.)

Stream gravels.

Min. Res. 1905, pp. 229-230.

1906, p. 255.

1907, pt. 1, p. 295.

\section{CLearwater COUNTy (formerly part of Nez Perce County).}

103. Burnt Creek. Au. (Pl.)

35 miles NE. Ahsahka, N. P. R. R.

Stream gravels.

Min. Res. 1905, p. 237.

1906, p. 261.

1907, pt. 1, p. 304.

1908 , pt. 1, p. 429

1909, pt. 1, p. 351.

104. Moose City. Au. (Pl.)

80 miles NE. Ahsahka, N. P. R. R.

Stream gravels.

105. Musselshell Creek (Weippe). Au, monazite. (D, Pl.)

25 miles E. Greer, N. P. R. R.

Granite, gneiss, and schist.

Veins, stream gravels.

Lindgren, W., A geological reconnaissance across the Bitterroot Range and

Clearwater Mountains: P. P. 27, 1904, p. 105.

Schrader, F. C., An occurrence of monazite in northern Idaho: Bull. 430, 1910, pp. 184-190.

106. Pierce. Au. (D, Pl.)

28 miles NE. Greer, N. P. R. R.

Granite, gneiss, and schist.

Veins, bench gravels. 
CLEARWATER COUNTY-Continued.

106. Pierce-Continued.

Lindgren, W., Mineral deposits of the Bitterroot Range and Clearwater Mountains: Bull. 213, 1903, pp. 66-70.

- A geological reconnaissance across the Bitterroot Range and Clearwater Mountains: P. P. 27, 1904, pp. 102-105.

Russell, I. C., Geology and water resources of Nez Perce County, Idaho, pt. 2: W. S. P. 54, 1901.

Min. Res. 1905, p. 237.

1906, pp. 261-262.

1907, pt. 1, p. 304.

1908, pt. 1, p. 429 .

1909 , pt. 1, p. 351.

\section{CUSTER COUNTY.}

44. Alder Creek (Lost River, White Knob). $\mathrm{Cu}, \mathrm{Au}, \mathrm{Ag}, \mathrm{Pb}$.

Mackay station, O. S. L. R. R.

Paleozoic sediments, granite, and porphyry.

Contact metamorphic.

Weed, W. H., The copper mines of the United States in 1905: Bull. 285, 1906, p. 108.

Min. Res. 1905, p. 230.

1906 , p. 255.

1907 , pt. 1, p. 295.

1908 , pt. 1, p. 422 .

1909 , pu 1, p. 346.

45. Bay Horse. $\mathrm{Ag}, \mathrm{Pb}, \mathrm{Cu}, \mathrm{Au}$.

76 miles NW. Mackay, O. S. L. R. R.

Paleozoic sediments cut by granite.

Veins and replacements.

Min. Res. 1887, p. 107.

1905 , p. 230.

1906, p. 256.

1908 , pt. 1, p. 422

1909 , pt. 1 , p. 346.

46. East Fork. $\mathrm{Pb}, \mathrm{Ag}$.

24 miles W. Mackay, O. S. L. R. R.

Min. Res. 1908, pt. 1, p. 422.

47. Loon Creek (Casto, Lost Packer). Au, Ag, Cu.

125 miles NW. Mackay, O. S. L. R. R.

Pre-Cambrian sediments cut by granite and capped by rhyolite.

Vein.

Umpleby, J. B., A preliminary account of the ore deposits of the Loon Creek district, Idaho: Bull. 530, 1912.

Weed, W. H., The copper mines of the United States in 1905: Bull. 285, 1906, p. 108.

Min. Res. 1906, p. 256.

1907, pt. 1, p. 295.

1908 , pt. 1, p. 422 .

1909 , pt. 1, p. 346.

48. Seafoam. Au, Ag.

109 miles NW. Ketchum, O. S. I. R. R. 
CUSTER COUNTY-Continued.

48. Seafoam-Continued.

Min. Res. 1905, p. 230.

1908 , pt. 1, p. 422.

1909 , pt. 1, p. 346 .

Top. sheet Bear Valley.

49. Sheep Mountain. Ag, Pb.

131 miles NW. Ketchum, O. S. L. R. R.

Granite and schist cut by diorite and quartz porphyry.

Replacements.

Eldridge, G. H., A geological reconnaissance across Idahn: Sixteenth Ann. Rept., pt. 2, 1895, p. 258.

Top. sheet Bear Valley.

50. Stanley Basin. Au, Ag. (D, Pl.)

89 miles NW. Ketchum, O. S. I. R. R.

Granite and porphyry.

Veins, stream gravels.

Min. Res. 1905, p. 230.

1906, p. 256.

1907, pt. 1, p. 296.

1908 , pt. 1, p. 422.

1909, pt. 1, p. 346.

Top. sheet Bear Valley.

51. Washington Basin. Au, Ag. 48 miles NW. Ketchum, O. S. L. R. R.

52. Yankee Fork (Custer). Au, Ag.

114 miles NW. Mackay, O. S. L. R. R.

Tertiary volcanics.

Veins.

Min. Res. 1905, p. 231.

1906, p. 256.

1907, pt. 1, p. 296.

1908 , pt. 1, pp. $421-422$.

1909 , pt. 1, p. 346 .

ELMORE COUNTY.

53. Atlanta. Au, Ag. (D, Pl.)

89 miles NE. Mountain Home, O. S. I. R. R.

Granite cut by quartz porphyry.

Veins, stream gravels.

Eldridge, G. H., A geological reconnaissance across Idahn: Sixteenth Ann.

Rept., pt. 2, 1895, pp. 253-257.

Min. Res. 1905, p. 231.

1906, p. 257.

1907, pt. 1, p. 297.

1908, pt. 1, pp. 423-424.

1909 , pt. 1, p. 347 .

Top. sheet Rocky Bar.

54. Black Warrior. Au, Ag.

99 miles NE. Mountain Home, O. S. I. R. R.

Granite cut by quartz porphyry.

Veins.

Min. Res. 1906, p. 256.

1907, pt. 1, p. 297.

1908, pt. 1, p. 423.

1909 , pt. 1, p. 347.

Top. sheet Rocky Bar. 
ELMORE COUNTY-Continued.

55. Highland Valley. Au, Ag. (Pl.)

25 miles ESE. Boise, O. S. L. R. R.

Stream gravels.

Min. Res. 1907, pt. 1, p. 297.

1908 , pt. 1, p. 423 .

1909 , pt. 1, p. 347

Top. sheet Idaho Basin.

56. Neal. $\mathrm{Au}, \mathrm{Ag}$.

25 miles SE. Boise, O. S. L. R. R.

Granite cut by granite and syenite porphyry and lamprophyre.

Veins.

Lindgren, W., The mining districts of Idaho Basin and Boise Ridge, Idaho: Eighteenth Ann. Rept., pt. 3, 1898, pp. 699-703.

Min. Res. 1905, p. 225.

1906, p. 250.

1907, pt. 1, p. 297.

1908 , pt. 1, p. 424 .

1909 , pt. 1, p. 347 .

Top. sheet Idaho Basin.

57. Pine Grove. Au (Ag).

45 miles NE. Mountain Home, O. S. L. R. R.

Veins (?).

Min. Res. 1905, p. 231.

1906, p. 257.

1907, pt. 1, pp. 297-298.

1908 , pt. 1, p. 424

1909 , pt. 1, p. 347

Top. sheet Camas Prairie.

58. Rocky Bar (Bear Creek). Au, Ag.

65 miles NE. Mountain Home, O. S. L. R. R.

Granite cut by diorite, diabase, and quartz porphyry.

Veins.

Eldridge, G. H., A geological reconnaissance across Idaho: Sixteenth Ann.

Rept., pt. 2, 1895, pp. 250-253.

Min. Res. 1907, pt. 1, p. 297.

1908 , pt. 1, p. 423.

1909 , pt. 1, p. 347 .

Top. sheet Rocky Bar.

FREMONT COUNTY.

59. Iittle Lost River (How). $\mathrm{Pb}, \mathrm{Ag}$.

25 miles NE. Arco, O. S. L. R. R.

Min. Res. 1908, pt. 1, p. 424.

1909 , pt. 1, p. 347.

60. Skull Canyon (Kaufman). $\mathrm{Cu}, \mathrm{Pb}, \mathrm{Ag}, \mathrm{Au}$.

50 miles W. Dubois, O. S. L. R. R.

Paleozoic sediments.

Replacements.

Weed, W. H., The copper mines of the United States in 1905: Bull. 285, 1906, p. 108.

Min. Res. 1905, p. 231.

1906, p. 257.

1907, pt. 1, p. 298.

1908, pt. 1, p. 424. 


\section{IDAHO COUNTY.}

61. Big Creek. $\mathrm{Au}, \mathrm{Ag}, \mathrm{Pb}, \mathrm{Cu}$.

60 miles W. Kooskia, N. P. R. R.

Veins.

Min. Res. 1907, pt. 1, p. 299. 1908 , pt. 1, p. 425. 1909 , pt. 1, p. 348 .

62. Camp Howard (White Bird). Cu, Au, Ag.

12 miles SSW. Grangeville, N. P. R. R.

Paleozoic sediments cut by diorite.

Veins.

Lindgren, W., A geological reconnaissance across the Bitterroot Range and Clearwater Mountains in Montana and Idaho: P. P. 27, 1904, p. 107.

Min. Res. 1907, pt. 1, p. 299. 1908 , pt. 1, p. 425.

63. Cottonwood Buttes. $\mathrm{Au}, \mathrm{Ag}, \mathrm{Cu}$.

Cottonwood station, N. P. R. R.

Pre-Tertiary sediments.

Veins.

Lindgren, W., A geological reconnaissance across the Bitterroot Range and Clearwater Mountains in Montana and Idaho: P. P. 27, 1904, pp. 106-107.

64. Crooks Corral. Au, Ag. (Pl.)

51 miles N. Evergreen, P. \& I. N. R. R.

Bench gravels.

Min. Res. 1907, pt. 1, p. 299. 1908, pt. 1, p. 425.

65. Dewey (Harpster). Au, Cu.

7 miles W. Grangeville, N. P. R. R.

Slates and schist cut by diorite.

Veins.

Lindgren, W., A geological reconnaissance across the Bitterroot Range and Clearwater Mountains in Montana and Idaho: P. P. 27, 1904, pp. 105-106.

66. Dixie. $\mathrm{Au}, \mathrm{Ag}$.

78 miles SE. Grangeville, N. P. R. R.

Granite.

Veins.

Lindgren, W., Mineral deposits of the Bitterroot Range and Clearwater Mountains, Montana: Bull. 213, 1903, pp. 66-70.

- A geological reconnaissance across the Bitterroot Range and Clearwater Mountains in Montana and Idaho: P. P. 27, 1904, p. 98.

Min. Res. 1905 , p. 232.

1906 , p. 258.

1907, pt. 1, p. 299

1908 , pt. 1, p. 425 .

1909 , pt. 1, p. 348 .

67. Elk City. Au, Ag. (D, Pl.)

52 miles SE. Grangeville, N. P. R. R.

Pre-Cambrian gneiss cut by granite.

Veins, stream gravels.

Lindgren, W., Mineral deposits of the Bitterroot Range and Clearwater Mountains, Montana: Bull. 213, 1903, pp. 66-70.

A geological reconnaissance across the .Bitterroot Range and Clearwater Mountains in Montana and Idaho: P. P. 27, 1904, pp. 91-96. 
IDAHO COUNTY-Continued.

67. Elk City-Continued.

Min. Res. 1905, p. 232.

1906, p. 258.

1907 , pt. 1, p. 299.

1908 , pt. 1, p. 425.

1909 , pt. 1, p. 348 .

68. Florence. Au. (D, Pl.)

42 miles SSE. Grangeville, N. P. R. R.

Granite.

Veins, stream gravels.

Lindgren, W., Mineral deposits of the Bitterroot Range and Clearwater Mountains, Montana: Bull. 213, 1903, pp. 66-70.

The gold and silver veins of Silver City, De Lamar, and other mining

districts in Idaho: Twentieth Ann. Rept., pt. 3, 1900, pp. 232-237.

Min. Res. 1905, p. 232.

1906 , p. 258.

1907 , pt. 1, p. 299.

1909 , pt. 1, p. 348.

69. Maggie. Au. (P1.)

8 miles E. Kooskia, N. P. R. R.

Stream gravels.

Min. Res. 1906, p. 258.

70. Marshall (Resort). Au, Ag. (D, PI.)

50 miles NE. Evergreen, P. \& I. N. R. R.

Granite.

Veins, stream gravels.

Lindgren, W., The gold and silver veins of Silver City, De Lamar, and other mining districts in Idaho: Twentieth Ann. Rept., pt. 3, 1900, p. 277.

Min. Res. 1905, p. 252.

1907, pt. 1, p. 300 .

1908 , pt. 1, p. 425.

1909, pt. 1, p. 348 .

71. Newsome. Au. (D, Pl.)

31 miles E. Grangeville, N. P. R. R.

Pre-Cambrian gneiss cut by granite.

Veins, terrace gravels.

Lindgren, W., Mineral deposits of the Bitterroot Range and Clearwater Mountains, Montana: Bull. 213, 1903, pp. 66-70.

A geological reconnaissance across the Bitterroot Range and Clear-

water Mountains in Montana and Idaho: P. P. 27, 1904, pp. 96-98.

Min. Res. 1905, p. 233.

1906, p. 258.

1907, pt. 1, p. 300.

1908 , pt. 1, p. 425 .

1909 , pt. 1, p. 348 .

72. Orogrande. Au, Ag. (D, PI.)

61 miles SE. Grangeville, N. P. R. R.

Pre-Cambrian gneiss cut by granite.

Veins, stream gravels.

Lindgren, W., A geological reconnaissance across the Bitterroot Range and

Clearwater Mountains in Montana and Idaho: P. P. 27, 1904, pp. 94-98.

Min. Res. 1905, p. 233.

1906 , pp. $425-426$.

1909, pt. 1, p. 349 . 
IDAHO COUNTY-Continued.

73. Profile. Au.

151 miles NE. Emmett, P. \& I. N. R. R.

Tertiary volcanics.

Veins and impregnations.

74. Robbins (Buffalo Hump). Au, Ag.

50 miles SE. Grangeville, N. P. R. R.

Granite, schist cut by granite dikes.

Veins.

Lindgren, W., Mineral deposits of the Bitterroot Range and Clearwater Mountains, Montana: Bull. 213, 1903, pp. 66-70.

A geological reconnaissance across the Bitterroot Range and Clearwater Mountains in Montana and Idaho: P. P. 27, 1904, pp. 99-102.

Min. Res. 1905, p. 233.

1906, pp. 258-259.

1907 , pt. 1, p. 300 .

1908 , pt. 1, p. 426 .

1909 , pt. 1, p. 349 .

75. Salmon River placers (Simpson). Au, Ag. (Pl.)

Stream gravels.

Min. Res. 1905, pp. 233-234.

1906, p. 259.

1907 , pt. 1, p. 300 .

1908 , pt. 1, p. 426

1909 , pt. 1, p. 349.

76. Thunder Mountain. Au, Ag.

139 miles NE. Emmett, P. \& I. N. R. R.

Tertiary volcanics.

Veins and impregnations.

Min. Res. 1905, p. 234.

1906, p. 259.

1907 , pt. 1, p. 300.

1908, pt. 1, p. 426.

1909 , pt. 1, p. 349 .

77. Warren. $\mathrm{Au}, \mathrm{Ag}(\mathrm{Cu})$. (D, Pl.)

67 miles NE. Evergreen, P. \& I. N. R. R.

Granite cut by lamprophyric dikes.

Veins.

Lindgren, W., The gold and silver veins of Silver City, De Lamar, and other mining districts in Idaho: Twentieth Ann. Rept., pt. 3, 1900, pp. 237-249.

Min. Res. 1905, p. 234.

1906, p. 259.

1907, pt. 1, pp. 300-301.

1908, pt. 1, p. 426.

1909 , pt. 1, p. 349 .

\section{BOOTENAI COUNTY.}

78. Camas Cove (Tyson). Au. (PI.)

18 miles SSE. St. Maries, C. M. \& P. S. R. R.

Stream gravels.

Min. Res. 1905, p. 234.

1907, pt. 1, p. 301.

1908, pt. 1, p. 426. 
79. Lakeview. $\mathrm{Ag}, \mathrm{Pb}$.

15 miles E. Athol, N. P. R. R.

Pre-Cambrian sediments.

Veins.

Calkins, F. C., and MacDonald, D. F., A geological reconnaissance in western Idaho and northwestern Montana: Bull. 384, 1909, pp. 42, 60, 62, 95-96.

Min. Res. 1905, p. 234. 1906 , p. 259.

Top. sheet Rathdrum.

80. Medimont. Ag.

Cataldo or Dudley station, N. P. R. R.

Algonkian sediments.

Veins.

Min. Res. 1905, p. 234.

\section{LATAH COUNTY.}

81. Gold Creek (Potlatch). Au. (PI.)

Station W. I. \& M. R. R.

Stream gravels.

Min. Res. 1905, p. 234.

1907, pt. 1, p. 301.

1908, pt. 1, p. 426.

82. Hoodoo (Blackbird). Au. (D, Pl.)

8 to 12 miles NNE. Harvard, W. I. \& M. R. R.

Min. Res. 1905, p. 234.

1907 , pt. 1, p. 301.

1908 , pt. 1, p. 426 .

83. Moscow. Au. (Pl, D.)

Station N. P. R. R.

Stream gravels.

Min. Res. 1905, p. 234.

1906, p. 259.

1907, pt. 1, p. 301

Top. sheet Pullman.

LEMHI COUNTY.

84. Blackbird. $\mathrm{Cu}, \mathrm{Au}, \mathrm{Ag}(\mathrm{Ni}, \mathrm{Co})$.

25 miles W. Noble, G. \& P. R. R.

Pre-Cambrian schist, cut by basic dikes and granite.

Veins and replacements.

85. Bluewing (Patterson Creek). Ag.

80 miles N. Mackay, O. S. L. R. R.

Granite (?).

Veins (?).

86. Carmen Creek. $\mathrm{Au}, \mathrm{Cu}$.

Station G. \& P. R. R.

Pre-Cambrian schists.

Veins.

Min. Res. 1907, pt. 1, p. 302

87. Eldorado (Geertson). Au.

10 miles NE. Baker, G. \& P. R. R.

Pre-Cambrian schists cut by basic dikes.

Veins. 


\section{IEMHI COUNTY-Continued.}

87. Eldorado (Geertson)-Continued.

Min. Res. 1.905, p. 236.

1908 , pt. 1, p. 427.

1909 , pt. 1, p. 350 .

88. Eureka. Au, Ag.

8 miles NW. Salmon, G. \& P. R. R.

Pre-Cambrian schists cut by granite.

Veins.

Min. Res. 1907, pt. 1, p. 302.

1908 , pt. 1, p. 427.

89. Forney (Gravel Range). Au, Ag. (D, Pl.)

46 miles SW. Salmon, G. \& P. R. R.

Tertiary volcanics.

Veins.

Min. Res. 1905, p. 236.

1907 , pt. 1, p. 302.

1908 , pt. 1, p. 427.

1909 , pt. 1 , p. 350 .

90. McDevitt. Au. (D, Pl.)

Sunfield station, G. \& P. R. R.

Pre-Cambrian schists.

Veins, stream gravels.

Min. Res. 1905, p. 236.

$$
1909 \text {, pt. 1, p. } 351 .
$$

91. Gibbonsville. Au, Ag. (D, P1.)

Station G. \& P. R. R.

Pre-Cambrian slates.

Veins.

Lindgren, W., Mineral deposits of the Bitterroot Range and Clearwater Mountains, Montana: Bull. 213, 1903, pp. 66-70.

- A geological reconnaissance across the Bitterroot Range and Clearwater Mountains in Montana and Idaho: P. P. 27, 1904, p. 90.

Min. Res. 1905, p. 236.

1906, p. 260.

1907, pt. 1, p. 302.

1908 , pt. 1, p. 427.

1909 , pt. 1, p. 350 .

92. Indian Creek (Ulysses). $\mathrm{Au}, \mathrm{Ag}, \mathrm{Cu}$.

17 miles WNW. Noble, G. \& P. R. R.

Pre-Cambrian schist cut by acidic dikes.

Veins.

Min. Res. 1905, p. 236.

1906, pp. 260-261.

1907 , pt. 1, p. 302 .

1908 , pt. 1, p. 427.

1909 , pt. 1, p. 350 .

93. Junction. $\mathrm{Pb}, \mathrm{Ag}$.

Station G. \& P. R. R.

Paleozoic sediments cut by acidic intrusions.

Veins.

Min. Res. 1907, pt. 1, p. 302.

1908 , pt. 1, p. 428.

1909, pt. 1, p. 350. 
LEMHI COUNTY-Continued.

94. Kirtley Creek. Au. (Pl, D.)

10 miles E. Sunfield, G. \& P. R. R.

Pre-Cambrian schists cut by basic dikes.

Veins, stream gravels.

Min. Res. 1907, pt. 1, pp. 302-303.

1908, pt. 1, p. 428.

95. Leesburg (Arnett Creek). Au. (Pl, D.)

16 miles W. Salmon, G. \& P. R. R.

Pre-Cambrian schist cut by granite.

Veins, stream gravels.

Min. Res. 1905, p. 236.

1907 , pt. 1, p. 303.

1908 , pt. 1 , p. 428

96. Mackinaw. Au, Ag. (Pl, D.)

20 miles NW. Salmon, G. \& P. R. R.

Pre-Cambrian complex cut by granite.

Veins, stream gravels.

Min. Ree. 1907, pt. 1, p. 303.

1908 , pt. 1, p. 428 .

1909 , pt. 1, p. 350 .

97. Mineral Hill (Shoup). Au. (D, Pl.)

13 miles W. Noble, G. \& P. R. R.

Gneiss cut by granite porphyry and diorite.

Veins, stream gravels.

Lindgren, W., A geological reconnaissance across the Bitterroot Range and Clearwater Mountains in Montana and Idaho: P. P. 27, 1904, pp. 66, 89-90.

Min. Res. 1905, p. 236.

1906, p. 261.

1907 , pt. 1, p. 303.

1908 , pt. 1, p. 428.

1909 , pt. 1, p. 351.

98. Parker Mountain. $\mathrm{Ag}, \mathrm{Au}$.

75 miles SW. Salmon, G. \& P. R. R.

Tertiary volcanics.

Veins.

Min. Res. 1905, p. 236.

1906, p. 261.

1907, pt. 1, pp. 303-304.

1908, pt. 1, p. 428.

99. Pratt Creek. $\mathrm{Au}, \mathrm{Ag}, \mathrm{Pb}$. ( $\mathrm{Pl}, \mathrm{D}$.

10 miles E. Baker, G. \& P. R. R.

Pre-Cambrian schist.

Veins, stream gravels.

Min. Res. 1908, pt. 1, p. 428.

100. Spring Mountain. $\mathrm{Pb}, \mathrm{Ag}, \mathrm{Cu}, \mathrm{Au}$.

38 miles SSE. Junction, G. \& P. R. R.; 60 miles NE. Mackay, O. S. L. R. R.

Paleozoic sediments cut by diorite

Veins and replacements.

Min. Res. 1887, p. 107.

1907 , pt. 1, p. 303.

1908 , pt. 1, p. 428.

1909, pt. 1, p. 351 . 
101. Texas Creek (Gilmore). $\mathrm{Pb}, \mathrm{Ag}, \mathrm{Au}$.

19 miles S. Junction, G. \& P. R. R.; 80 miles NNW. Dubois, O. S. L. R. R.

Paleozoic sediments cut by basic dikes.

Veins and replacements.

Min. Res. 1905, pp. 235, 236.

1906, pp. 261, 446.

1907, pt. 1, p. 303.

1908 , pt. 1, p. 428.

1909 , pt. 1, p. 351.

102. Yellowjacket. Au. (D, Pl.)

58 miles WSW. Salmon, G. \& P. R. R.

Schist (pre-Cambrian?) cut by rhyolite and andesite porphyry and minette.

Veins.

Eldridge, G. H., A geological reconnaissance across Idaho: Sixteenth Ann.

Rept., pt. 2, 1895, pp. 259-264

Min. Res. 1905, p. 236.

1906, p. 261.

1907, pt. 1, p. 304.

1908 , pt. 1, p. 428 .

1909 , pt. 1, p. 351 .

[Note.-For Nos. 103-106, see Clearwater County, pp. 165-166.]

OWYHEE COURTY.

107. Carson (War Eagle, Silver City, Florida Mountain). Ag, Au.

23 miles SSW. Murphy, I. N. R. R.

Granite and Tertiary volcanics.

Veins.

Lindgren, W., The gold and silver veins of Silver City, De Lamar, and other mining districta in Idaho: Twentieth Ann. Rept., pt. 3, 1900, pp. 147-157.

Min. Res. 1905, pp. 237-238.

1906, pp. 262-263.

1907 , pt. 1, p. 305 .

1908 , pt. 1 , pp. $429-430$.

1909 , pt. 1, p. 352 .

Top. sheet Silver City.

Folio 104, 1904.

108. De Lamar. Au, Ag.

31 miles SSW. Murphy, I. N. R. R.

Tertiary volcanics.

Veins.

Eldridge, G. H., A geological reconnaissance across Idaho: Sixteenth Ann. Rept., pt. 2, 1895, pp. 271-274.

Lindgren, W., The gold and silver veins of Silver ('ity, De Lamar, and other mining districts in Idaho: Twentieth Ann. Rept., pt. 3, 1900, pp. 106-147.

Min. Res. 1907, pt. 1, p. 305.

1908 , pt. 1, pp. $429-430$.

Top. sheet Silver City

Folio 104, 1904.

109. Flint. Ag, Au.

40 miles SSW. Murphy, I. N. R. R.

Granite and diorite.

Veins. 
OWYAEE COUNTY-Continued.

109. Flint-Continued.

Lindgren, W., The gold and silver veins of Silver City, De Lamar, and other mining districts in Idaho: Twentieth Ann. Rept., pt. 3, 1900, pp. 187-199. Min. Res. 1909, pt. 1, p. 352.

110. Mammoth. Au, Ag.

33 miles SSW. Murphy, I. N. R. R.

Granite.

Veins.

Lindgren, W., The gold and silver veins of Silver City, De Lamar, and other mining districts in Idaho: Twentieth Ann. Rept., pt. 3, 1900, p. 188.

111. Snake River placers. Au. (PI.)

Stream gravels.

Min. Res. 1907, pt. 1, p. 306. 1908, pt. 1, p. 430.

Top. sheets Bisuka, Silver City.

Folio 104, 1904.

112. South Mountain. $\mathrm{Pb}, \mathrm{Ag}, \mathrm{Cu}$.

46 miles SSW. Murphy, I. N. R. R.

Schist, diorite, and limestone.

Contact metamorphic, veins.

Lindgren, W., The gold and silver veins of Silver City, De Lamar, and other mining districts in Idaho: Twentieth Ann. Rept., pt. 3, 1900, p. 188.

Min. Res. 1906, p. 446.

1907 , pt. 1, pp. 305-306.

\section{SHOSHONE COUNTY.}

113. Bald Mountain (Monitor). $\mathrm{Cu}, \mathrm{Au}$.

$5 \frac{1}{2}$ miles W. Saltese, Mont., N. P. R. R., C. M. \& P. S. R. R.

Pre-Cambrian sediments cut by diabase.

Veins.

Collier, A. J., Ore deposits in the St. Joe River basin, Idaho: Bull. 285, 1906, pp. 135-136.

Pardee, J. T., Geology and mineralization of the upper St. Joe River basin, Idaho: Bull. 470, 1911, pp. 39-61.

114. Beaver (Cœur d'Alene). Pb, Ag, Cu, Au. (D, Pl.)

4 miles NW. Burke, N. P. R. R.

Pre-Cambrian sediments.

Veins.

Ransome, F. L., Ore deposits of the Cœur d'Alene district, Idaho: Bull. 260, 1905, pp. 274-303.

Ransome, F. L., and Calkins, F. C., The geology and ore deposits of the Cour d'Alene district, Idaho: P. P. 62, 1908.

Min. Res. 1905, p. 240.

1906, pp. 264, $445,446$.

1907, pt. 1, p. 308.

1908, pt. 1, p. 431.

1909 , pt. 1, pp. 353-354.

Top. sheet Cœur d'Alene special.

115. Black Prince. $\mathrm{Cu}$.

St. Joe station, C. M. \& P. S. R. R.

Pre-Cambrian quartzite cut by granite.

Veins.

Collier, A. J., Ore deposits in the St. Joe River basin, Idaho: Bull. 285, 1906, p. 136. 


\section{SHOSHONE COUNTY-Continued.}

115. Black Prince-Continued.

Pardee, J. T., Geology and mineralization of the upper St. Joe River basin, Idaho: Bull. 470, 1911, pp. 39-61.

116. Eagle (Murray, Cœur d'Alene). $\mathrm{Au}(\mathrm{Pb}, \mathrm{Ag})$.

Paragon station, I. N. R. R.

Pre-Cambrian sediments cut by acidic intrusives.

Veins.

Lindgren, W., A geological reconnaissance across the Bitterroot Range and Clearwater Mountains in Montana and Idaho: P. P. 27, 1904, pp. 108-111.'

Ransome, F. L., Ore deposits of the Coeur d'Alene district, Idaho: Bull. 260, 1905, pp. 274-303.

Ransome, F. L., and Calkins, F. C., The geology and ore deposits of the Cœur d'Alene district, Idaho: P. P. 62, 1908.

Min. Res. 1905, p. 240.

1906, pp. 264, 445, 446.

1907 , pt. 1, p. 308 .

1908, pt. 1, p. 431.

1909 , pt. 1, pp. $354,579$.

Top. sheet Cour d'Alene special.

117. Evolution (Cour d'Alene). $\mathrm{Pb}, \mathrm{Ag}, \mathrm{Cu}(\mathrm{Au})$.

Kellogg station, N. P. R. R.

Pre-Cambrian sediments.

Veins.

Ransome, F. L., Ore deposits of the Cœur d'Alene district, Idaho: Bull. 260, 1905, pp. 274-303.

Ransome, F. L., and Calkins, F. C., The geology and ore deposits of the Cœur d'Alene district, Idaho: P. P. 62, 1908.

Min. Res. 1905, p. 240.

1906, pp. 264, 445, 446 .

1908 , pt. 1, p. 432 .

1909 , pt. 1, p. 354.

Top. sheet Cour d'Alene special.

118. Hunter (Mullan, Cour d'Alene). $\mathrm{Pb}, \mathrm{Ag}, \mathrm{Cu}, \mathrm{Au}$.

Mullan station, N. P. R. R.

Pre-Cambrian sediments.

Veins.

Ransome, F. L., Ore deposits of the Cœur d'Alene district, Idaho: Bull. 260, 1905, pp. 274-303.

Ransome, F. L., and Calkins, F. C., The geology and ore deposits of the Cœur d'Alene district, Idaho: P. P. 62, 1908.

Min. Res. 1905, p. 240.

1906, pp. 264, 445, 446.

1907 , pt. 1, pp. 308-309.

1908, pt. 1, pp. 207-208, 432, 433.

1909 , pt. 1, pp. 354-355.

Top. sheet Coeur d'Alene special.

119. Leland (Burke, Cour d'Alene). $\mathrm{Pb}, \mathrm{Ag}, \mathrm{Au}, \mathrm{Cu}, \mathrm{Zn}$.

Burke station, N. P. R. R.

Pre-Cambrian sediments cut by monzonite.

Veins.

Ransome, F. L., Ore deposits of the Cœur d'Alene district, Idaho: Bull. 260, 1905, pp. 274-303.

$21528^{\circ}-$ Bull. $507-12-12$ 
SHOSHONE COUNTY-Continued.

119. Leland (Burke, Cour d'Alene)-Continued.

Ransome, F. L., and Calkins, F. C., The geology and ore deposits of the Cour d'Alene district, Idaho: P. P. 62, 1908.

Min. Res. 1905, pp. 240-250.

1906 , pp. 264-265, 445-446.

1907, pt. 1, pp. 309-310.

1908 , pt. 1, p. 433.

1909 , pt. 1, pp. 355-356.

Top. sheet Cour d'Alene special.

120. Pine Creek. $\mathrm{Pb}, \mathrm{Ag}, \mathrm{Zn}$.

12 miles SSE. Kingston, N. P. R. R.

Pre-Cambrian sediments.

Veins.

Calkins, F. C., and MacDonald, D. F., A geological reconnaissance in northern

Idaho and northwestern Montana: Bull. 384, 1909, pp. 94-95.

121. Placer Center (Wallace, Cour d'Alene). $\mathrm{Pb}, \mathrm{Ag}, \mathrm{Cu}, \mathrm{Zn}, \mathrm{W}$.

Wallace station, N. P. R. R.

Pre-Cambrian sediments cut by monzonite.

Veins.

Lindgren, W., A geological reconnaissance across the Bitterroot Range and Clearwater Mountains: P. P. 27, 1904, pp. 108-111.

Ransome, F. L., Ore deposits of the Cœur d'Alene district, Idaho: Bull. 260, 1905, pp. 274-303.

Ransome, F. L., and Calkins, F. C., The geology and ore deposits of the Cœur d'Alene district, Idaho: P. P. 62, 1908.

Min. Res. 1905, p. 241.

1906, pp. $265,445-446$.

1907 , pt. 1, p. 310.

1908 , pt. 1, pp. $434,723$.

1909 , pt. 1, p. 356 .

Top. sheet Cour d'Alene special.

122. Slate Creek. $\mathrm{Pb}, \mathrm{Ag}$.

15 miles S. Wallace, N. P. R. R.

Pre-Cambrian sediments cut by acidic intrusives.

Replacements and veins.

Collier, A. J., Ore deposits of the St. Joe River basin, Idaho: Bull. 285, 1906, pp. 133-134.

123. Omitted.

124. St. Jö. Au. (Pl.)

25 miles W. Iron Mountain, Mont., N. P. R. R., C. M. \& P. S. R. R.

Stream gravels.

Calkins, F. C., and Jones, E. L., jr., Geology of the St. Joe-Clearwater region, Idaho: Bull. 530, 1912.

Collier, A. J., Ore deposits of the St. Joe River basin, Idaho: Bull. 285, 1906, p. 137.

Pardee, J. T., Geology and mineralization of the upper St. Joe River basin, Idaho: Bull. 470, 1911, pp. 39-61.

Min. Res. 1909, pt. 1, p. 356.

125. St. Regis (Ccour d'Alene). Au, Cu.

Lookout station, N. P. R. R.

Pre-Cambrian sediments cut by acidic dikes.

Veins. 
SHOSHONE coUNTY-Continued.

125. St. Regis (Cour d'Alene)-Continued.

Ransome, F. L., Ore deposits of the Cœur d'Alene district, Idaho: Bull. 260, 1905 , pp. 274-303.

Ransome, F. L., and Calkins, F. C., The geology and ore deposits of the Cœur d'Alene district, Idaho: P. P. 62, 1908.

Min. Res. 1905, p. 241.

1906, pp. 266, 445-446.

1907 , pt. 1, p. 310.

Top. sheet Cœur d'Alene special.

126. Summit (Cœur d'Alene). $\mathrm{Au}, \mathrm{Pb}, \mathrm{Cu}, \mathrm{Ag}$.

10 miles SE. Paragon, I. N. R. R.

Pre-Cambrian sediments.

Veins.

Ransome, F. L., Ore deposits of the Cœur d'Alene district, Idaho: Bull. 260, 1905, pp. 274-303.

Ransome, F. L., and Calkins, F. C., The geology and ore deposits of the Cœur d'Alene district, Idaho: P. P. 62, 1908.

Min. Res. 1905, p. 241.

1906, pp. 266, 445-446.

1907, pt. 1, pp. 310-311, 712 .

1908, pt. 1, p. 434 .

Top. sheet Cœur d'Alene special.

127. Yreka (Wardner, Cour d'Alene). $\mathrm{Pb}, \mathrm{Ag}, \mathrm{Au}, \mathrm{Cu}$.

Kellogg station, N. P. R. R.

Pre-Cambrian sediments.

Veins.

Lindgren, W., A geological reconnaissance across the Bitterroot Range and Clearwater Mountains in Montana and Idaho: P. P. 27, 1904, pp. 108-111.

Ransome, F. L., Ore deposits of the Cœur d'Alene district, Idaho: Bull. 260, 1906, pp. 274-303.

Ransome, F. L., and Calkins, F. C., The geology and ore deposits of the Cœur d'Alene district, Idaho: P. P. 62, 1908.

Min. Res. 1885 , p. 387.

1886 , p. 146.

1887, pp. 107-109.

1888, pp. 88-89.

1905 , p. 241.

1906, pp. 266, 445, 446.

1908, pt. 1, p. 434 .

1909 , pt. 1, p. 356-357.

Top. sheet Cœur d'Alene special.

\section{WASHINGTON AND ADAMS COUNTIES.}

128. Black Lake. Au. (D, Pl.)

40 miles NNW. Council, P. \& I. N. R. R.

Min. Res. 1905, p. 242.

1906, p. 267.

129. Heath. $\mathrm{Cu}, \mathrm{Ag}, \mathrm{Au}$.

24 miles NW. Cambridge, P. \& I. N. R. R.

Triassic (?) sediments cut by diorite (?).

Contact metamorphic (?).

Lindgren, W., 'The gold and silver veins of Silver City, De Lamar, and other mining districts in Idaho: Twentieth Ann. Rept., pt. 3, 1900, pp. 253-254, 
WASHINGTON AND ADAMS COUNTIES-Continued.

129. Heath-Continued.

Min. Res. 1882, p. 229.

1906, p. 267.

130. Meadows. Au. (Pl.)

16 miles NE. Evergreen, P. \& I. N. R. R.

Min. Res. 1905, p. 242.

1906, p. 267.

131. Mineral. $\mathrm{Pb}, \mathrm{Ag}, \mathrm{Cu}, \mathrm{Au}$.

29 miles NNE. Huntington, Oreg., O. W. R. R. \& N. Co.

Greenstones, quartz diorite.

Replacements, veins.

Lindgren, W., The gold belt of the Blue Mountains of Oregon: Twentysecond Ann. Rept., pt. 2, 1901, pp. 754-756.

132. Monroe Creek (Weiser). Au. (D, Pl.)

10 miles N. Weiser, O. S. L. R. R., O. W. R. R. \& N. Co.

Top. sheet Weiser.

133. Placer Basin. Au.

37 miles NNE. Council, P. \& I. N. R. R.

Veins.

Lindgren, W., The gold and silver veins of Silver City, De Lamar, and other mining districts in Idaho: Twentieth Ann. Rept., pt. 3, 1900, p. 253.

134. Seven Devils. $\mathrm{Cu}, \mathrm{Au}, \mathrm{Ag}$.

37 miles NW. Council, P. \& I. N. R. R.

Triassic (?) sediments cut by diorite.

Contact metamorphic.

Lindgren, W., The gold and silver veins of Silver City, De Lamar, and other mining districts in Idaho: Twentieth Ann. Rept., pt. 3, 1900, p. 249.

Weed, W. H., The copper mines of the United States in 1905: Bull. 285, 1906, pp. 108-109.

Min. Res. 1898 (Twentieth Ann. Rept., pt. 6), pp. 182-184.

1905 , p. 242.

1906, p. 267.

1907, pt. 1, p. 312 .

1908 , pt. 1, p. 435.

1909 , pt. 1, p. 357. 


\section{MONTANA.}

Eighteen of the mountain counties of Montana produce all of the metallic wealth of that State. In this region there are 107 mining districts. Gold is the predominant metal in 68 of these districts, lead in 15, copper in 11, silver in 7, zinc in 1 , and iron in 1 . In 4 camps the predominant metal is uncertain.

Distribution of the predominant metals produced in the mining di trict s of Montana.

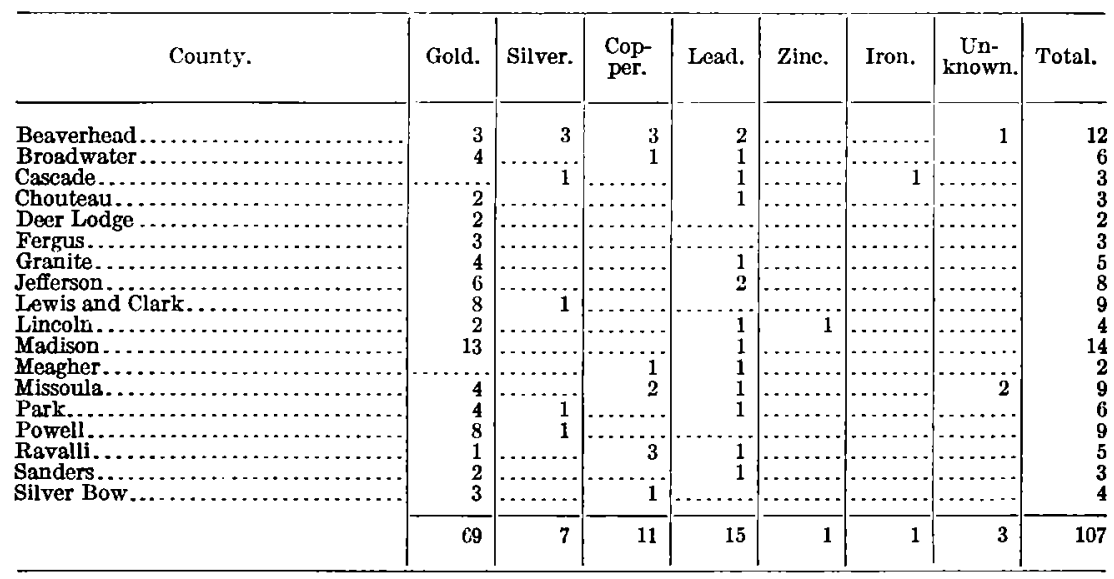

MINING DISTRICTS IN MONTANA.

[See Pl. VIII. Additional references will be found in Mineral Resources for 1910 and 1911.]

0a. Ajax. $\mathrm{Pb}, \mathrm{Ag}$.

BEAVERHEAD COUNTY.

1. Argenta. $\mathrm{Pb}, \mathrm{Ag}, \mathrm{Au}, \mathrm{Cu}$.

25 miles WNW. Dillon, O. S. L. R. R.

Paleozoic sediments cut by granite.

Contact metamorphic, veins.

Min. Res. 1906, p. 273.

1907 , pt. 1, p. 320.

1908, pt. 1, p. 445.

Top. sheet Dillon.

1909 , pt. 1, p. 367.

1a. Bald Mountain. Au, Ag.

Granite.

Veins.

2. Bannack. $\mathrm{Au}, \mathrm{Ag}, \mathrm{Cu} . \quad(\mathrm{Pl}, \mathrm{D}$.

40 miles WSW. Dillon, O. S. L. R. R.

Paleozoic sediments cut by syenite.

Contact metamorphic.

Stream gravels, veins.

Min. Res. 1908, pt. 1, p. 445.

Top. sheet Dillon.

1909 , pt. 1, p. 367. 
BEAVERHEAD COUNTY-Continued.

3. Beaverhead (Dark Horse). $\mathrm{Cu}, \mathrm{Ag}, \mathrm{Au}$.

Grant station, G. \& P. R. R.

Pre-Cambrian sediments cut by diorite.

Veins.

Min. Res. 1908, pt. 1, p. 445.

1909 , pt. 1 , p. 367.

4. Bluewing (Dillon). $\mathrm{Ag}, \mathrm{Pb}, \mathrm{Au}, \mathrm{Cu}$.

35 miles WNW. Dillon, O. S. L. R. R.

Paleozoic sediments cut by Tertiary igneous rocks.

Veins.

Min. Res. 1907, pt. 1, p. 320 .

1908 , pt. 1, p. 445.

1909 , pt. 1, p. 367 .

Top. sheet Dillon.

5. Bryant (Hecla). $\mathrm{Ag}, \mathrm{Cu}, \mathrm{Pb}, \mathrm{Au}, \mathrm{Mo}$.

10 miles W. Willis, O. S. L. R. R.

Paleozoic sediments cut by granite.

Replacements and contact metamorphic.

Min. Res. 1882, p. 311.

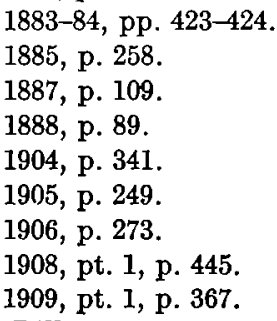

Top. sheet Dillon.

6. Elkhorn. $\mathrm{Cu}, \mathrm{Ag}, \mathrm{Pb}(\mathrm{Au})$.

45 miles $\mathrm{SW}$. Dillon, O. S. L. R. R.

Granite.

Veins.

Min. Res. 1908, pt. 1, p. 446.

1909 , pt. 1, p. 367.

6a. Mount Torrey.

Top. sheet Dillon.

7. Polaris. $\mathrm{Au}, \mathrm{Ag}, \mathrm{Cu}$.

40 miles W. Apex, 60 miles NW. Dillon, O. S. L. R. R.

Paleozoic sediments cut by granite.

Contact metamorphic (?).

Min. Res. 1908, pt. 1, p. 446.

Top. sheet Dillon.

8. Utopia. $\mathrm{Cu}, \mathrm{Ag}, \mathrm{Au}$.

10 miles W. Melrose, O. S. L. R. R.

Paleozoic sediments cut by granite.

Contact metamorphic.

Min. Res. 1907, pt. 1, p. 320.

1908 , pt. 1, p. 446

1909 , pt. 1, p. 367.

Top. sheet Dillon.

9. Vipond. $\mathrm{Ag}, \mathrm{Pb}, \mathrm{Au}, \mathrm{Cu}$.

11 miles SW. Divide, O. S. L. R. R.

Pre-Cambrian complex, Paleozoic sediments cut by granite and aplite. 


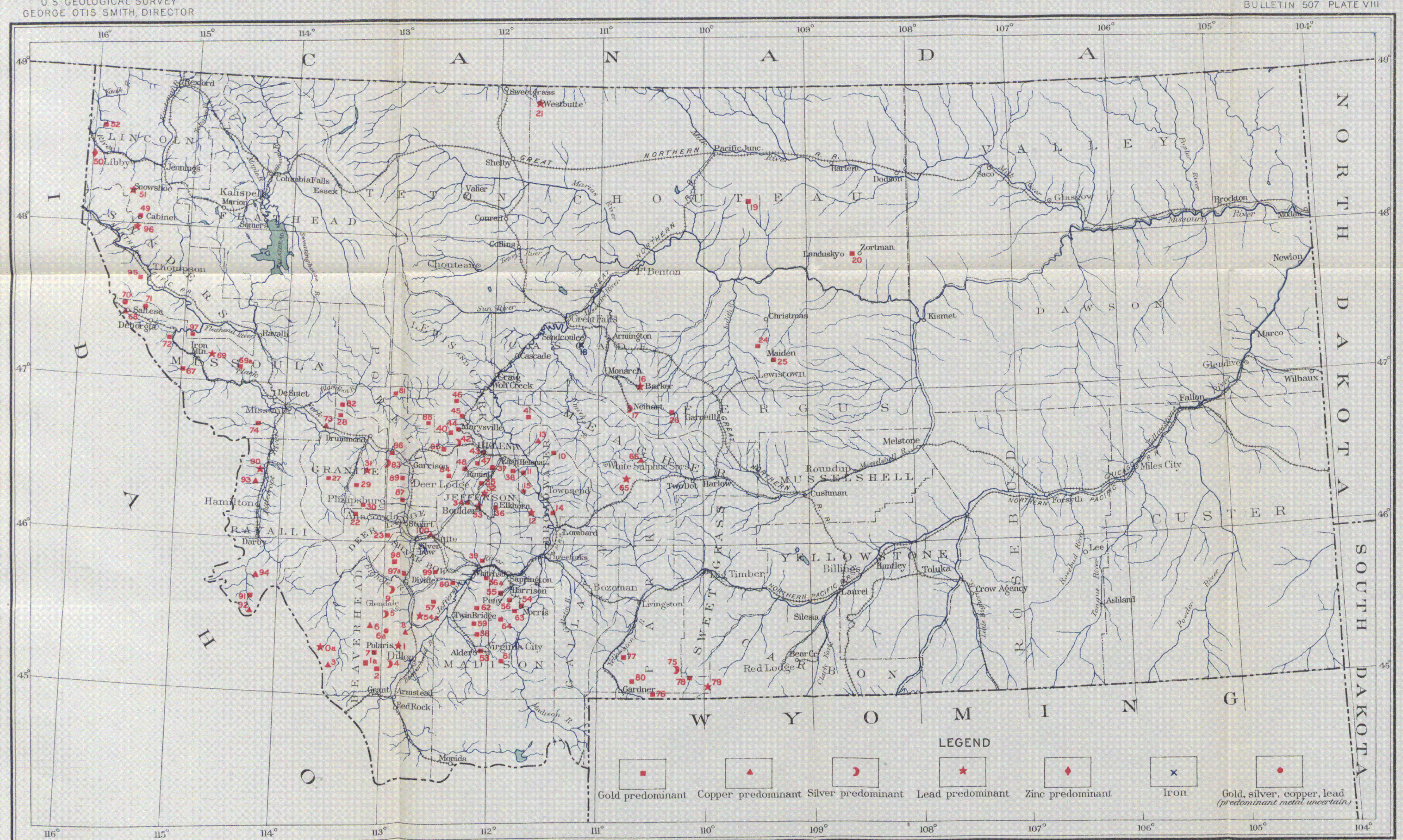

LIST OF MINING DISTRICTS

BEAVERHEAP COUNTY MAOISON COUNTY

Aal
A.renta
Bagd Mountain

Bannack
Beaverhead (Dark Horse)

Bryant (Hecla)
Elyton

E. Elkhorn Torrey

(w)

Polaris
Utopia
Vipond
Vipond

59. Sheridan (Mill Creek
Wisconsin)

Backer (Diamond City) ${ }^{60}$. Silver Stat

Beaver Creet (Winston)
Cedar Plain (Radersburg)

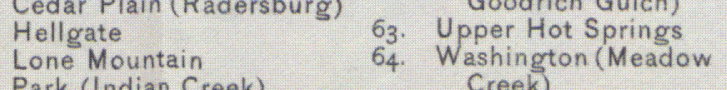

CASCADE COUNTY

MEAGHER COUNTY

Barker
Montana (Neihart)
Sand cowath

65. Castle Mountain
66. Musselshell
(Copperopelis)

UTEAU COUNT

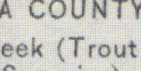

Learpaw Wockies (Landushy,
Litle Rortman, Whitcomb)
Wost Butte

68. Deneek, Superior)

$\begin{array}{lll}\text { West Butte } & \text { Whitcomb) } & \text { 6. Iron Mountain } \\ \text { Gga. Ninemile creek (Kenned) }\end{array}$

DEER LODGE COUNTY

Georgetown (Cable)

Packer Creek
R. Rock Island
St. Regis (Deer Creek,

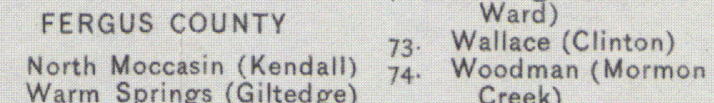

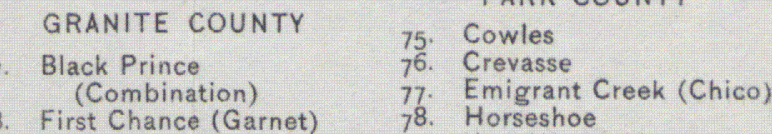

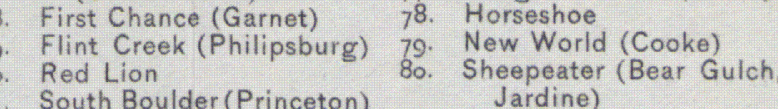

South Boulder (Princeton)

Amazon
Bulder

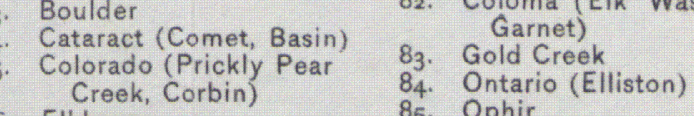

8.. Big Blackfoot (Helmville,

Elkhorn
W7. Lulch (Clancy.
Warm Springs)

8. McClellan Creek
39. Whitehall (Cardwell)

LEWIS AND CLARK COUNTY

8.
85. Oppir
86. Pioner

Racertack Creek
Wasticton Gulch (Finn
Zooell (Deer Lodge)

RAVALLI COUNTY

go. Curlew Hughes Creek (Alta)

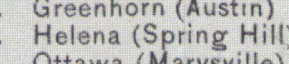

93. Pleasant View
94. Slate Creek

Poorman
Stemple (Fool Hen)

Vaughn (Rimini)
LINCOLN COUNTY

Cabinet
Callahan Creek (Grou

- Libby (S Snowshoe)

SANOERS COUNTY
Prospect Creek

96. Silver Butte (Vermilion
Cabinet

97. Spring Gulch
SILVER BOW COUS

ja. Fleecer Mountain
German Gulch

98. German Gulch
99. Highand Siberia)
90. Summit Valley (Butte)

MAP OF MONTANA. SHOWING LOCATION OF MINING DISTRICTS

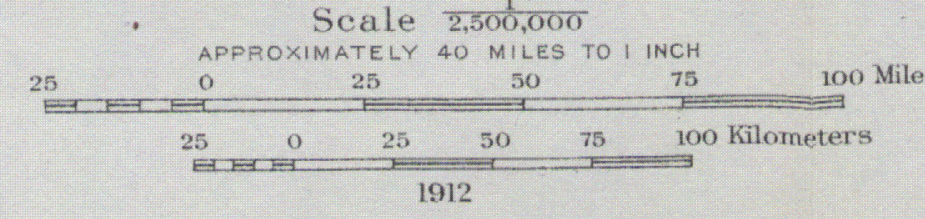


geaverhead coUNTy-Continted.

9. Vipond-Continued.

Veins.

Min. Res. 1908, pt. 1, p. 446.

1909 , pt. 1, p. 367.

Top. sheet Dillon.

\section{BROADWATER COTNTY.}

10. Backer (Diamond City). $\mathrm{Au}, \mathrm{Ag}, \mathrm{Cu}, \mathrm{Pb} . \quad(\mathrm{D}, \mathrm{Pl}$.)

14 miles N. Townsend, N. P. R. R.

Pre-Cambrian slates.

Veins, stream gravels.

Min. Res. 1905, p. 249.

1906, p. 273.

1908 , pt. 1, pp. 446-447.

1909 , pt. 1, p. 368 .

Top. sheet Fort Logan.

11. Beaver Creek (Winston). $\mathrm{Au}, \mathrm{Pb}, \mathrm{Ag}, \mathrm{Cu}$. (D, PI.)

Winston station, N. P. R. R.

Granite and andesite.

Veins, stream gravele.

Stone, R. W., Geologic relation of ore deposits in the Elkhorn Mountains,

Montana: Bull. 470, 1911, pp. 84-86.

Min. Res. 1905, p. 249.

1906, pp. 273, 274.

1907 , pt. 1, p. 320 .

1908 , pt. 1, p. 447.

1909, pt. 1, p. 368 .

Top. sheet Fort Logan.

12. Cedar Plain (Radersburg). $\mathrm{Pb}, \mathrm{Ag}, \mathrm{Cu}, \mathrm{Au}$.

11 miles W. Toston, N. P. R. R.

Andesite porphyry and Tertiary lake beds.

Veins.

Stone, R. W., Geologic relation of ore deposits in the Elkhorn Mountains, Montana: Bull. 470, 1911, pp. 93-96.

Min. Res. 1905, p. 249.

1906 , p. 274.

1908, pt. 1, p. 447.

1909 , pt. 1, p. 369.

Top. sheet Fort Logan.

13. Hellgate. $\mathrm{Cu}, \mathrm{Ag}, \mathrm{Pb}, \mathrm{Au}$.

12 miles N. Winston, N. P. R. R.

Min. Res. 1907, pt. 1, pp. 320-321.

Top. sheet Fort Logan.

14. Lone Mountain. $\mathrm{Au}, \mathrm{Ag}, \mathrm{Cu}$.

Toston station, N. P. R. R.

Top. sheet Fort Logan.

15. Park (Indian Creek). $\mathrm{Au}, \mathrm{Ag}, \mathrm{Cu}, \mathrm{Pb}$.

10 miles W. Townsend, N. P. R. R.

Andesite and diorite.

Veins.

Stone, R. W., Geologic relation of ore deposite in the Elkhorn Mountains, Montana: Bull. 470, 1911, pp. 89-91.

Min. Res. 1905, p. 249.

1906, p. 274.

1907, pt. 1., p. 321 . 
BROADWATER COUNTY-Continued.

15. Park (Indian Creek)-Continued.

Min. Res. 1908, pt. 1, p. 447.

1909 , pt. 1 , p. 369.

Top. sheet Fort Iogan.

\section{CASCADE COUNTY.}

16. Barker. $\mathrm{Pb}, \mathrm{Ag}$.

Station G. N. R. R.

Granite and Paleozoic sediments cut by porphyry.

Contact metamorphic, replacements, veins.

Weed, W. H., Geology of the Little Belt Mountains, Montana: Twentieth Ann. Rept., pt. 3, 1900, pp. 344-370, 441-446.

Min. Res. 1883-84, p. 424.

1909 , pt. 1, pp. 369-370.

Top. sheet Fort Benton.

Folio 55, 1899.

17. Montana (Neihart). $\mathrm{Ag}, \mathrm{Au}, \mathrm{Pb}(\mathrm{Cu})$.

Station G. N. R. R.

Pre-Cambrian gneiss, schist and Algonkian sandstone cut by rhyolite, diorite, and minette dikes.

Veins.

Harder, E. C., Manganese deposits of the United States: Bull. 427, 1910, pp. 148-149.

Weed, W. H., Geology of the Little Belt Mountains, Montana: Twentieth Ann. Rept., pt. 3, 1900, pp. 371-440.

Min. Res. 1905, p. 249.

1906, p. 274.

1907 , pt. 1, pp. 321-322.

1908, pt. 1, p. 448.

1909 , pt. I, pp. 369-370.

Top. sheet Little Belt Mountains.

Folio 56, 1899.

18. Sand Coulee. Fe.

12 miles SE. Great Falls, N. P. R. R., G. N. R. R.

Min. Res. 1888, p. 34.

1897 (Nineteenth Ann. Rept., pt. 6), p. 56.

Top. sheet Great Falls.

\section{CHOUTEAU COUNTT.}

19. Bearpaw. Au, Ag, $\mathrm{Pb}$.

45 miles S. Chinook, G. N. R. R.

Cretaceous sediments and Tertiary volcanics.

Veins.

Calhoun, F. H. H., The Montana lobe of the Keewatin ice sheet: P. P. 50, 1906.

Pepperberg, L. J., Notes on the minerai deposits of the Bearpaw Mountains, Montana: Bull. 430, 1910, pp. 135-146.

20. Iittle Rockies (Landusky, Zortman; Whitcomb). Au, Ag.

45-50 miles SSW. Dodson, G. N. R. R.

Pre-Cambrian schists, Paleozoic sediments domed by acidic Tertiary porphyries.

Replacements.

Emmons, W. H., Gold deposits of the Little Rocky Mountains, Montana: Bull. 340, 1908, pp. 96-116. 
chodteat cotrty-Continued.

20. Little Rockies (Landusky, Zortman, Whitcomb)-Continued.

Min. Res. 1905, p. 250.

1906, p. 275.

1907 , pt. 1, p. 322

1908 , pt. 1, p. 448

1909 , pt. 1, p. 370 .

21. West Butte. $\mathrm{Pb}, \mathrm{Ag}, \mathrm{Au}$.

54 miles NW. Chester, G. N. R. R.

Min. Res. 1908, pt. 1, p. 448.

\section{DEER LODGE COUNTY.}

22. Georgetown (Cable). Au, Cu, Ag.

$7 \frac{1}{2}$ miles WNW. Brown's siding, B. A. \& P. R. R.

Paleozoic sediments cut by granite.

Replacement, veins.

Emmons, W. H., The Granite-Bimetallic and Cable mines, Philipsburg quadrangle, Montana: Bull. 315, 1907, pp. 45-54.

Min. Res. 1898 (Twentieth Ann. Rept., pt. 6), p. 55.

1905 , p. 250.

1906, p. 275.

1907 , pt. 1, p. 322 .

1908 , pt. 1, p. 449

1909 , pt. 1, p. 370 .

Top. sheet Philipsburg.

23. Heber. $\mathrm{Au}, \mathrm{Ag}, \mathrm{Pb}, \mathrm{Cu} . \quad(\mathrm{D}, \mathrm{Pl}$.)

10 miles W. Gregson, B. A. \& P. R. R.

Min. Res. 1906, p. 275.

1907, pt. 1, p. 323 .

Top. sheet Helena.

\section{FERGUS COUNTY.}

24. North Moccasin (Kendall). Au, Ag.

20 miles N. Lewiston, C. M. \& P. S. R. R.

Paleozoic and Mesozoic sedimente cut by acidic intrusives.

Replacements.

Weed, W. H., and Pirsson, L. V., Geology and mineral resources of the Judith Mountains, Montana: Eighteenth Ann. Rept., pt. 3, 1898, pp. 437-616.

Min. Res. 1905, p. 250.

1906 , p. 276.

1907, pt. 1, p. 323.

1908 , pt. 1 , pp. $449-450$.

1909 , pt. 1, p. 371.

25. Warm Springs (Giltedge). $\mathrm{Au}, \mathrm{Pb}, \mathrm{Ag}, \mathrm{Cu}$.

15 miles NE. Lewiston, C. M. \& P. S. R. R.

Paleozoic and Mesozoic sediments cut by intrusives.

Replacements.

Weed, W. H., and Pirsson, L. V., Geology and mineral resources of the Judith Mountains, Montana: Eighteenth Ann. Rept., pt. 3, 1898, pp. 437-616.

Min. Res. 1898 (Twentieth Ann. Rept., pt. 6), pp. 56-57.

1905, pp. 250-251.

1906, p. 276.

1907, pt. 1, p. 323.

1908, pt. 1, p. 450.

1909, pt. 1, p. 371. 
FERGUS COUSTY-Continued.

26. Yogo. $\mathrm{Au}, \mathrm{Ag}, \mathrm{Cu}, \mathrm{Pb}$ :

30 miles WSW. Philbrook, G. N. R. R.

Paleozoic sediments cut by minettes.

Contact metamorphic.

Weed, W. H., Geology of the Little Belt Mountains, Montana: Twentieth Ann. Rept., pt. 3, 1900, pp. 317-333, 447-461.

\section{GRANITE COUNTY.}

27. Black Prince (Combination). $\mathrm{Au}, \mathrm{Cu}$.

15 miles SW. Hall, N. P. R. R.

Pre-Cambrian sediments.

Veins.

Top. sheet Sapphire.

28. First Chance (Garnet). $\mathrm{Au}, \mathrm{Ag}, \mathrm{Cu}, \mathrm{Pb} . \quad$ (D, Pl.)

11 miles N. Bearmouth, N. P. R. R., C. M. \& P. S. R. R.

Paleozoic sediments cut by granite.

Veins, stream gravels.

Min. Res. 1905, p. 251.

1906, p. 276.

1907, pt. 1, p. 324

1908 , pt. 1, p. 451.

1909 , pt. 1, p. 372 .

29. Flint Creek (Philipsburg). $\mathrm{Au}, \mathrm{Ag}, \mathrm{Pb}, \mathrm{Cu}$.

Station N. P. R. R.

Pre-Cambrian and Paleozoic sediments cut by granite.

Veins, contact metamorphic.

Emmons, W. H., The Granite-Bimetallic and Cable mines, Philipsburg quadrangle, Montana: Bull. 315, 1907, pp. 36-45.

Min: Res. 1905, p. 251.

1906, p. 277.

1907 , pt. 1, pp. 324-325.

1908 , pt. 1, p. 451 .

1909 , pt. 1, p. 372 .

Top. sheet Philipsburg.

30. Red Lion. Au, Ag.

25 miles NW. Anaconda, B. A. \& P. R. R.

Paleozoic sediments cut by granite.

Veins and replacements.

Min. Res. 1905, p. 252.

1906, p. 277.

1908 , pt. 1, p. 451.

Top. sheet Philipsburg.

31. South Boulder (Princeton). $\mathrm{Pb}, \mathrm{Ag}, \mathrm{Au}, \mathrm{Cu}$.

12 miles ESE. Flint, N. P. R. R.

Paleozoic sediments cut by granite.

Veins.

Min. Res. 1905, p. 252.

1906, p. 277.

1907 , pt. 1, p. 325

1908 , pt. 1, p. 451

1909 , pt. 1, p. 372

Top. sheet Philipsburg. 


\section{NEFFERSON COUTTY.}

32. Amazon. $\mathrm{Pb}, \mathrm{Ag}, \mathrm{Au}, \mathrm{Cu}$.

Station G. N. R. R.

Granite.

Veins.

Min. Res. 1908, pt. 1, p. 452.

1909, pt. 1, p. 373 .

Top. sheets Boulder, Helena.

33. Boulder. $\mathrm{Pb}, \mathrm{Ag}, \mathrm{Au}$. (D, Pl.)

Station G. N. R. R.

Granite cut by rhyolite.

Veins.

Weed, W. H., Mineral-vein formation at Boulder Hot Springs, Mont.: Twenty-first Ann. Rept., pt. 2, 1900, pp. 227-255

Min. Res. 1906, p. 278.

1907, pt. 1, p. 326 .

1908, pt. 1, p. 452.

1909 , pt. 1 , p. 373 .

Top. sheets Boulder, Helena.

34. Cataraet (Comet, Basin). Au, Ag, Pb, Cu. (D, Pl.)

Basin station, G. N. R. R.

Andesite cut by granite, both cut by dacite.

Veins.

Min. Res. 1904, p. 233.

1905, p. 252.

1906, p. 278.

1907, pt. 1, p. 326 .

1908, pt. 1, p. 452.

1909, pt. 1, pp. 373-374.

Top. sheets Boulder, Helena.

35. Colorado (Prickly Pear Creek, Corbin). $\mathrm{Au}, \mathrm{Ag}, \mathrm{Cu}, \mathrm{Pb}$.

Corbin station, G. N. R. R.

Andesite cut by granite, both cut by dacite.

Veins.

Stone, R. W., Geologic relation of ore deposits in the Elkhorn Mountaing, Montana: Bull. 470, 1911, p. 89

Min. Res. 1883-84, p. 423.

1905, pp. $252-253$.

1906, pp. 278-279.

1907 , pt. 1, p. 326 .

1908, pt. 1, p. 453.

1909, pt. 1, p. 374.

Top. sheets Boulder, Helena.

36. Elkhorn. $\mathrm{Au}, \mathrm{Ag}, \mathrm{Pb}, \mathrm{Cu}$.

Station G. N. R. R.

Paleozoic sediments cut by diorite, gabbro, and quartz monzonite.

Replacements, contact metamorphic.

Stone, R. W., Geologic relation of ore deposits in the Elkhorn Mountaine, Montana: Bull. 470, 1911, pp. 96-98.

Weed, W. H., and Barrell, J., Geology and ore deposits of Elkhorn mining district, Jefferson County, Mont.: Twenty-second Ann. Rept., pt. 2, 1900, pp. 399-550.

Min. Res. 1905, p. 253.

1906, p. 279.

1907 , pt. 1, p. 327. 
JEFFERSON COUNTY-Continued.

36. Elkhorn-Continued.

Min. Res. 1908, pt. 1, p. 453.

1909 , pt. 1 , p. 374 .

Top. sheet Fort Logan.

37. Lump Gulch (Clancy, Warm Springs). $\mathrm{Au}, \mathrm{Ag}, \mathrm{Pb}, \mathrm{Cu}$.

Clancy station, G. N. R. R.

Granite.

Vein.

Stone, R. W., Geologic relation of ore deposits in the Elkhorn Mountains, Montana: Bull. 470, 1911, pp. 79-80.

Min. Res. 1905, p. 253.

1906, p. 279.

1907 , pt. 1, p. 327.

1908 , pt. 1 , p. 453.

1909, pt. 1, pp. 374-375.

Top. sheets Boulder, Fort Logan.

38. McClellan Creek. $\mathrm{Au}, \mathrm{Ag}, \mathrm{Cu}, \mathrm{Mo}$.

9 miles NE. Clancy, G. N. R. R.

Andesite cut by granite.

Veins.

Stone, R. W., Geologic relation of ore deposits in the Elkhorn Mountains, Montana: Bull. 470, 1911, pp. 86-87.

39. Whitehall (Cardwell). Au, Ag. (D, Pl.)

Station N. P. R. R.

Pre-Cambrian and Paleozoic sediments cut by andesite porphyry.

Veins.

Min. Res. 1908, pt. 1, p. 453.

Top. sheet Dillon. 1909 , pt. 1, p. 375 .

\section{IEWIS AND CLARK COUNTY.}

40. Bald Butte (Marysville). $\mathrm{Au}, \mathrm{Ag}, \mathrm{Pb}, \mathrm{Cu}$.

2 miles W. Marysville, N. P. R. R.

Pre-Cambrian sediments cut by granite.

Veins.

Barrell, J., Geology of the Marysville mining district, Montana: P. P. 57, 1907.

Weed, W. H., Gold mines of the Marysville district, Montana: Bull. 213, 1903, pp. 88-89.

Min. Res. 1906, p. 280.

1907 , pt. 1, p. 328.

1909 , pt. 1, p. 375 .

Top. sheet Helena.

41. Dry Gulch (York). Au, Pb, Ag. (D, Pl.)

20 miles NE. Helena, G. N. R. R., N. P. R. R.

Min. Res. 1906, p. 280.

1909 , pt. 1, p. 376.

Top. sheet Fort Logan.

42. Greenhorn (Austin). Ag, $\mathrm{Au}, \mathrm{Pb}, \mathrm{Cu}$.

Austin station, N. P. R. R.

Pre-Cambrian and Paleozoic sediments cut by granite.

Veins.

Min. Res. 1908, pt. 1, p. 454.

1909 , pt. 1, p. 376.

Top. sheet Helena. 
LEWIS ATD CLARK COUNTY-Continued.

43. Helona (Spring Hill). $\mathrm{Au}, \mathrm{Pb}, \mathrm{Ag}, \mathrm{Cu}$. (D, PI.)

Station N. P. R. R., G. N. R. R.

Pre-Cambrian and Paleozoic sediments cut by granite.

Veins.

Min. Res. 1883-84, pp. 422-423.

1885, pp. 257-258.

1887 , p. 109.

1888 , p. 89.

1905 , p. 253.

1906 , p. 280.

1907 , pt. 1, p. 329.

1909 , pt. 1, p. 376.

Top. sheets Helena, Helena special.

44. Ottawa (Marysville). $\mathrm{Au}, \mathrm{Ag}, \mathrm{Cu}, \mathrm{Pb}$.

Marysville station, N. P. R. R.

Pre-Cambrian sediments cut by granite.

Veins.

Weed, W. H., Gold mines of the Marysville district, Montana: Bull. 213, 1903, pp. 88-89.

Min. Res. 1905, p. 253.

1906, p. 280.

1907, pt. 1, p. 328 .

1908 , pt. 1, p. 454

1909 , pt. 1 , p. 376 .

Top. sheets Helena, Marysville special.

45. Poorman. $\mathrm{Au}, \mathrm{Ag}(\mathrm{Cu})$.

Silver station, G. N. R. R.

Min. Res. 1907, pt. 1, p. 329.

1909 , pt. 1, p. 376.

Top. sheets Helena, Marysville special.

46. Stemple (Fool Hen). Au, Ag, $\mathrm{Pb}, \mathrm{Cu}$.

25 miles NW. Silver, G. N. R. R.

Sediments cut by granite.

Veins.

Min. Res. 1905, p. 254.

1906, p. 280.

1907, pt. 1, p. 329.

1908, pt. 1, p. 454 .

Top. sheet Helena. 1909 , pt. 1 , p. 376 .

47 Unionville. $\mathrm{Au}, \mathrm{Ag}(\mathrm{Cu}, \mathrm{Pb})$.

5 miles SSW. Helena, G. N. R. R., N. P. R. R.

Paleozoic sediments cut by granite

Veins.

Min. Res. 1883-84, p. 423.

1905, p. 254.

1906, p. 280.

1907, pt. 1, p. 329 .

1908 , pt. 1, p. 454.

1909 , pt. 1, p. 376.

Top. sheets Helena, Boulder, Helena special.

48 Vaughn (Rimini). $\mathrm{Au}, \mathrm{Ag}, \mathrm{Pb}$.

Rimini station, N. P. R. R.

Granite capped by rhyolite. 
LEWIS AND CLARK COUNTY-Continued.

48. Vaughn (Rimini)-Continued.

Veins.

Min. Res. 1906, p. 281.

1907, pt. 1, p. 329 .

1908 , pt. 1, p. 454.

1909 , pt. 1, p. 376 .

Top. sheet Boulder.

49. Cabinet. Au.

\section{LINCOLN COUNTY.}

25 miles SSE. Libby, G. N. R. R.; 15 miles NNE. Trout, N. P. R. R.

Pre-Cambrian sediments.

Veins.

Calkins, F. C., and MacDonald, D. F., A geological reconnaissance in northern Idaho and northwestern Montana: Bull. 384, 1909, pp. 105-106.

MacDonald, D. F., Economic features of northern Idaho and northwestern Montana: Bull, 285, 1906, p. 50.

50. Callahan Creek (Grouse Mountain). $\mathrm{Zn}, \mathrm{Pb}$.

8 miles S. Troy, G. N. R. R.

Pre-Cambrian sediments cut by basic dikes.

Veins, contact metamorphic.

Calkins, F. C., and MacDonald, D. F., A geological reconnaissance in northern Idaho and northwestern Montana: Bull. 384, 1909, pp. 99-102.

MacDonald, D. F., Economic features of northern Idaho and northwestern Montana: Bull. 285, 1906, p. 48.

51. Iibby (Snowshoe). Pb, Ag, Au. (D, Pl.)

18 miles S. Libby, G. N. R. R.

Pre-Cambrian sediments.

Veins, stream gravels.

Calkins, F. C., and MacDonald, D. F., A geological reconnaissance in northern Idaho and northwestern Montana: Bull. 384, 1909, pp. 103-105.

Schrader, F. C., Gold-bearing ground moraine in northwestern Montana: Bull. 470, 1911, pp. 70-74.

Min. Res. 1905, p. 251.

1906, p. 276.

1907, pt. 1, p. 324.

1908, pt. 1, p. 450 .

1909, pt. 1, p. 377 .

52. Sylvanite. Au.

18 miles NNW. Troy, G. N. R. R.

Pre-Cambrian sediments.

Veins.

Calkins, F. C., and MacDonald, D. F., A geological reconnaissance in northern Idaho and northwestern Montana: Bull. 384, 1909, pp. 102-103.

MacDonald, D. F., Economic features of northern Idaho and northwestern Montana: Bull. 285, 1906, pp. 49-50.

Min. Res. 1909, pt. 1, p. 377.

\section{MADISON COUNTY.}

53. Alder Gulch (Virginia City, Fairweather). Au, Ag. (Pl.)

12 miles E. Alder, N. P. R. R.

Stream gravels.

Min. Res. 1906, p. 281.

1907, pt. 1, pp. 330-331.

1908 , pt. 1 , pp. $455-456$.

1909, pt. 1, p. 378. 
MAdsor coUrTY-Continued.

53. Alder Gulch (Virginia City, Fairweather)-Continued.

Top. sheets Three Forks, Dillon.

Folio 24, 1896.

54. Lower Hot Springs (Norris). $\mathrm{Au}, \mathrm{Ag} . \quad(\mathrm{Pb}, \mathrm{Cu}$.

Station N. P. R. R.

Pre-Cambrian gneiss.

Veins.

Min. Res. 1905, p. 254.

1906, p. 281.

1907 , pt. 1, p. 331 .

1908, pt. 1, p. 456 .

1909 , pt. 1, p. 378 .

Top. sheet Three Forks.

Folio 24, 1896.

54a. McCartney Mountain. $\mathrm{Pb}, \mathrm{Ag}$.

Quartzite and slates cut by granite.

Veins.

Top. sheet Dillon.

55. Mineral Hill (Pony). $\mathrm{Au}, \mathrm{Cu}, \mathrm{Ag}, \mathrm{Pb}, \mathrm{Zn}$.

Pony station, N. P. R. R.

Pre-Cambrian gneiss cut by granite.

Veins.

Min. Res. 1905, pp. 254-255.

1906, p. 282.

1907, pt. 1, p. 331.

1908 , pt. 1, p. 456.

1909 , pt. 1, p. 378.

Top. sheet Three Forks.

Folio 24, 1896.

56. Norwegian (Whitehall). $\mathrm{Au}, \mathrm{Ag}, \mathrm{Cu}, \mathrm{Pb}$.

5 miles SE. Pony, N. P. R. R.

Pre-Cambrian gneiss.

Veins.

Min. Res. 1905, p. 255.

1907 , pt. 1, p. 332.

1908 , pt. 1, p. 456.

1909, pt. 1, p. 379.

Top. sheet Three Forks.

Folio 24, 1896.

56a. Point of Rocks (Mammoth). $\mathrm{Au}, \mathrm{Ag}, \mathrm{Cu}, \mathrm{Pb}$.

12 miles SE. Whitehall, N. P. R. R.

Pre-Cambrian and Paleozoic sediments cut by quartz monzonite.

Veins.

Top. sheet Dillon.

57. Rabbit. $\mathrm{Au}, \mathrm{Ag}, \mathrm{Pb}, \mathrm{Cu}$.

12 miles NW. Twin Bridges, N. P. R. R.

Pre-Cambrian complex cut by aplite.

Veins.

Min. Res. 1905, p. 255.

1906, p. 282.

1907, pt. 1, p. 331.

1908 , pt. 1, p. 456.

1909 , pt. 1, p. 379.

Top. sheet Dillon. 
MADISON COUNTY-Continued.

58. Ramshorn (Union). $\mathrm{Au}, \mathrm{Ag}, \mathrm{Cu}, \mathrm{Pb}$.

8 miles E. Laurin, N. P. R. R.

Pre-Cambrian schist and sediments cut by granite.

Veins.

Min. Res. 1908, pt. 1, p. 456.

1909 , pt. 1, p. 379

Top. sheet Dillon.

59. Sheridan (Mill Creek, Wisconsin). $\mathrm{Au}, \mathrm{Pb}, \mathrm{Ag}, \mathrm{Cu}$.

6 miles E. Sheridan station, N. P. R. R.

Pre-Cambrian schist and sediments cut by granite.

Veins.

Min. Res. 1905, p. 255.

1908 , pt. 1, p. 456

1909 , pt. 1, p. 379

Top. sheet Dillon.

60. Silver Star. $\mathrm{Au}, \mathrm{Pb}, \mathrm{Cu}, \mathrm{Ag}$.

4 miles NW. Silver Star siding, N. P. R. R.

Pre-Cambrian schist and Paleozoic sediments cut by quartz monzonite.

Veins and contact metamorphic.

Min. Res. 1905, p. 255.

1906 , p. 282.

1907, pt. 1, p. 331.

1908 , pt. 1, pp. $456-457$.

1909 , pt. 1, p. 379 .

Top. sheet Dillon.

61. Summit. $\mathrm{Au}, \mathrm{Ag}, \mathrm{Pb}$.

20 miles SE. Alder, N. P. R. R.

Pre-Cambrian complex.

Veins.

Min. Res. 1905, p. 255.

1906, p. 282.

1907 , pt. 1, p. 332 .

1908 , pt. 1, p. 457

1909, pt. 1, p. 379

Top. sheet Three Forks.

Folio 24, 1896.

62. Tidal Wave (Bear Gulch, Goodrich Gulch). $\mathrm{Au}, \mathrm{Pb}, \mathrm{Ag}, \mathrm{Cu}$.

10 miles E. Twin Bridges, N. P. R. R.

Pre-Cambrian and Paleozoic sediments cut by granite.

Veins and contact metamorphic.

Min. Res. 1906, p. 283.

1907, pt. 1, p. 332 .

1908, pt. 1, p. 457

1909, pt. 1, p. 379

Top. sheet Dillon.

63. Upper Hot Springs. $\mathrm{Au}, \mathrm{Pb}, \mathrm{Ag}$.

8 miles W. Norris, N. P. R. R.

Granite porphyry cut by aplite.

Veins.

Min. Res. 1905, p. 255.

1906, p. 283.

1907, pt. 1, p. 332 .

1908 , pt. 1, p. 457

1909 , pt. 1 , p. 380 . 


\section{MaDISON COUNTY-Continued.}

63. Upper Hot Springs-Continued.

Top. sheet Three Forks.

Folio 24, 1896.

64. Washington (Meadow Creek). Au, Pb, Ag.

12 miles SW. Norris, N. P. R. R.

Pre-Cambrian complex cut by granite and andesite.

Veins.

Min. Res. 1905, p. 255.

1906, p. 283.

1907, pt. 1, p. 332.

1908, pt. 1, p. 457.

1909 , pt. 1, p. 380 .

Top. sheet Three Forks.

Folio 24, 1896.

\section{MEAGHER COUNTY.}

65. Castle Mountain. $\mathrm{Pb}, \mathrm{Ag}, \mathrm{Cu}$.

7 miles W. Lennep, C. M. \& P. S. R. R.

Pre-Cambrian and Paleozoic sediments cut by granite and diorite.

Replacements.

Harder, E. C., Manganese depositg of the United States: Bull. 427, 1910, pp. 148-149.

Weed, W. H., and Pirsson, L. V., Geology of the Castle Mountain mining district, Montana: Bull. 139, 1896.

Min. Res. 1893, p. 131.

1894 (Sixteenth Ann. Rept., pt. 3), pp. 417-418.

1906, p. 333.

1908 , pt. 1, p. 457.

Top. sheet Little Belt Mountains.

Folio 56, 1899.

66. Musselshell (Copperopolis). $\mathrm{Cu}, \mathrm{Au}, \mathrm{Ag}$.

14 miles NW. Martinsdale, C. M. \& P. S. R. R.

Pre-Cambrian and Paleozoic sediments cut by granite and diorite.

Replacements.

Weed, W. H., and Pirsson, L. V., Geology of the Castle Mountain mining district, Montana: Bull. 139, 1896.

Min. Res. 1906, p. 283.

1908 , pt. 1, p. 457.

1909 , pt. 1 , p. 380.

Top. sheet Little Belt Mountains.

Folio 56, 1899.

\section{MISSOULA COUNTY.}

67. Cedar Creek (Trout Creek, Superior). Au. (Pl.)

15 miles SW. Superior, C. M. \& P. S. R. R.

Stream gravels.

Min. Res. 1905, p. 256.

1908 , pt. 1, p. 458.

1909 , pt. 1, pp. 380-381.

68. Denemora (Big Elk). $\mathrm{Cu}, \mathrm{Au}, \mathrm{Ag}, \mathrm{Pb}$.

Saltese station, C. M. \& P. S. R. R.

Pre-Cambrian sediments cut by acidic dikes.

Replacements and veins.

$21528^{\circ}-$ Bull. 507-12-13 
MISSOULA CoUNTY-Continued.

69. Iron Mountain. $\mathrm{Pb}, \mathrm{Ag}$.

22 miles NNW. Hudson, N. P. R. R., C. M. \& P. S. R. R.

Pre-Cambrian sediments.

Veins.

Calkins, F. C., and MacDonald, D. F., A geological reconnaissance in northern Idaho and northwestern Montana: Bull. 384, 1909, p. 107.

Min. Res. 1908, pt. 1, p. 458. 1909 , pt. 1, p. 381.

69a. Ninemile Creek (Kennedy Creek). Au. (Pl.)

12 miles NW. Huson, N. P. R. R., C. M. \& P. S. R. R.

Glacial gravels (?)

Schrader, F. C., Gold-bearing ground moraine in northwestern Montana: Bull. 470, 1911, pp. 62-74.

Min. Res. 1909, pt. 1, p. 381.

70. Packer Creek.

5 miles N. Saltese, C. M. \& P. S. R. R.

Pre-Cambrian sediments cut by acidic intrusives.

Replacements.

71. Rock Island.

8 miles N. Henderson, N. P. R. R.

Pre-Cambrian sediments cut by acidic intrusives.

Replacements and veins.

72. St. Regis (Deer Creek, Ward). Au. (D, Pl.)

St: Regis station, N. P. R. R., C. M. \& P. S. R. R.

Min. Res. 1907, pt. 1, p. 333.

1909, pt. 1, p. 381.

73. Wallace (Clinton). $\mathrm{Cu}, \mathrm{Au}, \mathrm{Ag}, \mathrm{Pb}$.

Clinton station, N. P. R. R.

Min. Res. 1905, p. 256.

1906, p. 283.

1907, pt. 1, p. 333.

Top. sheet Bonner.

74. Woodman (Mormon Creek). $\mathrm{Au}, \mathrm{Cu}$.

11 miles W. Lolo, N. P. R. R.

Pre-Cambrian sediments cut by diorite.

Veins.

Lindgren, W., Mineral deposits of the Bitterroot Range and Clearwater Mountains, Montana: Bull. 213, 1903, pp. 66-70.

A geological reconnaissance across the Bitterroot Range and Clearwater Mountains, in Montana and Idaho: P. P. 27, 1904, p. 86.

75. Cowles. Ag.

\section{PARK COUNTY.}

50 miles ENE. Gardiner, N. P. R. R.

Pre-Cambrian gneiss, gabbro, diorite.

Veins.

Top. sheet Livingston.

Folio 1, 1894.

76. Crevasse. $\mathrm{Au}, \mathrm{Ag}$.

10 miles ESE. Gardiner, N. P. R. R.

Pre-Cambrian gneiss.

Veins.

Min. Res. 1905, p. 256.

1906, p. 284.

1907, pt. 1, p. 333. 
PARK COUNTY-Continued.

76. Crevasse-Continued.

Top. shset Livingston.

Folio 1, 1894.

77. Emigrant Creek (Chico). Au. (D, Pl.)

5 miles SSE. Fridley, N. P. R. R.

Andesite, dacite.

Veins.

Min. Res. 1908, pt. 1, p. 458.

Top. sheet Livingston.

Folio 1, 1894.

78. Horseshoe. $\mathrm{Au}$.

60 miles E. Gardiner, N. P. R. R.

Pre-Cambrian gneiss cut by andesite and dacite.

Veins.

Top. sheet Livingston.

Folio 1, 1894.

79. New World (Cooke). $\mathrm{Pb}, \mathrm{Ag}, \mathrm{Cu}, \mathrm{Au}$.

65 miles E. Gardiner, N. P. R. R.

Basic lavas (?).

Veins.

Min. Res. 1906, p. 283.

1908 , pt. 1, p. 458

80. Sheepeater (Bear Gulch, Jardine). Au, Ag, W. (D, Pl.)

6 miles NE. Gardiner, N. P. R. R.

Pre-Cambrian gneiss, andesite, dacite.

Veine, stream gravels.

Min. Res. 1905, p. 256.

1906, p. 284.

1907 , pt. 1, p. 333.

1908, pt. 1, p. 458.

Top. sheet Livingston.

Folio 1, 1894.

POWELL COUNTY.

81. Big Blackfoot (Helmville). Au. (PI.)

22 miles NNE. Drummond, N. P. R. R., C. M. \& P. S. R. R.

Stream gravels.

Min. Res. 1909, pt. 1, p. 382.

Top. sheet Helena.

82. Coloma (Elk, Washoe, Garnet). $\mathrm{Au}, \mathrm{Ag}, \mathrm{Cu}$.

15 miles N. Bearmouth, 29 miles E. Bonner, N. P. R. R.

Paleozoic sediments cut by granite.

Veins.

Min. Res. 1905, p. 257.

1908 , pt. 1, p. 459.

1909, pt. 1, p. 382.

Top, sheet Bonner.

83. Gold Creek. $\mathrm{Ag}, \mathrm{Pb}, \mathrm{Au}, \mathrm{Cu}$. (Pl, D.)

10 miles SSW. Gold Creek, N. P. R. R., C. M. \& P. S. R. R.

Granite.

Veins, stream gravels.

Min. Res. 1906, p. 277.

1907, pt. 1, p. 325. 
POWELL COUNTY-Continued.

84. Ontario (Elliston). $\mathrm{Au}, \mathrm{Ag}, \mathrm{Pb}$.

Elliston station, N. P. R. R.

Paleozoic and Mesozoic sediments, andesite cut by granite.

Veins.

Min. Res. 1905, p. 257.

1906 , p. 284.

1907 , pt. 1, p. 334

1909 , pt. 1, p. 382 .

Top. sheets Boulder, Helena.

85. Ophir. $\mathrm{Au}, \mathrm{Ag}, \mathrm{Cu}, \mathrm{Pb}$.

9 miles N. Avon, N. P. R. R.

Paleozoic eediments cut by granite.

Contact metamorphic.

Min. Res. 1908, pt. 1, p. 459. 1909, pt. 1, p. 382 .

Top. sheet Helena.

86. Pioneer. Au, Ag. (Pl.)

Gold Creek station, N. P. R. R.

Stream gravele.

Min. Res. 1908, pt. 1, p. 459. 1909 , pt. 1, p. 382.

Top. sheet Helena.

87. Racetrack Creek. Au. (Pl.)

Station N. P. R. R.

Stream gravels.

Top. sheet Helena.

88. Washington Gulch (Finn). $\mathrm{Au}, \mathrm{Ag}, \mathrm{Pb}, \mathrm{Cu} . \quad$ (D, Pl.)

15 miles NNW. Avon, N. P. R. R.

Min. Res. 1907, pt. 1, p. 334.

1908 , pt. 1, p. 459

1909 , pt. 1, p. 382 .

Top. sheet Helena.

89. Zozell (Deer Lodge). $\mathrm{Au}, \mathrm{Ag}, \mathrm{Pb}$.

9 miles W. Deer Lodge, N. P. R. R., C. M. \& P. S. R. R.

Cretaceous andesites.

Veins.

Min. Res. 1905, p. 257.

1906 , p. 284.

1907 , pt. 1, p. 334 .

1908 , pt. 1, p. 459

1909 , pt. 1, p. 383 .

Top. sheet Helena.

90. Curlew. Pb, Ag.

\section{RAVALLI COUNTY.}

5 miles W. Victor, N. P. R. R.

Paleozoic sediments.

Veins.

Lindgren, W., Mineral deposits of the Bitterroot Range and Clearwater Mountains, Montana: Bull. 213, 1903, pp. 66-70.

- A geological reconnaissance across the Bitterroot Range and Clearwater Mountains in Montana and Idaho: P. P. 27, 1904, pp. 86-87.

Min. Res. 1906, p. 284.

1907 , pt. 1, p. 334 .

Top. sheet Hamilton. 
RAVALII COUNTY-Continued.

91. Hughes Creek (Alta). Au, Sn. (D, Pl.)

36 miles S. Darby, N. P. R. R.

Quartzite.

Stream gravels, veins.

Lindgren, W., Mineral deposits of the Bitterroot Range and Clearwater Mountains, Montana: Bull. 213, 1903, pp. 66-70.

— A geological reconnaissance across the Bitterroot Range and Clearwater Mountains in Montana and Idaho: P. P. 27, 1904, pp. 88-89.

Min. Res. 1909, pt. 1, p. 383.

92. Mineral Point. $\mathrm{Cu}, \mathrm{Ag}, \mathrm{Pb}$.

40 miles S. Darby, N. P. R. R.

Schists, granite, and gneise.

Veins.

Lindgren, W., Mineral deposits of the Bitterroot Range and Clearwater Mountains, Montana: Bull. 213, 1903, pp. 66-70.

A geological reconnaissance across the Bitterroot Range and Clearwater Mountains in Montana and Iduho: P. P. 27, 1904, p. 89.

93. Pleasant View. $\mathrm{Cu}, \mathrm{Ag}$.

5 miles W. Stevensville, N. P. R. R.

Granite.

Veins.

Lindgren, W., Mineral deposits of the Bitterroot Range and Clearwater Mountaine, Montana: Bull. 213, 1903, pp. 66-70.

$-\Lambda$ geological reconnaiseance acruss the Bitterroot Range and Clearwater Mountains in Montana and Idaho: P. P. 27, 1904, p. 87.

Top. sheet Hamilton.

94. Slate Creek. $\mathrm{Cu}, \mathrm{Ag}$.

30 miles $S$. Darby, N. P. R. R.

Granite porphyry.

Veins.

Lindgren, W., Mineral deposits of the Bitterroot Range and Clearwater Mountains, Montana: Bull, 213, 1903, pp. 66-70.

A geological reconnaissance across the Bitterroot Range and Clearwater Mountains in Montana and Idaho: P. P. 27, 1904, p. 88

SANDERS COUNTY.

95. Prospect Creek. $\mathrm{Au}, \mathrm{Ag}, \mathrm{Pb}$.

9 miles W. Thompson, N. P. R. R.

Pre-Cambrian sediments cut by basic dikee.

Veins.

Calkins, F. C., and MacDonald, D. F., A geological reconnaissance in northern Idaho and northwestern Montana: Bull. 384, 1909, p. 106.

MacDunald, D. F., Economic features of northern Idaho and northwestern Montana: Bull. 285, 1906, pp. 51-52.

Min. Res. 1908, pt. 1, p. 460.

96. Silver Butte (Vermilion, Cabinet). $\mathrm{Pb}, \mathrm{Ag}$.

10 miles N. Trout Creek, N. P. R. R., C. M. \& P. S. R. R.

Pre-Cambrian sediments.

Veins.

Calkins, F. C., and MacDonald, D. F., A geological reconnaiseance in northern Idaho and northwestern Montana: Bull. 384, 1909, p. 105.

97. Spring Gulch. $\mathrm{Au}, \mathrm{Cu}, \mathrm{Pb}, \mathrm{Ag}$.

10 miles E. St. Regis, N. P. R. R., C. M. \& P. S. R. R.

Min. Res. 1908, pt. 1, pp. 459-460.

1909, pt. 1, p. 383. 


\section{SILVER BOW COUTTY.}

97a. Fleecer Mountain. Au, Ag, Cu.

Paleozoic sediments cut by granite and andesite.

Veins, contact metamorphic.

Min. Res. 1909, pt. 1, p. 384.

98. German Gulch. $\mathrm{Au}, \mathrm{Ag}$.

15 miles W. Silver Bow, N. P. R. R.

Pre-Cambrian sediments cut by diorite.

Disseminated.

Min. Res. 1909, pt. 1, p. 384.

99. Highland (Siberia). $\mathrm{Au}, \mathrm{Ag}$.

20 miles ENE. Divide, O. S. L. R. R.

Pre-Cambrian and Paleozoic sediments cut by quartz monzonite.

Replacements and contact metamorphic.

100. Summit Valley (Butte). $\mathrm{Cu}, \mathrm{Ag}, \mathrm{Au}, \mathrm{Zn}$.

Station C. M. \& P. S. R. R., G. N. R. R., N. P. R. R,, O. S. L. R. R.,

Quartz monzonite cut by aplite, rhyolite.

Veins.

Harder, E. C., Manganese deposits of the United States: Bull. 380, 1909, pp. 273-274; Bull. 427, 1910, pp. 132-133.

Peters, E. D., Mines and reduction works of Butte City: Min. Res. 1883-84, pp. 374-396.

Weed, W. H., Ore deposits at Butte, Mont.: Bull. 213, 1902, pp. 170-180.

112-115

Min. Res. 1882, pp. 224-225.

1883-84, pp. 336-340.

1885 , pp. 216,349 .

1886, p. 117.

1888 , pp. $57-58$.

1891, pp. 90-99.

1900, pp. 157-158.

1904, pp. 232-233.

1905, pp. 257-259, 350-352.

1906, pp. 285-287, 399-401, 478.

1907, pt. 1, pp. 105, 334-337, 609-611, 713.

1908, pt. 1, pp. 211-212, 460-461, 722 .

1909, pt. 1, pp. 170, 384-386.

Top. sheets Helena, Butte special.

Folio 38, 1897. 


\section{NEVADA.}

In Nevada there are 182 mining districts in the 15 counties credited with the metallic wealth of the State. In 105 of these districts gold is the most valuable metal produced, in 38 silver, in 17 copper, in 14 lead, in 2 tungsten, in 1 iron, in 1 manganese, and in 1 zinc. Quicksilver was formerly mined exclusively in one locality. Two districts in the State are classed as uncertain-that is, the predominant metal is not known.

Distribution of the predominant metals produced in the mining districts of Nevada.

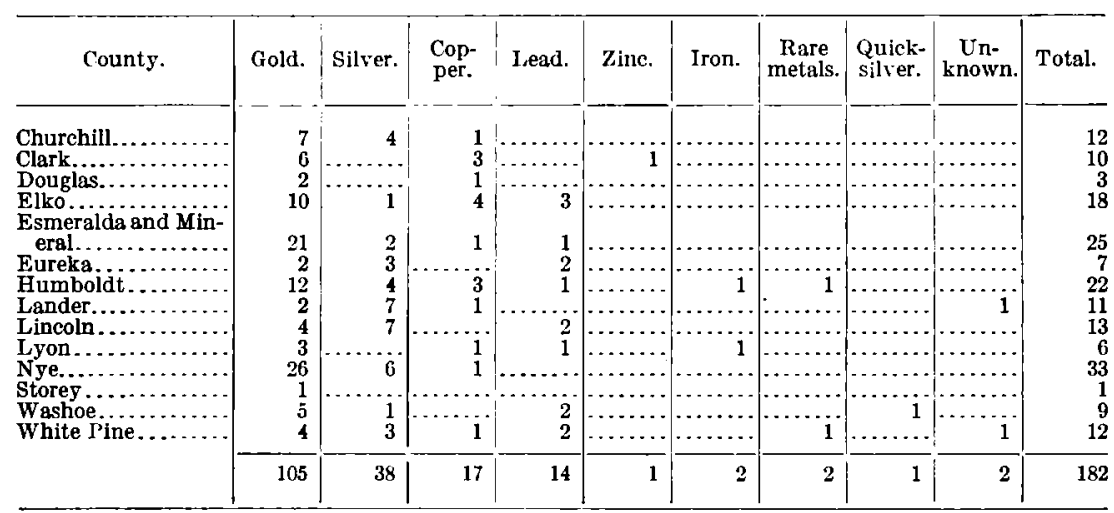

\section{MINING DISTRICTS IN NEVADA.}

[See Pl. IX. Additional references will be found in Mineral Resources for 1910 and 1911.]

\section{CHURCHILL COUNTY.}

1. Alpine. Ag, Au.

79 miles W. Fallon, S. P. R. R.

2. Bernice. Au, $\mathrm{Ag}, \mathrm{Sb}$.

60 miles ENE. Fallon, 75 miles SE. Lovelocks, S. P. R. R.

Min. Res. 1892, p. 260.

1906 , p. 512.

3. Boyer (Cottonwood Canyon). Au, $\mathrm{Ag}, \mathrm{Ni}$, Co.

65 miles SE. Lovelocks, S. P. R. R.

Triassic sediments cut by diorite capped by Tertiary volcanics.

Veins.

Ransome, F. I., Notes on some mining districts in Humboldt County, Nev.:

Bull. 414, 1909, pp. 55-58.

Min. Res. 1882, p. 404.

1883-84, p. 539.

1885 , pp. $299,361$.

1886, p. 171.

1887, p. 109.

1908, pt. 1, p. 470 . 
4. Eagleville (Hot Springs). Au, Ag. 64 miles SE. Fallon, S. P. R. R. Min. Res. 1908, pt. 1, p. 470.

Top. sheet Carson Sink.

5. Eastgate. Au, Ag. 90 miles SE. Fallon, S. P. R. R.

Min. Res. 1905, p. 266. 1906, p. 293. 1908 , pt. 1, p. 470.

6. Fairview. $\mathrm{Au}, \mathrm{Ag}$. 42 miles SSE. Fallon, S. P. R. R. Tertiary volcanics.

Veins.

Min. Res. 1905, p. 266.

1906, p. 293.

1907, pt. 1, pp. 344-345.

1908 , pt. 1, p. 471 .

1909, pt. 1, p. 394

Top. sheet Carson Sink.

7. I. X. I. $\mathrm{Ag}, \mathrm{Au}$.

35 miles SE. Lovelocks, S. P. R. R.

Min. Res. 1906, p. 293.

Top. sheet Carson Sink.

8. Jessup. Au.

35 miles S. Lovelocks, 10 miles NW. White Plains, S. P. R. R.

Min. Res. 1908, pt. 1, p. 471. 1909, pt. 1, p. 394.

Top. sheet Carson Sink.

9. West Gate. $\mathrm{Ag}, \mathrm{Pb}, \mathrm{Au}$.

54 miles ESE. Fallon, S. P. R. R.

Min. Res. 1905, p. 266.

Top. sheet Carson Sink.

10. White Cloud (Coppereid). $\mathrm{Cu}(\mathrm{Zn}, \mathrm{Fe})$.

35 miles SE. Lovelocks, S. P. R. R.

Triassic sediments cut by granite and diorite and capped by Tertiary volcanics.

Replacements, contact metamorphic.

Ransome, F. L., Notes on some mining districts in Humboldt County, Nev.: Bull. 414, 1909, pp. 59-62.

Min. Res. 1908, pt. 1, p. 472.

1909 , pt. 1, p. 394

11. White Plains. $\mathrm{Au}, \mathrm{Ag}$.

7 miles WSW. White Plains, 5 miles W. Huxley, S. P. R. R.

Min. Res. 1908, pt. 1, p. 471.

1909, pt. 1, p. 394.

Top. sheet Carson Sink.

12. Wonder (Hercules). Ag, Au.

40 miles ESE. Fallon, S. P. R. R.

Tertiary volcanics.

Veins.

Min. Res. 1905, p. 266.

1906, p. 293.

1907, pt. 1, p. 345 .

1908, pt. 1, p. 471-472. 


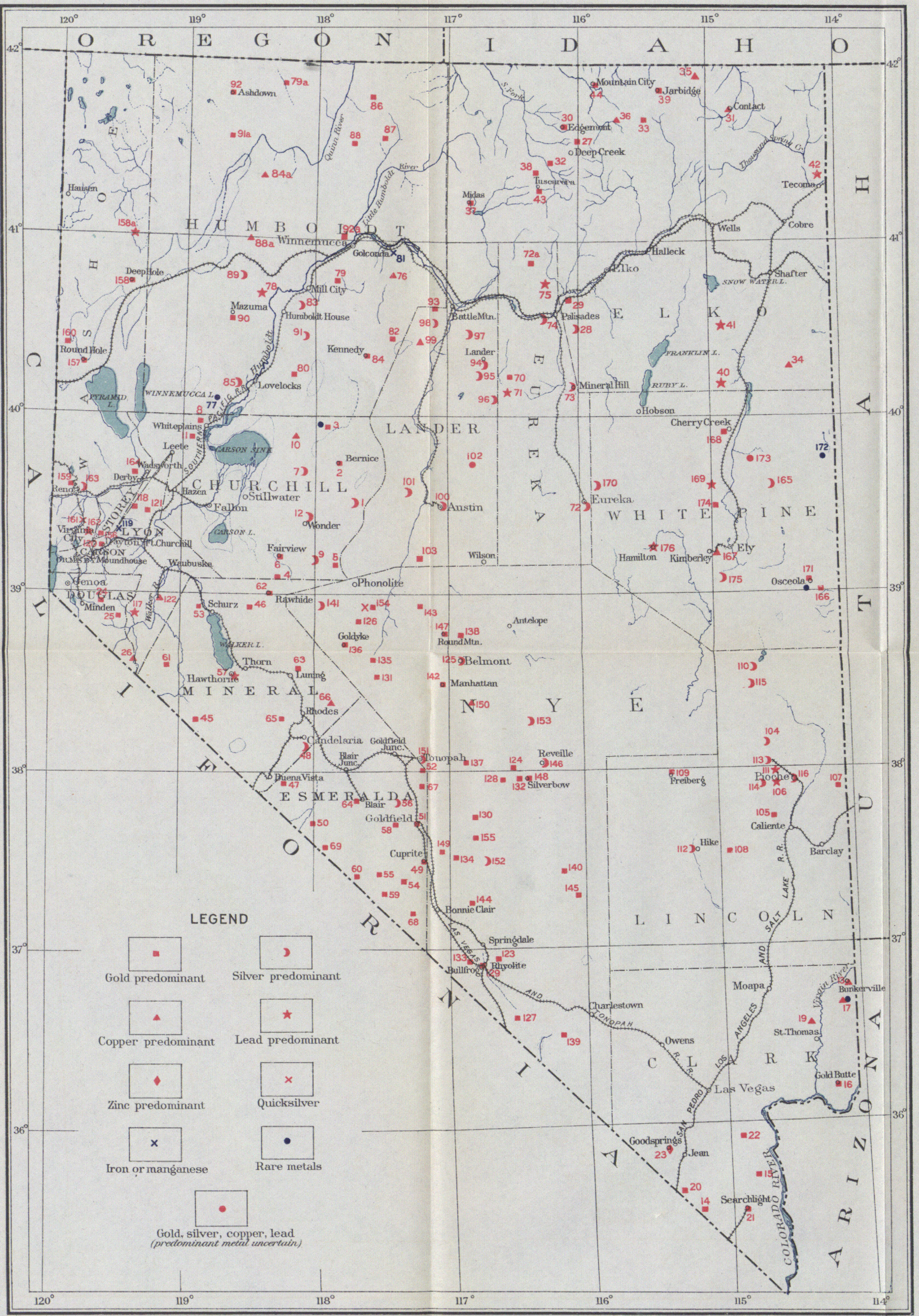

MAP OF NEVADA, SHOWING LOCATION OF MINING DISTRICTS
LIST OF MINING DISTRICTS

CHURCHILL COUNTY 9' Star (Rye Patch,

$\begin{array}{ll}\text { Alpine } & \text { gia. Varyville } \\ \text { Bernice } & \text { ga. Wisberg Ast }\end{array}$

$\begin{array}{ll}\text { Boyer (Cottonwood } & \text { 92. Vicksburg (Ashdown) } \\ \text { Canyon) } & 92 \text {. Winnemucca (Barrett Springs) }\end{array}$

EAgleville (Hot Springs) LANER COUNTY

Eastgate

I. X. L.

$\begin{array}{ll}\text { West Gate } & 95 . \text { Campbell (Lander) } \\ \text { White Cloud (Coppereid) } & \text { 96. Cortez (Mill Canyon) } \\ \text { White Plains } & \text { 97. Dean (Lewis, Mud }\end{array}$

Springs)
g. Kimberles (Hil

CLARK COUNTY 98. Galena (Copper Basin,

Bunkerville (Copper King)

Crescent (Copperkin

$\begin{array}{ll}\text { Eldorado Canyon (Nelson) } & \text { 102. Spencer } \\ \text { 103. Washington (Kingston) }\end{array}$

Logan (St.
Lyons

Searchlight

Yellow Pine (Good

Springs)

DOUGLAS COUNTY

Gardnerville

Wellington

ELKO COUNTY

Aura (Columbia)
Bullion (Railroad)

29. Carlin

Cornucopia
Cornwall Basin

Dolly Varden

Elk Mountains

Gold Basin (Rowland)
Gold Circle (Midas,

Summit)
Good Hope

Jarbidge

Spruce Mey

Tecoma

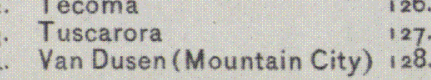

SSMERALDA AND MINERAL 129.

45. Aurora (Cambridge)

47. Buena Vista (Oneota)

9. Cuprite

Gold Mountain

Granite
Hornsilver (Lime Point)

Lida (Tule Canyon)

Lone Mountain
7. Lucky Boy (Hawthorne)

Oriental Wash

Palmetto

Pine Grove

Rawhide (Regent)
Santa Fe (Luning)

Silver Peakinineral Ridge)
Silver Star (Mina, Marietta)

LINCOLN COUNTY

104. Bristol
105. Chief
106. Comet

107. Eagle Valley (Fay, State

108. Ferguson (Delamar)

108. Ferguson
109. Freiberg

11i. Highland Valley

12. Hiko

14. Jackrabbit

115. Patterson

LYON COUNTY

7. Pine Nut

18. Ramsey

19. Red Mountain
120. Silver City (Paimyra

121. Tallapoosa
122. Yerington (Mason)

MINERAL COUNTY

NYE COUNTY

Bare Mountai

Bellehelen
Belmont (Philadelphia)

Berlin (Elsworth)

Big Dune

Bullfrog (Rhyolite)

. Gold Bar (Happy

Hooligan)
Gold Crater

Goldyke (Atwood)

7. Hannapah

(Concordia Green Isle)

Kawich (Gold Reed)

41. Lodi Valley (Marble)

Millett

Monte Cristo
Oak Spring

Reveille

Round Mountain

Silverbow Mountain

Superior

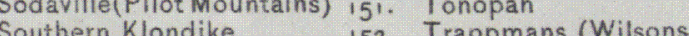

Southern Klondike 152 . Trappmans (Wilsons)

Windypah (Fesler)

EUREKA COUNTY

4. Unell

nion (lone)

Mill Canyon (Buckhorn)

Cortez (Bullion Hill, Mt.

STOREY COUNTY

Eureka (Pinto, Prospect)

WASHOE COUNTY

Mineral Hill

Safford

HUMBOLDT COUNTY

Adelaide (Gold Run)

Browns

79. Central (Chafey, Dun Glen)

Golconda

2. Gold Banks
$8_{3}$. Humboldt (Imlay)

4. Kennedy

85. Lovelock

87. Spring Creek ( Valley)

38. Rebel Creek

88a. Red Butte
89. Rosebud

Seven Troughs (Verno
Mazuma, Farrell)
Cottonwood (Round

Hole)
8. Deep Hole

8a. Leadville

Steamboat Springs
Washoe (Galena, Jumbo) 3edekind WHITE PINE COnghouse) 5. Aurum (Silver Canyon,

166. Black Horse

167. Ely (Robinson)
68. Gold Canyon (Cherry Creek)

69. Nunter ( 70. Newark (Strawberry)

171. Osceola (Tungsten)

172. Regan

173. Schellbourne
175. Ward (Taylor)
176. White Pine (Hamilton)

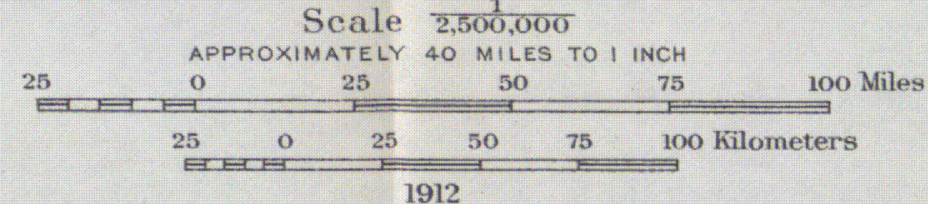




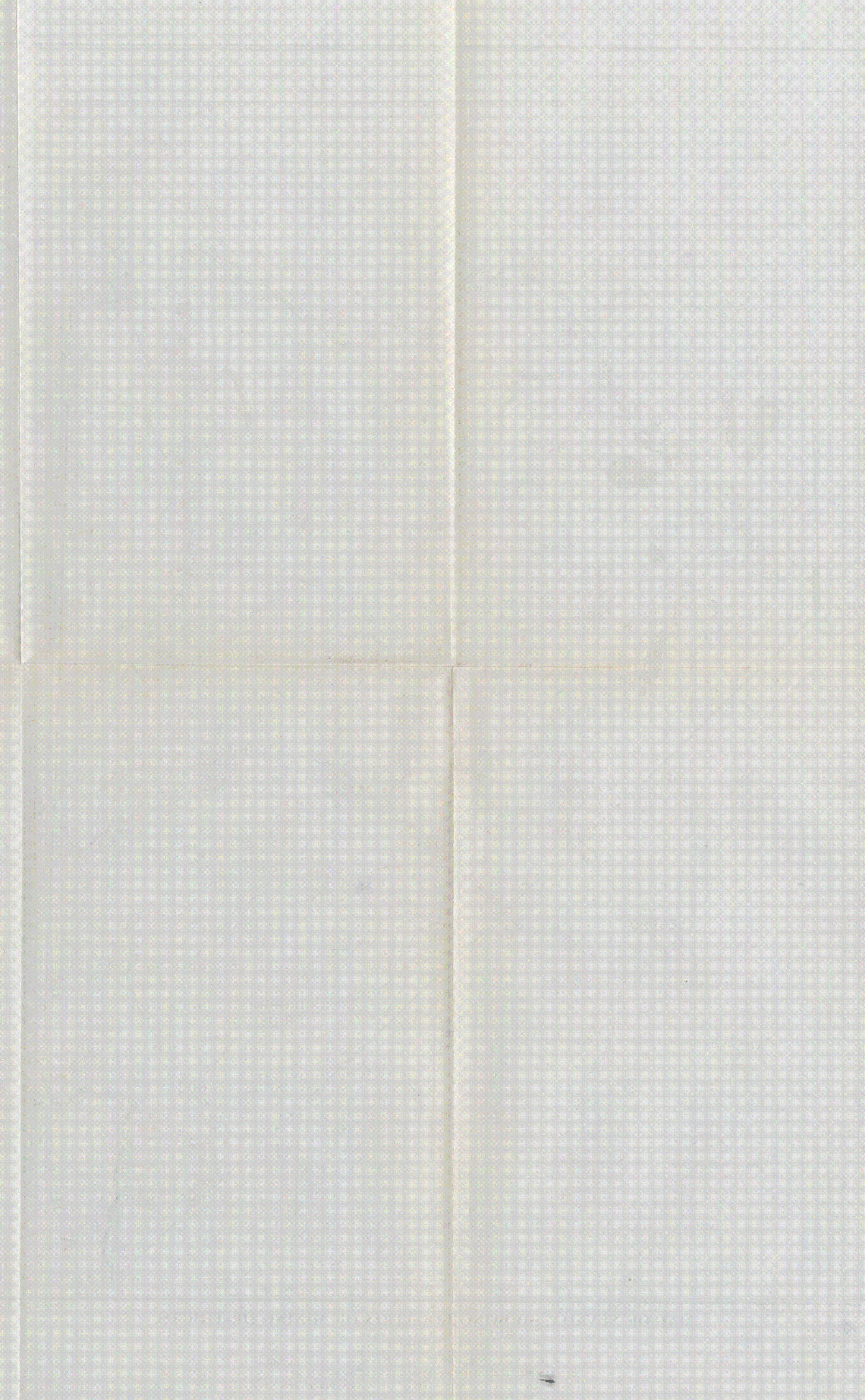


GHURCHILL COUNTY-Continued.

12. Wonder (Hercules)-Continued.

Min. Res. 1909, pt. 1, p. 394.

Top. sheet Carson Sink.

\section{CLARK COUNTY.}

13. Bunkerville (Copper King). $\mathrm{Cu}, \mathrm{Au}, \mathrm{Ag}$.

38 miles ENE. Moapa, S. P. L. A. \& S. L. R. R.

Paleozoic and Tertiary sediments cut by Tertiary lavas.

Veins.

Spurr, J. E., Geology of Nevada south of the fortieth parallel: Bull. 208, 1903, pp. 131-132.

Min. Res. 1906, p. 560.

1907, pt. 1, p. 365.

1908 , pt. 1, pp. 472, 736, 783.

1909 , pt. 1, p. 395.

Top. sheet St. Thomas.

14. Crescent. $\mathrm{Au}, \mathrm{Ag}, \mathrm{Pb}$.

6 mile E. Nipton, S. P. L. A. \& S. L. R. R.

Pre-Cambrian granite, quartzite cut by acidic and basic dikes.

Veins.

Ransome, F. L., Preliminary account of Goldfield, Bullfrog, and other mining districts in southern Nevada: Bull. 303, 1907, p. 79.

Min. Res. 1905, p. 270.

1906, pp. 296-297.

1907 , pt. 1, pp. $365-366$.

1908 , pt. 1, p. 472.

1909, pt. 1, p. 395.

15. Eldorado Canyon (Nelson). Au, Ag.

24 miles NNE. Searchlight, B. \& S. R. R.

Pre-Cambrian granite and gneise cut by acidic intrusives and capped by Tertiary volcanics.

Veins.

Ransome, F. L., Preliminary account of Goldfield, Bullfrog, and other mining districts in southern Nevada: Bull. 303, 1907, pp. 63-76.

Min. Res. 1905, p. 270.

1907, pt. 1, p. 366.

1908, pt. 1, p. 473.

1909 , pt. 1, p. 395 .

Top. sheet Camp Mohave.

16. Gold Butte. Au.

51 miles SE. Moapa, S. P. L. A. \& S. L. R. R.

Pre-Cambrian complex cut by acidic and basic dikes.

Veins.

Min. Res. 1905, p. 270.

1906, p. 297.

1907 , pt. 1, p. 367.

1908 , pt. 1 , p. 472.

1909 , pt. 1, p. 395.

Top. sheet St. Thomas.

17. Great Eastern. $\mathrm{Cu}, \mathrm{Ni}, \mathrm{Co}, \mathrm{Pt}$.

28 miles E. Moapa, S. P. L. A. \& S. L. R. R.

Pre-Cambrian gneiss and granite cut by acidic and basic dikes.

Disseminated.

Bancroft, H., Platinum in southeastern Nevada: Bull. 430, 1910, pp. 192-199. 
CLAR K COUNTY-Continued.

17. Great Eastern-Continued.

Spurr, J. E., Geology of Nevada south of the fortieth parallel: Bull. 208, 1903, pp. 131-132.

Min. Res. 1908, pt. 1, p. 783.

18. Omitted.

Top. sheet St. Thomas.

19. Logan (St. Thomas). Cu, Ag.

26 miles SE. Moapa, S. P. L. A. \& S. L. R. R.

Paleozoic and Mesozoic sediments capped by Tertiary volcanics.

Veins.

Spurr, J. E., Geology of Nevada south of the fortieth parallel: Bull. 208, 1903, pp. 136-138.

Min. Res. 1902, p. 141.

1908, pt. 1, p. 473 .

Top. sheet St. Thomas.

20. Lyons. Au.

15 miles SE. Jean, S. P. L. A. \& S. L. R. R.

Granite.

Veins.

Min. Res. 1907, pt. 1, p. 367.

21. Searchlight. $\mathrm{Au}, \mathrm{Ag}, \mathrm{Cu}, \mathrm{Pb}$.

Station B. \& S. R. R.

Pre-Cambrian complex cut by quartz monzonite and capped by Tertiary volcanics.

Veins.

Ransome, F. L., Preliminary account of Goldfield, Bullfrog, and other mining districts in southern Nevada: Bull. 303, 1907, pp. 63-76.

Min. Res. 1905, p. 270.

1906, p. 296-297.

1907 , pt. 1, pp. 368-369.

1908 , pt. 1 , p. $473-474$.

1909 , pt. 1, pp. 395-396.

Top. sheet Camp Mohave.

22. Vincent (Railroad Pass). Au, Ag.

12 miles E. Erie, S. P. L. A. \& S. L. R. R.

Top. sheet Camp Mohave.

23. Yellow Pine (Goodsprings). $\mathrm{Zn}, \mathrm{Pb}, \mathrm{Cu}, \mathrm{Au}, \mathrm{Ag}$.

8 miles NW. Jean, S. P. L. A. \& S. L. R. R.

Paleozoic sediments, cut by dikes.

Replacement.

Bain, II. F., A Nevada zinc deposit: Bull. 285, 1906, pp. 166-169.

Spurr, J. E., Geology of Nevada south of the fortieth parallel: Bull. 208, 1903, pp. 164-180.

Min. Res. 1905, p. 270.

1906, pp. $296,478$.

1907 , pt. 1, p. 369.

1908 , pt. 1, p. 474.

1909 , pt. 1 , pp. $396-397$.

DOUGLAS COUNTY.

24. Gardnerville. Au. (D, Pl.)

3 miles S. Minden, V. \& T. R. R.

Diorite, Tertiary volcanics and lake beds.

Veins. 
DOUGLAS COUNTY_Continued.

24. Gardnerville-Continued.

Spurr, J. E., Geology of Nevada south of the fortieth parallel: Bull. 208, 1903, pp. 120-126.

Min. Res., 1905, p. 266.

1907, pt. 1, p. 346 .

1908, p.. 1, p. 475.

Top. sheet Markleeville.

25. Red Canyon. $\mathrm{Au}, \mathrm{Ag}, \mathrm{Pb}$.

18 miles SE. Minden, V. \& T. R. R.

Granite and Tertiary volcanics.

Veins.

Spurr, J. E., Geology of Nevada south of the fortieth parallel: Bull. 208, 1903, pp. 120-126.

Min. Res. 1908, pt. 1, p. 475.

Top. sheets Markleeville, Wellington.

26. Wellington. $\mathrm{Cu}$.

31 miles SSE. Minden, V. \& T. R. R.

Tertiary volcanics.

Veins.

Spurr, J. E., Geology of Nevada south of the fortieth parallel: Bull. 208, 1903, pp. 120-126.

Min. Res. 1908, pt. 1, p. 475.

Top. sheet Wellington.

\section{ELKO COUNTY.}

27. Aura (Columbia). $\mathrm{Au}, \mathrm{Ag}(\mathrm{Pb}, \mathrm{Zn})$.

79 miles NNW. Elko, S. P. R. R.

Paleozoic sediments cut by granodiorite.

Veins.

Emmons, W. H., A reconnaissance of some mining camps in Elko, Lander, and Eureka counties, Nev.: Bull. 408, 1910, pp. 71-74.

Min. Res. 1906, p. 293.

1907, pt. 1, p. 346.

1909, pt. 1, p. 398.

28. Bullion (Railioad). $\mathrm{Ag}, \mathrm{Au}, \mathrm{Cu}, \mathrm{Pb}, \mathrm{Zn}$.

27 miles SSW. Elko, 12 miles SE. Palisade, S. P. R. R., W. P. R. R.

Paleozoic sediments cut by granodiorite.

Replacements, contact metamorphic, veins.

Emmons, W. H., A reconnaissance of some mining camps in Elko, Lander, and Eureka counties, Nev.: Bull. 408, 1910, pp. 88-95.

Weed, W. H., The copper mines of the United States in 1905: Bull. 285, 1906, p. 116.

Min. Res. 1906, p. 293.

1908, pt. 1, p. 477.

1909 , pt. 1 , pp. $399-400$.

29. Carlin. Au, Ag. (D, Pl.)

Station S. P. R. R., W. P. R. R.

Paleozoic sediments cut and capped by Tertiary volcanico.

Veins, replacements.

Min. Res. 1908, pt. 1, p. 476.

30. Centennial (Edgemont). Au, Ag (Pb).

92 miles NNW. Elko, S. P. R. R., W. P. R. R.

Paleozoic sediments.

Veins. 


\section{ELTKO COUNT Y-Continued.}

30. Centennial (Edgemont)-Continued.

Emmons, W. H., A reconnaissance of some mining camps in Elko, Lander, and Eureka counties, Nev : Bull. 408, 1910, pp. 75-79.

Min. Res. 1905, p. 266.

1906 , p. 293.

1907 , pt. 1, p. 346.

1908 , pt. 1, p. 476.

31. Contact. $\mathrm{Cu}, \mathrm{Ag}$. 1909 , pt. 1, p. 398.

89 miles NW. Tecoma, S. P. R. R.

Paleozoic sediments cut by granite.

Contact metamorphic, veins.

Schrader, F. C., A reconnaissance of the Jarbidge, Contact, and Elk Mountain mining districts, Elko County, Nev.: Bull. 497, 1912.

Min. Res. 1908, pt. 1, p. 476. 1909, pt. 1, p. 398.

32. Cornucopia. Au, Ag.

58 miles NNW. Elko, S. P. R. R., W. P. R. R.

Tertiary volcanics.

Veins.

Emmons, W. H., A reconnaissence of some mining camps in Elko, Lander, and Eureka counties, Nev.: Bull. 408, 1910, p. 63.

33. Cornwall Basin (Charleston). $\mathrm{Au}, \mathrm{Cu}$.

95 miles NNE. Elko, 50 miles NNW. Deeth, S. P. R. R., W. P. R. R.

Paleozoic sediments cut by granite.

Contact metamorphic, veins.

Min. Res. 1908, pt. 1, p. 476.

34. Dolly Varden. $\mathrm{Cu}, \mathrm{Pb}, \mathrm{Ag}$.

20 miles E. Mizpah, N. N. R. R.

Min. Res. 1908, pt. 1, p. 476.

1909 , pt. 1, p. 398.

35. Elk Mountain. $\mathrm{Cu}, \mathrm{Au}, \mathrm{Sb}$.

90 miles SSE. Twin Falls, Idaho, O. S. L. R. R.

Paleozoic sediments cut by granite.

Contact metamorphic.

Schrader, F. C., A reconnaiseance of the Jarbidge, Contact, and Elk Mountain mining districts, Elko County, Nev.: Bull. 497, 1912.

36. Gold Basin (Rowland). Cu, Au.

85 miles S. Twin Falls, Idaho, O. S. L. R. R.

37. Gold Circle (Midas, Summit). Au, Ag.

48 miles ENE. Golconda, S. P. R. R., W. P. R. R.

Tertiary volcanics.

Veins.

Emmons, W. H., A reconnaissance of some mining camps in Elko, Lander, and Eureka counties, Nev.: Bull. 408, 1910, pp. 48-57.

Min. Res. 1907, pt. 1, p. 348.

1908 , pt. 1 , p. 477.

1909 , pt. 1, p. 399 .

38. Good Hope. $\mathrm{Au}, \mathrm{Ag}$.

55 miles NW. Elko, S. P. R. R., W. P. R. R.

Tertiary volcanics.

Veins.

Emmons, W. H., A reconnaissance of some mining camps in Elko, Lander, and Eureka counties, Nev.: Bull. 408, 1910, p. 65. 
ELKO COU NTT $\mathbf{Y}$-Continued.

39. Jarbidge. Au, Ag.

95 miles S. Twin Falls, Idaho, O. S. L. R. R.; 74 miles N. Elko, S. P. R. R., W. P. R. R.

Tertiary volcanics.

Veins.

Schrader, F. C., A reconnaissance of the Jarbidge, Contact, and Elk Mountain mining districts, Elko County, Nev.: Bull. 497, 1912.

Min. Res. 1909, pt. 1, p. 399.

40. Ruby Valley. $\mathrm{Pb}, \mathrm{Ag}$.

I5 miles W. Currie, N. N. R. R.

Min. Res. 1908, pt. 1, p. 477.

41. Spruce Mountains. $\mathrm{Pb}, \mathrm{Cu}, \mathrm{Ag}, \mathrm{Au}$.

58 miles SE. Wells, S. P. R. R.; 15 miles NW. Mizpah, N. N. R. R.; 15 miles S. Jasper, W. P. R. R.

Paleozoic sediments cut by rhyolite.

Replacements.

Min. Res. 1905, p. 266.

1906, p. 293.

1907, pt. 1, p. 347.

1908 , pt. 1, p. 477.

1909, pt. 1, p. 400.

42. Tecoma. $\mathrm{Pb}, \mathrm{Ag}, \mathrm{Cu}, \mathrm{Au}$.

10 miles NNE. Tecoma, S. P. R. R.

Min. Res. 1908, pt. 1, p. 477.

43. Tuscarora. Au, Ag. 1909 , pt. 1, p. 400.

50 miles NW. Elko, S. P. R. R., W. P. R. R.

Tertiary volcanics.

Veins.

Emmons, W. H., A reconnaissance of some mining camps in Elko, Lander, and Eureka counties, Nev.: Bull. 408, 1910, pp. 57-62.

Min. Res. 1905, p. 266.

1906, p. 293.

1907, pt. 1, p. 347.

1908, pt. 1, p. 478.

1909 , pt. 1, p. 400 .

44. Van Dusen (Mountain City). $\mathrm{Au}, \mathrm{Ag}(\mathrm{Pb}, \mathrm{Zn})$.

102 miles N. Elko, S. P. R. R., W. P. R. R.

Paleozoic sediments cut by granodiorite and capped by Tertiary volcanics. Veins.

Emmons, W. H., A reconnaissance of some mining camps in Elko, Lander, and Eureka counties, Nev.: Bull. 408, 1910, pp. 80-84.

Min. Res. 1905, p. 266.

1906, pp. 293, 346.

1907, pt. 1, p. 476 .

ESMERALDA AND MINERAL COUNTIES.

45. Aurora (Cambridge). Au, Ag.

37 miles $\mathrm{SW}$. Thorne, S. P. R. R

Tertiary volcanics.

Veins.

Spurr, J. E., Geology of Nevada south of the fortieth parallel: Bull. 208, 1903, pp. 117-118.

Min. Res. 1905, p. 267. 
ESHERALDA AND MINERAL COUNTIES-Continued.

45. Aurora (Cambridge)-Continued.

Min. Res. 1906, p. 294.

1907 , pt. 1, p. 349.

1908, pt. 1, p. 479.

1909 , pt. 1, pp. $401-402$.

Top. sheet Hawthorne.

46. Bovard. Au, Ag.

28 miles E. Schurz, S. P. R. R.

Tertiary volcanics.

Veins.

Spurr, J. E., Geology of Nevada south of the fortieth parallel: Bull. 208, 1903, pp. 106-107.

Min. Res. 1908, pt. 1, p. 485.

Top. sheet Hawthorne.

47. Buena Vista (Oneota). $\mathrm{Au}, \mathrm{Ag}, \mathrm{Pb}, \mathrm{Cu}, \mathrm{Zn}$.

Station, S. P. R. R.

Tertiary volcanics.

Veins.

Spurr, J. E., Geology of Nevada south of the fortieth parallel: Bull. 208. 1903, pp. 206-212.

Min. Res. 1905, p. 268.

1907, pt. 1, p. 349 .

1908 , pt. 1, p. 479

1909 , pt. 1, p. 402.

48. Columbus (Candelaria). $\mathrm{Ag}, \mathrm{Au}, \mathrm{Pb}$.

Station, S. P. R. R.

Paleozoic sediments, Tertiary volcanics.

Veins.

Spurr, J. E., Geology of Nevada south of the fortieth parallel: Bull. 208, 1903, pp. 113-114.

Min. Res. 1906, p. 294.

1909 , pt. 1, p. 406.

Top. sheet Hawthorne.

49. Cuprite. Au, Ag.

Station, B. G. R. R.

Cambrian sediments cut by dikes of diorite and Tertiary volcanics.

Veins and replacements.

Ball, S. H., Notes on the ore deposits of southwestern Nevada and eastern California: Bull. 285, 1906, pp. 59-61; A geologic reconnaissance in southwestern Nevada and eastern California: Bull. 308, 1907, pp. 46, 69-71.

Min. Res. 1908, pt. 1, p. 483.

Top. sheet Lida.

50. Dyer. Au, Ag.

28 miles S. Coaldale. T. \& G. R. R.

Paleozoic sediments cut by granite.

Veins.

Spurr, J. E., Ore deposits of the Silver Peak quadrangle, Nevada: Bull. 225, 1904, p. 115; P. P. 55, 1906, pp. 84-85.

Top. sheet Silver Peak.

51. Goldfield. Au, Ag.

Station, T. \& G. R. R., B. G. R. R., L. V. \& T. R. R.

Tertiary volcanics underlain by Cambrian sediments cut by granite.

Veins, replacements. 
ESMERALDA AND MTHERAL COUNTIES-Continued.

51. Goldfield-Continued.

Ball, S. H., A geologic reconnaissance in southwestern Nevada and eastern California: Bull. 308, 1907, pp. 28, 32-34, 71-76, 107.

Ransome, F. L., Preliminary account of Goldfield, Bullfrog, and other mining districts in southern Nevada: Bull. 303, 1907, pp. 7-39.

—. The geology and ore deposits of Goldfield, Nev.: P. P. 66, 1909.

Spurr, J. E., Notes on the geology of the Goldfields district, Nevada: Bull. 225,1904 , pp. 118-119.

The ores of Goldfield, Nev.: Bull. 260, 1905, pp. 132-139.

Min. Res. 1905, p. 267.

1906, pp. 293-294.

1907 , pt. 1 , pp. 349-353, 391-394.

1908 , pt. 1 , pp. $479-482,714$.

1909 , pt. 1 , pp. $402-405$.

Top. sheets Lida, Goldfield special.

52. Gold Mountains. Au, Ag.

7 miles S. Tonopah, T. \& G. R. R.

Tertiary volcanics.

Veins.

Spurr, J. E., Ore deposits of Tonopah and neighboring districts, Nevada: Bull. 213, 1903, p. 87.

Top. sheet Lida.

53. Granite. $\mathrm{Au}, \mathrm{Cu}$.

8 miles W. Schurz, S. P. R. R.

Granite capped by Tertiary volcanics.

Veins.

Spurr, J. E., Geology of Nevada south of the fortieth parallel: Bull. 208, 1903, pp. 115, 117, 118.

Min. Res. 1907, pt. 1, p. 355.

Top. sheet Hawthorne.

54. Hornsilver (Lime Point). Au, $\Lambda \mathrm{g}, \mathrm{Pb}$.

16 miles SW. Cuprite, B. G. R. R.

Granite, Tertiary volcanics.

Veins.

Ball, S. H., Notes on the ore deposits of southwestern Nevada and eastern California: Bull. 285, 1906, p. 62.

Ransome, F. L., The Hornsilver district, Nevada: Bull. 380, 1909, pp. 41-43.

Min. Res. 1907, pt. 1, p. 354.

1908 , pt. 1, p. 483.

Top. sheet Lida.

1909 , pt. 1, p. 405 .

55. Lida (Tule Canjon). Au, Ag, $\mathrm{Pb}, \mathrm{C} u$.

30 miles SW. Goldfield, T. \& G. R. R., B. G. R. R., L. V. \& T. R. R.

Cambrian sediments cut by quartz monzonite, and capped by Tertiary volcanics.

Veins and impregnations.

Ball, S. H., Notes on the ore deposits of southwestern Nevada and eastern California: Bull. 285, 1906, pp. 61-62.

A geologic reconnaissance in southwestern Nevada and eastern California: Bull. 308, 1907, pp. 25, 31, 45, 57, 60-65.

Spurr, J. E., Geology of Nevada south of the fortieth parallel: Bull. 208, 1903, pp. 184-186.

Min. Res. 1906, p. 294.

1907 , pt. 1, p. 354 . 
ESMERALDA AND MINERAL COUNTIES-Continued.

55. Lida (Tule Canjon)-Continued.

Min. Res. 1908, pt. 1, p. 483.

Top. sheet Lida. 1909 , pt. 1, p. 405.

56. Lone Mountains. $\mathrm{Ag}, \mathrm{Pb}, \mathrm{Cu}, \mathrm{Au}$.

14 miles NNW. Goldfield, T. \& G. R. R.

Cambrian sediments cut by granite and diorite, capped by Tertiary volcanics.

Veins and replacements.

Ball, S. H., Notes on the ore deposits of southwestern Nevada and eastern California: Bull. 285, 1906, pp. 57-58.

- A geologic reconnaissance in southwestern Nevada and eastern California: Bull. 308, 1907, pp. 28, 31, 46, 51-55.

Spurr, J. E., Ore deposits of the Silver Peak quadrangle, Nevada: Bull. 225, 1904, pp. 114-115; P. P. 55, 1905, pp. 75-83.

Min. Res. 1906, p. 294.

Top. sheet Lida.

1908 , pt. 1, p. 483.

1909 , pt. 1, p. 405.

57. Lucky Boy (Hawthorne). $\mathrm{Pb}, \mathrm{Ag}$.

7 miles SW. Thorne, S. P. R. R.

Cambrian sediments cut by granite and diorite.

Veins.

Min. Res. 1907, pt. 1, p. 353.

1908 , pt. 1, pp. $482,483$.

1909 , pt. 1, p. 405 .

Top. sheet Hawthorne.

58. Montezuma. $\mathrm{Au}, \mathrm{Ag}, \mathrm{Pb}, \mathrm{Cu}$.

7 miles W. Goldfield, T. \& G. R. R., B. G. R. R., L. V. \& T. R. R.

Cambrian sediments cut by granite and diorite and capped by Tertiary volcanics.

Veins and replacements.

Ball, S. H., Notes on the ore deposits of southwestern Nevada and eastern California: Bull. 285, 1906, pp. 58-59.

- A geologic reconnaissance in southwestern Nevada and eastern California: Bull. 308, 1907, pp. 63-64.

Min. Res. 1905, pp. 267-268.

Top. sheet Lida. 1908 , pt. 1, p. 484

59. Oriental Wash. $\mathrm{Au}, \mathrm{Ag}, \mathrm{Cu}$.

45 miles SW. Goldfield, T. \& G. R. R., B. G. R. R., I. V. \& T. R. R.

Cambrian sediments cut by granite.

Veins.

Ball, S. H., Notes on the ore deposits of southwestern Nevada and eastern California: Bull. 285, 1906, pp. 64-65.

- A geologic reconnaissance in southwestern Nevada and eastern Cali. fornia: Bull. 308, 1907, pp. 45, 190-191.

Top. sheet Lida.

60. Palmetto. $\mathrm{Au}, \mathrm{Ag}, \mathrm{Pb}$.

45 miles SW. Goldfield, T. \& G. R. R., B. G. R. R., L. V. \& T. R. R.

Paleozoic sediments cut by granite.

Veins, contact metamorphic.

Spurr, J. E., Ore deposits of the Silver Peak quadrangle, Nevada: Bull. 225, 1904, p. 116; P. P. 55, 1905, pp. 92-96.

Min. Res. 1907, pt. 1, p. 355 .

1908, pt. 1, p. 484.

Top. sheet Silver Peak. 
ESMERALDA AND MINERAL COUNTIES-Contlnued.

61. Pine Grove. Au, Ag.

30 miles S. Yerington, N. C. B. R. R.

Min. Res. 1907, pt. 1, p. 359.

1908, pt. 1, p. 484

1909, pt. 1, p. 406.

Top. sheet Hawthorne.

62. Rawhide (Regent). Au, Ag.

28 miles E. Schurz, S. P. R. R.

Tertiary volcanics.

Veins.

Min. Res. 1907, pt. 1, p. 356.

1908 , pt. 1, pp. $484-485$.

1909 , pt. 1, p. 406 .

Top. sheet Hawthorne.

63. Santa $\mathrm{Fe}$ (Luning). $\mathrm{Au}, \mathrm{Cu}, \mathrm{Ag}, \mathrm{Pb}, \mathrm{Sb}$.

Station S. P. R. R.

Min. Res. 1882, p. 230.

1908, pt. 1, p. 484

1909 , pt. 1, pp. 406-407.

Top. sheet Hawthorne.

64. Silver Peak (Mineral Ridge). Au, Ag.

Blair station, S. P. R. R.

Paleozoic sediments cut by granite and diorite, capped by Tertiary volcanics.

Veins.

Spurr, J. E., Ore deposits of Tonopah and neighboring districts, Nevada: Bull. 213, 1902, pp. 85-86.

- Ore deposits of the Silver Peak quadrangle, Nevada: Bull. 225, 1904, pp. 112-114; P. P. 55, 1905, pp. 34-74.

- Geology of Nevada south of the fortieth parallel: Bull. 208, 1903, pp. 184-186.

Min. Res. 1905, p. 268.

1906, p. 294.

1907, pt. 1, pp. 354-355.

1908 , pt. 1, p. 486.

1909, pt. 1, p. 407.

Top. sheet Silver Peak.

65. Silver Star (Mina, Marietta). $\mathrm{Au}, \mathrm{Cu}, \mathrm{Ag}, \mathrm{Pb}$.

10 miles W. Mina, S. P. R. R.

Min. Res. 1905, p. 268.

1906, p. 294.

1907, pt. 1, p. 354 .

1908, pt. 1, pp. 484, 486.

1909 , pt. 1, p. 407.

Top. sheet Hawthorne.

66. Sodaville (Pilot Mountains). $\mathrm{Cu}$.

18 miles E. Sodaville, S. P. R. R.

Tertiary sediments cut by granite and capped by basalt.

Veins.

Spurr, J. E., Geology of Nevada south of the fortieth parallel: Bull. 208, 1903, pp. 103-105.

Weed, W. H., The copper mines of the United States in 1905: Bull. 285, 1906, p. 116.

Min. Res. 1882, p. 230.

$21528^{\circ}-$ Bull. $507-12-14$ 
ESMERALDA AND MINERAL COUNTIES-Continued.

66. Sodaville (Pilot Mountains)-Continued.

Min. Res. 1898 (Twentieth Ann. Rept., pt. 6), p. 185.

1904, p. 375.

1905 , p. 268.

1908, pt. 1, p. 484.

Top. sheet Tonopah.

67. Southern Klondike. $\mathrm{Au}, \mathrm{Ag}, \mathrm{Pb}$.

14 miles S. Tonopah, T. \& G. R. R.

Pre-Cambrian sediments cut by granite and capped by Tertiary volcanics.

Veins.

Spurr, J. E., Ore deposits of Tonopah and neighboring districts, Nevada: Bull. 213, 1903, pp. 86-87.

Ball, S. H., Notes on the ore deposits of southwestern Nevada and eastern California: Bull. 285, 1906, p. 58.

- A geologic reconnaissance in southweetern Nevada and eastern California: Bull. 308, 1907, pp. 28, 32, 33, 44-49, 77-81.

Min. Res. 1905, p. 268.

1906, p. 284.

1909 , pt. 1, p. 405.

Top. sheet Lida.

68. Tokop (Gold Mountains). $\mathrm{Au}, \mathrm{Ag}, \mathrm{Cu}$.

15 miles W. Bonnie Clare, B. G. R. R., L. V. \& T. R. R.

Cambrian sediments cut by granite and capped by Tertiary volcanics.

Veins.

Ball, S. H., Notes on the ore deposits of southwestern Nevada and eastern California: Bull. 285, 1906, pp. 63-64.

- A geologic reconnaissance in southwestern Nevada and eastern California: Bull. 308, 1907, pp. 188-189.

Ransome, F. L., Preliminary account of Goldfield, Bullfrog, and other mining districts in southern Nevada: Bull. 303, 1907, pp. 80-81.

Min. Res. 1905, p. 267.

1906, p. 482.

1909 , pt. 1, p. 405.

Top. sheet Lida.

69. Windypah (Fesler). Au, Ag.

25 miles SW. Blair, S. P. R. R.

Paleozoic sediments cut by granite.

Veins.

Spurr, J. E., Ore deposits of the Silver Peak quadrangle, Nevada: Bull. 225, 1904, pp. 115-116; P. P. 55, 1905, pp. 85-92.

Top. sheet Silver Peak.

EUREKA COUNTY.

70. Mill Canyon (Buckhorn). Au.

35 miles SSW. Palisade, S. P. R. R., W. P. R. R.

Granodiorite.

Veins.

Emmons, W. H., A reconnaissance of some mining camps in Elko, Lander, and Eureka counties, Nev.: Bull. 408, 1910, p. 106.

Min. Res. 1909, pt. 1, p. 408.

71. Cortez (Bullion Hill, Mount Tenabo). $\mathrm{Pb}, \mathrm{Ag}, \mathrm{Au}, \mathrm{Cu}, \mathrm{Zn}$.

30 miles S. Beowawe, S. P. R. R., W. P. R. R.

Paleozoic sediments cut by granite and porphyries and capped by Tertiary volcanics.

Replacements, veins. 
EURERA COUNTY-Continued.

71. Cortez (Bullion Hill, Mount Tenabo)-Continued.

Emmons, W. H., A reconnaissance of some mining camps in Elko, Lander, and Eureka counties, Nev.: Bull. 408, 1910, pp. 100-110.

Weed, W. H., The copper mines of the United States in 1905: Bull. 285, 1906, p. 116.

Min. Res. 1905, p. 269.

1906 , p. 295.

1907, pt. 1, p. 358.

1908 , pt. 1, p. 487.

1909 , pt. 1, p. 408.

72. Eureka (Pinto, Prospect, Ruby Hill). $\mathrm{Ag}, \mathrm{Pb}, \mathrm{Au}, \mathrm{Cu}, \mathrm{Zn}, \mathrm{Fe}$.

Station E. \& P. R. R.

Paleozoic sediments, granite porphyry, rhyolite.

Replacements, veins.

Curtis, J. S., Abstract of report on the mining geology of the Eureka district, Nevada: Fourth Ann. Rept., 1884, pp. 221-251.

Silver-lead deposits of Eureka, Nev.: M. VII, 1884.

Hague, A., Abstract of the report on the geology of the Eureka district, Nevada: Third Ann. Rept., 1883, pp. 237-290.

Geology of the Eureka district, Nevada: M. XX, 1892.

Harder, E. C., Manganese deposits of the United States: Bull. 380, 1909, p. 274.

Spurr, J. E., Geology of Nevada south of the fortieth parallel: Bull. 208, 1903, pp. 81-84.

Walcott, C. D., Paleontology of the Eureka district: M. VIII, 1884.

Min. Res. 1882, p. 309.

1883-84, pp. $418-419,462-473$.

1885 , p. 250.

1886 , p. 143.

1887, p. 104.

1888 , p. 86.

1905, p. 268.

1906, pp. 295, 450 .

1907, pt. 1, p. 357 .

1908 , pt. 1, p. 487.

72a. Iynn. Au.

1909, pt. 1, p. 409.

20 miles NW. Carlin, S. P. R. R., W. P. R. R.

Tertiary volcanice.

Veins.

Emmons, W. H., A reconnaissance of some mining camps in Elko, Lander, and Eureka counties, Nev.: Bull. 408, 1910, p. 87.

Min. Res. 1909, pt. 1, p. 409.

73. Mineral Hill. $\mathrm{Ag}, \mathrm{Pb}, \mathrm{Au}$.

5 miles SW. Mineral, E. \& P. R. R.

Paleozoic sediments cut by porphyry.

Replacements.

Emmons, W. H., A reconnaissance of some mining camps in Elko, Lander, and Eureka counties, Nev.: Bull. 408, 1910, pp. 95-99.

Min. Res. 1905, pp. 268-269.

1908, pt. 1, p. 287.

74. Safford. Ag.

6 miles W. Palisade, S. P. R. R., W. P. R. R.

Tertiary volcanics. 
74. Safford-Continued.

Veins.

Emmons, W. H., A reconnaissance of some mining camps in Elko, Lander, and Eureka counties, Nev.: Bull. 408, 1910, pp. 110-112.

Min. Res. 1909, pt. 1, p. 410.

75. Schroeder (Maggie Creek). $\mathrm{Pb}, \mathrm{Ag}, \mathrm{Au}, \mathrm{Sb}$.

9-15 miles NNW. Carlin, S. P. R. R., W. P. R. R.

Paleozoic sediments and Tertiary volcanics.

Veins, replacements.

Emmons, W. H., A reconnaissance of some mining camps in Elko, Lander, and Eureka counties, Nev.: Bull. 408, 1910, pp. 86-88.

Min. Res. 1908, pt. 1, p. 487.

1909, pt. 1, p. 410.

HUMBOLDT COUNTY.

76. Adelaide (Gold Run). $\mathrm{Cu}, \mathrm{Ag}, \mathrm{Au}$.

11 miles S. Golconda, S. P. R. R., W. P. R. R.

Triassic sediments cut by granite.

Replacement, contact metamorphic.

Ransome, F. L., Notes on some mining districts in Humboldt County, Nev.: Bull. 414, 1909, pp. 62-63.

Min. Res. 1899 (Twentieth Ann. Rept., pt. 6), p. 185.

1907 , pt. 1, p. 359.

1908, pt. 1, pp. 489-490.

77. Browns. W. 1909 , pt. 1, p. 412 .

2 miles W. Browns station, S. P. R. R.

Sediments cut by granite.

Contact metamorphic.

Min. Res. 1908, pt. 1, p. 724 .

78. Cedar. $\mathrm{Pb}, \mathrm{Ag}$.

1909 , pt. 1, p. 580 .

20 miles W. Mill City, S. P. R. R., W. P. R. R.

79. Central (Chafey, Dun Glen). Au, Ag, Pb.

10 miles E. Mill City, S. P. R. R., W. P. R. R.

Jurassic sediments, Tertiary volcanics.

Veins.

Ransome, F. L., Notes on some mining districts in Humboldt County, Nev.:

Bull. 414, 1909, pp. 49-52.

Min. Res. 1908, pt. 1, p. 490.

1909 , pt. 1, p. 411.

79a. Disaster. Au. (D, PI.)

100 miles NNW. Winnemucca, S. P. R. R., W. P. R. R., W. P. R. R.

80. Fitting (American Canyon). $\mathrm{Au}, \mathrm{Ag}, \mathrm{Hg}(\mathrm{Pb}, \mathrm{Cu}, \mathrm{Zn}$ ).

28 miles NE. Lovelocks, S. P. R. R., W. P. R. R.

Triassic sediments cut by granodiorite, capped by Tertiary volcanics.

Veins.

Ransome, F. L., Notes on some mining districts in Humboldt County, Nev.:

Bull. 414, 1909, p. 35.

Min. Res. 1883-84, p. 643.

1905, p. 269.

1907, pt. 1, p. 359.

1908, pt. 1, p. 489.

1909 , pt. 1 , pp. $411-412,554$. 


\section{EUuBordT CoUNTY-Continued.}

81. Golconda. Mn.

2 miles ESE. Golconda, S. P. R. R., W. P. R. R.

Tertiary sediments and volcanics.

Bedded lenses.

Harder, E. C., Manganese deposits of the United States: Bull. 380, 1909, p. 270 ; Bull. 427,1910 , pp. 153-157.

Min. Res. 1885, p. 349.

1886, p. 197.

1907 , pt. 1, p. 100.

82. Gold Banks. Au, Ag.

40 miles SSE. Winnemucca, S. P. R. R., W. P. R. R.

Triassic sediments cut by granite.

Veins.

Ransome, F. L., Notes on some mining districts in Humboldt County, Nev.: Bull. 414, 1909, p. 48.

Min. Res. 1907, pt. 1, p. 362 .

1908 , pt. 1, p. 489 .

83. Humboldt (Imlay). $\mathrm{Ag}, \mathrm{Au}, \mathrm{Cu}, \mathrm{Pb}$.

8 miles S. Mill City, S. P. R. R.

Jurassic sediments cut by granite and capped by Tertiary volcanics.

Veins.

Ransome, F. L., Notes on some mining districts in Humboldt County, Nev.: Bull. 414, 1909, p. 46.

Min. Res. 1905, p. 269.

1907 , pt. 1 , p. 360 .

84. Kennedy. Au, Ag.

$$
1908 \text {, pt. 1, p. } 490 .
$$

70 miles S. Winnemucca, S. P. R. R., W. P. R. R.

Triassic sediments cut by granite and capped by Tertiary volcanics.

Veins.

Ransome, F. L., Notes on some mining districts in Humboldt County, Nev.:

Bull. 414, 1909, pp. 52-54.

Min. Res. 1905, p. 269.

1907, pt. 1, p. 362.

1908 , pt. 1, p. 490.

1909 , pt. 1, p. 412.

84a. Jackson Creek. Cu.

75 miles NNW. Humboldt, S. P. R. R.; 30 miles N. Sulphur, W. P. R. R.

Granite and limestone.

Veins, contact metamorphic.

85. Lovelocks. $\mathrm{Ag}, \mathrm{Au}$.

10 miles W. Lovelocks, S. P. R. R.

Min. Res. 1908, pt. 1, p. 490.

86. National. $\mathrm{Au}, \mathrm{Ag}, \mathrm{Sb}$.

74 miles N. Winnemucca, S. P. R. R., W. P. R. R.

Tertiary volcanics.

Veins.

Min. Res. 1908, pt. 1, p. 490.

1909, pt. 1, p. 412 .

Top. sheet Paradise.

87. Spring City (Paradise Valley). Au, Ag.

44 miles NNE. Winnemucca, S. P. R. R., W. P. R. R.

Mesozoic metamorphosed slaten. 
HUMBOLDT COUNTY-Continued.

87. Spring City (Paradise Valley)-Continued.

Veins.

Min. Res. 1907, pt. 1, p. 362.

1908 , pt. 1, p. 490.

1909, pt. 1, p. 412 .

Top. sheet Paradise.

88. Rebel Creek (Willow Creek). $\mathrm{Au}, \mathrm{Ag}, \mathrm{Cu}$.

54 miles N. Winnemucca, S. P. R. R., W. P. R. R.

Mesozoic metamorphosed slates, granite.

Veins.

Min. Res. 1907, pt. 1, p. 362.

1908 , pt. 1, p. 490.

Top. sheet Paradise.

88a. Red Butte. Cu.

45 miles NW. Humboldt, S. P. R. R.; 15 miles N. Sulphur, W. P. R. R.

Gabbro and aplite.

Veins, disseminations.

Ransome, F. L., Notes on some mining districts in Humboldt County, Nev.: Bull. 414, 1909, p. 27.

89. Rosebud. Ag, Au, sulphur.

28 miles NW. Humboldt House, 35 miles NW. Mill City, S. P. R. R.; 52 miles W. Winnemucca, S. P. R. R., W. P. R. R.; Sulphur station, W. P. R. R.

Jurassic sediments and Tertiary volcanics.

Veins.

Adams, G. I., The Rabbit Hole sulphur mines near Humboldt House, Nev.: Bull. 225, 1904, pp. 497-500.

Ransome, F. L., Notes on some mining districts in Humboldt County, Nev.: Bull. 414, 1909, pp. 25-26.

Min. Res. 1906, p. 295.

1907 , pt. 1, p. 362 .

1908, pt. 1, p. 490.

1909 , pt. 1, p. 413.

90. Seven Troughs (Vernon, Mazuma, Farrel). $\mathrm{Au}, \mathrm{Ag}, \mathrm{Cu}, \mathrm{Pb}$.

32 miles NW. Lovelocks, S. P. R. R.

Jurassic sediments cut by granite and capped by Tertiary volcanics.

Veins.

Ransome, F. L., Notes on some mining districts in Humboldt County, Nev.:

Bull. 414, 1909, pp. 14-25.

Min. Res. 1906, p. 295.

1907 , pt. 1, pp. $360-362$.

1908 , pt. 1, pp. $490-491$.

1909 , pt. 1, p. 413.

91. Star (Rye Patch, Unionville). Ag, $\mathrm{Pb}, \mathrm{Au}, \mathrm{Cu}, \mathrm{Zn}, \mathrm{Sb}, \mathrm{Ni}, \mathrm{Co}$

14 miles S. Mill City, 10 miles E. Rye Patch, S. P. R. R.

Triassic sediments and Tertiary volcanics.

Replacements and veins.

Ransome, F. L., Notes on some mining districts in Humboldt County, Nev.:

Bull. 414, 1909, pp. 41-49.

Min. Res. 1905, p. 269.

1907 , pt. 1, p. 360 .

1908 , pt. 1, p. 489.

1909 , pt. 1 , pp. $413-414$. 


\section{HUMBOLDT COURTY-Continued.}

91a. Varyville. Au. (PI.)

120 miles NW. Winnemucca, S. P. R. R., W. P. R. R.

92. Viclesburg (Ashdown). Au, Ag.

120 miles NW. Winnemucca, S. P. R. R., W. P. R. R.

Min. Res. 1905, p. 269.

1906 , p. 295.

1908 , pt. 1, p. 489.

1909 , pt. 1, p. 411 .

Top. sheet Disaster.

92a. Winnemucca (Barrett Springs). Au, Ag.

5 miles WNW. Winnemucca, S. P. R. R., W. P. R. R.

Mesozoic metamorphosed slates, diorite.

Veins.

Min. Res. 1909, pt. 1, p. 414.

LANDER COUNTY.

93. Battle Mountain. Au, Ag.

10 miles W. Battle Mountain, S. P. R. R., W. P. R. R.

Tertiary volcanics.

Veins.

Min. Res. 1908, pt. 1, p. 492.

1909 , pt. 1, p. 415

94. Bullion (Tenabo, Lander). $\mathrm{Ag}, \mathrm{Au}, \mathrm{Pb}, \mathrm{Cu}$.

30 miles SE. Battle Mountain, 25 miles SE. Beowawe, S. P. R. R., W. P. R. R.

Paleozoic sediments capped by Tertiary volcanics.

Veins.

Emmone, W. H., A reconnaissance of some mining camps in Elko, Lander, and Eureka counties, Nev.: Bull. 408, 1910, pp. 118-119.

Min. Res. 1906, p. 296.

1907 , pt. 1 , p. 364 .

1909 , pt. 1, p. 415.

95. Campbell (Lander). Ag, $\mathrm{Pb}, \mathrm{Cu}$.

38 miles SE. Battle Mountain, S. P. R. R., W. P. R. R.

Paleozoic sediments capped by Tertiary volcanics.

Veins.

Emmons, W. H., A reconnaissance of some mining camps in Elko, Lander, and Eureka counties, Nev.: Bull. 408, 1910, pp. 118-119.

Min. Res. 1906, p. 286.

96. Cortez (Mill Canyon). $\mathrm{Ag}, \mathrm{Au}, \mathrm{Cu}, \mathrm{Pb}$.

35 miles S. Beowawe, S. P. R. R., W. P. R. R.

Paleozoic sediments cut by acidic and basic rocks.

Replacements, veins.

Emmons, W. H., A reconnaiseance of some mining camps in Elko, Lander, and Eureka counties, Nev.: Bull. 408, 1910, pp. 100-106.

Min. Res. 1908, pt. 1, p. 492.

1909 , pt. 1, p. 415.

97. Dean (Lewis, Mud Springs). Ag, Au.

18-22 miles SE. Battle Mountain, S. P. R. R., W. P. R. R.

Paleozoic sediments cut by granite and andesite.

Veins.

Emmons, W. H., A reconnaissance of some mining camps in Elko, Lander, and Eureka counties, Nev.: Bull. 408, 1910, pp. 119-126.

Min. Res. 1909, pt. 1, p. 415. 
LANDER COUNTY-Continued.

98. Galena (Copper Basin, Bannock). $\mathrm{Ag}, \mathrm{Au}, \mathrm{Pb}, \mathrm{Cu}, \mathrm{Zn}$.

12 miles SW. Battle Mountain, S. P. R. R., W. P. R. R.

Tertiary volcanics.

Veins.

Min. Res. 1882, pp. 230, 438-439.

$1883-84$, p. 643.

1905 , p. 269.

1907, pt. 1, p. 364 .

1908 , pt. 1, p. 492.

1909 , pt. 1, p. 415.

99. Kimberley (Hill Top). $\mathrm{Cu}, \mathrm{Pb}, \mathrm{Au}, \mathrm{Ag}$.

20 miles SW. Battle Mountain, S. P. R. R., W. P. R. R.

Paleozoic sediments cut by diorite and andesite.

Veins.

Min. Res. 1909, pt. 1, p. 415.

100. Reese River (Austin). $\mathrm{Ag}, \mathrm{Au}, \mathrm{Pb}, \mathrm{Cu}, \mathrm{Zn}$.

Station N. C. R. R.

Paleozoic sediments cut by granite and capped by Tertiary volcanics.

Veins.

Spurr, J. E., Geology of Nevada south of the fortieth parallel: Bull. 208, 1903, pp. 93-97.

Min. Res. 1905, p. 269.

1906, p. 296.

1907 , pt. 1, p. 363.

1908 , pt. 1, p. 492.

101. Skookum. $\mathrm{Ag}, \mathrm{Au}$. 1909, pt. 1, p. 414.

9 miles NW. Austin, N. C. R. R.

Paleozoic sediments cut by granite.

Veins, contact metamorphic.

102. Spencer.

Min. Res. 1907, pt. 1, p. 363.

NE. Austin, N. C. R. R.

Paleozoic sediments, Tertiary volcanics.

103. Washington (Kingston). Au (?).

30 miles SSW. Austin, N. C. R. R.

Paleozoic sediments cut by granite.

Min. Res. 1909, pt. 1, p. 415.

\section{LINCOLN COUNTY.}

104. Bristol. $\mathrm{Ag}, \mathrm{Cu}, \mathrm{Au}, \mathrm{Pb}$.

20 miles NW. Pioche, S. P. L. A. \& S. L. R. R.

Paleozoic sediments cut by rhyolite.

Replacements and veins.

Spurr, J. E., Geology of Nevada south of the fortieth parallel: Bull. 208, 1903, p. 42.

Min. Res. 1908, pt. 1, pp. 493-494.

1909 , pt. 1, pp. $416-417$

105. Chief. Au.

8 miles NNW. Caliente, S. P. L. A. \& S. L. R. R.

Paleozoic sediments cut by basic dikes.

Veins.

Spurr, J. E., Geology of Nevada south of the fortieth parallel: Bull. 208, 1903, pp. 37-48. 
105. Chief-Continued.

Min. Res. 1909, pt. 1, p. 416.

Top. sheet Pioche.

106. Comet. $\mathrm{Pb}, \mathrm{Ag}$.

12 miles W. Pioche, S. P. L. A. \& S. L. R. R.

Paleozoic sediments cut by porphyry.

Veins and replacements.

Spurr, J. E., Geology of Nevada south of the fortieth parallel: Bull. 208, 1903, pp. 151-152.

Top. sheet Pioche.

107. Eagle Valley (Fay, State $\operatorname{Line}$ ). Au, Ag.

21 miles NW. Modena, Utah, S. P. L. A. \& S. L. R. R.

Tertiary volcanics.

Veins.

Spurr, J. E., Geology of Nevada south of the fortieth parallel: Bull. 208, 1903, pp. 134-135.

Min. Res. 1905, p. 270.

1907, pt. 1, pp. 366-367.

1908, pt. I, p. 493.

1909 , pt. 1, p. 416.

Top. sheet Pioche.

108. Ferguson (Delamar). Au, Ag.

30 miles WSW. Caliente, S. P. L. A. \& S. L. R. R.

Paleozoic quartzite cut by basic dikes.

Replacements and veins.

Min. Res. 1905, p. 270.

1906, p. 296.

1908 , pt. 1, p. 493

1909 , pt. 1, p. 416.

Top. sheet Pioche.

109. Freiberg. $\mathrm{Au}, \mathrm{Ag}$.

75 miles W. Pioche, S. P. L. A. \& S. L. R. R.

Rhyolite (?).

Veins.

Spurr, J. E., Geology of Nevada south of the fortieth parallel: Bull. 208, 1903 , pp. 76-77.

110. Geyser. $\mathrm{Ag}, \mathrm{Au}, \mathrm{Pb}$.

81 miles S. Ely, N. N. R. R.

Spurr, J. E., Geology of Nevada south of the fortieth parallel: Bull. 208, 1903 , pp. 47-54.

111. Highland Valley. $\mathrm{Pb}, \mathrm{Ag}, \mathrm{Au}, \mathrm{Cu}$.

7 miles WNW. Pioche, S. P. L. A. \& S. L. R. R.

Paleozoic sediments cut by dikes.

Veins, replacements.

Spurr, J. E., Geology of Nevada south of the fortieth parallel: Bull. 208, 1903, pp. 38-45.

Min. Res. 1908, pt. 1, p. 493.

1909 , pt. 1, pp. $416-417$.

Top. sheet Pioche.

112. Hiko. $\mathrm{Ag}, \mathrm{Pb}$.

60 miles W. Caliente, S. P. L. A. \& S. I. R. R.

Paleozoic sediments.

Veins (?).

Spurr, J. E., Geology of Nevada south of the fortieth parallel: Bull. 208, 1903, pp. 152-153. 


\section{IINCOLN COUNTY-Continued.}

113. Jackrabbit. $\mathrm{Ag}, \mathrm{Au}, \mathrm{Pb}$.

15 miles NW. Pioche, S. P. L. A. \& S. L. R. R.

Paleozoic sediments.

Replacements.

Min. Res. 1907, pt. 1, p. 368.

1908, pt. 1, p. 493.

1909, pt. 1, pp. 416-417.

114. Lone Mountain. $\mathrm{Ag}, \mathrm{Pb}$.

16 miles W. Pioche, S. P. L. A. \& S. L. R. R.

Paleozoic sediments (?).

Veins, replacements.

Top. sheet Pioche.

115. Patterson. Ag.

50 miles SSE. Ely, N. N. R. R.

Spurr, J. E., Geology of Nevada south of the fortieth parallel: Bull, 208, 1903 , p. 40.

116. Pioche. $\mathrm{Ag}, \mathrm{Pb}, \mathrm{Au}, \mathrm{Cu}, \mathrm{Zn}, \mathrm{Mn}$.

Station S. P. L. A. \& S. L. R. R.

Paleozoic sediments cut by quartz porphyry.

Replacement, veins.

Harder, E. C., Manganese deposits of the United States: Bull. 380, 1909, p. 274.

Spurr, J. E., Geology of Nevada eouth of the fortieth parallel: Bull. 208, 1903, pp. 38-45.

Min. Res. 1905, p. 270.

1906, p. 296.

1907, pt. 1, p. 367.

1908, pt. 1, p. 493.

1909 , pt. 1, pp. 416-417.

Top. sheet Pioche.

\section{LYON COUNTY.}

117. Pine Nut. $\mathrm{Pb}, \mathrm{Ag}(\mathrm{Au})$.

22 miles SW. Wabuska, S. P. R. R.

Tertiary volcanics.

Veins.

Spurr, J. E., Geology of Nevada south of the fortieth parallel: Bull. 208, 1903, pp. 120-123.

Top. sheet Wabuska.

118. Ramsey. $\mathrm{Au}, \mathrm{Ag}$.

17 miles S. Clarks, S. P. R. R.

Tertiary volcanics.

Veins.

Hill, J. M., Notes on the economic geology of the Ramsey, Talapoosa, and White Horse mining districts in Lyon and Washoe counties, Nev.: Bull. 470, 1911, pp. 106-107.

Min. Res. 1907, pt. 1, p. 370.

1908 , pt. 1, p. 494.

1909 , pt. 1, p. 418.

Top. sheet Wabuska.

119. Red Mountain. Fe.

17 miles NE. Dayton, S. P. R. R.

Triassic sediments cut by granite. 
IFoN coUNTr-Continued.

119. Red Mountain-Continued.

Contact metamorphic.

Harder, E. C., Iron ores near Dayton, Nev.: Bull. 430, 1910, pp. 240-246.

Top. sheet Wabuska.

120. Silver City (Palmyra, Devils Gate). Au, Ag.

Station V. \& T. R. R

Tertiary volcanics.

Veins.

Min. Res. 1905, p. 271.

1906, p. 297.

1907, pt. 1, p. 359.

1908, pt. 1, p. 494.

1909 , pt. 1, p. 417.

Top. sheet Carson.

121. Talapoosa. $\mathrm{Au}, \mathrm{Ag}(\mathrm{Cu})$.

18 miles S. Fernley, S. P. R. R.

Tertiary volcanics.

Veins.

Hill, J. M., Notes on the economic geology of the Ramsey, Talapoosa, and White Horse mining districts, in Lyon and Washoe counties Nev.: Bull. 470, 1911, p. 108.

Top. sheet Wabuska.

122. Yerington (Mason). $\mathrm{Cu}(\mathrm{Pb}, \mathrm{Au}, \mathrm{Ag})$.

Station N. C. B. R. R.

Triassic sediments cut by granite.

Contact metamorphic.

Ransome, F. L., The Yerington copper district, Nevada: Bull. 380, 1909, pp. 99-119.

Spurr, J. E., Geology of Nevada south of the fortieth parallel: Bull. 208, 1903, p. 117.

Min. Res. 1905, p. 271.

1906, pp. 297, 401.

1907 , pt. 1, pp. 611,370 .

1908, pt. 1, pp. 213, 494.

1909 , pt. 1, pp. $408,418$.

Top. sheet Wellington.

MINERAL COUNTY. (See ESMERALDA.)

123. Bare Mountain. Au, Hg.

\section{NYE COUNTY.}

7 miles NE. Beatty, B. G. R. R.

Paleozoic sediments cut by monzonite porphyry and Tertiary volcanics.

Veins.

Ball, S. H., Notes on the ore deposits of southwestern Nevada and eastern California: Bull. 285, 1906, pp. 71-72.

A geologic reconnaissance in southwestern Nevada and eastern California: Bull. 308, 1907, pp. 153-157.

Top. sheet Furnace Creek.

124. Bellehelen. Au, Ag.

50 miles E. Tonopah, T. \& G. R. R.

Tertiary volcanics.

Veins.

Spurr, J. E., Geology of Nevada south of the fortieth parallel: Bull. 208, 1903, p. 181. 
NYE COUNTY-Continued.

124. Bellehelen-Continued.

Min. Res. 1907, pt. 1, p. 372 .

1908 , pt. 1, p. 496.

1909 , pt. 1, p. 419.

125. Belmont (Philadelphia). $\mathrm{Ag}, \mathrm{Pb}, \mathrm{Cu}(\mathrm{Hg})$.

72 miles NNE. Tonopah, T. \& G. R. R.

Paleozoic sediments cut by granite.

Veins.

Becker, G. F., Geology of the quicksilver deposits of the Pacific slope: M. XIII, 1888, p. 385.

Spurr, J. E., Geology of Nevada south of the fortieth parallel: Bull. 208, 1903, pp. 89-93.

126. Berlin (Elsworth). $\mathrm{Au}, \mathrm{Ag}(\mathrm{Cu}, \mathrm{Pb}, \mathrm{Zn})$.

40 miles NE. Luning, S. P. R. R.

Tertiary volcanics.

Veins.

Spurr, J. E., Geology or Nevada south of the fortieth parallel: Bull. 208, 1903, pp. 99-103.

Min. Res. 1907, pt. 1, p. 372.

1908 , pt. 1, p. 496.

1909 , pt. 1, p. 419.

Top. sheet Tonopah.

127. Big Dune. Au, Cu.

8 miles E. Leeland, T. \& T. R. R.

Cambrian sediments.

Veins.

Ball, S. H., A geologic reconnaissance in southwestern Nevada and eastern California: Bull. 308, 1907, p. 175.

Top. sheet Furnace Creek.

128. Blakes Camp. Au, Ag.

50 miles NE. Goldfield, T. \& G. R. R., B. G. R. R., L. V. \& T. R. R.

Tertiary volcanics.

Veins.

Ball, S. H., Notes on the ore deposits of southwestern Nevada and eastern California: Bull. 285, 1906, p. 66.

- A geologic reconnaissance in southwestern Nevada and eastern California: Bull. 308, 1907, p. 110.

129. Bullfrog (Rhyolite). Au, Ag.

Station B. G. R. R., T. \& G. R. R.

Tertiary volcanics.

Veins.

Ransome, F. L., Preliminary account of Goldfield, Bullfrog, and other mining districts in southern Nevada: Bull. 303, 1907, pp. 40-62. 1910. Geology and ore deposits of the Bullfrog district, Nevada: Bull. 407,

Min. Res. 1905, p. 272.

1906, p. 298.

1907, pt. 1, pp. 372, 394-398.

1908 , pt. 1 , pp. $496-497$.

1909 , pt. 1, pp. 419-420.

Top. sheets Furnace Creek, Bullfrog special.

130. Cactus Springs. Au.

24 miles E. Goldfield, B. G. R. R., L. V. \& T. R. R. 
NYE COUNTY-Continued.

130. Cactus Springs-Continued.

Tertiary volcanics.

Veins.

Ball, S. H., Notes on the ore deposits of southwestern Nevada and eastern California: Bull. 285, 1906, p. 68 .

- _ A geologic reconnaissance of southwestern Nevada and eastern California: Bull. 308, 1907, pp. 46, 89, 92-96.

Min. Res. 1909, pt. 1, p. 420.

131. Cloverdale. Au, Ag.

32 miles E. Luning, S. P. R. R.

Tertiary volcanics.

Veins.

Min. Res. 1905, p. 275.

1908 , pt. 1, p. 497.

1909 , pt. 1, p. 420 .

Top. sheet Tonopah.

132. Eden. Au.

70 miles E. Tonopah, T. \& G. R. R.

Tertiary volcanics.

Veins.

Ball, S. H., Notes on the ore deposits of southwestern Nevada and eastern California: Bull. 285, 1906, p. 66.

- A geologic reconnaissance in southwestern Nevada and eastern California: Bull. 308, 1907, pp. 110-111.

Top. sheet Kawich.

133. Gold Bar (Happy Hooligan). Au, Ag.

3 miles W. Bullfrog, B. G. R. R., L. V. \& T. R. R.

Paleozoic sediments, Tertiary rhyolites.

Veins.

Ball, S. H., Notes on the ore deposits of southwestern Nevada and eastern California: Bull. 285, 1906, p. 72.

A geologic reconnaissance in southwestern Nevada and eastern California: Bull. 308, 1907, p. 178.

Top. sheet Furnace Creek.

134. Gold Crater. $\mathrm{Au}, \mathrm{Cu}$.

27 miles SE. Goldfield, T. \& G. R. R., B. G. R. R., L. V. \& T. R. R.

Tertiary volcanics.

Veins.

Ball, S. H., Notes on the ore deposits of southwestern Nevada and eastern California: Bull. 285, 1906, pp. 69-70.

A geologic reconnaissance in southwestern Nevada and eastern California: Bull. 308, 1907, pp. 139-140.

Top. sheet Kawich.

135. Golden. Au, Ag.

40 miles NE. Sodaville, S. P. R. R.

Spurr, J. E., Geology of Nevada south of the fortieth parallel: Bull. 208, 1903, pp. 93-97.

Min. Res. 1907, pt. 1, p. 373.

136. Goldyke (Atwood). $\mathrm{Au}, \mathrm{Ag}, \mathrm{W}, \mathrm{Cu}, \mathrm{Pb}$.

32 miles NE. Luning, S. P. R. R.

Spurr, J. E., Geology of Nevada south of the fortieth parallel: Bull. 208, 1903, pp. 93-97. 
NYE COUNTY-Continued.

136. Goldyke (Atwood)-Continued.

Min. Res. 1907, pt. 1, pp. 372-373.

1908 , pt. 1 , pp. 497,724 .

Top. sheet Tonopah.

1909 , pt. 1, p. 420.

137. Hannapah. $\mathrm{Au}, \mathrm{Ag}$.

20 miles E. Tonopah, T. \& G. R. R.

Tertiary volcanics.

Veins.

Spurr, J. E., Ore deposits of Tonopah and neighboring districts, Nevada: Bull. 213,1903, p. 87.

Min. Res. 1908, pt. 1, p. 499. 1909 , pt. 1, p. 420.

138. Jefferson Canjon (Concordia, Green Isle). Au, Ag.

70 miles NNE. Tonopah, T. \& G. R. R.

Paleozoic sediments cut by porphyry.

Veins.

139. Johnnie. Au, Ag.

14 miles SSE. Amargosa, L. V. \& T. R. R.

Paleozoic sediments.

Veins.

Min. Res. 1907, pt. 1, p. 374

1908 , pt. 1, p. 497.

1909 , pt. 1 , pp. $420-421$.

Top. sheet Furnace Creek.

140. Kawich (Gold Reed). Au.

54 miles E. Goldfield, T. \& G. R. R., B. G. R. R., L. V. \& T. R. R.

Monzonite porphyry and rhyolite.

Veins.

Ball, S. H., Notes on the ore deposits of southwestern Nevada and eastern California: Bull. 285, 1906, pp. 67-68.

A geologic reconnaissance in southwestern Nevada and eastern Califormia: Bull. 308, 1907, pp. 108-113.

Spurr, J. E., Geology of Nevada south of the fortieth parallel: Bull. 208, 1903, p. 181.

Top. sheet Kawich.

141. Lodi Valley (Marble). Ag, Au, Pb.

63 miles NNE. Luning, S. P. R. R.

Min. Res. 1905, p. 252.

1906, p. 299.

1908 , pt. 1, p. 497

1909 , pt. 1 , p. 421 .

Top. sheet Tonopah.

142. Manhattan. Au, Ag. (D, PI.)

53 miles N. Tonopah, T. \& G. R. R.

Paleozoic sediments cut by granite and diorite and capped by Tertiary volcanics.

Veins and replacements.

Emmons, W. H., and Garrey, G. H., Notes on the Manhattan district: Bull. 303, 1907, pp. 84-92.

Min. Res. 1905 , p. 272.

1906, p. 299.

1907 , pt. 1, pp. $374,398$. 
NYE COUNTY-Continued.

142. Manhattan-Continued.

Min. Res. 1908, pt. 1, p. 498.

1909 , pt. 1, p. 421.

Top. sheet Tonopah.

143. Milletts. $\mathrm{Au}, \mathrm{Ag}, \mathrm{Pb}$.

105 miles N. Tonopah, T. \& G. R. R.

Spurr, J. E., Geology of Nevada south of the fortieth parallel: Bull. 208, 1903, pp. 93-97.

Min. Res. 1908, pt. 1, p. 498.

1909 , pt. 1, p. 421.

Top. sheet Tonopah.

144. Monte Cristo. Au.

20 miles ENE. Bonnie Claire, T. \& G. R. R.

Paleozoic sediments cut by granite and Tertiary volcanics.

Veins.

Ball, S. H., A geologic reconnaissance of southwestern Nevada and eastern California: Bull. 308, 1907, pp. 141-142.

Top. sheet Kawich.

145. Oak Springs. Au.

80 miles SW. Caliente, S. P. T. A. \& S. L. R. R.

Paleozoic sediments cut by granite and capped by Tertiary volcanics.

Veins.

Ball, S. H., Notes on the ore deposits of southwestern Nevada and eastern California: Bull. 285, 1906, pp. 70-71.

A geologic reconnaissance of southwestern Nevada and eastern California: Bull. 308, 1907, pp. 44, 45, 122-130.

Top. sheet Kawich.

146. Reveille. $\mathrm{Ag}, \mathrm{Pb}$.

67 miles E. Tonopah, T. \& G. R. R.

Paleozoic sediments cut by dikes.

Veins.

Ball, S. H., A geologic reconnaissance in southwestern Nevada and eastern California: Bull. 308, 1907, pp. 114-117.

Spurr, J. E., Geology of Nevada south of the fortieth parallel: Bull. 208, 1903, pp. 161-163.

Min. Res. 1905, p. 272.

1908, pt. 1, p. 498.

147. Round Mountain. Au, Ag, W. (D, Pl.)

74 miles N. Tonopah, T. \& G. R. R.

Tertiary volcanics.

Veins, wash gravels.

Ransome, F. L., Round Mountain, Nev.: Bull. 380, 1909, pp. 44-47.

Min. Res. 1906, pp. 298-299.

1907, pt. 1, pp. 373-374.

1908, pt. 1, pp. $498,499,724$.

1909 , pt. 1, p. 421.

Top. sheet Tonopah.

148. Silverbow. $\mathrm{Au}, \mathrm{Ag}$.

46 miles E. Goldfield, T. \& G. R. R.

Tertiary volcanics.

Veins.

Ball, S. H., Notes on the ore deposits of southwestern Nevada and eastern California: Bull. 285, 1906, pp. 65-66. 
NYE COUNTY-Continued.

148. Silverbow-Continued.

Ball, S. B., A geologic reconnaissance in southwestern Nevada and eastern California: Bull. 308, 1907, pp. 109-110.

Min. Res. 1907, pt. 1, p. 374.

1908 , pt. 1, p. 499.

Top. sheet Kawich.

149. Stonewall Mountain. Au.

17 miles SSE. Goldfield, T. \& G. R. R., B. G. R. R., L. V. \& T. R. R.

Paleozoic sediments cut by granite, capped by Tertiary volcanics.

Veins.

Ball, S. H., Notes on the ore deposits of southwestern Nevada and eastern California: Bull. 285, 1906, p. 70.

- A geologic reconnaissance in southwestern Nevada and eastern California: Bull. 308, 1907, pp. 28, 32, 33, 46, 47, 48, 83, 84-87, 88-99, 109, $133,136$.

Top. sheet Lida.

150. Superior. Cu.

35 miles NNE. Tonopah, T. \& G. R. R.

151. Tonopah. Ag. Au, W.

Station T. \& G. R. R.

Tertiary volcanics.

Veins.

Spurr, J. E., Ore deposits of Tonopah and neighboring districts, Nevada: Bull. 213, 1903, pp. 81-87.

The ore deposits of Tonopah, Nev. (preliminary report): Bull. 219, 1903.

- Preliminary report on the ore deposits of Tonopah, Nev.: Bull. 225, 1904, pp. 88-110.

- Developments at Tonopah, Nev., during 1904: Bull. 260, 1905, pp. 140-144.

- Geology of the Tonopah mining district, Nevada: P. P. 42, 1905.

Min. Res. 1905, p. 272.

1906, p. 298.

1907, pt. 1, pp. 375-378, 385-391.

1908 , pt. 1 , pp. $499-501$.

1909 , pt. 1, pp. $422-424$.

Top. sheets Tonopah, Tonopah special.

152. Trappmans (Wilsons). Ag, Au.

34 miles SE. Goldfield, T. \& G. R. R., B. G. R. R., L. V. \& T. R. R.

Granite and Tertiary volcanics.

Veins.

Ball, S. H., Notes on the ore deposits of southwestern Nevada and eastern California: Bull. 285, 1906, pp. 68-69.

- A geologic reconnaissance in southwestern Nevada and eastern California: Bull. 308, 1907, pp. 138-139.

Top. sheet Kawich.

153. Tybo (Hot Creek). Ag, $\mathrm{Au}, \mathrm{Pb}$.

111 miles ENE. Tonopah, T. \& G. R. R.

Paleozoic sediments and Tertiary volcanics.

Veins, replacements.

Spurr, J. E., Geology of Nevada south of the fortieth parallel: Bull. 208, 1903, pp. 84-87.

Min. Res. 1908, pt. 1, p. 497. 
ITE OOUNTY_Continued.

154. Union (Ione). $\mathrm{Hg}, \mathrm{Au}, \mathrm{Ag}, \mathrm{Pb}$.

60 miles S. Austin, N. C. R. R.

Tertiary volcanics.

Veins.

Min. Res. 1908, pt. 1, p. 692.

1909 , pt. 1, pp. $420,555$.

Top. sheet Tonopah.

155. Wellington (O'Briens). $\mathrm{Au}, \mathrm{Ag}$.

20 miles E. Cuprite, B. G. R. R.

Tertiary volcanics.

Veins.

Ball, S. H., Notes on the ore deposits of southwestern Nevada and eastern California: Bull. 285, 1906, p. 68 .

- - A geologic reconnaissance in southwestern Nevada and eastern California: Bull. 308,1907, pp. 46, 48, 95.

Top. sheet Kawich.

\section{STOREY COUNTY.}

156. Comstock. Au, Ag.

Virginia City station, V. \& T. R. R.

Diorite and Tertiary volcanics.

Veins.

Becker, G. F., Geology of the Comstock lode and the Washoe district: M. III, 1882.

Lord, E., Comstock mining and miners: M. IV, 1883.

Min. Res. 1905, pp. 272-273.

1906, p. 299.

1907 , pt. 1, pp. 378-381.

1908 , pt. 1, pp. 501-502.

1909 , pt. 1, pp. $425-426$.

Top. sheet Carson.

\section{WASHOI COUNTY.}

157. Cottonwood (Round Hole). Au.

10 miles NW. Puna, W. P. R. R.

Min. Res. 1908, pt. 1, p. 503.

Top. sheet Granite Range.

158. Deep Hole. Au.

Renard station, W. P. R. R.

Min. Res. 1908, pt. 1, p. 503.

Top. sheet Granite Range.

158a. Leadville. $\mathrm{Pb}, \mathrm{Ag}$.

100 miles NE. Amedee, 'Cal., N. C. \& O. R. R.; 50 miles NNW. Phil, W. P. R. R.

Tertiary volcanics.

Veins.

159. Peavine. $\mathrm{Au}(\mathrm{Ag}, \mathrm{Cu})$.

10 miles N. Reno, S. P. R. R., V. \& T. R. R.

Schists and granite.

Veins.

Min. Res. 1905, p. 273.

1906, p. 299.

1907, pt. 1, p. 382 .

1908, pt. 1, p. 503 .

21528 ${ }^{\circ}-$ Bull. 507-12-15 
WASHOE COUNTY-Contlnued.

159. Peavine-Continued.

Min. Res. 1909, pt. 1, p. 426.

Top. sheet Reno.

160. Sheephead. Au.

15 miles W. Renard, W. P. R. R.

Min. Res. 1907, pt. 1, p. 382.

Top. sheet Granite Range.

161. Steamboat Springs. Hg.

Station V.\& T. R. R.

Tertiary volcanics.

Impregnations.

Becker, G. F., Geology of the quicksilver deposits of the Pacific slope:

M. XIII, 1888, pp. 331-353; Eighth Ann. Rept., 1889, p. 979.

162. Washoe (Galena, Jumbo). $\mathrm{Pb}, \mathrm{Au}, \mathrm{Ag}, \mathrm{Zn}$.

5 miles W. Virginia City, V. \& T. R. R.

Granite.

Veins.

Becker, G. F., A summary of the geology of the Comstock lode and the Washoe district: Second Ann. Rept., 1882, pp. 291-330.

Min. Res. 1906, p. 299.

1907, pt. 1, p. 382.

1909 , pt. 1, p. 426 .

Top. sheet Carson.

163. Wedekind. $\mathrm{Ag}, \mathrm{Au}$.

4 miles NE. Reno, S. P. R. R.

Tertiary volcanics.

Veins and replacements.

Min. Res. 1909, pt. 1, p. 426.

Top. sheet Reno.

164. White Horse (Olinghouse). $\mathrm{Au}, \mathrm{Ag}$.

9 miles W. Wadsworth, S. P. R. R.

Tertiary volcanics.

Veins.

Hill, J. M., Notes on the economic geology of the Ramsey, Talapoosa, and White Horse mining districts, in Lyon and Washoe counties, Nev $\cdot$ Bull. 470, 1911, pp. 104-106.

Min. Res. 1905, p. 273.

1906, pp. 299-300.

1907, pt. 1, p. 382 .

1908 , pt. 1, p. 503 .

1909 , pt. 1, p. 426.

Top. sheet Wadsworth.

\section{WHITE PINE COONTY.}

165. Aurum (Silver Canyon, Siegel). Ag ( $\mathrm{Au})$.

36 miles SE. Cherry Creek, 10 miles E. Melvin, N. N. R. R.

Paleozoic sediments (?).

Veins and replacements.

Spurr, J. E., Geology of Nevada south of the fortieth parallel: Bull. 208, 1903 , pp. 38-47.

Min. Res. 1908, pt. 1, p. 383.

166. Black Horse. $\mathrm{Au}(\mathrm{Ag})$.

49 miles SE. Ely, N. N. R. R.

Paleozoic sediments cut by granite porphyry. 
WHITE PINE COUNTY-Continued.

166. Black Horse-Continued.

Veins.

Min. Res. 1905, p. 274.

1906, p. 300.

1907 , pt. 1, p. 383.

1909 , pt. 1, p. 428.

167. Ely (Robinson). $\mathrm{Cu}, \mathrm{Au}, \mathrm{Ag}, \mathrm{Pb}$.

Station N. N. R. R.

Paleozoic sediments cut by monzonite porphyry.

Disseminated, veins, contact metamorphic.

Spurr, J. E., Geology of Nevada south of the fortieth parallel: Bull. 208, 1903, pp. 47-54.

Weed, W. H., The copper mines of the United States in 1905: Bull. 285, 1906, pp. 116, 117.

Min. Res. 1905, pp. 274, 355.

1906 , p. 300.

1907, pt. 1, pp. 383-384, 612.

1908, pt. 1, pp. 212-213, 504-505.

1909 , pt. 1, pp. 170, 428-429.

Top. sheet Ely special.

168. Gold Canyon (Cherry Creek). Au, Ag.

5 miles W. Cherry Creek, N. N. R. R.

Paleozoic sediments.

Veins.

Spurr, J. E., Geology of Nevada south of the fortieth parallel: Bull. 208, 1903 , pp. $47-54$.

Min. Res. 1905, p. 273.

1906, p. 303.

1907 , pt. 1, p. 382 .

1908 , pt. 1, p. 504.

1909 , pt. 1, p. 428.

169. Hunter. $\mathrm{Pb}, \mathrm{Cu}, \mathrm{Ag}$.

10 miles SW. Granite, N. N. R. R.

Paleozoic sediments (?).

Veins (?).

Spurr, J. E., Geology of Nevada south of the fortieth parallel: Bull. 208, 1903, pp. 47-54.

Min. Res. 1906, p. 300.

170. Newark (Strawberry). Ag, Pb, Cu, Au.

29 miles N NE. Eureka, E. \& P. R. R.

Paleozoic sediments (?).

Veins (?).

Spurr, J. E., Geology of Nevada south of the fortieth parallel: Bull. 208, 1903 , pp. 77-84.

Min. Res. 1905, p. 274.

1906 , p. 300.

1907 , pt. 1, p. 384.

1908 , pt. 1, p. 505 .

171. Osceola (Tungsten). Au, W.

40 miles SE. Ely, N. N. R. R.

Paleozoic sediments cut by granite porphyry.

Veins. 
WHITE PINE COUNTY-Continued.

171. Osceola (Tungsten)-Continued.

Spurr, J. E., Geology of Nevada south of the fortieth parallel: Bull. 208, 1903, pp. 25-36.

Weeks, F. B., An occurrence of tungsten ore in eastern Nevada: Twentyfirst Ann. Rept., pt. 6, 1901, pp. 319-320.

Geology and mineral resources of the Osceola mining district, White Pine County, Nev.: Bull. 340, 1908, pp. 117-133.

- Tungsten deposits in the Snake Range, White Pine County, eastern Nevada: Idem, pp. 263-270.

Min. Res. 1899 (Twenty-first Ann. Rept., pt. 6), pp. 301, 319-320.

1905 , p. 274.

1907, pt. 1, p. 383.

1908, pt. 1 , pp. 505,725 .

172. Regan. W, Au. 1909 , pt. 1 , pp. $430,579-580$.

50 miles E. Cherry Creek, N. N. R. R.

Granite and porphyry (?).

Veins.

173. Schellbourne.

18 miles SE. Cherry Creek, N. N. R. R.

Spurr, J. E., Geology of Nevada south of the fortieth parallel: Bull. 208, 1903, pp. 38-47.

174. Steptoe (Granite). $\mathrm{Au}, \mathrm{Ag}, \mathrm{Pb}$.

5 miles W. Granite, N. N. R. R.

Paleozoic sediments.

Veins.

Spurr, J. E., Geology of Nevada south of the fortieth parallel: Bull. 208, 1903 , pp. 47-54.

Min. Res. 1908, pt. 1, p. 504.

1909 , pt. 1, p. 428.

175. Ward (Taylor). Ag, $\mathrm{Pb}$.

16 miles S. Ely, N. N. R. R.

Paleozoic sediments cut by monzonite porphyry.

Veins, contact metamorphic.

Spurr, J. E., Geology of Nevada south of the fortieth parallel: Dull. 208, 1903 , pp. $47-54$.

Min. Res. 1907, pt. 1, p. 384.

1908 , pt. 1 , pp. 505-506.

1909 , pt. 1, p. 430.

176. White Pine (Hamilton). $\mathrm{Pb}, \mathrm{Ag}, \mathrm{Cu}, \mathrm{Au}, \mathrm{Zn}$.

30 miles W. Ely, N. N. R. R.

Paleozoic sediments cut by granodiorite and monzonite.

Veins, replacements.

Spurr, J. E., Geology of Nevada south of the fortieth parallel: Bull. 208, 1903, pp. 61-68.

Min. Res. 1905, pp. 273-274.

1906, p. 300.

1908, pt. 1, p. 505.

1909, pt. 1, p. 430. 


\section{NEW MEXICO.}

In the State of New Mexico there are at the present time 15 counties in which mining is carried on. In these counties 85 mining districts are recognized by the United States Geological Survey. In 27 of these districts copper is the principal metal produced, in 26 gold predominates, in 16 silver, in 11 lead, in 2 zinc, and in 3 iron.

Distribution of the predominant metals produced in the mining districts of New Mexico.

\begin{tabular}{|c|c|c|c|c|c|c|c|}
\hline County. & Gold. & Silver. & Copper. & Lead. & Zinc. & Iron. & Total \\
\hline 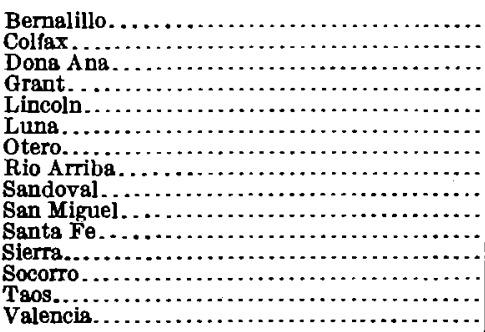 & $\begin{array}{r}{ }_{3} \\
4 \\
2 \\
2 \\
4 \\
4 \\
1 \\
1 \\
1 \\
1 \\
2 \\
2 \\
4 \\
2 \\
\ldots \ldots . .\end{array}$ & 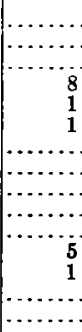 & $\begin{array}{r}1 \\
1 \\
1 \\
5 \\
1 \\
\cdots \\
2 \\
3 \\
2 \\
3 \\
1 \\
1 \\
3 \\
2 \\
2\end{array}$ & 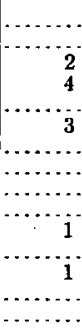 & 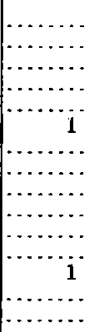 & $\left.\begin{array}{r}\cdots \\
\cdots \\
\cdots\end{array}\right]$ & $\begin{array}{r}1 \\
4 \\
5 \\
20 \\
6 \\
5 \\
3 \\
4 \\
3 \\
4 \\
5 \\
8 \\
11 \\
4 \\
2\end{array}$ \\
\hline & 26 & 16 & 27 & 11 & 2 & 3 & 85 \\
\hline
\end{tabular}

\section{MINING DISTRICTS IN NEW MEXICO.}

[See Pl. X. Acditional references will be found in Mineral Resources fer 1910 and 1911.]

\section{BERNALILLO COUNTY.}

1. Tijeras Canyon. Cu.

18 miles NE. Albuquerque, A. T. \& S. F. R. R.

Granite.

Veins.

Lindgren, W., The ore deposits of New Mexico: P. P. 68, 1910, p. 163.

Min. Res. 1905, p. 279.

1909, pt. 1, p. 435.

Top. sheet San Pedro.

\section{COLFAX COUNTY.}

2. Cimarroncito. Au.

12 miles S. Ute Park, St. L. R. M. \& P. R. R.

Carboniferous and Cretaceous sediments cut by monzonite.

Contact metamorphic.

Lindgren, W., The ore deposite of New Mexico: P. P. 68, 1910, p. 105.

3. Moreno (Elizabethtown). $\mathrm{Au}, \mathrm{Ag}, \mathrm{Cu}$. (D, Pl.)

12 miles W. Ute Park, St. L. R. M. \& P. R. R.

Paleozoic and Cretaceous sediments cut by monzonite porphyry.

Veins, contact metamorphic, wash gravels.

Lindgren, W., The ore deposits of New Mexico: P. P. 68, 1910, pp. 18, 32, $36,41,51-54,57-59,60,75,92-105$.

Min. Res. 1905, p. 279.

1906, p. 305.

1907 , pt. 1, p. 405.

1908 , pt. 1, p. 511. 
COLFAX COUNTY-Continued.

4. Ponil. Au. (Pl.)

Station St. L. R. M. \& P. R. R.

Wash gravels.

Lindgren, W., The ore deposits of New Mexico: P. P. 68, 1910, pp. 75, 92, 97. Min. Res. 1905, p. 279. 1907 , pt. 1, p. 405.

5. Ute Creek (Baldy). Au. (D, Pl.)

6 miles N. Ute Park, St. L. R. M. \& P. R. R.

Paleozoic and Cretaceous sediments cut by monzonite.

Veins, wash gravels.

Lindgren, $W$., The ore deposits of New Mexico: P. P. 68, 1910, pp. 97, 101-105, 348.

Min. Res. 1906, p. 279.

1907 , pt. 1, p. 405.

1908 , pt. 1, p. 511 .

DONA ANA COUNTY.

6. Black Mountain (Kent): Au, Ag.

25 miles NE. Las Cruces, A. T. \& S. F. R. R.

Paleozoic sediments cut by monzonite.

Contact metamorphic, veins.

Lindgren, W., The ore deposits of New Mexico: P. P. 68, 1910, pp. 86, 87, 209.

Min. Res. 1905, p. 279.

Top. sheet Las Cruces.

7. Hembrillo. $\mathrm{Cu}$.

45 miles NNE. Las Cruces, A. T. \& S. F. R. R.

Lindgren, W., The ore deposits of New Mexico: P. P. 68, 1910, p. 205.

8. Modoc. $\mathrm{Pb}, \mathrm{Ag}$.

20 miles W. Las Cruces, A. T. \& S. F. R. R.

Paleozoic sediments cut by andesite.

Contact metamorphic.

Lindgren, W., The ore deposits of New Mexico: P. P. 68, 1910, pp. 51-53, $57,58,60-64,205-213$.

Min. Res. 1905, p. 279.

Top. sheet Las Cruces.

9. Organ. $\mathrm{Pb}, \mathrm{Ag}, \mathrm{Cu}$.

15 miles NE. Las Cruces, A. T. \& S. F. R. R., stage line.

Paleozoic sediments cut by quartz monzonite.

Veins, contact metamorphic, replacements.

Keyes, C. R., Geology and underground water conditions of the Jornada del Muerto, New Mexico: W. S. P. 123, 1905, p. 23.

Lindgren, W., and Graton, L. C., A reconnaissance of the mineral deposits in New Mexico: Bull. 285, 1906, pp. 74-86.

Lindgren, W., The ore deposits of New Mexico: P. P. 68, 1910, pp. 51-53, $57,58,60-64,205-213$.

Weed, W. H., The copper mines of the United States in 1905: Bull. 285, 1906, p. 118.

Min. Res. 1905, p. 279.

1906 , p. 306.

1907 , pt. 1, p. 405.

1908 , pt. 1, p. 512 .

1909 , pt. 1, p. 436.

Top. sheet Las Cruces. 
LIST OF MINING DISTRICTS BERNALILLO COUNTY SANDOVAL COUNTY

Tijeras Canyon 48. Cochiti (Bland)

COLFAX COUNTY 49. Cuba (Nacimien

50. Placitas

Ponil

Ute Creek (Baldy)

DONA ANA COUNTY

SAN MIGUEL COUNTY

Mombrillo

9. Organ

GRANT COUNTY

Apache No. 2 (Anderson)

Black Hawk

Burro Mountains (Cow

Springs)

Central

Chloride Flat (Silver City) SIERRA COUNTY

Fierro 60 Caballos Mountains

Georgetown (Mimbres) 6t. Chloride (Apache, Cuchil

Granite Gap (San Simon) Negro)

. Hachita (Eureka,Sylvanite) 62 . Herm

64. Kingston (Black Range)

Pinos Altos

25. Pyramid (Lordsburg)

Red Hill

27. Santa Rita (Hanover)

Steeplerock

Virginia (Lordsburg)

LINCOLN COUNTY

Cedar Creek (Alto)

a. Estey (Oscuro)

3. Gallinas
Jicarilla

Nogal (Bonita, Parsons)

Whiteoaks

LUNA COUNTY

6. Cooks Peak

37. Florida Mountain

Fremont

Tres Hermanas
Victorio (Gage)

OTERO COUNTY

5. Kingston (B
5. Lake Valley

66. Pittsburg (Shandon)

67. Tierra Blanca (Bromide No. 1)

SOCORRO COUNTY

68. Canyoncito

69. Cooney (Mogollon)

70. Omitted

71. Jones Camp

73. Magdalena (Kelly)

74. Mill Canyon (Hop

Canyon)

75. Rosedale

76. San Andres

78. Silver Mountain. (Water Canyon)

79. Socorro

Highrolls

Jarilla (Orogrande, Silver

Hill, Brice)

Tularosa

RIO ARRIBA COUNTY

TAOS COUNTY

o. Anchor (La Belle)

81. Omitted

82. Picuris (Copper Hill)

8. Red River

Abiquiu

4. Bromide

Hopewell (Headistone)

\section{VALENCIA COUNTY}

5. Copperton (Zuni

Mountains)

MAP OF NEW MEXICO, SHOWING LOCATION OF MINING DISTRICTS

Scale $\frac{1}{2,500 ; 000}$

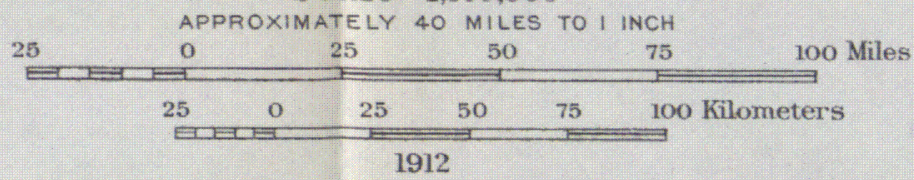



10. Texas Creek. $\mathrm{Au}, \mathrm{Ag}$.

21 miles NE. Las Cruces, A. T. \& S. F. R. R., stage.

Quartz monzonite.

Veins.

Lindgren, W., The ore deposits of New Mexico: P. P. 68, 1910, pp. 51-53, $57,58,60,64,205-213$.

Min. Res. 1905, p. 279.

1906 , p. 512.

GRANT COUNTY.

11. Apache No. 2 (Anderson). $\mathrm{Cu}(\mathrm{Au}, \mathrm{Ag}, \mathrm{W})$.

6 miles SSE. Hachita, E. P. \& S. W. R. R.

Paleozoic sediments cut by quartz monzonite.

Contact metamorphic.

Lindgren, W., The ore deposits of New Mexico: P. P. 68, 1910, pp. 51-53, $55-56,78,343-344$.

Weed, W. H., The copper mines of the United States in 1905: Bull. 285, 1906, p. 118.

Min. Res. 1906, p. 307.

1907 , pt. 1, p. 407.

1.908 , pt. 1 , pp. $513-726$.

1909 , pt. 1, p. 437 .

12. Black Hawk. Ag.

15 miles W. Silver City, A. T. \& S. F. R. R.

Gneiss cut by monzonite and diorite.

Veins.

Lindgren, W., The ore deposits of New Mexico: P. P. 68, 1910, pp. 57-58, 61, 324-325.

13. Burro Mountains (Cow Springs). Cu.

12 miles SW. Silver City, A. T. \& S. F. R. R., stage.

Pre-Cambrian granite and diorite cut by monzonite.

Veins, replacements, and stockworks.

Lindgren, W., The ore deposits of New Mexico: P. P. 68, 1910, pp. 17, 19, $27,28,38,61,62,321-324$.

Paige, S., Metalliferous ore deposits near the Burro Mountains, Grant County, N. Mex.: Bull. 470, 1911, pp. 131-150.

Weed, W. H., The copper mines of the United States in 1905: Bull. 285, 1906 , p. 118.

Min. Res. 1905, p. 280.

1906 , pp. 305, 307, 402.

1907, pt. 1, pp. 407,613 .

1908, pt. 1, pp. 214, 513.

1909 , pt. 1, pp. $170,437$.

Top. sheet Silver City.

14. Carpenter. $\mathrm{Pb}, \mathrm{Zn}$.

12 miles E. Fierro, A. T. \& S. F R. R.

Paleozoic sediments cut by granite porphyry and capped by rhyolite and andesite.

Veins.

Lindgren, W., The ore deposits of New Mexico: P. P. 68, 1910, pp. 37, 64, 65, 218, 268, 272.

15. Central. $\mathrm{Cu}, \mathrm{Pb}, \mathrm{Ag}(\mathrm{Zn})$.

7 miles E. Silver City, A. T. \& S. F. R. R., stage.

Cretaceous sediments cut by quartz monzonite porphyry. 
GRANT COURTY-ContInued.

15. Central-Continued.

Veins.

Lindgren, W., The ore deposits of New Mexico: P. P. 68, 1910, pp. 57, 61, 317-318.

Weed, W. H., The copper mines of the United States in 1905: Bull. 285, 1906, p. 118.

Min. Res. 1905, p. 280.

1906 , p. 307.

1907 , pt. 1, pp. 407-408.

1908 , pt. 1, p. 513 .

1909 , pt. 1, p. 437.

Top. sheets Silver City, Santa Rita special.

16. Chloride Flat (Silver City). $\mathrm{Ag}, \mathrm{Pb}$.

Station A. T. \& S. F. R. R.

Paleozoic sediments cut by quartz monzonite porphyry.

Replacements.

Lindgren, W., The ore deposits of New Mexico: P. P. 68, 1910, pp. 18, 26, $38,63-65,301-305$.

Min. Re日. 1905, p. 280.

1906, p. 307.

1907 , pt. 1, p. 408.

1908 , pt. 1 , p. 514.

1909 , pt. 1, p. 437

Top. sheet Silver City.

17. Fierro. $\mathrm{Fe}, \mathrm{Cu}$.

Station A. T. \& S. F. R. R.

Paleozoic sediments cut by quartz monzonite porphyry.

Contact metamorphic.

Lindgren, W., The ore deposits of New Mexico: P. P. 68, 1910, pp. 47, 55, 296, 306, 312-313.

Paige, S., The Hanover iron-ore deposite, New Mexico: Bull. 380, 1909, pp. 199-214.

Min. Res. 1907, pt. 1, pp. 407-408.

1908 , pt. 1, p. 513.

Top. sheet Silver City.

18. Georgetown (Mimbres). $\mathrm{Ag}(\mathrm{Pb})$.

11 miles NE. Santa Rita, A. T. \& S. F. R. R., stage.

Paleozoic sediments cut by quartz monzonite porphyry.

Veins and replacements.

Lindgren, W., The ore deposits of New Mexico: P. P. 68, 1910, pp. 18, 30, $38,62,63-65,306-307,318-319$.

Top. sheet Silver City.

19. Gold Hill. Au.

12 miles NE. Lordsburg, S. P. R. R.

Pre-Cambrian gneiss cut by granite and diabase.

Veins.

Lindgren, W., The ore deposits of New Mexico: P. P. 68, 1910, pp. 49, 50-51, $86,326-327$.

20. Granite Gap (San Simon). $\mathrm{Pb}, \mathrm{Ag}(\mathrm{Cu}, \mathrm{Au})$.

$4 \frac{1}{2}$ miles S. Steins, S. P. R. R.; 12 miles N. Antelope, E. P. \& S. W. R. R.

Granite, Paleozoic sediments cut by granite porphyry.

Replacements, contact metamorphic. 
GRANT GOUNTY-Continued.

20. Granite Gap (San Simon)-Continued.

Lindgren, W., The ore deptsits of New Mexico: P. P. 68, 1910, pp. 51-53, $62,63-64,329-331,340$.

Min. Res. 1905, p. 280.

1906 , p. 307.

1909 , pt. 1, p. 437.

21. Hachita (Eureka, Sylvanite). $\mathrm{Pb}, \mathrm{Ag}, \mathrm{Cu}, \mathrm{Au}$.

12 miles SW. Hachita, E. P. \& S. W. R. R., stage.

Paleozoic sediments cut by quartz monzonite and kersantite.

Veins, contact metamorphic.

Lindgren, W., The ore deposits of New Mexico: P. P. 68, 1910, pp. 18, 55, $56,57-58,59,60,335-343$.

Min. Res. 1905, p. 280.

1906, p. 307.

1907 , pt. 1, pp. 407,408 .

1908 , pt. 1, p. 514 .

1909 , pt. 1, p. 437.

22. Kimball (Steins Pass). $\mathrm{Ag}, \mathrm{Au}, \mathrm{Cu}$.

Steins station, S. P. R. R.

Diorite porphyry and rhyolite.

Veins.

Lindgren, W., The ore deposits of New Mexico: P. P. 68, 1910, pp. 68, 69-70, 328-329.

Min. Res. 1905, p. 280.

1906, p. 307.

1907 , pt. 1, p. 408.

1909 , pt. 1, p. 438.

.23. Lone Mountain. Ag.

6 miles E. Silver City, A. T. \& S. F. R. R.

Paleozoic sediments cut by quartz monzonite and granite porphyry.

Replacements.

Lindgren, W., The ore deposita of New Mexico: P. P. 68, 1910, pp. 30, 38, $62,63-64,65,320$.

24. Pinos Altos. $\mathrm{Cu}, \mathrm{Au}, \mathrm{Pb}, \mathrm{Ag}, \mathrm{Zn}$. (D, Pl.)

8 miles NNE. Silver City, A. T. \& S. F. R. R., stage.

Paleozoic sediments cut by granodiorite and diorite.

Veine, replacements.

Lindgren, W., The ore deposits of New Mexico: P. P. 68, 1910, pp. 18, 35, $38,43,57-58,59,61,62,64,75,297-301$.

Paige, S., The ore deposits near Pinos Altos, N. Mex.: Bull. 470, 1911, pp. 109-125.

Min. Res. 1905, p. 280.

1907 , pt. 1, p. 408.

1908 , pt. 1, pp. 514-515.

1909 , pt. 1, p. 438.

Top. sheet Silver City.

25. Pyramid (Lordsburg). Ag, Cu, Mo.

8 miles S. Lordsburg, S. P. R. R.

Diorite cut by andesite.

Veins.

Lindgren, W., The ore deposits of New Mexico: P. P. 68, 1910, pp. 39, $57-58,60-61,332-335$. 
GRANT COUNTY-Continued.

25. Pyramid (Lordsburg)-Continued.

Weed, W. H., The copper mines of the United States in 1905: Bull. 285, 1906, p. 118.

Min. Res. 1905, p. 280.

1906, p. 307.

1907, pt. 1, p. 408.

1908 , pt. 1 , p. 514

1909 , pt. 1, p. 437.

26. Red Hill. $\mathrm{Pb}, \mathrm{Ag}, \mathrm{Au}, \mathrm{Cu}$.

15 miles S. Playas, E. P. \& S. W. R. R.

Min. Res. 1905, p. 280.

1906, p. 307.

1907 , pt. 1, p. 408

1908 , pt. 1, p. 515 .

1909 , pt. 1, p. 438.

27. Santa Rita (Hanover). Cu, Fe.

Station A. T. \& S. F. R. R.

Paleozoic and Cretaceous sediments cut by quartz monzonite porphyry and capped by rhyolite.

Veins, contact metamorphic.

Birkinbine, J., Iron ores of the United States: Eighteenth Ann. Rept., pt. 5, 1897, pp. 48-50.

Lindgren, W., The ore deposits of New Mexico: P. P. 68, 1910, pp. 3, 5, 18, $38,41-43,51-53,55,57-58,305,317$.

Paige, S., The Hanover iron-ore deposits, New Mexico: Bull. 380, 1909, pp. 199-214.

Weed, W. H., The copper mines of the United States in 1905: Bull. 285, 1906, p. 118.

Min. Res. 1882, pp. 147-148, 225-226.

1883-84, p. 285.

1887, p. 76.

1906, pp. 402-403.

1907, pt. 1, pp. 408, 613 .

1908 , pt. 1, pp. 214, 513-514.

1909 , pt. 1, pp. 170, 437.

Top. sheets Silver City, Santa Rita special.

28. Steeplerock. Au, Ag.

16 miles NE. Duncan, Ariz., A. \& N. M. R. R.

Diorite porphyry and rhyolite.

Veins.

Lindgren, W., The ore deposits of New Mexico: P. P. 68, 1910, pp. 68, 69-71, $327-328$.

Min. Res. 1907, pt. 1, p. 408.

29. Telegraph. Ag. 1908 , pt. 1, p. 515 .

25 miles NW. Silver City, A. T. \& S. F. R. R.

Pre-Cambrian granite and gneiss and Paleozoic sediments.

Veins.

Lindgren, W., The ore deposits of New Mexico: P. P. 68, 1910, pp. 38, 57-58, $61,325-326$.

30. Virginia (Lordsburg). Ag, $\mathrm{Cu}$ (Au).

3 miles SSW. Lordsburg, S. P. R. R.

Diorite cut by andesite. 
GRANT COUNTT-Continued.

30. Virginis (Lordsburg)-Continued.

Veins.

Lindgren, W., The ore deposits of New Mexico: P. P. 68, 1910, pp. 39, 57-58, 60-61, 332-335.

Weed, W. H., The copper mines of the United States in 1905: Bull. 285, 1905, p. 118.

Min. Res. 1906, p. 307.

1908 , pt. 1, p. 514.

1909 , pt. 1, pp. $437-438$.

\section{IINCOLN COUNTY.}

31. Cedar Creek (Alto). Ag (Cu, Au).

10 miles W. Capitan, E. P. \& S. W. R. R.

Min. Res. 1908, pt. 1, p. 515.

1909 , pt. 1, p. 438.

31a. Estey (Oscuro). Cu.

15 miles WNW. Oscuro, E. P. \& S. W. R. R.

"Red Beds."

Disseminated.

Lindgren, W., The ore deposits of New Mexico: P. P. 68, 1910, pp. 77, 78, $201,202$.

Lee, W. T., and Girty, G. H., The Manzano group of the Rio Grande valley, New Mexico: Bull. 389, 1909.

Min. Res. 1907, pt. 1, p. 413.

1908 , pt. 1, p. 519.

32. Gallinas. Au. (Pl.)

5 miles W. Corona, E. P. \& S. W. R. R.

Wash gravels.

Lindgren, W., The ore deposits of New Mexico: P. P. 68, 1910, p. 176.

Min. Res. 1909, pt. 1, p. 438.

33. Jicarilla. Au, Ag. (Pl, D.)

8 miles ESE. Ancho, E. P. \& S. W. R. R.

Quartz monzonite cut by diorite.

Veins.

Lindgren, W., The ore deposits of New Mexico: P. P. 68, 1910, pp. 39, 51-53, $57-58,59,60,75,176,183-184$.

Min. Res. 1907, pt. 1, p. 409.

1908 , pt. 1 , p. 515.

1909 , pt. 1, p. 438.

34. Nogal (Bonita, Parsons). Au, Ag.

12-20 miles ESE. Carrizozo, E. P. \& S. W. R. R., stage.

Monzonite porphyry and diorite.

Veins.

Lindgren, W., The ore deposits of New Mexico: P. P. 68, 1910, pp. 39, 57-58, $75,175-179$.

Min. Res. 1905, pp. 280-281.

1906 , p. 308.

1907, pt. 1, p. 409

1908 , pt. 1 , p. 515

1909 , pt. 1, p. 438.

35. Whiteoaks. Au, Ag. (D, Pl.)

12 miles NE. Carrizozo, E. P. \& S. W. R. R.

Cretaceous sediments cut by monzonite. 


\section{InTCOLN COUNTY-Continued.}

35. Whiteoaks-Continued.

Veins.

Lindgren, W., The ore deposits of New Mexico: P. P. 68, 1910, pp. 19, 39, $57-58,60,66,175-176,179-182$.

Min. Res. 1905, p. 280.

1906 , p. 308.

1907 , pt. 1, p. 409.

1908, pt. 1, pp. 515-516, 727.

1909 , pt. 1, p. 439.

LUNA COUNTY.

36. Cooks Peak. $\mathrm{Pb}, \mathrm{Ag} . \mathrm{Au}, \mathrm{Zn}$.

17 miles W. Nutt, A. T. \& S. F. R. R.

Paleozoic sediments cut by granodiorite porphyry.

Replacements.

Lindgren, W., The ore deposits of New Mexico: P. P. 68, 1910, pp. 37, 39, 62, 63-64, 287-289.

Min. Res. 1905, p. 281.

1906, p. 308.

1907 , pt. 1, p. 409.

1908 , pt. 1, p. 516.

1909 , pt. 1, p. 439.

Top. sheet Deming.

37. Florida Mountains. $\mathrm{Ag}, \mathrm{Pb}(\mathrm{Cu}, \mathrm{Au})$.

12 miles SE. Deming, S. P. R. R., A. T. \& S. F. R. R.

Granite porphyry, andesite, and limestone.

Replacements.

Lindgren, W., The ore deposits of New Mexico: P. P. 68, 1910, pp. 27, 28, 30, 62, 225, 287, 289-290.

Min. Res. 1905, p. 281.

1906 , p. 308.

1907 , pt. 1, p. 409.

1908 , pt. 1, p. 516.

Top. sheet Deming.

38. Fremont. $\mathrm{Pb}, \mathrm{Ag}$.

15 miles SE. Hachita, E. P. \& S. W. R. R.

Paleozoic sediments cut by granite porphyry and kersantite.

Veins and replacement.

Lindgren, W., The ore deposits of New Mexico: P. P. 68, 1910, pp. 58, 60, 345-348.

Min. Res. 1909, pt. 1, p. 439.

39. Tres Hermanas. $\mathrm{Zn}, \mathrm{Pb}, \mathrm{Au}, \mathrm{Ag}$.

15 miles NE. Hermanas, E. P. \& S. W, R. R.; 25 miles S. Deming, S. P. R. R. Paleozoic sediments cut by quartz syenite porphyry.

Veins, contact metamorphic.

Lindgren, W., The ore deposits of New Mexico: P. P. 68, 1910, pp. 38, 41, $51,55,57-58,287,292-295$.

The Tres Hermanas mining district, New Mexico: Bull. 380, 1909, pp. 123-128.

Min. Res. 1905, p. 281.

1906, p. 308.

1908 , pt. 1, p. 516 .

1909 , pt. 1, p. 439. 
40. Victorio (Gage). $\mathrm{Pb}, \mathrm{Ag}, \mathrm{Au}, \mathrm{W}$.

Station S. P. R. R.

Limestone capped by andesite.

Veins, replacements.

Lindgren, W., The ore deposits of New Mexico: P. P. 68, 1910, pp. 18, 43, $62,63,64,287,290-292$.

Min. Res. 1906, p. 308. 1907, pt. 1, p. 409 1908, pt. 1, pp. 516, 726.

Top. sheet Deming.

\section{OTERO COUNTY.}

41. Highrolls. $\mathrm{Cu}, \mathrm{Ag}$.

2 miles N. Mountain Park, E. P. \& S. W. R. R.

Min. Res. 1908, pt. 1, p. 516 . 1909 , pt. 1, p. 439.

42. Jarilla (Orogrande, Silver Hill, Brice). $\mathrm{Au}, \mathrm{Ag}, \mathrm{Cu}(\mathrm{Pb}) . \quad(\mathrm{D}, \mathrm{Pl}$.

Brice station, E. P. \& S. W. R. R.

Paleozoic sediments cut by monzonite porphyry.

Contact metamorphic and veins.

Lindgren, W., The ore deposits of New Mexico: P. P. 68, 1910, pp. 19, 35, $56,60,75,184-187$.

Weed, W. H., The copper mines of the United States in 1905: Bull. 285, 1906, p. 118.

Min. Res. 1905, p. 281.

1906, p. 309.

1907, pt. 1, pp. 410, 614 .

1908 , pt. 1, pp. 516-517.

43. Tularosa. Cu, Ag. 1909 , pt. 1, p. 439.

13 miles ENE. Monterey, E. P. \& S. W. R. R.

Paleozoic and Mesozoic sediments, diorite.

Veins, disseminations.

Lindgren, W., The ore deposits of New Mexico: P. P. 68, 1910, pp. 77, 78, 184, 187-190.

Min. Res. 1905, p. 281.

1906, p. 309.

1907, pt. 1, p. 410.

1908 , pt. 1, p. 517.

1909 , pt. 1, p. 439.

RIO ARRIBA COUNTY.

44. Abiquiu. $\mathrm{Cu}$.

19 miles NW. Chamita, D. \& R. G. R. R., stage,

"Red Beds."

Disseminations.

Emmons, S. F., The copper in the red beds of the Colorado Plateau region:

Bull. 260, 1905, p. 225.

Lindgren, W., The ore deposits of New Mexico; P. P. 68, 1910, pp. 75, 77, $124,149$.

Min. Res. 1905, p. 282.

45. Bromide. $\mathrm{Cu}, \mathrm{Au}, \mathrm{Ag}$.

12 miles W. Tres Piedras, D. \& R, G, R. R.

Pre-Cambrian schist. 
RIO ARRIBA OOUNTY-Continued.

45. Bromide-Continued.

Veins.

Lindgren, W., The ore deposits of New Mexico: P. P. 68, 1910, pp. 49, 50, $51,124,126,130-133$.

Min. Res. 1905, pp. 281-282.

1907, pt. 1, p. 410 .

1908 , pt. 1, p. 517.

46. Gallina. $\mathrm{Cu}, \mathrm{Pb}, \mathrm{Ag}$.

65 miles WNW. Chamita, D. \& R. G. R. R.

"Red Beds."

Disseminated.

Lindgren, W., The ore deposits of New Mexico: P. P. 68, 1910, pp. 147-148.

Min. Res. 1908, pt. 1, p. 517.

47. Hopewell (Headstone). Au, Ag, Cu. (D, Pl.)

20 miles WNW. Tres Piedras, D. \& R. G. R. R.

Pre-Cambrian schists.

Veins, wash gravels.

Lindgren, W., The ore deposite of New Mexico: P. P. 68, 1910, pp. 27, 28, $49,50,51,75,124-130$.

Min. Res. 1905, p. 281.

1907, pt. 1, p. 410.

1908 , pt. 1, p. 517.

\section{SATDOVAL COUNTY.}

48. Cochiti (Bland). Au, Ag.

27 miles WNW. Domingo, A. T. \& S. F. R. R.

Monzonite and rhyolite porphyry.

Veins, replacements.

Lindgren, W., The ore deposits of New Mexico: P. P. 68, 1910, pp. 68, 69, $70,71,141,150-158$.

Min. Res. 1905, p. 282.

1907, pt. 1, p. 411 .

Top. sheet Jemez.

49. Cuba (Nacimiento Mountains). Cu.

88 miles NNW. Alameda, A. T. \& S. F. R. R., stage to Jemez Springs.

"Red Beds."

Disseminations.

Emmons, S. F., The copper in the red beds of the Colorado Plateau region: Bull. 260, 1905, p. 225.

Lindgren, W., The ore deposits of New Mexico: P. P. 68, 1910, pp. 77, 78, 143-147.

Weed, W. H., The copper mines of the United States in 1905: Bull. 285, 1906, p. 118.

Min. Res. 1882, pp. 225-226.

1906 , p. 309.

Top. sheet Jemez.

50. Placitas. Cu.

8 miles ESE. Bernalillo, A. T. \& S. F. R. R.

Paleozoic sediments.

Replacements.

Lindgren, W., The ore deposits of New Mexico: P. P. 68, 1910, p. 141.

Min. Res. 1907, pt. 1, p. 411.

Top. sheet San Pedro. 
SAT MIGUEL COUNTY.

51. Cooper (Pecos). $\mathrm{Cu}, \mathrm{Au}, \mathrm{Ag}$.

21 miles NNE. Glorieta, A. T. \& S. F. R. R.

Pre-Cambrian schists.

Disseminated.

Lindgren, W., The ore deposits of New Mexico: P. P. 68, 1910, pp. 112-114.

Min. Res. 1905, p. 282.

1906 , p. 309.

1907 , pt. 1, p. 411.

Top. sheet Santa Fe.

52. Omitted.

53. Mineral Hill. $\mathrm{Cu}$.

20 miles WNW. Las Vegas, A. T. \& S. F. R. R.

Top. sheet Las Vegas.

54. Rociada. $\mathrm{Au}, \mathrm{Ag}, \mathrm{Cu}(\mathrm{Pb}, \mathrm{Zn})$.

29 miles NNW. Las Vegas, A. T. \& S. F. R. R.

Pre-Cambrian gneiss and schist and Paleozoic sediments.

Veins.

Lindgren, W., The ore deposits of New Mexico: P. P. 68, 1910, pp. 27, 49, $50,51,109,114-116$.

Top. sheet Las Vegas.

55. Tecolote (Salitre, San Pablo). Cu.

12 miles SE. Las Vegas, 10 miles N. Bernal, A. T. \& S. F. R. R.

Upper Carboniferous and Cretaceous sediments.

Disseminated.

Lindgren, W., The ore deposits of New Mexico: P. P. 68, 1910, pp. 77, 109, 116-123.

Min. Res. 1905, p. 282.

1906, p. 309.

1907 , pt. 1, p. 411.

Top. sheets Las Vegas, Bernal.

SANTA FE COUNTY.

56. Cerrillos. $\mathrm{Pb}, \mathrm{Ag}, \mathrm{Cu}, \mathrm{Au}$.

Station A. T. \& S. F. R. R.

Cretaceous sediments cut by monzonite porphyry.

Veins.

Clarke, F. W., and Diller, J. S., Turquoise from New Mexico: Bull. 42, 1887, p. 39.

Lindgren, W., The ore deposits of New Mexico: P. P. 68, 1910, pp. 35, 36, $39,57,58,60,163-167$.

Min. Res. 1905, p. 282.

1906, p. 309.

1907, pt. 1, p. 411.

1908 , pt. 1, p. 517.

1909 , pt. 1, pp. $439-440$.

Top. sheet San Pedro.

57. Glorieta. Fe.

2 miles S. Fox Siding, A. T. \& S. F. R. R.

Cretaceous sandstone.

Beds.

Lindgren, W., The ore deposits of New Mexico: P. P. 68, 1910, pp. 77, 107, 111-112. 
SANTA FE COUNTY-Continued.

58. New Placers. Au. (Pl.)

20 miles S. Cerrillos, A. T. \& S. F. R. R.

Wash gravels.

Lindgren, W., The ore deposits of New Mexico: P. P. 68, 1910, pp. 17, 75, $76,163,164,170-175$.

Min. Res. 1905, p. 282.

1906, p. 309.

1907, pt. 1, pp. 411, 613.

1908 , pt. 1, p. 517.

Top. sheet San Pedro.

59. Old Placers (Ortiz, Dolores). Au. (D, Pl.)

15 miles S. Cerrillos, A. T. \& S. F. R. R.

Monzonite porphyry and diorite.

Contact metamorphic, veins.

Lindgren, W., The ore deposits of New Mexico: P. P. 68, 1910, pp. 17, 75, $76,167-169$.

Top. sheet San Pedro.

59a. San Pedro. $\mathrm{Cu}, \mathrm{Au}, \mathrm{Ag}, \mathrm{Pb}$.

20 miles S. Cerrillos, A. T. \& S. F. R. R.

Paleozoic and Cretaceous sediments cut by porphyry.

Contact metamorphic, veins.

Lindgren, W., The ore deposits of New Mexico: P. P. 68, 1910, pp. 51-53, $55,57-58,60,62,64,171-174$.

\section{SIERRA COUNTY.}

60. Caballos Mountains. $\mathrm{Cu}, \mathrm{Ag}, \mathrm{Pb}(\mathrm{Au}, \mathrm{V})$. (D, Pl.)

15 miles NW. Rincon, A. T. \& S. F. R. R.

Carboniferous sediments.

Veins, disseminations.

Hess, F. L., Vanadium in the Sierra de los Caballos, New Mexico: Bull. 530, 1912.

Lindgren, W., The ore deposits of New Mexico: P. P. 68, 1910, pp. 27, 28, $29,30,74,218,229,238,283-285$.

61. Chloride (Apache, Cuchillo Negro). Ag, $\mathrm{Cu}, \mathrm{Au}, \mathrm{Pb}$.

51 miles WNW. Engle, A. T. \& S. F. R. R., stage.

Paleozoic sediments capped by andesite and rhyolite.

Veins.

Lindgren, W., The ore deposits of New Mexico: P. P. 68, 1910, pp. 18, 55, 68-71, 218, 260-266.

Min. Res. 1905, pp. 282-283.

1906, pp. 309-310.

1907 , pt. 1, p. 412 .

62. Hermosa (Palomas). $\mathrm{Ag}, \mathrm{Pb}$.

35 miles W. Engle, A. T. \& S. F. R. R.

Paleozoic sediments capped by andesite and rhyolite.

Veins and replacements.

Lindgren, W., The ore deposits of New Mexico: P. P. 68, 1910, pp. 18, 55, 62-67, 77, 218, 232, 233, 267-268.

Min. Res. 1905, p. 283.

1908, pt. 1, p. 518.

1209 , pt. 1, p. 440 . 
SIERRA COUNTY-Continued.

63. Hillsboro (Las Animas). $\mathrm{Au}, \mathrm{Ag}, \mathrm{Cu}, \mathrm{V}, \mathrm{Mg}, \mathrm{Fe} . \quad(\mathrm{D}, \mathrm{Pl}$.)

18 miles N. Lake Valley, A. T. \& S. F. R. R.

Paleozoic sediments cut by monzonite porphyry and capped by andesite.

Veins and replacements.

Lindgren, W., The ore deposits of New Mexico: P. P. 68, 1910, pp. 18, 30, $31,37,39,43,62,64,65,68,69,70,75,214,218,227-228,238,239,272-276$, 277.

Weed, W. H., The copper mines of the United States in 1905: Bull. 285, 1906, p. 118.

Min. Res. 1887, p. 76.

1905 , p. 282.

1906, p. 310 .

1907, pt. 1, p. 412 .

1908 , pt. 1, p. 518 .

1909, pt. 1, p. 440.

64. Kingston (Black Range). $\mathrm{Ag}, \mathrm{Pb}, \mathrm{Cu}$.

27 miles NNW. Lake Valley, A. T. \& S. F. R. R.

Pre-Cambrian granite, gneiss, and schist, flanked by Paleozoic sediments and capped by andesite and rhyolite.

Replacements, veins.

Harder, E. C., Manganese deposits of the United States: Bull. 380, 1909, p. 274.

Lindgren, W., The ore deposits of New Mexico: P. P. 68, 1910, pp. 18, 31, $34,37,62,65,218,224,225,226,228,232,245,268-270$.

Min. Res. 1905, p. 283.

1907 , pt. 1, p. 412 .

1908 , pt. 1, p. 518.

1909 , pt. 1, p. 440.

65. Lake Valley. $\mathrm{Ag}, \mathrm{Pb}, \mathrm{Mn}, \mathrm{Au}, \mathrm{Cu}$.

Station A. T. \& S. F. R. R.

Paleozoic sediments capped by andesite and rhyolite.

Replacement, veins.

Lindgren, W., The ore deposits of New Mexico: P. P. 68, 1910, pp. 18, 30, $31,43,62,63,64,66,218,227,228,276-282$.

Min. Res. 1905, p. 283.

1909 , pt. 1, p. 440.

66. Pittsburg (Shandon). Au. (Pl.)

15 miles NW. Rincon, A. T. \& S. F. R. R.

Wash gravels.

Lindgren, W., The ore deposits of New Mexico: P. P. 68, 1910, pp. 19, 218, 282-284.

Min. Res. 1905, p. 282.

1906, p. 310.

1909 , pt. 1, p. 440

67. Tierra Blanca (Bromide No. 1). Ag, $\mathrm{Pb}, \mathrm{Au}$.

15 miles NW. Lake Valley, A. T. \& S. F. R. R.

Paleozoic sediments capped by andesite and rhyolite and cut by monzonite.

Replacement, veins.

Lindgren, W., The ore deposits of New Mexico: P. P. 68, 1910, pp. 37, 64, 66, 218, 268-271.

Min. Res. 1905, p. 283.

1906, p. 310.

$21528^{\circ}-$ Bull, $507-12-16$ 
68. Canyoncito. $\mathrm{Pb}, \mathrm{Cu}$.

\section{SOCORRO COUNTY.}

7 miles SE. La Joya, A. T. \& S. F. R. R.

Pre-Cambrian granite, gneiss, and schist, Paleozoic sediments cut by aplite. Veins.

Lindgren, W., The ore deposits of New Mexico: P. P. 68, 1910, pp. 74, 218, $240-241$.

69. Cooney (Mogollon). $\mathrm{Au}, \mathrm{Ag}, \mathrm{Cu}$.

85 miles NW. Silver City, A. T. \& S. F. R. R.

Tertiary volcanics.

Veins.

Lindgren, W., The ore deposits of New Mexico: P. P. 68, 1910, pp. 18, 42, $43,44,47,68,69-71,191-204$.

Weed, W. H., The copper mines in the United States in 1905: Bull. 285, 1906, p. 118.

Min. Res. 1905, p. 284.

1906, pp. 310-311.

1907 , pt. 1, pp. $412-413$.

1908 , pt. 1 , pp. $518-519$.

70. Omitted. 1909 , pt. 1, p. 441.

71. Jones Camp. Fe.

47 miles E. San Antonio, A. T. \& S. F. R. R.

Paleozoic limestone cut by monzonite.

Contact metamorphic.

Lindgren, W., The ore deposits of New Mexico: P. P. 68, 1910, pp. 51, 53, $203,204$.

Min. Res. 1882, pp. 147-148.

72. Hansonburg. $\mathrm{Cu}$.

28 miles E. San Antonio, A. T. \& S. F. R. R.

Paleozoic sediments.

Disseminated.

Lindgren, W., The ore deposits of New Mexico: P. P. 68, 1910, pp. 77, 203.

73. Magdalena (Kelly). $\mathrm{Zn}, \mathrm{Pb}, \mathrm{Au}, \mathrm{Ag}, \mathrm{Cu}$.

Station A. T. \& S. F. R. R.

Schist overlain by crystalline limestone, shale, and quartzite; Paleozoic sediments cut by granite, monzonite, quartz monzonite porphyry, and diabase.

Replacements.

Dutton, C. E., Mount Taylor and the Zuñi Plateau: Sixth Ann. Rept., 1885, p. 194.

Lindgren, W., The ore deposits of New Mexico: P. P. 68, 1910, pp. 18, 19, $47,51-53,55,214,218,220,241-258$.

Weed, W. H., The copper mines of the United States in 1905: Bull. 285, 1906.

Min. Res. 1883-84, p. 425.

1885 , p. 258.

1886 , p. 146.

1887 , p. 110.

1888, p. 89.

1904, p. 276.

1905 , p. 283.

1906, pp. 311, 403, 476.

1907, pt. 1, p. 413.

1908 , pt. 1, p. 519.

1909 , pt. 1, p. 441. 
SOCORRO COUNTY-Continued.

74. Mill Canyon (Hop Canyon). $\mathrm{Au}, \mathrm{Ag}, \mathrm{Cu}$.

6 miles S. Magdalena, A. T. \& S. F. R. R.

Tertiary volcanics.

Veins.

Lindgren, W., The ore deposits of New Mexico: P. P. 68, 1910, p. 258.

75. Rosedale Au, Ag.

30 miles SW. Magdalena, A. T. \& S. F. R. R.

Tertiary volcanics.

Veins.

Lindgren, W., The ore deposits of New Mexico: P. P. 68, 1910, pp. 68, 70, 218, 259-260.

Min. Res. 1905, p. 284.

1907, pt. 1, p. 413 .

1908, pt. 1, p. 519.

1909 , pt. 1, p. 441 .

76. San Andreas. $\mathrm{Cu}, \mathrm{Pb}, \mathrm{Bi}$.

20 miles E. Aleman, A. T. \& S. F. R. R.

Paleozoic sediments.

Veins.

Lindgren, W., The ore deposits of New Mexico: P. P. 68, 1910, pp. 35, 74, $205,234$.

Min. Res. 1908, pt. 1, p. 713.

77. San Lorenzo. Cu.

20 miles NW. Socorro, A. T. \& S. F. R. R.

Tertiary volcanics (?).

Veins (?).

Lindgren, W., The ore deposits of New Mexico: P. P. 68, 1910, p. 241.

78. Silver Mountain (Water Canyon). $\mathrm{Au}, \mathrm{Ag}, \mathrm{Cu}, \mathrm{Pb}$.

15 miles SE. Magdalena, A. T. \& S. F. R. R.

Paleozoic sediments cut by porphyry.

Contact metamorphic.

Lindgren, W., The ore deposits of New Mexico: P. P. 68, 1910, pp. 36, 37, $242,245,246,247,258$.

79. Socorro. $\mathrm{Ag}, \mathrm{Au}$.

2 miles NW. Socorro, A. T. \& S. F. R. R.

Andesite tuff.

Veins.

Lindgren, W., The ore deposits of New Mexico: P. P. 68, 1910, pp. 69-71, 218, 239-240.

Top. sheet Socorro.

80. Anchor (La Belle). Au, Ag.

TAOS COUNTY.

30 miles NNW. Ute Park, St. L. R. M. \& P. R. R.

Quartz monzonite and diorite porphyry.

Veins.

Lindgren, W., The ore deposits of New Mexico: P. P. 68, 1910, pp. 60, 82, $88,89$.

81. Omitted.

82. Picuris (Copper Fill). $\mathrm{Cu}, \mathrm{Ag}, \mathrm{Au} . \quad$ (D, Pl.)

12 miles ENE. Embudo, D. \& R. G. R. R.

Pre-Cambrian granite and schists.

Veins.

Lindgren, W., The ore deposits of New Mexico: P. P. 68, 1910, pp. 27, 28, $49-51,82,89-91$. 
TAOS COUNTY-Continued.

83. Red River. Au, Ag.

29 miles NW. Ute Park, St. L. R. M. \& P. R. R.

Pre-Cambrian granite and schist cut by monzonite porphyry, rhyolite, and andesite.

Veins.

Lindgren, W., The ore deposits of New Mexico: P. P. 68, 1910, pp. 35, 36, $42,43,68,69,70,71,82,84-88$.

Min. Res. 1906, p. 312.

1907 , pt. 1, p. 413.

84. Twining (Rio Hondo). $\mathrm{Cu}, \mathrm{Au}, \mathrm{Pb}, \mathrm{Ag}, \mathrm{Sb} . \quad$ (D, Pl.)

34 miles NE. Barranca, D. \& R. G. R. R.

Pre-Cambrian granite and schist cut by monzonite.

Veins, wash gravels.

Lindgren, W., The ore deposits of New Mexico: P. P. 68, 1910, pp. 75, 83,84 .

Min. Res. 1906, p. 312.

1907, pt. 1, p. 413.

\section{VALENCIA COUNTY.}

85. Copperton (Zuni Mountains). Cu.

23 miles W. Grant, A. T. \& S. F. R. R.

Pre-Cambrian granite and granite porphyry overlain by Paleozoic and Mesozoic sediments.

Veins, disseminated.

Lindgren, W., The ore deposits of New Mexico: P. P. 68, 1910, pp. 28, 77, 134, 135, 137-140.

Dutton, C. E., Mount Taylor and the Zuñi Plateau: Sixth Ann. Rept., 1885, pp. 105-198.

Top. sheet Wingate.

86. Manzano. Cu.

Mountain station, A. T. \& S. F. R. R.

Paleozoic and Mesozoic sediments.

Disseminated.

Lindgren, W., The ore deposits of New Mexico: P. P. 68, 1910, pp. 134, 163. 


\section{OREGON.}

In the State of Oregon there are 73 mining districts in 16 counties. Gold is the predominant mineral in 63 of these districts, and almost all of these contain some placer deposits. In 7 districts copper predominates, in 1 silver, and in 2 quicksilver.

Distribution of the predominant metals produced in the mining districts of Oregon.

\begin{tabular}{|c|c|c|c|c|c|}
\hline County. & Gold. & Silver. & Copper. & $\begin{array}{l}\text { Quick- } \\
\text { silver. }\end{array}$ & Total. \\
\hline 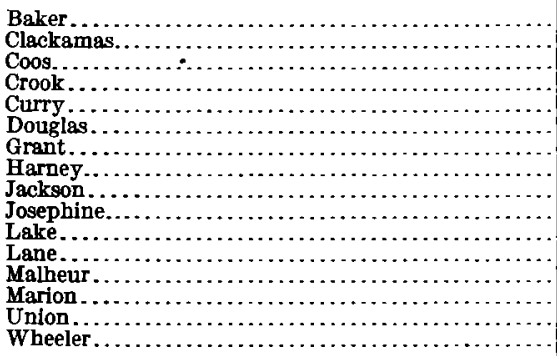 & \begin{tabular}{r|}
15 \\
3 \\
1 \\
5 \\
7 \\
6 \\
1 \\
6 \\
10 \\
1 \\
3 \\
2 \\
1 \\
1 \\
1
\end{tabular} & 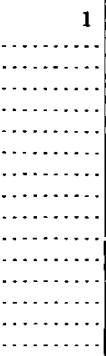 & 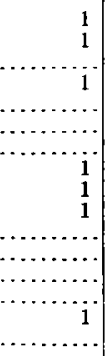 & 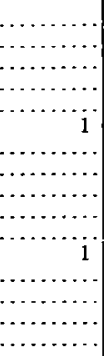 & $\begin{array}{r}17 \\
1 \\
3 \\
2 \\
5 \\
8 \\
6 \\
2 \\
7 \\
11 \\
1 \\
4 \\
2 \\
1 \\
2 \\
1\end{array}$ \\
\hline & $c 3$ & 1 & 7 & 2 & 73 \\
\hline
\end{tabular}

MINING DISTRICTS IN OREGON.

[See PI. XI. Additional references will be found in Mineral Resources for 1910 and 1911.]

\section{BAEER COUNTY.}

1. Bonanza (Geiser). Au, Ag. (D, Pl.)

8 miles NW. Whitney, S. V. R. R.

Argillites cut by granodiorite and capped by Tertiary volcanics.

Veins.

Lindgren, W., The gold belt of the Blue Mountains of Oregon: Twentysecond Ann. Rept., pt. 2, 1901, pp. 697-704.

Min. Res. 1906, p. 316.

1907 , pt. 1, p. 423.

1908 , pt. 1, p. 528.

Top. sheet Sumpter.

2. Buck Gulch. Au. (Pl.)

7 miles W. Sumpter, S. V. R. R.

Stream gravels.

Pardee, J. T., Placer gravels of the Sumpter and Granite districts, eastern Oregon: Bull. 430, 1910, pp. 59-65.

Top. sheet Sumpter.

3. Burkemont. Cu.

20 miles N. Baker City, O. W. R. R. \& N. Co.

Amygdaloidal basalt.

Disseminations.

Top. sheet Baker City. 
BAERR COUNTY-Continued.

4. Cable Cove. Au, $\mathrm{Ag}, \mathrm{Cu}$.

14 miles N. Sumpter, S. V. R. R.

Granite.

Veins.

Lindgren, W., The gold belt of the Blue Mountains of Oregon: Twentysecond Ann. Rept., pt. 2, 1901, pp. 671-676.

Pardee, J. T., Placer gravels of the Sumpter and Granite districts, eastern Oregon: Bull. 430, 1910, pp. 59-65.

Min. Res. 1906, p. 316.

1907 , pt. 1, p. 423.

1908 , pt. 1, p. 528 .

Top. sheet Burkemont.

5. Conner Creek. Au, Ag. (D, Pl.)

19 miles N. Huntington, O. W. R. R. \& N. Co.

Argillites.

Veins and stream gravels.

Lindgren, W., The gold belt of the Blue Mountains of Oregon: Twentysecond Ann. Rept., pt. 2, 1901, pp. 756-759.

Min. Res. 1906, p. 316.

1907 , pt. 1, p. 423.

6. Cornucopia. Au, Ag. 1908 , pt. 1, p. 528 .

62 miles NE. Baker City, O. W. R. R. \& N. Co.

Triassic (?) sediments cut by granite.

Veins.

Lindgren, W., The gold belt of the Blue Mountains of Oregon: Twentysecond Ann. Rept., pt. 2, 1901, pp. 740-745.

Min. Res. 1906, p. 316.

1907, pt. 1, pp. $422-423$.

1908 , pt. 1, pp. 527-528.

1909 , pt. 1, p. 447.

7. Cracker Creek (Sumpter). Au, Ag. (D, Pl.)

Sumpter station, S. V. R. R.

Paleozoic sediments and greenstones cut by granite.

Veins.

Lindgren, W., The gold belt of the Blue Mountains of Oregon: Twentysecond Ann. Rept., pt. 2, 1901, pp. 645-671.

Pardee, J. T., Faulting and vein structure in the Cracker Creek gold district, Baker County, Oreg.: Bull. 380, 1909, pp. 85-93.

Min. Res. 1905, p. 290.

1906 , p. 316.

1907 , pt. 1, pp. 422,423 .

1908 , pt. 1, p. 527.

1909, pt. 1, p. 447.

Top. sheet Sumpter.

8. Ellkhorn. Au, Ag.

14 miles NW. Baker City, O. W. R. R. \& N. Co.

Argillites cut by granodiorite and diorite.

Veins.

Lindgren, W., The gold belt of the Blue Mountains of Oregon: Twentysecond Ann. Rept., pt. 2, 1901, pp. 645-649.

Min. Res. 1905, p. 291.

1907, pt. 1, p. 422 .

Top. sheet Sumpter. 
1. Stice
1. Stictue

Virtue

CLACKAMAS COUNTY CoOs COUNTY

Eden

Randoloph
CROOK COUNTY
Howard (Ochooo, Boliv

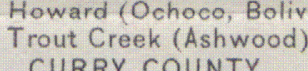

Beach placers (Denmark,
Harbor, Gold Creek, etc.)

Chetco

Rowert Bugte
Sises River

SOUGLAS COUNTY

Bohemia Crackerjach
Dothan

Dothan
Drew

Nugget (Myrtle Creek) Olalla

Riddles
Starvout (Green Mountain)

GRANT
Alamo
Alanyon
Cand

. Canyon

Granite
Quartzburg (Comer)
Susanville (Galena)

HARNEY COUNTY

Harney
Pueblo (Denio)

JACKSON COUNTY

Applegate

Blue Ledge
Draper

Graper Hill (Foots Creek. Creek)

1. Jacksonville OSEPHINE COUNTY Althouse

Gralice Jumporf Joe

Louse Creek (Granite H Lower Grave Creek Mt. Reuber

Wald Creek (Golden)
Wo COUNTY LAKE COUNTY
Coyote Hills LANE COU
Back Butte Black Bute
Bue River
Buhenia (Ch 
BAEER COUNTY-Continued.

9. Greenhorn. Au, Ag.

14 miles WNW. Whitney, S. V. R. R.

Paleozoic sediments cut by granodiorite and diorite.

Veins.

Lindgren, W., The gold belt of the Blue Mountains of Oregon: Twentysecond Ann. Rept., pt. 2, 1901, pp. 692-697.

Min. Res. 1906, p. 317.

Top. sheet Sumpter.

10. Iron Dyke (Homestead). $\mathrm{Ag}, \mathrm{Cu}, \mathrm{Pb}, \mathrm{Au}$.

72 miles NE. Baker City, O. W. R. R. \& N. Co.

Triassic greenstones.

Diseminations.

Lindgren, W., The gold belt of the Blue Mountains of Oregon: Twentysecond Ann. Rept., pt. 2, 1901, pp. 748-750.

Min. Res. 1907, pt. 1, p. 423.

1908 , pt. 1, p. 528.

11. Pocahontas (Auburn, Minersville). Au. (D, Pl.)

6 miles NW. Baker City, O. W. R. R. \& N. Co.

Paleozoic argillites cut by diorite.

Veins, stream gravels.

Lindgren, W., The gold belt of the Blue Mountains of Oregon: Twentysecond Ann. Rept., pt. 2, 1901, pp. 649-654.

Min. Res. 1909, pt. 1, p. 447.

12. Rye Valley (Mormon Basin). Au, Ag. (D, Pl.)

12 miles S. Durkee, O. W. R. R. \& N. Co.

Paleozoic argillites, granite and granodiorite.

Veins, stream gravels.

Lindgren, W., The gold belt of the Blue Mountains of Oregon: Twentysecond Ann. Rept., pt. 2, 1901, p. 767.

Min. Res. 1906, p. 316.

1907 , pt. 1, p. 423

1908, pt. 1, p. 528 .

1909, pt. 1, p. 447.

13. Sanger. $\mathrm{Au}, \mathrm{Ag}$. ( $\mathrm{Pl}, \mathrm{D}$.)

18 miles NE. Baker City, O. W. R. R. \& N. Co.

Triassic sediments.

Veins.

Lindgren, W., The gold belt of the Blue Mountains of Oregon: Twentysecond Ann. Rept., pt. 2, 1901, p. 738.

Weed, W. H., The copper mines of the United States in 1905: Bull. 285, 1906, p. 119.

Min. Res. 1907, pt. 1, p. 423.

1908 , pt. 1 , p. 528.

14. Sparta. Au, Ag. (D, Pl.)

27 miles ENE. Baker City, O. W. R. R. \& N. Co.

Granite.

Veins.

Lindgren, W., The gold belt of the Blue Mountains of Oregon: Twentysecond Ann. Rept., pt. 2, 1901, pp. 733-739.

Min. Res. 1907, pt. 1, p. 423.

15. Stice. $\mathrm{Au}, \mathrm{Ag}$. (Pl, D.)

18 miles S. Salisbury, S. V. R. R.

Paleozoic argillites cut and capped by Tertiary volcanics.

Veins. 
Baker coUNTY-Continued.

15. Stice-Continued.

Lindgren, W., The gold belt of the Blue Mountains of Oregon: Twentysecond Ann. Rept., pt. 2, 1901, pp. 773-776.

Min. Reg. 1907, pt. 1, p. 423.

Top. sheet Baker City.

16. Virtue. $\mathrm{Au}, \mathrm{Ag}$.

10 miles ESE. Baker City, O. W. R. R \& N. Co.

Paleozoic argillites cut by diorite.

Veins.

Lindgren, W., The gold belt of the Blue Mountains of Oregon: Twentysecond Ann. Rept., pt. 2, 1901, pp. 721-726.

Min. Res. 1899 (Twenty-first Ann. Rept., pt. 6), p. 301.

1906 , p. 316.

1907 , pt. 1, p. 422.

1908, pt. 1, p. 526.

1909 , pt. 1, p. 447.

Top. sheet Baker City.

17. Weatherby. Au, Ag. (D, Pl.)

Station O. W. R. R. \& N. Co.

Paleozoic sediments cut by granite and diorite.

Veins.

Lindgren, W., The gold belt of the Blue Mountains of Oregon: Twentysecond Ann. Rept., pt. 2, 1901, p. 762.

Min. Res. 1907, pt. 1, pp. 423-424.

1908 , pt. 1 , p. 528 .

18. Chena Creek. $\mathrm{Cu}, \mathrm{Au}$.

\section{ClaCKamas COURTY.}

31 miles W. Boring, P. R. R. L. \& P. Co.

19. Eden. Au. (Pl.)

coos COUNTY.

24 miles W. Dothan, S. P. R. R.

Stream gravels.

20. Myrtle Point (Johnson). Au, Ag. (D, Pl.)

59 miles WSW. Roseburg, S. P. R. R.

Stream gravels.

Diller, J. S., A geological reconnaissance in northwestern Oregon: Seventeenth Ann. Rept., pt. 1, 1896, pp. 519-520.

Min. Res. 1907, pt. 1, p. 424.

1909 , pt. 1, p. 447.

Top. sheet Coos Bay.

Folio 73, 1901.

21. Randolph. Au, Pt. (Pl.)

83 miles W. Roseburg, S. P. R. R.

Beach placers.

Min. Res. 1887, p. 142.

Top. sheet Coos Bay.

Folio 73, 1901.

CROOK COUNTY.

22. Howard (Ochoco, Bolivar). Au, Ag, Hg. (D, Pl.)

80 miles S. Shaniko, O. W. R. R. \& N. Co.

Min. Res. 1906, pp. 494-495.

1908 , pt. 1, pp. 691-692.

23. Trout Creek (Ashwood). $\mathrm{Cu}, \mathrm{Zn}, \mathrm{Au}, \mathrm{Ag}$.

26 miles S. Shaniko, O. W. R. R. \& N. Co. 


\section{CURRY COUNTY.}

24. Beach placers (Denmark, Harbor, Gold Creek, etc.). Au, Pt. (Pl.)

100-150 miles SW. Roseburg, S. P. R. R.

Beach gravels.

Day, D. T., and Richards, R. H., Black sands from placer mines: Bull. 285, 1906, pp. 150-164.

Kemp, J. F., The geological relations and distribution of platinum and associated metals: Bull. 193, 1902, pp. 51-53.

Min. Res. 1887, p. 142.

1905, pp. 1175-1258.

25. Chetco. Au, Pt. (Pl.) 1909 , pt. 1, p. 448.

124 miles SW. Grants Pass, S. P. R. R.

Beach gravels.

Day, D. T., and Richards, R. H., Black sands from placer mines: Bull. 285, 1906, pp. 150-164.

Min. Res. 1905, pp. 291, 1175-1258.

1906, p. 316.

1907, pt. 1, p. 424.

26. Lower Rogue (Mile Creek). Au. (Pl.)

52 miles SW. Dothan, S. P. R. R.

Stream gravels.

Top. sheet Port Orford.

Folio 89, 1903.

27. Rusty Butte. Au. (Pl.)

84 miles SW. Dillard, S. P. R. R.

Stream gravels.

Top. sheet Port Orford.

Folio 89, 1903.

28. Sixes River. Au. (Pl.)

78 miles SW. Dillard, S. P. R. R.

Stream gravels.

Top. sheet Port Orford.

Folio 89, 1903.

DOUGLAS COURTY.

29. Bohemia. $\mathrm{Au}, \mathrm{Ag}(\mathrm{Zn}, \mathrm{Pb}, \mathrm{Cu})$.

20 miles ESE. Wildwood, 12 miles SE. Disston, O. \& S. E. R. R.

Tertiary volcanice.

Veins.

Diller, J. S., The Bohemia mining region of western Oregon: Twentieth Ann. Rept., pt. 3, 1900, pp. 7-64.

MacDonald, D. F., Notes on the Bohemia mining district, Oregon: Bull. 380,1909 , pp. $80-84$.

Min. Res. 1907, pt. 1, p. 708.

1908 , pt. 1, p. 533 .

30. Crackerjack (Cow Creek). Au. (Pl.)

8 miles E. Glendale, S. P. R. R.

Stream gravels.

Diller, J. S., The mines of the Riddles quadrangle, Oregon: Bull. 340, 1908, p. 148.

Min. Res. 1907, pt. 1, p. 424.

31. Dothan. Au. (Pl.)

Station S. P. R. R.

Stream gravels.

Min. Res. 1905, p. 291.

1906, p. 316. 
32. Drew. Hg, Au. (D, Pl.)

33 miles E. Riddles, S. P. R. R.

Min. Res. 1908, pt. 1, p. 692.

33. Nugget (Myrtle Creek). Au, Ag, Cu. (D, Pl.)

$12 \frac{1}{2}$ miles E. Myrtle Creek, S. P. R. R.

Cretaceous sediments, metagabbro, and serpentine.

Veins, disseminations, stream gravels.

Min. Res. 1905, p. 291.

1907 , pt. 1, p. 424.

1908, pt. 1, p. 529 .

1909 , pt. 1, p. 448 .

Top. sheet Roseburg.

Folio 49, 1898.

34. Olalla. Au. (P1.)

9 miles WSW. Dillard, S. P. R. R.

Stream gravels.

Min. Res. 1909, pt. 1, p. 448.

35. Riddles. $\mathrm{Au}, \mathrm{Cu}, \mathrm{Ni}$. (D, Pl.)

Station S. P. R. R.

Mesozoic sediments cut by basic dikes.

Disseminated.

Clarke, F. W., Some nickel ores from Oregon: Bull. 60, 1890, pp. 21-26.

Diller, J. S., and Kay, G. F., The mines of the Riddles quadrangle, Oregon: Bull. 340, 1909, p. 148.

Kay, G. F., Nickel deposits of Nickel Mountain, Oregon: Bull. 315, 1907, pp. 120-127.

Min. Res. 1882, pp. 403-404.

1886 , p. 171.

1887, pp. 127-128.

1888 , p. 109.

1892, pp. 170-177.

1905 , p. 408.

Top. sheet Riddles.

36. Starvout (Green Mountain). Au. (Pl.)

25 miles E. Glendale, S. P. R. R.

Stream gravels.

Diller, J. S., and Kay, G. F., The mines of the Riddles quadrangle, Oregon:

Bull. 340,1908 , pp. 148, 152 .

Min. Res. 1908 , pt. 1, p. 529.

Top. sheet Riddles.

\section{GRANT COUNTY.}

37. Alamo. $\mathrm{Au}, \mathrm{Ag}$. (D, Pl.

20 miles WNW. Whitney, S. V. R. R.

Paleozoic argillites cut by diorite.

Veins, stream gravels.

Lindgren, W., The gold belt of the Blue Mountains of Oregon: Twentysecond Ann. Rept., pt. 2, 1901, pp. 688-692.

Top. sheet Sumpter.

38. Canjon. Au. (D, Pl.)

30 miles SW. Austin, S. V. R. R.

Paleozoic argillites, serpentine, and diabase.

Veins, stream gravels.

Lindgren, W., The gold belt of the Blue Mountains ot Oregon: Twentysecond Ann. Rept., pt. 2, 1901, pp. 712-720. 
GRANT COUWTY-Continued.

38. Canyon-Continued.

Min. Res. 1905, p. 291.

1907 , pt. 1, p. 424.

39. Crane Creek. Au. (Pl.)

20 miles NW. Sumpter, S. V. R. R.

Stream gravels.

Pardee, J. T., Placer gravels of the Sumpter and Granite districts, eastern Oregon: Bull. 430, 1910, pp. 59-65.

Top. sheet Sumpter.

40. Granite. Au, Ag. (D, P1.)

14 miles WNW. Sumpter, S. V. R. R.

Paleozoic argillites cut by diorite and granodiorite and capped by Tertiary volcanics.

Veins.

Lindgren, W., The gold belt of the Blue Mountains of Oregon: Twentysecond Ann. Rept., pt. 2, 1901, pp. 677-688.

Pardee, J. T., Placer gravels of the Sumpter and Granite districte, eastern Oregon: Bull. 430, 1910, pp. 59-65.

Min. Res. 1905, p. 291.

1906, p. 317.

1907, pt. 1, pp. 424-425.

1908 , pt. 1, pp. 529-530.

1909 , pt. 1, p. 448.

Top. sheet Sumpter.

41. Quartzburg (Comer). $\mathrm{Au}, \mathrm{Ag}, \mathrm{Cu}, \mathrm{Pb}(\mathrm{Co})$.

18 miles SW. Austin, S. V. R. R.

Paleozoic argillites and greenstones cut by diorite and diabase.

Veins.

Lindgren, W., The gold belt of the Blue Mountains of Oregon: Twentysecond Ann. Rept., pt. 2, 1901, pp. 708-712.

Weed, W. H., The copper mines of the United States in 1905: Bull. 285 1906, p. 119.

Min. Res. 1904, p. 314.

1905, pp. 291, 409.

1906, p. 317.

1907 , pt. 1, p. 425.

1908 , pt. 1, pp. 529-530.

Top. sheet Sumpter.

42. Susanville (Galena). Au, Ag, Pb. (D, Pl.)

27 miles NW. Austin, S. V. R. R.

Paleozoic argillites and greenstones cut by granite and capped by basalt.

Veins.

Lindgren, $W$. ., The gold belt of the Blue Mountains of Oregon: Twentysecond Ann. Rept., pt. 2, 1901, pp. 705-708.

Min. Res. 1906, p. 317.

1908, pt. 1, pp. 529-530.

1909 , pt. 1, p. 448.

43. Harney. Au. (Pl.)

HARNEY COUNTY.

117 miles WSW. Vale, O. S. L. R. R.

Min. Res. 1908, pt. 1, p. 530.

1909 , pt. 1, p. 448

44. Pueblo (Denio). Cu.

111 miles NW. Winnemucca, Nev., S. P. R. R., W. P. R. R.

Granite (?). 
HARNET COUTTY-Continued.

44. Pueblo (Denio)-Continued.

Waring, G. A., Geology and water resources of the Harney Basin region, Ore-

45. Omitted. gon: W. S. P. 231, 1909.

46. Applegate. Au. (D, Pl.)

JACKSON COUNTY.

14 miles SW. Jacksonville, R. R. V. R. R.

Paleozoic sediments and greenstones.

Stream gravels, veins.

Diller, J. S., and Kay, G. F., Mineral resources of the Grants Pass quadrangle and bordering districts, Oregon: Bull. 380, 1909, pp. 48-79.

Min. Res. 1907, pt. 1, p. 425.

1908 , pt. 1 , pp. $520-531$.

1909 , pt. 1, p. 448.

Top. sheets Grants Pass, Ashland.

47. Ashland. Au. (Pl, D.)

Station S. P. R. R.

Paleozoic sediments, granodiorite, and greenstones.

Stream gravels and veins.

Min. Res. 1907, pt. 1, p. 425. 1908 , pt. 1, p. 531.

Top. sheet Ashland.

48. Blue Ledge. $\mathrm{Cu}, \mathrm{Au}$.

33 miles SSW. Jacksonville, R. R. V. R. R.

Granodiorite (?).

Veins (?).

Top. sheet Ashland.

49. Draper. Au, Ag.

10 miles S. Gold Hill, S. P. R. R.

Greenstones and slates.

Veins.

Diller, J. S., and Kay, G. F., Mineral resources of the Grants Pass quadrangle and bordering districts, Oregon: Bull. 380, 1909, pp. 48-79.

Min. Res. 1908, pt. 1, p. 531.

Top. sheet Grants Pass.

50. Gold Fill (Foots Creek, Galls Creek, Sardine Creek). Au. (D, Pl.)

Station S. P. R. R.

Greenstones and slates.

Veins, stream gravels.

Diller, J. S., and Kay, G. F., Mineral resources of the Grants Pass quadrangle and bordering districts, Oregon: Bull. 380, 1909, pp. 48-79.

Min. Res. 1905, p. 291.

1906 , p. 317.

1907 , pt. 1, pp. 425-426.

1908 , pt. 1, pp. $530-531$

1909 , pt. 1, p. 448.

Top. sheets Grants Pass, Ashland.

51. Jacksonville. Au, Ag. (D, Pl.)

Station R. R. V. R. R.

Greenstones and slates.

Veins, stream gravels.

Diller, J. S., and Kay, G. F., Mineral resources of the Grants Pass quadrangle and bordering districts, Oregon: Bull. 380, 1909, pp. 48-79. 
JACKSON COUNTY-Continued.

51. Jacksonville-Continued.

Min. Res. 1905, p. 291.

1906, p. 317.

1907 , pt. 1, p. 426.

1908, pt. 1, p. 531.

Top. sheets Ashland, Grants Pass.

52. Pleasant Valley (Evans Creek). Au. (PI.)

20 miles N. Gold Hill, S. P. R. R

Stream gravels.

Diller, J. S., and Kay, G. F., The mines of the Riddles quadrangle, Oregon:

Bull. 340, 1908, pp. 150-151.

Min. Res. 1906, p. 531.

Top. sheet Riddles.

JOSEPHINE COUNTY.

53. Althouse. Au. (D, Pl.)

35 miles SSW. Grants Pass, S. P. R. R.

Greenstones.

Stream gravels, veins.

Diller, J. S., and Kay, G. F., Mineral resources of the Grants Pass quadrangle and bordering districts, Oregon: Bull. 380, 1909, pp. 48-79.

Min. Res. 1907, pt. 1, p. 426.

1908 , pt. 1, p. 532.

Top. sheet Grants Pass.

54. Applegate. Au. (Pl.)

14 miles SSE. Grants Pass, S. P. R. R.

Stream gravels.

Diller, J. S., and Kay, G. F., Mineral resources of the Grants Pass quadrangle and bordering districts, Oregon: Bull. 380, 1909, pp. 48-79.

Min. Res. 1907, pt. 1, p. 426.

1908, pt. 1, pp. 532-533.

Top. sheet Grants Pass.

55. Galice. $\mathrm{Au}, \mathrm{Ag}, \mathrm{Cu}$. (D, Pl.)

16 miles NW. Merlin, S. P. R. R.

Argillites cut by diorite.

Veins.

Min. Res. 1906, p. 318.

1907 , pt. 1, p. 426.

1908 , pt. 1, pp. 532-533.

1909 , pt. 1, p. 449.

56. Greenback (Upper Grave Creek). Au, Ag, Cu. (Pl, D.)

9 miles E. Leland, S. P. R. R.

Serpentine, greenstone, Paleozoic sediments.

Stream gravels, veins.

Diller, J. S., and Kay, G. F., The mines of the Riddles quadrangle, Oregon:

Bull. 340, 1908, pp. 140-142, 149-150.

Min. Res. 1905, p. 292.

1906, p. 318.

1907 , pt. 1, p. 426.

1908 , pt. 1, pp. 532-533.

1909, pt. 1, p. 449.

Top. sheet Riddles.

57. Jumpoff Joe. Au, Ag. (D, Pl.)

8 miles N. Grants Pass, S. P. R. R.

Greenstone and serpentine. 
JOSEPHINE COUNTY-Continued.

57. Jumpoff Joe-Continued.

Stream gravels, veins.

Diller, J. S., and Kay, G. F., The mines of the Riddles quadrangle, Oregon:

Bull. 340, 1908, pp. 143-145, 150.

Min. Res. 1905, p. 292.

1906 , p. 318.

1907 , pt. 1, p. 426.

1908 , pt. 1, pp. 532-533.

Top. sheet Riddlee.

58. Kerby. Au. (Pl.)

30 miles SSW. Grants Pass, S. P. R. R.

Stream gravels.

Diller, J. S., and Kay, G. F., Mineral resources of the Grants Pass quadrangle and bordering districts, Oregon: Bull. 380, 1909, pp. 48-79.

Min. Res. 1907, pt. 1, p. 426.

1908 , pt. 1, pp. 532-533.

59. Louse Creek (Granite Hill). Au. (D, Pl.)

5 miles N. Grants Pass, S. P. R. R.

Greenstone, Berpentine, granodiorite.

Veins and stream gravels.

Diller, J. S., and Kay, G. F., The mines of the Riddles quadrangle, Oregon: Bull. 340, 1908, p. 142.

Mineral resources of Grants Pass quadrangle and bordering districts

Oregon: Bull. 380, 1909, pp. 48-79.

Min. Res. 1905, p. 292.

1906 , p. 318.

1908 , pt. 1, pp. 532-533.

Top. sheets Grant Pass, Riddles.

60. Lower Grave Creek. Au. (D, Pl.)

10 miles $W$. Leland, S. P. R. R.

Mesozoic slates and greenstones.

Veins, terrace and stream gravels.

Min. Res. 1906, p. 318.

1907 , pt. 1, p. 427.

1908, pt. 1, pp. 532-533.

61. Mount Reuben. $\mathrm{Au}, \mathrm{Cu}$. (Pl, D.)

24 miles SSW. Dothan, S. P. R. R.

Veins.

62. Waldo. $\mathrm{Cu}, \mathrm{Au}, \mathrm{Ag}$. (Pl, D.)

38 miles SSW. Grants Pass, S. P. R. R.

Gabbro and serpentine.

Terrace and stream gravels, stockworks.

Diller, J. S., and Kay, G. F., Mineral resources of the Grante Pass quadrangle and bordering districts, Oregon: Bull. 380, 1909, pp. 48-79.

Weed, W. H., The copper mines of the United States in 1905: Bull. 285, 1906, p. 118.

Min. Res. 1887, p. 142.

1905, p. 292.

1906, pp. 318, 409.

1907 , pt. 1, p. 427.

1908 , pt. 1, pp. 532-533.

1909 , pt. 1, p. 449. 


\section{JOSEPEINE COUNTY-Continued.}

63. Wolf Creek (Golden). Au, Cu. (D, Pl.)

Station S. P. R. R.

Greenstone.

Stockworks, veins, terrace and stream gravels.

Diller, J. S., and Kay, G. F., The mines of the Riddles quadrangle, Oregon: Bull. 340, 1908, pp. 143, 149.

Min. Res. 1907, pt. 1, p. 427.

1908 , pt. 1, pp. 532-533.

1909, pt. 1, p. 449.

Top. sheet Riddles.

\section{LAKE COUNTY.}

64. Coyote Hills. Au.

61 miles N. Alturas, Cal., N. C. \& O. R. R.

Tertiary volcanics.

Veins.

65. Black Butte. Hg.

\section{IANE COUNTY.}

17 miles S. Cottage Grove, S. P. R. R.

Tertiary volcanics.

Veins, stockworks.

Min. Res. 1908, pt. 1, p. 691.

1909 , pt. 1, p. 555 .

66. Blue River. $\mathrm{Au}, \mathrm{Ag}(\mathrm{Pb}, \mathrm{Cu})$.

45 miles E. Springfield, S. P. R. R.

Tertiary volcanics, chiefly rhyolite.

Veins.

Diller, J. S., The Bohemia mining region of western Oregon: Twentieth Ann.

Rept., pt. 3, 1900, pp. 7-64.

Min. Res. 1905, p. 292.

1906 , p. 318.

67. Bohemia (Champion). $\mathrm{Au}, \mathrm{Ag}, \mathrm{Zn}, \mathrm{Pb}, \mathrm{Cu}$ (Hg).

14 miles S. Disston, O. \& S. E. R. R.

Tertiary volcanics.

Veins and disseminations.

Diller, J. S., The Bohemia mining region of western Oregon: Twentieth Ann.

Rept., pt. 3, 1900, pp. 7-64.

MacDonald, D. F., Notes on the Bohemia mining district, Oregon: Bull. 380, 1909, pp. 80-84.

Min. Res. 1905, pp. 292, 398.

1906, p. 319.

1908, pt. 1, p. 533.

1909 , pt. 1, p. 450 .

68. Fall Creek. Au, Ag.

15 miles ESE. Springfield, S. P. R. R.

MARION COUNTY.

69. Santiam (Elkhorn). $\mathrm{Au}, \mathrm{Ag}, \mathrm{Pb}, \mathrm{Cu}$.

14 miles NE. Gates, S. P. R. R.

\section{MALHEUR COUNTY.}

70. Humboldt. Au. (D, Pl.)

20 miles W. Huntington, O. W. R. R. \& N. Co.

Argillites cut by granodiorite.

Veins, stream gravels. 
MALHEUR COUNTY-Continued.

70. Humboldt-Continued.

Lindgren, W., The gold belt of the Blue Mountains of Oregon: Twentysecond Ann. Rept., pt. 2, 1901, pp. 770-773.

Min. Res. 1909, pt. 1, p. 450.

71. Malheur (Mormon Basin). Au, Ag. (D, Pl.)

22 miles SW. Durkee, O. W. R. R. \& N. Co.

Paleozoic argillites cut by diorite.

Veins.

Lindgren, W., The gold belt of the Blue Mountains of Oregon: Twentysecond Ann. Rept., pt. 2, 1901, pp. 770-773.

Min. Res. 1905, p. 292.

1907 , pt. 1, pp. $423,427$.

1908, pt. 1, p. 533.

1909 , pt. 1, p. 450.

UNION COUNTY.

72. Camp Carson. Au, Ag, $\mathrm{Pb}, \mathrm{Cu}$. (Pl, D.)

20 miles W. North Powder, O. W. R. R. \& N. Co.

Granite.

Veins, stream gravels.

Lindgren, W., The gold belt of the Blue Mountains of Oregon: Twentysecond Ann. Rept., pt. 2, 1901, pp. 676-677.

73. Medical Springs. $\mathrm{Cu}(\mathrm{Au}, \mathrm{Ag})$.

22 miles SSE. Union, C. R. R. of 0 .

Paleozoic sediments cut by diorite.

Contact metamorphic.

Weed, W. H., The copper mines of the United States in 1905: Bull. 285, 1906, p. 119.

WHEELER COUNTY.

74. Spanish Gulch. Au. (P1, D.)

110 miles SE. Shaniko, O. W. R. R. \& N. Co.

Min. Res. 1905, p. 293. 


\section{SOUTH DAKOTA.}

The Black Hills cover parts of three counties in western South Dakota. In this region there are 26 mining districts. In 14 districts the principal values are in gold, in 3 lead predominates, in 2 copper, in 1 silver, in 4 rare metals, and in 2 iron.

Distribution of the predominant metals produced in the mining districts of South Dakota.

\begin{tabular}{|c|c|c|c|c|c|c|c|}
\hline County. & Gold. & Silver. & Copper. & Lead. & Iron. & $\begin{array}{c}\text { Rare } \\
\text { metals. }\end{array}$ & Total. \\
\hline \multirow{3}{*}{ 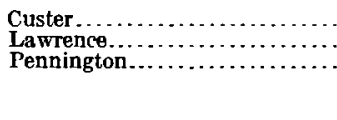 } & $\begin{array}{l}4 \\
5\end{array}$ & 1 & & 2 & 1 & & \\
\hline & 5 & $\ldots \ldots \ldots$ & 2 & 1 & 1 & 2 & 11 \\
\hline & 14 & 1 & 2 & 3 & 2. & 4 & 26 \\
\hline
\end{tabular}

\section{MINING DISTRICTS IN SOUTH DAKOTA.}

[See Pl. XII. Additional references will be found in Mineral Resources for 1910 and 1911.]

1. Chilkoot. $\mathrm{Au}, \mathrm{Cu}$.

\section{CUSTER COUNTY.}

10 miles E. Custer, C. B. \& Q. R. R.

Pre-Cambrian complex (?).

Veins, impregnations (?).

Top. sheet Hermosa.

2. Custer. Au. (D, Pl.)

5 miles S. Custer, C. B. \& Q. R. R.

Pre-Cambrian complex.

Veins, impregnations.

Min. Res. 1907, pt. 1, p. 429.

1908 , pt. 1, p. 535

1909 , pt. 1, p. 452 .

Top. sheet Harney Peak.

3. French Creek. Au.

Custer station, C. B. \& Q. R. R.

Pre-Cambrian complex (?).

Veins, impregnations (?).

Min. Res. 1905, p. 295.

Top. sheets Harney Peak, Hermosa.

4. Iron Mountains. $\mathrm{Fe}, \mathrm{Ag}, \mathrm{Pb}$.

12 miles ENE. Custer, C. B. \& Q. R. R.

Pre-Cambrian complex.

Veins, impregnations.

Top. sheet Hermosa.

5. Junction. Au. (D, Pl.)

10 miles NW. Custer, C. B. \& Q. R. R.

Pre-Cambrian complex.

Veins, impregnations.

Top. sheet Harney Peak.

21528 - Bull. $507-12-17$ 
6. Spokane. $\mathrm{Ag}, \mathrm{Pb}$.

8 miles W. Hermosa, C. \& N. W. R. R.

Pre-Cambrian complex.

Veins, impregnations.

Top. sheet Hermosa.

\section{LAWRENCE COUNTY.}

7. Carbonate. $\mathrm{Pb}, \mathrm{Ag}, \mathrm{Au}$.

2 miles E. Maurice, C. B. \& Q. R. R.

Carboniferous sediments cut by porphyry.

Replacements and veins.

Irving, J. D., Ore deposits of the northern Black Hills, South Dakota: Bull 225, 1904, pp. 123-140.

Irving, J. D., and Emmons, S. F., Economic resources of the northern Black Hills: P. P. 26, 1904.

Min. Res. 1907, pt. 1, p. 431.

Top. sheet Spearfish.

8. Deadwood. $\mathrm{Au}, \mathrm{Ag}, \mathrm{W}$. (D, Pl.)

Deadwood station, C. B. \& Q. R. R., C. \& N. W. R. R.

Algonkian schists and Cambrian sediments.

Veins and quartz-bearing conglomerate.

Irving, J. D., Ore deposits of the northern Black Hills, South Dakota: Bull. 225, 1904, pp. 123-140.

Irving, J. D., and Emmons, S. F., Economic resources of the northern Black Hills: P. P. 26, 1904.

Top. sheet Sturgis.

9. Elk Mountain (Ragged Top). Au.

10 miles S. Spearfish, C. B. \& Q. R. R.

Carboniferous limestone cut by phonolite.

Veins.

Irving, J. D., Ore deposits of the northern Black Hills, South Dakota: Bull. 225,1903, pp. 123-140.

Irving, J. D., and Emmons, S. F., Economic resources of the northern Black Hills: P. P. 26, 1904.

Min. Res. 1906, p. 322.

Top. sheet Spearfish.

10. Galena (Strawberry Gulch). Pb, Ag, Au. (D, Pl.)

4 miles NNW. Elk City, 2 miles E. siding, C. B. \& Q. R. R.

Algonkian schists cut by porphyry.

Impregnation.

Irving, J. D., Ore deposits of the northern Black Hills, South Dakota: Bull. 225,1904, pp. 123-140.

Irving, J. D., and Emmons, S. F., Economic resources of the northern Black Hills: P. P. 26, 1904.

O'Harra, C. C., Mineral resources of South Dakota: Bull. South Dakota Geol. Survey No. 3, 1902, pp. 60-62.

Min. Res. 1887, p. 110.

1906, pp. 321-322.

1907, pt. 1, p. 431.

1908 , pt. 1, p. 538 .

Top. sheet Sturgis.

11. Nigger Hill (Tinton). Sn, W. (D, Pl.)

9 miles W. Terry, C. B. \& Q. R. R.

Algonkian schists cut by pegmatite. 


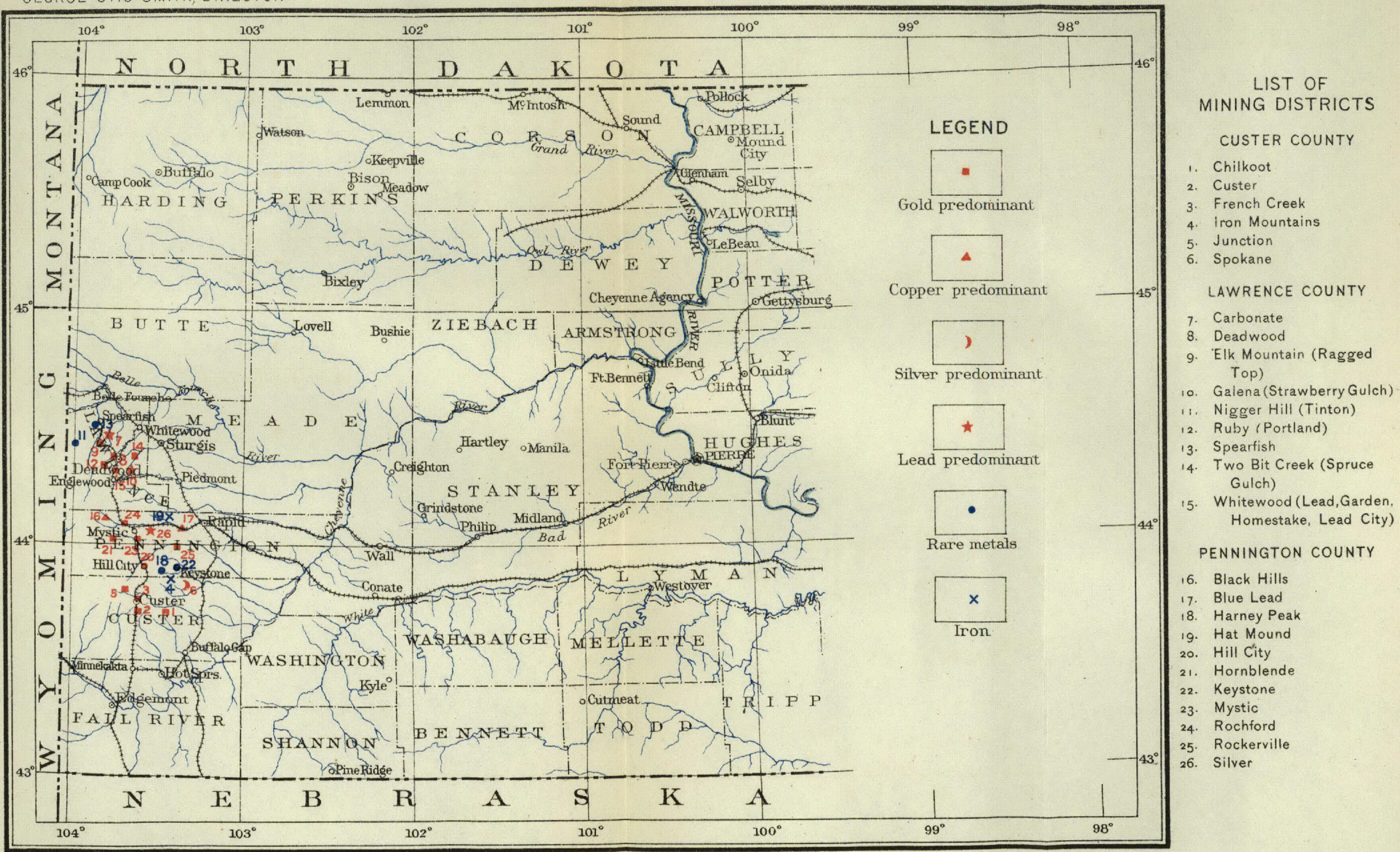

MAP OF PART OF SOUTH DAKOTA, SHOWING LOCATION OF MINING DISTRICTS

$$
\begin{aligned}
& \text { Scale } \frac{1}{2,500,000} \\
& \text { IPPROXIMATELY } 40 \text { MILES TO I INCH }
\end{aligned}
$$


LAWRENCE COUNTY-ContInued.

11. Nigger Hill (Tinton)-Continued.

Veins (?).

Irving, J. D., Ore deposite of the northern Black Hills, South Dakota: Bull. 225, 1904, pp. 123-140.

Hess, F. L., and Graton, L. C., The occurrence and distribution of tin: Bull. 260, 1905, pp. 164-165.

Hess, F. L., Tin, tungsten, and tantalum deposits of South Dakota: Bull. 380,1909 , pp. 131-163.

O'Harra, C. G., Mineral resources of South Dakota: Bull. South Dakota Geol. Survey No. 3, 1902, pp. 42, 43, 62-72.

Todd, J. E., A preliminary report on the geology of South Dakota: Bull. South Dakota Geol. Survey No. 1, 1895, p. 149.

Min. Res. 1883-84, p. 613.

1885, pp. 370-371.

1887, pp. 134-136.

1888, p. 148.

1894 (Sixteenth Ann. Rept., pt. 3), p. 530.

1903, p. 336.

1909 , pt. 1, p. 588.

12. Ruby (Portland). Au, Ag.

6 miles NW. Englewood, C. B. \& Q. R. R.

Cambrian sediments cut by porphyry.

Veins.

Irving, J. D., and Emmone, S. F., Economic resources of the northern Black Hills: P. P. 26, 1904.

Min. Res. 1906, pp. 321-322.

13. Spearflsh. Sn.

Station C. B. \& Q. R. R.

Pegmatite.

Top. sheet Spearfish.

O'Harra, C. C., Mineral resources of South Dakota: Bull. South Dakota Geol. Survey No. 3, 1902, pp. 43-45.

14. Two Bit Creek (Spruce Gulch). Au, Ag. (D, Pl.)

5 miles E. Deadwood, C. B. \& Q. R. R., G. N. R. R.

Cambrian sediments cut by acidic intrusives.

Veins.

Irving, J. D., and Emmons, S. F., Economic resources of the northern Black Hills: P. P. 26, 1904.

Min. Res. 1907, pt. 1, p. 431.

Top. sheet Sturgis.

15. Whitewood (Lead, Garden, Homestake, Lead City). Au, Ag, W.

Station C. B. \& Q. R. R., G. N. R. R.

Algonkian schists cut by acidic intrusives.

Veins and impregnations.

Irving, J. D., Ore deposits of the northern Black Hills, South Dakota: Bull. 225,1904 , pp. 123-140.

Irving, J. D., and Emmons, S. F., Economic resources of the northern Black Hills: P. P. 26, 1904.

Hess, F. L., Tin, tungsten, and tantalum deposits of South Dakota: Bull. 380,1909 , pp. 131-163.

O'Harra, C. C., Mineral resources of South Dakota: Bull. South Dakota Geol. Survey No. 3, 1902, pp. 11, 41.

Todd, J. E., A preliminary report on the geology of South Dakota: Bull.

South Dakota Geol. Survey No. 1, 1895, pp. 143-147. 
LAWRETCE COURTY-Continued.

15. Whitewood (Lead, Garden, Homestake, Lead City)-Continued.

Min. Res. 1904, pp. 331-333.

1905 , p. 295.

1906, p. 321.

1907 , pt. 1, pp. $429-431$.

1908 , pt. 1 , pp. $536-538$.

1909 , pt. 1, p. 453 .

Top. sheet Spearfish.

\section{PENMINGTON COUNTY.}

16. Black Hills. Cu.

10 miles W. Rochford, C. B. \& Q. R. R.

Pre-Cambrian schists.

Veins and impregnations.

17. Blue Lead. $\mathrm{Cu}$.

Big Bend station, R. C. B. H. \& W. R. R.

- Pre-Cambrian schists.

Veins and impregnations.

O'Harra, C. C., Mineral resources of South Dakota: Bull. South Dakota Geol. Survey No. 3, 1902, pp. 53-56.

18. Harney Peak. Sn, W, lithium.

7 miles SSE. Hill City, C. B. \& Q. R. R.

Pegmatite.

Rolker, C. M., Production of tin in various parts of the world: Sixteenth Ann. Rept., pt. 3, 1895, pp. 530-535.

Hess, F. L., and Graton, L. C., The occurrence and distribution of tin: Bull. 260, 1905, pp. 164-165.

Hess, F. L., Tin, tungsten, and tantalum deposits of South Dakota: Bull. 380, 1909, pp. 131-163.

O'Harra, C. C., Mineral resources of South Dakota: Bull. South Dakota Geol. Survey No. 3, 1902, pp. 62-72.

Todd, J. E., A preliminary report on the geology of South Dakota: Bull. South Dakota Geol. Survey No. 1, 1895, pp. 149-150.

Min. Res. 1883-84, pp. 611-613.

1885 , p. 370.

1887, pp. 136-138.

1888, pp. 144-148.

1889-90, pp. 120-121.

1891, p. 164.

1894 (Sixteenth Ann. Rept., pt. 3), pp. 530-535.

1903, p. 336.

1906, p. 323.

1908, pt. 1, pp. 721-722, 749.

1909 , pt. 1, p. 588.

Top. sheet Harney Peak

19. Hat Mound. Fe.

6 miles ENE. Pactola, R. C. B. H. \& W. R. R.

20. Hill City. Au. (D, Pl.)

Station C. B. \& Q. R. R.

Pre-Cambrian complex.

Stream gravels, veins.

Hess, F. L., Tin, tungsten, and tantalum deposits of South Dakota: Bull. 380,1909 , pp. 131-163. 
PENMTNGTON COUNTY-Continued.

20. Hill City-Continued.

Min. Res. 1907, pt. 1, p. 432.

1908, pt. 1, p. 538 .

1909, pt. 1, p. 454

Top. sheet Harney Peak.

21. Hornblende. $\mathrm{Au}, \mathrm{Ag}$.

10 miles SW. Rochford, C. B. \& Q. R. R.

Pre-Cambrian complex.

Veins and impregnations.

Min. Res. 1905, p. 297.

1907, pt. 1, p. 432.

1908, pt. 1, p. 539.

1909, pt. 1, p. 454 .

22. Keystone. Sn, Au, Ag. (D, Pl.)

10 miles ESE. Hill City, C. B. \& Q. R. R.

Pre-Cambrian complex cut by pegmatite.

Veins and lenses.

Min. Res. 1883-84, pp. 602-611.

1885, p. 370.

1887, pp. 136-138.

1888, pp. 149-151

1905 , p. 297.

1906, p. 323.

1907, pt. 1, p. 432.

1908 , pt. 1, p. 538.

1909, pt. 1, p. 454.

Top. sheet Hermosa.

23. Mystic. Au. (D, Pl.)

Station C. B. \& Q. R. R.

Pre-Cambrian complex.

Veins, impregnations.

Min. Res. 1908, pt. 1, p. 538.

24. Rochford. Au.

Station C. B. \& Q. R. R.

Pre-Cambrian complex.

Veins, impregnations.

Min. Res. 1907, pt. 1, p. 432.

25. Rockerville. Au, Cu. (D, Pl.)

14 miles S. Rapid City, R. C. B. H. \& W. R. R., C. \& N. W. R. R.

Pre-Cambrian complex.

Veins, impregnations.

O'Harra, C. C., Mineral resources of South Dakota: Bull. South Dakota Geol.

Survey No. 3, 1902, pp. 45-46.

Min. Res. 1908, pt. 1, p. 539.

Top. sheet Hermosa.

26. Silver. $\mathrm{Pb}, \mathrm{Ag}, \mathrm{Sb}$.

Silver City station, R. C. B. H. \& W. R. R.

Pre-Cambrian complex.

Veins, impregnations. 


\section{TEXAS.}

In the trans-Pecos country of Texas there are only three counties in which metal mining is carried on to any extent. Eleven districts are known to the Geological Survey. Four of these produce silver as the predominant metal, three quicksilver, two copper, one zinc, and one tin.

Distribution of the predominant metals produced in the mining districts of Texas.

\begin{tabular}{|c|c|c|c|c|c|c|}
\hline County. & Silver. & Copper. & $\begin{array}{l}\text { Quick- } \\
\text { silver. }\end{array}$ & Zinc. & $\begin{array}{c}\text { Rare } \\
\text { metals. }\end{array}$ & Total. \\
\hline \multirow[t]{2}{*}{ 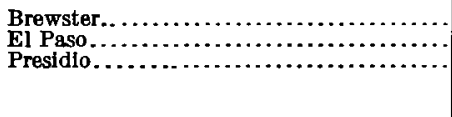 } & $\begin{array}{l}1 \\
2 \\
1\end{array}$ & 2 & $\begin{array}{l}\text { 3 } \\
\ldots \ldots+\cdots \\
\cdots\end{array}$ & $\begin{array}{c}\cdots \\
\cdots\end{array}$ & $i$ & \\
\hline & 4 & 2 & 3 & 1 & 1 & 11 \\
\hline
\end{tabular}

\section{MINING DISTRICTS IN TEXAS.}

[See Pl. XIII. Additional references will be found in Mineral Resources for 1910 and 1911.]

\section{BREWSTER COUTTY.}

1. Alpine. Ag, $\mathrm{Pb}$.

Station S. P. R. R.

Min. Res. 1908, pt. 1, p. 540.

Top. sheet Alpine.

2. Chisos and Study Butte. Hg.

120 miles SE. Marfa, S. P. R. R.

Cretaceous sediments cut by Tertiary volcanics.

Veins.

Min. Res. 1905, pp. 394-397.

1906, pp. 493-494.

1907 , pt. 1 , pp. $680-682$.

Top. sheet Chisos Mountains.

3. Mariscal. $\mathrm{Hg}$.

115 miles SE. Marathon, S. P. R. R.

Cretaceous sediments cut by Tertiary intrusives.

Veins.

Min. Res. 1894 (Sixteenth Ann. Rept., pt. 3), pp. 601-604.

1905, pp. 394-397.

1906 , pp. $493-494$.

1907 , pt. 1, pp. $680-682$.

Top. sheet Chisos Mountains.

4. Terlingua. Hg.

108 miles SSE. Marfa, S. P. R. R.

Cretaceous sediments cut and capped by Tertiary volcanics.

Veins, replacements.

Hillebrand, W. F., and Schaller, W. T., Mercury minerals from Terlingua, Tex.: Bull. 405, 1909. 


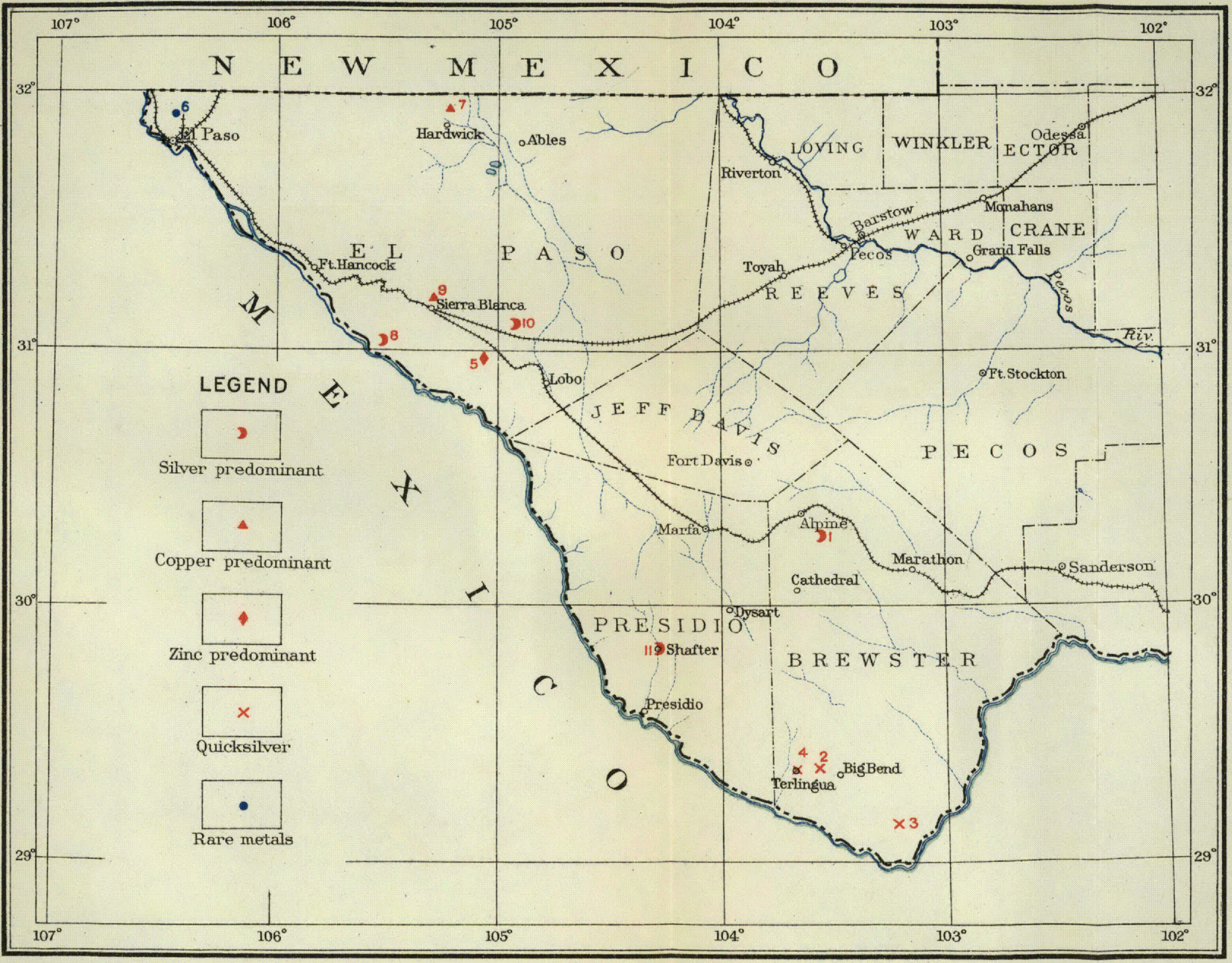

LIST OF

MINING DISTRICTS

BREWSTER COUNTY

Alpine

Chisos and Study Butte

Mariscal

Terlingua

EL PASO COUNTY

Eagle Mountain

El Paso (Franklin Mts.)

Guadalupe Mountains

Quitman Mts. (Bonanza)

Sierra Blanca (Lascar)

Sierra Diablo (Hazel)

PRESIDIO COUNTY

1. Shafter

MAP OF WESTERN TEXAS, SHOWING LOCATION OF MINING DISTRICTS

Scale $\frac{1}{2,500,000}$

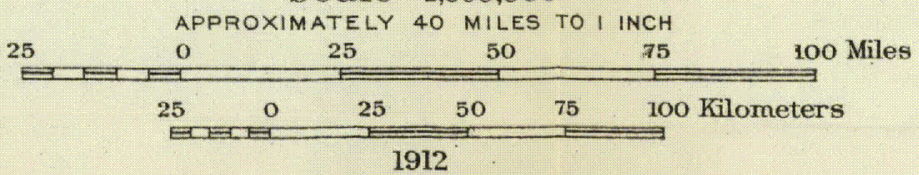



BREWSTER CoURTY-Continued.

4. Terlingua-Continued.

Min. Res. 1894 (Sixteenth Ann. Rept., pt. 3), pp. 601-604.

1899 (Twenty-first Ann. Rept., pt. 6), pp. 278-280.

1905, pp. 394-397.

1906, pp. 493-494.

1907, pt. 1, pp. $680-682$.

1909 , pt. 1 , pp. 555-556.

Top. sheets Terlingua, Terlingua special.

EL PASO COUNTY.

5. Eagle Mountains. Zn.

5 miles SW. Torbet, S. P. R. R.

Carboniferous sediments.

Min. Res. 1908, pt. 1, p. 541.

Top. sheet Eagle Mountains.

6. E1 Paso (Franklin Mountains). Sn.

10 miles N. El Paso, A. T. \& S. F. R. R., S. P. R. R., E. P. S. W. R. R.

Granite.

Veins.

Richardson, G. B., Tin in the Franklin Mountains, Texas: Bull. 285, 1906, pp. 146-149.

Weed, W. H., The El Paso tin deposits: Bull. 178, 1901.

Tin deposits at El Paso, Tex.: Bull. 213, 1903, pp. 99-102.

Min. Res. 1905, p. 305.

1906 , p. 544 .

1908 , pt. 1, p. 541 .

1909 , pt. 1, p. 589 .

Top. sheet El Paso.

Folio 166, 1909.

7. Guadalupe Mountains. Cu.

65 miles NNE. Sierra Blanca, S. P. R. R.

Paleozoic sediments.

Disseminations.

Min. Res. 1905, p. 305.

1907, pt. 1, p. 433.

1908 , pt. 1, p. 541 .

8. Quitman Mountains (Bonanza). $\mathbf{A g}, \mathrm{Pb}, \mathbf{Z n}$ 。

20 miles SW. Sierra Blanca, S. P. R. R.

Granite, limestone, greenstone.

Vein.

Min. Res. 1908, pt. 1, p. 541.

Top. sheet Sierra Blanca.

9. Sierra Blanca (Lascar). $\mathrm{Cu}, \mathrm{Pb}, \mathrm{Ag}$.

Allamore station, T. \& P. R. R.

Pre-Cambrian sediments, porphyry.

Contact metamorphic.

Min. Res. 1908, pt. 1, p. 541.

1909 , pt. 1, p. 455.

Top. sheet Sierra Blanca.

10. Sierra Diablo (Hazel). $\mathrm{Ag}, \mathrm{Cu}, \mathrm{Pb}$.

10 miles NW. Allamore, T. \& P. R. R.

Pre-Cambrian sediments.

Veins and impregnations. 
EL PASO CoUNTY-Continued.

10. Sierra Diablo (Hazel)-Continued.

Min. Res. 1905, p. 305.

1908 , pt. 1, p. 541.

1909 , pt. 1, p. 455 .

Top. sheet Sierra Blanca.

PRESIDIO COUNTY.

11. Shafter. $\mathrm{Ag}(\mathrm{Pb}, \mathrm{Au})$.

44 miles SSW. Marfa, S. P. R. R.

Carboniferous sediments cut by porphyry.

Replacements.

Min. Res. 1905, p. 305.

1906, p. 334 .

1907 , pt. 1, p. 433.

1908 , pt. 1, pp. 539,541

1909 , pt. 1, p. 455.

Top. sheet Shafter. 


\section{UTAH.}

Twenty-one counties of Utah contain 86 mining districts which are producing according to statistics collected by the United States Geological Survey. Gold is the predominant metal in 22 districts, silver in 20, copper in 15, lead in 14, and iron in 6 . The predominant metal in 6 districts is not certain, and of 3 camps producing rare metals 2 have deposits of uranium and vanadium and 1 antimony.

Distribution of the predominant metals produced in the mining districts of Utah.

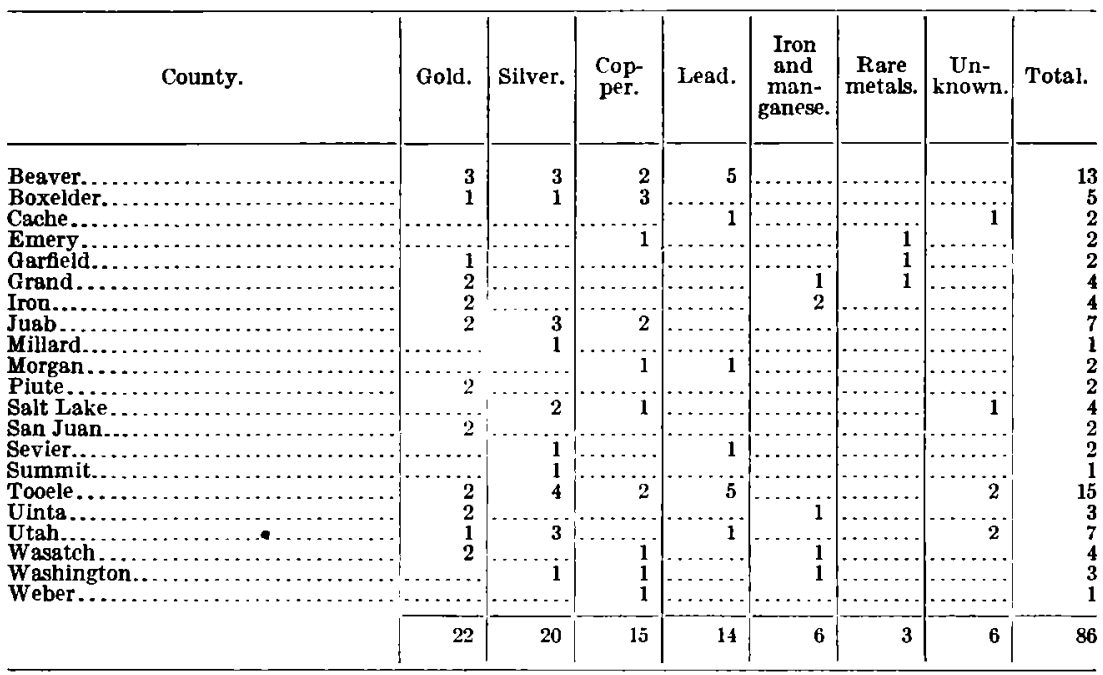

\section{MINING DISTRICTS IN UTAH.}

[See Pl. XIV. Additional references will be found in Mineral Resources for 1910 and 1911.]

\section{BEAVER COUNTY.}

1. Beaver Lake. $\mathrm{Ag}, \mathrm{Cu}, \mathrm{Pb}$.

12 miles W. Smyths, S. P. L. A. \& S. L. R. R.

Paleozoic sediments cut by monzonite.

Disseminations, replacements.

Min. Res. 1905, p. 315. 1907, pt. 1, p. 444

Top. sheet Frisco special.

2. Bradshaw. $\mathrm{Au}, \mathrm{Ag}, \mathrm{Pb}, \mathrm{Fe}$.

10 miles E. Milford, S. P. L. A. \& S. L. R. R.

Paleozoic sediments cut by acidic intrusives.

Replacements, veins. 
BEAVER COUNTY-Continued.

2. Bradshaw-Continued.

Min. Res. 1908, pt. 1, p. 554 .

1909 , pt. 1, p. 446

Top. sheet Beaver.

3. Granite. $\mathrm{Pb}, \mathrm{Ag}, \mathrm{Cu}, \mathrm{Bi}$.

25 miles ENE. Milford, S. P. L. A. \& S. L. R. R.

Paleozoic limestone cut by acidic intrusives.

Contact metamorphic.

Min. Res. 1883-84, pp. 654-655. 1907, pt. 1, p. 444

Top. sheet Beaver.

4. Indian Peak. $\mathrm{Pb}, \mathrm{Ag}$.

45 miles NW. Lund, S. P. L. A. \& S. L. R. R.

Tertiary volcanics.

Veins.

Min. Res. 1907, pt. 1, p. 444.

5. Lincoln (Jarloose). $\mathrm{Pb}, \mathrm{Ag}, \mathrm{Cu}, \mathrm{Au}, \mathrm{Zn}$.

20 miles SE. Milford, S. P. L. A. \& S. L. R. R.

Paleozoic sediments cut by acidic intrusives, Tertiary volcanics.

Replacements.

Min. Res. 1907, pt. 1, p. 444.

1908 , pt. 1, p. 554.

1909 , pt. 1, p. 466.

Top. sheet Beaver.

6. Newton. Au, Ag.

55 miles E. Milford, S. P. L. A. \& S. L. R. R.

Veins.

Min. Res. 1908, pt. 1, p. 554 .

1909 , pt. 1, p. 466.

Top. sheet Beaver.

7. North Star. $\mathrm{Ag}, \mathrm{Au}, \mathrm{Cu}, \mathrm{Pb}, \mathrm{Zn}$.

12 miles SW. Milford, S. P. L. A. \& S. L. R. R.

Paleozoic sediments cut by monzonite.

Replacements.

8. Pine Grove. Au, Ag.

40 miles NW. Lund, S. P. L. A. \& S. L. R. R.

Paleozoic sediments cut by porphyry.

Replacements.

9. Preuss (Newhouse). Cu, Au, Ag.

Newhouse station, S. P. L. A. \& S. L. R. R.

Paleozoic sediments cut by monzonite.

Impregnations, replacements, contact metamorphic.

Emmons, S. F., The Cactus copper mine, Utah: Bull. 260, 1905, pp. 242-248.

Weed, W. H., The copper mines of the United States in 1905: Bull. 285,

1906, pp. 121-122.

Min. Res. 1904, p. 276.

1905 , p. 315.

1906, pp. 341-343.

1907, pt. 1, pp. 444-445, 619.

1908, pt. 1, pp. 218, 554.

1909, pt. 1, p. 466.

Top. sheet Frisco special. 


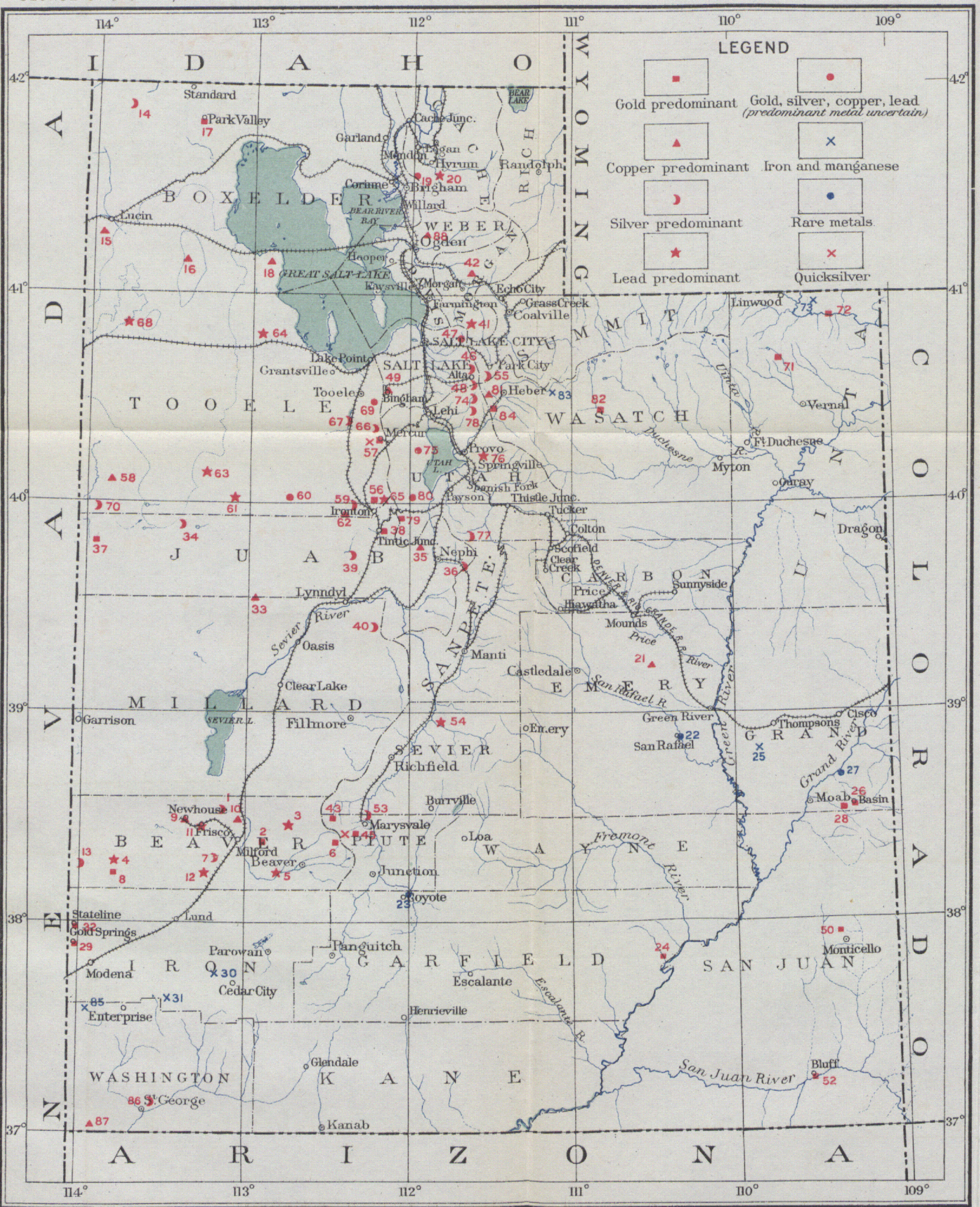

LIST OF MINING DISTRICTS

BEAVER COUNTY

Beaver Lake

Bradshaw

Granite

Indian Peak

Lincoln (Jarloose)

Newton

North Star

Pine Grove

Preuss (Newhouse)

Rocky

Washington

BOXELDER COUNTY

Ashbrook

Lucin

Newfoundland

Park Valley

Promontor

CACHE COUNTY

9. Boxelder

20. Paradise (La Plata)

EMERY COUNTY

Emery (Lost Springs)

2. San Rafael

GARFIELD COUNTY

Coyote Creek

24. White Canyon (Hite) GRAND COUNTY

5. Little Grand

Richardson

28. Wilson Mesa

\section{IRON COUNTY}

Gold Springs

Iron Springs

1. Pinto Iron

2. Stateline

\section{JUAB COUNTY}

Detroit (Joy)

Fish Springs

5. Mona

6. Mount Nebo

Spring Creek

8. Tintic

West Tintic

MILLARD COUNTY

Leamington (Oak City)

MORGAN COUNTY

1. Argenta (Mill Creek)

Morgan

PIUTE COUNTY

3. Kimberly (Gold Mtn.)

4. Omitted

45. Ohio (Marysvale, Gold Mountains)
SALT LAKE COUNTY

46. Big Cottonwood

47. Hot Springs

48. Little Cottonwood (Alta)

49. West Mountain (Bingham)

SAN JUAN COUNTY

5. Blue Mountains

(Monticello)

1. Omitted

52. Bluff

\section{SEVIER COUNTY}

53. Henry

54. Salina Creek

SUMMIT COUNTY

55. Uinta (Park City)

TOOELE COUNTY

56. Blue Bells

57. Camp Floyd (Mercur)

58. Clifton (Gold Hill)

59. Columbia

6o. Desert

6i. Dugway

62. Erikson

63. Granite Mountains

64. Lakeside

65. North Tintic

65. North

67. Rush Valley (Stockton)

68. Silver Islet

69. Tooele

70. Willow Springs

UINTA COUNTY

71. Carbonate

72. Green River (Cub Creek)

73. Spring Cree

UTAH COUNTY

74. American Fork

75. Lehi

76. Provo

77. Santaquin

78. Silver Lak

79. Tintic

8. Utah

\section{WASATCH COUNTY}

81. Blue Ledge

82. North Fork

83. Rhodes Platea

(Woodland)

84. Snake Creek

WASHINGTON COUNTY

85. Bull Valley

86. Harrisburg (Silver Reef)

87. Tutsagubet

WEBER COUNTY

88. Sierra Madre

MAP OF UTAH, SHOWING LOCATION OF MINING DISTRICTS

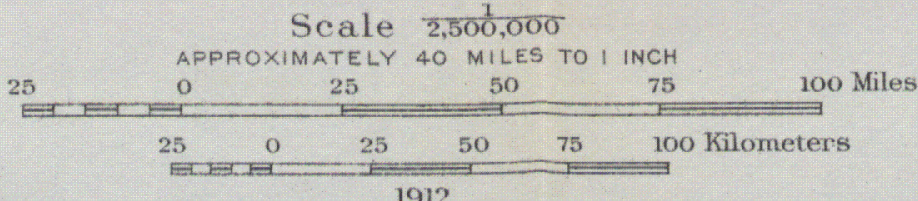



BEAVER COUNTY-Continued.

10. Rocky. $\mathrm{Cu}, \mathrm{Au}, \mathrm{Ag}, \mathrm{Fe}$.

10 miles NW. Milford, S. P. L. A. \& S. L. R. R.

Paleozoic sediments cut by monzonite.

Contact metamorphic.

11. Frisco. $\mathrm{Pb}, \mathrm{Cu}, \mathrm{Ag}, \mathrm{Au}, \mathrm{Zn}$.

Station S. P. I. A. \& S. L. R. R.

Paleozoic sediments cut by monzonite and capped by Tertiary volcanics.

Replacements, veins.

Min. Res. 1882, pp. 308-309.

1883-84, pp. 416-418.

1888 , p. 59.

1904, p. 276.

1905, p. 315.

1906, pp. 341-343, 408.

1907 , pt. 1, pp. 218, 444-445, 619.

1908 , pt. 1, pp. 554-555.

1909 , pt. 1, pp. 172, 466-467.

Top. sheet Frisco special.

12. Star. $\mathrm{Pb}, \mathrm{Ag}, \mathrm{Cu}, \mathrm{Au}, \mathrm{Zn}$.

20 miles SSW. Milford, S. P. L. A. \& S. L. R. R.

Paleozoic sediments cut by quartz monzonite.

Replacements, veins.

Min. Res. 1905, p. 315.

1906, pp. $343,476$.

1907 , pt. 1, pp. 445-446.

1908 , pt. 1, p. 555 .

1909 , pt. 1, p. 467.

13. Washington. $\mathrm{Ag}, \mathrm{Cu}, \mathrm{Pb}, \mathrm{Au}$.

50 miles NW. Lund, S. P. L. A. \& S. L. R. R.

Tertiary volcanics.

Veins.

BOXELDER COUNTY.

14. Ashbrook. Ag, Au.

83 miles S. Burley, Idaho, O. S. L. R. R.

15. Lucin. $\mathrm{Cu}(\mathrm{Au}, \mathrm{Ag}, \mathrm{Pb})$.

8 miles E. Tecoma, S. P. R. R.

Paleozoic sediments.

Replacements.

Min. Res. 1903, p. 343.

1907 , pt. 1, p. 446.

1908 , pt. 1 , pp. 555-556.

1909 , pt. 1, pp. 172, 467.

16. Newfoundland. $\mathrm{Cu}, \mathrm{Bi}$.

45 miles ESE. Lucin, S. P. R. R.

Min. Res. 1904, p. 375.

17. Park Valley. $\mathrm{Au}, \mathrm{Ag}, \mathrm{Cu}, \mathrm{Pb}$.

13 miles NW. Kelton, S. P. R. R.

Min. Res. 1905, p. 316.

1906 , p. 343.

1907 , pt. 1, p. 446.

1908, pt. 1, p. 556.

1909 , pt. 1, p. 467. 
BOXELDER COUNTY-Continued.

18. Promontory. Cu, Ag.

Lakeside station, S. P. R. R.

Min. Res. 1907, pt. 1, p. 464.

19. Boxelder.

CACHE COUNTT.

10 miles SSW. Wellsville, O. S. L. R. R.

20. Paradise (La Plata). $\mathrm{Pb}, \mathrm{Ag}$.

5 miles S. Hyrum, O. S. L. R. R.

Min. Res. 1908, pt. 1, p. 556.

\section{EMERY COUNTY.}

21. Emery (Lost Springs). $\mathrm{Cu}, \mathrm{Pb}, \mathrm{Au}, \mathrm{Ag}$.

10 miles W. Woodside, D. \& R. G. R. R.

Min. Res. 1906, p. 344.

Top. sheet Price River.

22. San Rafael. V, Cu.

18 miles SW. Green River, D. \& R. G. R. R.

Jurassic and Triassic sediments.

Disseminations.

Boutwell, J. M., Vanadium and uranium in Utah: Bull. 260, 1905, pp. 208-210.

Hess, F. L., Carnotite near Green River, Utah, Bull. 530, 1912.

Min. Res. 1904, p. 344. 1907 , pt. 1, p. 446.

Top. sheet San Rafael.

23. Coyote Creek. Sb.

GARFIELD COUNTY.

38 miles SSE. Marysvale, D. \& R. G. R. R.

Paleozoic and Mesozoic sediments capped by Tertiary volcanics.

Disseminations.

Richardson, G. B., Antimony in southern Utah: Bull. 340, 1908, pp. 253-256.

Min. Res. 1883-84, pp. 643-644.

1906 , pp. 511-512.

1907 , pt. 1, p. 708 .

Top. sheet Escalante.

24. White Canyon (Hite). Au. (Pl.)

85 miles SW. Richfield, D. \& R. G. R. R.

Stream gravels.

Min. Res. 1906, p. 344.

1908 , pt. 1, p. 556.

1909 , pt. 1, p. 467.

Top. sheet Henry Mountains.

25. Little Grande. Mn.

\section{GRAND COUNTY.}

10 miles S. Little Grande, D. \& R. G. R. R.

Triassic sediments.

Replacements.

Harder, E. C., Manganese deposits of the United States: Bull. 427, 1910, pp. $145-146$.

Min. Res. 1907, pt. 1, p. 100.

1908 , pt. 1, p. 152.

Top. sheet La Sal. 


\section{GRAND COUNTY-Continued.}

26. Miners Basin. $\mathrm{Au}, \mathrm{Ag}, \mathrm{Cu}$.

45 miles S. Cisco, D. \& R. G. R. R.

Monzonite porphyry.

Veins, stockworks.

Hill, J. M., Notes on the northern La Sal Mountains, Grand County, Utah: Bull. 530, 1912.

Min. Res. 1905, p. 316.

1907 , pt. 1, p. 446.

1908 , pt. 1, p. 556.

1909 , pt. 1 , pp. $467-468$.

Top. sheet La Sal.

27. Richardson. V.

27 miles S. Cisco, D. \& R. G. R. R.

Triassic and Cretaceous sediments.

Replacements.

Boutwell, J. M., Vanadium and uranium in Utah: Bull. 260, 1905, pp. 203-207.

Hill, J. M., Notes on the northern La Sal Mountains, Grand County, Utah:

Bull. 530, 1912.

Min. Res. 1904, p. 343.

Top. sheet La Sal.

28. Wilson Mesa. Au. (Pl.)

49 miles SE. Thompsons, D. \& R. G. R. R.

Glacial (?) gravels.

Hill, J. M., Notes on the northern La Sal Mountains, Grand County, Utah:

Bull. 530, 1912.

Min. Res. 1906, p. 344.

1908, pt. 1, p. 556

1909 , pt. 1, p. 468.

Top. sheet La Sal.

\section{IRON COENTY}

29. Gold Springs. Au, Mn.

17 miles NW. Modena, S. P. L. A. \& S. L. R. R.

Tertiary volcanics.

Veins.

Harder, E. C., Manganese deposits of the United States: Bull. 380, 1909, p. 274.

Min. Res. 1906, pp. 344-345.

1907 , pt. 1, pp. $100,447$.

1908 , pt. 1, p. 557.

1909 , pt. 1, p. 468

Top. sheet St. George.

30. Fron Springs. Fe.

22 miles SSE. Lund, S. P. L. A. \& S. I. R. R.

Paleozoic and Cretaceous sediments cut by andesite porphyry.

Contact metamorphic.

Leith, C. K., Iron ores in southern Utah: Bull. 225, 1904, pp. 229-237.

- Iron ores of the western United States and British Columbia: Bull. 285, 1906, p. 199.

Leith, C. K., and Harder, E. C., The iron ores of the Iron Springs district, southern Utah: Bull. 338, 1908.

Min. Res. 1883-84, p. 288.

Top. sheets St. George, Iron Springs special. 
31. Pinto Iron. Fe.

32 miles SSE. Modena, S. P. L. A. \& S. L. R. R.

Paleozoic sediments cut by andesite.

Contact metamorphic.

Leith, C. K., Iron ores in southern Utah: Bull. 225, 1904, pp. 229-237.

_- Iron ores of the western United States and British Columbia: Bull. 285, 1906, p. 199.

Min. Res. 1883-84, p. 288.

Top. sheet St. George.

32. Stateline. $\mathrm{Au}, \mathrm{Ag}, \mathrm{Pb}$.

17 miles NNW. Modena, S. P. L. A. \& S. L. R. R.

Tertiary volcanics.

Veins.

Min. Res. 1906, p. 345.

1907 , pt. I, p. 447.

1908, pt. 1, p. 557.

1909, pt. 1, p. 468.

\section{JUAB COUNTY.}

33. Detroit (Joy). Cu, $\mathrm{Au}, \mathrm{Ag}, \mathrm{Mn}, \mathrm{Bi}$.

35 miles NNW. Oasis, S. P. L. A. \& S. L. R. R.

Paleozoic sediments cut by granite.

Contact metamorphic.

Harder, E. C., Manganese deposits of the United States: Bull. 380, 1909, p. 273 ; Bull. 427, 1910, p. 157.

Min. Res. 1905, p. 317.

1907 , pt. 1, p. 100.

1908, pt. 1, p. 154.

1909 , pt. 1, p. 468.

34. Fish Springs. $\mathrm{Ag}, \mathrm{Pb}, \mathrm{Au}$.

65 miles NW. Oasis, S. P. L. A. \& S. L. R. R.

Paleozoic sediments cut by porphyry.

Replacements (?).

Min. Res. 1905, p. 316.

1906, p. 345.

1907 , pt. 1, p. 447.

1908 , pt. 1, p. 557.

1909 , pt. 1, p. 468.

Top. sheet Fish Springs.

35. Mona. $\mathrm{Cu}, \mathrm{Au}, \mathrm{Ag}(\mathrm{Sb})$.

Mona station S. P. L. A. \& S. L. R. R.

Top. sheets Manti, Tintic special.

Folio 65, 1899.

36. Mount Nebo. $\mathrm{Ag}, \mathrm{Pb}$.

10 miles N. Nephi, S. P. L. A. \& S. L. R. R., D. \& R. G. R. R.

Min. Res. 1907, pt. 1, p. 447.

1908 , pt. 1, p. 557.

1909 , pt. 1, p. 468.

Top. sheet Manti.

37. Spring Creek. Au, Ag.

105 miles NW. Oasis, S. P. L. A. \& S. L. R. R.

Min. Res. 1905, p. 317.

Top. sheet Fish Springs. 
38. Tintic. $\mathrm{Au}, \mathrm{Ag}, \mathrm{Pb}, \mathrm{Cu}$.

Station S. P. L. A. \& S. L. R. R., D. \& R. G. R. R.

Paleozoic sediments cut by monzonite.

Replacements and veins.

Tower, G. W., and Smith, G. O., Geology and mining industry of the Tintic district, Utah: Nineteenth Ann. Rept., pt. 3, 1899, pp. 601-776.

Min. Res. 1905, pp. 317-320, 355.

1906, pp. 345-348, 407-408, 449.

1907, pt. 1, pp. 447-452, 618-619.

1908, pt. 1, pp. 217, 557-561.

1909 , pt. 1 , pp. $172,468-473$.

Top. sheet Tintic special.

Folio 65, 1900.

39. West Tintic. $\mathrm{Ag}, \mathrm{Pb}, \mathrm{Fe}$.

15 miles SW. Tintic Junction, S. P. L. A. \& S. L. R. R., D. \& R. G. R. R.

Paleozoic sediments cut by monzonite.

Replacements and veins.

Tower, G. W., and Smith, G. O., Geology and mining industry of the Tintic district, Utah: Nineteenth Ann. Rept., pt. 3, 1899, pp. 601-767.

Min. Res. 1883-84, p. 289. 1909, pt. 1, p. 473.

Top. sheet Sevier Desert.

\section{MILLARD COUNTY.}

40. Leamington (Oak City). $\mathrm{Ag}, \mathrm{Pb}, \mathrm{Au}$.

12 miles S. Leamington, S. P. L. A. \& S. L. R. R.

Min. Res. 1905, p. 320.

1906 , p. 348 .

1909, pt. 1, p. 473.

Top. sheet Sevier Desert.

\section{MORGAN COUNTY.}

41. Argenta (Mill Creek). $\mathrm{Pb}, \mathrm{Ag}, \mathrm{Au}, \mathrm{Fe}$.

20 miles S. Morgan, U. P. R. R.

Min. Res. 1883-84, p. 288.

1905 , p. 320 .

1906, p. 348.

1907 , pt. 1, p. 453.

1908, pt. 1, p. 561 .

1909 , pt. 1 , p. 473.

Top. sheet Salt Lake.

42. Morgan. $\mathrm{Cu}, \mathrm{Ag}$.

10 miles N. Morgan, U. P. R. R.

Min. Res. 1907, pt. 1, p. 453.

1908 , pt. 1 , p. 561 .

\section{PIUTE COUNTY.}

43. Kimberly (Gold Mountain). Au, Ag.

15 miles SW. Sevier, D. \& R. G. R. R.

Tertiary volcanics.

Veins.

Lindgren, W., The Annie Laurie mine, Piute County, Utah: Bull. 285, 1906, pp. 87-90.

Min. Res. 1909 , pt. 1, p. 473.

Top. sheet Beaver. 
PIUTE CoUNTY-Continued.

44. Omitted.

45. Ohio (Marysvale, Mount Baldy). $\mathrm{Au}, \mathrm{Ag}, \mathrm{Cu}, \mathrm{Pb}, \mathrm{Hg}$.

Station D. \& R. G. R. R.

Tertiary volcanics and Triassic sediments.

Veins.

Butler, B. S., and Gale, H. S., Alunite, a newly discovered deposit near Marysvale, Utah: Bull. 511, 1912.

Lindgren, W., The Annie Laurie mine, Piute County, Utah: Bull. 285, 1906, pp. 87-90.

Min. Res. 1905, p. 320.

1906, p. 348.

1907, pt. 1, p. 453.

1908 , pt. 1, p. 561 .

1909 , pt. 1, p. 473.

Top. sheet Beaver.

\section{SALT LAKE COUNTY.}

46. Big Cottonwood. Ag, $\mathrm{Pb}, \mathrm{Au}, \mathrm{Cu}, \mathrm{Zn}$.

Sandy station, D. \& R. G. R. R.

Paleozoic sediments cut by granite.

Replacements, veins, contact metamorphic.

Min. Res. 1885, p. 249.

1905 , p. 321.

1906, pp. 348-349, 444.

1907 , pt. 1, pp. $453-454$.

1908, pt. 1, pp. 561-562.

1909 , pt. 1, pp. $473-474$.

Top. sheets Salt Lake, Cottonwood special.

47. Hot Springs.

10 miles N. Le Grande, D. \& R. G. R. R.

Top. sheet Salt Lake.

48. Little Cottonwood (Alta). $\mathrm{Ag}, \mathrm{Pb}, \mathrm{Au}, \mathrm{Cu}, \mathrm{Mn}, \mathrm{Fe}$.

16 miles E. Sandy, D. \& R. G. R. R., S. P. L. A. \& S. L. R. R.

Paleozoic sediments cut by granite.

Replacements, veins.

Weed, W. H., The copper mines of the United States in 1905: Bull. 285, 1906, p. 121.

Hess, F. I., Some molybdenum deposits in Maine, Utah, and California: Bull. 340, 1908, pp. 239-240.

Min. Res. 1885, p. 249.

1905 , pp. $320-321$.

1906 , pp. 348-349, 449.

1907 , pt. 1, pp. $453-454$.

1908. pt. 1, pp. 561-562.

1909 , pt. 1, pp. $473-474$.

Top. sheets Salt Lake, Cottonwood special.

49. West Mountain (Bingham). $\mathrm{Cu}, \mathrm{Au}, \mathrm{Ag}, \mathrm{Pb}$ (Zn, Fe, Mn).

Bingham Canyon, D. \& R. G. R. R.

Paleozoic sediments cut by monzonite.

Replacements, disseminations, veins.

Boutwell, J. M., Ore deposits of Bingham, Utah: Bull. 213, 1903, pp. 105122; Bull. 260, 1905, pp. 236-241.

Boutwell, J. M., Keith, A., and Emmons, S. F., Economic geology of the Bingham mining district, Utah: P. P. 38, 1905. 
BALT LAKE COUNTY-Contlnued.

49. West Mountain (Bingham)-Continued.

Weed, W. H., The copper mines of the United States iz 1905: Bull. 285, 1906, pp. 121-122.

Min. Res. 1883-84, pp. 416-418.

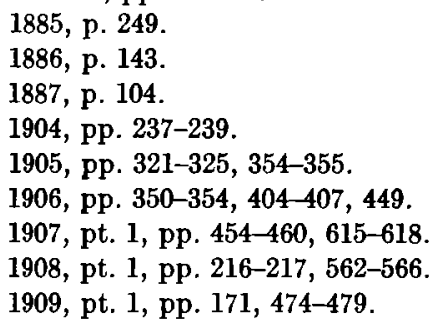

Top. sheets Bingham mining map, Tooele Vallev.

\section{SAT JUAT COUNTY.}

50. Blue Mountains (Monticello). $\mathrm{Au}, \mathrm{Ag}, \mathrm{Cu}$.

95 miles SSE. Thompson's, 75 miles W. Placerville, Colo., R. G. S. R. R.

Min. Res. 1908, pt. 1, pp. 566, 741.

51. Omitted.

Top. sheet Abajo.

52. Bluff. Au, Ag. (Pl.)

On San Juan and Grand rivere, 70 miles W. Cortez, Colo., R. G. S. R. R.

Stream gravels.

Min. Res. 1906, p. 354.

1908 , pt. 1, p. 566 .

Top. sheets Henry Mountains, Abajo.

53. Henry. Ag, Au.

EEVIER CoUNTy.

5 miles SE. Sevier, D. \& R. G. R. R.

Tertiary volcanics.

Veins.

Min. Res. 1905, p. 325.

1906, p. 354.

1908 , pt. 1 , pp. $561,567$.

Top. sheet Beaver.

54. Salina Creek. $\mathrm{Pb}, \mathrm{Cu}(\mathrm{Ag})$.

Salina station, D. \& R. G. R. R.

Min. Ree. 1908, pt. 1, p. 567.

Top. sheet Fish Lake.

gUYurT coonT.

55. Uinta (Park City). $\mathrm{Ag}, \mathrm{Pb}, \mathrm{Au}, \mathrm{Cu}, \mathrm{Zn}$.

Station D. \& R. G. R. R., U. P. R. R.

Pre-Cambrian and Paleozoic sediments cut by granodiorite and andesite.

Veins, replacements, and contact metamorphic.

Boutwell, J. M., Progress report on the Park City mining district, Utah: Bull. 213, 1903, pp. 31-40; Bull. 225, 1904, pp. 141-150; Bull. 260, 1905, pp. 150-153.

- and Woolsey, L. H., Geology and ore deposits of the Park City district, Utah: P. P. 77, 1912.

Weed, W. H., The copper mines of the United States in 1905: Bull. 285, 1906, pp. 121-122.

$21528^{\circ}-$ Bull. $507-12-18$ 
SUMuIT COURTY-Continued.

55. Uinta (Park City)-Continued.

Min. Res. 1885, p. 249.

1886 , p. 143.

1887 , p. 104.

1888 , p. 86.

1905, pp. 325-328.

1906, pp. 354-357, 449, 476.

1907 , pt. 1, pp. $460-463$.

1908 , pt. 1, pp. 567-570.

1909, pt. 1, pp. 479-481.

Top. sheets Salt Lake, Coalville, Park City special.

TOOELE COUNTY.

56. Blue Bells. $\mathrm{Au}(\mathrm{Ag}, \mathrm{Pb})$.

Lofgreen station, S. P. L. A. \& S. L. R. R.

Top. sheet Tooele Valley.

57. Camp Floyd (Mercur). $\mathrm{Au}, \mathrm{Ag}, \mathrm{Pb}(\mathrm{Hg})$.

Mercur station, S. L. \& M. R. R.

Paleozoic sediments cut by porphyry.

Replacements.

Becker, G. F., Geology of the quicksilver deposits of the Pacific slope: M. XIII, 1888, p. 386 .

Spurr, J. E., Economic geology of the Mercur mining district, Utah: Sixteenth Ann. Rept., pt. 2, 1896, pp. 343-455.

Min. Res. 1905, pp. 328, 329, 398.

1906, pp. 358-359, 494.

1907, pt. 1, pp. 464-465, 682 .

1908, pt. 1, pp. 570-571.

1909 , pt. 1 , pp. $482-483$.

Top. sheet Tooele Valley.

58. Clifton (Gold Hill). $\mathrm{Cu}, \mathrm{Au}, \mathrm{Ag}, \mathrm{Pb}$.

90 miles NE. Cherry Creek, Nev., N. N. R. R.

Paleozoic sediments cut by porphyry.

Contact metamorphic.

Min. Res. 1905, p. 317.

1906, p. 359.

1908 , pt. 1, p. 571.

1909 , pt. 1, p. 483.

59. Columbia. $\mathrm{Ag}, \mathrm{Pb}$.

Lofgreen station, S. P. I. A. \& S. L. R. R.

Min. Res. 1908, pt. 1, p. 572.

1909, pt. 1, p. 483.

60. Desert.

Top. sheet Sevier Desert.

45 miles SW. Center, S. P. L. A. \& S. L. R. R.

Top. sheet Tooele Valley.

61. Dugway. $\mathrm{Pb}, \mathrm{Cu}, \mathrm{Ag}$.

76 miles WSW. Center, S. P. L. A. \& S. L. R. R.

Paleozoic sediments cut by porphyry.

Min. Res. 1905, p. 317.

1906, p. 359.

62. Erikson. Cu.

25 miles SSW. Center, S. P. L. A. \& S. L. R. R.

Granite. 
TOOELE COUNTY-Continued.

62. Erikson-Continued.

Veins.

Top. sheet Sevier Desert.

63. Granite Mountains. $\mathrm{Pb}, \mathrm{Ag}$.

85 miles W. Center, S. P. L. A. \& S. I. R. R.

Paleozoic sediments cut by porphyry (?).

Veins (?).

Min. Res. 1905, p. 317.

64. Lakeside. $\mathrm{Pb}, \mathrm{Ag}$.

Dell station, W. P. R. R.

Min. Res. 1905, p. 317.

Top. sheet Tooele Valley.

65. North Tintic. $\mathrm{Pb}, \mathrm{Ag}, \mathrm{Zn}(\mathrm{Au})$.

5 miles N. Eureka, D. \& R. G. R. R.

Paleozoic sediments.

Replacements and veins.

Tower, G. W., and Smith, G. O., Geology and mining industry of the Tintic district, Utah: Nineteenth Ann. Rept., pt. 3, 1899, pp. 601-767.

Min. Res. 1905, p. 330.

1906 , pp. 359-360, 476 .

1907 , pt. 1, p. 465 .

1909 , pt. 1 , pp. $483-484$.

Top. sheets Sevier Desert, Tintic special.

Folio 65, 1900.

66. Ophir. $\mathrm{Ag}, \mathrm{Au}, \mathrm{Pb}, \mathrm{Cu}, \mathrm{Zn}(\mathrm{Fe})$.

8 miles E. St. John, S. P. L. A. \& S. L. R. R.

Paleozoic sediments cut by monzonite.

Veins and replacements.

Min. Res. 1885, p. 249.

1905, pp. 328,330 .

1906, pp. 360, 408.

1907, pt. 1, p. 465.

1908, pt. 1, p. 572 .

1909 , pt. 1, p. 484.

Top. sheet Tooele Valley.

67. Rush Valley (Stockton). $\mathrm{Ag}, \mathrm{Pb}, \mathrm{Au}(\mathrm{Cu}, \mathrm{Zn}, \mathrm{Fe}$ ).

Stockton station, S. P. L. A. \& S. L. R. R.

Paleozoic sediments cut by acidic and basic dikes.

Veins, replacements.

Min. Res. 1905, pp. 328, 330.

1906, p. 360 .

1907, pt. 1, pp. 465-466.

1908 , pt. 1, p. 572 .

1909 , pt. 1, p. 484.

Top. sheet Tooele Valley.

68. Silver Islet. $\mathrm{Pb}, \mathrm{Cu}, \mathrm{Ag}$.

9 miles N. Wendover, W. P. R. R.

Limestone.

Replacements.

Min. Res. 1908, pt. 1, p. 572.

69. Tooele.

1909 , pt. 1, p. 484

Station S. P. L. A. \& S. L. R. R.

Top. sheet Tooele Valley. 
70. Willow Springs. Ag, $\mathrm{Pb}$. 80 miles NE. Cherry Creek, Nev., N. N. R. R.

Top. sheet Fish Springe.

\section{UIHTA COUNTY.}

71. Carbonate. $\mathrm{Au}, \mathrm{Ag}, \mathrm{Cu}$. 82 miles NNW. Dragoon, U. R. R.

Top. sheets Marsh Peak, Ashley.

72. Green Rivêr (Cub Creek). Au. (Pl.) 70 miles SE. Carter, Wyo., U. P. R. R.

Stream gravels.

Min. Res. 1905, pp. 360-361. 1908 , pt. 1, p. 572. 1909 , pt. 1, p. 448.

Top. sheet Ashley.

73. Spring Creek. Fe.

70 miles SE. Carter, Wyo., U. P. R. R.

Jurassic and Cretaceous sediments.

Bedded.

Boutwell, J. M.; Iron ores in the Uinta Mountains, Utah: Bull. 225, 1904, p. 225.

Top. sheet Ashley.

\section{UTAH COUNTY.}

74. American Fork. $\mathrm{Ag}, \mathrm{Au}, \mathrm{Pb}, \mathrm{Cu}(\mathrm{Zn})$.

25 miles NE. American Fork, D. \& R. G. R. R., S. P. L. A. \& S. L. R. R.

Paleozoic sediments.

Replacements.

Min. Res. 1905, p. 330.

1906 , p. 361.

1907 , pt. 1, pp. $466-467$.

1908 , pt. 1, p. 573 .

1909 , pt. 1, p. 485 .

75. Lehi.

Top. sheets Salt Lake, Cottonwood special.

15 miles S. Lehi, D. \& R. G. R. R., S. P. I. A. \& S. L. R. R.

Top. sheet Salt Lake.

76. Provo. $\mathrm{Pb}, \mathrm{Ag}$.

Provo station, D. \& R. G. R. R., S. P. L. A. \& S. L. R. R.

Top. sheet Salt Lake.

77. Santaquin. $\mathrm{Ag}, \mathrm{Pb}$.

Station S. P. L. A. \& S. L. R. R

Min. Res. 1909, pt. 1, p. 485.

Top. sheet Manti.

78. Silver Lake. $\mathrm{Ag}, \mathrm{Pb}$.

15 miles E. American Fork, D. \& R. G. R. R., S. P. L. A. \& S. L. R. R.

Top. sheet Salt Lake.

79. Tintic. $\mathrm{Au}, \mathrm{Ag}, \mathrm{Pb}$.

Eureka station, D. \& R. G. R. R.

Paleozoic sediments cut by monzonite.

Replacements and veins.

Harder, E. C., Manganese deposits of the United States: Bull. 380, 1909, p. 279.

Tower, G. W., and Smith, G. O., Geology and mining industry of the Tintic district, Utah: Nineteenth Ann. Rept., pt. 3, 1899, pp. 601-767. 
UTAH COUXTY-Continued.

79. Tintic-Continued.

Weed, W. H., The copper mines of the United States in 1905: Bull. 285, 1906, p. 121.

Min. Res. 1882, pp. 228-229.

$$
\begin{aligned}
& 1887, \text { p. } 104 . \\
& 1905, \text { p. } 330 . \\
& 1907 \text {, pt. } 1, \text { p. } 466 . \\
& 1908, \text { pt. } 1, \text { p. } 572 . \\
& 1909 \text {, pt. 1, p. } 485 .
\end{aligned}
$$

Top. sheets Manti, Tintic special.

80. Utah.

Folio 65, 1900.

10 miles N. Elberta, D. \& R. G. R. R.

Top. sheets Tooele Valley, Salt Lake.

WASATCH COUNTY.

81. Blue Ledge. $\mathrm{Cu}, \mathrm{Ag}, \mathrm{Au}$.

5 miles W. Heber, D. \& R. G. R. R.

Top. sheet Salt Lake.

82. North Fork. $\mathrm{Au}, \mathrm{Ag}, \mathrm{Cu}$.

40 miles SE. Park City, D. \& R. G. R. R.

Paleozoic sediments.

Min. Res. 1905, p. 328.

Top. sheets Uinta, Hayden Peak.

83. Rhodes Plateau (Woodland). Fe.

30 miles ESE. Park City, 30 miles ENE. Heber, D. \& R. G. R. R.

Paleozoic sediments.

Replacements.

Boutwell, J. M., Iron ores of the Uinta Mountains, Utah: Bull. 225, 1904, pp. 221-228.

Top. sheet Coalville.

84. Bnake Creek. $\mathrm{Au}, \mathrm{Ag}, \mathrm{Pb}, \mathrm{Zn}, \mathrm{Cu}$.

10 miles SW. Heber, D. \& R. G. R. R.

Top. sheets Salt Lake, Coalville.

85. Bull Valley. Fe.

\section{WASHINGTON COUNTY.}

28 miles S. Modena, S. P. L. A. \& S. L. R. R.

Paleozoic sediments cut and capped by Tertiary lavas.

Contact metamorphic.

Leith, C. K., and Harder, E. C., The iron ores of the Iron Springs district, southern Utah: Bull. 338, 1908, p. 90.

Min. Res. 1908, pt. 1, p. 573.

Top. sheet St. George.

86. Hamisburg (Silver Reef). Ag.

75 miles SE. Modena, S. P. L. A. \& S. L. R. R.

"Red Beds."

Impregnations.

Emmons, S. F., The copper in the red beds of the Colorado Plateau region: Bull. 260, 1905, p. 228.

Min. Res. 1905, pp. 330-331.

1907 , pt. 1, p. 467.

1908 , pt. 1, p. 573.

1909 , pt. 1, p. 485.

Top. sheet St. George 


\section{WASHINGTON COUNTY-Continued.}

87. Tutsagubet. $\mathrm{Cu}, \mathrm{Pb}, \mathrm{Ag}(\mathrm{Au})$.

60 miles SSE. Acoma, Nev., S. P. L. A. \& S. L. R. R.

Paleozoic limestone.

Replacements.

Weed, W. H., The copper mines of the United States in 1905: Bull. 285, 1906, p. 121.

Min. Res. 1906, pp. 361-362, 408.

1907 , pt. 1, p. 467.

1909, pt. 1, p. 485 .

Top. sheet St. George.

\section{WEBER COUNTY.}

88. Sierra Madre. $\mathrm{Cu}, \mathrm{Au}, \mathrm{Ag}, \mathrm{Pb}, \mathrm{Fe}$.

10 miles N. Ogden, U. P. R. R., S. P. R. R., D.\& R. G. R. R., O. S. L. R. R. Pre-Cambrian granite and gneiss, Paleozoic eediments.

Veins.

Min. Res. 1905, p. 331.

1906, pp. 343-344.

1908, pt. 1, p. 556 . 


\section{WASHINGTON.}

In Washington 59 mining districts are scattered through 15 counties. In 31 of these districts the gold values are highest, in 11 copper is most valuable, in 6 lead, in 5 silver, and in 3 iron. There is one camp producing antimony, one tungsten, and one tin and tungsten, all classed as rare-metal districts.

Distribution of the predominant metals produced in the mining districts of Washington.

\begin{tabular}{|c|c|c|c|c|c|c|c|}
\hline County. & Gold. & Silver. & $\begin{array}{l}\text { Cop- } \\
\text { per. }\end{array}$ & Lead. & Iron. & $\begin{array}{c}\text { Rare } \\
\text { metals. }\end{array}$ & Total. \\
\hline 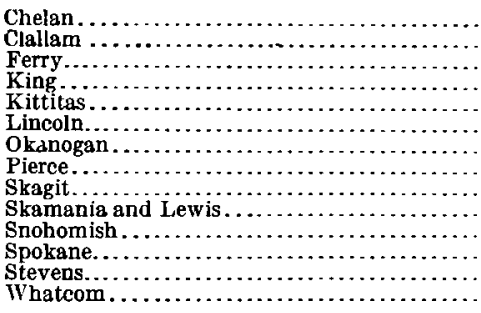 & 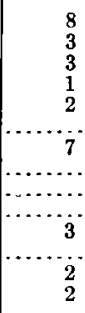 & 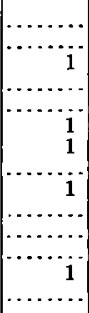 & 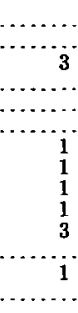 & 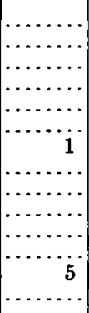 & 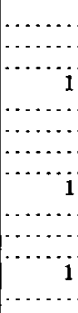 & 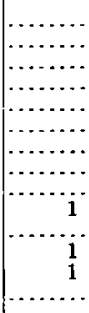 & $\begin{array}{r}8 \\
3 \\
7 \\
2 \\
2 \\
1 \\
10 \\
1 \\
3 \\
2 \\
6 \\
1 \\
11 \\
2\end{array}$ \\
\hline & 31 & 5 & 11 & 6 & 3 & 3 & 59 \\
\hline
\end{tabular}

MINING DISTRICTS IN WASHINGTON.

[See Pl. XV. Additional references wi!l be found in Mineral Resources for 1910 and 1911.]

\section{CHELAN COUNTT.}

1. Bridge Creek (Stehelin). Au, Ag.

100 miles N. Wenatchee, G. N. R. R.

Granite, gneiss, and schist.

Veins.

Ann. Rept. Washington Geol. Survey, vol. 1, 1902, p. 92.

Top. sheet Stehekin.

2. Entiat. $\mathrm{Au}, \mathrm{Ag}$.

20 miles N.,Wenatchee, G. N. R. R.

Schists.

Veins.

Russell, I. C., A preliminary paper on the geology of the Cascade Mountains in northern Washington: Twentieth Ann. Rept., pt. 2, 1900, p. 209. Min. Res. 1907, pt. 1, p. 473.

Top. sheet Chelan.

3. Horseshoe Basin (Pershall). $\mathrm{Au}, \mathrm{Ag}, \mathrm{Pb}, \mathrm{Cu}$.

140 miles N. Wenatchee, G. N. R. R.

Min. Res. 1904, p. 340.

Top. sheet Stehekin. 
4. Lakeside. A-1.

40 miles N. Wenatchee, G. N. R. R.

Min. Res. 1907, pt. 1, p. 473.

1908 , pt. 1, p. 578.

Top. sheet Chelan.

5. Leavenworth. Au, Ag. (D, Pl.)

Station G. N. R. R.

Min. Res. 1907, pt. 1, p. 473.

1908 , pt. 1, p. 577.

Top. sheet Chiwaukum.

6. Peshastin (Blewett). Au, Ag. (D, Pl.)

12 miles S. Dryden, G. N. R. R.

Paleozoic sediments, peridotite, granodiorite.

Veins.

Ann. Rept. Washington Geol. Survey, vol. 1, 1902, pp. 94-98.

Ruseell, I. C., A preliminary paper on the geology of the Cascade Mountains in northern Washington: Twentieth Ann. Rept., pt. 2, 1900, pp. 208-209.

Smith, G. O., Gold mining in central Washington: Bull. 213, 1903, p. 76.

Weaver, C. E., Geology and ore deposits of the Blewett mining district:

Bull. Washington Geol. Survey No. 6, 1911.

Min. Res. 1905, p. 336.

Top. sheets Chiwaukum, Mount Stuart.

Folio 108, 1904.

7. Railroad Creek. Au, Ag, Cu, Mo.

87 miles N. Wenatchee, G. N. R. R.

Gneiss and schist cut by granite.

Veins.

Ann. Rept. Washington Geol. Survey, vol. 1, 1902, pp. 91-94.

Top. sheet Stehekin.

8. Wenatchee. Au.

7 miles W. Wenatchee, G. N. R. R.

Sediments cut by rhyolite.

Veins.

Min. Res. 1908, pt. 1, p. 578.

CLALIAY COUNTY.

9. Ozette River. Au. (Pl.)

120 miles W. Port Townsend, P. T. S. R. R.

Beach placers.

Arnold, R., Gold placers of the coast of Washington: Bull. 260, 1905, pp. 154-157.

Min. Res. 1908, pt. 1, p. 578.

10. Shishi Beach. Au. (Pl.)

120 miles E. Port Townsend, P. T. S. R. R.

Beach gravels.

Arnold, R., Gold placers of the coast of Washington: Bull, 260, 1905, pp. 154-157.

11. Yellow Banks. Au. (Pl.)

130 miles W. Port Townsend, P. T. S. R. R.

Beach gravels.

Arnold, R., Gold placers of the coast of Washington: Bull. 260, 1905, pp. 154-157. 
CHELAN COUNTY
Bridge Creek (Stehekin)

Entiat

4. Lakeside

5. Leavenworth

7. Railroad Creek

CLALLAM COUNTY

o. Shishi Beach

Yellow Banks

Columbia River (placers)

4. Danville
5. Lone Star

15. Meteor (Covada)
16. Mepubic (Eureka)

8. Sanpoil KING COUNTY

19. Money Creek (Miller Creek)

KITTITAS COUNT

20. Fish Lake

LEWIS COUNTY, (See Skam
LINCOLN COUNTY

2. Crystal

Conconully (Ruby)

Myers Creek (Chesaw)

2. Nighthawk

6. Osoyoos Lake (Oroville,
7. Palmer Mountain

Squaw Creek (Methow)

29. Twisp

PPERCE COUNTY
2. Carbon River

SKAGIT Coun
Bald Mountain

Bald Mountain

SKAMAANIA and LEWIS COUNTIES

5. Mineral
6. St. Helens

SNOHOMISH COUNTY

37. Garrington

39. Index

41. Silver Creek
4. Stilaguamish (Silverton)

43. Silver Hill

44. Bossburg

45. Deep Creek Lake

47. Deer Park
48. Deer Trail

. Metaline

50. Meyers Falls

5. Northport

53. Orient

Wharcom county

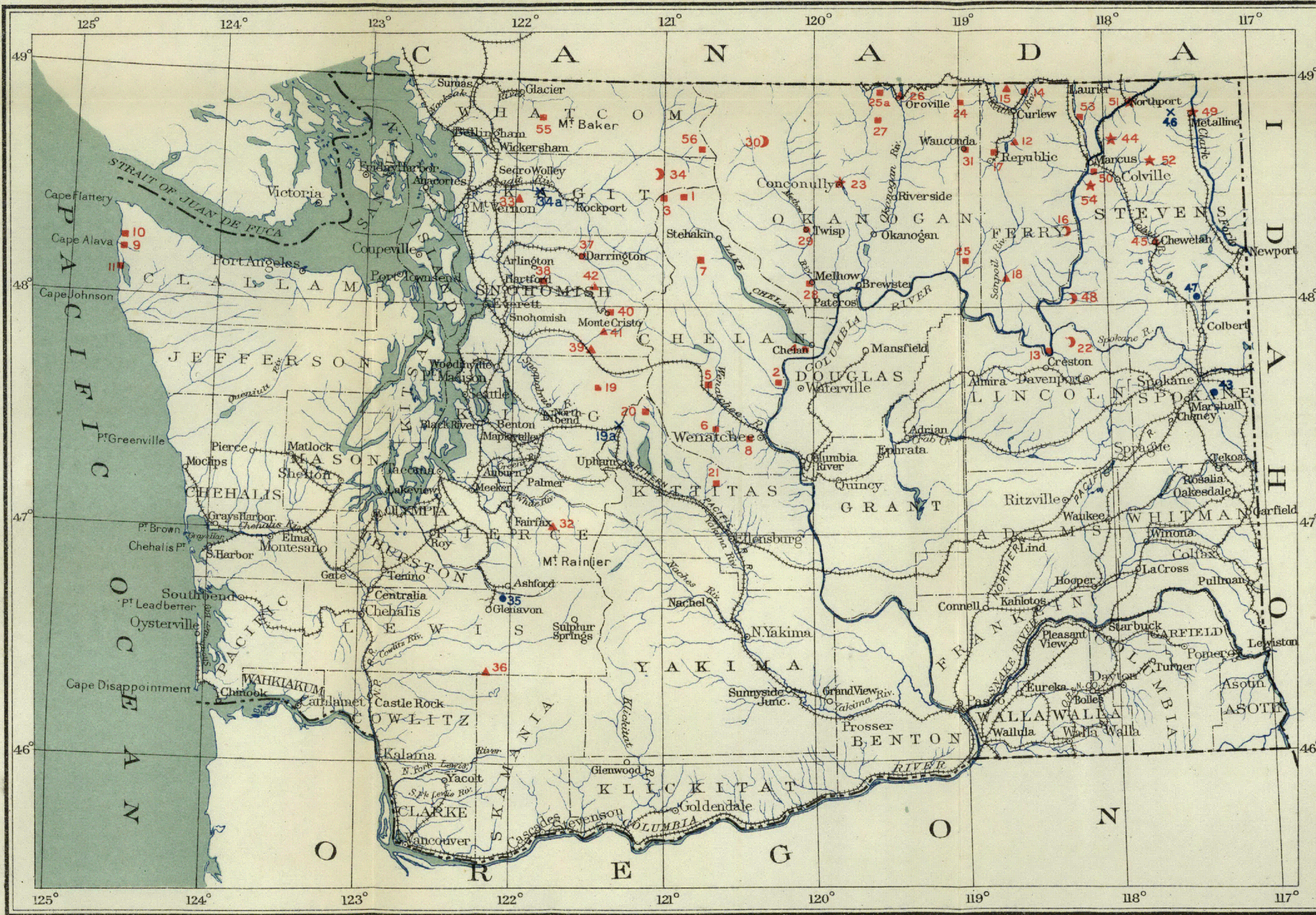

LEGEND

.

$\Delta$

Copper predominant
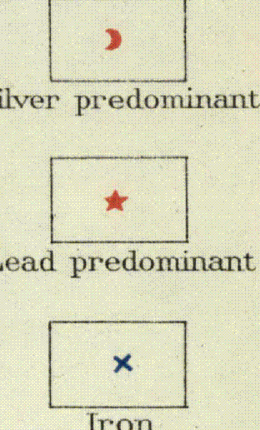

Snoqualmie Pass

SKAGIT COUNT
34a. Hamilton

MAP OF WASHINGTON, SHOWING LOCATION OF MINING DISTRICTS

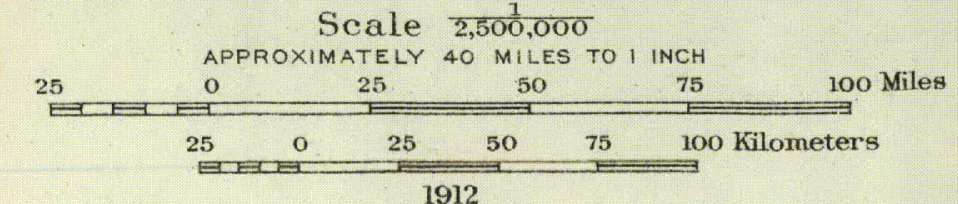




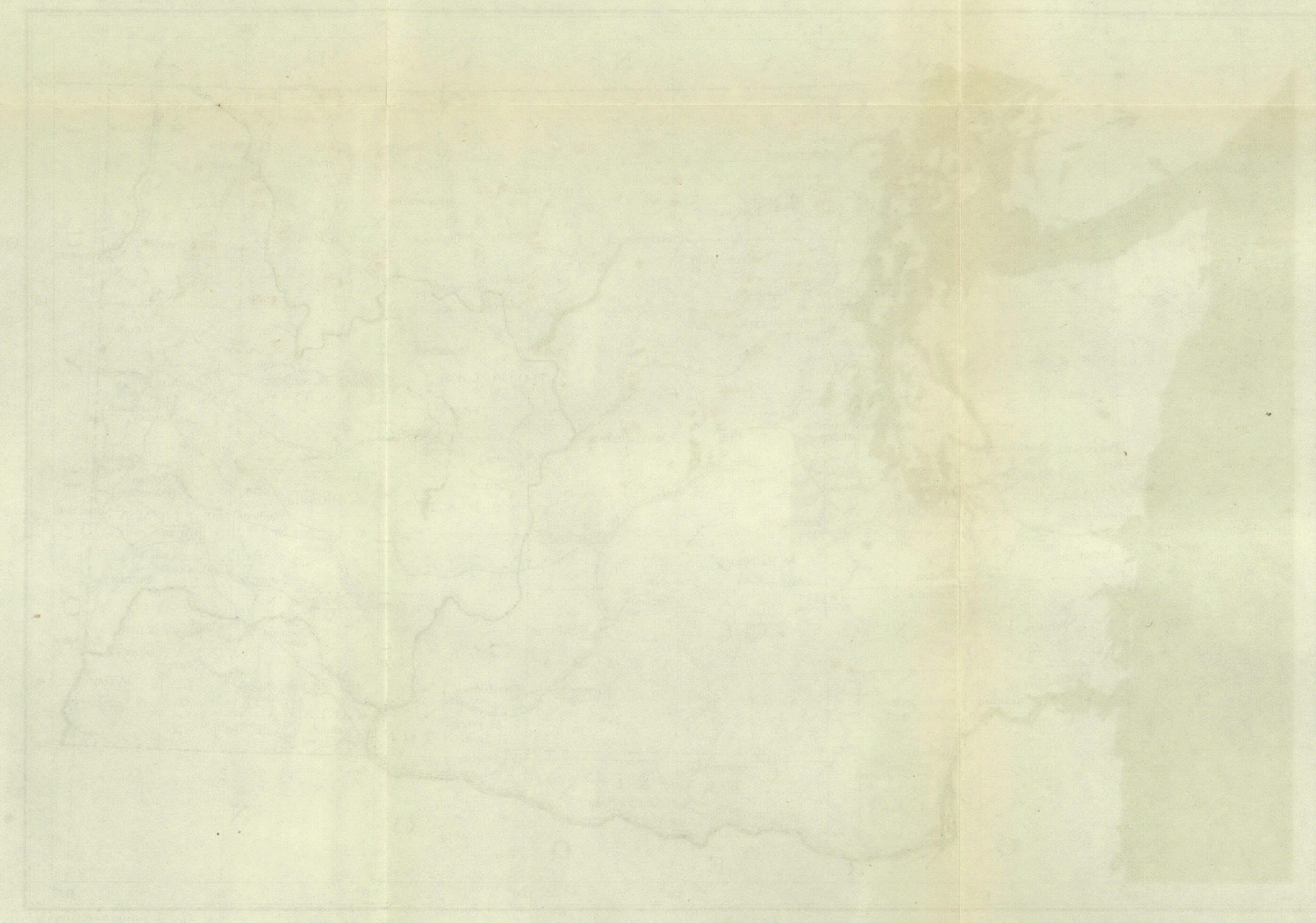


12. Belcher. Cu.

Belcher siding, G. N. R. R.

Paleozoic sediments cut by quartz monzonite.

Replacements, contact metamorphic.

Weed, W. H., The copper mines of the United States in 1905: Bull. 285, 1906, p. 125.

Top. sheet Republic.

13. Columbia River. Au. (Pl.)

11 miles NE. Creston, N. P. R. R.

Stream gravels.

Collier, A. J., Gold-bearing river sands of northeastern Washington: Bull. 315, 1907, pp. 56-70.

14. Danville. $\mathrm{Au}, \mathrm{Cu}, \mathrm{Ag}$.

Station G. N. R. R.

Paleozoic sediments and greenstones.

Replacements and veins.

Min. Res. 1907, pt. 1, p. 473.

1908 , pt. 1, p. 578 .

Top. sheet Republic.

15. Lone Star. $\mathrm{Cu}$.

6 miles W. Danville, G. N. R. R.

Paleozoic sediments cut by quartz monzonites.

Veins and replacements.

Top. sheet Republic.

16. Meteor (Covada). $\mathrm{Ag}, \mathrm{Pb}, \mathrm{Sb}$.

34 miles SSW. Meyers Falle, G. N. R. R.

Metamorphosed Paleozoic sediments cut by diorite.

Veins.

Min. Res. 1907, pt. 1, p. 473.

1908 , pt. 1, p. 578.

17. Republic (Eureka). $\mathrm{Au}, \mathrm{Ag}$.

Station G. N. R. R.

Tertiary volcanics.

Veins.

Ann. Rept. Washington Geol. Survey, vol. 1, 1902, pp. 52-60.

Umpleby, J. B., Geology and ore deposits of Republic mining district:

Bull. Washington Geol. Survey No. 1, 1910.

Min. Res. 1905, p. 356.

1906, p. 366.

1907, pt. 1, pp. 473-474.

1908, pt. 1, p. 578.

Top. sheet Republic.

18. Sanpoil. $\mathrm{Cu}, \mathrm{Pb}, \mathrm{Ag}, \mathrm{Ni}$.

30 miles N. Wilbur, N. P. R. R.

Metamorphosed Paleozoic sediments cut by quartz monzonite.

Veins, replacements.

\section{EING COUNTY.}

19. Money Creek (Miller Creek). $\mathrm{Au}, \mathrm{Ag}, \mathrm{Pb}, \mathrm{Cu}, \mathrm{Sb}$.

Berlin station, G. N. R. R.

Granite.

Veins.

Ann. Rept. Washington Geol. Survey, vol. 1, 1902, pp. 120-124. 
KING COUNTY-Continued.

19. Money Creek (Miller Creek)—Continued.

Min. Res. 1905, p. 336.

1906 , p. 367 .

Top. sheet Skykomish.

19a. Snoqualmie Pass. Fe.

Snoqualime Pass, C. M. \& P: S. R. R.

Marble, limestone, and granite.

Bedded.

Ann. Rept. Washington Geol. Survey, vol. 1, 1902, pp. 241-243.

\section{RITTITAS COUNTY.}

20. Fish Lake. $\mathrm{Au}, \mathrm{Ag}, \mathrm{Cu}$.

40 miles N. Roslyn, N. P. R. R.

Min. Res. 1905, p. 336.

Top. sheet Skykomish.

21. Swauk (Liberty). Au, Ag, Fe. (D, PI.)

15 miles ENE. Clealum, N. P. R. R.

Tertiary sediments cut by basalt.

Veins.

Ann. Rept. Washington Geol. Survey, vol. 1, 1902, pp. 125-127, 243-252.

Russell, I. C., A preliminary paper on the geology of the Cascade Mountains

in northern Washington: Twentieth Ann. Rept., pt. 2, 1900, pp. 206-208.

Smith, G. O., Gold mining in central Washington: Bull. 213, 1903, pp. 76-80.

Min. Res. 1905, p. 336.

1906 , p. 367.

1907 , pt. 1, p. 474

1908 , pt. 1, p. 579.

Top. sheet Mount Stuart.

Folio 106, 1904.

LEWIS COUNTY. (Seo Skamanla.)

LINCOLN COUNTY.

22. Crystal. $\mathrm{Ag}, \mathrm{Pb}$.

15 miles N. Davenport, N. P. R. R.

Schists and slates.

Veins.

Ann. Rept. Washington Geol. Survey, vol. 1, 1902, p. 60.

oganogan COUTTY.

23. Conconully (Ruby). $\mathrm{Pb}, \mathrm{Ag}, \mathrm{Au}, \mathrm{Cu}$.

26 miles S. Nighthawk, G. N. R. R.

Granite, schist, and gneiss cut by dikes.

Veins.

Ann. Rept. Washington Geol. Survey, vol. 1, 1902, pp. 70-72.

Smith, G. O., and Calkins, F. C., A geological reconnaissance across the Cascade Range near the forty-ninth parallel: Bull. 235, 1904, pp. 94-95.

Min. Res. 1907, pt. 1, p. 475.

1908 , pt. 1 , p. 580.

Top. sheet Chopaka.

24. Myers Creek (Chesaw). Au, Ag, Cu. (D, Fl.)

5 miles S. Myncaster, Canada, G. N. R. R.

Paleozoic sediments and greenstones cut by granite and syenite.

Veins, contact metamorphic.

Ann. Rept. Washington Geol. Survey, vol. 1, 1902, pp. 64-66. 
ORATOGAN COUNTY-Continued.

24. Myers Creek (Chesaw)-Continued.

Umpleby, J. B., Geology and ore deposits of the Myers Creek mining district:

Bull. Washington Geol. Survey No. 5, 1911, pp. 11-52.

Min. Res. 1906, p. 367.

1907, pt. 1, p. 474.

1908 , pt. 1, p. 579 .

Top. sheet Osoyoos.

25. Moses (Nespelem, Park City). Au, Ag, Pb, Cu.

37 miles N. Almira, N. P. R. R.

Veins.

Ann. Rept. Washington Geol. Survey, vol. 1, 1902, p. 72.

Min. Res. 1908, pt. 1, p. 579.

25a. Nighthawk. $\mathrm{Au}, \mathrm{Ag}$.

Station G. N. R. R.

Paleozoic sediments and greenstones cut by granite.

Veins.

Umpleby, J. B., Geology and ore deposits of the Oroville-Nighthawk mining district: Bull. Washington Geol. Survey No. 5, 1911, pp. 55-107.

26. Osoyoos Lake (Oroville). $\mathrm{Cu}, \mathrm{Au}$.

Oroville station, G. N. R. R.

Paleozoic sediments and greenstones cut by andesite.

Veins, disseminations.

Umpleby, J. B., Geology and ore deposits of the Oroville-Nighthawk mining district: Bull. Washington Geol. Survey No. 5, 1911, pp. 55-107.

Min. Res. 1907, pt. 1, p. 474.

Top. sheet Osoyoos.

27. Palmer Mountains (Wannacut Lake, Loomis). Au, Cu, Ag.

14 miles S. Nighthawk, G. N. R. R.

Greenstones and Paleozoic sediments cut by granite.

Veins.

Ann. Rept. Washington Geol. Survey, vol. 1, 1902, pp. 66-70.

Smith, G. O., and Calkins, F. C., A geologic reconnaissance across the Cascade Range near the forty-ninth parallel: Bull. 235, 1904, p. 95.

Min. Res. 1906, p. 367.

1908, pt. 1, pp. 580, 723.

Top. sheet Chopaka.

28. Squaw Creek (Methow). Au, Ag, Cu, Sb.

53 miles N. Mansfield, 73 miles NNE. Wenatchee, G. N. R. R.

Gneiss cut by granite.

Veins.

Ann. Rept. Washington Geol. Survey, vol. 1, 1902, pp. 75-78.

Min. Res. 1906, p. 512.

1907, pt. 1, p. 475.

1908 , pt. 1, p. 579 .

Top. sheet Methow.

29. Twisp. $\mathrm{Au}, \mathrm{Ag}, \mathrm{Cu}$.

97 miles N. Wenatchee, G. N. R. R.

Ann. Rept. Washington Geol. Survey, vol. 1, 1902, pp. 74-75.

Top. sheet Methow.

30. Upper Methow. Ag, Cu, Au.

119 miles N. Wenatchee, G. N. R. R.

Gneiss and schist cut by granite.

Veins.

Ann. Rept. Washington Geol. Survey, vol. 1, 1902, pp. 73-74. 
31. Wauconda. $\mathrm{Au}, \mathrm{Ag}$.

19 miles NW. Republic, G. N. R. R.

Paleozoic sediments cut by granite.

Veins.

Ann. Rept. Washington Geol. Survey, vol. 1, 1902, pp. 62-64.

32. Carbon River. $\mathrm{Cu}, \mathrm{Au}$.

PIERCE COUNTY.

Fairfax station, N. P. R. R.

Syenite.

Veins.

Ann. Rept. Washington Geol. Survey, vol. 1, 1902, pp. 127-131.

33. Bald Mountains. $\mathrm{Cu}, \mathrm{Au}$.

SRAGIT COUNTY.

10 miles S. Lymon, G. N. R. R.

Schist and slate cut by diorite.

Veins.

Ann. Rept. Washington Geol. Survey, vol. 1, 1902, pp. 89-90.

Top. sheet Mount Vernon.

34. Thunder Creek. Ag, $\mathrm{Au}$.

36 miles W. Rockport, G. N. R. R.

Veins.

Ann. Rept. Washington Geol. Survey, vol. 1, 1902, p. 90.

34a. Hamilton. Fe.

Hamilton station, G. N. R. R.

Sandstones, limestones, shale, and slate.

Beds.

Ann. Rept. Washington Geol. Survey, vol. 1, 1902, pp. 235-241.

35. Mineral. As.

SKAMANTA AND LEWIS COUNTIES.

Station T. E. R. R.

Min. Res. 1904, p. 372.

36. St. Helens. $\mathrm{Cu}, \mathrm{Ag}, \mathrm{Pb}$.

41 miles E. Castlerock, N. P. R. R.

Syenite cut by porphyry.

Veins.

Ann. Rept. Washington Geol. Survey, vol. 1, 1902, pp. 131-135.

Min. Res. 1905, p. 337.

\section{SNOHOMISH COUNTY.}

97. Darrington. $\mathrm{Au}, \mathrm{Ag}, \mathrm{Cu}$.

Station N. P. R. R.

Schist, slate, serpentine.

Veins.

Ann. Rept. Washington Geol. Survey, vol. 1, 1902, pp. 99-101.

Russell, I. C., A preliminary paper on the geology of the Cascade Mountains in northern Washington: Twentieth Ann. Rept., pt. 2, 1900, p. 209. Min. Res. 1908, pt. 1, p. 580.

38. Granite Falls. $\mathrm{Au}, \mathrm{Ag}, \mathrm{Cu}$.

Station N. P. R. R.

Min. Res. 1906, p. 367.

39. Index. $\mathrm{Cu}, \mathrm{Ag}, \mathrm{Au}$.

1907, pt. 1, p. 475.

Station G. N. R. R.

Granite cut by basic dikes. 
39. Index-Continued.

Veins.

Ann. Rept. Washington Geol. Survey, vol. 1, 1902, pp. 114-120.

Weaver, C. E., Geology and ore deposite of the Index mining district: Bull. Washington Geol. Survey No. 7, 1912.

Weed, W. H., The copper mines of the United States in 1905: Bull. 285, 1906, p. 123.

Min. Res. 1907, pt. 1, p. 475. 1908 , pt. 1, p. 580 .

Top. sheet Skykomish.

40. Monte Cristo. $\mathrm{Au}, \mathrm{Ag}, \mathrm{Cu}, \mathrm{As}$.

Station N. P. R. R.

Mesozoic granites and Tertiary volcanic日.

Veins.

Ann. Rept. Washington Geol. Survey, vol. 1, 1902, pp. 108-111.

Spurr, J. E., The ore deposits of Monte Cristo, Wash.: Twenty-second Ann. Rept., pt. 2, 1901, pp. 777-866.

Min. Res. 1905, p. 337.

1907 , pt. 1, p. 475 .

Top. sheet Skykomish.

41. Silver Creek. $\mathrm{Cu}, \mathrm{Au}, \mathrm{Ag}, \mathrm{Pb}$.

8 miles SW. Monte Cristo, N. P. R. R.

Granite and Tertiary volcanics.

Veins.

Ann. Rept. Washington Geol. Survey, vol. 1, 1902, pp. 111-114.

Spurr, J. E., The ore deposits of Monte Cristo, Wash.: Twenty-second Ann. Rept., pt. 2, 1901, pp. 777-866.

Top. sheet Skykomish.

42. Stilaguamish (Silverton). $\mathrm{Cu}, \mathrm{Au}, \mathrm{Ag}, \mathrm{Pb}$.

Station N. P. R. R.

Granite, diorite.

Veins.

Ann. Rept. Washington Geol. Survey, vol. 1, 1902, pp. 101-108.

Min. Res. 1906, p. 367.

Top. sheet Glacier Peak.

43. Silver Hill. Sn, W.

SPOKANE COUNTY.

12 miles SSE. Spokane, N. P. R. R., G. N. R. R.

Pre-Cambrian gneiss and schist cut by acidic and basic dikes and capped by basalt.

Veins.

Collier, A. J., Tin ore at Spokane, Wash.: Bull. 340, 1908, pp. 295-305.

Min. Res. 1907, pt. 1, p. 725 .

1908 , pt. 1, p. 723 .

Top. sheet Spokane.

STEVENS COUNTY.

44. Bossburg. Pb, Ag.

Station G. N. R. R.

Paleozoic sediments.

Replacement, veins.

Ann. Rept. Washington Geol. Survey, vol. 1, 1902, p. 45.

Min. Res. 1907, pt. 1, p. 476.

1908 , pt. 1, pp. 580-581. 
45. Chewelah. $\mathrm{Cu}, \mathrm{Ag}, \mathrm{Pb}, \mathrm{Zn}, \mathrm{Au}, \mathrm{Fe}$.

Station G. N. R. R.

Paleozoic sediments cut by diorite and other basic intrusives.

Veins.

Ann. Rept. Washington Geol. Survey, vol. 1, 1902, p. 45.

Weed, W. H., The copper mines of the United States in 1905: Bull. 285, 1906, p. 123.

Min. Res. 1905, p. 337.

1907 , pt. 1, p. 476.

1908 , pt. 1 , pp. 580-581.

46. Deep Creek Lake. Fe.

12 miles SE. Northport, G. N. R. R.

Granite, Paleozoic sediments.

Bedded.

Leith, C. K., Iron ores of the western United States and British Columbia: Bull. 285, 1906, p. 195.

47. Deer Park. W.

5 miles NE. Loon Lake, 10 miles N. Deer Park, G. N. R. R.

Granite and metamorphosed Paleozoic sediments.

Lenses and veins.

Ann. Rept. Washington Geol. Survey, vol. 1, 1902, p. 46.

Bancroft, H., Notes on tungsten deposits near Deer Park, Wash.: Bull. 430, 1910, pp. 214-216.

Min. Res. 1905, pp. 411-412. 1909 , pt. 1, p. 580.

48. Deer Trail. $\mathrm{Ag}, \mathrm{Pb}, \mathrm{W}, \mathrm{Au}, \mathrm{Cu}$.

18 miles WSW. Springdale, G. N. R. R.

Paleozoic sediments cut by acidic and basic dikes.

Veins.

Ann. Rept. Washington Geol. Survey, vol. 1, 1902, pp. 46-50.

Min. Res. 1904, p. 331.

1905, p. 337.

1906, p. 367.

1907, pt. 1, p. 476

1908, pt. 1, pp. 581, 723.

49. Metaline. $\mathrm{Pb}, \mathrm{Zn}, \mathrm{Ag}$.

1909 , pt. 1, p. 580.

Station I. \& W. N. R. R.

Paleozoic sediments.

Disseminated and replacements.

Bancroft, H., Lead and zinc deposits in the Metaline mining district, northeastern Washington: Bull. 470, 1911, pp. 188-200.

Min. Res. 1906, p. 451. 1908 , pt. 1, p. 581.

50. Mejers Falls. $\mathrm{Au}, \mathrm{Ag}, \mathrm{Cu}$.

Station G. N. R. R.

Paleozoic sediments cut by porphyry.

Veins.

Ann. Rept. Washington Geol. Survey, vol. 1, 1902, p. 44.

Min. Res. 1905, p. 337.

1907, pt. 1, p. 476.

1908, pt. 1, pp. 580-581. 
STEVEYS courTY-Continued.

51. Northport. $\mathrm{Pb}, \mathrm{Ag}, \mathrm{Zn}$.

Station G. N. R. R.

Paleozoic sediments cut by acidic and basic dikes.

Veins, replacements.

Ann. Rept. Washington Geol. Survey, vol. 1, 1902, pp. 43-44.

Min. Res. 1906, pp. 367, 451.

1907, pt. 1, p. 476 .

52. Old Dominion (Colville). $\mathrm{Pb}, \mathrm{Ag}$.

7 miles E. Colville, G. N. R. R.

Paleozoic sediments cut by granite.

Contact metamorphic.

Min. Res. 1908, pt. 1, pp. 580-581.

53. Orient. $\mathrm{Au}, \mathrm{Ag}, \mathrm{Cu}$.

Station G. N. R. R.

Tertiary volcanics.

Veins, impregnations.

Min. Res. 1905, p. 337.

1908, pt. 1, p. 581.

54. Silver Queen. $\mathrm{Pb}, \mathrm{Ag}$.

8 miles S. Meyers Falle, G. N. R. R.

Paleozoic sediments cut by granite.

Replacement veins.

WHATCOM COUNTY.

55. Mount Baker. Au, Ag, Cu.

12 miles ESE. Glacier, B. B. \& B. C. R. R.

Metamorphosed sediments cut by porphyry.

Veins.

Ann. Rept. Washington Geol. Survey, vol. 1, 1902, p. 79.

Smith, G. O., and Calkins, F. C., A geological reconnaissance across the Cascade Range near the forty-ninth parallel: Bull. 235, 1904, pp. 95-96.

Min. Res. 1905, p. 337.

1906 , p. 368.

1907 , pt. 1, p. 477.

56. Slate Creek (Barron). Au, Ag. (Pl.)

60 miles NE. Rockport, G. N. R. R.

Stream gravels.

Ann. Rept. Washington Geol. Survey, vol. 1, 1902, pp. 83-88.

Russell, I. C., A preliminary paper on the geology of the Cascade Mountains in northern Washington: Twentieth Ann. Rept., pt. 2, 1900, p. 209.

Min. Res. 1906, p. 368.

1907, pt. 1 , p. 477.

1908 , pt. 1, pp. 581-582. 


\section{WYOMING.}

In the State of Wyoming 11 counties are producers of metallic minerals and 36 mining districts are recognized by the United States Geological Survey. In 19 of these districts copper is the principal metal produced and in 13 gold values predominate. Three districts are assigned to iron, but in one of these chromium is mined exclusively. One locality is in the prospect stage and its predominant metal is uncertain.

Distribution of the predominant metals produced in the mining districts of Wyoming.

\begin{tabular}{|c|c|c|c|c|c|}
\hline County. & Gold. & Copper. & Iron. & $\underset{\text { Unown. }}{\text { Un. }}$ & Total. \\
\hline 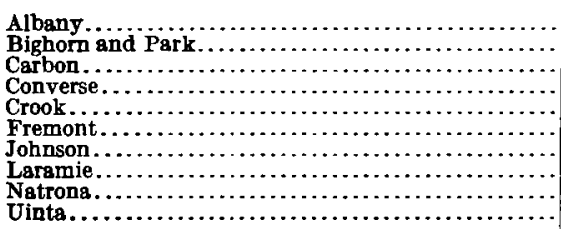 & 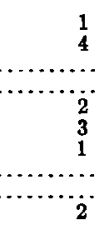 & $\begin{array}{r}\mathbf{4} \\
\mathbf{1} \\
\mathbf{4} \\
\mathbf{1} \\
\mathbf{3} \\
\mathbf{3} \\
\mathbf{1} \\
\mathbf{3} \\
\mathbf{1} \\
\mathbf{1}\end{array}$ & 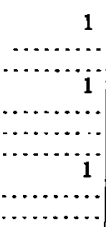 & 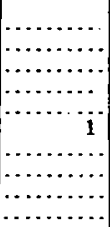 & $\begin{array}{l}6 \\
5 \\
4 \\
4 \\
2 \\
2 \\
7 \\
2 \\
4 \\
1 \\
1 \\
3\end{array}$ \\
\hline & 13 & 19 & 3 & 1 & 36 \\
\hline
\end{tabular}

MINING DISTRICTS IN WYOMING.

[See Pl. XVI. Additional references will be found in Mineral Resourees for 1910 and 1911.]

ALBANY COUHTY.

1. Centennial (La Plata). Au.

Station L. H. P. \& P. R. R.

Min. Res. 1906, p. 369.

1907 , pt. 1, p. 479.

1908, pt. 1, p. 584

Top. sheet Medicine Bow.

2. Douglas Creek (Holmes). Cu, Au, Ag, Pt, Pd. (D, Pl.)

10 miles WNW. Albany, L. H. P. \& P. R. R.

Gneise and quartzite cut by basic dikes.

Veins.

Ann. Rept. State Geologist Wyoming, 1904, pp. 23-24.

Ann. Rept. Territorial Geologist Wyoming, 1887, pp. 69-70.

Beeler, H. C., Mineral and allied resources of Albany County, Wyo., Wyoming Geol. Survey, 1906, pp. 13-54.

Emmons, S. F., Platinum in copper ores in Wyoming: Bull. 213, 1903, pp. 94-97.

Jamison, C. E., Mineral resources of Wyoming, Wyoming Geol. Survey, 1911, pp. 28-29.

Mining in the Grand Encampment copper district, Wyoming Geol. Survey, 1905. 
LIST OF

MINING DISTRICTS

ALBANY COUNTY

Centennial (La Plata)
Douglas Creek (Holmes)

Horse Creek

Laramie Peak (Esterbrook)

BIGHORN AND PARK

Bald Mountain

South Fork

Sunlight

River (Kirwin)

CARBON COUNTY

Encampment (Battle,
Purgatory. Three Forks)

Rankin

CONVERSE COUNTY

6. Deer Creek

CROOK COUNTY

8. Bear Lodge

FREMONT COUNTY

20. Atlantic

Birdseye (Copper

2. De Pass

3. Lewiston

25. South Pass
6. Willow Creek

JOHNSON COUNTY

7. Bull Camp

LARAMIE COUNTY

29. Hartville (Sunrise, Platte

Canyon)
Fish Creek

31. Rawhide Buttes

NATRONA COUNTY

33. Casper Mountain

PARK COUNTY

(See Bighorn)

UINTA COUNTY

34. Coxcomb

35. Snake River placers

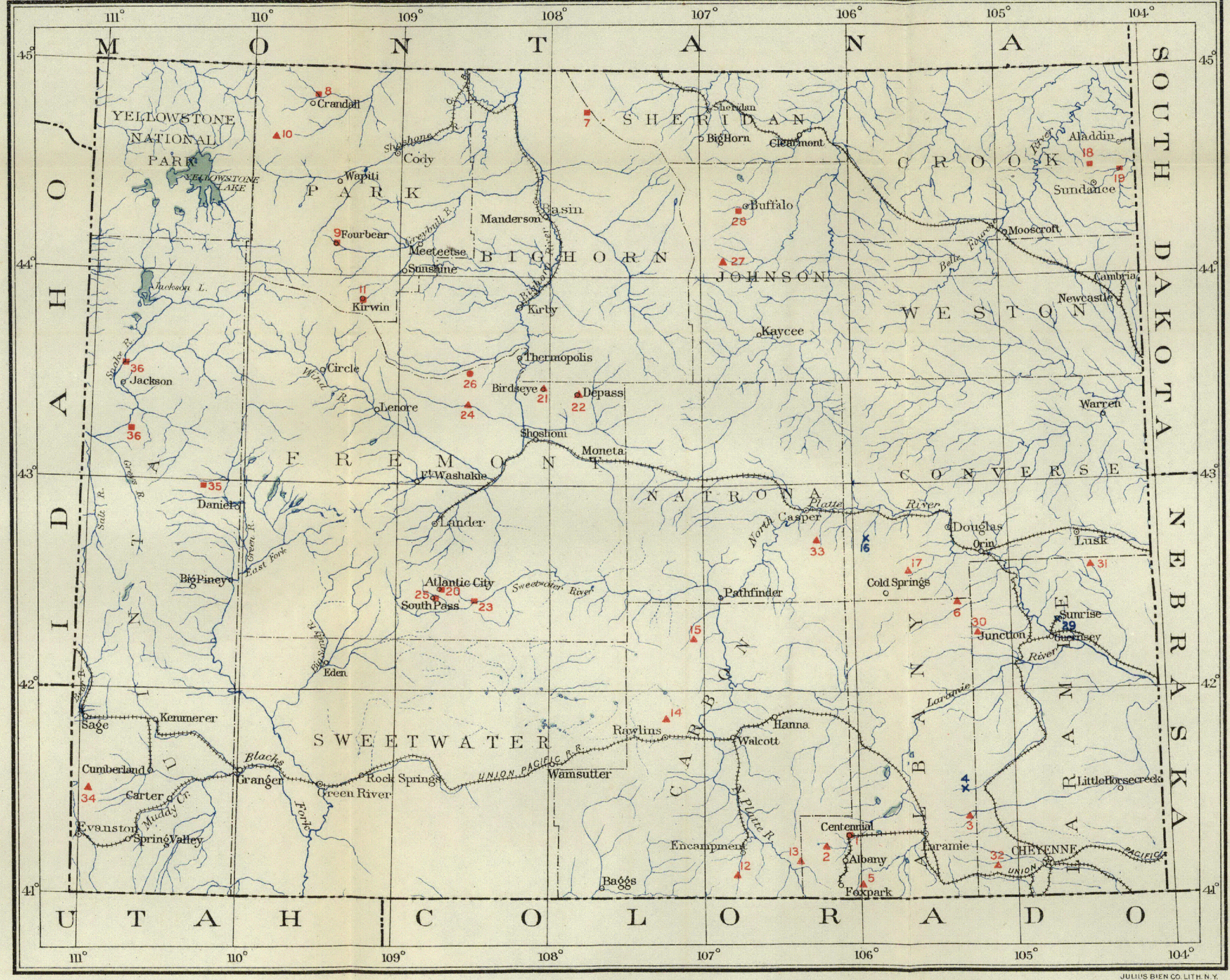

MAP OF WYOMING, SHOWING LOCATION OF MINING DISTRICTS

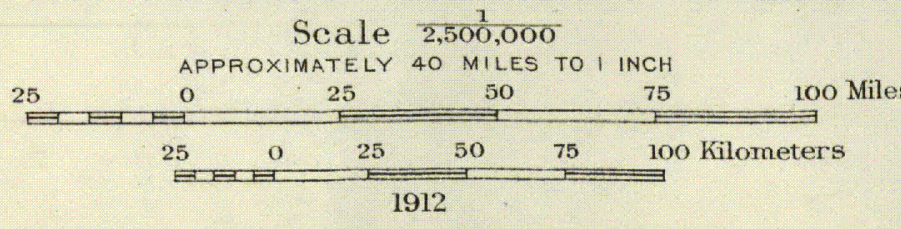

LEGEND

.

Gold predominant

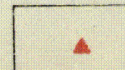

Copper predominant

$x$

Iron and chromium $\cdot$

Gold, silver, copper, lead
precolominant melat uncercain 


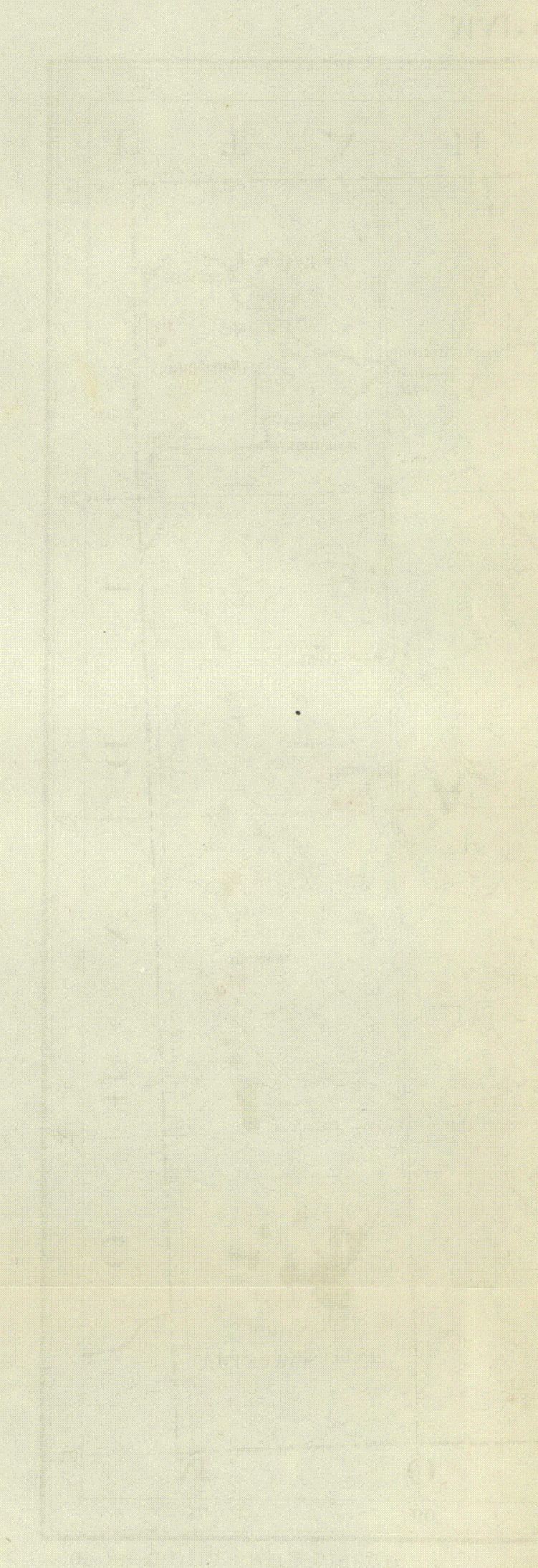


ALBANY COUNTY-Continued.

2. Douglas Creek (Holmes)-Continued.

Min. Res. 1902, pp. 244-250.

1905 , p. 339.

.1906, pp. 369-370.

1907, pt. 1, p. 479

1908, pt. 1, p. 584.

1909 , pt. 1, p. 493.

Top. sheet Medicine Bow.

3. Horse Creek. Cu.

Station C. \& S. R. R.

Pre-Cambrian schist and granite.

Veins (?).

Min. Res. 1882, pp. 229-230.

1906, p. 370.

1908 , pt. 1, p. 584.

Top. sheet Sherman.

Folio 173, 1910.

4. Iron Mountain. Fe, Ti.

8 miles west of Iron Mountain, C. \& S. R. R.

Pre-Cambrian complex.

Lenticular masses and dikes.

Kemp, J. F., The titaniferous iron ores of the Adirondacks: Nineteenth Ann. Rept., pt. 3, 1899, p. 420.

Ball, S. H., Titaniferous iron ore of Iron Mountain, Wyo.: Bull. 315, 1907, pp. 206-212.

Top. sheet Sherman.

Folio 173, 1910.

5. Jelm Mountains. $\mathrm{Cu}, \mathrm{Au}, \mathrm{Ag}$.

33 miles SW. Laramie, U. P. R. R.

Pre-Cambrian complex.

Veins.

Ann. Rept. State Geologist Wyoming, 1904, pp. 24-25.

Ann. Rept. Territorial Geologist Wyoming, 1886, pp. 17-18.

Beeler, H. C., Mineral and allied resources of Albany County, Wyo., Wyoming Geol. Survey, 1906, pp. 54-58.

Min. Res. 1907, pt. 1, p. 479 .

1908, pt. 1, p. 584 .

Top. sheet Laramie.

Folio 173, 1910.

6. Laramie Peak (Esterbrook). Cu.

22 miles S. Douglas, C. B. \& Q. R. R.

Granite.

Veins.

Ann. Rept. State Geologist Wyoming, 1904, pp. 24-25.

Beeler, H. C., Mineral and allied resources of Albany County, Wyo., Wyoming Geol. Survey, 1906, pp. 60-70.

The North Laramie Peak copper district, Wyoming Geol. Survey, 1904.

Min. Res. 1907, pt. 1, p. 480.

1908 , pt. 1, p. 584 .

BIGHORN AND PARK COUNTIES.

7. Bald Mountain. Au. (Pl.)

30 miles E. Kane, C. B. \& Q. R. R.

Cambrian sediments.

21528 ${ }^{\circ}$ - Bull. $507-12-19$ 


\section{BIGHORN AND PARK COUNTIES-Continued.}

\section{Bald Mountain-Continued.}

Free gold in conglomerate.

Eldridge, G. H., A geological reconnaissance in northwest Wyoming: Bull. 119,1894 , p. 66.

Darton, N. H., Geology of the Bighorn Mountains: P. P. 51, 1906, pp. 112-113.

Fisher, C. A., Geology and water resources of the Bighorn Basin, Wyoming:

P. P. 53, 1907, p. 60.

Top. sheet Bald Mountain.

Folio 141, 1906.

8. Clark Fork. Au. (Pl.)

23 miles SSW. Belfry, N. P. R. R.

Stream gravels.

Fisher, C. A., Geology and water resources of Bighorn Basin, Wyoming: P. P. 53, 1907, p. 60 .

Top. sheet Crandall.

Folio 52, 1899.

9. South Fork. $\mathrm{Au}, \mathrm{Ag}, \mathrm{Cu}$.

52 miles SW. Cody, C. B. \& Q. R. R.

Tertiary volcanics.

Veins.

Top. sheet Ishawooa.

Folio 52, 1899.

10. Sunlight. $\mathrm{Cu}, \mathrm{Au}, \mathrm{Ag}$.

95 miles ESE. Gardiner, N. P. R. R.

Tertiary volcanics.

Veins.

Ann. Rept. State Geologist Wyoming, 1904, pp. 25-26.

Min. Res. 1905, p. 340.

Top. sheet Crandall.

Folio 52, 1899.

11. Wood River (Kirwin). Au, Ag, $\mathrm{Pb}, \mathrm{Cu}$.

76 miles SSW. Cody, 97 miles WSW. Basin, C. B. \& Q. R. R.

Ann. Rept. State Geologist Wyoming, 1904, pp. 25-26.

Beeler, H. C., Wyoming mines, 1907, Wyoming Geol. Survey, 1908, pp. 19-21.

Min. Res. 1905, p. 340.

1906 , p. 370.

1907, pt. 1, p. 481.

1908 , pt. 1, p. 585 .

1909, pt. 1, p. 494.

Top. sheet Kirwin.

\section{CARBON COUNTY.}

12. Encampment (Battle, Purgatory, Three Forks). $\mathrm{Cu}, \mathrm{Ag}, \mathrm{Au}$.

Station S. \& E. R. R.

Pre-Cambrian complex.

Stockworks and veins.

Ann. Rept. State Geologist Wyoming, 1904, pp. 26-31.

Beeler, H. C., Wyoming mines and minerals in brief, Wyoming Geol. Survey. 1904, pp. 9-11.

Jamieson, C. E., Mineral resources of Wyoming, Wyoming Geol. Survey, 1911, p. 28.

- Mining in the Grand Encampment copper district, Wyoming Geol. Survey, 1905. 
CARBON COUNTY-Continued.

12. Encampment (Battle, Purgatory, Three Forks)-Continued.

Spencer, A. C., Mineral resources of the Encampment copper region, Wyoming: Bull. 213, 1903, pp. 158-162.

The copper deposits of the Encampment district, Wyoming: P. P. $25,1904$.

Weed, W. H., The copper mines of the United States in $1905 ;$ Bull. 285, 1906, pp. 123-124.

Min. Res. 1905, p. 340.

1906, pp. 370,410 .

1907 , pt. 1, pp. $480,621,622$.

1908 , pt. 1, pp. $219,584-585$.

1909, pt. 1, p. 493.

Top. sheet Encampment special.

13. French Creek. Cu, Au.

30 miles E. Encampment, S. \& E. R. R.

Pre-Cambrian complex cut by basic dikes.

Top. sheet Medicine Bow.

14. Rankin. $\mathrm{Cu}$.

10 miles N. Rawlins, U. P. R. R.

15. Seminole. $\mathrm{Cu}, \mathrm{Fe}$.

60 miles N. Rawlins, U. P. R. R.

Schists and quartzites.

Lenses.

Ann. Rept. Territorial Geologist Wyoming, 1886, p. 10.

Ann. Rept. Territorial Geologist Wyoming, 1887, pp. 70-72.

Ann. Rept. Territorial Geologist Wyoming, 1890, pp. 61-63.

Min. Res. 1882, p. 147.

1883-84, p. 285.

16. Deer Creek. Cr.

CONVERSE COUNTY.

15 miles SW. Glenrock, C. \& N. W. R. R.

Serpentine.

Lenses.

Min. Res. 1908, pt. 1, p. 766.

17. Warbonnet. $\mathrm{Cu}$.

25 miles SW. Douglas, C. \& N. W. R. R.

Ann. Rept. State Geologist Wyoming, 1904, pp. 31-32.

Beeler, H. C., The North Laramie Peak copper district, Wyoming Geol. Survey, 1904.

18. Bear Lodge. Au, Ag. (D, Pl.)

CROOK COUNTY.

15 miles SW. Aladdin, W. \& M. R. R. R.

Cretaceous and Tertiary sediments cut by Tertiary volcanics.

Min. Res. 1906, p. 370.

Top. sheet Aladdin.

Folio 128, 1905.

19. Hurricane. Au. (Pl.)

20 miles S. Aladdin, W. \& M. R. R. R.; 20 miles W. Spearfish, S. Dak., C. B. \& Q. R. R.

Wash gravels.

Min. Res. 1906, pp. 370-371.

1907 , pt. 1, p. 481.

1908 , pt. 1 , p. 585 .

1909 , pt. 1, p. 494. 
CROOK COUKTY-Continued.

19. Eurricane-Continued.

Top. sheet Sundance.

Folio 127, 1905.

FREMONT OOUNTY.

20. Atlantic. Au. (D, Pl.)

34 miles S. Lander, W. \& N. W. R. R

Pre-Cambrian complex.

Veins.

Ann. Rept. State Geologist Wyoming, 1904, pp. 34-36.

Ann. Rept. Territorial Geologist Wyoming, 1886, pp. 12-15.

-Beeler, H. C., A brief review of the South Pass gold district, Fremont County, Wyo., Wyoming Geol. Survey, 1904.

Wyoming mines, 1908, Wyoming Geol. Survey, 1909, pp. 21-25.

Jamieson, C. E., Geology and mineral resources of a portion of Fremont County, Wyo., Wyoming Geol. Survey, 1911, pp. 75, 80.

Mineral resources of Wyoming, Wyoming Geol. Survey, 1911, pp. 24-26.

Min. Res. 1905, p. 341.

1906, p. 371.

1907 , pt. 1, p. 482.

1908 , pt. 1, p. 586 .

1909 , pt. 1, p. 449.

21. Birdseye (Copper Mountain). Cu, Ag.

17 miles N. Shoshone, W. \& N. W. R. R.

Pre-Cambrian granite and Paleozoic sediments cut by basic dikes.

Veins.

Beeler, H. C., Wyoming mines, 1908, Wyoming Geol. Survey, 1909, pp. 17-19.

Darton, N. H., Geology of the Bighorn Mountains: P. P. 51, 1906, p. 113.

Jamieson, C. E., Mineral resources of Wyoming, Wyoming Geol. Survey, 1911, p. 29.

Min. Res. 1906, p. 371.

1907, pt. 1, p. 481.

1908 , pt. 1, p. 586.

22. De Pass. $\mathrm{Cu}, \mathrm{Ag}$.

20 miles NNE. Shoshone, W. \& N. W. R. R.

Paleozoic sediments.

Darton, N. H., Geology of the Bighorn Mountains: P. P. 51, 1906, p. 113.

Min. Res. 1907, pt. 1, p. 481.

23. Lewiston. Au. (Pl.)

44 miles S. Lander, W. \& N. W. R. R.

Min. Res. 1905, p. 341.

1907, pt. 1, p. 482.

24. Owl Creek. $\mathrm{Cu}, \mathrm{Au}, \mathrm{Ag}$.

45 miles NW. Riverton, W. \& N. W. R. R.

Pre-Cambrian granite and schist.

Veins.

Ann. Rept. State Geologist Wyoming, 1904, pp. 33-34.

Ann. Rept. Territorial Geologist Wyoming, 1886, pp. 12-15.

Beeler, H. C., Wyoming mines, 1908, Wyoming Geol. Survey, 1908, pp. 16-17.

25. South Pass. Au. (D, Pl.)

39 miles S. Lander, W. \& N. W. R. R.

Pre-Cambrian complex.

Veins.

Ann. Rept. State Geologist Wyoming, 1904, pp. 33-34. 
FREMONT COUNTY-Continued.

25. South Pass-Continued.

Ann. Rept. Territorial Geologist Wyoming, 1886, pp. 12-15.

Beeler, H. C., Wyoming mines and minerals in brief, Wyoming Geol. Survey, 1904, pp. 7-8.

A brief review of the South Pass gold district, Fremont County, Wyo., Wyoming Geol. Survey, 1904.

Wyoming mines, 1908, Wyoming Geol. Survey, 1909, pp. 21-25.

Jamieson, C. E., Geology and mineral resources of a portion of Fremont County, Wyo., Wyoming Geol. Survey, 1911, pp. 75-80.

Min. Res. 1905, p. 341.

1906, p. 371.

1907, pt. 1, p. 482.

1908 , pt. 1, p. 586.

26. Willow Creek. 1909 , pt. 1, p. 449.

22 miles WSW. Kerby, C. B. \& Q. R. R.

Pre-Cambrian granite and schist.

Veins.

Beeler, H. C., Wyoming mines, 1908, Wyoming Geol. Survey, 1909, p. 19.

Min. Res. 1907, pt. 1, p. 482.

1909 , pt. 1, p. 494

27. Bull Camp. Cu.

\section{JOHNSON COUNTY.}

69 miles SW. Clearmont, C. B. \& Q. R. R.

Granite cut by diabase.

Veins.

Ann. Rept. State Geologist Wyoming, 1904, p. 37.

Darton, N. H., Geology of the Bighorn Mountains: P. P. 51, 1906, p. 114.

Min. Res. 1906, p. 371.

1907 , pt. 1, p. 482.

1908 , pt. 1, p. 586.

1909 , pt. 1, p. 494

Top. sheet Fort McKinney.

Folio 142, 1906.

28. Kelley Creek (Buffalo). Au. (P1.)

35 miles SW. Clearmont, C. B. \& Q. R. R.

Cambrian conglomerate.

Gold in conglomerate.

Min. Res. 1906, p. 371.

1907 , pt. 1 , p. 482

Top. sheet Fort McKinney.

Folio 142, 1906.

\section{LARAMIE COUNTY.}

29. Hartville (Sunrise, Platte Cańyon). $\mathrm{Fe}(\mathrm{Cu})$.

Stations C. B. \& Q. R. R.

Pre-Cambrian complex and Paleozoic sediments.

Replacements.

Ann. Rept. State Geologist Wyoming, 1904, p. 37.

Ann. Rept. Territorial Geologist Wyoming, 1886, pp. 19-26.

Ann. Rept. Territorial Geologist Wyoming, 1887, pp. 66-70.

Ann. Rept. Territorial Geologist Wyoming, 1890, pp. 51-61.

Ball, S. H., Copper depositis of the Hartville uplift, Wyoming: Bull. 315, 1907, pp. 93-107. 
IARAMIE COUNTY-Continued.

29. Hartville (Sunrise, Platte Canjon)-Continued.

Ball, S. H., The Hartville iron-ore range, Wyoming: Bull. 315, 1907, pp. 190-205.

Beeler, H. C., Wyoming mines, 1908, Wyoming Geol. Survey, 1909, pp. 29-33.

Jamieson, C. E., Mineral resources of Wyoming, Wyoming Geol. Survey, 1911, pp. 27-28.

Leith, C. K., Iron ores of the western United States and British Columbia: Bull. 285, 1906, pp. 194-195.

Min. Res. 1882, p. 147.

$$
\begin{aligned}
& 1883-84, \text { pp. } 229,285,342,758-759 . \\
& 1885, \text { p. } 342 . \\
& 1888 \text {, } 76 . \\
& 1889, \text { p. } 59 . \\
& 1905, \text { p. } 76 . \\
& \text { 1907, pt. } 1 \text {, p. } 482 . \\
& \text { 1908, pt. 1, pp. } 109-110,585 . \\
& 1909, \text { pt. } 1 \text {, p. } 493 .
\end{aligned}
$$

Top. sheet Hartville.

Folio 91, 1903.

30. Fish Creek. Cu.

39 miles W. Junction, C. B. \& Q. R. R.

31. Rawhide Buttes. $\mathrm{Cu}, \mathrm{Au}, \mathrm{Ag}$.

34 miles NNW. Torrington, C. B. \& Q. R. R.

Granite, schist.

Veins.

Ann. Rept. Territorial Geologist Wyoming, 1887, pp. 62-66.

Min. Res. 1907, pt. 1, p. 482.

1908, pt. 1, p. 585 .

1909 , pt. 1, p. 498.

32. Silver Crown (Hecla). $\mathrm{Cu}, \mathrm{Au}, \mathrm{Ag}$.

5 miles N. Granite Canyon, U. P. R. R.

Pre-Cambrian schists and granite.

Veins, impregnations.

Ann. Rept. State Geologist Wyoming, 1904, p. 37.

Ann. Rept. Territorial Geologist Wyoming, 1886, pp. 7-10.

Ann. Rept. Territorial Geologist Wyoming, 1887, p. 69.

Min. Res. 1908, pt. 1, p. 585.

Top. sheet Sherman.

Folio 173, 1910.

MATRONA COUNTY.

33. Casper Mountain. $\mathrm{Cu}$.

25 miles S. Casper, W. \& N. W. R. R.

Min. Res. 1905, p. 341.

1906, p. 371.

PARK COUNTY. (Seo BIghorn.)

34. Cockscomb. $\mathrm{Cu}$.

UINTA COUNTY.

25 miles N. Evanston, U. P. R. R.

"Red Beds."

Disseminated.

Veatch, A. C., Geography and geology of a portion af southwestern

Wyoming: P. P. 56, 1907, p. 163. 
WYOMING.

UITTA COUNTY-Continued.

35. Horse Creek. Au, Ag. (D, Pl.)

114 miles N. Kemmerer, U. P. R. R.

Jurassic slates and limestones.

Disseminated.

Schultz, A. R., Gold developments in central Uinta County, Wyo.: Bull. 315, 1907, pp. 74-75.

36. Snake River placers (Hoback, Pine Bar). Au, Ag. (Pl.)

140 miles E. St. Anthony, Idaho, O. S. L. R. R.

Stream and terrace gravels.

Schultz, A. R., Gold developments in central Uinta County, Wyo.: Bull. 315, 1907, pp. 71-88.

Min. Res. 1906, p. 371.

1907, pt. 1, p. 482. 


\section{AIPHABETIC LIST OF MINING DISTRICTS.}

In the following list all the districts shown on Plates III to XVI are named in alphabetic order. In the State column "California, $N$ " indicates the northern counties of California (Pl. IV), and "California, S" the southern counties (Pl. V). The numbers agree with those used in the plates and in the preceding lists.

Mining districts in the western United States.

\begin{tabular}{|c|c|c|c|c|c|}
\hline Mining district. & State. & No. & Mining district. & State. & No. \\
\hline A. & & & Ashcrof & Colorado. & \\
\hline Abiquiu..... & New Mexic & 44 & $\begin{array}{l}\text { Ash } \\
\text { Ash }\end{array}$ & $\begin{array}{l}\text { Nevada... } \\
\text { Oregon }\end{array}$ & 92 \\
\hline & California, & 77 & $\begin{array}{l}\text { Ashland... } \\
\text { Ash Peak }\end{array}$ & $\begin{array}{l}\text { Oregon.... } \\
\text { Arizona... }\end{array}$ & $\begin{array}{l}47 \\
28\end{array}$ \\
\hline del & Nevado... & $\begin{array}{r}179 \\
76\end{array}$ & Ashwood. & Oregon... & 23 \\
\hline tert & Califormia, & 120 & Aspen... & Colorado. & 99 \\
\hline & California, & 47 & AtI & $\begin{array}{l}\text { Idaho....... } \\
\text { W voming }\end{array}$ & $\begin{array}{l}53 \\
20\end{array}$ \\
\hline gu & Arizona... & $\begin{array}{r}35 \\
104\end{array}$ & Atolia. & California, & $\begin{array}{r}20 \\
124\end{array}$ \\
\hline & Montana. & $\begin{array}{r}104 \\
03\end{array}$ & Atwood. & Nevada... & 136 \\
\hline jo. & Artzons.. & 66 & & California, & 102 \\
\hline 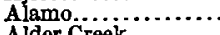 & Oregon.. & 37 & $\begin{array}{l}\text { Auburm } \\
\text { Auld.. }\end{array}$ & $\begin{array}{l}\text { Oregon...... } \\
\text { California, }\end{array}$ & 113 \\
\hline Alde & $\begin{array}{l}\text { Idah } \\
\text { Mon }\end{array}$ & $\begin{array}{l}44 \\
53\end{array}$ & Aura... & Nevada.... & 27 \\
\hline Alice & & $\mathbf{2 3}$ & Au & .... do.... & 45 \\
\hline Ile & California, & 143 & $\begin{array}{l}\text { Aur } \\
\text { Aus }\end{array}$ & Montana.... & $\begin{array}{r}165 \\
42\end{array}$ \\
\hline & $\ldots . . d$ & 185 & Aus & Nevada.. & 100 \\
\hline Alpi & Colorado. & $\begin{array}{l}1 \\
8\end{array}$ & Aztec... & Arizona... & 101 \\
\hline $\mathrm{pi}$ & Neva & 1 & Azusa.. & California, $\mathrm{s}$ & \\
\hline $\begin{array}{l}\text { Alpi } \\
\text { Alta }\end{array}$ & $\begin{array}{l}\text { Texa } \\
\text { Mont }\end{array}$ & $\begin{array}{l}1 \\
91\end{array}$ & B. & & \\
\hline Alta & Utah.... & 48 & & & \\
\hline & & 53 & Baboquiva & Arizona.. & \\
\hline Alt & & 31 & & & 114 \\
\hline Altc & Cal & 185 & $\mathrm{Bac}$ & Mon & \\
\hline & Cali & 121 & $\mathrm{Bac}$ & $\mathrm{Cal}$ & \\
\hline & & 10 & $\mathrm{Ba}$ & .....do... & 67 \\
\hline Am & Califo & 48 & $\mathrm{Ba}$ & .....do... & 121 \\
\hline & & 32 & & & \\
\hline $\mathrm{Am}$ & $\mathbf{N}$ & 80 & & & \\
\hline$A \pi$ & Uta & 74 & & $\mathrm{Ca}$ & \\
\hline $\mathbf{H}$ & iia, $N$ & 138 & & & \\
\hline & Ari & 67 & & & \\
\hline & New 1 & 80 & & & \\
\hline & & 11 & & & \\
\hline & Calif & 20 & & & \\
\hline An & Col & 117 & & Califormi & \\
\hline A & Idah & 9 & & & \\
\hline & New Mexico . & 61 & & Cali & \\
\hline No. & & 11 & & Mo & \\
\hline & Colo & 44 & & & \\
\hline AT & Oreg & 46 & & $\mathrm{Ca}$ & 16 \\
\hline A & & 54 & & Idaho $\ldots . . . .$. & \\
\hline & Arizona. & 27 & & Nevada.... & \\
\hline & & 1 & intain & & 12 \\
\hline & & 41 & & & \\
\hline Arg & Col & 19 & & $\mathrm{ia}, \mathrm{S} . .$. & 15 \\
\hline & & 68 & Springs & & 92 \\
\hline Creek. & Idaho .......... & 95 & n.......... & Washington... & \\
\hline Aron & California, S. & 122 & Bas & Montana....... & \\
\hline Arras & .....do.......... & 72 & & & \\
\hline Arro & ...do..... & 123 & Battle Mountair & $\mathrm{Col}$ & \\
\hline Asht & Utah ..... & 14 & Battle Mountair & $\mathrm{Ner}$ & \\
\hline
\end{tabular}


Mining districts in the western United States-Continued.

\begin{tabular}{|c|c|c|c|c|c|}
\hline Mining district. & State. & No. & Mining district. & State. & No. \\
\hline Bay Hors & Idaho.. & 45 & Bodie. & Californi & 88 \\
\hline Bayles... & Californis & 122 & Bohemia. & Oregon. & 29 \\
\hline Beach & Oregon.. & 24 & Bohemia. & .....do.. & 67 \\
\hline Bear Creek. & Idaho.... & 58 & Boise....... & Idaho... & 2 \\
\hline $\begin{array}{l}\text { Bear Gulch. } \\
\text { Bear Gulch. }\end{array}$ & Montana & $\begin{array}{l}62 \\
80\end{array}$ & Bolivar.... & Oregon. & 22 \\
\hline Bear Lake.. & Idaho... & 5 & Bonanza.. & Texas.. & \\
\hline Bear Lodge & Wyoming & 18 & Bonita... & New Mexic & 34 \\
\hline Bearpaw.. & Montana. & 19 & Bossburg. & Washingto & 44 \\
\hline Beaver... & Idaho.... & 114 & Boulder.. & Montana.. & 33 \\
\hline Besver C & California, & 152 & Boundary C & Arizona. & 42 \\
\hline Beave & Montana. & 11 & Bovard..... & Nevada. & 46 \\
\hline Beav & ....do... & 3 & Box Canyor & Colorado & 50 \\
\hline Bea & Utah... & 1 & Boxelder.. & Utah... & 19 \\
\hline Belcher... & Washing & 12 & Boyer.... & Nevada. & 3 \\
\hline Bells Unior & California & 76 & Bradshaw... & Utah... & 2 \\
\hline Bellehelen. & Nevada. & 124 & Bradshaw M & Arizona. & 119 \\
\hline Belmont. - & & 125 & Brandy City. & California, & 139 \\
\hline Ben Hur. & Californis & 69 & Breckenridge & Colorado. & 124 \\
\hline Bensc & Arizona. & 11 & Brice....... & New Me: & 42 \\
\hline Bentley.. & & 41 & Bridge Creek & Washin & \\
\hline Berlin... & Nevada. & 126 & Bright wood & Californi & 126 \\
\hline Berni & & 2 & Bristol.... & Nevada. & 104 \\
\hline Bevan.... & Colorado. & 124 & Bromide... & New Me & 45 \\
\hline Beveridge. & California & 11 & Bromide N & ....do. do. & 67 \\
\hline Big Bar... & California & 186 & Browns.... & Nevada & 77 \\
\hline Big 1 & Arizona & 105 & Brov & Californis & 216 \\
\hline Big Cottonw & Utah.. & 46 & Brownsville & .....do. & 45 \\
\hline reek.. & Idaho... & 61 & Brownsville. & ......do. & 217 \\
\hline Big & California, & 49 & Bryant ..... & Montana & 5 \\
\hline Big Dry Cre & .....do... & 1 & Buckeye... & California, & 137 \\
\hline Big Dune.. & Nevada. & 127 & Buck Guich & Oregon... & 2 \\
\hline Big 1 & Montana & 68 & Buckhorn .. & Nevada. & 70 \\
\hline Bfgh & Arizona. & 132 & Buckskin... & Colorado & 88 \\
\hline Big P & California & 12 & Buena Vista. & Novada. & 47 \\
\hline Big Oak F lat & California & 209 & Buffalo....... & Wyoming & 28 \\
\hline am.... & Utab.... & 49 & Buffalo Hump & Idaho.... & 74 \\
\hline Birdseye... & Wyoming & 21 & Bull Camp.. & Wyomin & 27 \\
\hline Hisbee..... & Arizons.. & 10 & Bullfrog.... & Nevada. & 129 \\
\hline Creel & Californis, & 13 & & Californis & 127 \\
\hline Black Bear. & California, & 173 & ion.. & Nevada. & 28 \\
\hline Blackbird. & Idaho.... & 82 & Bullic & .... do. & 94 \\
\hline Blackbird. & ....do.. & & H Hill. & & 71 \\
\hline Black Butte. & Oregon.. & 65 & Bull Valley.. & Utah... & 85 \\
\hline Black Canyon & Arizona. - & 106 & Bully Choop. & California, 1 & 187 \\
\hline Black Diamor & Californiz, & 123 & y Hill... & ....do.... & 124 \\
\hline Blackfoot.. & Montana. & 81 & Bunker Hill & Arizona & 29 \\
\hline Blackhawk... & California, s & 125 & Bunker Hill. & & 83 \\
\hline Black Hawk. & New Mexic & 12 & Bunkerville. & Nevada. & 13 \\
\hline Black Hills... & Arizona... & 107 & Burke....... & Idaho. & 119 \\
\hline Black Hills. . . & South Dakot & 16 & Burl & Oregon. & 3 \\
\hline Black Hornet & Idaho........ & & it Creek.. & Idaho..... & 103 \\
\hline Black 苗o & Nevadi. & 166 & Burnt Ranch. & Californis, & 199 \\
\hline Black Lake... & Idaho.... & 128 & Burro Mountains & New Mexic & 13 \\
\hline Black Mountain & California, & 50 & Butte............ & Montans... & 100 \\
\hline Black Mou & New Mexico & & Butte Valley.... & California, N.... & 105 \\
\hline Black Pri & Idaho... & 115 & & & \\
\hline Black Prin & Montana. & 27 & C. & & \\
\hline Black Ran & New Mexic & 64 & & & \\
\hline Black Ro & Arizona... & 108 & Cababi..... & Arizona.. & 70 \\
\hline Blacktail. & Idaho.... & 39 & Caballos Mountains... & New Mexi & 60 \\
\hline Black War & Arizona.. & 18 & tet................... & Montana. & 49 \\
\hline Black War & Idaho.... & 54 & & $\ldots$...do... & 96 \\
\hline Blake....... & Colorado. & 115 & $\mathrm{Cab}$ & .....do.. & 22 \\
\hline Blakes Camp & Nevada... & 128 & Cable Cove.. & oregon. & 4 \\
\hline Bland...... & New Mexico & 48 & Cactus Springs. & Nevada..... & 130 \\
\hline Blewett........ & Washington & 6 & Calico...... & California, $\mathrm{s}$ & 128 \\
\hline Blind Spring & California & 87 & $\mathrm{Ca}$ & Arizona..... & 1 \\
\hline ksburg.. & Californi: & 56 & mia.. & Colorado... & 68 \\
\hline Blue Bells... & Utah...... & 56 & California....... & .....do... & 71 \\
\hline Blue Canyon & California, $j$ & 92 & Callahan Creek. & Montana.. & 50 \\
\hline Blue Lead. . . & South Dakot & 17 & hans ...... & California, N.... & 154 \\
\hline Ledge... & California, $\mathbf{N}$ & 153 & Calumet.... & Colorado.......... & 41 \\
\hline edge. & Oregon... & 48 & & Idaho. & 10 \\
\hline Blue Lodge. ... . & Utah.... & 81 & as Cove. & .... do... & 78 \\
\hline Blue Mountain. & Colorado. & 111 & Cambridge.. & Nevada. & 45 \\
\hline Blue Moumtains & Utah....... & 50 & Campbell.. & ....do.. & 05 \\
\hline Blue Ravine.... & California, $\mathrm{N}$ & 117 & Camp Bird. & Colorado. & 84 \\
\hline Blue Ridge... & Colorado.... & 45 & Camp Carson. & Oregon.... & 72 \\
\hline Blue River. & Oregon.... & 66 & Camp Floyd.... & Utah... & 57 \\
\hline Bluewing.. & Idaho... & 85 & Camp Howard. & Idaho......... & 62 \\
\hline Bluewing........ & Montane.... & 4 & Campo Seco..... & California, N........ & 21 \\
\hline & & & Camptonville.... & & 218 \\
\hline
\end{tabular}


Mining districts in the western United States-Continued.

\begin{tabular}{|c|c|c|c|c|c|}
\hline Mining district. & State. & No. & Mining district. & State. & No. \\
\hline Canada Hill & California, N......... & 93 & Clark Fork & Wyoming. & 8 \\
\hline Candelaria.. & Nevada............. & 48 & Clear Creek. & California, $\mathbf{N}$.... & 156 \\
\hline Canon City. & Colorado & 36 & Clear Lake. & $\therefore$ do $\ldots . . .$. & 63 \\
\hline Canyon.... & Oregon. & 38 & Cleors.... & Colorad & 10 \\
\hline Canyon City & California, & 188 & Cifton. & Utah... & 58 \\
\hline Canyoncito. & New Mexícc & 68 & Clinton. & Montan & 73 \\
\hline Carbonate. & South Dakot & 7 & Cloverdale. & Californis & 178 \\
\hline Carbonate. & Utah...... & 71 & Cloverdale. & Nevada. & 131 \\
\hline Carbon $\mathbf{k}$ & Washing & 32 & Cloverpatch & California & 90 \\
\hline Cardwell .... & Montana & 39 & Clyde..... & Idaho ... & 14 \\
\hline Cargo Muchac & California & 7 & Coal Creek. & Colorado & 100 \\
\hline Caríbou . . . . . & Colorado & $\begin{array}{r}3 \\
00\end{array}$ & $\cdots \cdots$ & Calíornia & 79 \\
\hline Carlin .. & Nevada & 29 & Cocnetop & Colorado & 82 \\
\hline $\begin{array}{l}\text { Carmen Cree } \\
\text { Carpenter. }\end{array}$ & $\begin{array}{l}\text { Idaho ......... } \\
\text { New Mexico. }\end{array}$ & $\begin{array}{l}80 \\
14\end{array}$ & Cochiti.... & Arizona & 6 \\
\hline Carrville.... & California, $\mathbf{N}$ & 189 & Cockscomb & Wyomi & 34 \\
\hline Carson. & Idaho... & 107 & & & $(114$ \\
\hline Carson Hill. & California & 26 & & & 116 \\
\hline Carters ..... & $\ldots$.... do.. & 213 & & & 117 \\
\hline Casa Gra & Arizons. & 92 & & & 118 \\
\hline Cascade _... & Colorado . & 22 & Coeur d'Alene ........ & Idaho. & 119 \\
\hline Casper Moun & Wyoming & 33 & & & 121 \\
\hline $\begin{array}{l}\text { Castle Dome. } \\
\text { Castle Mounte }\end{array}$ & $\begin{array}{l}\text { Arizona. } \\
\text { Montana }\end{array}$ & $\begin{array}{r}124 \\
65\end{array}$ & & & $\begin{array}{l}125 \\
126\end{array}$ \\
\hline Castle Peak. & Californis & 89 & & & 127 \\
\hline Casto ....... & Idaho... & 47 & Coffee.. & California, I & 190 \\
\hline Cataract & Montana & 34 & Coloma. & ....do. & 38 \\
\hline Cave Canyor & California & 129 & Coloma... & Montana & 82 \\
\hline Care Creek. & Arizona .. & 36 & Colorado... & $\ldots$ do ... & 35 \\
\hline Cebolla ....... & Colorado & 51 & Colridge... & California, $\mathrm{N}$ & 191 \\
\hline Cecilville ... & California, $\mathbf{N}$ & 155 & Columbia.. & ....do do & 211 \\
\hline $\begin{array}{l}\text { Cedar. } \\
\text { Cedar. }\end{array}$ & $\begin{array}{l}\text { Calilornia, } \mathrm{S} \text {. } \\
\text { Colorado. }\end{array}$ & $\begin{array}{r}75 \\
122\end{array}$ & $\begin{array}{l}\text { Columbia. } \\
\text { Columbia. }\end{array}$ & $\begin{array}{l}\text { Colorad } \\
\text { Nevada }\end{array}$ & $\begin{array}{l}96 \\
27\end{array}$ \\
\hline Cedar... & Nevada. & $\begin{array}{r}122 \\
78\end{array}$ & Columbia.. & Utah.... & 59 \\
\hline Cedar Creek & Montana & 67 & Columbia Riv & Washington. & 13 \\
\hline Cedar Creek. & New Mexico & 31 & Columbine.. & Colorado.... & 107 \\
\hline Cedar Plain. & Montana.... & 12 & Columbus. & Nevada.... & 48 \\
\hline Cedar Valley & Arizona... & 43 & Colville.... & Washingt & 52 \\
\hline Centennial... & Nevada... & 30 & Combination & Montana. & 27 \\
\hline Centennial. . & Wyoming. & 1 & Comer.. & Oregon. . & 41 \\
\hline Centerville. & Idăho ..... & 26 & Comet. . & Montana & 34 \\
\hline Central ..... & Colorado... & 1 & Comet... & $\ldots \ldots$ & 106 \\
\hline Central. & $\ldots$ do . . . . . & 42 & Comobabi & Arizona. & 70 \\
\hline Central. & Nevada. & 79 & Comstock. . & Nevada. & 156 \\
\hline Central .... & New Mexico & 15 & Conconully & Washing & 23 \\
\hline Central Benit & California. $\mathrm{S}$ & 118 & Concordia. & Nevada... & 138 \\
\hline Central City. & Colorado... & 42 & Congress.... & Arizona... & 110 \\
\hline Cerbat....... & Arizona... & 44 & Conner Creel & Idaho.... & 42 \\
\hline Cerrillos .... & New Mex & 56 & Conner Creek $\ldots \ldots$ & Oregon.. & 5 \\
\hline Cerro Gordo & California, & 14 & Cons olldated-Mont- & Colorado... & 89 \\
\hline Chafey ...... & Nevada. & 79 & gomery. & & \\
\hline Chalk Creek & Colorado. & 9 & Contact... & Nevada. & 31 \\
\hline Champion ... & Oregon.... & 67 & Cooke... & Montana & 79 \\
\hline Charlestown. & Nevada... & 33 & Cooks $\mathrm{Pe}$ & New Mexico . . . . . . . . . . & 36 \\
\hline Chemehuevis & Arizona.... & 45 & Coolin... & Idaho ... . & 41 \\
\hline Chena Creek. & Oregon.. & 18 & Cooney . & New Mex & 69 \\
\hline Cherokee... & California, & 13 & Cooper. & $\ldots$ do. & 51 \\
\hline Cherry Creek & Arizona... & 109 & Copper Basin & Arizona... & 111 \\
\hline Cherry Creek......... & Nevads.... & 168 & Copper Basin......... & Nevada.. & 98 \\
\hline Chesaw...... & Washington & 24 & Copper Creek & Arizo & \\
\hline Chetco ..... & Oregon .... & 25 & copper ureek & ATIZ & 83 \\
\hline Chewelah. . & Washingtor & 45 & Coppereid :- & Nevada .... & 10 \\
\hline Chico... & Montana .... & 77 & Copper Bill.. & New Mexicc & 82 \\
\hline Chief ... & Nevada..... & 105 & Copper King........ & Nevada. & 13 \\
\hline Chilkoot.... & South Dakot & 1 & Copper Mountain. ... & Arizons... & 31 \\
\hline Ching Flat .. & California, $\mathbf{N}$ & $\mathbf{5 7}$ & Copper Mountain. . . . . & Wyoming. & 21 \\
\hline Chinese Cam & $\ldots$... do ..... & 210 & Copperopolis......... & California, $\mathbf{N} \ldots \ldots \ldots$ & 22 \\
\hline Chips Flat.. & $\ldots$.... do... & 143 & Copperopolis.. & Montana... & 66 \\
\hline Chisos...... & Texas... & 2 & Copper Ridge. & Colorado & 104 \\
\hline Chloride. & Arizona ... & 46 & Copperton. & New Mexico. & 85 \\
\hline Chloride... & New Mexico. & 61 & Copper World ......... & California, S....... & 141 \\
\hline Chloride Cliff . & California, S. & 15 & Corbin............... & Montana . . . . . . . . . . . & 35 \\
\hline Chloride Flat.. & New Mexíco. & 16 & Cornucopia & Nerada... & 32 \\
\hline Christmas... & Arizona ...... & 19 & Cornucopia. & Oregon.. & 6 \\
\hline Chuckwalla. & Californis, S. & 108 & Cornwall Basin... & & 33 \\
\hline Church Meado & California, $\mathbf{N}$. & 140 & Corons........... & California, S..... & 109 \\
\hline Cienega............ & Arjzona...... & 125 & Corral....... & Colorado.............. & 22 \\
\hline Cims .......... & California, S.. & 130 & Corral Creek . . . . . . . . & $\ldots$. . do ..... & 46 \\
\hline Cimarroncito. & New Mexico.... & 2 & Cortez....... & Nevada.. & 71 \\
\hline Clancy . . . . . . & Montana . . . . . & 37 & Cortez.. & da & 96 \\
\hline Clark........ & Arizona ..... & 30 & Cotopaxi. & Colorado. & 37 \\
\hline Clark Fork. & Idaho........ & 36 & Cottäge Gro & & 157 \\
\hline
\end{tabular}


Mining districts in the western United States-Continued.

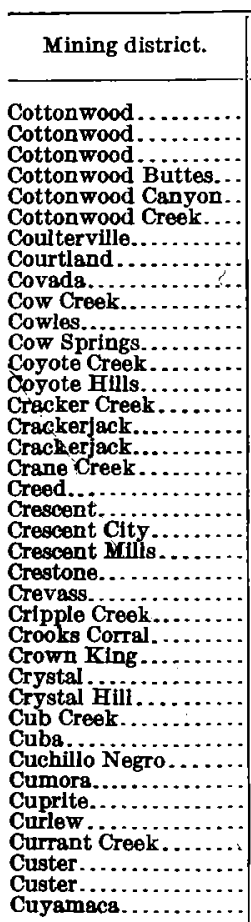

D.

Daggett. .

Dairy Farm

Damaseus.

Danville...

Darrington. ...........

Darwin.

Daulton.

Daylight.

Desdwood.

Dead wood

Deadwood.

Dean.

Decatur.

Dedrlck.

Deep Cregi

Deep Hole

Deep Sprtngs.

Deer Creek.

Deer Creek.

Deerlodge.

Deer Park.

Deer Trail

De Lamar

Delamar.

Del Rio .............

Democrat

Denemora

Denmark.

Denny .............

De Pass...............

Desert.

Detroit

Devils Gate

Dewey.

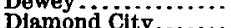

Dlamond Creek........

Dillon.

Disaster

Dixle.

\begin{tabular}{|c|c|c|c|c|}
\hline State. & No. & Mining district. & State. & No. \\
\hline Californ & 131 & Dobbins. & Californis & 219 \\
\hline Colorado & 11 & Dodge... & ....do.. & 195 \\
\hline Nevada. & 157 & Dogtown & Californ & 91 \\
\hline Idaho. . & 63 & Dolly Varden. & Nevada & 34 \\
\hline Nevada & 3 & Dolores. . & New Me & 59 \\
\hline Californ & 158 & Dome... & Idaho.: & 11 \\
\hline ....do. & 68 & Domingo & Colorado & 51 \\
\hline ........ & 2 & Dorleska. & Californi & 196 \\
\hline Washir & 16 & Dos Cabe & Arizona. & 3 \\
\hline$\ldots \ldots \ldots$ & 30 & Dos Palm & Callfornia & 110 \\
\hline$\ldots .$. & 75 & Dothan. . & Oregon... & 31 \\
\hline ......... & 13 & Douglas & $\ldots \ldots$ & 97 \\
\hline$\ldots$ & $\begin{array}{l}23 \\
64\end{array}$ & Douglas F & Wyoming. & $\begin{array}{r}2 \\
30\end{array}$ \\
\hline & 7 & Douglas $\mathrm{N}$ & Colorado & 105 \\
\hline Californi & 132 & Downievilie... & Californfa & 141 \\
\hline Oregon. & 30 & Dragoon....... & Arizona. & 4 \\
\hline ...do. & 39 & Draper. & Oregon. & 49 \\
\hline Colored & 78 & Drew.... & ...do.. & 32 \\
\hline Nevada. & 14 & Dripping & Arizona. & 20 \\
\hline Californ & 34 & Dry Gulch.... & Montana & 41 \\
\hline & 106 & Dry Lake..... & Calffornis & 133 \\
\hline Colorado & 114 & Drytown. & Californis, & 9 \\
\hline Montan & 76 & Dugway. & Utah... & 61 \\
\hline Colorad & 130 & Dulzura. & Californi & 171 \\
\hline Idaho.. & 64 & Dumont.. & Colorado & 24 \\
\hline$\ldots \ldots . .$. & 118 & $\ldots \ldots \ldots$ & .......... & 79 \\
\hline . & 22 & Dunton. & $\ldots \ldots$ & 31 \\
\hline Colorado & 116 & Duquesne & Arizona. & 102 \\
\hline Utah.... & 72 & Dutch Flat. & Callforni & 96 \\
\hline New Me & 49 & Dutro........ & Callfornia, $\mathrm{S} . \ldots . . .$. & 103 \\
\hline ...do.. & 61 & Dyer......... & Nevada............. & 50 \\
\hline Idaho . - & 42 & & & \\
\hline Nevada. & 49 & E. & & \\
\hline Montana. & 90 & Eagle.... & Idaho. & 116 \\
\hline Colorado. & 38 & Eagle Mountain & Californi & 114 \\
\hline Idaho .... & 52 & Eagle Mountains.. & Texas... & 5 \\
\hline South D & 170 & Eagle River...... & Colorado... & 35 \\
\hline Caniornl & & Eagle Valley .. & Nevada.... & 107 \\
\hline & & East Beaver................ & Colorad & 131 \\
\hline Califor & 128 & East Fork $\ldots \ldots \ldots$ & Idaho... & 46 \\
\hline Californ & 94 & Eastgate...... & Nevada. & 5 \\
\hline ...do. & 95 & East Mancos... & Colorado. & 79 \\
\hline Washin & 14 & East Shore.... & Idaho.... & 36 \\
\hline Montan & $\mathbf{3}$ & Eastman Gulch. & Californi & 198 \\
\hline Washing & 37 & Echo Canyon... & Callfornia & 19 \\
\hline Californi & 16 & Eden . . & $\ldots \ldots \ldots$ & 132 \\
\hline ...do do & 80 & Eden. . & Oregon. & 19 \\
\hline & 17 & Edgem & $\mathrm{Ne}$ & 30 \\
\hline Callforn & 159 & Edmanton. . & $\mathbf{N} \ldots \ldots \ldots$ & 108 \\
\hline ....do & 192 & Ehrenberg . . . & Arizona. . & 135 \\
\hline Idaho.: & 27 & Eldorado... & California & 29 \\
\hline South D & 8 & Eldorado. & .... do... & 39 \\
\hline$\ldots . .$. & 97 & Eldorado..... & ........ & 87 \\
\hline lorado & 101 & Eldorado Canyon. . & Nevada. .... & 15 \\
\hline California & 193 & Eldorado Pass. & Arizona. & 47 \\
\hline Washington & 46 & Elizabethtown .... & California, $\bar{\lambda}$ & 30 \\
\hline Neva & 158 & lizabethtown ....... & New Mexico.......... & 3 \\
\hline iffornto & 18 & Elk...... & Montang. ............. & 82 \\
\hline Montana. & 72 & Elk City. & Ida & 67 \\
\hline $\mathbf{w}$ & 16 & Elkhort & $\mathrm{Co}$ & 106 \\
\hline Mont & 89 & Elkhorn. & Idaho.. & 12 \\
\hline Washingto & 47 & Elkhorn. & Montana & 6 \\
\hline & 48 & Elkhorn. & .....do... & 36 \\
\hline Idabo. & 108 & Elkhorn... & Oregon. & 8 \\
\hline Nevada. & 108 & Ik Mountain. . & Colorado. & 69 \\
\hline Arizona & 112 & intain... & Nevada. . & 35 \\
\hline Color & 22 & Elk Mountain... & cots. ...... & \\
\hline Mont & 68 & Ellison........ & Arizona. & 21 \\
\hline Oregon & 44 & Elliston.. & Montana. & 84 \\
\hline & 24 & El Paso............. & Texas... & \\
\hline Callfornia & 194 & Elsworth......... & Nevada. & 126 \\
\hline Wyoming. & 22 & Ely....... & $\ldots$ do.. & 167 \\
\hline Utah.... & 60 & Embargo........... & Colorado. & 102 \\
\hline & 33 & ery ............ & Utah........ & 21 \\
\hline & 120 & Emigrant...... & California, S. & 20 \\
\hline Idaho.. & 65 & Emigrant Creek.... & Montana..... & 77 \\
\hline Montana. & 10 & Empire............ & Arizons.... & 71 \\
\hline California, $\mathbf{N}$. & 35 & Empire......... & Colorado. . & 26 \\
\hline Montana.... & 4 & Empire....... & $\ldots$ do... & \\
\hline rada. & $79 a$ & Encampment........ & Wyoming. & 12 \\
\hline & & & & \\
\hline
\end{tabular}


Mining districts in the western United States-Continued.

\begin{tabular}{|c|c|c|c|c|c|}
\hline Mining district. & State. & No. & Mining district. & State. & No. \\
\hline Erikson & Utah... & 62 & Gallina. & New Mexico & 46 \\
\hline Escondido. & California, $\mathrm{S}$ & 172 & Gallinas. & .....do...... & 32 \\
\hline Esmeralda. & California, $\mathbf{N}$ & 23 & Galls Creel & Oregon. & 50 \\
\hline Esterbrool & Wyoming... & 6 & Garden.... & South D & 15 \\
\hline Estey .... & New Mexico & $31 \mathrm{a}$ & Garden Valley.... . . . . & Californi & 42 \\
\hline $\operatorname{tna} .$. & California, $\mathrm{N}$ & 160 & Gardnerville......... & Nevada. & 24 \\
\hline Eureka. & Arizona : & 113 & Garlie Spri & California & 134 \\
\hline Eureka. & California, $\mathrm{N} \ldots \ldots \ldots$ & 142 & Garlock .. & ...do... & 51 \\
\hline ....... & Colorado.......... & 118 & Garnet.............. & Montang & $\begin{array}{l}28 \\
82\end{array}$ \\
\hline $\begin{array}{l}\text { Eureka. . . } \\
\text { Eureks. . }\end{array}$ & Nevada... & $\begin{array}{l}88 \\
72\end{array}$ & $\begin{array}{l}\text { Garnet.... } \\
\text { Gazelle.... }\end{array}$ & California. & $\begin{array}{r}82 \\
162\end{array}$ \\
\hline Eureka. & New Mexic & 21 & Geertson. & Idaho.... & 87 \\
\hline Eureka. & Washington. & 17 & Geiser... & Oregon. & \\
\hline Evans Cre & Oregon.... & 52 & Genesee Valley . . . . . & Californi & $10 \%$ \\
\hline Evergreen & Colorado & 66 & Georgetown......... & ...do.. & A3 \\
\hline Evolution & Idaho... & 117 & Georgetown $\ldots \ldots \ldots$ & Colorad & 20 \\
\hline & & & $\begin{array}{l}\text { Georgetown } \\
\text { German Gu }\end{array}$ & New M & $\begin{array}{l}22 \\
18 \\
98\end{array}$ \\
\hline Fairplay & California, & 40 & Geyser.... & Nevada & 110 \\
\hline Fairv & Nevada.. & 6 & Geyserville & Californ & 179 \\
\hline Fairweath & Montana. & 53 & Gibbonsvill & Idaho.. & 91 \\
\hline Fall Creek & Oregon. & 68 & Gibsonville & Caliform & 145 \\
\hline Farrell.. & Nevada & 80 & Gilman. . . & Colorado... & 33 \\
\hline Fay. & $\ldots$ do. & 107 & Gilmore... & Idaho... & 101 \\
\hline Ferg & .....do. & 108 & Gilta $\ldots \ldots \ldots \ldots \ldots$ & Californi & 163 \\
\hline $\begin{array}{l}\text { Fesler. } \\
\text { Fierro. }\end{array}$ & New Mexico & $\begin{array}{l}69 \\
17\end{array}$ & Giltedge $\ldots \ldots \ldots \ldots \ldots$ & Montar & 25 \\
\hline Fine Go & California, S & $\begin{array}{l}17 \\
81\end{array}$ & $\begin{array}{l}\text { Gleason Mountam } \ldots \ldots \\
\text { Globe.............. }\end{array}$ & $\begin{array}{l}\text { Californ } \\
\text { Arizona }\end{array}$ & $\begin{array}{l}76 \\
22\end{array}$ \\
\hline Finger $\mathrm{R}$ & ...do. & 188 & Globe. & Californi & 189 \\
\hline Finn... & Montan & 88 & Glorieta.. & New $\mathbf{M e}$ & 57 \\
\hline First & $\ldots$ do. & 28 & Golconda. & Nevade & 81 \\
\hline & Wyon & 30 & Gold Banks & $190 \mathrm{~s}$ & 82 \\
\hline Fish Lake & Washingto & 20 & Gold Bar ... & ...do. & $13 \overline{3}$ \\
\hline Fish Springs & California, S & 41 & Gold Basin . & Arizona & 48 \\
\hline Fish Springs. & Utah........ & 34 & Gold Basin. & Nevada. & 36 \\
\hline Fitting ....... & Nevada.... & 80 & Goldbelt... & California, & 22 \\
\hline Flagstaff & California, $\mathrm{N}$. & 41 & Gold Brick. & Colorado... & 54 \\
\hline Fleecer Mount & Montana . . . . & $97 a$ & Gold Bug. . . . & Arizona. & 49 \\
\hline Flint ....... & Idaho.... & 109 & Gold Butte... & Nevada. & 16 \\
\hline Flint & Montane & 29 & old Canyo & .....do.... & 168 \\
\hline Flore & Idaho. & 68 & 1 Circle & .....do... & 37 \\
\hline orida orida $_{\text {ountain }}$ & $\begin{array}{l}\text { Colorado } \\
\text { Idaho... }\end{array}$ & $\begin{array}{r}72 \\
107\end{array}$ & $\begin{array}{l}\text { Gold Crater. } \\
\text { Gold Creek. }\end{array}$ & jodo. & $\begin{array}{r}134 \\
81\end{array}$ \\
\hline $\begin{array}{l}\text { Florida Mountain. ... . } \\
\text { Florida Mountains... . }\end{array}$ & New Mexico & $\begin{array}{r}107 \\
37\end{array}$ & Gold Creek... & $\begin{array}{l}\text { Ioano... } \\
\text { Montana }\end{array}$ & $\begin{array}{l}81 \\
83\end{array}$ \\
\hline Folsom. . . . . . . . & California, $\mathbf{N}$ & 117 & Gold Creek ... & Oregon.. & 24 \\
\hline Fool Hen. & Montana.... & 46 & Golden ....... & Colorado & 67 \\
\hline Foots Creek. & Oregon... & 50 & Golden.... & Nevada. & 135 \\
\hline Forbestown. & Califormia, $\mathbf{N}$ & 14 & Golden.... & Oregon.. & 63 \\
\hline Forest... & ....do....... & 143 & oldfield.. & Nevada. & 51 \\
\hline Fore & $\ldots$ do. & 97 & Gold Fork. & Idaho. & 28 \\
\hline For & ...do. & 161 & Gold Hill. & Colorado & 2 \\
\hline$\overline{\mathbf{F}}$ & Idaho. & 89 & Gold Hill. & New Mexi & 19 \\
\hline ort Hall & ...do... & 4 & Gold Hill. & Oregon.... & 50 \\
\hline (T) & California & 173 & Gold Hill... & Utah. . . & 58 \\
\hline Francis (?)... & Arizona. & 14 & Gold Mountair & ....do. & 43 \\
\hline Franklin Mountains. . & Texas. - & 6 & Gold Mountain & Arizona. & 84 \\
\hline Frazier Mountain... & California, S. & 193 & Gold Mountair & Nevada. & 52 \\
\hline Fre & New Mexico. & 38 & Gold Mountain & ....do... & 68 \\
\hline French Cor & California, N.. & 80 & Gold Reed.... & Nevada... & 140 \\
\hline French Creek. & South Dakota. & 3 & Gold Road ... & Arizona... & 50 \\
\hline French Creek & Wyoming & 13 & Gold Run ... & California, $\mathbf{N} \ldots \ldots \ldots$ & 96 \\
\hline French Gulch & California, $\mathrm{N}$ & 125 & d Run... & 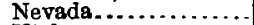 & 76 \\
\hline Fre & Nevada..... & 109 & Springs. & Utah..... & 29 \\
\hline Fri & Colorado. & 125 & dyke. .... & Nevada. . & 136 \\
\hline Frisco .... & Utah...... & 11 & Goler...... & California, S....... & 52 \\
\hline Frying Pan & Colorado.............. & 97 & Good Hope. & Nevada................ & \\
\hline Fulford...... & & 34 & Goodrich Gule & 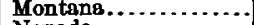 & 62 \\
\hline Furnace C & California, S...... & 21 & Goodsprings. . & Novada. . & 23 \\
\hline Furnier... & California, N......... & 144 & Goodzears Bar & Califormia, $\mathbf{N} . . . .$. & 141 \\
\hline & & & Gore Range. . . & Colorado........... & 110 \\
\hline G. & & & Gottville.... & California, N ....... & 164 \\
\hline Gage. & New Mexico. & 40 & $\begin{array}{l}\text { Grand Canyon . . . . . } \\
\text { Grand Island........ }\end{array}$ & $\begin{array}{l}\text { Arizona. } \\
\text { Colorado }\end{array}$ & \\
\hline Galena. & Colorado... & 61 & Grand Lake. . & ....do... & 47 \\
\hline Galena. & Idaho... & 13 & Granite...... & ....... & 12 \\
\hline Galena. & Nevada & 98 & inite... & Nerada. & \\
\hline lena. & ....do... & 162 & Granite...... & ....do.................. & 174 \\
\hline Galena. & Oregon.. & 42 & Granite....... & Oregon........... & 40 \\
\hline Galena. & South Dakota. . . . & 10 & Granite........ & 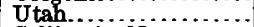 & \\
\hline Galice. . & Oregon........... & 55 & Granite Basin. & California, $\mathbf{N} \ldots \ldots \ldots$ & 110 \\
\hline Galiuro............... & 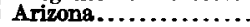 & 84 & Granite Falls......... & Washington............. & \\
\hline
\end{tabular}


Mining districts in the western United States-Continued.

\begin{tabular}{|c|c|c|c|c|c|}
\hline Mining dist & & No. & Mining district. & & No. \\
\hline Aranite & New Me & 20 & Hazel. & Texas. & 10 \\
\hline $\cos$ & Oregon. & 59 & Headston & New $\mathrm{Me}$ & 47 \\
\hline Granite & Utah... & 63 & Heath... & Idaho.. & 129 \\
\hline ranitevi & $\ldots \ldots$ & 81 & Heber. & Montan & 23 \\
\hline & $\ldots \ldots$ & 39 & Fecla. & ....do.. & 5 \\
\hline Gr & Californi & 135 & Hecla... & Wyomi & 32 \\
\hline $\begin{array}{l}\text { Grapevine } \\
\text { Grass Vall }\end{array}$ & Californi & $\begin{array}{l}23 \\
84\end{array}$ & Hedges. & Californ & $\begin{array}{r}7 \\
137\end{array}$ \\
\hline Hens & Idaho... & 89 & Helena... & California, & 201 \\
\hline & Colorado & 28 & Helena. & Montana.. & 43 \\
\hline & ....do.. & 76 & Hellgate. & ....do... & 13 \\
\hline & Californi & 180 & Helmville & ....do. & 81 \\
\hline & Nevada. & 17 & Helvetia. . & Arizona & 74 \\
\hline & $\ldots \ldots$ & 72 & $\ldots . .$. & New Mex & 7 \\
\hline$\cdots$ & Californis & 64 & Henley ... & California, I & 158 \\
\hline$\cdots$ & $\ldots \ldots$ & $\mathbf{5 6}$ & Henry $\ldots$ & Utah...... & 53 \\
\hline - & $\ldots \ldots \ldots$ & 39 & Herault . . & California, $\mathrm{N}$ & 121 \\
\hline ho & , & $\begin{array}{r}42 \\
9\end{array}$ & $\cdots \cdots$ & Nevada .... & 12 \\
\hline ins & $\ldots \ldots$ & 53 & Hig & Mow & $\begin{array}{l}62 \\
99\end{array}$ \\
\hline Is & $\ldots \ldots$ & 138 & Highland & Idaho. & 1 \\
\hline lee & rizons & 32 & Highland & ....do. & 29 \\
\hline reen Mou & liforn & 69 & Highland & ....do. & 55 \\
\hline reen Moun & $\ldots \ldots$ & 54 & Eighland & Novada. & 111 \\
\hline een Mou & $\ldots \ldots . .$. & 36 & Highrolls. . & New Mexic & 41 \\
\hline $\operatorname{een} \mathbf{R i}$ & $\ldots . .$. & 72 & Hiko.... & Nevada. & 112 \\
\hline $\operatorname{sen} V$ & $\ldots \ldots$ & 23 & Bildreth.. & California & 83 \\
\hline$t$ & $\ldots \ldots$ & 106 & Bill City.. & South Dakota. . ....... & 20 \\
\hline & $\ldots \ldots \ldots$ & 21 & Hillsboro.. & New Mexico & 63 \\
\hline & rizons & 60 & Hill Top... & Nevada. & 99 \\
\hline ceenwoo & Californ & 42 & Hite. ..... & Utah.... & 24 \\
\hline & Colorad & 42 & Hite Cove. & Califormia, $\mathbf{N}$. & 70 \\
\hline$\therefore \ldots$ & $\ldots$ do... & 20 & Hoag . . . . . . & $\ldots$.......... & 75 \\
\hline izaly Fl & California & 44 & Hoback. . & Wyoming . . . . . . . . & 36 \\
\hline Grizaly & ..do... & 86 & Holcomb. & California, S......... & 138 \\
\hline$\ldots$ & Monta & 50 & Holmes. . - & Wyoming.... & 2 \\
\hline ub Gu & Californi & 82 & Holy Cross.. & Colorado ... & 35 \\
\hline antain. & Texas. . & 7 & Homer...... & California, S.......... & 92 \\
\hline Guert & Oaliforni & 180 & $\ldots .$. & South Dakota......... & 15 \\
\hline Gunsight. - & $\begin{array}{l}\text { Arizona } \\
\text { Californi }\end{array}$ & $\begin{array}{l}73 \\
24\end{array}$ & Homestead . . & Oregon $\ldots \ldots \ldots \ldots$ & 10 \\
\hline Gwin... & Calluorma & 24 & ( & $\begin{array}{l}\text { Idaho } \ldots \text {, } \\
\text { Californis, } \mathbf{N} \ldots\end{array}$ & $\begin{array}{l}82 \\
58\end{array}$ \\
\hline & & & 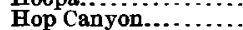 & New Mexico . . . . . . . & 00 \\
\hline nita. & New Me & 21 & Hopewell. . . . . . . . . . & & 47 \\
\hline Hac & rizons. & 51 & Hornblende & South Dakota..... & 21 \\
\hline $\mathbf{B}$ & orac & 107 & Hornbrook. & California, N..... . & 158 \\
\hline & ho. & 18 & Hornitos.. & . do & 71 \\
\hline H & & 90 & Hornsilver & Nevada................ & 54 \\
\hline & Californis & 165 & Hors & Wyoming.............. & \\
\hline & Idaho.. & 14 & Horse Cree & (1. 010 & 35 \\
\hline $\mathbf{H}$ & Nevada. & 176 & Horsesho & Colorado... & 92 \\
\hline & $\ldots$ do... & 137 & Horsesho & Montana... & 78 \\
\hline Ha & New Mex & 27 & Horseshoe Basin... & Washington. & 3 \\
\hline & & 72 & Hotaling..... & California, N......... & 98 \\
\hline & Califorr & 166 & Hot Cl & Nevada............... & 153 \\
\hline $\mathbf{H} \mathbf{z}$ & Nevada. & 133 & $\mathbf{H o}$ & $\ldots \ldots$ do $\ldots \ldots \ldots \ldots$ & 4 \\
\hline $\mathbf{H}$ & Oregon. & 24 & Ings. & Utah..... & 47 \\
\hline & Arizons. & 126 & Ho & Oregon . & 22 \\
\hline crabble & Colorado & 29 & Howe. & Idaho..... & 59 \\
\hline Ion...... & rado & 48 & Howes C & Colorado... & 73 \\
\hline & & 43 & Huachuca. & Arizona.... & 5 \\
\hline & South Da & 18 & & & \\
\hline ter. & Idaho... & 65 & & & \\
\hline lahal & Arizona. & 127 & Hualpai & do. & 52 \\
\hline & & 118 & & & 56 \\
\hline & olorido & 69 & & & \\
\hline & California & 24 & Huerfano. & Colorado. & 64 \\
\hline & Utah..... & 86 & 百v & Montana . & 91 \\
\hline in & Califormia & 126 & Humboldt. . & Nevada. . . . . . . . . . & 83 \\
\hline W & Arizona. - & 93 & mboldt. . & Oregon... & 70 \\
\hline & California, & 136 & 豆 & Arizona. & 12 \\
\hline $\log 2$ a 1 & & $\mathbf{5}$ & & Idaho... & 118 \\
\hline & rado. & 91 & He & Nevada... & 169 \\
\hline rille & & 29 & ricane. & Wyoming. & 10 \\
\hline & Arizona.: & 114 & Hydraulie........... & Colorado............. & 89 \\
\hline t Moun & South Dakota. & 19 & & & \\
\hline vilah.. & California, S... & 55 & 1. & & \\
\hline wkins Creek. & California, N . . & 199 & & & \\
\hline l-imanillo & $\because \pi_{\text {dodo.... }}$ & 167 & Tbex....... & California, S........... & 139 \\
\hline vthorne... & Nevada.: & 57 & & & 26 \\
\hline To & . & $\begin{array}{r}66 \\
200\end{array}$ & lababo & & \\
\hline
\end{tabular}


Mining districts in the western United States-Continued.

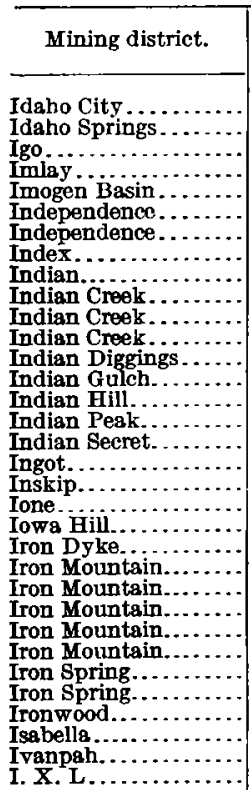

J.

Jackrabbit.

Jackrabbit..............

Jackson

Jackson

Jackson Creek

Jacksonville

Jacksonville

Jacobs Gulch............

Jacobs Lake.

Jamestown

Jamison.

Jarbidge

Jardine.

Jarilla.

Jarloose

Jefferson Canyon.

Jelm Mountains.

Jenny Lind...

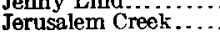

Jessup.

Jicarilla

Johnnie

Johnson.

Johnson.

Johnsville.

Jordan .

Joy........

Julian.

Junction.

Junction ...............

Junction City.........

K.

Kaibab Plateau.......

Kane Springs.........

Kaufman.

Kawich.

Kearsarge.

Keeler.

Keith

Kelley.

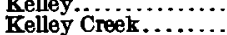

Kelley Creek......................

Kelsey.

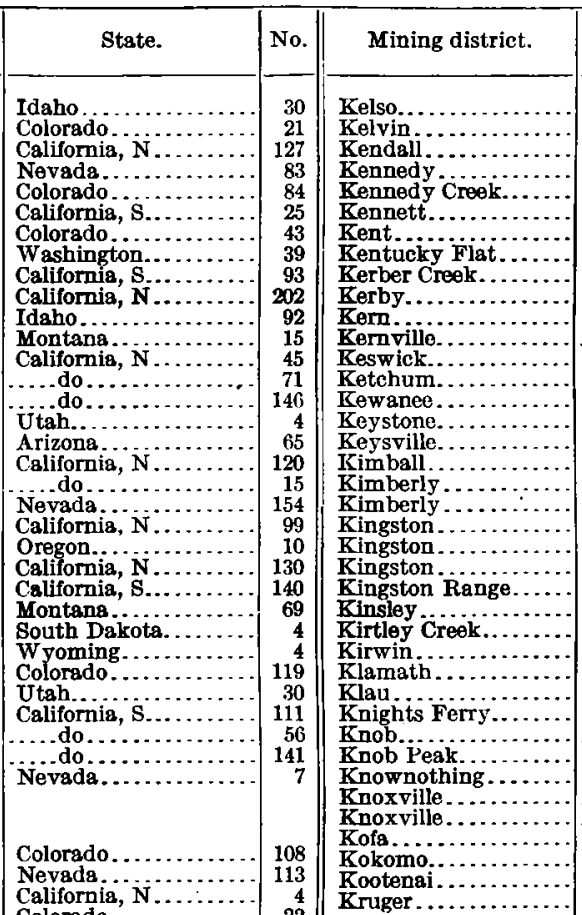

Colorado...............

Nevada...

California, N ...

Oregon................. 51

Idaho................. 31

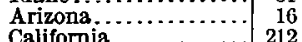

California, N.......... 111

Nevada.............. 39

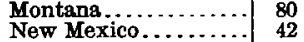

Utah . . . . . . . . . . . . .

Nevada...

W yoming.

California, N......................... 125

Nevada...............

New Mexico.

Nevada..

Arizona.

Oregon..................

California, N............ 111

California, S.............

New Mexico

California, $\$$...........

Utah...

California, S............. 174

Nevada................. 162

Oregon...............

South Dakota. ......

California, $\mathbf{N}$...........

Arizona

Californig $\mathrm{s}^{2} . . . . .$.

Idaho.

Nevada.

California, s

.... do . . . . . . . . . . . . .

wyoming.

Now Mexico

New Mexico.
California, N
L.

La Belle..

La Fortuna.............

Laguna.

La Joya................

Lake.

.

Lakeside...

Lakeside...........

Lake Valle

Lakeview

Lamoine .............

Lander..................

Lander.

Landusky ...............

La Panza.

Ia Plata.

La Plata.

La Plata .........

La Plata.

La Port

Laramie Peak...

Larkin. .

La Sal................

Las Animas.

Lascar.......

I,atrobe.

Lava Beds.

Lava Creek

La Veta.

Lay.

Lead

Lead City

Lead ville.

Leamington

Lee

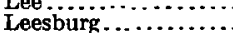

Lees Camp..............

Lehi.

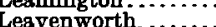

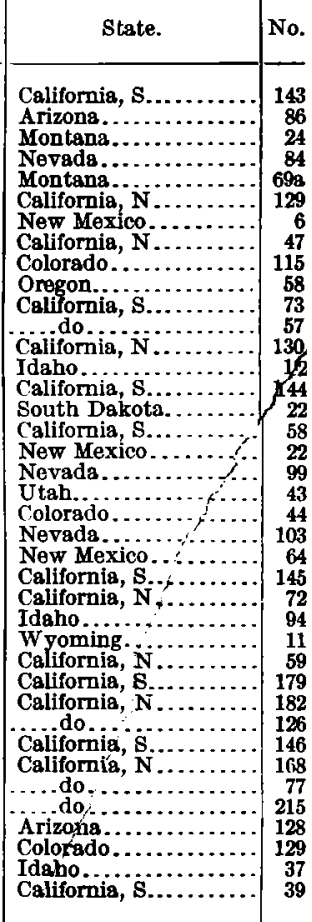

New Mexico......... 80

A rizong

California, N.......... 12

Colorado................ 62

California, S........... 97

Jtah............... 64

Washington........... 4

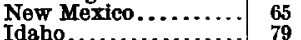

Idaho ............... 79

....do............... 5

Nevada.................. 94

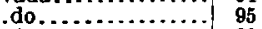

Montana.............. 20

California, s............ 180

Colorado............. 13

Ū..do.................

W yoming..............

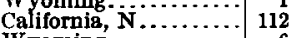

Wyoming . $\ldots \ldots \ldots .6$

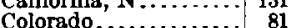

Now Mexico............. 6

New Mexico...........
Texas

California, N............ 100

California, S............ 148

California, S............ 147

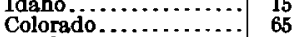

....do.................. 24

South Dakota......... 15

.... do ............... 15

Colorado................

Nevada............... 1589

Utah.................. 40

Califomin 5

Idaho

Calfornia, s.............

Utah................... 
Mining districts in the western United States-Continued.

\begin{tabular}{|c|c|c|c|c|c|}
\hline listrict. & Sta & No. & Mining district. & State. & No. \\
\hline & Idaho. . & 119 & Man & Califor & \\
\hline Lev & Calformis & 3 & Manz & New & 86 \\
\hline Lev & $\begin{array}{l}\text { Nevada } \\
\text { Califormia }\end{array}$ & $\begin{array}{r}97 \\
198\end{array}$ & Marble & Nevade & 141 \\
\hline & Wy yoming. . . . . . $\ldots$ & $\begin{array}{r}198 \\
23\end{array}$ & $\begin{array}{l}\text { Marie } \\
\text { Marip }\end{array}$ & Califor & $\begin{array}{l}65 \\
73\end{array}$ \\
\hline & Montana. & 51 & Mar & Texas. & \\
\hline & Vash & 21 & Mars & Idaho. & 70 \\
\hline & & $\mathbf{5 5}$ & Martin. & ....do & 15 \\
\hline & $\cdots$ & 113 & Marys & Utah. & 45 \\
\hline $\mathbf{I}$ & $\ldots$ & 54 & Mary & Califor & 220 \\
\hline I & Color & 23 & Marys & Montan & 40 \\
\hline Ut & “ ügdo.. & 98 & Mary & & 44 \\
\hline & $\begin{array}{l}\text { Utah... } \\
\text { Californ }\end{array}$ & 5 & Mas & Nevad & 122 \\
\hline & Ainjo & $\begin{array}{r}129 \\
48\end{array}$ & $\mathrm{Ma}$ & Callifo & 98 \\
\hline & C & 120 & May & .....do. & $\begin{array}{l}04 \\
79\end{array}$ \\
\hline I & $\cdots$ & 25 & May & ....do. & 181 \\
\hline $\mathrm{LI}$ & $\cdots$ & 59 & $\mathrm{May}$ & A rizon & 54 \\
\hline$\cdots$ & $\ldots \ldots$ & 20 & $\mathbf{M a z}$ & ......... & 90 \\
\hline ttle WOo & $\ldots \ldots$ & $\begin{array}{l}16 \\
17\end{array}$ & $\begin{array}{l}\text { MeCartne } \\
\text { McClellan }\end{array}$ & $\ldots \ldots \ldots$ & $\begin{array}{r}54 a \\
38\end{array}$ \\
\hline & Californ & 91 & McConnic & Arizon & $\begin{array}{l}38 \\
55\end{array}$ \\
\hline & \#do... & 2 & MeCra & do. & 43 \\
\hline Iodi & Nevada. & 141 & MeD & Idaho & 90 \\
\hline Logan. & ...do. & 19 & MeIntyre. & & \\
\hline $\begin{array}{l}\text { Logto } \\
\text { Lone }\end{array}$ & $\begin{array}{l}\text { Califorr } \\
\text { Colorad }\end{array}$ & 49 & McLe & $\ldots \ldots \ldots$ & 120 \\
\hline Lone & $\begin{array}{l}\text { Colorad } \\
\text { Montan }\end{array}$ & $\begin{array}{l}31 \\
14\end{array}$ & $\begin{array}{l}\text { Meadow } \\
\text { Meadow }\end{array}$ & $\ldots \ldots \ldots$. & 64 \\
\hline & New Mex & $\begin{array}{l}14 \\
23\end{array}$ & Mes & (n........ & $\begin{array}{r}83 \\
130\end{array}$ \\
\hline 0 & Nevada. & 114 & Me & Califor & 108 \\
\hline 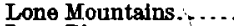 & & 56 & $\mathrm{Me}$ & & 73 \\
\hline $\mathbf{I}$ & Califor & 30 & $\mathbf{M e}$ & Idabo. & 80 \\
\hline Lon & Arizo & 33 & MeI & California, & 113 \\
\hline Lon & Wash & 15 & Melc & California, & 26 \\
\hline Lon & Californ & 149 & . & Utah........ & 57 \\
\hline Lon & ..... do. & 59 & $\mathrm{Me}$ & . . . . . & 175 \\
\hline & ....do do & 31 & $\operatorname{line}$. & $\ldots$ & 49 \\
\hline$\ldots$ & Washin & 27 & If... & . . . . . . . . & 32 \\
\hline$\ldots .$. & Idaho & $\begin{array}{l}47 \\
25\end{array}$ & Meteor... & $\ldots \ldots \ldots$ & 16 \\
\hline$\cdots$ & Nêw & $\begin{array}{l}25 \\
30\end{array}$ & Meth & $\ldots \ldots \ldots$ & 28 \\
\hline ros & Califor & 105 & $\mathrm{Me}$ & 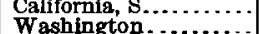 & \\
\hline & Califor & 118 & 1 & $\ldots \ldots \ldots$ & $\begin{array}{l}50 \\
25\end{array}$ \\
\hline $\mathrm{F}$ & Califar & 186 & ite... & & $\begin{array}{l}25 \\
38\end{array}$ \\
\hline st 1 & Arizon & 53 & $\operatorname{gan} \mathbf{B}$ & Callfornl & 117 \\
\hline & & 24 & $\operatorname{gan} \mathrm{B}$ & do & 101 \\
\hline 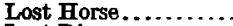 & Ifforn & 112 & 1 & Nerada & 37 \\
\hline & Id & 44 & . & Californ & 6 \\
\hline & Utah. & 21 & Creek. & Oregon. & 26 \\
\hline 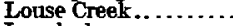 & $\ldots \ldots \ldots \ldots$ & 59 & $\cdots$ & $\ldots \ldots \ldots$ & 70 \\
\hline & & 85 & $\mathbf{M i}$ & & 96 \\
\hline $\mathbf{L}$ & Callfornis & 36 & $\mathrm{Mi}$ & Now Me & 74 \\
\hline & ....do.. & 82 & 1 Creek. & Californi & 3 \\
\hline Creek. & Oregon. & 60 & Mill Creek & $\ldots \ldots \ldots$ & 59 \\
\hline prings... & Montana & 54 & Mill Creek & $\ldots \ldots \ldots \ldots$ & 41 \\
\hline & & 26 & Miller Creek & $\ldots . .$. & 19 \\
\hline & & 120 & Mil & vada. & 143 \\
\hline cin. & Utah. . & 15 & Mimbres. & New Mex & 18 \\
\hline ck & Nevada & 57 & Mina . . . . & vada. & 65 \\
\hline $\mathbf{L}$ & Montana & 37 & rets. & $\ldots \ldots$ & 84 \\
\hline $\mathbf{L}$ & Callfornia & 92 & & $\ldots \ldots$ & 131 \\
\hline uning & Nevada. & 63 & Mir & $\ldots \ldots \ldots$ & 35 \\
\hline & . . . do . . & $72 a$ & al Cre & & \\
\hline & ...do... & 20 & Mineral Hill & \#do... & 87 \\
\hline & & & & ldaho. & 18 \\
\hline & & & eral Hi & do. & 97 \\
\hline & & & - & & \\
\hline & Idgho.... & 96 & ral Hill & . . . & \\
\hline 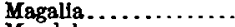 & Callfornia, N .... & 16 & al Hil & Ne & 53 \\
\hline & New Mexico.... . & 73 & Mineral $\overline{\mathrm{K}}$ in & Califo & 190 \\
\hline Mggri & Idaho.. & 69 & Mineral Par & AI & 56 \\
\hline & & 75 & eral Poir & $\ldots \ldots$ & 92 \\
\hline & Colorado & 4 & $\mathrm{ral} \mathrm{R}$ & ada. & 64 \\
\hline & .....do... & 64 & $\mathrm{Ba}$ & & 26 \\
\hline te.. & .do.. & 66 & & lifornia & 204 \\
\hline thet & gon. & 71 & $\overline{\mathbf{M}} \mathbf{i}$ & & 11 \\
\hline & $\mathrm{Ar}$ & 85 & $\mathbf{M}$ & Callfornia, $\mathbf{N}$. & 143 \\
\hline ammoth. & California, S... & 97 & & Colorado ............. & 124 \\
\hline & Idaho......... & 110 & & $\ldots \ldots \ldots$ & 57 \\
\hline noth. & Montana & $56 a$ & & & 31 \\
\hline $\tan$. & Colorado. & 74 & & New Mexico.......... & \\
\hline attan. & Nevada.... & $\begin{array}{r}142 \\
41\end{array}$ & - & & 69 \\
\hline
\end{tabular}


Mining districts in the western United States-Continued.

\begin{tabular}{|c|c|c|c|c|c|}
\hline Mining district. & State. & No. & Mining district. & Btate. & No. \\
\hline Mokelumne Hill.: & Californis, $\mathbf{N}$ & 27 & Newmire. . & Colorado & 120 \\
\hline 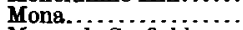 & Utah......... & 35 & New Placers & New Mexi & 58 \\
\hline Monarch-Garfield. . & Colorsdo & 14 & Newsome......... & Idaho.... & 71 \\
\hline Money Creek...... & Washington & 19 & Newton... & Utah... & 6 \\
\hline Monitor . . . . . . . & $\ldots . . .$. & 113 & Newtown. & Californi & 51 \\
\hline Monroe Creek. & ....do. & 132 & Newville & ...do. do. & 183 \\
\hline Montana. . . . . & Colorad & 24 & New World & Montan & 79 \\
\hline Montana. & Montan & 17 & New York & Californi & 151 \\
\hline Monte Cristo... & Nevada & 144 & Nigger Hill. & South Dak & 11 \\
\hline Monte Cristo... & Washin & 40 & Nightha & Washing & $25 \mathbf{a}$ \\
\hline Monte Negro... . & Colifornia & 114 & Nimshew.. & Californis & 17 \\
\hline Monte Negro . . . . & $\ldots \ldots \ldots$ & 167 & Ninemile Creek. . . . . . . & Montana & 69 \\
\hline Montezuma . . . . . & $\cdots \ldots \ldots$ & 75 & Nogal. & New Me & $\$ 34$ \\
\hline Montezuma & Colorad & 126 & Nogales & Arizons & 95 \\
\hline Montezuma . & Nevada & 58 & Nolton. & California, & 166 \\
\hline & $\begin{array}{l}\text { Utah..... } \\
\text { California }\end{array}$ & 50 & Norris ....... & Montana . & 54 \\
\hline $\begin{array}{l}\text { Monument Peak.. } \\
\text { Moore Creek....... }\end{array}$ & $\begin{array}{l}\text { California } \\
\text { Idaho.... }\end{array}$ & $\begin{array}{r}148 \\
25\end{array}$ & $\begin{array}{l}\text { North Bloomfield. .... } \\
\text { North Columbia..... }\end{array}$ & $\begin{array}{l}\text { California } \\
\text { do... do... }\end{array}$ & $\begin{array}{l}85 \\
86\end{array}$ \\
\hline Moose City ........ & .... do.... & 104 & North Fork. . . . . . & Californi & 85 \\
\hline Mooyis Y aak & $\ldots$ do. & 38 & North Fork. & Utah... & 82 \\
\hline Morenci .... . & Arizona. & 31 & North Moccasin . . . . . & Montan & 24 \\
\hline Moreno. & New Mex & 3 & Northport........... & Washing & 51 \\
\hline Morgan. . . & Utah.... & 42 & North San Ju & Californis & 87 \\
\hline Mormon Basin. . & Oregon. & 12 & North Star - - & Utah....... & 7 \\
\hline Mormon Basin . . & ...do... & 71 & North Tintic & ..do..... & 65 \\
\hline Mormon Creek . . & Montan: & 74 & Norwegian. . & Montans . . . & 56 \\
\hline $\begin{array}{l}\text { Morongo.. } \\
\text { Moscow... }\end{array}$ & Idaho.... & $\begin{array}{r}149 \\
83\end{array}$ & Nugget..... & Uregon..... & 33 \\
\hline Moses.... & Washingtou & 25 & 0 . & & \\
\hline Mosquito. & Colorado.... & $\mathbf{9 3}$ & & & \\
\hline Mountain City.... & Nevada. & 44 & Oak Bar. & California & 169 \\
\hline Mount Baker. . . & Washing & 55 & Oak City ... & Utah.... & 40 \\
\hline Mount Baldy. . & Utah... & 45 & Oak Creek. & Colorado & 109 \\
\hline Mount Bullion... & Californis & 73 & Oak Springs & Nevada. & 145 \\
\hline Mount Diablo... & r...do... & 33 & Oat Hill... & California & 78 \\
\hline Mount Nebo..... & Utah.... & 39 & O'Briens... & Nevada.... & 155 \\
\hline Mount Pisgah. & Idaho. & 7 & Oceanic .... & Calliformia, & 181 \\
\hline Mount Pleasant & California & 150 & Ocean View & ...do.... & 182 \\
\hline Mount Raymond & California & 84 & Ochoco.... & Oregon..... & 22 \\
\hline Mount Reuben . - & Oregon... & 61 & Ogilby $\ldots .$. & Californis, \& & 7 \\
\hline Mount Tenabo. & $\begin{array}{l}\text { Nevada.. } \\
\text { Montana. }\end{array}$ & 71 & 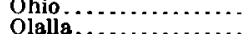 & Utah........ & 45 \\
\hline Mount Torrey .... & $\begin{array}{l}\text { Montana. } \\
\text { Colorado. }\end{array}$ & $\begin{array}{r}6 \mathbf{a} \\
121\end{array}$ & $\begin{array}{l}\text { Olalla..... } \\
\text { Old Baidy. }\end{array}$ & Oregon.. & 34 \\
\hline $\begin{array}{l}\text { Mount Wilson... } \\
\text { Mowry . . . . . }\end{array}$ & $\begin{array}{l}\text { Colorado. } \\
\text { Arizone. . }\end{array}$ & $\begin{array}{r}121 \\
94\end{array}$ & $\begin{array}{l}\text { Old Baldy } \ldots . . . . . . . \\
\text { old Dominion. } \ldots\end{array}$ & $\begin{array}{l}\text { Arizona ..... } \\
\text { Washington }\end{array}$ & $\begin{array}{l}70 \\
52\end{array}$ \\
\hline $\begin{array}{l}\text { Mowry -....... } \\
\text { Mud Springs. }\end{array}$ & Arizona. & $\begin{array}{l}94 \\
97\end{array}$ & Old Hat. . . . . . . . . . & $\begin{array}{l}\text { Washington. } \\
\text { Arizona..... }\end{array}$ & 88 \\
\hline Muldoon..... & Idaho... & 17 & old Placers............ & New Mexic & 59 \\
\hline Mullan.. & .....do.... & 118 & Oleta......... & California, $\mathbf{N}$ & 7 \\
\hline Murphy & Californis & 23 & Olinghouse. & Nevada..... & 164 \\
\hline Muriay. . . & Idaho... & 116 & Olive...... & Arizona . - & 77 \\
\hline Music Mountains & Arizona. & 58 & O'Mears... & California, $\mathrm{N}$ & 170 \\
\hline Musselshell ...... & Montana & 66 & Omega.... & $\ldots \ldots d 0 . . . \ldots$. & 90 \\
\hline Musselshell Creeh & Idaho... & 105 & Oneota... & Nevada. & 47 \\
\hline Myers Creek..... & Washingtc & 24 & Ono.... & California, I & 128 \\
\hline Myrtle Creek. & Oregon.. & 33 & Ontario. & Montang.. & 84 \\
\hline Myrtle Point & ....do... & 20 & Onward & California, 8 & 115 \\
\hline Mystic.... & South Dakota........ & $\mathbf{2 3}$ & Ophir... & California, I & 102 \\
\hline $\mathbf{N}$ & & & $\begin{array}{l}\text { Ophir... } \\
\text { Ophir.. }\end{array}$ & Colorado.. & $\begin{array}{r}119 \\
85\end{array}$ \\
\hline & & & Ophir.. & Utah..... & 66 \\
\hline Nacimiento Mountains & New Mex & 49 & Oracle. & Arizona. & 88 \\
\hline Napa Consolidated . & Californis & 79 & Ord.... & Californis, & 152 \\
\hline ville..... & ...do... & 50 & Organ ... & New Mexic & 9 \\
\hline National. & Nevada. & 86 & Orick.... & Californis, 1 & 60 \\
\hline Naturita.. & Colorado & 82 & Orient. ...... & Washington. & 53 \\
\hline & Idaho... & 56 & Oriental Was & Nevada.... & 59 \\
\hline Neder & Colorado & 5 & Orleans..... & California, & 61 \\
\hline dle Mountains & & 72 & Oro Blanco. & Arizona.. & 96 \\
\hline dles.......... & Californis & 150 & Oro Fino... & California, 1 & 171 \\
\hline y.. & Idaho.... & 21 & Oro Fino.... & Colorado... & 71 \\
\hline & Montana & 17 & Oro Grande. & California, S & 153 \\
\hline n... & Nevada. & 15 & Orogrande. & Idabo....... & 72 \\
\hline lem.: & Washingt & 25 & Orogrande. & New Mexico & 42 \\
\hline $\mathrm{da} \mathrm{Cit}$ & California & 84 & Oroville... & Californis, $\mathbf{N}$ & 18 \\
\hline evada.... & Colorado. & 42 & Oroville. & Washington. & 26 \\
\hline ew Alma & California & 119 & Ortiz... & New Mexico. & 59 \\
\hline rrk... & Nevada.. & 170 & Usceola. . & Nevada...... & 171 \\
\hline berry & California, S.. & 147 & Oscaro . . . . & New Mexico. & $31 \mathrm{a}$ \\
\hline Coso. & . do... . . . . & 32 & Osoyoos Lake & Washington. & 26 \\
\hline ewfoundland & Utah.... & 16 & Ottawa....... & Montana.... & 44 \\
\hline lewhall...... & Californis, $\mathrm{S}$.. & 72 & Owl Creek. . & Wyoming. & 24 \\
\hline 10utse- & Utah.............. & 9 & Owl Holes... & California, S. & 154 \\
\hline 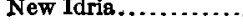 & California, $8 . . . \ldots \ldots \ldots$ & 119 & Ozette River......... & Washington............ & \\
\hline
\end{tabular}


Mining districts in the western United States-Continued.

\begin{tabular}{|c|c|c|c|c|c|}
\hline Mining district. & State. & No. & Mining district. & State. & No. \\
\hline & & & Placerville. & California & 54 \\
\hline Paciflc... & Califormia, I & 52 & $\begin{array}{l}\text { Placerville... } \\
\text { Placitas ..... }\end{array}$ & $\begin{array}{l}\text { Colorado......... } \\
\text { New Mexico.... }\end{array}$ & $\begin{array}{r}120 \\
50\end{array}$ \\
\hline Packer Cre & Montana... & 70 & Planet....... & Arieona.......... & 130 \\
\hline Paiute & $\begin{array}{l}\text { California, } s \\
\text { Washington }\end{array}$ & 25 & $\begin{array}{l}\text { Platoro ........ } \\
\text { Platte Canyon }\end{array}$ & $\begin{array}{l}\text { Colorado.. } \\
\text { Wyoming. }\end{array}$ & 27 \\
\hline $\begin{array}{l}\text { Paimer sot } \\
\text { Palmetto.. }\end{array}$ & $\begin{array}{l}\text { Washington. } \\
\text { Arizona..... }\end{array}$ & 97 & Pleasant Vallcy ........ & Oregon.... & 52 \\
\hline Palm & Nevada. & 60 & Pleasant View........ & Montana. & 93 \\
\hline Palm & & 120 & Plomo....... & Colorado . . & $28 a$ \\
\hline $\begin{array}{l}\text { Palor } \\
\text { Panar }\end{array}$ & $\begin{array}{l}\text { New Mexic } \\
\text { Californis, }\end{array}$ & $\begin{array}{l}62 \\
33\end{array}$ & Plymouth.. & California, i & $\begin{array}{r}131 \\
9\end{array}$ \\
\hline $\operatorname{tanac}$ & ....do.............. & 120 & Pocahontas. & Oregon..... & 11 \\
\hline arad & Arizona. & 1 & Point of Rocks. & Montana... & $56 a$ \\
\hline trad & Utah... & 20 & Poison Spring. . & California, s & $\begin{array}{r}34 \\
148\end{array}$ \\
\hline $8+$ & $\begin{array}{l}\text { Nevada... } \\
\text { Californis. }\end{array}$ & $\begin{array}{l}87 \\
48\end{array}$ & $\begin{array}{l}\text { Poker Flat. } \\
\text { Polaris..... }\end{array}$ & $\begin{array}{l}\text { Camborna, } \\
\text { Arizona... }\end{array}$ & $\begin{array}{l}148 \\
128\end{array}$ \\
\hline & Colorado. & 63 & Polaris.... & Montana... & 7 \\
\hline & Montans. . & 15 & Pollasky .. & California, 8 & 4 \\
\hline Park Gity & Utah......... & 55 & Ponil.... & New Mexico & $\begin{array}{r}4 \\
55\end{array}$ \\
\hline Park Gity ... & $\begin{array}{l}\text { Washingt } \\
\text { Idaho.... }\end{array}$ & $\begin{array}{l}25 \\
98\end{array}$ & Poorman. & modo.... & 45 \\
\hline Park Valley. & Utah..... & 17 & Portersville & California, & 191 \\
\hline Parsons $\ldots$ & New Mex & 34 & $\begin{array}{l}\text { Portland... } \\
\text { Port Winc. }\end{array}$ & South Dako & 12 \\
\hline Patagoni & $\begin{array}{l}\text { Arizona... } \\
\text { California }\end{array}$ & $\begin{array}{l}98 \\
99\end{array}$ & Potholes... & Californis, 8 & $\begin{array}{r}149 \\
10\end{array}$ \\
\hline atterson. & Nevada. & 115 & Potlatch.: & Idaho....... & 81 \\
\hline Patterson & Idaho... & 85 & $\begin{array}{l}\text { Potter Rid } \\
\text { Poverty Hi }\end{array}$ & California, & 86 \\
\hline Payette Riv & ....do... & $\begin{array}{l}31 \\
23\end{array}$ & $\begin{array}{l}\text { Poverty Hil } \\
\text { Pratt Creek. }\end{array}$ & $\begin{array}{l}\text { Callornis, } \\
\text { Idaho..... }\end{array}$ & $\begin{array}{r}150 \\
99\end{array}$ \\
\hline $\begin{array}{l}\text { Payson.. } \\
\text { Pearce... }\end{array}$ & $\begin{array}{l}\text { Arızona. } \\
\text {.....do... }\end{array}$ & $\begin{array}{r}23 \\
7\end{array}$ & Preston Pea & California, i & 172 \\
\hline Pearl.... & Colorado. & 75 & Preuss...... & Utah ........ & 9 \\
\hline Pearl.... & Idaho... & $\mathbf{3 5}$ & Prickly Per & Montana. & 35 \\
\hline & Nevada. & 159 & Priest Lake & Idabo..... & 41 \\
\hline Peck... & Arizona.... & 115 & Profile... & Idaho..... & $\begin{array}{l}31 \\
73\end{array}$ \\
\hline Pecos & New Mexic & 51 & Promonto & Utah.... & 18 \\
\hline $\begin{array}{l}\text { Pend Oreille } \\
\text { Perfumo Car }\end{array}$ & $\begin{array}{l}\text { Idaho ..... } \\
\text { California, }\end{array}$ & $\begin{array}{r}39 \\
183\end{array}$ & Prospect. & Nevada. . & 72 \\
\hline Perigo...... & Colorado.... & 43 & Prospect Cr & Montana .... & 95 \\
\hline Perris... & California, & 116 & Providence Mou & Callorn18, s & $\begin{array}{l}163 \\
126\end{array}$ \\
\hline Pershall & $\begin{array}{l}\text { Washing } \\
\text { Colorado }\end{array}$ & $\mathbf{3}$ & Provo . . . . . . . . & Ütah...... & 76 \\
\hline Peshastin.... & Washington. & 6 & Pueblo.... & Oregon..... & 44 \\
\hline Phluadelphia & Nevado..... & 125 & Pyramid .. & New Mexico... & 25 \\
\hline Thilipsburg. & & 29 & & & \\
\hline Plcacho .... & Callfornia, & 8 & Q. & & \\
\hline $\begin{array}{l}\text { Picuris...... } \\
\text { Pieplant... }\end{array}$ & $\begin{array}{l}\text { New Mexu } \\
\text { Colorado. }\end{array}$ & $\begin{array}{l}82 \\
58\end{array}$ & & & \\
\hline Pierce...... & Idaho.... & 106 & Quartzburg. & Idaho.... & 32 \\
\hline Pike...... & California & 147 & Quartz Creek & Colorado. & $\begin{array}{l}41 \\
55\end{array}$ \\
\hline & & 59 & Quartz Mounts & California, $\mathrm{N}$ & 74 \\
\hline Pilot Hill..... & California, N & 53 & Quartz Mountej & Colorado.. & 42 \\
\hline $\begin{array}{l}\text { Pilot Mount } \\
\text { Pimg }\end{array}$ & $\begin{array}{l}\text { Nevada. } \\
\text { Arizona. }\end{array}$ & 66 & Quartzsite...... & Arizona .... & 131 \\
\hline $\begin{array}{l}\text { Pima ........ } \\
\text { Pinacate... }\end{array}$ & $\begin{array}{l}\text { Arizona ..... } \\
\text { Californis, } \mathrm{s} \text {. }\end{array}$ & $\begin{array}{r}77 \\
178\end{array}$ & Queen City. & California, $\mathbf{N}$ & 149 \\
\hline & $\begin{array}{l}\text { Callormis, s. } \\
\text { Colorado.... }\end{array}$ & & Queens.... & Colorado... & 20 \\
\hline $\begin{array}{l}\text { Pine } \\
\text { Pine Bar................. }\end{array}$ & Wyoming.. & 36 & Quijotos & Arizona.... & 78 \\
\hline Pine Creek. & Idaho..... & 40 & & & \\
\hline Pine Creek. & ...do... & 120 & $\mathbf{R}$. & & \\
\hline Pine Flat... & California, $\mathrm{N}$ & 181 & n. & & \\
\hline Pine Grove. & $\ldots \ldots$ do......... & 8 & Rabbit. & Montana. & 57 \\
\hline Pine Grove. & Idaho... & 57 & Racetraci & 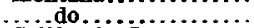 & 87 \\
\hline Pine Grove. . & Nevada.. & 61 & nacher. & California, s. & 62 \\
\hline Pine Grove.. & $U \operatorname{tah} . . . . . . .$. & 8 & Radersburg.. & Montans... & 12 \\
\hline Pine Mounta & California, S.. & 18 & Rag & South Dako & 9 \\
\hline Pine Nut. & Nevada....... & 117 & Ral & Nevada.... & 28 \\
\hline Pine Peak . . & California, S. & 182 & Railrood Creek & Washington. & 7 \\
\hline Pine Valley. & $z_{\text {d do }}$ & 176 & Railroad Flat. & California, $\mathbf{N}$ & 28 \\
\hline Pinos Altos. & New Mexico. & 24 & pad Pass. & Nevada..... & 22 \\
\hline Pinto......... & Nevada... & 72 & on Divide & California, $\mathbf{N}$ & $1 \overline{03}$ \\
\hline Pinto Creek. & Ariz & 26 & Ran & Nevada. & 118 \\
\hline Pinto Iron.. . & & 31 & Ra & Montana & 58 \\
\hline Pinyon Mountain. & Callfornia, s & 117 & lph... & Oregon....... & 21 \\
\hline Pioche.............. & Nevada..... & 116 & Randsburg. . & California, $\mathrm{S}$ & 63 \\
\hline Pioneer.... & Arizona.... & 89 & 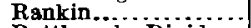 & Wyoming... & 14 \\
\hline Pioneer.. & Colorado. & 32 & Rattlesnake Divide... & California, $\mathbf{N}$. & 37 \\
\hline Pion & & $\begin{array}{l}86 \\
33\end{array}$ & Rawhide . . . . . . . . . & a.do. do. & 212 \\
\hline Pioneerville & Idaho.... & 33 & Rawhide . & Nevada. & 62 \\
\hline $\begin{array}{l}\text { PIru Creek } \\
\text { Pittsburg.. }\end{array}$ & $\begin{array}{l}\text { Californis, s } \\
\text { New Mexico }\end{array}$ & 194 & Rawhide Buttes....... & Wyoming.. & 31 \\
\hline $\begin{array}{l}\text { Pittsbu } \\
\text { Piute I }\end{array}$ & $\begin{array}{l}\text { New Mexico } \\
\text { California, }\end{array}$ & $\begin{array}{l}66 \\
61\end{array}$ & eek & Arizona... & 86 \\
\hline $\begin{array}{l}\text { Piute Moun } \\
\text { Placer Basir }\end{array}$ & $\begin{array}{l}\text { Californla, } \\
\text { Idaho.... }\end{array}$ & $\begin{array}{r}61 \\
133\end{array}$ & $\begin{array}{l}\text { Rebel Cree } \\
\text { Red Butte }\end{array}$ & vada.. & 88 \\
\hline $\begin{array}{l}\text { Placer Basin........... } \\
\text { Placer Center...... }\end{array}$ & Idaho....... & $\begin{array}{l}133 \\
121\end{array}$ & $\begin{array}{l}\text { Red Butte.................. } \\
\text { Red Canyon......... }\end{array}$ & 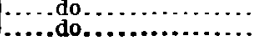 & $\begin{array}{r}88 a \\
25\end{array}$ \\
\hline
\end{tabular}


Mining districts in the western United States-Continued.

\begin{tabular}{|c|c|c|c|c|c|}
\hline Mining district. & State. & No. & Mining distriet. & & No. \\
\hline kea U1 & Colorado & 33 & Salina Creek.. & Utah. & \\
\hline Reddin & California, & 133 & Balitre........ & New Me & \\
\hline Red Gol & Colorado. & 109 & Salmon Ri & Idaho. & \\
\hline Red Gulc & & 40 & San Andreas.. & New Mez & \\
\hline Red Hill. & New Me & 26 & San Antonio. & Californi & 155 \\
\hline Red Lion & Montana & 30 & $\ldots .$. & Arizons. & 19 \\
\hline Red Mo & $\ldots . .$. & 85 & $\ldots \ldots$ & Arizons & 100 \\
\hline$\cdots$ & .......... & 119 & $\ldots$ & Colorad & \\
\hline & - & 83 & $\ldots .$. & Montana & \\
\hline$\cdots$ & A & 99 & San Domingo. & Arizona. & \\
\hline & Californi & 64 & Ban Emigdio. & Californi & \\
\hline Reese $\mathbf{F}$ & Nevada. & 100 & & & \\
\hline Regan. & ....do. & 172 & San Francisco. & Arizona & \\
\hline$\ldots$ & do. & 62 & & & \\
\hline$\ldots$ & Califf & 85 & Sanger & Or & \\
\hline$\ldots$ & . . . . . . & 17 & $\operatorname{San} \mathrm{Jac}$ & Californ & \\
\hline$\cdots$ & Idaho. & 70 & San Lo & New Me. & \\
\hline$\ldots$ & Nevada & 146 & San Luis & Californi & \\
\hline Rhodes Pl & Utah... & 83 & $\operatorname{San} \mathrm{Ma}$ & ..do do & 17 \\
\hline Rhyolite. & $\ldots \ldots$ & 129 & San Pablo & New Me & \\
\hline Rice & ........ & 177 & San P & . & \\
\hline & ....... & 27 & Sanpoi & Washin & \\
\hline $\mathbf{R}$ & A & 122 & San Rafael. & Utah. & \\
\hline co. & Colorado & 32 & San Si & New Me & \\
\hline$\ldots$ & Oregon. & 35 & $\ldots .$. & Nevada & \\
\hline$\ldots$ & $\ldots \ldots$ & 48 & $\ldots$ & Califor & \\
\hline$\ldots$ & ........ & 79 & $\ldots \ldots$ & ...do. & \\
\hline & .......... & 184 & $\ldots .$. & Arizan & \\
\hline$\ldots$ & (2) & 84 & San & Utah. & \\
\hline & $\mathrm{Ar}$ & 90 & Bant & New Me & \\
\hline & $\ldots . .$. & 15 & $\ldots .$. & zona & \\
\hline$\ldots$ & & 99 & $\cdots$ & Californ & 18 \\
\hline & $\ldots \ldots \ldots$ & 74 & & & \\
\hline & ....... & $16 \overline{7}$ & San V & iffornit & 17 \\
\hline & Col & 83 & $\operatorname{San} \mathbf{X}$ & Arizouda. & \\
\hline$\ldots$ & Soutl & 24 & Sarato & Califormia, S & \\
\hline$\ldots$ & $\ldots \ldots$ & 54 & $\ldots$. & gon..... & \\
\hline & Colorado & 56 & $\ldots$ & $\mathrm{Cg}$ & \\
\hline & $\ldots .$. do & 110 & 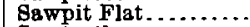 & C & \\
\hline & Ide & 35 & & & \\
\hline$\ldots$ & ......... & 25 & $\ldots . .$. & California, & \\
\hline Isl & Mo & 71 & $\ldots$ & ....do. & 15 \\
\hline & Utah & 10 & $\ldots$ & Nevada & 17 \\
\hline & Idaho & 58 & $\ldots$ & .....do. & \\
\hline$\ldots$ & Califor & 173 & & Califor & \\
\hline & S....... & 65 & $\ldots$. & Califo & 17 \\
\hline & $\ldots . . . .$. & $\mathbf{2 8}$ & & Idaho. & \\
\hline & $\mathrm{Ne}$ & 89 & ght. & Nerada & \\
\hline $\mathbf{R}$ & New Mex & 75 & & California, & \\
\hline $\mathbf{R}$ & Idsho & 19 & & Color & \\
\hline $\mathbf{R}$ & $\mathrm{C}$ & 29 & . & & \\
\hline Rol & $\mathbf{C}$ & 88 & . & Idaho & 13 \\
\hline$\ldots . .$. & Neva & 157 & $\ldots .$. & $\mathrm{Ne}$ & \\
\hline and Mou & $\ldots$. do. & 147 & Wount & Californis, & 15 \\
\hline wland. . & ... do.. & 36 & $\ldots \ldots$ & Taxes. & \\
\hline & Colorado & $\mathbf{5 3}$ & & New M & \\
\hline $\mathbf{R}$ & & 57 & & Califormis, 1 & 13 \\
\hline $\mathbf{R} 1$ & South & 1 & $\ldots \ldots$ & $\ldots \ldots \ldots$ & \\
\hline & W ashington........ & 23 & & Ho & \\
\hline Ruby & Nerada. & 72 & lead... & $\mathrm{Ne}$ & 16 \\
\hline Ruby Valley & & 40 & Mountsin ...... & Idaho. & \\
\hline Rush Valley & Utah.. & 67 & Ranch. & Caltornia, I & \\
\hline Russ.... & Califor & 35 & iden..... & Mont & \\
\hline Russell & Colorado.. & 2 & $\ldots \ldots$ & Color & \\
\hline sell. & .....do. & 42 & & & \\
\hline sty 1 & & 27 & pring & in, $\mathbf{N}$ & 3 \\
\hline Rye Patch & Neva & 91 & hishi Beach. & Washin & \\
\hline Rye Va & Oregon. & 12 & Shotgun Creek........ & Califormin, $\mathbf{N}, \ldots$. & 13 \\
\hline Ryne.. & Califormia & 33 & 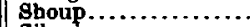 & Idaho............. & 9 \\
\hline & & & & Montan & \\
\hline $\mathbf{S}$ & & & & Nevada & 16 \\
\hline Secramento & Colored & 94 & Bierra Blanca...$\ldots$ & Texas................ & \\
\hline Baddle & Ari & 10 & errs Cit & & $\begin{array}{l}15 \\
15\end{array}$ \\
\hline & Nerad & 74 & lerra Diablo....... & & \\
\hline & & & lerrs Madre. . . . . . . . . & Utah..... & \\
\hline Eele & Washingt & 36 & & Arizons.... & \\
\hline & Idaho.... & 124 & & Califormis, 8.... & 15 \\
\hline & Californi & 177 & Silver.... & Arizons...$\ldots \ldots$ & 132 \\
\hline & & 149 & Sllver $\ldots . .$. & Bouth Dakota... . . . . . & 2 \\
\hline & $108110 . .$. & 125 & Bilver Bell... & 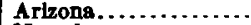 & 8 \\
\hline Regis. & Montana & 72 & Bilverbow...... & Nerads.... & 14 \\
\hline & & & & & \\
\hline
\end{tabular}


Mining districts in the western United States-Continued.

\begin{tabular}{|c|c|c|c|c|c|}
\hline Mining district. & State. & No. & Mining district. & Etato. & No. \\
\hline silve & Nevada. & 165 & Spring $\mathbf{H i}$ & montans & 43 \\
\hline .. & Idaho. . & 107 & Spring $\mathrm{M}$ & Idaho... & 100 \\
\hline ilve & Nevada. & 120 & pruce Gu & South $\mathbf{D}$ & 14 \\
\hline & ....... & 16 & Spruce $\mathbf{M o}$ & Nevada. & 41 \\
\hline & lorado & 29 & Squaw & Arizona & 116 \\
\hline & ashing & 41 & uaw & Californ & 129 \\
\hline ilve. & yomin & 32 & 8 & Washin & 28 \\
\hline Silve & Arizons & $\mathbf{8 2}$ & $\ldots$ & Californ & 127 \\
\hline Silve & New $\mathrm{Me}$ & 42 & ley. & Arizona. & 34 \\
\hline Silver & $\ldots \ldots$. & 43 & anley 1 & Idaho. . & 50 \\
\hline liver & ....... & 68 & $\mathbf{r} \ldots \ldots$ & Nevada & 91 \\
\hline Sikve & rizona & 91 & ar... & Utah. & 12 \\
\hline Silye & $\operatorname{tah} .$. & 78 & arvou & Oregon. & 36 \\
\hline Silve & iforni & $\mathbf{3}$ & $8 \mathrm{Li}$ & vada. & 107 \\
\hline Silve! & lifornis & 158 & teline & Utah... & 32 \\
\hline ilver Mo & $\ldots \ldots$ & 78 & $\cdots$ & $\ldots . .$. & 120 \\
\hline Ive' & $\ldots \ldots \ldots$ & 64 & .. & $\ldots .$. & 113 \\
\hline ilver & & 20 & pring & $\mathbf{N}$ & 161 \\
\hline Iver & & $\overline{54}$ & Rock & $\mathrm{rad}$ & 76 \\
\hline Ulver & Californ & 125 & - & Californ & 160 \\
\hline ive & Utah... & 86 & - & New Me & 28 \\
\hline .. & $\ldots \ldots$ & 60 & eins I & $\ldots$ do. & 22 \\
\hline ( & $\ldots$ & 65 & heki & Washin & 1 \\
\hline & & 42 & la.. & Californ & 136 \\
\hline Simp & I & 75 & mple. & Mon & 46 \\
\hline ies & & 28 & nt.... & Californ & 212 \\
\hline S & $\ldots$ & 38 & $\cdots$ & Nevada. & 174 \\
\hline & $\ldots$ & 101 & $\ldots$ & $\ldots$. & 15 \\
\hline & ... & 111 & $\ldots$ & $\ldots \ldots$ & 42 \\
\hline & & 60 & & h... & 67 \\
\hline & Californi & 205 & ekto & zona & 61 \\
\hline & Idaho... & 122 & $\ldots$ & Idaho. & 42 \\
\hline & Montan & 94 & Lountain. - & Nevada & 149 \\
\hline+- & Washin & 56 & & & 170 \\
\hline ate $]$ & Californ & 68 & Julch. & South I & 10 \\
\hline to & ....do. & 122 & .. & California, 8 & 161 \\
\hline Slater & Colorado & 106 & & Colorado... & 6 \\
\hline & do... & 112 & Bank. . . & Celifornia, I & 65 \\
\hline & California & 221 & Creek... & .....do. & 32 \\
\hline & 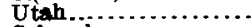 & 84 & _..... & $\ldots \ldots$ & 176 \\
\hline ng & Colorad & $12 \hat{0}$ & 8 & Califor & 69 \\
\hline & & & & Idaho.. & 126 \\
\hline & & & $\mathbf{S}$ & Montan & 61 \\
\hline Snake & Idaho. & 21 & & Nevada.... & 37 \\
\hline & & 43 & $\ldots$ & Idaho...... & 33 \\
\hline & & (111 & & Mo & 100 \\
\hline lacers. . & $\mathrm{V}$ & 36 & & $\ldots .$. & 103 \\
\hline & Colc & 86 & & $\mathrm{O}$ & \\
\hline & Monta & 51 & light & Wyoming. & 10 \\
\hline Sn & Californis & 194 & & California, 1 & 105 \\
\hline $\mathbf{S}$ & Colorado & 122 & & Colorado... & 78 \\
\hline & New Me & 79 & $\cdots$ & & 2 \\
\hline & $\mathbf{A}$ & 66 & & $\mathbf{M g}$ & 67 \\
\hline & Ideho. & 22 & & Nevada... & 150 \\
\hline & Californ & 77 & & Oregon.... & 42 \\
\hline & Califormig & 175 & $k \ldots \ldots . . .$. & California, & 10 \\
\hline ins... & & $\mathbf{7 5}$ & $\mathbf{r} \ldots \ldots \ldots$ & Colorado... & 128 \\
\hline & & 213 & & & 133 \\
\hline $\mathrm{Sc}$ & Color & 17 & & California, S & 39 \\
\hline Bo & & 31 & auk.. & Washington & 21 \\
\hline ndike... & & 67 & edes P & California, $N$ & 206 \\
\hline reek.... & Califormi: & 127 & camore & California, S......... & \\
\hline South Fork. & & $\mathbf{g}$ & Sy Ivanite... & Montana.... & 52 \\
\hline South Fork & & 51 & Sylpanite................... & New Mexico. & 21 \\
\hline South Moun & Idaho. & 112 & & & \\
\hline uth Pas & Wyomin & 25 & Table Mountain. & California, B...... & 106 \\
\hline ish $\mathrm{Di}$ & Califormia & 55 & Table Rock..... & Celifornia, $\mathbf{N} \ldots \ldots$ & 148 \\
\hline - & Oregon ... & 74 & Talapoos & Nevada............... & 121 \\
\hline $\mathbf{R}$ & & 115 & & & \\
\hline & lifornis & 159 & & & \\
\hline & Ore & 14 & Taylors & California, N... & 116 \\
\hline $\mathbf{S}$ & Colc & 30 & Tecolot. & New Mexico...... & 5 \\
\hline & Ith Dal & 13 & 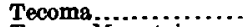 & Nevada... & \\
\hline & Nevada. & 102 & Tecopa Mountain..... & California & 3 \\
\hline & Californis & 89 & Telegraph............... & New Mex & \\
\hline & & 23 & Tellúride............. & & \\
\hline & South Dak & $\mathbf{6}$ & Temescal Mountains.. & California, S.... & $117 \mathrm{a}$ \\
\hline IIIJE Giny & Nevada... & 87 & Temperance Flat.... & 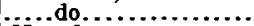 & \\
\hline & Colorado.. & 113 & 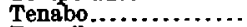 & Nevada.. & 9 \\
\hline & Utah....... & 37 & Tenmile.............. & Colorado.. & 129 \\
\hline & & 73 & Tennessee Pass........ & $\ldots \ldots \ldots \ldots$ & 69 \\
\hline & & & & & \\
\hline
\end{tabular}


Mining districts in the western United States-Continued.

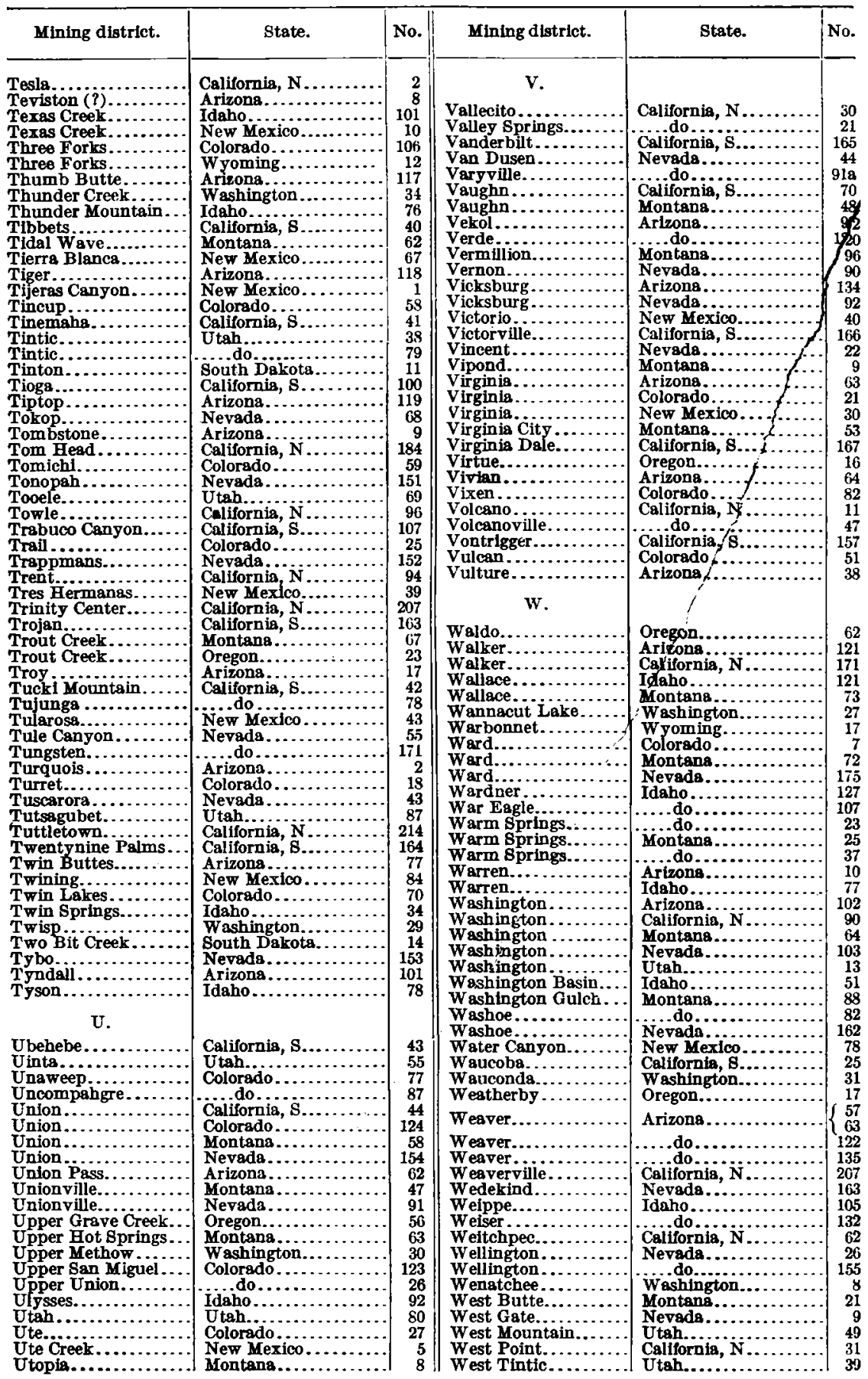


Mining districts in the western United States-Continued.

\begin{tabular}{|c|c|c|c|c|c|}
\hline Mining distriet. & State. & No. & Mining district. & Stste. & No. \\
\hline West view. & Idaho... & $\mathbf{3 5}$ & Winchester (?) ...... & Arizona. & 12 \\
\hline Westville.... & California, & 104 & 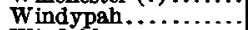 & Nevada. & 69 \\
\hline West Walker & California, & 101 & 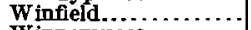 & Colorado & $1 \mathbf{a}$ \\
\hline Wheatland. & California, & 222 & Winnemuccs........... & Nerada. & 923 \\
\hline Whetstone... & Arizona... & 11 & Winston................. & Montana. . & 11 \\
\hline Whipple Moun & California, $\mathrm{S}$ & 148 & W inthrop. & California, & 124 \\
\hline Whisky Diggin & California, $\mathrm{N}$ & 145 & Wisconsin. - & Montana.. & 59 \\
\hline Whitcomb... & Montena... & 20 & Wolf Creek.. & Oregon... & 63 \\
\hline White Bird. & Idaho..... & 62 & Wolverine............ & Colorado & 47 \\
\hline White Canyor & Utah.... & 24 & 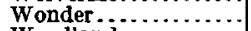 & Nevada. & 12 \\
\hline Clou & rada. & 10 & Woodland.. & Utah.... & 83 \\
\hline Earth.......... & orado. & 60 & Woodman... & Montana. & 74 \\
\hline White Gold Basin... & California, & 9 & Wood River & Wyoming. & 11 \\
\hline Whitehall. . & Montana. & 39 & Woody .... & California, $\mathrm{S}$. & 71 \\
\hline White & $\ldots$.... do... & 56 & Wrightson. & Arizona..... & 103 \\
\hline Hills. & Arizona. & 65 & & & \\
\hline WI & rado. & 41 & Y. & & \\
\hline White Horse & Nevada.: & 164 & Yankee... & Coloradc & 23 \\
\hline Whitehouse - & California, & 137 & Yankee For & Idaho... & 52 \\
\hline teogks... & New Mexí & $\begin{array}{r}35 \\
109\end{array}$ & Yankee Bill.. & Californis & 19 \\
\hline Peak. & ifornis & 102 & Yellow Banks. & Washington. & 11 \\
\hline $\begin{array}{l}\text { White Picach } \\
\text { Whitepine... }\end{array}$ & $\stackrel{A}{C}$ & $\begin{array}{r}123 \\
59\end{array}$ & Yellowjacke & Idaho........ & 102 \\
\hline White Pine.. & Nevada. & 176 & Yellow Pine.. & Nevada. & 23 \\
\hline White Plains. & & 11 & wston & $\ldots \ldots \ldots$ & 13 \\
\hline River. & California, & 192 & Yering & $\begin{array}{l}\text { Nevada. } \\
\text { Montana }\end{array}$ & 122 \\
\hline Whitewood... & South Dako & 15 & York... & ....do.. & 41 \\
\hline $\begin{array}{l}\text { Whitlock..... } \\
\text { Wickenburg. }\end{array}$ & Californis, $\mathbf{N}$ & 73 & You Bet. & California, $\mathbf{N}$. & 91 \\
\hline $\begin{array}{l}\text { Wickenburg. } \\
\text { Wildrose. . . }\end{array}$ & California, S... & 49 & Yreka... & . do....... & 167 \\
\hline Willow..... & . do ...... & 46 & Yuhe Besin & Idaho... & 127 \\
\hline Willow Creek. & Idaho ... & 35 & Y uba Basin.. & Caluornus, $N \ldots \ldots \ldots$ & \\
\hline ow Creek.. & Nevada. . . & 88 & $\mathbf{Z}$. & & \\
\hline Willow Creek... & Wyoming. & 26 & & Montans & \\
\hline Willow Springs. & Utah.... & 70 & Zortman.... & Montans....... & 20 \\
\hline $\begin{array}{l}\text { Wilson Mesa.... } \\
\text { Wilsons......... }\end{array}$ & & 28 & Zozell......... & . do $\ldots \ldots \ldots$ & 89 \\
\hline Wilsons....... & & 152 & Zuni Mountains...... & New Mexico............ & 85 \\
\hline
\end{tabular}


